\title{
FRACTIONAL CALCULUS OF WEYL ALGEBRA AND FUCHSIAN DIFFERENTIAL EQUATIONS
}

\author{
TOSHIO OSHIMA
}

\begin{abstract}
We give a unified interpretation of confluences, contiguity relations and Katz's middle convolutions for linear ordinary differential equations with polynomial coefficients and their generalization to partial differential equations. The integral representations and series expansions of their solutions are also within our interpretation. As an application to Fuchsian differential equations on the Riemann sphere, we construct a universal model of Fuchsian differential equations with a given spectral type, in particular, we construct single ordinary differential equations without apparent singularities corresponding to the rigid local systems, whose existence was an open problem presented by Katz. Furthermore we obtain an explicit solution to the connection problem for the rigid Fuchsian differential equations and the necessary and sufficient condition for their irreducibility. We give many examples calculated by our fractional calculus.
\end{abstract}

\section{Contents}

1. Introduction

2. Fractional operations

2.1. Weyl algebra 12

2.2. Laplace and gauge transformations and reduced representatives 13

2.3. Examples of ordinary differential operators

2.4. Ordinary differential equations 20

2.5. Okubo normal form and Schlesinger canonical form 22

3. Confluences 24

3.1. Regular singularities 24

3.2. A confluence 28

3.3. Versal additions 29

3.4. Versal operators 30

4. Series expansion 33

5. Contiguity relation 35

6. Fuchsian differential equation and generalized Riemann scheme 37

6.1. Generalized characteristic exponents 37

6.2. Tuples of partitions 41

6.3. Conjugacy classes of matrices 43

6.4. Realizable tuples of partitions 44

7. Reduction of Fuchsian differential equations 48

8. Deligne-Simpson problem 58

Key words and phrases. Fuchsian systems, middle convolution, hypergeometric functions, special functions, Weyl algebra.

2000 Mathematics Subject Classification. Primary 34M35; Secondary 34M40, 34M15

Supported by Grant-in-Aid for Scientific Researches (A), No. 20244008, Japan Society of Promotion of Science

Toshio Oshima, Graduate School of Mathematical Sciences, University of Tokyo, 7-3-1, Komaba, Meguro-ku, Tokyo 153-8914, Japan

e-mail address : oshima@ms.u-tokyo.ac.jp. 
8.1. Fundamental lemmas 58

8.2. Existence theorem 60

8.3. Divisible spectral types 65

8.4. Universal model 67

8.5. Simply reducible spectral type 69

9. A Kac-Moody root system

9.1. Correspondence with a Kac-Moody root system

9.2. Fundamental tuples 81

10. Expression of local solutions 84

11. Monodromy 87

11.1. Middle convolution of monodromies 88

11.2. Scott's lemma and Katz's rigidity 93

12. Reducibility 96

12.1. Direct decompositions 96

12.2. Reduction of reducibility 101

13. Shift operators 109

13.1. Construction of shift operators and recurrence relations 109

13.2. Relation to reducibility

13.3. Polynomial solutions 118

14. Connection problem

14.1. Connection formula 118

14.2. An estimate for large exponents

14.3. Zeros and poles of connection coefficients

15. Examples 136

15.1. Basic tuples 137

15.2. Rigid tuples 140

15.3. Jordan-Pochhammer family 143

15.4. Hypergeometric family 147

15.5. Even/Odd family

15.6. Trigonometric identities

15.7. Rigid examples of order at most $4 \quad 159$

15.8. Other rigid examples with a small order

15.9. Submaximal series and minimal series 169

15.10. Appell's hypergeometric functions 181

15.11. Okubo and Risa/Asir 187

16. Further problems 188

16.1. Multiplicities of spectral parameters 188

16.2. Schlesinger canonical form 188

16.3. Apparent singularities 188

16.4. Irregular singularities $\quad 189$

16.5. Special parameters

16.6. Shift operators

16.7. Several variables

16.8. Other problems

17. Appendix 191

References 


\section{INTRODUCTION}

Gauss hypergeometric functions and the functions in their family, such as Bessel functions, Whittaker functions, Hermite functions, Legendre polynomials and Jacobi polynomials etc. are the most fundamental and important special functions (cf. EMO, Wa, WW]). Many formulas related to the family have been studied and clarified together with the theory of ordinary differential equations, the theory of holomorphic functions and relations with other fields. They have been extensively used in various fields of mathematics, mathematical physics and engineering.

Euler studied the hypergeometric equation

$$
x(1-x) y^{\prime \prime}+(c-(a+b+1) x) y^{\prime}-a b y=0
$$

with constant complex numbers $a, b$ and $c$ and he got the solution

$$
F(a, b, c ; x):=\sum_{k=0}^{\infty} \frac{a(a+1) \cdots(a+k-1) \cdot b(b+1) \cdots(b+k-1)}{c(c+1) \cdots(c+k-1) \cdot k !} x^{k} .
$$

The series $F(a, b, c ; x)$ is now called Gauss hypergeometric series or function and Gauss proved the Gauss summation formula

$$
F(a, b, c ; 1)=\frac{\Gamma(c) \Gamma(c-a-b)}{\Gamma(c-a) \Gamma(c-b)}
$$

when the real part of $c$ is sufficiently large. Then in the study of this function an important concept was introduced by Riemann. That is the Riemann scheme

$$
\left\{\begin{array}{ccc}
x=0 & 1 & \infty \\
0 & 0 & a \\
1-c & c-a-b & b
\end{array}\right\}
$$

which describes the property of singularities of the function and Riemann proved that this property characterizes the Gauss hypergeometric function.

The equation (1.1) is a second order Fuchsian differential equation on the Riemann sphere with the three singular points $\{0,1, \infty\}$. One of the main purpose of this paper is to generalize these results to the general Fuchsian differential equation on the Riemann sphere. In fact, our study will be applied to the following three kinds of generalizations.

One of the generalizations of the Gauss hypergeometric family is the hypergeometric family containing the generalized hypergeometric function ${ }_{n} F_{n-1}(\alpha, \beta ; x)$ or the solutions of Jordan-Pochhammer equations. Some of their global structures are concretely described as in the case of the Gauss hypergeometric family.

The second generalization is a class of Fuchsian differential equations such as the Heun equation which is of order 2 and has 4 singular points in the Riemann sphere. In this case, there appear accessory parameters. The global structure of the generic solution is quite transcendental and the Painleve equation which describes the deformations preserving the monodromies of solutions of the equations with an apparent singular point is interesting and has been quite deeply studied and now it becomes an important field of mathematics.

The third generalization is a class of hypergeometric functions of several variables, such as Appell's hypergeometric functions (cf. AK]), Gelfand's generalized hypergeometric functions (cf. [G] ) and Heckman-Opdam's hypergeometric functions (cf. $\mathrm{HO}$ ). The author and Shimeno $\mathrm{OS}$ studied the ordinary differential equations satisfied by the restrictions of Heckman-Opdam's hypergeometric function on singular lines through the origin and we found that some of the equations belong to the even family classified by Simpson [Si], which is now called a class of rigid differential equations and belongs to the first generalization in the above. 
The author's original motivation related to the study in this note is a generalization of Gauss summation formula, namely, to calculate a connection coefficient for a solution of this even family, which is solved in $\$ 14$ as a direct consequence of the general formula (1.23) of certain connection coefficients described in Theorem 14.6 . This paper is the author's first step to a unifying approach for these generalizations and the recent development in general Fuchsian differential equations described below with the aim of getting concrete and computable results. In this paper, we will avoid intrinsic arguments and results if possible and hence the most results can be implemented in computer programs. Moreover the arguments in this paper will be understood without referring to other papers.

Rigid differential equations are the differential equations which are uniquely determined by the data describing the local structure of their solutions at the singular points. From the point of view of the monodromy of the solutions, the rigid systems are the local systems which are uniquely determined by local monodromies around the singular points and Katz [Kz] studied rigid local systems by defining and using the operations called middle convolutions and additions, which enables us to construct and analyze all the rigid local systems. In fact, he proved that any irreducible rigid local system is transformed into a trivial equation $\frac{d u}{d z}=0$ by successive application of the operations. In another word, any irreducible rigid local system is obtained by successive applications of the operations to the trivial equation because the operations are invertible.

The arguments there are rather intrinsic by using perverse sheaves. DettweilerReiter [DR, DR2] interprets Katz's operations on monodromy generators and those on the systems of Fuchsian differential equations of Schlesinger canonical form

$$
\frac{d u}{d x}=\sum_{j=1}^{p} \frac{A_{j}}{x-c_{j}} u
$$

with constant square matrices $A_{1}, \ldots, A_{p}$.

Here $A_{j}$ are called the residue matrices of the system at the singular points $x=c_{j}$, which describe the local structure of the solutions. For example, the eigenvalues of the monodromy generator at $x=c_{j}$ are $e^{2 \pi \sqrt{-1} \lambda_{1}}, \ldots, e^{2 \pi \sqrt{-1} \lambda_{n}}$, where $\lambda_{1}, \ldots, \lambda_{n}$ are eigenvalues of $A_{j}$. The residue matrix of the system at $x=\infty$ equals $A_{0}:=-\left(A_{1}+\cdots+A_{p}\right)$. These operations are useful also for non-rigid Fuchsian systems.

Related to the Riemann-Hilbert problem, there is a natural problem to determine the condition on matrices $B_{0}, B_{1}, \ldots, B_{p}$ of Jordan canonical form such that there exists an irreducible system of Schlesinger canonical form with the residue matrices $A_{j}$ conjugate to $B_{j}$ for $j=0, \ldots, p$, respectively. An obvious necessary condition is the equality $\sum_{j=0}^{p}$ Trace $B_{j}=0$. A similar problem for monodromy generators, namely its multiplicative version, is equally formulated. The latter is called a mutiplicative version and the former is called an additive version. Kostov [ $\mathrm{Ka}$, Ko2 called them Deligne-Simpson problems and gave an answer under a certain genericity condition. We note that the addition is a kind of a gauge transformation

$$
u(x) \mapsto(x-c)^{\lambda} u(x)
$$

and the middle convolution is essentially an Euler transformation or a transformation by an Riemann-Liouville integral

$$
u(x) \mapsto \frac{1}{\Gamma(\mu)} \int_{c}^{x} u(t)(x-t)^{\mu-1} d t
$$

or a fractional derivation. 
Crawley-Boevey $\mathrm{CB}$ found a relation between the Deligne-Simpson problem and representations of certain quivers and gave an explicit answer for the additive Deligne-Simpson problem in terms of a Kac-Moody root system.

Yokoyama Yo2 defined operations called extensions and restrictions on the systems of Fuchsian ordinary differential equations of Okubo normal form

$$
(x-T) \frac{d u}{d x}=A u .
$$

Here $A$ and $T$ are constant square matrices such that $T$ are diagonalizable. He proved that the irreducible rigid system of Okubo normal form is transformed into a trivial equation $\frac{d u}{d z}=0$ by successive applications of his operations if the characteristic exponents are generic.

The relation between Katz's operations and Yokoyama's operations is clarified by O7] and it is proved there that their algorithms of reductions of Fuchsian systems are equivalent and so are those of the constructions of the systems.

These operations are quite powerful and in fact if we fix the number of accessory parameters of the systems, they are connected into a finite number of fundamental systems (cf. O6, Proposition 8.1 and Theorem 10.2] and Proposition 9.13), which is a generalization of the fact that the irreducible rigid Fuchsian system is connected to the trivial equation.

Hence it is quite useful to understand how does the property of the solutions transform under these operations. In this point of view, the system of the equations, the integral representation and the monodromy of the solutions are studied by [DR, DR2, HY] in the case of the Schlesinger canonical form. Moreover the equation describing the deformation preserving the monodromy of the solutions doesn't change, which is proved by $[\mathrm{HF}$. In the case of the Okubo normal form the corresponding transformation of the systems, that of the integral representations of the solutions and that of their connection coefficients are studied by [O2], [Ha] and [Yo3], respectively. These operation are explicit and hence it will be expected to have explicit results in general Fuchsian systems.

To avoid the specific forms of the differential equations, such as Schlesinger canonical form or Okubo normal form and moreover to make explicit calculations easier under the transformations, we introduce certain operations on differential operators with polynomial coefficients in $\$ 2$. The operations in $\$ 2$ enables us to equally handle equations with irregular singularities or systems of equations with several variables.

The ring of differential operators with polynomial coefficients is called a Weyl algebra and denoted by $W[x]$ in this paper. The endomorphisms of $W[x]$ do not give a wide class of operations and Dixmier Dix conjectured that they are the automorphisms of $W[x]$. But when we localize coordinate $x$, namely in the ring $W(x)$ of differential operators with coefficients in rational functions, we have a wider class of operations.

For example, the transformation of the pair $\left(x, \frac{d}{d x}\right)$ into $\left(x, \frac{d}{d x}-h(x)\right)$ with any rational function $h(x)$ induces an automorphism of $W(x)$. This operation is called a gauge transformation. The addition in DR, DR2 corresponds to this operation with $h(x)=\frac{\lambda}{x-c}$ and $\lambda, c \in \mathbb{C}$, which is denoted by $\operatorname{Ad}\left((x-c)^{\lambda}\right)$.

The transformation of the pair $\left(x, \frac{d}{d x}\right)$ into $\left(-\frac{d}{d x}, x\right)$ defines an important automorphism $\mathrm{L}$ of $W[x]$, which is called a Laplace transformation. In some cases the Fourier transformation is introduced and it is a similar transformation. Hence we may also localize $\frac{d}{d x}$ and introduce the operators such as $\lambda\left(\frac{d}{d x}-c\right)^{-1}$ and then the transformation of the pair $\left(x, \frac{d}{d x}\right)$ into $\left(x-\lambda\left(\frac{d}{d x}\right)^{-1}, \frac{d}{d x}\right)$ defines an endomorphism in this localized ring, which corresponds to the middle convolution or an Euler transformation or a fractional derivation and is denoted by $\operatorname{Ad}\left(\partial^{-\lambda}\right)$ 
or $m c_{\lambda}$. But the simultaneous localizations of $x$ and $\frac{d}{d x}$ produce the operator $\left(\frac{d}{d x}\right)^{-1} \circ x^{-1}=\sum_{k=0}^{\infty} k ! x^{-k-1}\left(\frac{d}{d x}\right)^{-k-1}$ which is not algebraic in our sense and hence we will not introduce such a microdifferential operator in this paper and we will not allow the simultaneous localizations of the operators.

Since our equation $P u=0$ studied in this paper is defined on the Riemann sphere, we may replace the operator $P$ in $W(x)$ by a suitable representative $\tilde{P} \in$ $\mathbb{C}(x) P \cap W[x]$ with the minimal degree with respect to $x$ and we put $\mathrm{R} P=\tilde{P}$. Combining these operations including this replacement gives a wider class of operations on the Weyl algebra $W[x]$. In particular, the operator corresponding to the addition is $\operatorname{RAd}\left((x-c)^{\lambda}\right)$ and that corresponding to the middle convolution is $\operatorname{RAd}\left(\partial^{-\mu}\right)$ in our notation. The operations introduced in $\oint 2$ correspond to certain transformations of solutions of the differential equations defined by elements of Weyl algebra and we call the calculation using these operations fractional calculus of Weyl algebra.

To understand our operations, we show that, in Example 2.8, our operations enables us to construct Gauss hypergeometric equations, the equations satisfied by airy functions and Jordan-Pochhammer equations and to give integral representations of their solutions.

In this paper we mainly study ordinary differential equations and since any ordinary differential equation is cyclic, namely, it is isomorphic to a single differential operator $P u=0$ (cf. $\$ 2.4$ ), we study a single ordinary differential equation $P u=0$ with $P \in W[x]$. In many cases, we are interested in a specific function $u(x)$ which is characterized by differential equations and if $u(x)$ is a function with the single variable $x$, the differential operators $P \in W(x)$ satisfying $P u(x)=0$ are generated by a single operator and hence it is naturally a single differential equation. A relation between our fractional calculus and Katz's middle convolution is briefly explained in $\$ 2.5$.

In $\$ 3.1$ we review fundamental results on Fuchsian ordinary differential equations. Our Weyl algebra $W[x]$ is allowed to have some parameters $\xi_{1}, \ldots$ and in this case the algebra is denoted by $W[x ; \xi]$. The position of singular points of the equations and the characteristic exponents there are usually the parameters and the analytic continuation of the parameters naturally leads the confluence of additions (cf. $\S 3.3$ ).

Combining this with our construction of equations leads the confluence of the equations. In the case of Jordan-Pochhammer equations, we have versal JordanPochhammer equations. In the case of Gauss hypergeometric equation, we have a unified expression of Gauss hypergeometric equation, Kummer equation and Hermite-Weber equation and get a unified integral representation of their solutions (cf. Example 3.5). After this section in this paper, we mainly study single Fuchsian differential equations on the Riemann sphere. Equations with irregular singularities will be discussed elsewhere.

In $\S$ and $\S$ 国 we examine the transformation of series expansions and contiguity relations of the solutions of Fuchsian differential equations under our operations.

The Fuchsian equation satisfied by the generalized hypergeometric series

$$
\begin{gathered}
{ }_{n} F_{n-1}\left(\alpha_{1}, \ldots, \alpha_{n}, \beta_{1}, \ldots, \beta_{n-1} ; x\right)=\sum_{k=0}^{\infty} \frac{\left(\alpha_{1}\right)_{k} \ldots\left(\alpha_{n}\right)_{k}}{\left(\beta_{1}\right)_{k} \ldots\left(\beta_{n-1}\right)_{n-1} k !} x^{k} \\
\text { with }(\gamma)_{k}:=\gamma(\gamma+1) \cdots(\gamma+k-1)
\end{gathered}
$$

is characterized by the fact that it has $(n-1)$-dimensional local holomorphic solutions at $x=1$, which is more precisely as follows. The set of characteristic exponents of the equation at $x=1$ equals $\left\{0,1, \ldots, n-1,-\beta_{n}\right\}$ with $\alpha_{1}+\cdots+\alpha_{n}=\beta_{1}+\cdots+\beta_{n}$ and those at 0 and $\infty$ are $\left\{1-\beta_{1}, \ldots, 1-\beta_{n-1}, 0\right\}$ and $\left\{\alpha_{1}, \ldots, \alpha_{n}\right\}$, respectively. Then if $\alpha_{i}$ and $\beta_{j}$ are generic, the Fuchsian differential equation $P u=0$ is uniquely 
characterized by the fact that it has the above set of characteristic exponents at each singular point 0 or 1 or $\infty$ and the monodromy generator around the point is semisimple, namely, the local solution around the singular point has no logarithmic term. We express this condition by the (generalized) Riemann scheme

$$
\left\{\begin{array}{ccc}
x=0 & 1 & \infty \\
1-\beta_{1} & {[0]_{(n-1)}} & \alpha_{1} \\
\vdots & & \vdots \\
1-\beta_{n-1} & & \alpha_{n-1} \\
0 & -\beta_{n} & \alpha_{n}
\end{array}\right\}, \quad[\lambda]_{(k)}:=\left(\begin{array}{c}
\lambda \\
\lambda+1 \\
\vdots \\
\lambda+k-1
\end{array}\right) .
$$

In particular, when $n=3$, the (generalized) Riemann scheme is

$$
\left\{\begin{array}{cccc}
x=0 & 1 & \infty & \\
1-\beta_{1} & (0 \\
1-\beta_{2} & \alpha_{1} & \\
0 & -\beta_{3} & \alpha_{2} & ; x
\end{array}\right\} .
$$

The corresponding usual Riemann scheme is obtained from the generalized Riemann scheme by eliminating (and ). Here $[0]_{(n-1)}$ in the above Riemann scheme means the characteristic exponents $0,1, \ldots, n-2$ but it also indicates that the corresponding monodromy generator is semisimple in spite of integer differences of the characteristic exponents. Thus the set of (generalized) characteristic exponents $\left\{[0]_{(n-1)},-\beta_{n}\right\}$ at $x=1$ is defined. Here we remark that the coefficients of the Fuchsian differential operator $P$ which is uniquely determined by the generalized Riemann scheme for generic $\alpha_{i}$ and $\beta_{j}$ are polynomial functions of $\alpha_{i}$ and $\beta_{j}$ and hence $P$ is naturally defined for any $\alpha_{i}$ and $\beta_{j}$ as is given by (15.21). Similarly the Riemann scheme of Jordan-Pochhammer equation of order $p$ is

$$
\begin{gathered}
\left\{\begin{array}{ccccc}
x=c_{0} & c_{1} & \cdots & c_{p-1} & \infty \\
{[0]_{(p-1)}} & {[0]_{(p-1)}} & \cdots & {[0]_{(p-1)}} & {\left[\lambda_{p}^{\prime}\right]_{(p-1)}} \\
\lambda_{0} & \lambda_{1} & \cdots & \lambda_{p-1} & \lambda_{p}
\end{array}\right\}, \\
\lambda_{0}+\cdots+\lambda_{p-1}+\lambda_{p}+(p-1) \lambda_{p}^{\prime}=p-1 .
\end{gathered}
$$

The last equality in the above is called Fuchs relation.

In $\S$ f we define the set of generalized characteristic exponents at a regular singular point of a differential equation $P u=0$. In fact, when the order of $P$ is $n$, it is the set $\left\{\left[\lambda_{1}\right]_{\left(m_{1}\right)}, \ldots,\left[\lambda_{k}\right]_{\left(m_{k}\right)}\right\}$ with a partition $n=m_{1}+\cdots+m_{k}$ and complex numbers $\lambda_{1}, \ldots, \lambda_{k}$. It means that the set of characteristic exponents at the point equals $\left\{\lambda_{j}+\nu ; \nu=0, \ldots, m_{j}-1\right.$ and $\left.j=1, \ldots, k\right\}$ and the corresponding monodromy generator is semisimple if $\lambda_{i}-\lambda_{j} \notin \mathbb{Z}$ for $1 \leq i<j \leq k$. In 86.1 we define the set of generalized characteristic exponents without the assumption $\lambda_{i}-\lambda_{j} \notin \mathbb{Z}$ for $1 \leq i<j \leq k$. Here we only remark that when $\lambda_{i}=\lambda_{1}$ for $i=1, \ldots, k$, it is also characterized by the fact that the Jordan normal form of the monodromy generator is defined by the dual partition of $n=m_{1}+\cdots+m_{k}$ together with the usual characteristic exponents.

Thus for a single Fuchsian differential equation $P u=0$ on the Riemann sphere which has $p+1$ regular singular points $c_{0}, \ldots, c_{p}$, we define a (generalized) Riemann scheme

$$
\left\{\begin{array}{ccccc}
x=c_{0} & c_{1} & \cdots & c_{p} & \\
{\left[\lambda_{0,1}\right]_{\left(m_{0,1}\right)}} & {\left[\lambda_{1,1}\right]_{\left(m_{1,1}\right)}} & \cdots & {\left[\lambda_{p, 1}\right]_{\left(m_{p, 1}\right)}} & \\
\vdots & \vdots & \vdots & \vdots & ; x \\
{\left[\lambda_{0, n_{0}}\right]_{\left(m_{0, n_{0}}\right)}} & {\left[\lambda_{1, n_{1}}\right]_{\left(m_{1, n_{1}}\right)}} & \cdots & {\left[\lambda_{p, n_{p}}\right]_{\left(m_{p, n_{p}}\right)}} &
\end{array}\right\}
$$


Here $n=m_{j, 1}+\cdots+m_{j, n_{j}}$ for $j=0, \ldots, p$ and $n$ is the order of $P$ and $\lambda_{j, \nu} \in \mathbb{C}$. The $(p+1)$-tuple of partitions of $n$, which is denoted by $\mathbf{m}=\left(m_{j, \nu}\right)_{\substack{j=0, \ldots, p \\ \nu=1, \ldots, n_{j}}}$, is called the spectral type of $P$ and the Riemann scheme (1.10). Here we note that the Riemann scheme (1.10) should always satisfy the Fuchs relation

$$
\begin{aligned}
\left|\left\{\lambda_{\mathbf{m}}\right\}\right| & :=\sum_{j=0}^{p} \sum_{\nu=1}^{n_{p}} m_{j, \nu} \lambda_{j, \nu}-\operatorname{ord} \mathbf{m}+\frac{1}{2} \operatorname{idx} \mathbf{m}=0, \\
\operatorname{idx} \mathbf{m}: & =\sum_{j=0}^{p} \sum_{\nu=1}^{n_{p}} m_{j, \nu}^{2}-(p-1) \operatorname{ord} \mathbf{m} .
\end{aligned}
$$

Here idx $\mathbf{m}$ coincides with the index of rigidity introduced by $\mathrm{Kz}$.

In 8 6, after introducing certain representatives of conjugacy classes of matrices and some notation and concepts related to tuples of partitions, we define that the tuple $\mathbf{m}$ is realizable if there exists a Fuchsian differential operator $P$ with the Riemann scheme (1.10) for generic complex numbers $\lambda_{j, \nu}$ under the condition (1.11). Furthermore, if there exists such an operator $P$ so that $P u=0$ is irreducible, we define that $\mathbf{m}$ is irreducibly realizable.

Lastly in $\$$, we examine the generalized Riemann schemes of the product of Fuchsian differential operators and the dual operators.

In $\$$ 词 we examine the transformations of the Riemann scheme under our operations corresponding to the additions and the middle convolutions, which define transformations within Fuchsian differential operators. The operations induce transformations of spectral types of Fuchsian differential operators, which keep the indices of rigidity invariant but change the orders in general. Looking at the spectral types, we see that the combinatorial aspect of the reduction of Fuchsian differential operators is parallel to that of systems of Schlesinger canonical form. In this section, we also examine the combination of these transformation and the fractional linear transformations.

As our interpretation of Deligne-Simpson problem introduced by Kostov, we examine the condition for the existence of a given Riemann scheme in $\$ 8$. We determine the conditions on $\mathbf{m}$ such that $\mathbf{m}$ is realizable and irreducibly realizable, respectively, in Theorem 8.13. Moreover if $\mathbf{m}$ is realizable, Theorem 8.13 gives an explicit construction of the universal Fuchsian differential operator

$$
\begin{gathered}
P_{\mathbf{m}}=\left(\prod_{j=1}^{p}\left(x-c_{j}\right)^{n}\right) \frac{d^{n}}{d x^{n}}+\sum_{k=0}^{n-1} a_{k}(x, \lambda, g) \frac{d^{k}}{d x^{k}}, \\
\lambda=\left(\lambda_{j, \nu}\right) \begin{array}{c}
j=0, \ldots, p \\
\nu=1, \ldots, n_{j}
\end{array}, \quad g=\left(g_{1}, \ldots, g_{N}\right) \in \mathbb{C}^{N}
\end{gathered}
$$

with the Riemann scheme (1.10), which has the following properties.

For fixed complex numbers $\lambda_{j, \nu}$ satisfying (1.11) the operator with the Riemann scheme (1.10) satisfying $c_{0}=\infty$ equals $P_{\mathbf{m}}$ for a suitable $g \in \mathbb{C}^{N}$ up to a left multiplication by an element of $\mathbb{C}(x)$ if $\lambda_{j, \nu}$ are "generic", namely,

(1.14) $(\Lambda(\lambda) \mid \alpha) \notin\left\{-1,-2, \ldots, 1-\left(\alpha \mid \alpha_{\mathbf{m}}\right)\right\}$ for any $\alpha \in \Delta(\mathbf{m})$ with $\left(\alpha \mid \alpha_{\mathbf{m}}\right)>1$

under the notation used in (1.21), or $\mathbf{m}$ is fundamental or simply reducible (cf. Definition 8.14 and s8.5), etc. Here $g_{1}, \ldots, g_{N}$ are called accessory parameters and if $\mathbf{m}$ is irreducibly realizable, $N=1-\frac{1}{2} \mathrm{idx} \mathbf{m}$. In particular, if there is an irreducible and locally non-degenerate (cf. Definition 11.8) operator $P$ with the Riemann scheme (1.10), then $\lambda_{j, \nu}$ are "generic".

The coefficients $a_{k}(x, \lambda, g)$ of the differential operator $P_{\mathbf{m}}$ are polynomials of the variables $x, \lambda$ and $g$. The coefficients satisfy $\frac{\partial^{2} a_{k}}{\partial g_{\nu}^{2}}=0$ and furthermore $g_{\nu}$ can 
be equal to suitable $a_{i_{\nu}, j_{\nu}}$ under the expression $P_{\mathbf{m}}=\sum a_{i, j} x^{i} \frac{d^{j}}{d x^{j}}$ and the pairs $\left(i_{\nu}, j_{\nu}\right)$ for $\nu=1, \ldots, N$ are explicitly given in the theorem.

The universal operator $P_{\mathbf{m}}$ is a classically well-known operator in the case of Gauss hypergeometric equation, Jordan-Pochhammer equation or Heun's equation etc. and the theorem assures the existence of such a good operator for any realizable tuple $\mathbf{m}$. We define the tuple $\mathbf{m}$ is rigid if $\mathbf{m}$ is irreducibly realizable and moreover $N=0$, namely, $P_{\mathbf{m}}$ is free from accessory parameters.

In particular, the theorem gives the affirmative answer for the following question. Katz asked a question in the introduction in the book [Kz] whether a rigid local system is realized by a single Fuchsian differential equation $P u=0$ without apparent singularities (cf. Corollary 12.12 iii)).

It is a natural problem to examine the Fuchsian differential equation $P_{\mathbf{m}} u=$ 0 with an irreducibly realizable spectral type $\mathbf{m}$ which cannot be reduced to an equation with a lower order by additions and middle convolutions. The tuple $\mathbf{m}$ with this condition is called fundamental.

The equation $P_{\mathbf{m}} u=0$ with an irreducibly realizable spectral type $\mathbf{m}$ can be transformed by the operation $\partial_{\max }$ (cf. Definition 7.6) into a Fuchsian equation $P_{\mathbf{m}^{\prime}} v=0$ with a fundamental spectral type $\mathbf{m}^{\prime}$. Namely, there exists a non-negative integer $K$ such that $P_{\mathbf{m}^{\prime}}=\partial_{\max }^{K} P_{\mathbf{m}}$ and we define $f \mathbf{m}:=\mathbf{m}^{\prime}$. Then it turns out that a realizable tuple $\mathbf{m}$ is rigid if and only if the order of $f \mathbf{m}$, which is the order of $P_{f \mathbf{m}}$ by definition, equals 1 . Note that the operator $\partial_{\max }$ is essentially a product of suitable operators $\operatorname{RAd}\left(\left(x-c_{j}\right)^{\lambda_{j}}\right)$ and $\operatorname{RAd}\left(\partial^{-\mu}\right)$.

In this paper we study the transformations of several properties of the Fuchsian differential equation $P_{\mathbf{m}} u=0$ under the additions and middle convolutions. If they are understood well, the study of the properties are reduced to those of the equation $P_{f \mathbf{m}} v=0$, which are of order 1 if $\mathbf{m}$ is rigid. We note that there are many rigid spectral types $\mathbf{m}$ and for example there are 187 different rigid spectral types $\mathbf{m}$ with ord $\mathbf{m} \leq 8$ as are given in $\$ 15.2$.

As in the case of the systems of Schlesinger canonical form studied by [CB], the combinatorial aspect of transformations of the spectral type $\mathbf{m}$ of the Fuchsian differential operator $P$ induced from our fractional operations is described in $\$ 9$ by using the terminology of a Kac-Moody root system $\left(\Pi, W_{\infty}\right)$. Here $\Pi$ is the fundamental system of a Kac-Moody root system with the following star-shaped Dynkin diagram and $W_{\infty}$ is the Weyl group generated by the simple reflections $s_{\alpha}$ for $\alpha \in \Pi$. The elements of $\Pi$ are called simple roots.

Associated to a tuple $\mathbf{m}$ of $(p+1)$ partitions of a positive integer $n$, we define an element $\alpha_{\mathbf{m}}$ in the positive root lattice (cf. $\S 9.1$, (9.5)):

$$
\begin{aligned}
& \Pi:=\left\{\alpha_{0}, \alpha_{j, \nu} ; j=0,1, \ldots, \nu=1,2, \ldots\right\}, \\
& W_{\infty}:=\left\langle s_{\alpha} ; \alpha \in \Pi\right\rangle, \\
& \alpha_{\mathbf{m}}:=n \alpha_{0}+\sum_{j=0}^{p} \sum_{\nu=1}^{n_{j}-1}\left(\sum_{i=\nu+1}^{n_{j}} m_{j, i}\right) \alpha_{j, \nu}, \\
&\left(\alpha_{\mathbf{m}} \mid \alpha_{\mathbf{m}}\right)=\operatorname{idx} \mathbf{m}
\end{aligned}
$$

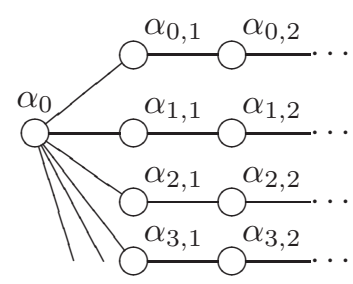

We can define a fractional operation on $P_{\mathbf{m}}$ which is compatible with the action of $w \in W_{\infty}$ on the root lattice (cf. Theorem 9.5):

$$
\begin{array}{ccr}
\left\{P_{\mathbf{m}}: \text { Fuchsian differential operators }\right\} & \rightarrow\left\{\left(\Lambda(\lambda), \alpha_{\mathbf{m}}\right) ; \alpha_{\mathbf{m}} \in \bar{\Delta}_{+}\right\} \\
\downarrow \text { fractional operations } & \circlearrowright & \downarrow W_{\infty} \text {-action, }+\tau \Lambda_{0, j}^{0} \\
\left\{P_{\mathbf{m}}: \text { Fuchsian differential operators }\right\} & \rightarrow & \left\{\left(\Lambda(\lambda), \alpha_{\mathbf{m}}\right) ; \alpha_{\mathbf{m}} \in \bar{\Delta}_{+}\right\} .
\end{array}
$$


Here $\tau \in \mathbb{C}$ and

$$
\begin{aligned}
& \Lambda^{0}:=\alpha_{0}+\sum_{\nu=1}^{\infty}(1+\nu) \alpha_{0, \nu}+\sum_{j=1}^{p} \sum_{\nu=1}^{\infty}(1-\nu) \alpha_{j, \nu}, \\
& \Lambda_{i, j}^{0}:=\sum_{\nu=1}^{\infty} \nu\left(\alpha_{i, \nu}-\alpha_{j, \nu}\right), \\
& \Lambda_{0}:=\frac{1}{2} \alpha_{0}+\frac{1}{2} \sum_{j=0}^{p} \sum_{\nu=1}^{\infty}(1-\nu) \alpha_{j, \nu}, \\
& \Lambda(\lambda):=-\Lambda_{0}-\sum_{j=0}^{p} \sum_{\nu=1}^{\infty}\left(\sum_{i=1}^{\nu} \lambda_{j, i}\right) \alpha_{j, \nu}
\end{aligned}
$$

and these linear combinations of infinite simple roots are identified with each other if their differences are in $\mathbb{C} \Lambda^{0}$. We note that

$$
\left|\left\{\lambda_{\mathbf{m}}\right\}\right|=\left(\Lambda(\lambda)+\frac{1}{2} \alpha_{\mathbf{m}} \mid \alpha_{\mathbf{m}}\right) \text {. }
$$

The realizable tuples exactly correspond to the elements of the set $\bar{\Delta}_{+}$of positive integer multiples of the positive roots of the Kac-Moody root system whose support contains $\alpha_{0}$ and the rigid tuples exactly correspond to the positive real roots whose support contain $\alpha_{0}$. For an element $w \in W_{\infty}$ and an element $\alpha \in \bar{\Delta}_{+}$we do not consider $w \alpha$ in the commutative diagram (1.16) when $w \alpha \notin \bar{\Delta}_{+}$.

Hence the fact that any irreducible rigid Fuchsian equation $P_{\mathbf{m}} u=0$ is transformed into the trivial equation $\frac{d v}{d x}=0$ by our invertible fractional operations corresponds to the fact that there exists $w \in W_{\infty}$ such that $w \alpha_{\mathbf{m}}=\alpha_{0}$ because $\alpha_{\mathbf{m}}$ is a positive real root. The monotone fundamental tuples of partitions correspond to $\alpha_{0}$ or the positive imaginary roots $\alpha$ in the closed negative Weyl chamber which are indivisible or satisfies $(\alpha \mid \alpha)<0$. A tuple of partitions $\mathbf{m}=\left(m_{j, \nu}\right)_{\substack{j=0, \ldots, p \\ \nu=1, \ldots, n_{j}}}$ is said to be monotone if $m_{j, 1} \geq m_{j, 2} \geq \cdots \geq m_{j, n_{j}}$ for $j=0, \ldots, p$. For example, we prove the exact estimate

$$
\text { ord } \mathbf{m} \leq 3|\operatorname{idx} \mathbf{m}|+6
$$

for any fundamental tuple $\mathbf{m}$ in $\$ 9.2$. Since we may assume

$$
p \leq \frac{1}{2}|\operatorname{idx} \mathbf{m}|+3
$$

for a fundamental tuple $\mathbf{m}$, there exist only finite number of monotone fundamental tuples with a fixed index of rigidity. We list the fundamental tuples of the index of rigidity 0 or -2 in Remark 8.9 or Proposition 8.10, respectively.

Our results in $\S 4$, $\$$ and $\$ 8$ give an integral expression and a power series expression of a local solution of the universal equation $P_{\mathbf{m}} u=0$ corresponding to the characteristic exponent whose multiplicity is free in the local monodromy. These expressions are in $\$ 10$.

In $\$ 11.1$ we review the monodromy of solutions of a Fuchsian differential equation from the view point of our operations. The theorems in this section are given by DR, DR2, Kz, Ko]. In $\$ 11.2$ we review Scott's lemma $\mathrm{Sg}$ and related results with their proofs, which are elementary but important for the study of the irreducibility of the monodromy.

In $\$ 2.1$ we examine the condition for the decomposition $P_{\mathbf{m}}=P_{\mathbf{m}^{\prime}} P_{\mathbf{m}^{\prime \prime}}$ of universal operators with or without fixing the exponents $\left\{\lambda_{j, \nu}\right\}$, which implies the reducibility of the equation $P_{\mathbf{m}} u=0$. In $\$ 12.2$ we study the value of spectral parameters which makes the equation reducible and obtain Theorem 12.10. In particular we have a necessary and sufficient condition on characteristic exponents so that the monodromy of the solutions of the equation $P_{\mathbf{m}} u=0$ with a rigid 
spectral type $\mathbf{m}$ is irreducible, which is given in Corollary 12.12 or Theorem 12.13 . When $m_{j, 1} \geq m_{j, 2} \geq \cdots$ for any $j \geq 0$, the condition equals

$$
(\Lambda(\lambda) \mid \alpha) \notin \mathbb{Z} \quad(\forall \alpha \in \Delta(\mathbf{m})) .
$$

Here $\Delta(\mathbf{m})$ denotes the totality of real positive roots $\alpha$ such that $w_{\mathbf{m}} \alpha$ are negative and $w_{\mathbf{m}}$ is the element of $W_{\infty}$ with the minimal length so that $\alpha_{0}=w_{\mathbf{m}} \alpha_{\mathbf{m}}$ (cf. Definition 9.8 and Proposition 9.9 v)). The number of elements of $\Delta(\mathbf{m})$ equals the length of $w_{\mathbf{m}}$, which is the minimal length of the expressions of $w_{\mathbf{m}}$ as products of simple reflections $s_{\alpha}$ with $\alpha \in \Pi$.

In $\S 13$ we construct shift operators between rigid Fuchsian differential equations with the same spectral type such that the differences of the corresponding characteristic exponents are integers. Theorem 13.3 gives a recurrence relation of certain solutions of the rigid Fuchsian equations, which is a generalization of the formula

$$
c(F(a, b+1, c ; x)-F(a, b, c ; x))=a x F(a+1, b+1, c+1 ; x)
$$

and moreover gives relations between the universal operators and the shift operators in Theorem 13.3 and Theorem 13.7. In particular, Thorem 13.7 gives a condition which assures that a universal operator is this shift operator.

The shift operators are useful for the study of Fuchsian differential equations when they are reducible because of special values of the characteristic exponents. Theorem 13.9 give a necessary condition and a sufficient condition so that the shift operator is bijective. In many cases we get a necessary and sufficient condition by this theorem. As an application of a shift operator we examine polynomial solutions of a rigid Fuchsian differential equation of Okubo type in $\S 13.3$.

In $\$ 14.1$ we study a connection problem of the Fuchsian differential equation $P_{\mathbf{m}} u=0$. First we give Lemma 14.2 which describes the transformation of a connection coefficient under an addition and a middle convolution. In particular, for the equation $P_{\mathbf{m}} u=0$ satisfying $m_{0, n_{0}}=m_{1, n_{1}}=1$, Theorem 14.4 says that the connection coefficient $c\left(c_{0}: \lambda_{0, n_{0}} \rightsquigarrow c_{1}: \lambda_{1, n_{1}}\right)$ from the local solution corresponding to the exponent $\lambda_{0, n_{0}}$ to that corresponding to $\lambda_{1, n_{1}}$ in the Riemann scheme (1.10) equals the connection coefficient of the reduced equation $P_{f \mathbf{m}} v=0$ up to the gamma factors which are explicitly calculated.

In particular, if the equation is rigid, Theorem 14.6 gives the connection coefficient as a quotient of products of gamma functions and an easier non-zero term. For example, when $p=2$, the easier term doesn't appear and the connection coefficient has the universal formula

$$
c\left(c_{0}: \lambda_{0, n_{0}} \rightsquigarrow c_{1}: \lambda_{1, n_{1}}\right)=\frac{\prod_{\nu=1}^{n_{0}-1} \Gamma\left(\lambda_{0, n_{0}}-\lambda_{0, \nu}+1\right) \cdot \prod_{\substack{\nu=1 \\ \mathbf{m}^{\prime} \oplus \mathbf{m}^{\prime \prime}=\mathbf{m} \\ m_{0, n_{0}}^{\prime}=m_{1, n_{1}}^{\prime \prime}=1}}^{n_{1}-1} \Gamma\left(\left|\left\{\lambda_{\mathbf{m}^{\prime}}\right\}\right|\right)}{\left.\prod_{1, \nu}-\lambda_{1, n_{1}}\right)} .
$$

Here the notation (1.11) is used and $\mathbf{m}=\mathbf{m}^{\prime} \oplus \mathbf{m}^{\prime \prime}$ means that $\mathbf{m}=\mathbf{m}^{\prime}+\mathbf{m}^{\prime \prime}$ with rigid tuples $\mathbf{m}^{\prime}$ and $\mathbf{m}^{\prime \prime}$. Moreover the number of gamma factors in the above denominator is equals to that of the numerator. The author conjectured this formula in 2007 and proved it in 2008 (cf. O6]). The proof in $\$ 14.1$ is different from the original proof, which is explained in \$14.3.

Suppose $p=2$, ord $\mathbf{m}=2, m_{j, \nu}=1$ for $0 \leq j \leq 2$ and $1 \leq \nu \leq 2$, Then (1.23) equals

$$
\frac{\Gamma\left(\lambda_{0,2}-\lambda_{0,1}+1\right) \Gamma\left(\lambda_{1,2}-\lambda_{1,1}\right)}{\Gamma\left(\lambda_{0,1}+\lambda_{1,2}+\lambda_{2,1}\right) \Gamma\left(\lambda_{0,1}+\lambda_{1,2}+\lambda_{2,2}\right)},
$$

which implies (1.3) under (1.4). 
The hypergeometric series $F(a, b, c ; x)$ satisfies $\lim _{k \rightarrow+\infty} F(a, b, c+k ; x)=1$ if $|x| \leq 1$, which obviously implies $\lim _{k \rightarrow+\infty} F(a, b, c+k ; 1)=1$. Gauss proves the summation formula (1.3) by this limit formula and the recurrence relation $F(a, b, c ; 1)=\frac{(c-a)(c-b)}{c(c-a-b)} F(a, b, c+1 ; 1)$. We have $\lim _{k \rightarrow+\infty} c\left(c_{0}: \lambda_{0, n_{0}}+k \rightsquigarrow c_{1}:\right.$ $\left.\lambda_{1, n_{1}}-k\right)=1$ in the connection formula (1.23) (cf. Corollary 14.7). This suggests a similar limit formula for a local solution of a general Fuchsian differential equation, which is given in $\$ 14.2$.

In $\$ 14.3$ we propose a procedure to calculate the connection coefficient (cf. Remark 14.19), which is based on the calculation of its zeros and poles. This procedure is different from the proof of Theorem 14.6 in $\$ 14.1$ and useful to calculate a certain connection coefficient between local solutions with multiplicities in eigenvalues of local monodromies. The coefficient is defined in Definition 14.17 .

In \$15 we show many examples which explain our fractional calculus in this paper and also give concrete results of the calculus. In $\$ 15.1$ we list all the fundamental tuples whose indices of rigidity are not smaller than -6 and in $\$ 15.2$ we list all the rigid tuples whose orders are not larger than 8 , most of which are calculated by a computer program okubo explained in $\$ 15.11$. In $\$ 15.3$ and $\S 15.4$ we apply our fractional calculus to Jordan-Pochhammer equations and the hypergeometric family, respectively, which helps us to understand our unifying study of rigid Fuchsian differential equations. In $\$ 15.5$ we apply our fractional calculus to the even/odd family classified by [Si] and most of the results there have been first obtained by the calculus.

In $\$ 15.7$, $\$ 15.8$ and $\$ 15.9$ we study the rigid Fuchsian differential equations of order not larger than 4 and those of order 5 or 6 and the equations belonging to 12 submaximal series classified by [Ro], respectively. Note that these 12 maximal series contain Yokoyama's list $|\mathrm{Yo}|$. In $\$ 15.9 .2$, we explain how we read the condition of irreducibility, connection coefficients, shift operators etc. of the corresponding differential equation from the data given in $\$ 15.7-\$ 15.9$. In $\$ 15.6$, we show some interesting identities of trigonometric functions as a consequence of the concrete value (1.23) of connection coefficients. We examine Appell's hypergeometric equations in $\$ 15.10$ by our fractional calculus, which will be further discussed in another paper.

In $\$ 16$ we give some problems to be studied related to the results in this paper.

In $\$ 17$ a theorem on Coxeter groups is given, which was proved by K. Nuida through a private communication between the author and Nuida. The theorem is useful for the study of the difference of various reductions of Fuchsian differential equations (cf. Proposition $9.9 \mathrm{v}$ )). The author greatly thanks Nuida for allowing the author to put the theorem with its proof in this paper.

The author express his sincere gratitude to Kazuo Okamoto and Yoshishige Haraoka for the guidance to the subjects developed in this paper and to Kazuki Hiroe for reading the manuscript of this paper.

\section{Fractional operations}

2.1. Weyl algebra. In this section we define several operations on a Weyl algebra. The operations are elementary or well-known but their combinations will be important.

Let $\mathbb{C}\left[x_{1}, \ldots, x_{n}\right]$ denote the polynomial ring of $n$ independent variables $x_{1}, \ldots, x_{n}$ over $\mathbb{C}$ and let $\mathbb{C}\left(x_{1}, \ldots, x_{n}\right)$ denote the quotient field of $\mathbb{C}\left[x_{1}, \ldots, x_{n}\right]$. The Weyl algebra $W\left[x_{1}, \ldots, x_{n}\right]$ of $n$ variables $x_{1}, \ldots, x_{n}$ is the algebra over $\mathbb{C}$ generated by $x_{1}, \ldots, x_{n}$ and $\frac{\partial}{\partial x_{1}}, \ldots, \frac{\partial}{\partial x_{n}}$ with the fundamental relation

$$
\left[x_{i}, x_{j}\right]=\left[\frac{\partial}{\partial x_{i}}, \frac{\partial}{\partial x_{j}}\right]=0, \quad\left[\frac{\partial}{\partial x_{i}}, x_{j}\right]=\delta_{i, j} \quad(1 \leq i, j \leq n) .
$$


We introduce a Weyl algebra $W\left[x_{1}, \ldots, x_{n}\right]\left[\xi_{1}, \ldots, \xi_{n}\right]$ with parameters $\xi_{1}, \ldots, \xi_{N}$ by

$$
W\left[x_{1}, \ldots, x_{n}\right]\left[\xi_{1}, \ldots, \xi_{N}\right]:=\mathbb{C}\left[\xi_{1}, \ldots, \xi_{N}\right] \underset{\mathbb{C}}{\otimes} W\left[x_{1}, \ldots, x_{n}\right]
$$

and put

$$
\begin{aligned}
W\left[x_{1}, \ldots, x_{n} ; \xi_{1}, \ldots, \xi_{N}\right] & :=\mathbb{C}\left(\xi_{1}, \ldots, \xi_{N}\right) \underset{\mathbb{C}}{\otimes} W\left[x_{1}, \ldots, x_{n}\right] \\
W\left(x_{1}, \ldots, x_{n} ; \xi_{1}, \ldots, \xi_{N}\right) & :=\mathbb{C}\left(x_{1}, \ldots, x_{n}, \xi_{1}, \ldots, \xi_{N}\right) \underset{\mathbb{C}\left[x_{1}, \ldots, x_{n}\right]}{\otimes} W\left[x_{1}, \ldots, x_{n}\right] .
\end{aligned}
$$

Here we have

$$
\begin{aligned}
{\left[x_{i}, \xi_{\nu}\right] } & =\left[\frac{\partial}{\partial x_{i}}, \xi_{\nu}\right]=0 \quad(1 \leq i \leq n, 1 \leq \nu \leq N) \\
{\left[\frac{\partial}{\partial x_{i}}, \frac{g}{f}\right] } & =\frac{\partial}{\partial x_{i}}\left(\frac{g}{f}\right) \\
& =\frac{\frac{\partial g}{\partial x_{i}} \cdot f-g \cdot \frac{\partial f}{\partial x_{i}}}{f^{2}} \quad\left(f, g \in \mathbb{C}\left[x_{1}, \ldots, x_{n}, \xi_{1}, \ldots, \xi_{N}\right]\right)
\end{aligned}
$$

and $\left[\frac{\partial}{\partial x_{i}}, f\right]=\frac{\partial f}{\partial x_{i}} \in \mathbb{C}\left[x_{1}, \ldots, x_{n}, \xi_{1}, \ldots, \xi_{N}\right]$.

For simplicity we put $x=\left(x_{1}, \ldots, x_{n}\right)$ and $\xi=\left(\xi_{1}, \ldots, \xi_{N}\right)$ and the algebras $\mathbb{C}\left[x_{1}, \ldots, x_{n}\right], \mathbb{C}\left(x_{1}, \ldots, x_{n}\right), W\left[x_{1}, \ldots, x_{n}\right]\left[\xi_{1}, \ldots, \xi_{N}\right], W\left[x_{1}, \ldots, x_{n} ; \xi_{1}, \ldots, \xi_{N}\right]$, $W\left(x_{1}, \ldots, x_{n} ; \xi_{1}, \ldots, \xi_{N}\right)$ etc. are also denoted by $\mathbb{C}[x], \mathbb{C}(x), W[x][\xi], W[x ; \xi]$, $W(x ; \xi)$ etc., respectively. Then

$$
\mathbb{C}[x, \xi] \subset W[x][\xi] \subset W[x ; \xi] \subset W(x ; \xi) .
$$

The element $P$ of $W(x ; \xi)$ is uniquely written by

$$
P=\sum_{\alpha=\left(\alpha_{1}, \ldots, \alpha_{n}\right) \in \mathbb{Z}_{\geq 0}^{n}} p_{\alpha}(x, \xi) \frac{\partial^{\alpha_{1}+\cdots+\alpha_{n}}}{\partial x_{1}^{\alpha_{1}} \cdots \partial x_{n}^{\alpha_{n}}} \quad\left(p_{\alpha}(x, \xi) \in \mathbb{C}(x, \xi)\right) .
$$

Here $\mathbb{Z}_{\geq 0}=\{0,1,2, \ldots\}$. Similar we will denote the set of positive integers by $\mathbb{Z}_{>0}$. If $P \in W(x ; \xi)$ is not zero, the maximal integer $\alpha_{1}+\cdots+\alpha_{n}$ satisfying $p_{\alpha}(x, \xi) \neq 0$ is called the order of $P$ and denoted by ord $P$. If $P \in W[x ; \xi], p_{\alpha}(x, \xi)$ are polynomials of $x$ with coefficients in $\mathbb{C}(\xi)$ and the maximal degree of $p_{\alpha}(x, \xi)$ as polynomials of $x$ is called the degree of $P$ and denoted by $\operatorname{deg} P$.

2.2. Laplace and gauge transformations and reduced representatives. First we will define some fundamental operations on $W[x ; \xi]$.

Definition 2.1. i) For a non-zero element $P \in W(x ; \xi)$ we choose an element $(\mathbb{C}(x, \xi) \backslash\{0\}) P \cap W[x ; \xi]$ with the minimal degree and denote it by $\mathrm{R} P$ and call it a reduced representative of $P$. If $P=0$, we put $\mathrm{R} P=0$. Note that $\mathrm{R} P$ is determined up to multiples by non-zero elements of $\mathbb{C}(\xi)$.

ii) For a subset $I$ of $\{1, \ldots, n\}$ we define an automorphism $\mathrm{L}_{I}$ of $W[x ; \xi]$ :

$$
\mathrm{L}_{I}\left(\frac{\partial}{\partial x_{i}}\right)=\left\{\begin{array}{ll}
x_{i} & (i \in I) \\
\frac{\partial}{\partial x_{i}} & (i \notin I)
\end{array}, \quad \mathrm{L}_{I}\left(x_{i}\right)=\left\{\begin{array}{ll}
-\frac{\partial}{\partial x_{i}} & (i \in I) \\
x_{i} & (i \notin I)
\end{array} \text { and } \mathrm{L}_{I}\left(\xi_{\nu}\right)=\xi_{\nu} .\right.\right.
$$

We put $\mathrm{L}=\mathrm{L}_{\{1, \ldots, n\}}$ and call $\mathrm{L}$ the Laplace transformation of $W[x ; \xi]$.

iii) Let $W_{L}(x ; \xi)$ be the algebra isomorphic to $W(x ; \xi)$ which is defined by the Laplace transformation

$$
\mathrm{L}: W(x ; \xi) \stackrel{\sim}{\rightarrow} W_{L}(x ; \xi) \stackrel{\sim}{\rightarrow} W(x ; \xi) .
$$

For an element $P \in W_{L}(x ; \xi)$ we define

$$
\mathrm{R}_{L}(P):=\mathrm{L}^{-1} \circ \mathrm{R} \circ \mathrm{L}(P) .
$$


Note that the element of $W_{L}(x ; \xi)$ is a finite sum of products of elements of $\mathbb{C}[x]$ and rational functions of $\left(\frac{\partial}{\partial x_{1}}, \ldots, \frac{\partial}{\partial x_{n}}, \xi_{1}, \ldots, \xi_{N}\right)$.

We will introduce an automorphism of $W(x ; \xi)$.

Definition 2.2 (gauge transformation). Fix an element $\left(h_{1}, \ldots, h_{n}\right) \in \mathbb{C}(x, \xi)^{n}$ satisfying

$$
\frac{\partial h_{i}}{\partial x_{j}}=\frac{\partial h_{j}}{\partial x_{i}} \quad(1 \leq i, j \leq n) .
$$

We define an automorphism $\operatorname{Adei}\left(h_{1}, \ldots, h_{n}\right)$ of $W(x ; \xi)$ by

$$
\begin{aligned}
\operatorname{Adei}\left(h_{1}, \ldots, h_{n}\right)\left(x_{i}\right) & =x_{i} & & (i=1, \ldots, n), \\
\operatorname{Adei}\left(h_{1}, \ldots, h_{n}\right)\left(\frac{\partial}{\partial x_{i}}\right) & =\frac{\partial}{\partial x_{i}}-h_{i} & & (i=1, \ldots, n), \\
\operatorname{Adei}\left(h_{1}, \ldots, h_{n}\right)\left(\xi_{\nu}\right) & =\xi_{\nu} & & (\nu=1, \ldots, N) .
\end{aligned}
$$

Choose functions $f$ and $g$ satisfying $\frac{\partial g}{\partial x_{i}}=h_{i}$ for $i=1, \ldots, n$ and put $f=e^{g}$ and

$$
\operatorname{Ad}(f)=\operatorname{Ade}(g)=\operatorname{Adei}\left(h_{1}, \ldots, h_{n}\right) .
$$

We will define a homomorphism of $W(x ; \xi)$.

Definition 2.3 (coordinate transformation). Let $\phi=\left(\phi_{1}, \ldots, \phi_{n}\right)$ be an element of $\mathbb{C}\left(x_{1}, \ldots, x_{m}, \xi\right)^{n}$ such that the rank of the matrix

$$
\Phi:=\left(\frac{\partial \phi_{j}}{\partial x_{i}}\right)_{\substack{1 \leq i \leq m \\ 1 \leq j \leq n}}
$$

equals $n$ for a generic point $(x, \xi) \in \mathbb{C}^{m+N}$. Let $\Psi=\left(\psi_{i, j}(x, \xi)\right)_{\substack{1 \leq i \leq n \\ 1 \leq j \leq m}}$ be an left inverse of $\Phi$, namely, $\Psi \Phi$ is an identity matrix of size $n$ and $m \geq n$. Then a homomorphism $T_{\phi}^{*}$ from $W\left(x_{1}, \ldots, x_{n} ; \xi\right)$ to $W\left(x_{1}, \ldots, x_{m} ; \xi\right)$ is defined by

$$
\begin{aligned}
T_{\phi}^{*}\left(x_{i}\right) & =\phi_{i}(x) & & (1 \leq i \leq n), \\
T_{\phi}^{*}\left(\frac{\partial}{\partial x_{i}}\right) & =\sum_{j=1}^{m} \psi_{i, j}(x, \xi) \frac{\partial}{\partial x_{j}} & & (1 \leq i \leq n) .
\end{aligned}
$$

If $m>n$, we choose linearly independent elements $h_{\nu}=\left(h_{\nu, 1}, \ldots, h_{\nu, m}\right)$ of $\mathbb{C}(x, \xi)^{m}$ for $\nu=1, \ldots, m-n$ such that $\psi_{i, 1} h_{\nu, 1}+\cdots+\psi_{i, m} h_{\nu, m}=0$ for $i=1, \ldots, n$ and $\nu=1, \ldots, m-n$ and put

$$
\mathcal{K}^{*}(\phi):=\sum_{\nu=1}^{m-n} \mathbb{C}(x, \xi) \sum_{j=1}^{m} h_{\nu, j} \frac{\partial}{\partial x_{j}} \in W(x ; \xi) .
$$

The meaning of these operations are clear as follows.

Remark 2.4. Let $P$ be an element of $W(x ; \xi)$ and let $u(x)$ be an analytic solution of the equation $P u=0$ with a parameter $\xi$. Then under the notation in Definitions 2.1 2.2, we have $(\mathrm{R} P) u(x)=(\operatorname{Ad}(f)(P))(f(x) u(x))=0$. Note that $\mathrm{R} P$ is defined up to the multiplications of non-zero elements of $\mathbb{C}(\xi)$.

If a Laplace transform

$$
\left(\mathcal{R}_{k} u\right)(x)=\int_{C} e^{-x_{1} t_{1}-\cdots-x_{k} t_{k}} u\left(t_{1}, \ldots, t_{k}, x_{k+1}, \ldots, x_{n}\right) d t_{1} \cdots d t_{k}
$$

of $u(x)$ is suitably defined, then $\left(\mathrm{L}_{\{1, \ldots, k\}}(\mathrm{R} P)\right)\left(\mathcal{R}_{k} u\right)=0$, which follows from the equalities $\frac{\partial \mathcal{R}_{k} u}{\partial x_{i}}=\mathcal{R}_{k}\left(-x_{i} u\right)$ and $0=\int_{C} \frac{\partial}{\partial t_{i}}\left(e^{-x_{1} t_{1}-\cdots-x_{k} t_{k}} u\left(t, x_{k+1}, \ldots\right)\right) d t=$ $-x_{i} \mathcal{R}_{k} u+\mathcal{R}_{k}\left(\frac{\partial u}{\partial t_{i}}\right)$ for $i=1, \ldots, k$. Moreover we have

$$
f(x) \mathcal{R}_{k} \mathrm{R} P u=f(x)\left(L_{\{1, \ldots, k\}}(\mathrm{R} P)\right)\left(\mathcal{R}_{k} u\right)=\left(\operatorname{Ad}(f) L_{\{1, \ldots, k\}}(\mathrm{R} P)\right)\left(f(x) \mathcal{R}_{k} u\right) .
$$


Under the notation of Definition 2.3, we have $T_{\phi}^{*}(P) u\left(\phi_{1}(x), \ldots, \phi_{n}(x)\right)=0$ and $Q u\left(\phi_{1}(x), \ldots, \phi_{n}(x)\right)=0$ for $Q \in \mathcal{K}^{*}(\phi)$.

Another transformation of $W[x ; \xi]$ based on an integral transformation frequently used will be given in Proposition 15.1.

We introduce some notation for combinations of operators we have defined.

Definition 2.5. Retain the notation in Definition 2.1 2.3 and recall that $f=e^{g}$ and $h_{i}=\frac{\partial g}{\partial x_{i}}$.

$$
\begin{aligned}
\operatorname{RAd}(f) & =\operatorname{RAde}(g)=\operatorname{RAdei}\left(h_{1}, \ldots, h_{n}\right):=\operatorname{R} \circ \operatorname{Adei}\left(h_{1}, \ldots, h_{n}\right), \\
\operatorname{AdL}(f) & =\operatorname{AdeL}(h)=\operatorname{AdeiL}\left(h_{1}, \ldots, h_{n}\right) \\
& :=\mathrm{L}^{-1} \circ \operatorname{Adei}\left(h_{1}, \ldots, h_{n}\right) \circ \mathrm{L}, \\
\operatorname{RAdL}(f) & =\operatorname{RAdeL}(h)=\operatorname{RAdeiL}\left(h_{1}, \ldots, h_{n}\right) \\
& :=\mathrm{L}^{-1} \circ \operatorname{RAdei}\left(h_{1}, \ldots, h_{n}\right) \circ \mathrm{L}, \\
\operatorname{Ad}\left(\partial_{x_{i}}^{\mu}\right) & :=\mathrm{L}^{-1} \circ \operatorname{Ad}\left(x_{i}^{\mu}\right) \circ \mathrm{L}, \\
\operatorname{RAd}\left(\partial_{x_{i}}^{\mu}\right) & :=\mathrm{L}^{-1} \circ \operatorname{RAd}\left(x_{i}^{\mu}\right) \circ \mathrm{L} .
\end{aligned}
$$

Here $\mu$ is a complex number or an element of $\mathbb{C}(\xi)$ and $\operatorname{Ad}\left(\partial_{x_{i}}^{\mu}\right)$ defines an endomorphism of $W_{L}(x ; \xi)$.

We will sometimes denote $\frac{\partial}{\partial x_{i}}$ by $\partial_{x_{i}}$ or $\partial_{i}$ for simplicity. If $n=1$, we usually denote $x_{1}$ by $x$ and $\frac{\partial}{\partial x_{1}}$ by $\frac{d}{d x}$ or $\partial_{x}$ or $\partial$. We will give some examples.

Since the calculation $\operatorname{Ad}\left(x^{-\mu}\right) \partial=x^{-\mu} \circ \partial \circ x^{\mu}=x^{-\mu}\left(x^{\mu} \partial+\mu x^{\mu-1}\right)=\partial+\mu x^{-1}$ is allowed, the following calculation is justified by the isomorphism (2.7):

$$
\begin{aligned}
\operatorname{Ad}\left(\partial^{-\mu}\right) x^{m}= & \partial^{-\mu} \circ x^{m} \circ \partial^{\mu} \\
= & \left(x^{m} \partial^{-\mu}+\frac{(-\mu) m}{1 !} x^{m-1} \partial^{-\mu-1}+\frac{(-\mu)(-\mu-1) m(m-1)}{2 !} x^{m-2} \partial^{-\mu-2}\right. \\
& \left.\quad+\cdots+\frac{(-\mu)(-\mu-1) \cdots(-\mu-m+1) m !}{m !} \partial^{-\mu-m}\right) \partial^{\mu} \\
= & \sum_{\nu=0}^{m}(-1)^{\nu}(\mu)_{\nu}\left(\begin{array}{c}
m \\
\nu
\end{array}\right) x^{m-\nu} \partial^{-\nu} .
\end{aligned}
$$

This calculation is in a ring of certain pseudo-differential operators according to Leibniz's rule. In general, we may put $\operatorname{Ad}\left(\partial^{-\mu}\right) P=\partial^{-\mu} \circ P \circ \partial^{\mu}$ for $P \in W[x ; \xi]$ under Leibniz's rule. Here $m$ is a positive integer and we use the notation

$$
(\mu)_{\nu}:=\prod_{i=0}^{\nu-1}(\mu+i), \quad\left(\begin{array}{c}
m \\
\nu
\end{array}\right):=\frac{\Gamma(m+1)}{\Gamma(m-\nu+1) \Gamma(\nu+1)}=\frac{m !}{(m-\nu) ! \nu !} .
$$

2.3. Examples of ordinary differential operators. In this paper we mainly study ordinary differential operators. We give examples of the operations we have defined, which are related to classical differential equations.

Example 2.6 $(n=1)$. For a rational function $h(x, \xi)$ of $x$ with a parameter $\xi$ we denote by $\int h(x, \xi) d x$ the function $g(x, \xi)$ satisfying $\frac{d}{d x} g(x, \xi)=h(x, \xi)$. Put $f(x, \xi)=e^{g(x, \xi)}$ and define

$$
\vartheta:=x \frac{d}{d x} .
$$

Then we have the following identities.

$$
\operatorname{Adei}(h) \partial=\partial-h=\operatorname{Ad}\left(e^{\int h(x) d x}\right) \partial=e^{\int h(x) d x} \circ \partial \circ e^{-\int h(x) d x},
$$




$$
\begin{aligned}
\operatorname{Ad}(f) x & =x, \quad \operatorname{AdL}(f) \partial=\partial, \\
\operatorname{Ad}(\lambda f) & =\operatorname{Ad}(f) \quad \operatorname{AdL}(\lambda f)=\operatorname{AdL}(f), \\
\operatorname{Ad}(f) \partial & =\partial-h(x, \xi) \Rightarrow \operatorname{AdL}(f) x=x+h(\partial, \xi), \\
\operatorname{Ad}\left((x-c)^{\lambda}\right) & =\operatorname{Ade}(\lambda \log (x-c))=\operatorname{Adei}\left(\frac{\lambda}{x-c}\right), \\
\operatorname{Ad}\left((x-c)^{\lambda}\right) x & =x, \quad \operatorname{Ad}\left((x-c)^{\lambda}\right) \partial=\partial-\frac{\lambda}{x-c}, \\
\operatorname{RAd}\left((x-c)^{\lambda}\right) \partial & =\operatorname{Ad}\left((x-c)^{\lambda}\right)((x-c) \partial)=(x-c) \partial-\lambda, \\
\operatorname{RAdL}\left((x-c)^{\lambda}\right) x & =L^{-1} \circ \operatorname{RAd}\left((x-c)^{\lambda}\right)(-\partial) \\
& =L^{-1}((x-c)(-\partial)+\lambda) \\
& =(\partial-c) x+\lambda=x \partial-c x+1+\lambda, \\
\operatorname{RAdL}\left((x-c)^{\lambda}\right) \partial & =\partial, \quad \operatorname{RAdL}\left((x-c)^{\lambda}\right)((\partial-c) x)=(\partial-c) x+\lambda, \\
\operatorname{Ad}\left(\partial^{\lambda}\right) \vartheta & =\operatorname{AdL}\left(x^{\lambda}\right) \vartheta=\vartheta+\lambda, \\
\operatorname{Ad}\left(e^{\frac{\lambda(x-c)^{m}}{m}}\right) x & =x, \quad \operatorname{Ad}\left(e^{\frac{\lambda(x-c)^{m}}{m}}\right) \partial=\partial-\lambda(x-c)^{m-1}, \\
\operatorname{RAdL}\left(e^{\frac{\lambda(x-c)^{m}}{m}}\right) x & = \begin{cases}x+\lambda(\partial-c)^{m-1} & (m \geq 1), \\
(\partial-c)^{1-m} x+\lambda \quad(m \leq-1), & (x-c)^{1-m} \partial .\end{cases} \\
T_{(x-c)^{m}}^{*}(x) & =(x-c)^{m}, \quad T_{(x-c)^{m}}^{*}(\partial)=\frac{1}{m}(x-c)
\end{aligned}
$$

Here $m$ is a non-zero integer and $\lambda$ is a non-zero complex number.

Some operations are related to Katz's operations defined by [Kz]. The operation $\operatorname{RAd}\left((x-c)^{\mu}\right)$ corresponds to the addition given in $[\mathrm{DR}$ and the operator

$$
m c_{\mu}:=\operatorname{RAd}\left(\partial^{-\mu}\right)=\operatorname{RAdL}\left(x^{-\mu}\right)
$$

corresponds to Katz's middle convolution and the Euler transformation or the Riemann-Liouville integral (cf. [Kh, §5.1]) or the fractional derivation

$$
\left(I_{c}^{\mu}(u)\right)(x)=\frac{1}{\Gamma(\mu)} \int_{c}^{x} u(t)(x-t)^{\mu-1} d t .
$$

Here $c$ is suitably chosen. In most cases, $c$ is a singular point of the multi-valued holomorphic function $u(x)$. The integration may be understood through an analytic continuation with respect to a parameter or in the sense of generalized functions. When $u(x)$ is a multi-valued holomorphic function on the punctured disk around $c$, we can define the complex integral

$$
\left(\tilde{I}_{c}^{\mu}(u)\right)(x):=\int^{(x+, c+, x-, c-)} u(z)(x-z)^{\mu-1} d z
$$

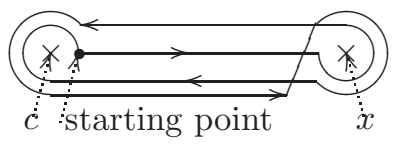

through Pochhammer contour $(x+, c+, x-, c-)$ along a double loop circuit (cf. WW, 12.43]). If $(z-c)^{-\lambda} u(z)$ is a meromorphic function in a neighborhood of the point $c$, we have

$$
\left(\tilde{I}_{c}^{\mu}(u)\right)(x)=\left(1-e^{2 \pi \lambda \sqrt{-1}}\right)\left(1-e^{2 \pi \mu \sqrt{-1}}\right) \int_{c}^{x} u(t)(x-t)^{\mu-1} d t .
$$


For example, we have

$$
\begin{aligned}
I_{c}^{\mu}\left((x-c)^{\lambda}\right) & =\frac{1}{\Gamma(\mu)} \int_{c}^{x}(t-c)^{\lambda}(x-t)^{\mu-1} d t \\
& =\frac{(x-c)^{\lambda+\mu}}{\Gamma(\mu)} \int_{0}^{1} s^{\lambda}(1-s)^{\mu-1} d s \quad(x-t=(1-s)(x-c)) \\
& =\frac{\Gamma(\lambda+1)}{\Gamma(\lambda+\mu+1)}(x-c)^{\lambda+\mu} \\
\tilde{I}_{c}^{\mu}\left((x-c)^{\lambda}\right) & =\frac{4 \pi^{2} e^{\pi(\lambda+\mu) \sqrt{-1}}}{\Gamma(-\lambda) \Gamma(1-\mu) \Gamma(\lambda+\mu+1)}(x-c)^{\lambda+\mu+1} .
\end{aligned}
$$

For $k \in \mathbb{Z}_{\geq 0}$ we have

$$
\tilde{I}_{c}^{\mu}\left((x-c)^{k} \log (x-c)\right)=\frac{-4 \pi^{2} k ! e^{\pi \lambda \sqrt{-1}}}{\Gamma(1-\mu) \Gamma(\mu+k+1)}(x-c)^{\mu+k+1} .
$$

We note that since

$$
\frac{d}{d t}\left(u(t)(x-t)^{\mu-1}\right)=u^{\prime}(t)(x-t)^{\mu-1}-\frac{d}{d x}\left(u(t)(x-t)^{\mu-1}\right)
$$

and

$$
\begin{aligned}
\frac{d}{d t}\left(u(t)(x-t)^{\mu}\right) & =u^{\prime}(t)(x-t)^{\mu}-u(t) \frac{d}{d x}(x-t)^{\mu} \\
& =x u^{\prime}(t)(x-t)^{\mu-1}-t u^{\prime}(t)(x-t)^{\mu-1}-\mu u(t)(x-t)^{\mu-1},
\end{aligned}
$$

we have

$$
\begin{aligned}
I_{c}^{\mu}(\partial u) & =\partial I_{c}^{\mu}(u), \\
I_{c}^{\mu}(\vartheta u) & =(\vartheta-\mu) I_{c}^{\mu}(u) .
\end{aligned}
$$

Remark 2.7. i) The integral (2.37) is naturally well-defined and the equalities (2.43) are valid if $\operatorname{Re} \lambda>1$ and $\lim _{x \rightarrow c} x^{-1} u(x)=0$. Depending on the definition of $I_{c}^{\lambda}$, they are also valid in many cases, which can be usually proved in this paper by analytic continuations with respect to certain parameters (for example, cf. (4.6)). Note that (2.43) is valid if $I_{c}^{\mu}$ is replaced by $\tilde{I}_{c}^{\mu}$ defined by (2.38).

ii) Let $\epsilon$ be a positive number and let $u(x)$ be a holomorphic function on

$$
U_{\epsilon, \theta}^{+}:=\left\{x \in \mathbb{C} ;|x-c|<\epsilon \text { and } e^{-i \theta}(x-c) \notin(-\infty, 0]\right\} .
$$

Suppose that there exists a positive number $\delta$ such that $\left|u(x)(x-c)^{-k}\right|$ is bounded on $\left\{x \in U_{\epsilon, \theta}^{+} ;|\operatorname{Arg}(x-c)-\theta|<\delta\right\}$ for any $k>0$. Note that the function $P u(x)$ also satisfies this estimate for $P \in W[x]$. Then the integration (2.37) is defined along a suitable path $C: \gamma(t)(0 \leq t \leq 1)$ such that $\gamma(0)=c, \gamma(1)=x$ and $|\operatorname{Arg}(\gamma(t)-c)-\theta|<\delta$ for $0<t<\frac{1}{2}$ and the equalities (2.43) are valid.

Example 2.8. We apply additions, middle convolutions and Laplace transformations to the trivial ordinary differential equation

$$
\frac{d u}{d x}=0
$$

which has the solution $u(x) \equiv 1$. 
i) (Gauss hypergeometric equation). Put

$$
\begin{aligned}
P_{\lambda_{1}, \lambda_{2}, \mu}: & =\operatorname{RAd}\left(\partial^{-\mu}\right) \circ \operatorname{RAd}\left(x^{\lambda_{1}}(1-x)^{\lambda_{2}}\right) \partial \\
& =\operatorname{RAd}\left(\partial^{-\mu}\right) \circ \operatorname{R}\left(\partial-\frac{\lambda_{1}}{x}+\frac{\lambda_{2}}{1-x}\right) \\
& =\operatorname{RAd}\left(\partial^{-\mu}\right)\left(x(1-x) \partial-\lambda_{1}(1-x)+\lambda_{2} x\right) \\
& =\operatorname{RAd}\left(\partial^{-\mu}\right)\left(\left(\vartheta-\lambda_{1}\right)-x\left(\vartheta-\lambda_{1}-\lambda_{2}\right)\right) \\
& =\operatorname{Ad}\left(\partial^{-\mu}\right)\left(\left(\vartheta+1-\lambda_{1}\right) \partial-(\vartheta+1)\left(\vartheta-\lambda_{1}-\lambda_{2}\right)\right) \\
& =\left(\vartheta+1-\lambda_{1}-\mu\right) \partial-(\vartheta+1-\mu)\left(\vartheta-\lambda_{1}-\lambda_{2}-\mu\right) \\
& =(\vartheta+\gamma) \partial-(\vartheta+\beta)(\vartheta+\alpha) \\
& =x(1-x) \partial^{2}+(\gamma-(\alpha+\beta+1) x) \partial-\alpha \beta
\end{aligned}
$$

with

$$
\left\{\begin{array}{l}
\alpha=-\lambda_{1}-\lambda_{2}-\mu \\
\beta=1-\mu \\
\gamma=1-\lambda_{1}-\mu
\end{array}\right.
$$

We have a solution

$$
\begin{aligned}
u(x) & =I_{0}^{\mu}\left(x^{\lambda_{1}}(1-x)^{\lambda_{2}}\right) \\
& =\frac{1}{\Gamma(\mu)} \int_{0}^{x} t^{\lambda_{1}}(1-t)^{\lambda_{2}}(x-t)^{\mu-1} d t \\
& =\frac{x^{\lambda_{1}+\mu}}{\Gamma(\mu)} \int_{0}^{1} s^{\lambda_{1}}(1-s)^{\mu-1}(1-x s)^{\lambda_{2}} d s \quad(t=x s) \\
& =\frac{\Gamma\left(\lambda_{1}+1\right) x^{\lambda_{1}+\mu}}{\Gamma\left(\lambda_{1}+\mu+1\right)} F\left(-\lambda_{2}, \lambda_{1}+1, \lambda_{1}+\mu+1 ; x\right) \\
& =\frac{\Gamma\left(\lambda_{1}+1\right) x^{\lambda_{1}+\mu}(1-x)^{\lambda_{2}+\mu}}{\Gamma\left(\lambda_{1}+\mu+1\right)} F\left(\mu, \lambda_{1}+\lambda_{2}+\mu, \lambda_{1}+\mu+1 ; x\right) \\
& =\frac{\Gamma\left(\lambda_{1}+1\right) x^{\lambda_{1}+\mu}(1-x)^{-\lambda_{2}}}{\Gamma\left(\lambda_{1}+\mu+1\right)} F\left(\mu,-\lambda_{2}, \lambda_{1}+\mu+1 ; \frac{x}{x-1}\right)
\end{aligned}
$$

of the Gauss hypergeometric equation $P_{\lambda_{1}, \lambda_{2}, \mu} u=0$ with the Riemann scheme

$$
\left\{\begin{array}{ccc}
x=0 & 1 & \infty \\
0 & 0 & 1-\mu \\
\lambda_{1}+\mu & \lambda_{2}+\mu & -\lambda_{1}-\lambda_{2}-\mu
\end{array} ; x\right\}
$$

which is transformed by the middle convolution $m c_{\mu}$ from the Riemann scheme

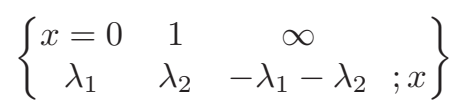

of $x^{\lambda_{1}}(1-x)^{\lambda_{2}}$. Here using Riemann's $P$ symbol, we note that

$$
\begin{aligned}
& P\left\{\begin{array}{ccc}
x=0 & 1 & \infty \\
0 & 0 & 1-\mu \\
\lambda_{1}+\mu & \lambda_{2}+\mu & -\lambda_{1}-\lambda_{2}-\mu
\end{array}\right\} \\
& =x^{\lambda_{1}+\mu} P\left\{\begin{array}{ccc}
x=0 & 1 & \infty \\
-\lambda_{1}-\mu & 0 & \lambda_{1}+1 ; x \\
0 & \lambda_{2}+\mu & -\lambda_{2}
\end{array}\right\} \\
& =x^{\lambda_{1}+\mu}(1-x)^{\lambda_{2}+\mu} P\left\{\begin{array}{ccc}
x=0 & 1 \\
-\lambda_{1}-\mu & -\lambda_{2}-\mu & \lambda_{1}+\lambda_{2}+\mu+1 ; x \\
0 & 0 & \mu
\end{array}\right\}
\end{aligned}
$$




$$
\begin{aligned}
& =x^{\lambda_{1}+\mu} P\left\{\begin{array}{cccc}
x=0 & 1 & \infty & x \\
-\lambda_{1}-\mu & \lambda_{1}+1 & 0 & ; \\
0 & -\lambda_{2} & \lambda_{2}+\mu & x-1
\end{array}\right\} \\
& =x^{\lambda_{1}+\mu}(1-x)^{-\lambda_{2}} P\left\{\begin{array}{cccc}
x=0 & 1 & \infty \\
-\lambda_{1}-\mu & \lambda_{1}+\lambda_{2}+1 & -\lambda_{2} & ; \\
0 & 0 & \mu & x-1
\end{array}\right\} .
\end{aligned}
$$

In general, the Riemann scheme and its relation to $m c_{\mu}$ will be studied in $\$$ and the symbol ' $P$ ' will be omitted for simplicity.

The function $u(x)$ defined by (2.47) corresponds to the characteristic exponent $\lambda_{1}+\mu$ at the origin and depends meromorphically on the parameters $\lambda_{1}, \lambda_{2}$ and $\mu$. The local solutions corresponding to the characteristic exponents $\lambda_{2}+\mu$ at 1 and $-\lambda_{1}-\lambda_{2}-\mu$ at $\infty$ are obtained by replacing $I_{0}^{\mu}$ by $I_{1}^{\mu}$ and $I_{\infty}^{\mu}$, respectively.

When we apply $\operatorname{Ad}\left(x^{\lambda_{1}^{\prime}}(x-1)^{\lambda_{2}^{\prime}}\right)$ to $P_{\lambda_{1}, \lambda_{2}, \mu}$, the resulting Riemann scheme is

$$
\left\{\begin{array}{ccc}
x=0 & 1 & \infty \\
\lambda_{1}^{\prime} & \lambda_{2}^{\prime} & 1-\lambda_{1}^{\prime}-\lambda_{2}^{\prime}-\mu \\
\lambda_{1}+\lambda_{1}^{\prime}+\mu & \lambda_{2}+\lambda_{2}^{\prime}+\mu & -\lambda_{1}-\lambda_{2}-\lambda_{1}^{\prime}-\lambda_{2}^{\prime}-\mu,
\end{array} ; x\right\} .
$$

Putting $\lambda_{1,1}=\lambda_{1}^{\prime}, \lambda_{1,2}=\lambda_{1}+\lambda_{1}^{\prime}+\mu, \lambda_{2,1}=\lambda_{2}^{\prime}, \lambda_{2,2}=\lambda_{2}+\lambda_{2}^{\prime}+\mu, \lambda_{0,1}=$ $1-\lambda_{1}^{\prime}-\lambda_{2}^{\prime}-\mu$ and $\lambda_{0,2}=-\lambda_{1}-\lambda_{2}-\lambda_{1}^{\prime}-\lambda_{2}^{\prime}-\mu$, we have the Fuchs relation

$$
\lambda_{0,1}+\lambda_{0,2}+\lambda_{1,1}+\lambda_{1,2}+\lambda_{2,1}+\lambda_{2,2}=1
$$

and the corresponding operator

$$
\begin{aligned}
P_{\lambda}= & x^{2}(x-1)^{2} \partial^{2}+x(x-1)\left(\left(\lambda_{0,1}+\lambda_{0,2}+1\right) x+\lambda_{1,1}+\lambda_{1,2}-1\right) \partial \\
& +\lambda_{0,1} \lambda_{0,2} x^{2}+\left(\lambda_{2,1} \lambda_{2,2}-\lambda_{0,1} \lambda_{0,2}-\lambda_{1,1} \lambda_{1,2}\right) x+\lambda_{1,1} \lambda_{1,2}
\end{aligned}
$$

has the Riemann scheme

$$
\left\{\begin{array}{ccc}
x=0 & 1 & \infty \\
\lambda_{0,1} & \lambda_{1,1} & \lambda_{2,1} \\
\lambda_{0,2} & \lambda_{1,2} & \lambda_{2,2}
\end{array}\right\}
$$

By the symmetry of the transposition $\lambda_{j, 1}$ and $\lambda_{j, 2}$ for each $j$, we have integral representations of other local solutions.

ii) (Airy equations). For a positive integer $m$ we put

$$
\begin{aligned}
P_{m} & :=\mathrm{L} \circ \operatorname{Ad}\left(e^{\frac{x^{m+1}}{m+1}}\right) \partial \\
& =\mathrm{L}\left(\partial-x^{m}\right)=x-(-\partial)^{m} .
\end{aligned}
$$

Thus the equation

$$
\frac{d^{m} u}{d x^{m}}-(-1)^{m} x u=0
$$

has a solution

$$
u_{j}(x)=\int_{C_{j}} \exp \left(\frac{z^{m+1}}{m+1}-x z\right) d z \quad(0 \leq j \leq m),
$$

where the path $C_{j}$ of the integration is

$$
C_{j}: z(t)=e^{\frac{(2 j-1) \pi \sqrt{-1}}{m+1}-t}+e^{\frac{(2 j+1) \pi \sqrt{-1}}{m+1}+t} \quad(-\infty<t<\infty) .
$$

Here we note that $u_{0}(x)+\cdots+u_{m}(x)=0$. The equation has the symmetry under the rotation $x \mapsto e^{\frac{2 \pi \sqrt{-1}}{m+1}} x$. 
iii) (Jordan-Pochhammer equation). For $\left\{c_{1}, \ldots, c_{p}\right\} \in \mathbb{C} \backslash\{0\}$ put

$$
\begin{aligned}
P_{\lambda_{1}, \ldots, \lambda_{p}, \mu}: & =\operatorname{RAd}\left(\partial^{-\mu}\right) \circ \operatorname{RAd}\left(\prod_{j=1}^{p}\left(1-c_{j} x\right)^{\lambda_{j}}\right) \partial \\
& =\operatorname{RAd}\left(\partial^{-\mu}\right) \circ \mathrm{R}\left(\partial+\sum_{j=1}^{p} \frac{c_{j} \lambda_{j}}{1-c_{j} x}\right) \\
& =\operatorname{RAd}\left(\partial^{-\mu}\right)\left(p_{0}(x) \partial+q(x)\right) \\
& =\partial^{-\mu+p-1}\left(p_{0}(x) \partial+q(x)\right) \partial^{\mu}=\sum_{k=0}^{p} p_{k}(x) \partial^{p-k}
\end{aligned}
$$

with

$$
\begin{aligned}
& p_{0}(x)=\prod_{j=1}^{p}\left(1-c_{j} x\right), \quad q(x)=p_{0}(x) \sum_{j=1}^{p} \frac{c_{j} \lambda_{j}}{1-c_{j} x}, \\
& p_{k}(x)=\left(\begin{array}{c}
-\mu+p-1 \\
k
\end{array}\right) p_{0}^{(k)}(x)+\left(\begin{array}{c}
-\mu+p-1 \\
k-1
\end{array}\right) q^{(k-1)}(x), \\
& \left(\begin{array}{l}
\alpha \\
\beta
\end{array}\right):=\frac{\Gamma(\alpha+1)}{\Gamma(\beta+1) \Gamma(\alpha-\beta+1)} \quad(\alpha, \beta \in \mathbb{C}) .
\end{aligned}
$$

We have solutions

$$
u_{j}(x)=\frac{1}{\Gamma(\mu)} \int_{\frac{1}{c_{j}}}^{x} \prod_{\nu=1}^{p}\left(1-c_{\nu} t\right)^{\lambda_{\nu}}(x-t)^{\mu-1} d t \quad\left(j=0,1, \ldots, p, c_{0}=0\right)
$$

of the Jordan-Pochhammer equation $P_{\lambda_{1}, \ldots, \lambda_{p}, \mu} u=0$ with the Riemann scheme

$$
\left\{\begin{array}{cccc}
x=\frac{1}{c_{1}} & \cdots & \frac{1}{c_{p}} & \infty \\
{[0]_{(p-1)}} & \cdots & {[0]_{(p-1)}} & {[1-\mu]_{(p-1)}} \\
\lambda_{1}+\mu & \cdots & \lambda_{p}+\mu & -\lambda_{1}-\cdots-\lambda_{p}-\mu
\end{array} ; x\right\} .
$$

Here and hereafter we use the notation

$$
[\lambda]_{(k)}:=\left(\begin{array}{c}
\lambda \\
\lambda+1 \\
\vdots \\
\lambda+k-1
\end{array}\right)
$$

for a complex number $\lambda$ and a non-negative integer $k$. If the component $[\lambda]_{(k)}$ is appeared in a Riemann scheme, it means the corresponding local solutions with the exponents $\lambda+\nu$ for $\nu=0, \ldots, k-1$ have a semisimple local monodromy when $\lambda$ is generic.

2.4. Ordinary differential equations. We will study the ordinary differential equation

$$
\mathcal{M}: P u=0
$$

with an element $P \in W(x ; \xi)$ in this paper. The solution $u(x, \xi)$ of $\mathcal{M}$ is at least locally defined for $x$ and $\xi$ and holomorphically or meromorphically depends on $x$ and $\xi$. Hence we may replace $P$ by R $P$ and we similarly choose $P$ in $W[x ; \xi]$.

We will identify $\mathcal{M}$ with the left $W(x ; \xi)$-module $W(x ; \xi) / W(x ; \xi) P$. Then we may consider (2.58) as the fundamental relation of the generator $u$ of the module $\mathcal{M}$.

The results in this subsection are standard and well-known but for our convenience we briefly review them. First note that $W(x ; \xi)$ is a (left) Euclidean ring: 
Let $P, Q \in W(x ; \xi)$ with $P \neq 0$. Then there uniquely exists $R, S \in W(x ; \xi)$ such that

$$
Q=S P+R \quad(\operatorname{ord} R<\operatorname{ord} P) .
$$

Hence we note that $\operatorname{dim}_{\mathbb{C}(x, \xi)}(W(x ; \xi) / W(x ; \xi) P)=\operatorname{ord} P$. We get $R$ and $S$ in (2.59) by a simple algorithm as follows. Put

$$
P=a_{n} \partial^{n}+\cdots+a_{1} \partial+a_{0} \text { and } Q=b_{m} \partial^{m}+\cdots+b_{1} \partial+b_{0}
$$

with $a_{n} \neq 0, b_{m} \neq 0$. Here $a_{n}, b_{m} \in \mathbb{C}(x, \xi)$. The division 2.59$)$ is obtained by the induction on ord $Q$. If ord $P>\operatorname{ord} Q,(2.59)$ is trivial with $S=0$. If $\operatorname{ord} P \leq \operatorname{ord} Q$, (2.59) is reduced to the equality $Q^{\prime}=S^{\prime} P+R$ with $Q^{\prime}=Q-a_{n}^{-1} b_{m} \partial^{m-n} P$ and $S^{\prime}=S-a_{n}^{-1} b_{m} \partial^{m-n}$ and then we have $S^{\prime}$ and $R$ satisfying $Q^{\prime}=S^{\prime} P+R$ by the induction because ord $Q^{\prime}<\operatorname{ord} Q$. The uniqueness of (2.59) is clear by comparing the highest order terms of (2.59) in the case when $Q=0$.

By the standard Euclid algorithm using the division (2.59) we have $M, N \in$ $W(x ; \xi)$ such that

$$
M P+N Q=U, P \in W(x ; \xi) U \text { and } Q \in W(x ; \xi) U .
$$

Hence in particular any left ideal of $W(x ; \xi)$ is generated by a single element of $W[x ; \xi]$, namely, $W(x ; \xi)$ is a principal ideal domain.

Definition 2.9. The operators $P$ and $Q$ in $W(x ; \xi)$ are defined to be mutually prime if one of the following equivalent conditions is valid.

$$
W(x ; \xi) P+W(x ; \xi) Q=W(x ; \xi),
$$

there exists $R \in W(x ; \xi)$ satisfying $R Q u=u$ for the equation $P u=0$,

$$
\left\{\begin{array}{l}
\text { the simultaneous equation } P u=Q u=0 \text { has not a non-zero solution } \\
\text { for a generic value of } \xi
\end{array}\right.
$$

Moreover we have the following.

$$
\text { Any left } W(x ; \xi) \text {-module } \mathcal{R} \text { with } \operatorname{dim}_{\mathbb{C}(x, \xi)} \mathcal{R}<\infty \text { is cyclic, }
$$

namely, it is generated by a single element. Hence any system of ordinary differential equations is isomorphic to a single differential equation under the algebra $W(x ; \xi)$.

To prove (2.65) it is sufficient to show that the direct sum $\mathcal{M} \oplus \mathcal{N}$ of $\mathcal{M}: P u=0$ and $\mathcal{N}: Q v=0$ is cyclic. In fact $M \oplus \mathcal{N}=W(x ; \xi) w$ with $w=u+(x-c)^{n} v \in$ $\mathcal{M} \oplus \mathcal{N}$ and $n=\operatorname{ord} P$ if $c \in \mathbb{C}$ is generic. For the proof we have only to show $\operatorname{dim}_{\mathbb{C}(x, \xi)} W(x ; \xi) w \geq m+n$ and we may assume that $P$ and $Q$ are in $W[x ; \xi]$ and they are of the form (2.60). Fix $\xi$ generically and we choose $c \in \mathbb{C}$ such that $a_{n}(c) b_{m}(c) \neq 0$. Since the function space $V=\left\{\phi(x)+(x-c)^{n} \varphi(x) ; P \phi(x)=\right.$ $Q \varphi(x)=0\}$ is of dimension $m+n$ in a neighborhood of $x=c, \operatorname{dim}_{W(x ; \xi)} W(x ; \xi) w \geq$ $m+n$ because the relation $R w=0$ for an operator $R \in W(x ; \xi)$ implies $R \psi(x)=0$ for $\psi \in V$.

Thus we have the following standard definition.

Definition 2.10. Fix $P \in W(x ; \xi)$ with ord $P>0$. The equation 2.58 is irreducible if and only if one of the following equivalent conditions is valid.

The left $W(x ; \xi)$-module $\mathcal{M}$ is simple.

The left $W(x ; \xi)$-ideal $W(x ; \xi) P$ is maximal.

$P=Q R$ with $Q, R \in W(x ; \xi)$ implies ord $Q \cdot \operatorname{ord} R=0$.

$\forall Q \notin W(x ; \xi) P, \exists M, N \in W(x ; \xi)$ satisfying $M P+N Q=1$.

$$
\left\{\begin{array}{l}
S T \in W(x ; \xi) P \text { with } S, T \in W(x ; \xi) \text { and ord } S<\text { ord } P \\
\Rightarrow S=0 \text { or } T \in W(x ; \xi) P .
\end{array}\right.
$$


The equivalence of the above conditions is standard and easily proved. The last condition may be a little non-trivial.

Suppose (2.70) and $P=Q R$ and ord $Q \cdot \operatorname{ord} R \neq 0$. Then $R \notin W(x ; \xi) P$ and therefore $Q=0$, which contradicts to $P=Q R$. Hence (2.70) implies (2.68).

Suppose (2.66), 2.69), $S T \in W(x ; \xi) P$ and $T \notin W(x ; \xi) P$. Then there exists $P^{\prime}$ such that $\{J \in W(x ; \xi) ; J T \in W(x ; \xi) P\}=W(x ; \xi) P^{\prime}$, ord $P^{\prime}=$ ord $P$ and moreover $P^{\prime} v=0$ is also simple. Since $S v=0$ with ord $S<\operatorname{ord} P^{\prime}$, we have $S=0$.

In general, a system of ordinary differential equations is defined to be irreducible if it is simple as a left $W(x ; \xi)$-module.

Remark 2.11. Suppose the equation $\mathcal{M}$ given in 2.58 is irreducible.

i) Let $u(x, \xi)$ be a non-zero solution of $\mathcal{M}$, which is locally defined for the variables $x$ and $\xi$ and meromorphically depends on $(x, \xi)$. If $S \in W[x ; \xi]$ satisfies $S u(x, \xi)=0$, then $S \in W(x ; \xi) P$. Therefore $u(x, \xi)$ determines $\mathcal{M}$.

ii) Suppose ord $P>1$. Fix $R \in W(x ; \xi)$ such that ord $R<\operatorname{ord} P$ and $R \neq 0$. For $Q \in W(x ; \xi)$ and a positive integer $m$, the condition $R^{m} Q u=0$ is equivalent to $Q u=0$. Hence for example, if $Q_{1} u+\partial^{m} Q_{2} u=0$ with certain $Q_{j} \in W(x ; \xi)$, we will allow the expression $\partial^{-m} Q_{1} u+Q_{2} u=0$ and $\partial^{-m} Q_{1} u(x, \xi)+Q_{2} u(x, \xi)=0$.

iii) For $T \notin W(x ; \xi) P$ we construct a differential equation $Q v=0$ satisfied by $v=T u$ as follows. Put $n=\operatorname{ord} P$. We have $R_{j} \in W(x ; \xi)$ such that $\partial^{j} T u=R_{j} u$ with ord $R_{j}<$ ord $P$. Then there exist $b_{0}, \ldots, b_{n} \in \mathbb{C}(x, \xi)$ such that $b_{n} R_{n}+\cdots+$ $b_{1} R_{1}+b_{0} R_{0}=0$. Then $Q=b_{n} \partial^{n}+\cdots+b_{1} \partial+b_{0}$.

2.5. Okubo normal form and Schlesinger canonical form. In this subsection we briefly explain the interpretation of Katz's middle convolution (cf. [Kz]) by DR and its relation to our fractional operations.

For constant square matrices $T$ and $A$ of size $n^{\prime}$, the ordinary differential equation

$$
\left(x I_{n^{\prime}}-T\right) \frac{d u}{d x}=A u
$$

is called Okubo normal form of Fuchsian system when $T$ is a diagonal matrix. Then

$$
m c_{\mu}\left(\left(x I_{n^{\prime}}-T\right) \partial-A\right)=\left(x I_{n^{\prime}}-T\right) \partial-\left(A+\mu I_{n^{\prime}}\right)
$$

for generic $\mu \in \mathbb{C}$, namely, the system is transformed into

$$
\left(x I_{n^{\prime}}-T\right) \frac{d u_{\mu}}{d x}=\left(A+\mu I_{n^{\prime}}\right) u_{\mu}
$$

by the operation $m c_{\mu}$. Hence for a solution $u(x)$ of (2.71), the Euler transformation $u_{\mu}(x)=I_{c}^{\mu}(u)$ of $u(x)$ satisfies (2.73).

For constant square matrices $A_{j}$ of size $m$ and the Schlesinger canonical form

$$
\frac{d v}{d x}=\sum_{j=1}^{p} \frac{A_{j}}{x-c_{j}} v
$$

of a Fuchsian system of the Riemann sphere, we have

$$
\left.\frac{d u}{d x}=\sum_{j=1}^{p} \frac{\tilde{A}_{j}}{x-c_{j}} u, \quad \tilde{A}_{j}:=j\right)\left(\begin{array}{lll}
A_{1} & \cdots & A_{p}
\end{array}\right) \text { and } u:=\left(\begin{array}{c}
\frac{v}{x-c_{1}} \\
\vdots \\
\frac{v}{x-c_{p}}
\end{array}\right) .
$$

Here $\tilde{A}_{j}$ are square matrices of size $p m$. The addition $\operatorname{Ad}\left(\left(x-c_{k}\right)^{\mu_{k}}\right)$ transforms $A_{j}$ into $A_{j}+\mu_{k} \delta_{j, k} I_{m}$ for $j=1, \ldots, p$ in the system (2.74). Putting

$$
A=\tilde{A}_{1}+\cdots+\tilde{A}_{p} \text { and } T=\left(\begin{array}{ccc}
c_{1} I_{n} & & \\
& \ddots & \\
& & c_{p} I_{n}
\end{array}\right),
$$


the equation (2.75) is equivalent to (2.71) with $n^{\prime}=p m$. Define square matrices of size $n^{\prime}$ by

$$
\begin{aligned}
\tilde{A} & :=\left(\begin{array}{llll}
A_{1} & & \\
& \ddots & \\
& & A_{p}
\end{array}\right), \\
\tilde{A}_{j}(\mu) & :=j)\left(\begin{array}{ccccccc}
j & & \\
A_{1} & \cdots & A_{j-1} & A_{j}+\mu & A_{j+1} & \cdots & A_{p}
\end{array}\right) .
\end{aligned}
$$

Then ker $\tilde{A}$ and $\operatorname{ker}(A+\mu)$ are invariant under $\tilde{A}_{j}(\mu)$ for $j=1, \ldots, p$ and therefore $\tilde{A}_{j}(\mu)$ induce endomorphisms of $V:=\mathbb{C}^{p m} /(\operatorname{ker} \tilde{A}+\operatorname{ker}(A+\mu))$, which correspond to square matrices of size $N:=\operatorname{dim} V$, which we put $\bar{A}_{j}(\mu)$, respectively, under a fixed basis of $V$. Then the middle convolution $m c_{\mu}$ of (2.74) is the system

$$
\frac{d w}{d x}=\sum_{j=1}^{p} \frac{\bar{A}_{j}(\mu)}{x-c_{j}} w
$$

of $\operatorname{rank} N$, which is defined and studied by DR, DR2. Here ker $\tilde{A} \cap \operatorname{ker}(A+\mu)=\{0\}$ if $\mu \neq 0$.

We define another realization of the middle convolution as in [05, §2]. Suppose $\mu \neq 0$. The square matrices of size $n^{\prime}$

$$
\left.A_{j}^{\vee}(\mu):=j\right)\left(\begin{array}{c}
j \\
A_{1} \\
\vdots \\
A_{j}+\mu \\
\vdots \\
A_{p}
\end{array}\right) \text { and } A^{\vee}(\mu):=A_{1}^{\vee}(\mu)+\cdots+A_{p}^{\vee}(\mu)
$$

satisfy

$$
\begin{aligned}
\tilde{A}\left(A+\mu I_{n^{\prime}}\right) & =A^{\vee}(\mu) \tilde{A}=\left(A_{i} A_{j}+\mu \delta_{i, j} A_{i}\right)_{\substack{1 \leq i \leq p \\
1 \leq j \leq p}} \in M\left(n^{\prime}, \mathbb{C}\right), \\
\tilde{A}\left(A+\mu I_{n^{\prime}}\right) \tilde{A}_{j}(\mu) & =A_{j}^{\vee}(\mu) \tilde{A}\left(A+\mu I_{n^{\prime}}\right) .
\end{aligned}
$$

Hence $w^{\vee}:=\tilde{A}\left(A+\mu I_{n^{\prime}}\right) u$ satisfies

$$
\begin{aligned}
\frac{d w^{\vee}}{d x} & =\sum_{j=1}^{p} \frac{A_{j}^{\vee}(\mu)}{x-c_{j}} w^{\vee}, \\
\sum_{j=1}^{p} \frac{A_{j}^{\vee}(\mu)}{x-c_{j}} & =\left(\frac{A_{i}+\mu \delta_{i, j} I_{m}}{x-c_{j}}\right)_{\substack{1 \leq i \leq p, 1 \leq j \leq p}}
\end{aligned}
$$

and $\tilde{A}\left(A+\mu I_{n^{\prime}}\right)$ induces the isomorphism

$$
\tilde{A}\left(A+\mu I_{n^{\prime}}\right): V=\mathbb{C}^{n^{\prime}} /\left(\mathcal{K}+\mathcal{L}_{\mu}\right) \stackrel{\sim}{\rightarrow} V^{\vee}:=\operatorname{Im} \tilde{A}\left(A+\mu I_{n^{\prime}}\right) \subset \mathbb{C}^{n^{\prime}} .
$$

Hence putting $\bar{A}_{j}^{\vee}(\mu):=\left.A_{j}^{\vee}(\mu)\right|_{V^{\vee}}$, the system (2.78) is isomorphic to the system

$$
\frac{d w^{\vee}}{d x}=\sum_{j=1}^{p} \frac{\bar{A}_{j}^{\vee}(\mu)}{x-c_{j}} w^{\vee}
$$


of rank $N$, which can be regarded as a middle convolution $m c_{\mu}$ of (2.74). Here

$$
w^{\vee}=\left(\begin{array}{c}
w_{1}^{\vee} \\
\vdots \\
w_{p}^{\vee}
\end{array}\right), \quad w_{j}^{\vee}=\sum_{\nu=1}^{p}\left(A_{j} A_{\nu}+\mu \delta_{j, \nu}\right)\left(u_{\mu}\right)_{\nu} \quad(j=1, \ldots, p)
$$

and if $v(x)$ is a solution of (2.74), then

$$
w^{\vee}(x)=\left(\sum_{\nu=1}^{p}\left(A_{j} A_{\nu}+\mu \delta_{j, \nu}\right) I_{c}^{\mu}\left(\frac{v(x)}{x-c_{\nu}}\right)\right)_{j=1, \ldots, p}
$$

satisfies (2.84).

Since any non-zero homomorphism between irreducible $W(x)$-modules is an isomorphism, we have the following remark (cf. $\S 2.4$ and $\$$ 国).

Remark 2.12. Suppose that the systems (2.74) and (2.84) are irreducible. Moreover suppose the system (2.74) is isomorphic to a single Fuchsian differential equation $P \tilde{u}=0$ as left $W(x)$-modules and the equation $m c_{\mu}(P) \tilde{w}=0$ is also irreducible. Then the system (2.84) is isomorphic to the single equation $m c_{\mu}(P) \tilde{w}=0$ because the differential equation satisfied by $I_{c}^{\mu}(\tilde{u}(x))$ is isomorphic to that of $I_{c}^{\mu}(Q \tilde{u}(x))$ for a non-zero solution $v(x)$ of $P \tilde{u}=0$ and an operator $Q \in W(x)$ with $Q \tilde{u}(x) \neq 0$ (cf. s; , Remark 7.4 iii) and Proposition 8.12).

In particular if the systems are rigid and their spectral parameters are generic, all the assumptions here are satisfied (cf. Remark 6.17 ii) and Corollary 12.12).

Yokoyama [Yo2] defines extension and restriction operations among the systems of differential equations of Okubo normal form. The relation of Yokoyama's operations to Katz's operations is clarified by [07, which shows that they are equivalent from the view point of the construction and the reduction of systems of Fuchsian differential equations.

\section{Confluences}

3.1. Regular singularities. In this subsection we review fundamental facts related to the regular singularities of the ordinary differential equations.

3.1.1. Characteristic exponents. The ordinary differential equation

$$
a_{n}(x) \frac{d^{n} u}{d x^{n}}+a_{n-1}(x) \frac{d^{n-1} u}{d x^{n-1}}+\cdots+a_{1}(x) \frac{d u}{d x}+a_{0}(x) u=0
$$

of order $n$ with meromorphic functions $a_{j}(x)$ defined in a neighborhood of $c \in \mathbb{C}$ has a singularity at $x=c$ if the function $\frac{a_{j}(x)}{a_{n}(x)}$ has a pole at $x=c$ for a certain $j$. The singular point $x=c$ of the equation is a regular singularity if it is a removable singularity of the functions $b_{j}(x):=(x-c)^{n-j} a_{j}(x) a_{n}(x)^{-1}$ for $j=0, \ldots, n$. In this case $b_{j}(c)$ are complex numbers and the $n$ roots of the indicial equation

$$
\sum_{j=0}^{n} b_{j}(c) s(s-1) \cdots(s-j+1)=0
$$

are called the charactersitic exponents of (3.1) at $c$.

Let $\left\{\lambda_{1}, \ldots, \lambda_{n}\right\}$ be the set of these characteristic exponents at $c$.

If $\lambda_{j}-\lambda_{1} \notin \mathbb{Z}_{>0}$ for $1<j \leq n$, then (3.1) has a unique solution $(x-c)^{\lambda_{1}} \phi_{1}(x)$ with a holomorphic function $\phi_{1}(x)$ in a neighborhood of $c$ satisfying $\phi_{1}(c)=1$.

Definition 3.1. The regular singularity and the characteristic exponents for the differential operator

$$
P=a_{n}(x) \frac{d^{n}}{d x^{n}}+a_{n-1}(x) \frac{d^{n-1}}{d x^{n-1}}+\cdots+a_{1}(x) \frac{d}{d x}+a_{0}(x)
$$


are defined by those of the equation (3.1), respectively. Suppose $P$ has a regular singularity at $c$. We say $P$ is normalized at $c$ if $a_{n}(x)$ is holomorphic at $c$ and

$$
a_{n}(c)=a_{n}^{(1)}(c)=\cdots=a_{n}^{(n-1)}(c)=0 \text { and } a_{n}^{(n)}(c) \neq 0 .
$$

In this case $a_{j}(x)$ are analytic and have zeros of order at least $j$ at $x=c$ for $j=0, \ldots, n-1$.

3.1.2. Local solutions. The ring of convergent power series at $x=c$ is denoted by $\mathcal{O}_{c}$ and for a complex number $\mu$ and a non-negative integer $m$ we put

$$
\mathcal{O}_{c}(\mu, m):=\bigoplus_{\nu=0}^{m}(x-c)^{\mu} \log ^{\nu}(x-c) \mathcal{O}_{c}
$$

Let $P$ be a differential operator of order $n$ which has a regular singularity at $x=c$ and let $\left\{\lambda_{1}, \cdots, \lambda_{n}\right\}$ be the corresponding characteristic exponents. Suppose $P$ is normalized at $c$. If a complex number $\mu$ satisfies $\lambda_{j}-\mu \notin\{0,1,2, \ldots\}$ for $j=1, \ldots, n$, then $P$ defines a linear bijective map

$$
P: \mathcal{O}_{c}(\mu, m) \stackrel{\sim}{\rightarrow} \mathcal{O}_{c}(\mu, m)
$$

for any non-negative integer $m$.

Let $\hat{\mathcal{O}}_{c}$ be the ring of formal power series $\sum_{j=0}^{\infty} a_{j}(x-c)^{j}\left(a_{j} \in \mathbb{C}\right)$ of $x$ at $c$. For a domain $U$ of $\mathbb{C}$ we denote by $\mathcal{O}(U)$ the ring of holomorphic functions on $U$. Put

$$
B_{r}(c):=\{x \in \mathbb{C} ;|x-c|<r\}
$$

for $r>0$ and

$$
\begin{aligned}
\hat{\mathcal{O}}_{c}(\mu, m) & :=\bigoplus_{\nu=0}^{m}(x-c)^{\mu} \log ^{\nu}(x-c) \hat{\mathcal{O}}_{c}, \\
\mathcal{O}_{B_{r}(c)}(\mu, m) & :=\bigoplus_{\nu=0}^{m}(x-c)^{\mu} \log ^{\nu}(x-c) \mathcal{O}_{B_{r}(c)} .
\end{aligned}
$$

Then $\mathcal{O}_{B_{r}(c)}(\mu, m) \subset \mathcal{O}_{c}(\mu, m) \subset \hat{\mathcal{O}}_{c}(\mu, m)$.

Suppose $a_{j}(x) \in \mathcal{O}\left(B_{r}(c)\right)$ and $a_{n}(x) \neq 0$ for $x \in B_{r}(c) \backslash\{c\}$ and moreover $\lambda_{j}-\mu \notin\{0,1,2, \ldots\}$, we have

$$
\begin{aligned}
& P: \mathcal{O}_{B_{r}(c)}(\mu, m) \stackrel{\sim}{\rightarrow} \mathcal{O}_{B_{r}(c)}(\mu, m), \\
& P: \hat{\mathcal{O}}_{c}(\mu, m) \stackrel{\sim}{\rightarrow} \hat{\mathcal{O}}_{c}(\mu, m) .
\end{aligned}
$$

The proof of these results are reduced to the case when $\mu=m=c=0$ by the translation $x \mapsto x-c$, the operation $\operatorname{Ad}\left(x^{-\mu}\right)$, and the fact $P\left(\sum_{j=0}^{m} f_{j}(x) \log ^{j} x\right)=$ $\left(P f_{m}(x)\right) \log ^{j} x+\sum_{j=0}^{m-1} \phi_{j}(x) \log ^{j} x$ with suitable $\phi_{j}(x)$ and moreover we may assume

$$
\begin{gathered}
P=\prod_{j=0}^{n}\left(\vartheta-\lambda_{j}\right)-x R(x, \vartheta), \\
x R(x, \vartheta)=x \sum_{j=0}^{n-1} r_{j}(x) \vartheta^{j} \quad\left(r_{j}(x) \in \mathcal{O}\left(B_{r}(c)\right)\right) .
\end{gathered}
$$

When $\mu=m=0,(3.11)$ is easy and $(3.10)$ and hence (3.6) are also easily proved by the method of majorant series (for example, cf. [O1]).

For the differential operator

$$
Q=\frac{d^{n}}{d x^{n}}+b_{n-1}(x) \frac{d^{n-1}}{d x^{n-1}}+\cdots+b_{1}(x) \frac{d}{d x}+b_{0}(x)
$$


with $b_{j}(x) \in \mathcal{O}\left(B_{r}(c)\right)$, we have a bijection

$$
\begin{aligned}
Q: \mathcal{O}\left(B_{r}(c)\right) & \stackrel{\sim}{\rightarrow} \mathcal{O}\left(B_{r}(c)\right) \oplus \mathbb{C}^{n} \\
\Psi & \Psi \\
u(x) & \mapsto \quad P u(x) \oplus\left(u^{(j)}(c)\right)_{0 \leq j \leq n-1}
\end{aligned}
$$

because $Q(x-c)^{n}$ has a regular singularity at $x=c$ and the characteristic exponents are $-1,-2, \ldots,-n$ and hence (3.10) assures that for any $g(x) \in \mathbb{C}[x]$ and $f(x) \in$ $\mathcal{O}\left(B_{r}(c)\right)$ there uniquely exists $v(x) \in \mathcal{O}\left(B_{r}(c)\right)$ such that $Q(x-c)^{n} v(x)=f(x)-$ $Q g(x)$.

If $\lambda_{\nu}-\lambda_{1} \notin \mathbb{Z}_{>0}$, the characteristic exponents of $R:=\operatorname{Ad}\left((x-c)^{-\lambda_{1}-1}\right) P$ at $x=c$ are $\lambda_{\nu}-\lambda_{1}-1$ for $\nu=1, \ldots, n$ and therefore $R=S(x-c)$ with a differential operator $R$ whose coefficients are in $\mathcal{O}\left(B_{r}(c)\right)$. Then there exists $v_{1}(x) \in \mathcal{O}\left(B_{r}(c)\right)$ such that $-S 1=S(x-c) v_{1}(x)$, which means $P\left((x-c)^{\lambda_{1}}\left(1+(x-c) v_{1}(x)\right)\right)=0$. Hence if $\lambda_{i}-\lambda_{j} \notin \mathbb{Z}$ for $1 \leq i<j \leq n$, we have solutions $u_{\nu}(x)$ of $P u=0$ such that

$$
u_{\nu}(x)=(x-c)^{\lambda_{\nu}} \phi_{\nu}(x)
$$

with suitable $\phi_{\nu} \in \mathcal{O}\left(B_{r}(c)\right)$ satisfying $\phi_{\nu}(c)=1$ for $\nu=1, \ldots, n$.

Put $k=\#\left\{\nu ; \lambda_{\nu}=\lambda_{1}\right\}$ and $m=\#\left\{\nu ; \lambda_{\nu}-\lambda_{1} \in \mathbb{Z}_{\geq 0}\right\}$. Then we have solutions $u_{\nu}(x)$ of $P u=0$ for $\nu=1, \ldots, k$ such that

$$
u_{\nu}(x)-(x-c)^{\lambda_{1}} \log ^{\nu-1}(x-c) \in \mathcal{O}_{B_{r}(c)}\left(\lambda_{1}+1, m-1\right) .
$$

If $\mathcal{O}_{B_{r}(c)}$ is replaced by $\hat{\mathcal{O}}_{c}$, the solution

$u_{\nu}(x)=(x-c)^{\lambda_{1}} \log ^{\nu-1}(x-c)+\sum_{i=1}^{\infty} \sum_{j=0}^{m-1} c_{\nu, i, j}(x-c)^{\lambda_{1}+i} \log ^{j}(x-c) \in \hat{\mathcal{O}}_{c}\left(\lambda_{1}, m-1\right)$

is constructed by inductively defining $c_{\nu, i, j} \in \mathbb{C}$. Since

$$
\begin{gathered}
P\left(\sum_{i=N+1}^{\infty} \sum_{j=0}^{m-1} c_{\nu, i, j}(x-c)^{\lambda_{1}+i} \log ^{j}(x-c)\right)=-P\left((x-c)^{\lambda_{1}} \log ^{\nu-1}(x-c)\right. \\
\left.+\sum_{i=1}^{N} c_{\nu, i, j}(x-c)^{\lambda_{1}+i} \log ^{j}(x-c)\right) \in \mathcal{O}_{B_{r}(c)}\left(\lambda_{1}+N, m-1\right)
\end{gathered}
$$

for an integer $N$ satisfying $\operatorname{Re}\left(\lambda_{\ell}-\lambda_{1}\right)<N$ for $\ell=1, \ldots, n$, we have

$$
\sum_{i=N+1}^{\infty} \sum_{j=0}^{m-1} c_{\nu, i, j}(x-c)^{\lambda_{1}+i} \log ^{j}(x-c) \in \mathcal{O}_{B_{r}(c)}\left(\lambda_{1}+N, m-1\right)
$$

because of (3.10) and 3.11), which means $u_{\nu}(x) \in \mathcal{O}_{B_{r}(c)}\left(\lambda_{1}, m\right)$.

3.1.3. Fuchsian differential equations. The regular singularity at $\infty$ is similarly defined by that at the origin under the coordinate transformation $x \mapsto \frac{1}{x}$. When $P \in W(x)$ and the singular points of $P$ in $\overline{\mathbb{C}}:=\mathbb{C} \cup\{\infty\}$ are all regular singularities, the operator $P$ and the equation $P u=0$ are called Fuchsian. Let $\overline{\mathbb{C}}^{\prime}$ be the subset of $\overline{\mathbb{C}}$ deleting singular points $c_{0}, \ldots, c_{p}$ from $\overline{\mathbb{C}}$. Then the solutions of the equation $P u=0$ defines a map

$$
\mathcal{F}: \overline{\mathbb{C}}^{\prime} \supset U:(\text { simply connected domain }) \mapsto \mathcal{F}(U) \subset \mathcal{O}(U)
$$

by putting $\mathcal{F}(U):=\{u(x) \in \mathcal{O}(U) ; P u(x)=0\}$. Put

$$
U_{j, \epsilon, R}= \begin{cases}\left\{x=c_{j}+r e^{\sqrt{-1} \theta} ; 0<r<\epsilon, R<\theta<R+2 \pi\right\} & \left(c_{j} \neq \infty\right) \\ \left\{x=r e^{\sqrt{-1} \theta} ; r>\epsilon^{-1}, R<\theta<R+2 \pi\right\} & \left(c_{j}=\infty\right) .\end{cases}
$$


For simply connected domains $U, V \subset \overline{\mathbb{C}}^{\prime}$, the map $\mathcal{F}$ satisfies

$$
\begin{aligned}
& \mathcal{F}(U) \subset \mathcal{O}(U) \text { and } \operatorname{dim} \mathcal{F}(U)=n, \\
& V \subset U \Rightarrow \mathcal{F}(V)=\left.\mathcal{F}(U)\right|_{V},
\end{aligned}
$$

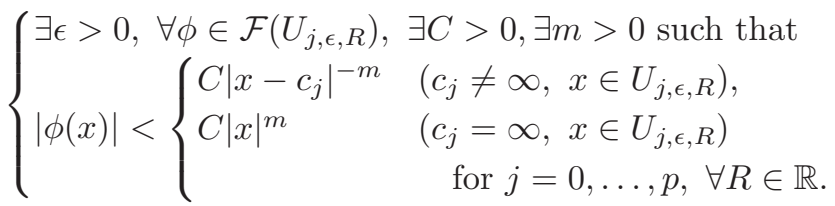

Then we have the bijection

$$
\begin{aligned}
\left\{\partial^{n}+\sum_{j=0}^{n-1} a_{j}(x) \partial^{j} \in W(x): \text { Fuchsian }\right\} & \stackrel{\sim}{\rightarrow} \quad\{\mathcal{F} \text { satisfying } \underset{\Psi}{(3.16)-(3.18)}\} \\
P & \mapsto \quad\{U \mapsto\{u \in \mathcal{O}(U) ; P u=0\}\} .
\end{aligned}
$$

Here if $\mathcal{F}(U)=\sum_{j=1}^{n} \mathbb{C} \phi_{j}(x)$,

$$
a_{j}(x)=(-1)^{n-j} \frac{\operatorname{det} \Phi_{j}}{\operatorname{det} \Phi_{n}} \text { with } \Phi_{j}=\left(\begin{array}{ccc}
\phi_{1}^{(0)}(x) & \cdots & \phi_{n}^{(0)}(x) \\
\vdots & \vdots & \vdots \\
\phi_{1}^{(j-1)}(x) & \cdots & \phi_{n}^{(j-1)}(x) \\
\phi_{1}^{(j+1)}(x) & \cdots & \phi_{n}^{(j+1)}(x) \\
\vdots & \vdots & \vdots \\
\phi_{1}^{(n)}(x) & \cdots & \phi_{n}^{(n)}(x)
\end{array}\right) .
$$

The elements $\mathcal{F}_{1}$ and $\mathcal{F}_{2}$ of the right hand side of (3.19) are naturally identified if there exists a simply connected domain $U$ such that $\mathcal{F}_{1}(U)=\mathcal{F}_{2}(U)$.

Let

$$
P=\partial^{n}+a_{n-1}(x) \partial^{n-1}+\cdots+a_{0}(x)
$$

be a Fuchsian differential operator with $p+1$ regular singular points $c_{0}=\infty, c_{1}, \ldots, c_{p}$ and let $\lambda_{j, 1}, \ldots, \lambda_{j, n}$ be the characteristic exponents of $P$ at $c_{j}$, respectively. Since $a_{n-1}(x)$ is holomorphic at $x=\infty$ and $a_{n-1}(\infty)=0$, there exists $a_{n-1, j} \in \mathbb{C}$ such that $a_{n-1}(x)=-\sum_{j=1}^{p} \frac{a_{n-1, j}}{x-c_{j}}$. For $c \in \mathbb{C}$ we have $x^{n}\left(\partial^{n}-c x^{-1} \partial^{n-1}\right)=$ $\vartheta^{n}-\left(c+\frac{n(n-1)}{2}\right) \vartheta^{n-1}+c_{n-2} \vartheta^{n-2}+\cdots+c_{0}$ with $c_{j} \in \mathbb{C}$. Hence we have

$$
\lambda_{j, 1}+\cdots+\lambda_{j, n}= \begin{cases}-\sum_{j=1}^{p} a_{n-1, j}-\frac{n(n-1)}{2} & (j=0), \\ a_{n-1, j}+\frac{n(n-1)}{2} & (j=1, \ldots, p),\end{cases}
$$

and the Fuchs relation

$$
\sum_{j=0}^{p} \sum_{\nu=1}^{n} \lambda_{j, \nu}=\frac{(p-1) n(n-1)}{2}
$$

Suppose $P u=0$ is reducible. Then $P=S R$ with $S, R \in W(x)$ so that $n^{\prime}=$ ord $R<n$. Since the solution $v(x)$ of $R v=0$ satisfies $P v(x)=0, R$ is also Fuchsian. Note that the set of $m$ characteristic exponents $\left\{\lambda_{j, \nu}^{\prime} ; \nu=1, \ldots, n^{\prime}\right\}$ of $R v=0$ at $c_{j}$ is a subset of $\left\{\lambda_{j, \nu} ; \nu=1, \ldots, n\right\}$. The operator $R$ may have other singular points $c_{1}^{\prime}, \ldots, c_{q}^{\prime}$ called apparent singular points where any local solutions at the points is analytic. Hence the set characteristic exponents at $x=c_{j}^{\prime}$ are $\left\{\lambda_{j, \nu}^{\prime} \quad \nu=1, \ldots, n^{\prime}\right\}$ such that $0 \leq \mu_{j, 1}<\mu_{j, 2}<\cdots<\mu_{j, n^{\prime}}$ and $\mu_{j, \nu} \in \mathbb{Z}$ for $\nu=1, \ldots, n^{\prime}$ and $j=1, \ldots, q$. Since $\mu_{j, 1}+\cdots+\mu_{j, n^{\prime}} \geq \frac{n^{\prime}\left(n^{\prime}-1\right)}{2}$, the Fuchs relation 
for $R$ implies

$$
\mathbb{Z} \ni \sum_{j=0}^{p} \sum_{\nu=1}^{n^{\prime}} \lambda_{j, \nu}^{\prime} \leq \frac{(p-1) n^{\prime}\left(n^{\prime}-1\right)}{2} .
$$

Fixing a generic point $q$ and pathes $\gamma_{j}$ around $c_{j}$ as in (11.25) and moreover a base $\left\{u_{1}, \ldots, u_{n}\right\}$ of local solutions of the equation $P u=0$ at $q$, we can define monodromy generators $M_{j} \in G L(n, \mathbb{C})$. We call the tuple $\mathbf{M}=\left(M_{0}, \ldots, M_{p}\right)$ the monodromy of the equation $P u=0$. The monodromy $\mathbf{M}$ is defined to be irreducible if there exists no subspace $V$ of $\mathbb{C}^{n}$ such that $M_{j} V \subset V_{j}$ for $j=0, \ldots, p$ and $0<\operatorname{dim} V<n$, which is equivalent to the condition that $P$ is irreducible.

Suppose $Q v=0$ is another Fuchsian differential equation of order $n$ with the same singular points. The monodromy $\mathbf{N}=\left(N_{0}, \ldots, N_{p}\right)$ is similarly defined by fixing a base $\left\{v_{1}, \ldots, v_{n}\right\}$ of local solutions of $Q v=0$ at $q$. Then

$$
\begin{aligned}
\mathbf{M} \sim \mathbf{N} & \stackrel{\text { def }}{\Leftrightarrow} \exists g \in G L(n, \mathbb{C}) \text { such that } N_{j}=g M_{j} g^{-1}(j=0, \ldots, p) \\
& \Leftrightarrow Q v=0 \text { is } W(x) \text {-isomorphic to } P u=0 .
\end{aligned}
$$

If $Q v=0$ is $W(x)$-isomorphic to $P u=0$, the isomorphism defines an isomorphism between their solutions and then $N_{j}=M_{j}$ under the bases corresponding to the isomorphism.

Suppose there exists $g \in G L(n, \mathbb{C})$ such that $N_{j}=g M_{j} g^{-1}$ for $j=0, \ldots, p$. The equations $P u=0$ and $Q u=0$ are $W(x)$-isomorphic to certain first order systems $U^{\prime}=A(x) U$ and $V^{\prime}=B(x) V$ of rank $n$, respectively. We can choose bases $\left\{U_{1}, \ldots, U_{n}\right\}$ and $\left\{V_{1}, \ldots, V_{n}\right\}$ of local solutions of $P U=0$ and $Q V=0$ at $q$, respectively, such that their monodromy generators corresponding $\gamma_{j}$ are same for each $j$. Put $\tilde{U}=\left(U_{1}, \ldots, U_{n}\right)$ and $\tilde{V}=\left(V_{1}, \ldots, V_{n}\right)$. Then the element of the matrix $\tilde{V} \tilde{U}^{-1}$ is holomorphic at $q$ and can be extended to a rational function of $x$ and then $\tilde{V} \tilde{U}^{-1}$ defines a $W(x)$-isomorphism between the equations $U^{\prime}=A(x) U$ and $V^{\prime}=B(x) V$.

Example 3.2 (apparent singularity). The differential equation

$$
x(x-1)(x-c) \frac{d y^{2}}{d x}+\left(x^{2}-2 c x+c\right) \frac{d y}{d x}=0
$$

is a special case of Heun's equation (8.19) with $\alpha=\beta=\lambda=0$ and $\gamma=\delta=1$. It has regular singularities at $0,1, c$ and $\infty$ and its Riemann scheme equals

$$
\left\{\begin{array}{cccc}
x=\infty & 0 & 1 & c \\
0 & 0 & 0 & 0 \\
0 & 0 & 0 & 2
\end{array}\right\} .
$$

The local solution at $x=c$ corresponding to the characteristic exponent 0 is holomorphic at the point and therefore $x=c$ is an apparent singularity, which corresponds to the zero of the Wronskian $\operatorname{det} \Phi_{n}$ in (3.20). Note that the equation (3.24) has the solutions 1 and $c \log x+(1-c) \log (x-1)$.

The equation (3.24) is not $W(x)$-isomorphic to Gauss hypergeometric equation if $c \neq 0$ and $c \neq 1$, which follows from the fact that $c$ is a modulus of the isomorphic classes of the monodromy. It is easy to show that any tuple of matrices $\mathbf{M}=$ $\left(M_{0}, M_{1}, M_{2}\right) \in G L(2, \mathbb{C})$ satisfying $M_{2} M_{1} M_{0}=I_{2}$ is realized as the monodromy of the equation obtained by applying a suitable addition $\operatorname{RAd}\left(x^{\lambda_{0}}(1-x)^{\lambda_{1}}\right)$ to a certain Gauss hypergeometric equation or the above equation.

3.2. A confluence. The non-trivial equation $(x-a) \frac{d u}{d x}=\mu u$ obtained by the addition $\operatorname{RAd}\left((x-a)^{\mu}\right) \partial$ has a solution $(x-a)^{\mu}$ and regular singularities at $x=c$ 
and $\infty$. To consider the confluence of the point $x=a$ to $\infty$ we put $a=\frac{1}{c}$. Then the equation is

$$
((1-c x) \partial+c \mu) u=0
$$

and it has a solution $u(x)=(1-c x)^{\mu}$.

The substitution $c=0$ for the operator $(1-c x) \partial+c \mu \in W[x ; c, \mu]$ gives the trivial equation $\frac{d u}{d x}=0$ with the trivial solution $u(x) \equiv 1$. To obtain a nontrivial equation we introduce the parameter $\lambda=c \mu$ and we have the equation

$$
((1-c x) \partial+\lambda) u=0
$$

with the solution $(1-c x)^{\frac{\lambda}{c}}$. The function $(1-c x)^{\frac{\lambda}{c}}$ has the holomorphic parameters $c$ and $\lambda$ and the substitution $c=0$ gives the equation $(\partial+\lambda) u=0$ with the solution $e^{-\lambda x}$. Here $(1-c x) \partial+\lambda=\operatorname{RAdei}\left(\frac{\lambda}{1-c x}\right) \partial=\operatorname{RAd}\left((1-c x)^{\frac{\lambda}{c}}\right) \partial$.

This is the simplest example of the confluence and we define a confluence of simultaneous additions in this subsection.

3.3. Versal additions. For a function $h(c, x)$ with a holomorphic parameter $c \in \mathbb{C}$ we put

$$
\begin{aligned}
h_{n}\left(c_{1}, \ldots, c_{n}, x\right) & :=\frac{1}{2 \pi \sqrt{-1}} \int_{|z|=R} \frac{h(z, x) d z}{\prod_{j=1}^{n}\left(z-c_{j}\right)} \\
& =\sum_{k=1}^{n} \frac{h\left(c_{k}, x\right)}{\prod_{1 \leq i \leq n, i \neq k}\left(c_{k}-c_{i}\right)}
\end{aligned}
$$

with a sufficiently large $R>0$. Put

$$
h(c, x):=c^{-1} \log (1-c x)=-x-\frac{c}{2} x^{2}-\frac{c^{2}}{3} x^{3}-\frac{c^{3}}{4} x^{4}-\cdots .
$$

Then

$$
(1-c x) h^{\prime}(c, x)=-1
$$

and

$$
\begin{aligned}
h_{n}^{\prime}\left(c_{1}, \ldots, c_{n}, x\right) \prod_{1 \leq i \leq n}\left(1-c_{i} x\right) & =-\sum_{k=1}^{n} \frac{\prod_{1 \leq i \leq n, i \neq k}\left(1-c_{i} x\right)}{\prod_{1 \leq i \leq n, i \neq k}\left(c_{k}-c_{i}\right)} \\
& =-x^{n-1} .
\end{aligned}
$$

The last equality in the above is obtained as follows. Since the left hand side of (3.29) is a holomorphic function of $\left(c_{1}, \ldots, c_{n}\right) \in \mathbb{C}^{n}$ and the coefficient of $x^{m}$ is homogeneous of degree $m-n+1$, it is zero if $m<n-1$. The coefficient of $x^{n-1}$ proved to be -1 by putting $c_{1}=0$. Thus we have

$$
\begin{aligned}
h_{n}\left(c_{1}, \ldots, c_{n}, x\right) & =-\int_{0}^{x} \frac{t^{n-1} d t}{\prod_{1 \leq i \leq n}\left(1-c_{i} t\right)}, \\
e^{\lambda_{n} h_{n}\left(c_{1}, \ldots, c_{n}, x\right)} & \circ\left(\prod_{1 \leq i \leq n}\left(1-c_{i} x\right)\right) \partial \circ e^{-\lambda_{n} h_{n}\left(c_{1}, \ldots, c_{n}, x\right)} \\
& =\left(\prod_{1 \leq i \leq n}\left(1-c_{i} x\right)\right) \partial+\lambda_{n} x^{n-1} \\
e^{\lambda_{n} h_{n}\left(c_{1}, \ldots, c_{n}, x\right)} & =\prod_{k=1}^{n}\left(1-c_{k} x\right)^{\frac{\lambda_{n}}{c_{k} \Pi_{1 \leq i \leq n}\left(c_{k}-c_{i}\right)}}
\end{aligned}
$$


Definition 3.3 (versal addition). We put

$$
\begin{aligned}
& \operatorname{AdV}_{\left(\frac{1}{c_{1}}, \ldots, \frac{1}{c_{p}}\right)}\left(\lambda_{1}, \ldots, \lambda_{p}\right):=\operatorname{Ad}\left(\prod_{k=1}^{p}\left(1-c_{k} x\right)^{\sum_{n=k}^{p} \frac{\lambda_{n}}{c_{k} \Pi_{1 \leq i \leq n}\left(c_{k}-c_{i}\right)}}\right) \\
&=\operatorname{Adei}\left(-\sum_{n=1}^{p} \frac{\lambda_{n} x^{n-1}}{\prod_{i=1}^{n}\left(1-c_{i} x\right)}\right) \\
& \operatorname{RAdV}_{\left(\frac{1}{c_{1}}, \ldots, \frac{1}{c_{p}}\right)}\left(\lambda_{1}, \ldots, \lambda_{p}\right)=\operatorname{R} \circ \operatorname{AdV}_{\left(\frac{1}{c_{1}}, \ldots, \frac{1}{c_{p}}\right)}\left(\lambda_{1}, \ldots, \lambda_{p}\right) .
\end{aligned}
$$

We call $\operatorname{RAdV}_{\left(\frac{1}{c_{1}}, \ldots, \frac{1}{c_{p}}\right)}\left(\lambda_{1}, \ldots, \lambda_{p}\right)$ a versal addition at the $p$ points $\frac{1}{c_{1}}, \ldots, \frac{1}{c_{p}}$.

Putting

$$
h(c, x):=\log (x-c),
$$

we have

$$
h_{n}^{\prime}\left(c_{1}, \ldots, c_{n}, x\right) \prod_{1 \leq i \leq n}\left(x-c_{i}\right)=\sum_{k=1}^{n} \frac{\prod_{1 \leq i \leq n, i \neq k}\left(x-c_{i}\right)}{\prod_{1 \leq i \leq n, i \neq k}\left(c_{k}-c_{i}\right)}=1
$$

and the conflunence of additions around the origin is defined by

$$
\begin{aligned}
& \operatorname{AdV}_{\left(a_{1}, \ldots, a_{p}\right)}^{0}\left(\lambda_{1}, \ldots, \lambda_{p}\right):=\operatorname{Ad}\left(\prod_{k=1}^{p}\left(x-a_{k}\right)^{\sum_{n=k}^{p} \prod_{\substack{1 \leq i \leq n_{n}^{\left(a_{k}-a_{i}\right)} \\
i \neq k}}^{\lambda_{p}}}\right) \\
& =\operatorname{Adei}\left(\sum_{n=1}^{p} \frac{\lambda_{n}}{\prod_{1 \leq i \leq n}\left(x-a_{i}\right)}\right), \\
& \operatorname{RAdV}_{\left(a_{1}, \ldots, a_{p}\right)}^{0}\left(\lambda_{1}, \ldots, \lambda_{p}\right)=\mathrm{R} \circ \operatorname{AdV}_{\left(a_{1}, \ldots, a_{p}\right)}^{0}\left(\lambda_{1}, \ldots, \lambda_{p}\right) .
\end{aligned}
$$

Remark 3.4. Let $g_{k}(c, x)$ be meromorphic functions of $x$ with the holomorphic parameter $c=\left(c_{1}, \ldots, c_{p}\right) \in \mathbb{C}^{p}$ for $k=1, \ldots, p$ such that

$$
g_{k}(c, x) \in \sum_{i=1}^{p} \mathbb{C} \frac{1}{1-c_{i} x} \text { if } 0 \neq c_{i} \neq c_{j} \neq 0 \quad(1 \leq i<j \leq p, 1 \leq k \leq p) .
$$

Suppose $g_{1}(c, x), \ldots, g_{p}(c, x)$ are linearly independent for any fixed $c \in \mathbb{C}^{p}$. Then there exist entire functions $a_{i, j}(c)$ of $c \in \mathbb{C}^{p}$ such that

$$
g_{k}(x, c)=\sum_{n=1}^{p} \frac{a_{k, n}(c) x^{n-1}}{\prod_{i=1}^{n}\left(1-c_{i} x\right)}
$$

and $\left(a_{i, j}(c)\right) \in G L(p, \mathbb{C})$ for any $c \in \mathbb{C}^{p}$ (cf. [03, Lemma 6.3]). Hence the versal addition is essentially unique.

3.4. Versal operators. If we apply a middle convolution to a versal addition of the trivial operator $\partial$, we have a versal Jordan-Pochhammer operator.

$$
\begin{aligned}
P & :=\operatorname{RAd}\left(\partial^{-\mu}\right) \circ \operatorname{RAdV}_{\left(\frac{1}{c_{1}}, \ldots, \frac{1}{c_{p}}\right)}\left(\lambda_{1}, \ldots, \lambda_{p}\right) \partial \\
& =\operatorname{RAd}\left(\partial^{-\mu}\right) \circ \mathrm{R}\left(\partial+\sum_{k=1}^{p} \frac{\lambda_{k} x^{k-1}}{\prod_{\nu=1}^{k}\left(1-c_{\nu} x\right)}\right) \\
& =\partial^{-\mu+p-1}\left(p_{0}(x) \partial+q(x)\right) \partial^{\mu}=\sum_{k=0}^{p} p_{k}(x) \partial^{p-k}
\end{aligned}
$$


with

$$
\begin{aligned}
& p_{0}(x)=\prod_{j=1}^{p}\left(1-c_{j} x\right), \quad q(x)=\sum_{k=1}^{p} \lambda_{k} x^{k-1} \prod_{j=k+1}^{p}\left(1-c_{j} x\right), \\
& p_{k}(x)=\left(\begin{array}{c}
-\mu+p-1 \\
k
\end{array}\right) p_{0}^{(k)}(x)+\left(\begin{array}{c}
-\mu+p-1 \\
k-1
\end{array}\right) q^{(k-1)}(x) .
\end{aligned}
$$

We naturally obtain the integral representation of solutions of the versal JordanPochhammer equation $P u=0$, which we show in the case $p=2$ as follows.

Example 3.5. We have the versal Gauss hypergeometric operator

$$
\begin{aligned}
P_{c_{1}, c_{2} ; \lambda_{1}, \lambda_{2}, \mu}:= & \operatorname{RAd}\left(\partial^{-\mu}\right) \circ \operatorname{RAdV}\left(\frac{1}{\left.c_{1}, \frac{1}{c_{2}}\right)}\left(\lambda_{1}, \lambda_{2}\right) \partial\right. \\
= & \operatorname{RAd}\left(\partial^{-\mu}\right) \circ \operatorname{RAd}\left(\left(1-c_{1} x\right)^{\frac{\lambda_{1}}{c_{1}}+\frac{\lambda_{2}}{c_{1}\left(c_{1}-c_{2}\right)}}\left(1-c_{2} x\right)^{\frac{\lambda_{2}}{c_{2}\left(c_{2}-c_{1}\right)}}\right) \\
= & \operatorname{RAd}\left(\partial^{-\mu}\right) \circ \operatorname{RAdei}\left(-\frac{\lambda_{1}}{1-c_{1} x}-\frac{\lambda_{2} x}{\left(1-c_{1} x\right)\left(1-c_{2} x\right)}\right) \partial \\
= & \operatorname{RAd}\left(\partial^{-\mu}\right) \circ \operatorname{R}\left(\partial+\frac{\lambda_{1}}{1-c_{1} x}+\frac{\lambda_{2} x}{\left(1-c_{1} x\right)\left(1-c_{2} x\right)}\right) \\
= & \operatorname{Ad}\left(\partial^{-\mu}\right)\left(\partial\left(1-c_{1} x\right)\left(1-c_{2} x\right) \partial+\partial\left(\lambda_{1}\left(1-c_{2} x\right)+\lambda_{2} x\right)\right) \\
= & \left(\left(1-c_{1} x\right) \partial+c_{1}(\mu-1)\right)\left(\left(1-c_{2} x\right) \partial+c_{2} \mu\right) \\
& +\lambda_{1} \partial+\left(\lambda_{2}-\lambda_{1} c_{2}\right)(x \partial+1-\mu) \\
= & \left(1-c_{1} x\right)\left(1-c_{2} x\right) \partial^{2} \\
& +\left(\left(c_{1}+c_{2}\right)(\mu-1)+\lambda_{1}+\left(2 c_{1} c_{2}(1-\mu)+\lambda_{2}-\lambda_{1} c_{2}\right) x\right) \partial \\
& +(\mu-1)\left(c_{1} c_{2} \mu+\lambda_{1} c_{2}-\lambda_{2}\right)
\end{aligned}
$$

whose solution is obtained by applying $I_{c}^{\mu}$ to

$$
K_{c_{1}, c_{2} ; \lambda_{1}, \lambda_{2}}(x)=\left(1-c_{1} x\right)^{\frac{\lambda_{1}}{c_{1}}+\frac{\lambda_{2}}{c_{1}\left(c_{1}-c_{2}\right)}}\left(1-c_{2} x\right)^{\frac{\lambda_{2}}{c_{2}\left(c_{2}-c_{1}\right)}}
$$

The equation $P u=0$ has the Riemann scheme

$$
\left.\left\{\begin{array}{ccc}
x=\frac{1}{c_{1}} & \frac{1}{c_{2}} & \infty \\
0 & 0 & 1-\mu \\
\frac{\lambda_{1}}{c_{1}}+\frac{\lambda_{2}}{c_{1}\left(c_{1}-c_{2}\right)}+\mu & \frac{\lambda_{2}}{c_{2}\left(c_{2}-c_{1}\right)}+\mu & -\frac{\lambda_{1}}{c_{1}}+\frac{\lambda_{2}}{c_{1} c_{2}}-\mu
\end{array}\right\} x\right\} .
$$

Thus we have the following well-known confluent equations

$$
\begin{array}{rlrl}
P_{c_{1}, 0 ; \lambda_{1}, \lambda_{2}, \mu} & =\left(1-c_{1} x\right) \partial^{2}+\left(c_{1}(\mu-1)+\lambda_{1}+\lambda_{2} x\right) \partial-\lambda_{2}(\mu-1), & & \text { (Kummer) } \\
K_{c_{1}, 0 ; \lambda_{1}, \lambda_{2}} & =\left(1-c_{1} x\right)^{\frac{\lambda_{1}}{c_{1}}+\frac{\lambda_{2}}{c_{1}^{2}} \exp \left(\frac{\lambda_{2} x}{c_{1}}\right),} & & \\
P_{0,0 ; 0,-1, \mu} & =\partial^{2}-x \partial+(\mu-1), & & \text { (Hermite) } \\
\operatorname{Ad}\left(e^{\frac{1}{4} x^{2}}\right) & P_{0,0 ; 0,1, \mu}=\left(\partial-\frac{1}{2} x\right)^{2}+x\left(\partial-\frac{1}{2} x\right)-(\mu-1) & \\
& =\partial^{2}+\left(\frac{1}{2}-\mu-\frac{x^{2}}{4}\right), & & \text { (Weber) } \\
K_{0,0 ; 0, \mp 1} & =\exp \left(\int_{0}^{x} \pm t d t\right)=\exp \left( \pm \frac{x^{2}}{2}\right) . &
\end{array}
$$


The solution

$$
\begin{aligned}
D_{-\mu}(x) & :=(-1)^{-\mu} e^{\frac{x^{2}}{4}} I_{\infty}^{\mu}\left(e^{-\frac{x^{2}}{2}}\right)=\frac{e^{\frac{x^{2}}{4}}}{\Gamma(\mu)} \int_{x}^{\infty} e^{-\frac{t^{2}}{2}}(t-x)^{\mu-1} d t \\
& =\frac{e^{\frac{x^{2}}{4}}}{\Gamma(\mu)} \int_{0}^{\infty} e^{-\frac{(s+x)^{2}}{2}} s^{\mu-1} d s=\frac{e^{-\frac{x^{2}}{4}}}{\Gamma(\mu)} \int_{0}^{\infty} e^{-x s-\frac{t^{2}}{2}} s^{\mu-1} d s \\
& \sim x^{-\mu} e^{-\frac{x^{2}}{4}}{ }_{2} F_{0}\left(\frac{\mu}{2}, \frac{\mu}{2}+\frac{1}{2} ;-\frac{2}{x^{2}}\right)=\sum_{k=0}^{\infty} x^{-\mu} e^{-\frac{x^{2}}{4}} \frac{\left(\frac{\mu}{2}\right)_{k}\left(\frac{\mu}{2}+\frac{1}{2}\right)_{k}}{k !}\left(-\frac{2}{x^{2}}\right)^{k}
\end{aligned}
$$

of Weber's equation $\frac{d^{2} u}{d x^{2}}=\left(\frac{x^{2}}{4}+\mu-\frac{1}{2}\right) u$ is called a parabolic cylinder function (cf. [WW, §16.5]). Here the above last line is an asymptotic expansion when $x \rightarrow$ $+\infty$.

The normal form of Kummer equation is obtained by the coordinate transformation $y=x-\frac{1}{c_{1}}$ but we also obtain it as follows:

$$
\begin{aligned}
P_{c_{1} ; \lambda_{1}, \lambda_{2}, \mu}:= & \operatorname{RAd}\left(\partial^{-\mu}\right) \circ \operatorname{R} \circ \operatorname{Ad}\left(x^{\lambda_{2}}\right) \circ \operatorname{AdV}_{\frac{1}{c_{1}}}\left(\lambda_{1}\right) \partial \\
= & \operatorname{RAd}\left(\partial^{-\mu}\right) \circ \mathrm{R}\left(\partial-\frac{\lambda_{2}}{x}+\frac{\lambda_{1}}{1-c_{1} x}\right) \\
= & \operatorname{Ad}\left(\partial^{-\mu}\right)\left(\partial x\left(1-c_{1} x\right) \partial-\partial\left(\lambda_{2}-\left(\lambda_{1}+c_{1} \lambda_{2}\right) x\right)\right) \\
= & (x \partial+1-\mu)\left(\left(1-c_{1} x\right) \partial+c_{1} \mu\right)-\lambda_{2} \partial+\left(\lambda_{1}+c_{1} \lambda_{2}\right)(x \partial+1-\mu) \\
= & x\left(1-c_{1} x\right) \partial^{2}+\left(1-\lambda_{2}-\mu+\left(\lambda_{1}+c_{1}\left(\lambda_{2}+2 \mu-2\right)\right) x\right) \partial \\
& +(\mu-1)\left(\lambda_{1}+c_{1}\left(\lambda_{2}+\mu\right)\right), \\
P_{0 ; \lambda_{1}, \lambda_{2}, \mu}= & x \partial^{2}+\left(1-\lambda_{2}-\mu+\lambda_{1} x\right) \partial+\lambda_{1}(\mu-1), \\
P_{0 ;-1, \lambda_{2}, \mu}= & x \partial^{2}+\left(1-\lambda_{2}-\mu-x\right) \partial+1-\mu \quad(\text { Kummer }), \\
K_{c_{1} ; \lambda_{1}, \lambda_{2}}(x):= & x^{\lambda_{2}}\left(1-c_{1} x\right)^{\frac{\lambda_{1}}{c_{1}}}, \quad K_{0 ; \lambda_{1}, \lambda_{2}}(x)=x^{\lambda_{2}} \exp \left(-\lambda_{1} x\right) .
\end{aligned}
$$

The Riemann scheme of the equation $P_{c_{1} ; \lambda_{1}, \lambda_{2}, \mu} u=0$ is

$$
\left\{\begin{array}{ccc}
x=0 & \frac{1}{c_{1}} & \infty \\
0 & 0 & 1-\mu \\
\lambda_{2}+\mu & \frac{\lambda_{1}}{c_{1}}+\mu & -\frac{\lambda_{1}}{c_{1}}-\lambda_{2}-\mu
\end{array}\right\}
$$

and the local solution at the origin corresponding to the characteristic exponent $\lambda_{2}+\mu$ is given by

$$
I_{0}^{\mu}\left(K_{c_{1} ; \lambda_{1}, \lambda_{2}}\right)(x)=\frac{1}{\Gamma(\mu)} \int_{0}^{x} t^{\lambda_{2}}\left(1-c_{1} t\right)^{\frac{\lambda_{1}}{c_{1}}}(x-t)^{\mu-1} d t .
$$

In particular, we have a solution

$$
\begin{aligned}
u(x) & =I_{0}^{\mu}\left(K_{0 ;-1, \lambda_{2}}\right)(x)=\frac{1}{\Gamma(\mu)} \int_{0}^{x} t^{\lambda_{2}} e^{t}(x-t)^{\mu-1} d t \\
& =\frac{x^{\lambda_{2}+\mu}}{\Gamma(\mu)} \int_{0}^{1} s^{\lambda_{2}}(1-s)^{\mu-1} e^{x s} d s \quad(t=x s) \\
& =\frac{\Gamma\left(\lambda_{2}+1\right) x^{\lambda_{2}+\mu}}{\Gamma\left(\lambda_{2}+\mu+1\right)}{ }_{1} F_{1}\left(\lambda_{2}+1, \mu+\lambda_{2}+1 ; x\right)
\end{aligned}
$$

of the Kummer equation $P_{0 ;-1, \lambda_{2}, \mu} u=0$ corresponding to the exponent $\lambda_{2}+\mu$ at the origin. If $c_{1} \notin(-\infty, 0]$ and $x \notin[0, \infty]$ and $\lambda_{2} \notin \mathbb{Z}_{\geq 0}$, the local solution at $-\infty$ 
corresponding to the exponent $-\lambda_{2}-\frac{\lambda_{1}}{c_{1}}-\mu$ is given by

$$
\begin{aligned}
& \frac{1}{\Gamma(\mu)} \int_{-\infty}^{x}(-t)^{\lambda_{2}}\left(1-c_{1} t\right)^{\frac{\lambda_{1}}{c_{1}}}(x-t)^{\mu-1} d t \\
& =\frac{(-x)^{\lambda_{2}}}{\Gamma(\mu)} \int_{0}^{\infty}\left(1-\frac{s}{x}\right)^{\lambda_{2}}\left(1+c_{1}(s-x)\right)^{\frac{\lambda_{1}}{c_{1}}} s^{\mu-1} d s \quad(s=x-t) \\
\frac{\lambda_{1}=-1}{c_{1} \rightarrow+0} & \frac{(-x)^{\lambda_{2}}}{\Gamma(\mu)} \int_{0}^{\infty}\left(1-\frac{s}{x}\right)^{\lambda_{2}} e^{x-s} s^{\mu-1} d s \\
& =\frac{(-x)^{\lambda_{2}} e^{x}}{\Gamma(\mu)} \int_{0}^{\infty} s^{\mu-1} e^{-s}\left(1-\frac{s}{x}\right)^{\lambda_{2}} d s \\
& \sim \sum_{n=0}^{\infty} \frac{\Gamma(\mu+n) \Gamma\left(-\lambda_{2}+n\right)}{\Gamma(\mu) \Gamma\left(-\lambda_{2}\right) n ! x^{n}}(-x)^{\lambda_{2}} e^{x}=(-x)^{\lambda_{2}} e^{x}{ }_{2} F_{0}\left(-\lambda_{2}, \mu ; \frac{1}{x}\right) .
\end{aligned}
$$

Here the above last line is an asymptotic expansion of a rapidly decreasing solution of the Kummer equation when $\mathbb{R} \ni-x \rightarrow+\infty$. The Riemann scheme of the equation $P_{0 ;-1, \lambda_{2}, \mu} u=0$ can be expressed by

$$
\left\{\begin{array}{ccc}
x=0 & \infty & (1) \\
0 & 1-\mu & 0 \\
\lambda_{2}+\mu & -\lambda_{2} & 1
\end{array}\right\}
$$

In general, the expression $\left\{\begin{array}{cccc}\infty & \left(r_{1}\right) & \cdots & \left(r_{k}\right) \\ \lambda & \alpha_{1} & \cdots & \alpha_{k}\end{array}\right\}$ with $0<r_{1}<\cdots<r_{k}$ means the existence of a solution $u(x)$ satisfying

$$
u(x) \sim x^{-\lambda} \exp \left(\sum_{\nu=1}^{k} \alpha_{\nu} \frac{x^{r_{\nu}}}{r_{\nu}}\right) \text { for }|x| \rightarrow \infty
$$

under a suitable restriction of $\operatorname{Arg} x$. Here $k \in \mathbb{Z}_{\geq 0}$ and $\lambda, \alpha_{\nu} \in \mathbb{C}$.

\section{SERIES EXPANSion}

In this section we review the Euler transformation and remark on its relation to middle convolutions.

First we note the following which will be frequently used:

$$
\begin{gathered}
\int_{0}^{1} t^{\alpha-1}(1-t)^{\beta-1} d t=\frac{\Gamma(\alpha) \Gamma(\beta)}{\Gamma(\alpha+\beta)}, \\
(1-t)^{-\gamma}=\sum_{\nu=0}^{\infty} \frac{(-\gamma)(-\gamma-1) \cdots(-\gamma-\nu+1)}{\nu !}(-t)^{\nu} \\
=\sum_{\nu=0}^{\infty} \frac{\Gamma(\gamma+\nu)}{\Gamma(\gamma) \nu !} t^{\nu}=\sum_{\nu=0}^{\infty} \frac{(\gamma)_{\nu}}{\nu !} t^{\nu} .
\end{gathered}
$$

The integral (4.1) converges if $\operatorname{Re} \alpha>0$ and $\operatorname{Re} \beta>0$ and the right hand side is meromorphically continued to $\alpha \in \mathbb{C}$ and $\beta \in \mathbb{C}$. If the integral in (4.1) is interpreted in the sense of generalized functions, (4.1) is valid if $\alpha \notin\{0,-1,-2, \ldots\}$ and $\beta \notin\{0,-1,-2, \ldots\}$. 
Euler transformation $I_{c}^{\mu}$ is sometimes expressed by $\partial^{-\mu}$ and as is shown in ( $\mathrm{Kh}$, $\S 5.1]$ ), we have

$$
\begin{aligned}
I_{c}^{\mu} u(x) & :=\frac{1}{\Gamma(\mu)} \int_{c}^{x}(x-t)^{\mu-1} u(t) d t \\
& =\frac{(x-c)^{\mu}}{\Gamma(\mu)} \int_{0}^{1}(1-s)^{\mu-1} u((x-c) s+c) d s, \\
I_{c}^{\mu} \circ I_{c}^{\mu^{\prime}} & =I_{c}^{\mu+\mu^{\prime}}, \\
I_{c}^{-n} u(x) & =\frac{d^{n}}{d x^{n}} u(x), \\
I_{c}^{\mu} \sum_{n=0}^{\infty} c_{n}(x-c)^{\lambda+n} & =\sum_{n=0}^{\infty} \frac{\Gamma(\lambda+n+1)}{\Gamma(\lambda+\mu+n+1)} c_{n}(x-c)^{\lambda+\mu+n} \\
& =\frac{\Gamma(\lambda+1)}{\Gamma(\lambda+\mu+1)} \sum_{n=0}^{\infty} \frac{(\lambda+1)_{n} c_{n}}{(\lambda+\mu+1)_{n}}(x-c)^{\lambda+\mu+n}, \\
I_{\infty}^{\mu} \sum_{n=0}^{\infty} c_{n} x^{\lambda-n} & =e^{\pi \sqrt{-1} \mu} \sum_{n=0}^{\infty} \frac{\Gamma(-\lambda-\mu+n)}{\Gamma(-\lambda+n)} c_{n} x^{\lambda+\mu-n} .
\end{aligned}
$$

Moreover the following equalities which follow from (2.47) are also useful.

$$
\begin{aligned}
I_{0}^{\mu} & \sum_{n=0}^{\infty} c_{n} x^{\lambda+n}(1-x)^{\beta} \\
= & \frac{\Gamma(\lambda+1)}{\Gamma(\lambda+\mu+1)} \sum_{m, n=0}^{\infty} \frac{(\lambda+1)_{m+n}(-\beta)_{m} c_{n}}{(\lambda+\mu+1)_{m+n} m !} x^{\lambda+\mu+m+n} \\
= & \frac{\Gamma(\lambda+1)}{\Gamma(\lambda+\mu+1)}(1-x)^{-\beta} \sum_{m, n=0}^{\infty} \frac{(\lambda+1)_{n}(\mu)_{m}(-\beta)_{m} c_{n}}{(\lambda+\mu+1)_{m+n} m !} x^{\lambda+\mu+n}\left(\frac{x}{x-1}\right)^{m} .
\end{aligned}
$$

If $\lambda \notin \mathbb{Z}_{<0}$ (resp. $\lambda+\mu \notin \mathbb{Z}_{\geq 0}$ ) and moreover the power series $\sum_{n=0}^{\infty} c_{n} t^{n}$ has a positive radius of convergence, the equalities (4.6) (resp, 4.7)) is valid since $I_{c}^{\mu}$ (resp. $\left.I_{\infty}^{\mu}\right)$ can be defined through analytic continuations with respect to the parameters $\lambda$ and $\mu$. Note that $I_{c}^{\mu}$ is an invertible map of $\mathcal{O}_{c}(x-c)^{\lambda}$ onto $\mathcal{O}_{c}(x-$ $c)^{\lambda+\mu}$ if $\lambda \notin\{-1,-2,-3, \ldots\}$ and $\lambda+\mu \notin\{-1,-2,-3, \ldots\}$.

Proposition 4.1. Let $\lambda$ and $\mu$ be complex numbers satisfying $\lambda \notin \mathbb{Z}_{<0}$. Differentiating the equality (4.6) with respect to $\lambda$, we have the linear map

$$
I_{c}^{\mu}: \mathcal{O}_{c}(\lambda, m) \rightarrow \mathcal{O}_{c}(\lambda+\mu, m)
$$

under the notation (3.5), which is also defined by (4.3) if $\operatorname{Re} \lambda>-1$ and $\operatorname{Re} \mu>0$. Here $m$ is a non-negative integer. Then we have

$$
I_{c}^{\mu}\left(\sum_{j=0}^{m} \phi_{j} \log ^{j}(x-c)\right)-I_{c}^{\mu}\left(\phi_{m}\right) \log ^{m}(x-c) \in \mathcal{O}(\lambda+\mu, m-1)
$$

for $\phi_{j} \in \mathcal{O}_{c}$ and $I_{c}^{\mu}$ satisfies (2.43). The map (4.9) is bijective if $\lambda+\mu \notin \mathbb{Z}_{<0}$. In particular for $k \in \mathbb{Z}_{\geq 0}$ we have $I_{c}^{\mu} \partial^{k}=\partial^{k} I_{c}^{\mu}=I_{c}^{\mu-k}$ on $\mathcal{O}_{c}(\lambda, m)$ if $\lambda-k \notin$ $\{-1,-2,-3, \ldots\}$.

Suppose that $P \in W[x]$ and $\phi \in \mathcal{O}_{c}(\lambda, m)$ satisfy $P \phi=0, P \neq 0$ and $\phi \neq 0$. Let $k$ and $N$ be non-negative integers such that

$$
\partial^{k} P=\sum_{i=0}^{N} \sum_{j \geq 0} a_{i, j} \partial^{i}((x-c) \partial)^{j}
$$


with suitable $a_{j, j} \in \mathbb{C}$ and put $Q=\sum_{i=0}^{N} \sum_{j \geq 0} c_{i, j} \partial^{i}((x-c) \partial-\mu)^{j}$. Then if $\lambda \notin\{N-1, N-2, \ldots, 0,-1, \ldots\}$, we have

$$
I_{c}^{\mu} \partial^{k} P u=Q I_{c}^{\mu}(u) \text { for } u \in \mathcal{O}_{c}(\lambda, m)
$$

and in particular $Q I_{c}^{\mu}(\phi)=0$.

Fix $\ell \in \mathbb{Z}$. For $u(x)=\sum_{i=\ell}^{\infty} \sum_{j=0}^{m} c_{i, j}(x-c)^{i} \log ^{j}(x-c) \in \mathcal{O}_{c}(\ell, m)$ we put $\left(\Gamma_{N} u\right)(x)=\sum_{\nu=\max \{\ell, N-1\}}^{\infty} \sum_{j=0}^{m} c_{i, j}(x-c)^{i} \log ^{j}(x-c)$. Then

$$
\left(\prod_{\ell-N \leq \nu \leq N-1}((x-c) \partial-\nu)^{m+1}\right) \partial^{k} P\left(u(x)-\left(\Gamma_{N} u\right)(x)\right)=0
$$

ane therefore

$$
\begin{aligned}
& \left(\prod_{\ell-N \leq \nu \leq N-1}((x-c) \partial-\mu-\nu)^{m+1}\right) Q I_{c}^{\mu}\left(\Gamma_{N} u\right) \\
& \quad=I_{c}^{\mu}\left(\prod_{\ell-N \leq \nu \leq N-1}((x-c) \partial-\nu)^{m+1}\right) \partial^{k} P u .
\end{aligned}
$$

In particular, $\prod_{\ell-N \leq \nu \leq N-1}((x-c) \partial-\mu-\nu)^{m+1} \cdot Q I_{c}^{\mu}\left(\Gamma_{N}(u)\right)=0$ if $P u=0$.

Suppose moreover $\lambda \notin \mathbb{Z}$ and $\lambda+\mu \notin \mathbb{Z}$ and $Q=S T$ with $S, T \in W[x]$ such that $x=c$ is not a singular point of the operator $S$. Then $T I_{c}^{\mu}(\phi)=0$. In particular,

$$
\left(\operatorname{RAd}\left(\partial^{-\mu}\right) P\right) I_{c}^{\mu}(\phi)=0 \text {. }
$$

Hence if the differential equation $\left(\operatorname{RAd}\left(\partial^{-\mu}\right) P\right) v=0$ is irreducible, we have

$$
W(x)\left(\operatorname{RAd}\left(\partial^{-\mu}\right) P\right)=\left\{T \in W(x) ; T I_{c}^{\mu}(\phi)=0\right\} .
$$

The statements above are also valid even if we replace $x-c, I_{c}^{\mu}$ by $\frac{1}{x}, I_{\infty}^{\mu}$, respectively.

Proof. It is clear that $(4.9)$ is well-defined and $(4.10)$ is valid. Then $(4.9)$ is bijective because of (4.6) and (4.10). Since (2.43) is valid when $m=0$, it is also valid when $m=1,2, \ldots$ by the definition of (4.9).

The equalities (4.6) and (4.7) assure that $Q I_{c}^{\mu}(\phi)=0$. Note that $T I_{c}^{\mu}(\phi) \in$ $\mathcal{O}(\lambda+\mu-N, m)$ with a suitable positive integer $N$. Since $\lambda+\mu-N \notin \mathbb{Z}$ and any solution of the equation $S v=0$ is holomorphic at $x=c$, the equality $S\left(T I_{c}^{\mu}(\phi)\right)=0$ implies $T I_{c}^{\mu}(\phi)=0$.

The remaining claims in the theorem are similarly clear.

Remark 4.2. i) Let $\gamma:[0,1] \rightarrow \mathbb{C}$ be a path such that $\gamma(0)=c$ and $\gamma(1)=x$. Suppose $u(x)$ is holomorphic along the path $\gamma(t)$ for $0<t \leq 1$ and $u(\gamma(t))=\phi(\gamma(t))$ for $0<t \ll 1$ with a suitable function $\phi \in \mathcal{O}_{c}(\lambda, m)$. Then $I_{c}^{\mu}(u)$ is defined by the integration along the path $\gamma$. In fact, if the path $\gamma(t)$ with $t \in[0,1]$ splits into the three paths corresponding to the decomposition $[0,1]=[0, \epsilon] \cup[\epsilon, 1-\epsilon] \cup[1-\epsilon, 1]$ with $0<\epsilon \ll 1$. Let $c_{1}=c, \ldots, c_{p}$ be points in $\mathbb{C}^{n}$ and suppose moreover $u(x)$ is extended to a multi-valued holomorphic function on $\mathbb{C} \backslash\left\{c_{1}, \ldots, c_{p}\right\}$. Then $I_{c}^{x}(u)$ also defines a multi-valued holomorphic function on $\mathbb{C} \backslash\left\{c_{1}, \ldots, c_{p}\right\}$.

ii) Proposition 4.1 is also valid if we replace $\mathcal{O}_{c}(\lambda, m)$ by the space of functions given in Remark 2.7 ii). In fact the above proof also works in this case.

\section{Contiguity Relation}

The following proposition is clear from Proposition 4.1. 
Proposition 5.1. Let $\phi(x)$ be a non-zero solution of an ordinary differential equation $P u=0$ with an operator $P \in W[x]$. Let $P_{j}$ and $S_{j} \in W[x]$ for $j=1, \ldots, N$ so that $\sum_{j=1}^{N} P_{j} S_{j} \in W[x] P$. Then for a suitable $\ell \in \mathbb{Z}$ we have

$$
\sum Q_{j}\left(I_{c}^{\mu}\left(\phi_{j}\right)\right)=0
$$

by putting

$$
\begin{aligned}
\phi_{j} & =S_{j} \phi, \\
Q_{j} & =\partial^{\ell-\mu} \circ P_{j} \circ \partial^{\mu} \in W[x], \quad(j=1, \ldots, N),
\end{aligned}
$$

if $\phi(x) \in \mathcal{O}(\lambda, m)$ with a non-negative integer $m$ and a complex number $\lambda$ satisfying $\lambda \notin \mathbb{Z}$ and $\lambda+\mu \notin \mathbb{Z}$ or $\phi(x)$ is a function given in Remark 2.7 ii). If $P_{j}=\sum_{k \geq 0, \ell \geq 0} c_{j, k, \ell} \partial^{k} \vartheta^{\ell}$ with $c_{j, k, \ell} \in \mathbb{C}$, then we can assume $\ell \leq 0$ in the above. Moreover we have

$$
\partial\left(I_{c}^{\mu+1}\left(\phi_{1}\right)\right)=I_{c}^{\mu}\left(\phi_{1}\right)
$$

Proof. Fix an integer $k$ such that $\partial^{k} P_{j}=\tilde{P}_{j}(\partial, \vartheta)=\sum_{i_{1}, i_{2}} c_{i_{1}, i_{2}} \partial^{i_{1}} \vartheta^{i_{2}}$ with $c_{i_{1}, i_{2}} \in$ $\mathbb{C}$. Since $0=\sum_{j=1}^{N} \partial^{k} P_{j} S_{j} \phi$, Proposition 4.1 proves $0=\sum_{j=1}^{N} I_{c}^{\mu}\left(\tilde{P}_{j}(\partial, \vartheta) S_{j} \phi\right)=$ $\sum_{j=1}^{N} \tilde{P}_{j}(\partial, \vartheta-\mu) I_{c}^{\mu}\left(S_{j} \phi\right)$, which implies the first claim of the proposition.

The last claim is clear from (4.4) and (4.5).

Corollary 5.2. Let $P(\xi)$ and $K(\xi)$ be non-zero elements of $W[x ; \xi]$. If we substitute $\xi$ and $\mu$ by generic complex numbers, we assume that there exists a solution $\phi_{\xi}(x)$ satisfying the assumption in the preceding proposition and that $I_{c}^{\mu}\left(\phi_{\xi}\right)$ and $I_{c}^{\mu}\left(K(\xi) \phi_{\xi}\right)$ satisfy irreducible differential equations $T_{1}(\xi, \mu) v_{1}=0$ and $T_{2}(\xi, \mu) v_{2}=$ 0 with $T_{1}(\xi, \mu)$ and $T_{2}(\xi, \mu) \in W(x ; \xi, \mu)$, respectively. Then the differential equation $T_{1}(\xi, \mu) v_{1}=0$ is isomorphic to $T_{2}(\xi, \mu) v_{2}=0$ as $W(x ; \xi, \mu)$-modules.

Proof. Since $K(\xi) \cdot 1-1 \cdot K(\xi)=0$, we have $Q(\xi, \mu) I_{c}^{\mu}\left(\phi_{\xi}\right)=\partial^{\ell} I_{c}^{\mu}\left(K(\xi) \phi_{\xi}\right)$ with $Q(\xi, \mu)=\partial^{\ell-\mu} \circ K(\xi) \circ \partial^{\mu}$. Since $\partial^{\ell} I_{c}^{\mu}\left(\phi_{\xi}\right) \neq 0$ and the equations $T_{j}(\xi, \mu) v_{j}=0$ are irreducible for $j=1$ and 2 , there exist $R_{1}(\xi, \mu)$ and $R_{2}(\xi, \mu) \in W(x ; \xi, \mu)$ such that $I_{c}^{\mu}\left(\phi_{\xi}\right)=R_{1}(\xi, \mu) Q(\xi, \mu) I_{c}^{\mu}\left(\phi_{\xi}\right)=R_{1}(\xi, \mu) \partial^{\ell} I_{c}^{\mu}\left(K(\xi) \phi_{\xi}\right)$ and $I_{c}^{\mu}\left(K(\xi) \phi_{\xi}\right)=$ $R_{2}(\xi, \mu) \partial^{\ell} I_{c}^{\mu}\left(K(\xi) \phi_{\xi}\right)=R_{2}(\xi, \mu) Q(\xi, \mu) I_{c}^{\mu}\left(\phi_{\xi}\right)$. Hence we have the corollary.

Using the proposition, we get the contiguity relations with respect to the parameters corresponding to powers of linear functions defining additions and the middle convolutions.

For example, in the case of Gauss hypergeometric functions, we have

$$
\begin{aligned}
u_{\lambda_{1}, \lambda_{2}, \mu}(x) & :=I_{0}^{\mu}\left(x^{\lambda_{1}}(1-x)^{\lambda_{2}}\right), \\
u_{\lambda_{1}, \lambda_{2}, \mu-1}(x) & =\partial u_{\lambda_{1}, \lambda_{2}, \mu}(x), \\
\partial u_{\lambda_{1}+1, \lambda_{2}, \mu}(x) & =(x \partial+1-\mu) u_{\lambda_{1}, \lambda_{2}, \mu}(x), \\
\partial u_{\lambda_{1}, \lambda_{2}+1, \mu}(x) & =((1-x) \partial+\mu-1) u_{\lambda_{1}, \lambda_{2}, \mu}(x) .
\end{aligned}
$$

Here Proposition 5.1 with $\phi=x^{\lambda_{1}}(1-x)^{\lambda_{2}},\left(P_{1}, S_{1}, P_{2}, S_{2}\right)=(1, x,-x, 1)$ and $\ell=1$ gives the above third identity.

Since $P_{\lambda_{1}, \lambda_{2}, \mu} u_{\lambda_{1}, \lambda_{2}, \mu}(x)=0$ with

$$
\begin{aligned}
P_{\lambda_{1}, \lambda_{2}, \mu}= & \left(x(1-x) \partial+\left(1-\lambda_{1}-\mu-\left(2-\lambda_{1}-\lambda_{2}-2 \mu\right) x\right) \partial\right. \\
& -(\mu-1)\left(\lambda_{1}+\lambda_{2}+\mu\right)
\end{aligned}
$$


as is given in Example 2.8, the inverse of the relation $u_{\lambda_{1}, \lambda_{2}, \mu-1}(x)=\partial u_{\lambda_{1}, \lambda_{2}, \mu}(x)$ is

$$
u_{\lambda_{1}, \lambda_{2}, \mu}(x)=-\frac{x(1-x) \partial+\left(1-\lambda_{1}-\mu-\left(2-\lambda_{1}-\lambda_{2}-2 \mu\right) x\right)}{(\mu-1)\left(\lambda_{1}+\lambda_{2}+\mu\right)} u_{\lambda_{1}, \lambda_{2}, \mu-1}(x) .
$$

The equalities $u_{\lambda_{1}, \lambda_{2}, \mu-1}(x)=\partial u_{\lambda_{1}, \lambda_{2}, \mu}(x)$ and (2.47) mean

$$
\begin{aligned}
& \frac{\Gamma\left(\lambda_{1}+1\right) x^{\lambda_{1}+\mu-1}}{\Gamma\left(\lambda_{1}+\mu\right)} F\left(-\lambda_{2}, \lambda_{1}+1, \lambda_{1}+\mu ; x\right) \\
& =\frac{\Gamma\left(\lambda_{1}+1\right) x^{\lambda_{1}+\mu-1}}{\Gamma\left(\lambda_{1}+\mu\right)} F\left(-\lambda_{2}, \lambda_{1}+1, \lambda_{1}+\mu+1 ; x\right) \\
& \quad+\frac{\Gamma\left(\lambda_{1}+1\right) x^{\lambda_{1}+\mu}}{\Gamma\left(\lambda_{1}+\mu+1\right)} \frac{d}{d x} F\left(-\lambda_{2}, \lambda_{1}+1, \lambda_{1}+\mu+1 ; x\right)
\end{aligned}
$$

and therefore $u_{\lambda_{1}, \lambda_{2}, \mu-1}(x)=\partial u_{\lambda_{1}, \lambda_{2}, \mu}(x)$ is equivalent to

$$
(\gamma-1) F(\alpha, \beta, \gamma-1 ; x)=(\vartheta+\gamma-1) F(\alpha, \beta, \gamma ; x) .
$$

The contiguity relations are very important for the study of differential equations. For example the author's original proof of the connection formula (1.23) announced in $\mathrm{O} 6$ is based on the relations (cf. $\$ 14.3$ ).

Some results related to contiguity relations will be given in $\S 13$ but we will not go further in this subject and it will be discussed in another paper.

\section{FuCHSIAN DifFERENTIAL EQUATION AND GENERALIZED RiEMANn SCHEME}

6.1. Generalized characteristic exponents. We examine the Fuchsian differential equations

$$
P=a_{n}(x) \frac{d^{n}}{d x^{n}}+a_{n-1}(x) \frac{d^{n-1}}{d x^{n-1}}+\cdots+a_{0}(x)
$$

with given local monodromies at regular singular points. For this purpose we first study the condition so that monodromy generators of the solutions of a Fuchsian differential equation is semisimple even when its exponents are not free of multiplicity.

Lemma 6.1. Suppose that the operator (6.1) defined in a neighborhood of the origin has a regular singularity at the origin. We may assume $a_{\nu}(x)$ are holomorphic at 0 and $a_{n}(0)=a_{n}^{\prime}(0)=\cdots=a_{n}^{(n-1)}(0)=0$ and $a_{n}^{(n)}(0) \neq 0$. Then the following conditions are equivalent for a positive integer $k$.

$$
\begin{array}{rlrl}
P & =x^{k} R & & \text { with a suitable holomorphic differential operator } R \\
& \text { at the origin, } \\
P x^{\nu} & =o\left(x^{k-1}\right) & & \text { for } \nu=0, \ldots, k-1, \\
P u & =0 & \text { has a solution } x^{\nu}+o\left(x^{k-1}\right) \text { for } \nu=0, \ldots, k-1, \\
P & =\sum_{j \geq 0} x^{j} p_{j}(\vartheta) & \text { with polynomials } p_{j} \text { satisfying } p_{j}(\nu)=0
\end{array}
$$

Proof. 6.2$) \Rightarrow 6.3) \Leftrightarrow(6.4)$ is clear.

Assume (6.3). Then $P x^{\nu}=o\left(x^{k-1}\right)$ for $\nu=0, \ldots, k-1$ implies $a_{j}(x)=x^{k} b_{j}(x)$ for $j=0, \ldots, k-1$. Since $P$ has a regular singularity at the origin, $a_{j}(x)=x^{j} c_{j}(x)$ for $j=0, \ldots, n$. Hence we have (6.2).

Since $P x^{\nu}=\sum_{j=0}^{\infty} x^{\nu+j} p_{j}(\nu)$, the equivalence $\left.6.30 \Leftrightarrow 6.5\right)$ is clear.

Definition 6.2. Suppose $P$ in (6.1) has a regular singularity at $x=0$. Under the notation 2.57 we define that $P$ has a (generalized) characteristic exponent $[\lambda]_{(k)}$ at $x=0$ if $x^{n-k} \operatorname{Ad}\left(x^{-\lambda}\right)\left(a_{n}(x)^{-1} P\right) \in W[x]$. 
Note that Lemma 6.1 shows that $P$ has a characteristic exponent $[\lambda]_{(k)}$ at $x=0$ if and only if

$$
x^{n} a_{n}(x)^{-1} P=\sum_{j \geq 0} x^{j} q_{j}(\vartheta) \prod_{0 \leq i<k-j}(\vartheta-\lambda-i)
$$

with polynomials $q_{j}(t)$. By a coordinate transformation we can define generalized characteristic exponents for any regular singular point as follows.

Definition 6.3 (generalized characteristic exponents). Suppose $P$ in (6.1) has regular singularity at $x=c$. Let $n=m_{1}+\cdots+m_{q}$ be a partition of the positive integer $n$ and let $\lambda_{1}, \ldots, \lambda_{q}$ be complex numbers. We define that $P$ has the (set of generalized) characteristic exponents $\left\{\left[\lambda_{1}\right]_{\left(m_{1}\right)}, \ldots,\left[\lambda_{q}\right]_{\left(m_{q}\right)}\right\}$ and the spectral type $\left\{m_{1}, \ldots, m_{q}\right\}$ at $x=c \in \mathbb{C} \cup\{\infty\}$ if there exist polynomials $q_{\ell}(s)$ such that

$$
(x-c)^{n} a_{n}(x)^{-1} P=\sum_{\ell \geq 0}(x-c)^{\ell} q_{\ell}((x-c) \partial) \prod_{\nu=1}^{q} \prod_{0 \leq i<m_{\nu}-\ell}\left((x-c) \partial-\lambda_{\nu}-i\right)
$$

in the case when $c \neq \infty$ and

$$
x^{-n} a_{n}(x)^{-1} P=\sum_{\ell \geq 0} x^{-\ell} q_{\ell}(\vartheta) \prod_{\nu=1}^{q} \prod_{0 \leq i<m_{\nu}-\ell}\left(\vartheta+\lambda_{\nu}+i\right)
$$

in the case when $c=\infty$. Here if $m_{j}=1,\left[\lambda_{j}\right]_{\left(m_{j}\right)}$ may be simply written as $\lambda_{j}$.

Remark 6.4. i) In Definition 6.3 we may replace the left hand side of 6.7) by $\phi(x) a_{n}(x)^{-1} P$ where $\phi$ is analytic function in a neighborhood of $x=c$ such that $\phi(c)=\cdots=\phi^{(n-1)}(c)=0$ and $\phi^{(n)}(c) \neq 0$. In particular when $a_{n}(c)=\cdots=$ $a_{n}^{(n)}(c)=0$ and $a_{n}(c) \neq 0, P$ is said to be normalized at the singular point $x=c$ and the left hand side of (6.7) can be replaced by $P$.

In particular when $c=0$ and $P$ is normalized at the regular singular point $x=0$, the condition $(6.7)$ is equivalent to

$$
\prod_{\nu=1}^{k} \prod_{0 \leq i<m_{\nu}-\ell}\left(s-\lambda_{\nu}-i\right) \mid p_{j}(s) \quad\left(\forall \ell=0,1, \ldots, \max \left\{m_{1}, \ldots, m_{k}\right\}-1\right)
$$

under the expression $P=\sum_{j=0}^{\infty} x^{j} p_{j}(\vartheta)$.

ii) In Definition 6.3 the condition that the operator $P$ has a set of generalized characteristic exponents $\left\{\lambda_{1}, \ldots, \lambda_{n}\right\}$ is equivalent to the condition that it is the set of the usual characteristic exponents.

iii) Any one of $\{\lambda, \lambda+1, \lambda+2\},\left\{[\lambda]_{(2)}, \lambda+2\right\}$ and $\left\{\lambda,[\lambda+1]_{(2)}\right\}$ is the set of characteristic exponents of

$$
P=(\vartheta-\lambda)(\vartheta-\lambda-1)(\vartheta-\lambda-2+x)+x^{2}(\vartheta-\lambda+1)
$$

at $x=0$ but $\left\{[\lambda]_{(3)}\right\}$ is not.

iv) Suppose $P$ has a holomorphic parameter $t \in B_{1}(0)$ (cf. (3.7)) and $P$ has regular singularity at $x=c$. Suppose the set of the corresponding characteristic exponents is $\left\{\left[\lambda_{1}(t)\right]_{\left(m_{1}\right)}, \ldots,\left[\lambda_{q}(t)\right]_{\left(m_{q}\right)}\right\}$ for $t \in B_{1}(0) \backslash\{0\}$ with $\lambda_{\nu}(t) \in \mathcal{O}\left(B_{1}(0)\right)$. Then this is also valid in the case $t=0$, which clearly follows from the definition.

When

$$
P=\sum_{\ell \geq 0} x^{-\ell} q_{\ell}((x-c) \partial) \prod_{\nu=1}^{q} \prod_{0 \leq i<m_{\nu}-\ell}\left((x-c) \partial-\lambda_{\nu}-i\right)
$$

we put

$$
P_{t}=\sum_{\ell \geq 0} x^{-\ell} q_{\ell}((x-c) \partial) \prod_{\nu=1}^{q} \prod_{0 \leq i<m_{\nu}-\ell}\left((x-c) \partial-\lambda_{\nu}-\nu t-i\right)
$$


Here $\lambda_{\nu} \in \mathbb{C}, q_{0} \neq 0$ and ord $P=m_{1}+\cdots+m_{q}$. Then the set of the characteristic exponents of $P_{t}$ is $\left\{\left[\tilde{\lambda}_{1}(t)\right]_{\left(m_{1}\right)}, \ldots,\left[\tilde{\lambda}_{q}(t)\right]_{\left(m_{q}\right)}\right\}$ with $\tilde{\lambda}_{j}(t)=\lambda_{j}+j t$. Since $\tilde{\lambda}_{i}(t)-$ $\tilde{\lambda}_{j}(t) \notin \mathbb{Z}$ for $0<|t| \ll 1$, we can reduce certain claims to the case when the values of characteristic exponents are generic. Note that we can construct local independent solutions which holomorphically depend on $t$ (cf. $\mathrm{O} 4$ ).

Lemma 6.5. i) Let $\lambda$ be a complex number and let $p(t)$ be a polynomial such that $p(\lambda) \neq 0$. Then for non-negative integers $k$ and $m$ we have the exact sequence

$$
0 \longrightarrow \mathcal{O}_{0}(\lambda, k-1) \longrightarrow \mathcal{O}_{0}(\lambda, m+k-1) \stackrel{p(\vartheta)(\vartheta-\lambda)^{k}}{\longrightarrow} \mathcal{O}_{0}(\lambda, m-1) \longrightarrow 0
$$

under the notation (3.5).

ii) Let $m_{1}, \ldots, m_{q}$ be non-negative integers. Let $P$ be a differential operator of order $n$ whose coefficients are in $\mathcal{O}_{0}$ such that

$$
P=\sum_{\ell=0}^{\infty} x^{\ell} r_{\ell}(\vartheta) \prod_{\nu=1}^{q} \prod_{0 \leq k<m_{\nu}-\ell}(\vartheta-k)
$$

with polynomials $r_{\ell}$. Put $m_{\max }=\max \left\{m_{1}, \ldots, m_{q}\right\}$ and suppose $r_{0}(\nu) \neq 0$ for $\nu=0, \ldots, m_{\max }-1$.

Let $\mathbf{m}^{\vee}=\left(m_{1}^{\vee}, \ldots, m_{m_{\text {max }}}^{\vee}\right)$ be the dual partition of $\mathbf{m}:=\left(m_{1}, \ldots, m_{q}\right)$, namely,

$$
m_{\nu}^{\vee}=\#\left\{j ; m_{j} \geq \nu\right\} .
$$

Then for $i=0, \ldots, m_{\max }-1$ and $j=0, \ldots, m_{i+1}^{\vee}-1$ we have the functions

$$
u_{i, j}(x)=x^{i} \log ^{j} x+\sum_{\mu=i+1}^{m_{\max }-1} \sum_{\nu=0}^{j} c_{i, j}^{\mu, \nu} x^{\mu} \log ^{\nu} x
$$

such that $c_{i, j}^{\mu, \nu} \in \mathbb{C}$ and $P u_{i, j} \in \mathcal{O}_{0}\left(m_{\max }, j\right)$.

iii) Let $m_{1}^{\prime}, \ldots, m_{q^{\prime}}^{\prime}$ be non-negative integers and let $P^{\prime}$ be a differential operator of order $n^{\prime}$ whose coefficients are in $\mathcal{O}_{0}$ such that

$$
P^{\prime}=\sum_{\ell=0}^{\infty} x^{\ell} r_{\ell}^{\prime}(\vartheta) \prod_{\nu=1}^{q} \prod_{0 \leq k<m_{\nu}^{\prime}-\ell}\left(\vartheta-m_{\nu}-k\right)
$$

with polynomials $q_{\ell}^{\prime}$. Then for a differential operator $P$ of the form (6.10) we have

$$
P^{\prime} P=\sum_{\ell=0}^{\infty} x^{\ell}\left(\sum_{\nu=0}^{\ell} r_{\ell-\nu}^{\prime}(\vartheta+\nu) r_{\nu}(\vartheta)\right) \prod_{\nu=1}^{q} \prod_{0 \leq k<m_{\nu}+m_{\nu}^{\prime}-\ell}(\vartheta-k) .
$$

Proof. i) The claim is easy if $(p, k)=(1,1)$ or $(\vartheta-\mu, 0)$ with $\mu \neq \lambda$. Then the general case follows from induction on $\operatorname{deg} p(t)+k$.

ii) Put $P=\sum_{\ell>0} x^{\ell} p_{\ell}(\vartheta)$ and $m_{\nu}^{\vee}=0$ if $\nu>m_{\max }$. Then for a non-negative integer $\nu$, the multiplicity of the root $\nu$ of the equation $p_{\ell}(t)=0$ is equal or larger than $m_{\nu+\ell+1}^{\vee}$ for $\ell=1,2, \ldots$ If $0 \leq \nu \leq m_{\max }-1$, the multiplicity of the root $\nu$ of the equation $p_{0}(t)=0$ equals $m_{\nu+1}^{\vee}$.

For non-negative integers $i$ and $j$, we have

$$
x^{\ell} p_{\ell}(\vartheta) x^{i} \log ^{j} x=x^{i+\ell} \sum_{0 \leq \nu \leq j-m_{i+\ell+1}^{\vee}} c_{i, j, \ell, \nu} \log ^{\nu} x
$$

with suitable $c_{i, j, \ell, \nu} \in \mathbb{C}$. In particular, $p_{0}(\vartheta) x^{i} \log ^{j} x=0$ if $j<m_{i}^{\vee}$. If $\ell>0$ and $i+\ell<m_{\max }$, there exist functions

$$
v_{i, j, \ell}=x^{i+\ell} \sum_{\nu=0}^{j} a_{i, j, \ell, \nu} \log ^{\nu} x
$$


with suitable $a_{i, j, \ell, \nu} \in \mathbb{C}$ such that $p_{0}(\vartheta) v_{i, j, \ell}=x^{\ell} p_{\ell}(\vartheta) x^{i} \log ^{j} x$ and we define a $\mathbb{C}$-linear map $Q$ by

$$
Q x^{i} \log ^{j} x=-\sum_{\ell=1}^{m_{\max }-i-1} v_{i, j, \ell}=-\sum_{\ell=1}^{m_{\max }-i-1} \sum_{\nu=0}^{j} a_{i, j, \ell, \nu} x^{i+\ell} \log ^{\nu} x,
$$

which implies $p_{0}(\vartheta) Q x^{i} \log ^{j} x=-\sum_{\ell=1}^{m_{\max }-i-1} x^{\ell} p_{\ell}(\vartheta) x^{i} \log ^{j}$ and $Q^{m_{\max }}=0$. Putting $T u:=\sum_{\nu=0}^{m_{\max }-1} Q^{\nu} u$ for $u \in \sum_{i=0}^{m_{\max }-1} \sum_{j=0}^{q-1} \mathbb{C} x^{i} \log ^{j} x$, we have

$$
\begin{aligned}
P T u & \equiv p_{0}(\vartheta) T u+\sum_{\ell=1}^{m_{\max }-1} x^{\ell} p_{\ell}(\vartheta) T u & & \bmod \mathcal{O}_{0}\left(m_{\max }, j\right) \\
& \equiv p_{0}(\vartheta)(1-Q) T u & & \bmod \mathcal{O}_{0}\left(m_{\max }, j\right) \\
& \equiv p_{0}(\vartheta)(1-Q)\left(1+Q+\cdots+Q^{m_{\max }-1}\right) u & & \bmod \mathcal{O}_{0}\left(m_{\max }, j\right) \\
& =p_{0}(\vartheta) u . & &
\end{aligned}
$$

Hence if $j<m_{i}^{\vee}, P T x^{i} \log ^{j} x \equiv 0 \bmod \mathcal{O}_{0}\left(m_{\max }, j\right)$ and $u_{i, j}(x):=T x^{i} \log ^{j} x$ are required functions.

iii) Since

$$
\begin{aligned}
& x^{\ell^{\prime}} r_{\ell^{\prime}}^{\prime}(\vartheta) \prod_{\nu=1}^{q} \prod_{0 \leq k^{\prime}<m_{\nu}^{\prime}-\ell^{\prime}}\left(\vartheta-m_{\nu}-k^{\prime}\right) \cdot x^{\ell} r_{\ell}(\vartheta) \prod_{\nu=1}^{q} \prod_{0 \leq k<m_{\nu}-\ell}(\vartheta-k) \\
& =x^{\ell+\ell^{\prime}} r_{\ell^{\prime}}^{\prime}(\vartheta+\ell) r_{\ell}(\vartheta) \prod_{\nu=1}^{q} \prod_{0 \leq k^{\prime}<m_{\nu}^{\prime}-\ell^{\prime}}\left(\vartheta-m_{\nu}-k^{\prime}+\ell\right) \prod_{0 \leq k<m_{\nu}-\ell}(\vartheta-k) \\
& =x^{\ell+\ell^{\prime}} r_{\ell^{\prime}}^{\prime}(\vartheta+\ell) r_{\ell}(\vartheta) \prod_{\nu=1}^{q} \prod_{0 \leq k<m_{\nu}+m_{\nu^{\prime}}-\ell-\ell^{\prime}}(\vartheta-k),
\end{aligned}
$$

we have the claim.

Definition 6.6 (generalized Riemann scheme). Let $P \in W[x]$. Then we call $P$ is Fuchsian in this paper when $P$ has at most regular singularities in $\mathbb{C} \cup\{\infty\}$. Suppose $P$ is Fuchsian with regular singularities at $x=c_{0}=\infty, c_{1}, \ldots, c_{p}$ and the functions $\frac{a_{j}(x)}{a_{n}(x)}$ are holomorphic on $\mathbb{C} \backslash\left\{c_{1}, \ldots, c_{p}\right\}$ for $j=0, \ldots, n$. Moreover suppose $P$ has the set of characteristic exponents $\left\{\left[\lambda_{j, 1}\right]_{\left(m_{j, 1}\right)}, \ldots,\left[\lambda_{j, n_{j}}\right]_{\left(m_{j, n_{j}}\right)}\right\}$ at $x=c_{j}$. Then we define the Riemann scheme of $P$ or the equation $P u=0$ by

$$
\left\{\begin{array}{cccc}
x=c_{0}=\infty & c_{1} & \cdots & c_{p} \\
{\left[\lambda_{0,1}\right]_{\left(m_{0,1}\right)}} & {\left[\lambda_{1,1}\right]_{\left(m_{1,1}\right)}} & \cdots & {\left[\lambda_{p, 1}\right]_{\left(m_{p, 1}\right)}} \\
\vdots & \vdots & \vdots & \vdots \\
{\left[\lambda_{0, n_{0}}\right]_{\left(m_{0, n_{0}}\right)}} & {\left[\lambda_{1, n_{1}}\right]_{\left(m_{1, n_{1}}\right)}} & \cdots & {\left[\lambda_{p, n_{p}}\right]_{\left(m_{p, n_{p}}\right)}}
\end{array}\right\} .
$$

Remark 6.7. The Riemann scheme (6.15) always satisfies the Fuchs relation (cf. (3.21)):

$$
\sum_{j=0}^{p} \sum_{\nu=1}^{n_{j}} \sum_{i=0}^{m_{j, \nu}-1}\left(\lambda_{j, \nu}+i\right)=\frac{(p-1) n(n-1)}{2} .
$$

Definition 6.8 (spectral type). In Definition 6.6 we put

$$
\mathbf{m}=\left(m_{0,1}, \ldots, m_{0, n_{0}} ; m_{1,1}, \ldots ; m_{p, 1}, \ldots, m_{p, n_{p}}\right),
$$

which will be also written as $m_{0,1} m_{0,2} \cdots m_{0, n_{0}}, m_{1,1} \cdots, m_{p, 1} \cdots m_{p, n_{p}}$ for simplicity. Then $\mathbf{m}$ is a $(p+1)$-tuple of partitions of $n$ and we define that $\mathbf{m}$ is the spectral type of $P$. 
If the set of (usual) characteristic exponents

$$
\Lambda_{j}:=\left\{\lambda_{j, \nu}+i ; 0 \leq i \leq m_{j, \nu}-1 \text { and } \nu=1, \ldots, n_{\nu}\right\}
$$

of the Fuchsian differential operator $P$ at every regular singular point $x=c_{j}$ are $n$ different complex numbers, $P$ is said to have distinct exponents.

Remark 6.9. We remark that the Fuchsian differential equation $\mathcal{M}: P u=0$ is irreducible (cf. Definition 2.10) if and only if the monodromy of the equation is irreducible.

If $P=Q R$ with $Q$ and $R \in W(x ; \xi)$, the solution space of the equation $Q v=0$ is a subspace of that of $\mathcal{M}$ and closed under the monodromy and therefore the monodromy is reducible. Suppose the space spanned by certain linearly independent solutions $u_{1}, \ldots, u_{m}$ is invariant under the monodromy. We have a non-trivial simultaneous solution of the linear relations $b_{m} u_{j}^{(m)}+\cdots+b_{1} u_{j}^{(1)}+b_{0} u_{j}=0$ for $j=1, \ldots, m$. Then $\frac{b_{j}}{b_{m}}$ are single-valued holomorphic functions on $\mathbb{C} \cup\{\infty\}$ excluding finite number of singular points. In view of the local behavior of solutions, the singularities of $\frac{b_{j}}{b_{m}}$ are at most poles and hence they are rational functions. Then we may assume $R=b_{m} \partial^{m}+\cdots+b_{0} \in W(x ; \xi)$ and $P \in W(x ; \xi) R$.

Here we note that $R$ is Fuchsian but $R$ may have a singularity which is not a singularity of $P$ and is an apparent singularity. For example, we have

$$
x(1-x) \partial^{2}+(\gamma-\alpha x) \partial+\alpha=\left(\frac{\gamma}{\alpha}-x\right)^{-1}(x(1-x) \partial+(\gamma-\alpha x))\left(\left(\frac{\gamma}{\alpha}-x\right) \partial+1\right)
$$

We also note that the equation $\partial^{2} u=x u$ is irreducible and the monodromy of its solutions is reducible.

6.2. Tuples of partitions. For our purpose it will be better to allow some $m_{j, \nu}$ equal 0 and we generalize the notation of tuples of partitions as in 06 .

Definition 6.10. Let $\mathbf{m}=\left(m_{j, \nu}\right)_{\substack{j=0,1, \ldots \\ \nu=1,2, \ldots}}$ be an ordered set of infinite number of non-negative integers indexed by non-negative integers $j$ and positive integers $\nu$. Then $\mathbf{m}$ is called a $(p+1)$-tuple of partitions of $n$ if the following two conditions are satisfied.

$$
\begin{aligned}
\sum_{\nu=1}^{\infty} m_{j, \nu}=n & (j=0,1, \ldots), \\
m_{j, 1}=n & (\forall j>p) .
\end{aligned}
$$

A $(p+1)$-tuple of partition $\mathbf{m}$ is called monotone if

$$
m_{j, \nu} \geq m_{j, \nu+1} \quad(j=0,1, \ldots, \nu=1,2, \ldots)
$$

and called trivial if $m_{j, \nu}=0$ for $j=0,1, \ldots$ and $\nu=2,3, \ldots$. Moreover $\mathbf{m}$ is called standard if $\mathbf{m}$ is monotone and $m_{j, 2}>0$ for $j=0, \ldots, p$. The greatest common divisor of $\left\{m_{j, \nu} ; j=0,1, \ldots, \nu=1,2, \ldots\right\}$ is denoted by $\operatorname{gcd} \mathbf{m}$ and $\mathbf{m}$ is called divisible (resp. indivisible) if $\operatorname{gcd} \mathbf{m} \geq 2(\operatorname{resp} \operatorname{gcd} \mathbf{m}=1)$. The totality of $(p+1)$-tuples of partitions of $n$ are denoted by $\mathcal{P}_{p+1}^{(n)}$ and we put

$$
\begin{aligned}
\mathcal{P}_{p+1} & :=\bigcup_{n=0}^{\infty} \mathcal{P}_{p+1}^{(n)}, \quad \mathcal{P}^{(n)}:=\bigcup_{p=0}^{\infty} \mathcal{P}_{p+1}^{(n)}, \quad \mathcal{P}:=\bigcup_{p=0}^{\infty} \mathcal{P}_{p+1}, \\
\operatorname{ord} \mathbf{m} & :=n \quad \text { if } \mathbf{m} \in \mathcal{P}^{(n)}, \\
\mathbf{1} & :=(1,1, \ldots)=\left(m_{j, \nu}=\delta_{\nu, 1}\right)_{\substack{j=0,1, \ldots \\
\nu=1,2, \ldots}} \in \mathcal{P}^{(1)},
\end{aligned}
$$




$$
\begin{aligned}
\operatorname{idx}\left(\mathbf{m}, \mathbf{m}^{\prime}\right) & :=\sum_{j=0}^{p} \sum_{\nu=1}^{\infty} m_{j, \nu} m_{j, \nu}^{\prime}-(p-1) \operatorname{ord} \mathbf{m} \cdot \operatorname{ord} \mathbf{m}^{\prime}, \\
\operatorname{idx} \mathbf{m} & :=\operatorname{idx}(\mathbf{m}, \mathbf{m})=\sum_{j=0}^{p} \sum_{\nu=1}^{\infty} m_{j, \nu}^{2}-(p-1) \operatorname{ord} \mathbf{m}^{2}, \\
\operatorname{Pidx} \mathbf{m} & :=1-\frac{\operatorname{idx} \mathbf{m}}{2} .
\end{aligned}
$$

Here ord $\mathbf{m}$ is called the order of $\mathbf{m}$. For $\mathbf{m}, \mathbf{m}^{\prime} \in \mathcal{P}$ and a non-negative integer $k, \mathbf{m}+k \mathbf{m}^{\prime} \in \mathcal{P}$ is naturally defined. Note that

$$
\begin{aligned}
\operatorname{idx}\left(\mathbf{m}+\mathbf{m}^{\prime}\right) & =\operatorname{idx} \mathbf{m}+\operatorname{idx} \mathbf{m}^{\prime}+2 \operatorname{idx}\left(\mathbf{m}, \mathbf{m}^{\prime}\right), \\
\operatorname{Pidx}\left(\mathbf{m}+\mathbf{m}^{\prime}\right) & =\operatorname{Pidx} \mathbf{m}+\operatorname{Pidx} \mathbf{m}^{\prime}-\operatorname{idx}\left(\mathbf{m}, \mathbf{m}^{\prime}\right)-1 .
\end{aligned}
$$

For $\mathbf{m} \in \mathcal{P}_{p+1}^{(n)}$ we choose integers $n_{0}, \ldots, n_{k}$ so that $m_{j, \nu}=0$ for $\nu>n_{j}$ and $j=0, \ldots, p$ and we will sometimes express $\mathbf{m}$ as

$$
\begin{aligned}
\mathbf{m} & =\left(\mathbf{m}_{0}, \mathbf{m}_{1}, \ldots, \mathbf{m}_{p}\right) \\
& =m_{0,1}, \ldots, m_{0, n_{0}} ; \ldots ; m_{k, 1}, \ldots, m_{p, n_{p}} \\
& =m_{0,1} \cdots m_{0, n_{0}}, m_{1,1} \cdots m_{1, n_{1}}, \ldots, m_{k, 1} \cdots m_{p, n_{p}}
\end{aligned}
$$

if there is no confusion. Similarly $\mathbf{m}=\left(m_{0,1}, \ldots, m_{0, n_{0}}\right)$ if $\mathbf{m} \in \mathcal{P}_{1}$. Here

$$
\mathbf{m}_{j}=\left(m_{j, 1}, \ldots, m_{j, n_{j}}\right) \text { and } \quad \text { ord } \mathbf{m}=m_{j, 1}+\cdots+m_{j, n_{j}} \quad(0 \leq j \leq p) .
$$

For example $\mathbf{m}=\left(m_{j, \nu}\right) \in \mathcal{P}_{3}^{(4)}$ with $m_{1,1}=3$ and $m_{0, \nu}=m_{2, \nu}=m_{1,2}=1$ for $\nu=1, \ldots, 4$ will be expressed by

$$
\mathbf{m}=1,1,1,1 ; 3,1 ; 1,1,1,1=1111,31,1111=1^{4}, 31,1^{4} .
$$

Let $\mathfrak{S}_{\infty}$ be the restricted permutation group of the set of indices $\{0,1,2,3, \ldots\}=$ $\mathbb{Z}_{\geq 0}$, which is generated by the transpositions $(j, j+1)$ with $j \in \mathbb{Z}_{\geq 0}$. Put $\mathfrak{S}_{\infty}^{\prime}=$ $\left\{\sigma \in \mathfrak{S}_{\infty} ; \sigma(0)=0\right\}$, which is isomorphic to $\mathfrak{S}_{\infty}$.

Definition 6.11. The transformation groups $S_{\infty}$ and $S_{\infty}^{\prime}$ of $\mathcal{P}$ are defined by

$$
\begin{aligned}
S_{\infty} & :=H \ltimes S_{\infty}^{\prime}, \\
S_{\infty}^{\prime} & :=\left\{\left(\sigma_{i}\right)_{i=0,1, \ldots} ; \sigma_{i} \in \mathfrak{S}_{\infty}^{\prime}, \sigma_{i}=1 \quad(i \gg 1)\right\}, \quad H \simeq \mathfrak{S}_{\infty}, \\
m_{j, \nu}^{\prime} & =m_{\sigma(j), \sigma_{j}(\nu)} \quad(j=0,1, \ldots, \nu=1,2, \ldots)
\end{aligned}
$$

for $g=\left(\sigma, \sigma_{1}, \ldots\right) \in S_{\infty}, \mathbf{m}=\left(m_{j, \nu}\right) \in \mathcal{P}$ and $\mathbf{m}^{\prime}=g \mathbf{m}$. A tuple $\mathbf{m} \in \mathcal{P}$ is isomorphic to a tuple $\mathbf{m}^{\prime} \in \mathcal{P}$ if there exists $g \in S_{\infty}$ such that $\mathbf{m}^{\prime}=g \mathbf{m}$. We denote by $s \mathbf{m}$ the unique monotone element in $S_{\infty}^{\prime} \mathbf{m}$.

Definition 6.12. For a tuple of partitions $\mathbf{m}=\left(m_{j, \nu}\right)_{\substack{1 \leq \nu \leq n_{j} \\ 0 \leq j \leq p}} \in \mathcal{P}_{p+1}$ and $\lambda=$ $\left(\lambda_{j, \nu}\right)_{\substack{1 \leq \nu \leq n_{j} \\ 0 \leq j \leq p}}$ with $\lambda_{j, \nu} \in \mathbb{C}$, we define

$$
\left|\left\{\lambda_{\mathbf{m}}\right\}\right|:=\sum_{j=0}^{p} \sum_{\nu=1}^{n_{j}} m_{j, \nu} \lambda_{j, \nu}-\operatorname{ord} \mathbf{m}+\frac{\mathrm{idx} \mathbf{m}}{2} .
$$

We note that the Fuchs relation (6.16) is equivalent to

$$
\left|\left\{\lambda_{\mathbf{m}}\right\}\right|=0
$$


because

$$
\begin{aligned}
\sum_{j=0}^{p} \sum_{\nu=1}^{n_{j}} \sum_{i=0}^{m_{j, \nu}-1} i & =\frac{1}{2} \sum_{j=0}^{p} \sum_{\nu=1}^{n_{j}} m_{j, \nu}\left(m_{j, \nu}-1\right)=\frac{1}{2} \sum_{j=0}^{p} \sum_{\nu=1}^{n_{j}} m_{j, \nu}^{2}-\frac{1}{2}(p+1) n \\
& =\frac{1}{2}\left(\operatorname{idx} \mathbf{m}+(p-1) n^{2}\right)-\frac{1}{2}(p+1) n \\
& =\frac{1}{2} \mathrm{idx} \mathbf{m}-n+\frac{(p-1) n(n-1)}{2} .
\end{aligned}
$$

6.3. Conjugacy classes of matrices. Now we review on the conjugacy classes of matrices. For $\mathbf{m}=\left(m_{1}, \ldots, m_{N}\right) \in \mathcal{P}_{1}^{(n)}$ and $\lambda=\left(\lambda_{1}, \ldots, \lambda_{N}\right) \in \mathbb{C}^{N}$ we define a matrix $L(\mathbf{m} ; \lambda) \in M(n, \mathbb{C})$ as follows, which is introduced and effectively used by O2 and $\mathrm{O} 6$ :

If $\mathbf{m}$ is monotone, then

$$
\begin{aligned}
& L(\mathbf{m} ; \lambda):=\left(A_{i j}\right)_{\substack{1 \leq i \leq N \\
1 \leq j \leq N}}, \quad A_{i, j} \in M\left(m_{i}, m_{j}, \mathbb{C}\right), \\
& A_{i j}= \begin{cases}\lambda_{i} I_{m_{i}} \\
I_{m_{i}, m_{j}}:=\left(\delta_{\mu \nu}\right)_{\substack{1 \leq \mu \leq n_{i} \\
1 \leq \nu \leq n_{j}}}=\left(\begin{array}{c}
I_{m_{j}} \\
0
\end{array}\right) & (i=j-1), \\
0 & (i \neq j, j-1) .\end{cases}
\end{aligned}
$$

Here $I_{m_{i}}$ denote the identity matrix of size $m_{i}$ and $M\left(m_{i}, m_{j}, \mathbb{C}\right)$ means the set of matrices of size $m_{i} \times m_{j}$ with components in $\mathbb{C}$ and $M(m, \mathbb{C}):=M(m, m, \mathbb{C})$.

For example

$$
L\left(2,1,1 ; \lambda_{1}, \lambda_{2}, \lambda_{3}\right):=\left(\begin{array}{cccc}
\lambda_{1} & 0 & 1 & \\
0 & \lambda_{1} & 0 & \\
& & \lambda_{2} & 1 \\
& & & \lambda_{3}
\end{array}\right) .
$$

If $\mathbf{m}$ is not monotone, we fix a permutation $\sigma$ of $\{1, \ldots, N\}$ so that $\left(m_{\sigma(1)}, \ldots, m_{\sigma(N)}\right)$ is monotone and put $L(\mathbf{m} ; \lambda)=L\left(m_{\sigma(1)}, \ldots, m_{\sigma(N)} ; \lambda_{\sigma(1)}, \ldots, \lambda_{\sigma(N)}\right)$.

When $\lambda_{1}=\cdots=\lambda_{N}=\mu, L(\mathbf{m} ; \lambda)$ may be simply denoted by $L(\mathbf{m}, \mu)$.

We denote $A \sim B$ for $A, B \in M(n, \mathbb{C})$ if and only if there exists $g \in G L(n, \mathbb{C})$ with $B=g A g^{-1}$.

When $A \sim L(\mathbf{m} ; \lambda), \mathbf{m}$ is called the spectral type of $A$ and denoted by $\operatorname{spc} A$ with a monotone $\mathbf{m}$.

Remark 6.13. i) If $\mathbf{m}=\left(m_{1}, \ldots, m_{K}\right) \in \mathcal{P}_{1}^{(n)}$ is monotone, we have

$$
A \sim L(\mathbf{m} ; \lambda) \Leftrightarrow \operatorname{rank} \prod_{\nu=1}^{j}\left(A-\lambda_{\nu}\right)=n-\left(m_{1}+\cdots+m_{j}\right) \quad(j=0,1, \ldots, K) .
$$

ii) For $\mu \in \mathbb{C}$, put

$$
(\mathbf{m} ; \lambda)_{\mu}=\left(m_{i_{1}}, \ldots, m_{i_{K}} ; \mu\right) \text { with }\left\{i_{1}, \ldots, i_{K}\right\}=\left\{i ; \lambda_{i}=\mu\right\} .
$$

Then we have

$$
L(\mathbf{m} ; \lambda) \sim \bigoplus_{\mu \in \mathbb{C}} L\left((\mathbf{m} ; \lambda)_{\mu}\right) .
$$

iii) Suppose $\mathbf{m}$ is monotone. Then for $\mu \in \mathbb{C}$

$$
\begin{aligned}
& L(\mathbf{m}, \mu) \sim \bigoplus_{j=1}^{m_{1}} J\left(\max \left\{\nu ; m_{\nu} \geq j\right\}, \mu\right), \\
& J(k, \mu):=L\left(1^{k}, \mu\right) \in M(k, \mathbb{C}) .
\end{aligned}
$$


iv) For $A \in M(n, \mathbb{C})$, we put $Z(A)=Z_{M(n, \mathbb{C})}(A):=\{X \in M(n, \mathbb{C}) ; A X=$ $X A\}$. Then

$$
\operatorname{dim} Z_{M(n, \mathbb{C})}(L(\mathbf{m}, \lambda))=m_{1}^{2}+m_{2}^{2}+\cdots
$$

v) (cf. O8, Lemma 3.1]). Let $\mathbf{A}(t):[0,1) \rightarrow M(n, \mathbb{C})$ be a continuous function. Suppose there exist a continuous function $\lambda=\left(\lambda_{1}, \ldots, \lambda_{K}\right):[0,1) \rightarrow \mathbb{C}^{K}$ such that $A(t) \sim L(\mathbf{m} ; \lambda(t))$ for $t \in(0,1)$. Then

$$
A(0) \sim L(\mathbf{m} ; \lambda(0)) \text { if and only if } \operatorname{dim} Z(A(0))=m_{1}^{2}+\cdots+m_{K}^{2} .
$$

Note that the Jordan canonical form of $L(\mathbf{m} ; \lambda)$ is easily obtained by $(6.35)$ and 6.36). For example, $L(2,1,1 ; \mu) \simeq J(3, \mu) \oplus J(1, \mu)$.

\subsection{Realizable tuples of partitions.}

Proposition 6.14. Let $P u=0$ be a differential equation of order $n$ which has a regular singularity at 0 . Let $\left\{\left[\lambda_{1}\right]_{\left(m_{1}\right)}, \ldots,\left[\lambda_{q}\right]_{\left(m_{q}\right)}\right\}$ be the corresponding set of the characteristic exponents. Here $\mathbf{m}=\left(m_{1}, \ldots, m_{q}\right)$ a partition of $n$.

i) Suppose there exists $k$ such that

$$
\begin{gathered}
\lambda_{1}=\lambda_{2}=\cdots=\lambda_{k}, \\
m_{1} \geq m_{2} \geq \cdots \geq m_{k}, \\
\lambda_{j}-\lambda_{1} \notin \mathbb{Z} \quad(j=k+1, \ldots, q) .
\end{gathered}
$$

Let $\mathbf{m}^{\vee}=\left(m_{1}^{\vee}, \ldots, m_{r}^{\vee}\right)$ be the dual partition of $\left(m_{1}, \ldots, m_{k}\right)$ (cf. (6.11)). Then for $i=0, \ldots, m_{1}-1$ and $j=0, \ldots, m_{i+1}^{\vee}-1$ the equation has the solutions

$$
u_{i, j}(x)=\sum_{\nu=0}^{j} x^{\lambda_{1}+i} \log ^{\nu} x \cdot \phi_{i, j, \nu}(x) .
$$

Here $\phi_{i, j, \nu}(x) \in \mathcal{O}_{0}$ and $\phi_{i, \nu, j}(0)=\delta_{\nu, j}$ for $\nu=0, \ldots, j-1$.

ii) Suppose

$$
\lambda_{i}-\lambda_{j} \neq \mathbb{Z} \backslash\{0\} \quad(0 \leq i<j \leq q) .
$$

In this case we say that the set of characteristic exponents $\left\{\left[\lambda_{1}\right]_{\left(m_{1}\right)}, \ldots,\left[\lambda_{q}\right]_{\left(m_{q}\right)}\right\}$ is distinguished. Then the monodromy generator of the solutions of the equation at 0 is conjugate to

$$
L\left(\mathbf{m} ;\left(e^{2 \pi \sqrt{-1} \lambda_{1}}, \ldots, e^{2 \pi \sqrt{-1}} \lambda_{q}\right)\right) .
$$

Proof. Lemma 6.5 ii) shows that there exist $u_{i, j}(x)$ of the form stated in i) which satisfy $P u_{i, j}(x) \in \mathcal{O}_{0}\left(\lambda_{1}+m_{1}, j\right)$ and then we have $v_{i, j}(x) \in \mathcal{O}_{0}\left(\lambda_{1}+m_{1}, j\right)$ such that $P u_{i, j}(x)=P v_{i, j}(x)$ because of (3.6). Thus we have only to replace $u_{i, j}(x)$ by $u_{i, j}(x)-v_{i, j}(x)$ to get the claim in i). The claim in ii) follows from that of $\mathrm{i}$ ).

Remark 6.15. i) Suppose $P$ is a Fuchsian differential operator with regular singularities at $x=c_{0}=\infty, c_{1}, \ldots, c_{p}$ and moreover suppose $P$ has distinct exponents. Then the Riemann scheme of $P$ is (6.15) if and only if $P u=0$ has local solutions $u_{j, \nu, i}(x)$ of the form

$$
u_{j, \nu, i}(x)=\left\{\begin{array}{c}
\left(x-c_{j}\right)^{\lambda_{j, \nu}+i}\left(1+o\left(\left|x-c_{j}\right|^{m_{j}, \nu-i-1}\right)\right) \\
\left(x \rightarrow c_{j}, i=0, \ldots, m_{j, \nu}-1, j=1, \ldots, p\right), \\
x^{-\lambda_{0, \nu}-i}\left(1+o\left(x^{-m_{0, \nu}+i+1}\right)\right) \\
\left(x \rightarrow \infty, i=0, \ldots, m_{0, \nu}\right) .
\end{array}\right.
$$

Moreover suppose $\lambda_{j, \nu}-\lambda_{j, \nu^{\prime}} \notin \mathbb{Z}$ for $1 \leq \nu<\nu^{\prime} \leq n_{j}$ and $j=0, \ldots, p$. Then

$$
u_{j, \nu, i}(x)= \begin{cases}\left(x-c_{j}\right)^{\lambda_{j, \nu}+i} \phi_{j, \nu, i}(x) & (1 \leq j \leq p) \\ x^{-\lambda_{0, \nu}-i} \phi_{0, \nu, i}(x) & (j=0)\end{cases}
$$


with $\phi_{j, \nu, i}(x) \in \mathcal{O}_{c_{j}}$ satisfying $\phi_{j, \nu, i}\left(c_{j}\right)=1$. In this case $P$ has the Riemann scheme (6.15) if and only if at the each singular point $x=c_{j}$, the set of characteristic exponents of the equation $P u=0$ equals $\Lambda_{j}$ in (6.17) and the monodromy generator of its solutions is semisimple.

ii) Suppose $P$ has the Riemann scheme (6.15) and $\lambda_{1,1}=\cdots=\lambda_{1, n_{1}}$. Then the monodromy generator of the solutions of $P u=0$ at $x=c_{1}$ has the eigenvalue $e^{2 \pi \sqrt{-1}} \lambda_{1,1}$ with multiplicity $n$. Moreover the monodromy generator is conjugate to the matrix $L\left(\left(m_{1,1}, \ldots, m_{1, n_{1}}\right), e^{2 \pi \sqrt{-1}} \lambda_{1,1}\right)$, which is also conjugate to

$$
J\left(m_{1,1}^{\vee}, e^{2 \pi \sqrt{-1} \lambda_{1,1}}\right) \oplus \cdots \oplus J\left(m_{1, n_{1}^{\prime}}^{\vee}, e^{2 \pi \sqrt{-1} \lambda_{1,1}}\right) .
$$

Here $\left(m_{1,1}^{\vee}, \ldots, m_{1, n_{1}^{\vee}}^{\vee}\right)$ is the dual partition of $\left(m_{1,1}, \ldots, m_{1, n_{1}}\right)$. A little weaker condition for $\lambda_{j, \nu}$ assuring the same conclusion is given in Proposition 11.9.

Definition 6.16 (realizable spectral type). Let $\mathbf{m}=\left(\mathbf{m}_{0}, \ldots, \mathbf{m}_{p}\right)$ be a $(p+1)$ tuple of partitions of a positive integer $n$. Here $\mathbf{m}_{j}=\left(m_{j, 1}, \ldots, m_{j, n_{j}}\right)$ and $n=$ $m_{j, 1}+\cdots+m_{j, n_{j}}$ for $j=0, \ldots, p$ and $m_{j, \nu}$ are non-negative numbers. Fix $p$ different points $c_{j}(j=1, \ldots, p)$ in $\mathbb{C}$ and put $c_{0}=\infty$.

Then $\mathbf{m}$ is a realizable spectral type if there exists a Fuchsian operator $P$ with the Riemann scheme 6.15) for generic $\lambda_{j, \nu}$ satisfying the Fuchs relation 6.16). Moreover in this case if there exists such $P$ so that the equation $P u=0$ is irreducible, which is equivalent to say that the monodromy of the equation is irreducible, then $\mathbf{m}$ is irreducibly realizable.

Remark 6.17. i) In the above definition $\left\{\lambda_{j, \nu}\right\}$ are generic if, for example, $0<$ $m_{0,1}<$ ord $\mathbf{m}$ and $\left\{\lambda_{j, \nu} ;(j, \nu) \neq(0,1), j=0, \ldots, p, 1 \leq \nu \leq n_{j}\right\} \cup\{1\}$ are linearly independent over $\mathbb{Q}$.

ii) It follows from the facts (cf. 3.22$)$ ) in $\$ 3.1$ that if $\mathbf{m} \in \mathcal{P}$ satisfies

$$
\begin{array}{r}
\left|\left\{\lambda_{\mathbf{m}^{\prime}}\right\}\right| \notin \mathbb{Z}_{\leq 0}=\{0,-1,-2, \ldots\} \text { for any } \mathbf{m}^{\prime}, \mathbf{m}^{\prime \prime} \in \mathcal{P} \\
\text { satisfying } \mathbf{m}=\mathbf{m}^{\prime}+\mathbf{m}^{\prime \prime} \text { and } 0<\text { ord } \mathbf{m}^{\prime}<\text { ord } \mathbf{m},
\end{array}
$$

the Fuchsian differential equation with the Riemann scheme (6.15) is irreducible. Hence if $\mathbf{m}$ is indivisible and realizable, $\mathbf{m}$ is irreducibly realizable.

Fix distinct $p$ points $c_{1}, \ldots, c_{p}$ in $\mathbb{C}$ and put $c_{0}=\infty$. The Fuchsian differential operator $P$ with regular singularities at $x=c_{j}$ for $j=1, \ldots, n$ has the normal form

$$
P=\left(\prod_{j=1}^{p}\left(x-c_{j}\right)^{n}\right) \partial^{n}+a_{n-1}(x) \partial^{n-1}+\cdots+a_{1}(x) \partial+a_{0}(x),
$$

where $a_{i}(x) \in \mathbb{C}[x]$ satisfy

$$
\begin{aligned}
\operatorname{deg} a_{i}(x) & \leq(p-1) n+i \\
\left(\partial^{\nu} a_{i}\right)\left(c_{j}\right) & =0 \quad(0 \leq \nu \leq i-1)
\end{aligned}
$$

for $i=0, \ldots, n-1$.

Note that the condition (6.44) (resp. 6.45)) corresponds to the fact that $P$ has regular singularities at $x=c_{j}$ for $j=1, \ldots, p$ (resp. at $x=\infty$ ).

Since $a_{i}(x)=b_{i}(x) \prod_{j=1}^{p}\left(x-c_{j}\right)^{i}$ with $b_{i}(x)=\sum_{r=0}^{(p-1)(n-i)} b_{i, r} x^{r} \in W[x]$ satisfying $\operatorname{deg} b_{i}(x) \leq(p-1) n+i-p i=(p-1)(n-i)$, the operator $P$ has the parameters $\left\{b_{i, r}\right\}$. The numbers of the parameters equals

$$
\sum_{i=0}^{n-1}((p-1)(n-i)+1)=\frac{(p n+p-n+1) n}{2}
$$


The condition $\left(x-c_{j}\right)^{-k} P \in W[x]$ implies $\left(\partial^{\ell} a_{i}\right)\left(c_{j}\right)=0$ for $0 \leq \ell \leq k-1$ and $0 \leq i \leq n$, which equals $\left(\partial^{\ell} b_{i}\right)\left(c_{j}\right)=0$ for $0 \leq \ell \leq k-1-i$ and $0 \leq i \leq k-1$. Therefore the condition

$$
\left(x-c_{j}\right)^{-m_{j, \nu}} \operatorname{Ad}\left(\left(x-c_{j}\right)^{-\lambda_{j, \nu}}\right) P \in W[x]
$$

gives $\frac{\left(m_{j, \nu}+1\right) m_{j, \nu}}{2}$ independent linear equations for $\left\{b_{\nu, r}\right\}$ since $\sum_{i=0}^{m_{j, \nu}-1}\left(m_{j, \nu}-\right.$ $i)=\frac{\left(m_{j, \nu}+1\right) m_{j, \nu}}{2}$. If all these equations have a simultaneous solution and they are independent except for the relation caused by the Fuchs relation, the number of the parameters of the solution equals

$$
\begin{aligned}
& \frac{(p n+p-n+1) n}{2}-\sum_{j=0}^{p} \sum_{\nu=1}^{n_{j}} \frac{m_{j, \nu}\left(m_{j, \nu}+1\right)}{2}+1 \\
& =\frac{(p n+p-n+1) n}{2}-\sum_{j=0}^{p} \sum_{\nu=1}^{n_{j}} \frac{m_{j, \nu}^{2}}{2}-(p+1) \frac{n}{2}+1 \\
& =\frac{1}{2}\left((p-1) n^{2}-\sum_{j=0}^{p} \sum_{\nu=1}^{n_{j}} m_{j, \nu}^{2}+1\right)=\operatorname{Pidx} \mathbf{m} .
\end{aligned}
$$

Remark 6.18 (cf. [06, §5]). Katz [Ka] introduced the index of rigidity of an irreducible local system by the number idx $\mathbf{m}$ whose spectral type equals $\mathbf{m}=$ $\left(m_{j, \nu}\right)_{\substack{j=0, \ldots, p \\ \nu=1, \ldots, n_{j}}}$ and proves idx $\mathbf{m} \leq 2$, if the local system is irreducible.

Assume the local system is irreducible. Then Katz [K] shows that the local system is uniquely determined by the local monodromies if and only if idx $\mathbf{m}=2$ and in this case the local system and the tuple of partition $\mathbf{m}$ are called rigid. If idx $\mathbf{m}>2$, the corresponding system of differential equations of Schleginger normal form

$$
\frac{d u}{d x}=\sum_{j=1}^{p} \frac{A_{j}}{x-a_{j}} u
$$

has 2 Pidx $\mathbf{m}$ parameters which are independent from the characteristic exponents and local monodromies. They are called accessory parameters. Here $A_{j}$ are constant square matrices of size $n$. The number of accessory parameters of the single Fuchsian differential operator without apparent singularities will be the half of this number 2 Pidx $\mathbf{m}$ (cf. Theorem 8.13 and $[\mathrm{Sz}]$ ).

Lastly in this subsection we calculate the Riemann scheme of the products and the dual of Fuchsian differential operators.

Theorem 6.19. Let $P$ be a Fuchsian differential operator with the Riemann scheme 6.15). Suppose $P$ has the normal form (6.43).

i) Let $P^{\prime}$ be a Fuchsian differential operator with regular singularities also at $x=c_{0}=\infty, c_{1}, \ldots, c_{p}$. Then if $P^{\prime}$ has the Riemann scheme

$$
\left\{\begin{array}{cc}
x=c_{0}=\infty & c_{j}(j=1, \ldots, p) \\
{\left[\lambda_{0,1}+m_{0,1}-(p-1) \operatorname{ord} \mathbf{m}\right]_{\left(m_{0,1}^{\prime}\right)}} & {\left[\lambda_{j, 1}+m_{j, 1}\right]_{\left(m_{j, 1}^{\prime}\right)}} \\
\vdots & \vdots \\
{\left[\lambda_{0, n_{0}}+m_{0, n_{0}}-(p-1) \operatorname{ord} \mathbf{m}\right]_{\left(m_{0, n_{0}}^{\prime}\right)}} & {\left[\lambda_{j, n_{j}}+m_{j, n_{j}}\right]_{\left(m_{j, n_{j}}^{\prime}\right)}}
\end{array}\right\}
$$


the Fuchsian operator $P^{\prime} P$ has the spectral type $\mathbf{m}+\mathbf{m}^{\prime}$ and the Riemann scheme

$$
\left\{\begin{array}{cccc}
x=c_{0}=\infty & c_{1} & \cdots & c_{p} \\
{\left[\lambda_{0,1}\right]_{\left(m_{0,1}+m_{0,1}^{\prime}\right)}} & {\left[\lambda_{1,1}\right]_{\left(m_{1,1}+m_{1,1}^{\prime}\right)}} & \cdots & {\left[\lambda_{p, 1}\right]_{\left(m_{p, 1}+m_{p, 1}^{\prime}\right)}} \\
\vdots & \vdots & \vdots & \vdots \\
{\left[\lambda_{0, n_{0}}\right]_{\left(m_{0, n_{0}}+m_{0, n_{0}}^{\prime}\right)}} & {\left[\lambda_{1, n_{1}}\right]_{\left(m_{1, n_{1}}+m_{1, n_{1}}^{\prime}\right)}} & \cdots & {\left[\lambda_{p, n_{p}}\right]_{\left(m_{p, n_{p}}+m_{1, n_{p}}^{\prime}\right)}}
\end{array}\right\}
$$

Suppose the Fuchs relation (6.32) for (6.15). Then the Fuchs relation for (6.49) is valid if and only if so is the Fuchs relation for (6.50).

ii) For $Q=\sum_{k \geq 0} q_{k}(x) \partial^{k} \in W(x)$, we define

$$
Q^{*}:=\sum_{k \geq 0}(-\partial)^{k} q_{k}(x)
$$

and the dual operator $P^{\vee}$ of $P$ by

$$
P^{\vee}:=a_{n}(x)\left(a_{n}(x)^{-1} P\right)^{*}
$$

when $P=\sum_{k=0}^{n} a_{k}(x) \partial^{k}$. Then the Riemann scheme of $P^{\vee}$ equals

$$
\left\{\begin{array}{cc}
x=c_{0}=\infty & c_{j}(j=1, \ldots, p) \\
{\left[2-n-m_{0,1}-\lambda_{0,1}\right]_{\left(m_{0,1}\right)}} & {\left[n-m_{j, 1}-\lambda_{j, 1}\right]_{\left(m_{j, 1}\right)}} \\
\vdots & \vdots \\
{\left[2-n-m_{0, n_{0}}-\lambda_{0, n_{0}}\right]_{\left(m_{0, n_{0}}\right)}} & {\left[n-m_{j, n_{j}}-\lambda_{j, n_{j}}\right]_{\left(m_{j, n_{j}}\right)}}
\end{array}\right\} .
$$

Proof. i) It is clear that $P^{\prime} P$ is a Fuchsian differential operator of the normal form if so is $P^{\prime}$ and Lemma 6.5 iii) shows that the characteristic exponents of $P^{\prime} P$ at $x=c_{j}$ for $j=1, \ldots, p$ are just as given in the Riemann scheme (5.50). Put $n=$ ord $\mathbf{m}$ and $n^{\prime}=\mathbf{m}^{\prime}$. We can also apply Lemma 6.5 iii) to $x^{-(p-1) n} P$ and $x^{-(p-1) n^{\prime}} P^{\prime}$ under the coordinate transformation $x \mapsto \frac{1}{x}$, we have the set of characteristic exponents as is given in $(6.50)$ because $x^{-(p-1)\left(n+n^{\prime}\right)} P^{\prime} P=\left(\operatorname{Ad}\left(x^{-(p-1) n}\right) x^{-(p-1) n^{\prime}} P^{\prime}\right)\left(x^{-(p-1) n}\right) P$.

The Fuchs relation for 6.49) equals

$$
\sum_{j=0}^{p} \sum_{\nu=1}^{n_{j}} m_{j, \nu}^{\prime}\left(\lambda_{j, \nu}+m_{j, \nu}-\delta_{j, 0}(p-1) \operatorname{ord} \mathbf{m}\right)=\operatorname{ord} \mathbf{m}^{\prime}-\frac{\mathrm{idx} \mathbf{m}^{\prime}}{2} .
$$

Since

$$
\sum_{j=0}^{p} \sum_{\nu=1}^{n_{j}} m_{j, \nu}^{\prime}\left(m_{j, \nu}-\delta_{j, 0}(p-1) \operatorname{ord} \mathbf{m}\right)=\operatorname{idx}\left(\mathbf{m}, \mathbf{m}^{\prime}\right),
$$

the condition is equivalent to

$$
\sum_{j=0}^{p} \sum_{\nu=1}^{n_{j}} m_{j, \nu}^{\prime} \lambda_{j, \nu}=\operatorname{ord} \mathbf{m}^{\prime}-\frac{\mathrm{idx} \mathbf{m}}{2}-\mathrm{idx}\left(\mathbf{m}, \mathbf{m}^{\prime}\right)
$$

and also to

$$
\sum_{j=0}^{p} \sum_{\nu=1}^{n_{j}}\left(m_{j, \nu}+m_{j, \nu}^{\prime}\right) \lambda_{j, \nu}=\operatorname{ord}\left(\mathbf{m}+\mathbf{m}^{\prime}\right)-\frac{\operatorname{idx}\left(\mathbf{m}+\mathbf{m}^{\prime}\right)}{2}
$$

under the condition (6.32). 
ii) We may suppose $c_{1}=0$. Then

$$
\begin{aligned}
a_{n}(x)^{-1} P & =\sum_{\ell \geq 0} x^{\ell-n} q_{\ell}(\vartheta) \prod_{\substack{1 \leq \nu \leq n_{1} \\
0 \leq i<m_{1, \nu}-\ell}}\left(\vartheta-\lambda_{1, \nu}-i\right), \\
a_{n}(x)^{-1} P^{\vee} & =\sum_{\ell \geq 0} q_{\ell}(-\vartheta-1) \prod_{\substack{1 \leq \nu \leq n_{1} \\
0 \leq i<m_{1, \nu}-\ell}}\left(-\vartheta-\lambda_{1, \nu}-i-1\right) x^{\ell-n} \\
& =\sum_{\ell \geq 0} x^{\ell-n} s_{\ell}(\vartheta) \prod_{\substack{1 \leq \nu \leq n_{1} \\
0 \leq i<m_{1, \nu}-\ell}}\left(\vartheta+\lambda_{1, \nu}+i+1+\ell-n\right) \\
& =\sum_{\ell \geq 0} x^{\ell-n} s_{\ell}(\vartheta) \prod_{\substack{1 \leq \nu \leq n_{1} \\
0 \leq j<m_{1, \nu}-\ell}}\left(\vartheta+\lambda_{1, \nu}-j+m_{1, \nu}-n\right)
\end{aligned}
$$

with suitable polynomials $q_{\ell}$ and $s_{\ell}$ such that $q_{0}, s_{0} \in \mathbb{C}^{\times}$. Hence the set of characteristic exponents of $P^{\vee}$ at $c_{1}$ is $\left\{\left[n-m_{1, \nu}-\lambda_{1, \nu}\right]_{\left(m_{1, \nu}\right)} ; \nu=1, \ldots, n_{1}\right\}$.

At infinity we have

$$
\begin{aligned}
a_{n}(x)^{-1} P & =\sum_{\ell \geq 0} x^{-\ell-n} q_{\ell}(\vartheta) \prod_{\substack{1 \leq \nu \leq n_{1} \\
0 \leq i<m_{0, \nu}-\ell}}\left(\vartheta+\lambda_{0, \nu}+i\right), \\
\left(a_{n}(x)^{-1} P\right)^{*} & =\sum_{\ell \geq 0} x^{-\ell-n} s_{\ell}(\vartheta) \prod_{\substack{1 \leq \nu \leq n_{0} \\
0 \leq i<m_{0, \nu}-\ell}}\left(\vartheta-\lambda_{0, \nu}-i+1-\ell-n\right) \\
& =\sum_{\ell \geq 0} x^{-\ell-n} s_{\ell}(\vartheta) \prod_{\substack{1 \leq \nu \leq n_{1} \\
0 \leq j<m_{0, \nu}-\ell}}\left(\vartheta-\lambda_{0, \nu}+j+2-n-m_{0, \nu}\right)
\end{aligned}
$$

with suitable polynomials $q_{\ell}$ and $s_{\ell}$ with $q_{0}, s_{0} \in \mathbb{C}^{\times}$and the set of characteristic exponents of $P^{\vee}$ at $c_{1}$ is $\left\{\left[2-n-m_{0, \nu}-\lambda_{0, \nu}\right]_{\left(m_{0, \nu}\right)} ; \nu=1, \ldots, n_{0}\right\}$

Example 6.20. The Riemann scheme of the dual $P_{\lambda_{1}, \ldots, \lambda_{p}, \mu}^{\vee}$ of Jordan-Pochhammer operator $P_{\lambda_{1}, \ldots, \lambda_{p}, \mu}^{\vee}$ given in Example 2.8 iii) is

$$
\left\{\begin{array}{cccc}
\frac{1}{c_{1}} & \cdots & \frac{1}{c_{p}} & \infty \\
{[1]_{(p-1)}} & \cdots & {[1]_{(p-1)}} & {[2-2 p+\mu]_{(p-1)}} \\
\lambda_{1}-\mu+p-1 & \cdots & -\lambda_{p}-\mu+p-1 & \lambda_{1}+\cdots+\lambda_{p}+\mu-p+1
\end{array}\right\} .
$$

\section{Reduction of Fuchsian differential EQuations}

Additions and middle convolutions introduced in $\$ 2$ are transformations within Fuchsian differential operators and we examine how their Riemann schemes change under the transformations.

Proposition 7.1. i) Let $P u=0$ be a Fuchsian differential equation. Suppose there exists $c \in \mathbb{C}$ such that $P \in(\partial-c) W[x]$. Then $c=0$.

ii) For $\phi(x) \in \mathbb{C}(x), \lambda \in \mathbb{C}, \mu \in \mathbb{C}$ and $P \in W[x]$, we have

$$
\begin{aligned}
& P \in \mathbb{C}[x] \operatorname{RAdei}(-\phi(x)) \circ \operatorname{RAdei}(\phi(x)) P, \\
& P \in \mathbb{C}[\partial] \operatorname{RAd}\left(\partial^{-\mu}\right) \circ \operatorname{RAd}\left(\partial^{\mu}\right) P .
\end{aligned}
$$

In particular, if the equation $P u=0$ is irreducible and $\operatorname{ord} P>1, \operatorname{RAd}\left(\partial^{-\mu}\right) \circ$ $\operatorname{RAd}\left(\partial^{\mu}\right) P=c P$ with $c \in \mathbb{C}^{\times}$.

Proof. i) Put $P=(\partial-c) Q$. Then there is a function $u(x)$ satisfying $Q u(x)=e^{c x}$. Since $P u=0$ has at most a regular singularity at $x=\infty$, there exist $C>0$ and $N>0$ such that $|u(x)|<C|x|^{N}$ for $|x| \gg 1$ and $0 \leq \arg x \leq 2 \pi$, which implies $c=0$. 
ii) This follows from the fact

$$
\begin{aligned}
& \text { Adei }(-\phi(x)) \circ \operatorname{Adei}(\phi(x))=\text { id, } \\
& \text { Adei }(\phi(x)) f(x) P=f(x) \operatorname{Adei}(\phi(x)) P \quad(f(x) \in \mathbb{C}(x))
\end{aligned}
$$

and the definition of $\operatorname{RAdei}(\phi(x))$ and $\operatorname{RAd}\left(\partial^{\mu}\right)$.

The addition and the middle convolution transform the Riemann scheme of the Fuchsian differential equation as follows.

Theorem 7.2. Let $P u=0$ be a Fuchsian differential equation with the Riemann scheme (6.15). We assume that $P$ has the normal form (6.43).

i) (addition) The operator $\operatorname{Ad}\left(\left(x-c_{j}\right)^{\tau}\right) P$ has the Riemann scheme

$$
\left\{\begin{array}{cccccc}
x=c_{0}=\infty & c_{1} & \cdots & c_{j} & \cdots & c_{p} \\
{\left[\lambda_{0,1}-\tau\right]_{\left(m_{0,1}\right)}} & {\left[\lambda_{1,1}\right]_{\left(m_{1,1}\right)}} & \cdots & {\left[\lambda_{j, 1}+\tau\right]_{\left(m_{j, 1}\right)}} & \cdots & {\left[\lambda_{p, 1}\right]_{\left(m_{p, 1}\right)}} \\
\vdots & \vdots & \vdots & \vdots & \vdots & \vdots \\
{\left[\lambda_{0, n_{0}}-\tau\right]_{\left(m_{0, n_{0}}\right)}} & {\left[\lambda_{1, n_{1}}\right]_{\left(m_{1, n_{1}}\right)}} & \cdots & {\left[\lambda_{j, n_{j}}+\tau\right]_{\left(m_{j, 1}\right)}} & \cdots & {\left[\lambda_{p, n_{p}}\right]_{\left(m_{p, n_{p}}\right)}}
\end{array}\right\} \text {. }
$$

ii) (middle convolution) Fix $\mu \in \mathbb{C}$. By allowing the condition $m_{j, 1}=0$, we may assume

$$
\mu=\lambda_{0,1}-1 \text { and } \lambda_{j, 1}=0 \text { for } j=1, \ldots, p
$$

and $\#\left\{j ; m_{j, 1}<n\right\} \geq 2$ and $P$ is of the normal form (6.43). Putting

$$
d:=\sum_{j=0}^{p} m_{j, 1}-(p-1) n,
$$

we suppose

$$
\begin{aligned}
& m_{j, 1} \geq d \text { for } j=0, \ldots, p, \\
& \left\{\begin{array}{l}
\lambda_{0, \nu} \notin\left\{0,-1,-2, \ldots, m_{0,1}-m_{0, \nu}-d+2\right\} \\
\text { if } m_{0, \nu}+\cdots+m_{p, 1}-(p-1) n \geq 2, m_{1,1} \cdots m_{p, 1} \neq 0 \text { and } \nu \geq 1,
\end{array}\right. \\
& \left\{\begin{array}{l}
\lambda_{0,1}+\lambda_{j, \nu} \notin\left\{0,-1,-2, \ldots, m_{j, 1}-m_{j, \nu}-d+2\right\} \\
\text { if } m_{0,1}+\cdots+m_{j-1,1}+m_{j, \nu}+m_{j+1,1}+\cdots+m_{p, 1}-(p-1) n \geq 2, \\
m_{j, 1} \neq 0,1 \leq j \leq p \text { and } \nu \geq 2 .
\end{array}\right.
\end{aligned}
$$

Then $S:=\partial^{-d} \operatorname{Ad}\left(\partial^{-\mu}\right) \prod_{j=1}^{p}\left(x-c_{j}\right)^{-m_{j, 1}} P \in W[x]$ and the Riemann scheme of $S$ equals

$$
\left\{\begin{array}{cccc}
x=c_{0}=\infty & c_{1} & \cdots & c_{p} \\
{[1-\mu]_{\left(m_{0,1}-d\right)}} & {[0]_{\left(m_{1,1}-d\right)}} & \cdots & {[0]_{\left(m_{p, 1}-d\right)}} \\
{\left[\lambda_{0,2}-\mu\right]_{\left(m_{0,2}\right)}} & {\left[\lambda_{1,2}+\mu\right]_{\left(m_{1,2}\right)}} & \cdots & {\left[\lambda_{p, 2}+\mu\right]_{\left(m_{p, 2}\right)}} \\
\vdots & \vdots & \vdots & \vdots \\
{\left[\lambda_{0, n_{0}}-\mu\right]_{\left(m_{0, n_{0}}\right)}} & {\left[\lambda_{1, n_{1}}+\mu\right]_{\left(m_{1, n_{1}}\right)}} & \cdots & {\left[\lambda_{p, n_{p}}+\mu\right]_{\left(m_{\left.p, n_{p}\right)}\right)}}
\end{array}\right\}
$$

More precisely, the condition (7.5) and the condition (7.6) for $\nu=1$ assure $S \in$ $W[x]$. In this case the condition (7.6) (resp. (7.7) for a fixed $j$ ) assures that the sets of characteristic exponents of $\bar{P}$ at $x=\infty$ (resp. $c_{j}$ ) are equal to the sets given in (7.8), respectively.

Here we have $\operatorname{RAd}\left(\partial^{-\mu}\right) \mathrm{R} P=S$, if

$$
\left\{\begin{array}{c}
\lambda_{j, 1}+m_{j, 1} \text { are not characteristic exponents of } P \\
\text { at } x=c_{j} \text { for } j=0, \ldots, p, \text { respectively, }
\end{array}\right.
$$

and moreover

$$
m_{0,1}=d \quad \text { or } \quad \lambda_{0,1} \notin\left\{-d,-d-1, \ldots, 1-m_{0,1}\right\} .
$$


Using the notation in Definition 2.5, we have

$$
\begin{gathered}
S=\operatorname{Ad}\left(\left(x-c_{1}\right)^{\lambda_{0,1}-2}\right)\left(x-c_{1}\right)^{d} T_{\frac{1}{x-c_{1}}}^{*}(-\partial)^{-d} \operatorname{Ad}\left(\partial^{-\mu}\right) T_{\frac{1}{x}+c_{1}}^{*} \\
\cdot\left(x-c_{1}\right)^{d} \prod_{j=1}^{p}\left(x-c_{j}\right)^{-m_{j, 1}} \operatorname{Ad}\left(\left(x-c_{1}\right)^{\lambda_{0,1}}\right) P
\end{gathered}
$$

under the conditions $(7.5)$ and

$$
\left\{\begin{array}{l}
\lambda_{0, \nu} \notin\left\{0,-1,-2, \ldots, m_{0,1}-m_{0, \nu}-d+2\right\} \\
\text { if } m_{0, \nu}+\cdots+m_{p, 1}-(p-1) n \geq 2, m_{1,1} \neq 0 \quad \text { and } \nu \geq 1 .
\end{array}\right.
$$

iii) Suppose ord $P>1$ and $P$ is irreducible in ii). Then the conditions (7.5), (7.6), (7.7) are valid. The condition (7.10) is also valid if $d \geq 1$.

All these conditions in ii) are valid if $\#\left\{j ; m_{j, 1}<n\right\} \geq 2$ and $\mathbf{m}$ is realizable and moreover $\lambda_{j, \nu}$ are generic under the Fuchs relation with $\lambda_{j, 1}=0$ for $j=1, \ldots, p$.

iv) Let $\mathbf{m}=\left(m_{j, \nu}\right)_{\substack{j=0, \ldots, p \\ \nu=1, \ldots, n_{j}}} \in \mathcal{P}_{p+1}^{(n)}$. Define d by (7.4). Suppose $\lambda_{j, \nu}$ are complex numbers satisfying (7.3). Suppose moreover $m_{j, 1} \geq d$ for $j=1, \ldots, p$. Defining $\mathbf{m}^{\prime} \in \mathcal{P}_{p+1}^{(n)}$ and $\lambda_{j, \nu}^{\prime}$ by

$$
\begin{aligned}
m_{j, \nu}^{\prime} & =m_{j, \nu}-\delta_{\nu, 1} d \quad\left(j=0, \ldots, p, \nu=1, \ldots, n_{j}\right), \\
\lambda_{j, \nu}^{\prime} & = \begin{cases}2-\lambda_{0,1} & (j=0, \nu=1), \\
\lambda_{j, \nu}-\lambda_{0,1}+1 & (j=0, \nu>1), \\
0 & (j>0, \nu=1), \\
\lambda_{j, \nu}+\lambda_{0,1}-1 & (j>0, \nu>1),\end{cases}
\end{aligned}
$$

we have

$$
\operatorname{idx} \mathbf{m}=\operatorname{idx} \mathbf{m}^{\prime}, \quad\left|\left\{\lambda_{\mathbf{m}}\right\}\right|=\left|\left\{\lambda_{\mathbf{m}^{\prime}}^{\prime}\right\}\right| .
$$

Proof. The claim i) is clear from the definition of the Riemann scheme.

ii) Suppose (7.5), (7.6) and (7.7). Then

$$
P^{\prime}:=\left(\prod_{j=1}^{p}\left(x-c_{j}\right)^{-m_{j, 1}}\right) P \in W[x] .
$$

Note that $\mathrm{R} P=P^{\prime}$ under the condition (7.9). Put $Q:=\partial^{(p-1) n-\sum_{j=1}^{p} m_{j, 1}} P^{\prime}$. Here we note that (7.5) assures $(p-1) n-\sum_{j=1}^{p} m_{j, 1} \geq 0$.

Fix a positive integer $j$ with $j \leq p$. For simplicity suppose $j=1$ and $c_{j}=0$. Since $P^{\prime}=\sum_{j=0}^{n} a_{j}(x) \partial^{j}$ with $\operatorname{deg} a_{j}(x) \leq(p-1) n+j-\sum_{j=1}^{p} m_{j, 1}$, we have

$$
x^{m_{1,1}} P^{\prime}=\sum_{\ell=0}^{N} x^{N-\ell} r_{\ell}(\vartheta) \prod_{\substack{1 \leq \nu \leq n_{0} \\ 0 \leq i<m_{0, \nu}-\ell}}\left(\vartheta+\lambda_{0, \nu}+i\right)
$$

and

$$
N:=(p-1) n-\sum_{j=2}^{p} m_{j, 1}=m_{0,1}+m_{1,1}-d
$$

with suitable polynomials $r_{\ell}$ such that $r_{0} \in \mathbb{C}^{\times}$. Suppose

$$
\prod_{\substack{1 \leq \nu \leq n_{0} \\ 0 \leq i<m_{0, \nu}-\ell}}\left(\vartheta+\lambda_{0, \nu}+i\right) \notin x W[x] \text { if } N-m_{1,1}+1 \leq \ell \leq N .
$$

Since $P^{\prime} \in W[x]$, we have

$$
x^{N-\ell} r_{\ell}(\vartheta)=x^{N-\ell} x^{\ell-N+m_{1,1}} \partial^{\ell-N+m_{1,1}} s_{\ell}(\vartheta) \text { if } \quad N-m_{1,1}+1 \leq \ell \leq N
$$


for suitable polynomials $s_{\ell}$. Putting $s_{\ell}=r_{\ell}$ for $0 \leq \ell \leq N-m_{1,1}$, we have

$$
\begin{aligned}
P^{\prime}= & \sum_{\ell=0}^{N-m_{1,1}} x^{N-m_{1,1}-\ell} s_{\ell}(\vartheta) \prod_{\substack{1 \leq \nu \leq n_{0} \\
0 \leq i<m_{0, \nu}-\ell}}\left(\vartheta+\lambda_{0, \nu}+i\right) \\
& +\sum_{\ell=N-m_{1,1}+1}^{N} \partial^{\ell-N+m_{1,1}} s_{\ell}(\vartheta) \prod_{\substack{1 \leq \nu \leq n_{0} \\
0 \leq i<m_{0, \nu}-\ell}}\left(\vartheta+\lambda_{0, \nu}+i\right) .
\end{aligned}
$$

Note that $s_{0} \in \mathbb{C}^{\times}$and the condition (7.17) is equivalent to the condition $\lambda_{0, \nu}+i \neq 0$ for any $\nu$ and $i$ such that there exists an integer $\ell$ with $0 \leq i \leq m_{0, \nu}-\ell-1$ and $N-m_{1,1}+1 \leq \ell \leq N$. This condition is valid if (7.6) is valid, namely, $m_{1,1}=0$ or

$$
\lambda_{0, \nu} \notin\left\{0,-1, \ldots, m_{0,1}-m_{0, \nu}-d+2\right\}
$$

for $\nu$ satisfying $m_{0, \nu} \geq m_{0,1}-d+2$. Under this condition we have

$$
\begin{aligned}
Q= & \sum_{\ell=0}^{N} \partial^{\ell} s_{\ell}(\vartheta) \prod_{1 \leq i \leq N-m_{1,1}-\ell}(\vartheta+i) \cdot \prod_{\substack{1 \leq \nu \leq n_{0} \\
0 \leq i<m_{0, \nu}-\ell}}\left(\vartheta+\lambda_{0, \nu}+i\right), \\
\operatorname{Ad}\left(\partial^{-\mu}\right) Q= & \sum_{\ell=0}^{N} \partial^{\ell} s_{\ell}(\vartheta-\mu) \prod_{\substack{1 \leq i \leq N-m_{1,1}-\ell\\
}}(\vartheta-\mu+i) \\
& \cdot \prod_{1 \leq i \leq m_{0,1}-\ell}(\vartheta+i) \cdot \prod_{\substack{2 \leq \nu \leq n_{0} \\
0 \leq i<m_{0, \nu}-\ell}}\left(\vartheta-\mu+\lambda_{0, \nu}+i\right)
\end{aligned}
$$

since $\mu=\lambda_{0,1}-1$. Hence $\partial^{-m_{0,1}} \operatorname{Ad}\left(\partial^{-\mu}\right) Q$ equals

$$
\begin{aligned}
& \sum_{\ell=0}^{m_{0,1}-1} x^{m_{0,1}-\ell} s_{\ell}(\vartheta-\mu) \prod_{1 \leq i \leq N-m_{1,1}-\ell}(\vartheta-\mu+i) \prod_{\substack{2 \leq \nu \leq n_{0} \\
0 \leq i<m_{0, \nu}-\ell}}\left(\vartheta-\mu+\lambda_{0, \nu}+i\right) \\
& +\sum_{\ell=m_{0,1}}^{N} \partial^{\ell-m_{0,1}} s_{\ell}(\vartheta-\mu) \prod_{1 \leq i \leq N-m_{1,1}-\ell}(\vartheta-\mu+i) \prod_{\substack{2 \leq \nu \leq n_{0} \\
0 \leq i<m_{0, \nu}-\ell}}\left(\vartheta-\mu+\lambda_{0, \nu}+i\right)
\end{aligned}
$$

and then the set of characteristic exponents of this operator at $\infty$ is

$$
\left\{[1-\mu]_{\left(m_{0,1}-d\right)},\left[\lambda_{0,2}-\mu\right]_{\left(m_{0,2}\right)}, \ldots,\left[\lambda_{0, n_{0}}-\mu\right]_{\left(m_{0, n_{0}}\right)}\right\} .
$$

Moreover $\partial^{-m_{0,1}-1} \operatorname{Ad}\left(\partial^{-\mu}\right) Q \notin W[x]$ if $\lambda_{0,1}+m_{0,1}$ is not a characteristic exponent of $P$ at $\infty$ and $-\lambda_{0,1}+1+i \neq m_{0,1}+1$ for $1 \leq i \leq N-m_{1,1}=m_{0,1}-d$, which assures $x^{m_{0,1}} s_{0} \prod_{1 \leq i \leq N-m_{1,1}}(\vartheta-\mu+i) \prod_{\substack{2 \leq \nu \leq n_{0} \\ 0 \leq i<m_{0, \nu}}}\left(\vartheta-\mu+\lambda_{1, \nu}+i\right) \notin \partial W[x]$.

Similarly we have

$$
\begin{aligned}
P^{\prime}= & \sum_{\ell=0}^{m_{1,1}} \partial^{m_{1,1}-\ell} q_{\ell}(\vartheta) \prod_{\substack{2 \leq \nu \leq n_{1} \\
0 \leq i<m_{1, \nu}-\ell}}\left(\vartheta-\lambda_{1, \nu}-i\right) \\
& +\sum_{\ell=m_{1,1}+1}^{N} x^{\ell-m_{1,1}} q_{\ell}(\vartheta) \prod_{\substack{2 \leq \nu \leq n_{1} \\
0 \leq i<m_{1, \nu}-\ell}}\left(\vartheta-\lambda_{1, \nu}-i\right),
\end{aligned}
$$




$$
\begin{aligned}
Q= & \sum_{\ell=0}^{m_{1,1}} \partial^{N-\ell} q_{\ell}(\vartheta) \prod_{\substack{2 \leq \nu \leq n_{1} \\
0 \leq i<m_{1, \nu}-\ell}}\left(\vartheta+\lambda_{1, \nu}-i\right) \\
& +\sum_{\ell=m_{1,1}+1}^{N} \partial^{N-\ell} q_{\ell}(\vartheta) \prod_{i=1}^{\ell-m_{1,1}}(\vartheta+i) \prod_{\substack{2 \leq \nu \leq n_{1} \\
0 \leq i<m_{1, \nu}-\ell}}\left(\vartheta-\lambda_{1, \nu}-i\right) . \\
\operatorname{Ad}\left(\partial^{-\mu}\right) Q= & \sum_{\ell=0}^{N} \partial^{N-\ell} q_{\ell}(\vartheta-\mu) \prod_{1 \leq i \leq \ell-m_{1,1}}(\vartheta-\mu+i) \\
& \cdot \prod_{\substack{2 \leq \nu \leq n_{1} \\
0 \leq i<m_{1, \nu}-\ell}}\left(\vartheta-\mu-\lambda_{1, \nu}-i\right)
\end{aligned}
$$

with $q_{0} \in \mathbb{C}^{\times}$. Then the set of characteristic exponents of $\partial^{-m_{0,1}} \operatorname{Ad}\left(p^{-\mu}\right) Q$ equals

$$
\left\{[0]_{\left(m_{1,1}-d\right)},\left[\lambda_{1,2}+\mu\right]_{\left(m_{1,2}\right)}, \ldots,\left[\lambda_{1, n_{1}}+\mu\right]_{\left(m_{1, n_{1}}\right)}\right\}
$$

if

$$
\prod_{\substack{2 \leq \nu \leq n_{1} \\ 0 \leq i<m_{1, \nu}-\ell}}\left(\vartheta-\mu-\lambda_{1, \nu}-i\right) \notin \partial W[x]
$$

for any integers $\ell$ satisfying $0 \leq \ell \leq N$ and $N-\ell<m_{0,1}$. This condition is satisfied if $(7.7)$ is valid, namely, $m_{0,1}=0$ or

$$
\begin{aligned}
& \lambda_{0,1}+\lambda_{1, \nu} \notin\left\{0,-1, \ldots, m_{1,1}-m_{1, \nu}-d+2\right\} \\
& \text { for } \nu \geq 2 \text { satisfying } m_{1, \nu} \geq m_{1,1}-d+2
\end{aligned}
$$

because $m_{1, \nu}-\ell-1 \leq m_{1, \nu}+m_{0,1}-N-2=m_{1, \nu}-m_{1,1}+d-2$ and the condition $\vartheta-\mu-\lambda_{1, \nu}-i \in \partial W[x]$ means $-1=\mu+\lambda_{1, \nu}+i=\lambda_{0,1}-1+\lambda_{1, \nu}+i$.

Now we will prove (7.11). Under the conditions, it follows from $(7.18)$ that

$$
\begin{aligned}
& \tilde{P}:=x^{m_{0,1}-N} \operatorname{Ad}\left(x^{\lambda_{0,1}}\right) \prod_{j=2}^{p}\left(x-c_{j}\right)^{-m_{j, 1}} P \\
& =x^{m_{0,1}+m_{1,1}-N} \operatorname{Ad}\left(x^{\lambda_{0,1}}\right) P^{\prime}
\end{aligned}
$$

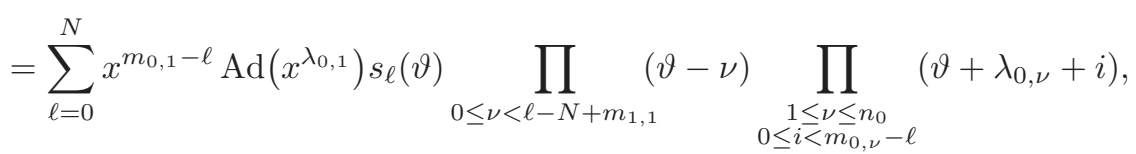

$$
\begin{aligned}
& \tilde{Q}:=(-\partial)^{N-m_{0,1}} T_{\frac{1}{x}}^{*} \tilde{P} \\
& =(-\partial)^{N-m_{0,1}} \sum_{\ell=0}^{N} x^{\ell-m_{0,1}} s_{\ell}\left(-\vartheta-\lambda_{0,1}\right) \prod_{0 \leq \nu<\ell-N+m_{1,1}}\left(-\vartheta-\lambda_{0,1}-\nu\right) \\
& \cdot \prod_{\substack{2 \leq \nu \leq n_{0} \\
0 \leq i<m_{0, \nu}-\ell}}\left(-\vartheta+\lambda_{0, \nu}-\lambda_{0,1}+i\right) \prod_{0 \leq i \leq m_{0,1}-\ell}(-\vartheta+i) \\
& =\sum_{\ell=0}^{N}(-\partial)^{N-\ell} s_{\ell}\left(-\vartheta-\lambda_{0,1}\right) \prod_{1 \leq i \leq \ell-m_{0,1}}(-\vartheta-i) \\
& \cdot \prod_{0 \leq \nu<\ell-N+m_{1,1}}\left(-\vartheta-\lambda_{0,1}-\nu\right) \prod_{\substack{2 \leq \nu \leq n_{0} \\
0 \leq i<m_{0, \nu}-\ell}}\left(-\vartheta+\lambda_{0, \nu}-\lambda_{0,1}+i\right)
\end{aligned}
$$


and therefore

$$
\begin{aligned}
& \operatorname{Ad}\left(\partial^{-\mu}\right) \tilde{Q}=\sum_{\ell=0}^{N}(-\partial)^{N-\ell} s_{\ell}(-\vartheta-1) \prod_{1 \leq i \leq \ell-m_{0,1}}\left(-\vartheta+\lambda_{0,1}-1-i\right) \\
& \cdot \prod_{0 \leq \nu<\ell-N+m_{1,1}}(-\vartheta-1-\nu) \prod_{\substack{2 \leq \nu \leq n_{0} \\
0 \leq i<m_{0, \nu}-\ell}}\left(-\vartheta+\lambda_{0, \nu}-1+i\right) .
\end{aligned}
$$

Since

$$
\begin{aligned}
(-\partial)^{N-\ell-m_{1,1}} \prod_{0 \leq \nu<\ell-N+m_{1,1}}(-\vartheta-1-\nu) & = \begin{cases}x^{\ell-N+m_{1,1}} & \left(N-\ell<m_{1,1}\right), \\
(-\partial)^{N-\ell-m_{1,1}} & \left(N-\ell \geq m_{1,1}\right),\end{cases} \\
& =x^{\ell-N+m_{1,1}} \prod_{0 \leq \nu<N-\ell-m_{1,1}}(-\vartheta+\nu),
\end{aligned}
$$

we have

$$
\begin{gathered}
\tilde{Q}^{\prime}:=(-\partial)^{-m_{1,1}} \operatorname{Ad}\left(\partial^{-\mu}\right) \tilde{Q}=\sum_{\ell=0}^{N} x^{\ell-N+m_{1,1}} \prod_{0 \leq \nu<N-\ell-m_{1,1}}(-\vartheta+\nu) \\
\cdot s_{\ell}(-\vartheta-1) \prod_{0 \leq \nu<\ell-m_{0,1}}\left(-\vartheta+\lambda_{0,1}-2-\nu\right) \prod_{\substack{2 \leq \nu \leq n_{0} \\
0 \leq i<<m_{0, \nu}-\ell}}\left(-\vartheta+\lambda_{0, \nu}-1+i\right)
\end{gathered}
$$

and

$$
\begin{gathered}
x^{m_{0,1}+m_{1,1}-N} \operatorname{Ad}\left(x^{\lambda_{0,1}-2}\right) T_{\frac{1}{x}}^{*} \tilde{Q}^{\prime}=\sum_{\ell=0}^{N} x^{m_{0,1}-\ell} \prod_{0 \leq \nu<\ell-m_{0,1}}(\vartheta-\nu) \cdot s_{\ell}\left(\vartheta-\lambda_{0,1}+1\right) \\
\cdot \prod_{0 \leq \nu<N-m_{1,1}-\ell}\left(\vartheta-\lambda_{0,1}+2+\nu\right) \prod_{\substack{2 \leq \nu \leq n_{0} \\
0 \leq i<m_{0, \nu}-\ell}}\left(\vartheta+\lambda_{0, \nu}-\lambda_{0,1}+1+i\right),
\end{gathered}
$$

which equals $\partial^{-m_{0,1}} \operatorname{Ad}\left(\partial^{-\mu}\right) Q$ because $\prod_{0<\nu<k}(\vartheta-\nu)=x^{k} \partial^{k}$ for $k \in \mathbb{Z}_{\geq 0}$.

iv) (Cf. Remark 9.4 ii) for another proof.) Since

$$
\begin{aligned}
\operatorname{idx} \mathbf{m}-\operatorname{idx} \mathbf{m}^{\prime} & =\sum_{j=0}^{p} m_{j, 1}^{2}-(p-1) n^{2}-\sum_{j=0}^{p}\left(m_{j, 1}-d\right)^{2}+(p-1)(n-d)^{2} \\
& =2 d \sum_{j=0}^{p} m_{j, 1}-(p+1) d^{2}-2(p-1) n d+(p-1) d^{2} \\
& =d\left(2 \sum_{j=0}^{p} m_{j, 1}-2 d-2(p-1) n\right)=0
\end{aligned}
$$

and

$$
\begin{aligned}
\sum_{j=0}^{p} \sum_{\nu=1}^{n_{j}} m_{j, \nu} \lambda_{j, \nu} & -\sum_{j=0}^{p} \sum_{\nu=1}^{n_{j}} m_{j, \nu}^{\prime} \lambda_{j, \nu}^{\prime} \\
& =m_{0,1}(\mu+1)-\left(m_{0,1}-d\right)(1-\mu)+\mu\left(n-m_{0,1}-\sum_{j=1}^{p}\left(n-m_{j, 1}\right)\right) \\
& =\left(\sum_{j=0}^{p} m_{j, 1}-d-(p-1) n\right) \mu-m_{0,1} d-\left(m_{0,1}-d\right)=d,
\end{aligned}
$$

we have the claim.

The claim iii) follows from the following lemma when $P$ is irreducible. 
Suppose $\lambda_{j, \nu}$ are generic in the sense of the claim iii). Put $\mathbf{m}=\operatorname{gcd}(\mathbf{m}) \overline{\mathbf{m}}$. Then an irreducible subspace of the solutions of $P u=0$ has the spectral type $\ell^{\prime} \overline{\mathbf{m}}$ with $1 \leq \ell^{\prime} \leq \operatorname{gcd}(\mathbf{m})$ and the same argument as in the proof of the following lemma shows iii).

The following lemma is known which follows from Scott's lemma (cf. $\$ .19$ ).

Lemma 7.3. Let $P$ be a Fuchsian differential operator with the Riemann scheme (6.15). Suppose $P$ is irreducible. Then

$$
\text { idx } \mathbf{m} \leq 2 .
$$

Fix $\ell=\left(\ell_{0}, \ldots, \ell_{p}\right) \in \mathbb{Z}_{>0}^{p+1}$ and suppose ord $P>1$. Then

$$
m_{0, \ell_{0}}+m_{1, \ell_{1}}+\cdots+m_{p, \ell_{p}}-(p-1) \text { ord } \mathbf{m} \leq m_{k, \ell_{k}} \text { for } k=0, \ldots, p .
$$

Moreover the condition

$$
\lambda_{0, \ell_{0}}+\lambda_{1, \ell_{1}}+\cdots+\lambda_{p, \ell_{p}} \in \mathbb{Z}
$$

implies

$$
m_{0, \ell_{0}}+m_{1, \ell_{1}}+\cdots+m_{p, \ell_{p}} \leq(p-1) \text { ord } \mathbf{m} .
$$

Proof. Let $M_{j}$ be the monodromy generators of the solutions of $P u=0$ at $c_{j}$, respectively. Then $\operatorname{dim} Z\left(M_{j}\right) \geq \sum_{\nu=1}^{n_{j}} m_{j, \nu}^{2}$ and therefore $\sum_{j=0}^{p} \operatorname{codim} Z\left(M_{j}\right) \leq$ $(p+1) n^{2}-\left(\operatorname{idx} \mathbf{m}+(p-1) n^{2}\right)=2 n^{2}-\mathrm{idx} \mathbf{m}$. Hence Corollary $11.12($ cf. (11.47) $)$ proves 7.19 ).

We may assume $\ell_{j}=1$ for $j=0, \ldots, p$ and $k=0$ to prove the lemma. By the map $u(x) \mapsto \prod_{j=1}^{p}\left(x-c_{j}\right)^{-\lambda_{j, 1}} u(x)$ we may moreover assume $\lambda_{j, \ell_{j}}=0$ for $j=1, \ldots, p$. Suppose $\lambda_{0,1} \in \mathbb{Z}$. We may assume $M_{p} \cdots M_{1} M_{0}=I_{n}$. Since $\operatorname{dim} \operatorname{ker} M_{j} \geq m_{j, 1}$, Scott's lemma (Lemma 11.11) assures (7.22).

The condition (7.20) is reduced to $\left(7.22\right.$ by putting $m_{0, \ell_{0}}=0$ and $\lambda_{0, \ell_{0}}=$ $-\lambda_{1, \ell_{1}}-\cdots-\lambda_{p, \ell_{p}}$ because we may assume $k=0$ and $\ell_{0}=n_{0}+1$.

Remark 7.4. i) Retain the notation in Theorem 7.2. The operation in Theorem 7.2 i) corresponds to the addition and the operation in Theorem 7.2 ii) corresponds to Katz's middle convolution (cf. [Kz), which are studied by $\sqrt{\mathrm{DR}}$ ) for the systems of Schlesinger canonical form.

The operation $c(P):=\operatorname{Ad}\left(\partial^{-\mu}\right) \partial^{(p-1) n} P$ is always well-defined for the Fuchsian differential operator of the normal form which has $p+1$ singular points including $\infty$. This corresponds to the convolution defined by Katz. Note that the equation $S v=0$ is a quotient of the equation $c(P) \tilde{u}=0$.

ii) Retain the notation in the previous theorem. Suppose the equation $P u=0$ is irreducible and $\lambda_{j, \nu}$ are generic complex numbers satisfying the assumption in Theorem 7.2. Let $u(x)$ be a local solution of the equation $P u=0$ corresponding to the characteristic exponent $\lambda_{i, \nu}$ at $x=c_{i}$. Assume $0 \leq i \leq p$ and $1<\nu \leq n_{i}$. Then the irreducible equations $\left(\operatorname{Ad}\left(\left(x-c_{j}\right)^{r}\right) P\right) u_{1}=0$ and $\left(\operatorname{RAd}\left(\partial^{-\mu}\right) \circ \operatorname{R} P\right) u_{2}=0$ are characterized by the equations satisfied by $u_{1}(x)=\left(x-c_{j}\right)^{r} u(x)$ and $u_{2}(x)=$ $I_{c_{i}}^{\mu}(u(x))$, respectively.

Moreover for any integers $k_{0}, k_{1}, \ldots, k_{p}$ the irreducible equation $Q u_{3}=0$ satisfied by $u_{3}(x)=I_{c_{i}}^{\mu+k_{0}}\left(\prod_{j=1}^{p}\left(x-c_{j}\right)^{k_{j}} u(x)\right)$ is isomorphic to the equation $\left(\operatorname{RAd}\left(\partial^{-\mu}\right) \circ\right.$ $\mathrm{R} \mathrm{P}) u_{2}=0$ as $W(x)$-modules (cf. $\S 2.4$ and $\S 5$ ).

Example 7.5 (exceptional parameters). The Fuchsian differential equation with the Riemann scheme

$$
\left\{\begin{array}{cccc}
x=\infty & 0 & 1 & c \\
{[\delta]_{(2)}} & {[0]_{(2)}} & {[0]_{(2)}} & {[0]_{(2)}} \\
2-\alpha-\beta-\gamma-2 \delta & \alpha & \beta & \gamma
\end{array}\right\}
$$


is a Jordan-Pochhammer equation (cf. Example 2.8 ii)) if $\delta \neq 0$, which is proved by the reduction using the operation $\operatorname{RAd}\left(\partial^{1-\delta}\right) \mathrm{R}$ given in Theorem $7.2 \mathrm{ii}$ ).

The Riemann scheme of the operator

$$
\begin{aligned}
P_{r}= & x(x-1)(x-c) \partial^{3} \\
& -\left((\alpha+\beta+\gamma-6) x^{2}-((\alpha+\beta-4) c+\alpha+\gamma-4) x+(\alpha-2) c\right) \partial^{2} \\
& -(2(\alpha+\beta+\gamma-3) x+(\alpha+\beta-2) c+\alpha+\gamma-2+r) \partial
\end{aligned}
$$

equals

$$
\left\{\begin{array}{cccc}
x=\infty & 0 & 1 & c \\
{[0]_{(2)}} & {[0]_{(2)}} & {[0]_{(2)}} & {[0]_{(2)}} \\
2-\alpha-\beta-\gamma & \alpha & \beta & \gamma
\end{array}\right\}
$$

which corresponds to a Jordan-Pochhammer operator when $r=0$. If the parameters are generic, $\operatorname{RAd}(\partial) P_{r}$ is Heun's operator 8.19 ) with the Riemann scheme

$$
\left\{\begin{array}{cccc}
x=\infty & 0 & 1 & c \\
2 & 0 & 0 & 0 \\
3-\alpha-\beta-\gamma & \alpha-1 & \beta-1 & \gamma-1
\end{array}\right\},
$$

which contains the accessory parameter $r$. This transformation doesn't satisfy (7.6) for $\nu=1$.

The operator $\operatorname{RAd}\left(\partial^{1-\alpha-\beta-\gamma}\right) P_{r}$ has the Riemann scheme

$$
\left\{\begin{array}{cccc}
x=\infty & 0 & 1 & c \\
\alpha+\beta+\gamma-1 & 0 & 0 & 0 \\
\alpha+\beta+\gamma & 1-\beta-\gamma & 1-\gamma-\alpha & 1-\alpha-\beta
\end{array}\right\}
$$

and the monodromy generator at $\infty$ is semisimple if and only if $r=0$. This transformation doesn't satisfy (7.6) for $\nu=2$.

Definition 7.6. Let

$$
P=a_{n}(x) \partial^{n}+a_{n-1}(x) \partial^{n-1}+\cdots+a_{0}(x)
$$

be a Fuchsian differential operator with the Riemann scheme (6.15). Here some $m_{j, \nu}$ may be 0 . Fix $\ell=\left(\ell_{0}, \ldots, \ell_{p}\right) \in \mathbb{Z}_{>0}^{p+1}$ with $1 \leq \ell_{j} \leq n_{j}$. Suppose

$$
\#\left\{j ; m_{j, \ell_{j}} \neq n \text { and } 0 \leq j \leq p\right\} \geq 2 \text {. }
$$

Put

$$
d_{\ell}(\mathbf{m}):=m_{0, \ell_{0}}+\cdots+m_{p, \ell_{p}}-(p-1) \text { ord } \mathbf{m}
$$

and

$$
\begin{aligned}
\partial_{\ell} P:= & \operatorname{Ad}\left(\prod_{j=1}^{p}\left(x-c_{j}\right)^{\lambda_{j, \ell_{j}}}\right) \prod_{j=1}^{p}\left(x-c_{j}\right)^{m_{j, \ell_{j}}-d_{\ell}(\mathbf{m})} \partial^{-m_{0, \ell_{0}}} \operatorname{Ad}\left(\partial^{1-\lambda_{0, \ell_{0}}-\cdots-\lambda_{p, \ell_{p}}}\right) \\
& \cdot \partial^{(p-1) n-m_{1, \ell_{1}}-\cdots-m_{p, \ell_{p}}} a_{n}^{-1}(x) \prod_{j=1}^{n}\left(x-c_{j}\right)^{n-m_{j, \ell_{j}}} \operatorname{Ad}\left(\prod_{j=1}^{p}\left(x-c_{j}\right)^{-\lambda_{j, \ell_{j}}}\right) P .
\end{aligned}
$$


If $\lambda_{j, \nu}$ are generic under the Fuchs relation or $P$ is irreducible, $\partial_{\ell} P$ is well-defined as an element of $W[x]$ and

$$
\begin{aligned}
\partial_{\ell}^{2} P= & P \text { with } P \text { of the form }(6.43) \\
\partial_{\ell} P \in & W(x) \operatorname{RAd}\left(\prod_{j=1}^{p}\left(x-c_{j}\right)^{\lambda_{j, \ell_{j}}}\right) \operatorname{RAd}\left(\partial^{1-\lambda_{0}, \ell_{0}-\cdots-\lambda_{p, \ell_{p}}}\right) \\
& \cdot \operatorname{RAd}\left(\prod_{j=1}^{p}\left(x-c_{j}\right)^{-\lambda_{j, \ell_{j}}}\right) P
\end{aligned}
$$

and $\partial_{\ell}$ gives a correspondence between differential operators of normal form (6.43). Here the spectral type $\partial_{\ell} \mathbf{m}$ of $\partial_{\ell} P$ is given by

$$
\partial_{\ell} \mathbf{m}:=\left(m_{j, \nu}^{\prime}\right) \underset{\substack{0 \leq j \leq p \\ 1 \leq \nu \leq n_{j}}}{\text { and }} m_{j, \nu}^{\prime}=m_{j, \nu}-\delta_{\ell_{j}, \nu} \cdot d_{\ell}(\mathbf{m})
$$

and the Riemann scheme of $\partial_{\ell} P$ equals

$$
\partial_{\ell}\left\{\lambda_{\mathbf{m}}\right\}:=\left\{\lambda_{\mathbf{m}^{\prime}}^{\prime}\right\} \quad \text { with } \quad \lambda_{j, \nu}^{\prime}= \begin{cases}\lambda_{0, \nu}-2 \mu_{\ell} & \left(j=0, \nu=\ell_{0}\right) \\ \lambda_{0, \nu}-\mu_{\ell} & \left(j=0, \nu \neq \ell_{0}\right) \\ \lambda_{j, \nu} & \left(1 \leq j \leq p, \nu=\ell_{j}\right) \\ \lambda_{0, \nu}+\mu_{\ell} & \left(1 \leq j \leq p, \nu \neq \ell_{j}\right)\end{cases}
$$

by putting

$$
\mu_{\ell}:=\sum_{j=0}^{p} \lambda_{j, \ell_{j}}-1 .
$$

It follows from Theorem 7.2 that the above assumption is satisfied if

$$
m_{j, \ell_{j}} \geq d_{\ell}(\mathbf{m}) \quad(j=0, \ldots, p)
$$

and

$$
\begin{aligned}
& \sum_{j=0}^{p} \lambda_{j, \ell_{j}+\left(\nu-\ell_{j}\right) \delta_{j, k}} \notin\left\{i \in \mathbb{Z} ;(p-1) n-\sum_{j=0}^{p} m_{j, \ell_{j}+\left(\nu-\ell_{j}\right) \delta_{j, k}}+2 \leq i \leq 0\right\} \\
& \quad \text { for } k=0, \ldots, p \text { and } \nu=1, \ldots, n_{k} .
\end{aligned}
$$

Note that $\partial_{\ell} \mathbf{m} \in \mathcal{P}_{p+1}$ is well-defined for a given $\mathbf{m} \in \mathcal{P}_{p+1}$ if (7.31) is valid. Moreover we define

$$
\begin{aligned}
\partial \mathbf{m} & :=\partial_{(1,1, \ldots)} \mathbf{m}, \\
\partial_{\max } \mathbf{m} & :=\partial_{\ell_{\max }(\mathbf{m})} \mathbf{m} \text { with } \\
\ell_{\max }(\mathbf{m})_{j} & :=\min \left\{\nu ; m_{j, \nu}=\max \left\{m_{j, 1}, m_{j, 2}, \ldots\right\}\right\}, \\
d_{\max }(\mathbf{m}) & :=\sum_{j=0}^{p} \max \left\{m_{j, 1}, m_{j, 2}, \ldots, m_{j, n_{j}}\right\}-(p-1) \operatorname{ord} \mathbf{m} .
\end{aligned}
$$

For a Fuchsian differential operator $P$ with the Riemann scheme (6.15) we define

$$
\partial_{\max } P:=\partial_{\ell_{\max }(\mathbf{m})} P \text { and } \partial_{\max }\left\{\lambda_{\mathbf{m}}\right\}=\partial_{\ell_{\max }(\mathbf{m})}\left\{\lambda_{\mathbf{m}}\right\} \text {. }
$$

A tuple $\mathbf{m} \in \mathcal{P}$ is called basic if $\mathbf{m}$ is indivisible and $d_{\max }(\mathbf{m}) \leq 0$.

Proposition 7.7 (linear fractional transformation). Let $\phi$ be a linear fractional transformation of $\mathbb{P}^{1}(\mathbb{C})$, namely there exists $\left(\begin{array}{ll}\alpha & \beta \\ \gamma & \delta\end{array}\right) \in G L(2, \mathbb{C})$ such that $\phi(x)=$ $\frac{\alpha x+\beta}{\gamma x+\delta}$. Let $P$ be a Fuchsian differential operator with the Riemann scheme (6.15).

We may assume $-\frac{\delta}{\gamma}=c_{j}$ with a suitable $j$ by putting $c_{p+1}=-\frac{\delta}{\gamma}, \lambda_{p+1,1}=0$ and 
$m_{p+1,1}=n$ if necessary. Fix $\ell=\left(\ell_{0}, \cdots \ell_{p}\right) \in \mathbb{Z}_{>0}^{p+1}$. If 7.31) and (7.32) are valid, we have

$$
\begin{aligned}
\partial_{\ell} P & \in W(x) \operatorname{Ad}\left((\gamma x+\delta)^{2 \mu}\right) T_{\phi^{-1}}^{*} \partial_{\ell} T_{\phi}^{*} P \\
\mu & =\lambda_{0, \ell_{0}}+\cdots+\lambda_{p, \ell_{p}}-1 .
\end{aligned}
$$

Proof. The claim is clear if $\gamma=0$. Hence we may assume $\phi(x)=\frac{1}{x}$ and the claim follows from (7.11).

Remark 7.8. i) Fix $\lambda_{j, \nu} \in \mathbb{C}$. If $P$ has the Riemann scheme $\left\{\lambda_{\mathbf{m}}\right\}$ with $d_{\max }(\mathbf{m})=$ $1, \partial_{\ell} P$ is well-defined and $\partial_{\max } P$ has the Riemann scheme $\partial_{\max }\left\{\lambda_{\mathbf{m}}\right\}$. This follows from the fact that the conditions (7.5), (7.6) and (7.7) are valid when we apply Theorem 7.2 to the operation $\partial_{\max }: P \mapsto \partial_{\max } P$.

ii) We remark that

$$
\begin{aligned}
\operatorname{idx} \mathbf{m} & =\operatorname{idx} \partial_{\ell} \mathbf{m}, \\
\operatorname{ord} \partial_{\max } \mathbf{m} & =\operatorname{ord} \mathbf{m}-d_{\max }(\mathbf{m}) .
\end{aligned}
$$

Moreover if idx $\mathbf{m}>0$, we have

$$
d_{\max }(\mathbf{m})>0
$$

because of the identity

$$
\left(\sum_{j=0}^{k} m_{j, \ell_{j}}-(p-1) \operatorname{ord} \mathbf{m}\right) \cdot \operatorname{ord} \mathbf{m}=\mathrm{idx} \mathbf{m}+\sum_{j=0}^{p} \sum_{\nu=1}^{n_{j}}\left(m_{j, \ell_{j}}-m_{j, \nu}\right) \cdot m_{j, \nu}
$$

If idx $\mathbf{m}=0$, then $d_{\max }(\mathbf{m}) \geq 0$ and the condition $d_{\max }(\mathbf{m})=0$ implies $m_{j, \nu}=m_{j, 1}$ for $\nu=2, \ldots, n_{j}$ and $j=0,1, \ldots, p$ (cf. Corollary 8.3).

iii) The set of indices $\ell_{\max }(\mathbf{m})$ is defined in (7.34) so that it is uniquely determined. It is sufficient to impose only the condition

$$
m_{j, \ell_{\max }(\mathbf{m})_{j}}=\max \left\{m_{j, 1}, m_{j, 2}, \ldots\right\} \quad(j=0, \ldots, p)
$$

on $\ell_{\max }(\mathbf{m})$ for the arguments in this paper.

Thus we have the following result.

Theorem 7.9. A tuple $\mathbf{m} \in \mathcal{P}$ is realizable if and only if $s \mathbf{m}$ is trivial (cf. Definitions 6.10 and 6.11) or $\partial_{\max } \mathbf{m}$ is well-defined and realizable.

Proof. We may assume $\mathbf{m} \in \mathcal{P}_{p+1}^{(n)}$ is monotone.

Suppose $\#\left\{j ; m_{j, 1}<n\right\}<2$. Then $\partial_{\max } \mathbf{m}$ is not well-defined. We may assume $p=0$ and the corresponding equation $P u=0$ has no singularities in $\mathbb{C}$ by applying a suitable addition to the equation and then $P \in W(x) \partial^{n}$. Hence $\mathbf{m}$ is realizable if and only if $\#\left\{j ; m_{j, 1}<n\right\}=0$, namely, $\mathbf{m}$ is trivial.

Suppose $\#\left\{j ; m_{j, 1}<n\right\} \geq 2$. Then Theorem 7.2 assures that $\partial_{\max } \mathbf{m}$ is realizable if and only if $\partial_{\max } \mathbf{m}$ is realizable.

In the next section we will prove that $\mathbf{m}$ is realizable if $d_{\max }(\mathbf{m}) \leq 0$. Thus we will have a criterion whether a given $\mathbf{m} \in \mathcal{P}$ is realizable or not by successive applications of $\partial_{\max }$.

Example 7.10. There are examples of successive applications of $s \circ \partial$ to monotone elements of $\mathcal{P}$ :

$$
\begin{aligned}
& \underline{1} 11, \underline{4} 11, \underline{4} 2, \underline{3} 3 \stackrel{15-2 \cdot 6=3}{\longrightarrow} 111, \underline{1} 11, \underline{2} 1 \stackrel{4-3=1}{\longrightarrow} 11, \underline{1} 1, \underline{1} 1 \stackrel{3-2=1}{\longrightarrow} 1,1,1 \text { (rigid) } \\
& \underline{2} 11, \underline{2} 11, \underline{1} 111 \stackrel{5-4=1}{\longrightarrow} 111, \underline{1} 11, \underline{1} 11 \stackrel{3-3=0}{\longrightarrow} 111,111,111 \text { (realizable, not rigid) } \\
& \underline{2} 11, \underline{2} 11, \underline{2} 11, \underline{3} 1 \stackrel{9-8=1}{\longrightarrow} 111, \underline{1} 11, \underline{1} 11, \underline{2} 1 \stackrel{5-6=-1}{\longrightarrow} \text { (realizable, not rigid) }
\end{aligned}
$$


$\underline{2} 2, \underline{2} 2, \underline{1111} \stackrel{5-4=1}{\longrightarrow} \underline{2} 1, \underline{2} 1, \underline{1} 11 \stackrel{5-3=2}{\longrightarrow} \times($ not realizablej

The numbers on the above arrows are $d_{(1,1, \ldots)}(\mathbf{m})$. We sometimes delete the trivial partition as above.

The transformation of the generalized Riemann scheme of the application of $\partial_{\max }^{k}$ is described in the following definition.

Definition 7.11 (Reduction of Riemann schemes). Let $\mathbf{m}=\left(m_{j, \nu}\right)_{\substack{j=0, \ldots, p \\ \nu=1, \ldots, n_{j}}} \in$ $\mathcal{P}_{p+1}$ and $\lambda_{j, \nu} \in \mathbb{C}$ for $j=0, \ldots, p$ and $\nu=1, \ldots, n_{j}$. Suppose $\mathbf{m}$ is realizable. Then there exists a positive integer $K$ such that

$$
\begin{gathered}
\operatorname{ord} \mathbf{m}>\operatorname{ord} \partial_{\max } \mathbf{m}>\operatorname{ord} \partial_{\max }^{2} \mathbf{m}>\cdots>\operatorname{ord} \partial_{\max }^{K} \mathbf{m} \\
\text { and } s \partial_{\max }^{K} \mathbf{m} \text { is trivial or } d_{\max }\left(\partial_{\max }^{K} \mathbf{m}\right) \leq 0 .
\end{gathered}
$$

Define $\mathbf{m}(k) \in \mathcal{P}_{p+1}, \ell(k) \in \mathbb{Z}, \mu(k) \in \mathbb{C}$ and $\lambda(k)_{j, \nu \in \mathbb{C}}$ for $k=0, \ldots, K$ by

$$
\begin{aligned}
\mathbf{m}(0) & =\mathbf{m} \text { and } \mathbf{m}(k)=\partial_{\max } \mathbf{m}(k-1) \quad(k=1, \ldots, K), \\
\ell(k) & =\ell_{\max }(\mathbf{m}(k)) \text { and } d(k)=d_{\max }(\mathbf{m}(k)),
\end{aligned}
$$

(7.46) $\left\{\lambda(k)_{\mathbf{m}(k)}\right\}=\partial_{\max }^{k}\left\{\lambda_{\mathbf{m}}\right\}$ and $\mu(k)=\lambda(k+1)_{1, \nu}-\lambda(k)_{1, \nu} \quad\left(\nu \neq \ell(k)_{1}\right)$.

Namely, we have

$$
\begin{aligned}
& \lambda(0)_{j, \nu}=\lambda_{j, \nu} \quad\left(j=0, \ldots, p, \nu=1, \ldots, n_{j}\right), \\
& \mu(k)=\sum_{j=0}^{p} \lambda(k)_{j, \ell(k)_{j}}-1, \\
& \lambda(k+1)_{j, \nu}= \begin{cases}\lambda(k)_{0, \nu}-2 \mu(k) & \left(j=0, \nu=\ell(k)_{0}\right), \\
\lambda(k)_{0, \nu}-\mu(k) & \left(j=0,1 \leq \nu \leq n_{0}, \nu \neq \ell(k)_{0}\right), \\
\lambda(k)_{j, \nu} & \left(1 \leq j \leq p, \nu=\ell(k)_{j}\right), \\
\lambda(k)_{j, \nu}+\mu(k) & \left(1 \leq j \leq p, 1 \leq \nu \leq n_{j}, \quad \nu \neq \ell(k)_{j}\right)\end{cases} \\
& =\lambda(k)_{j, \nu}+\left((-1)^{\delta_{j, 0}}-\delta_{\nu, \ell(k)_{j}}\right) \mu(k), \\
& \left\{\lambda_{\mathbf{m}}\right\} \stackrel{\partial_{\ell(0)}}{\longrightarrow} \cdots \longrightarrow\left\{\lambda(k)_{\mathbf{m}(k)}\right\} \stackrel{\partial_{\ell(k)}}{\longrightarrow}\left\{\lambda(k+1)_{\mathbf{m}(k+1)}\right\} \stackrel{\partial_{\ell(k+1)}}{\longrightarrow} \cdots .
\end{aligned}
$$

\section{Deligne-Simpson Problem}

In this section we give an answer for the existence and the construction of Fuchsian differential equations with given Riemann schemes and examine the irreducibility for generic spectral parameters.

8.1. Fundamental lemmas. First we prepare two lemmas to construct Fuchsian differential operators with a given spectral type.

Definition 8.1. For $\mathbf{m}=\left(m_{j, \nu}\right)_{\substack{j=0, \ldots, p \\ 1 \leq \nu \leq n_{j}}} \in \mathcal{P}_{p+1}^{(n)}$, we put

$$
\begin{aligned}
N_{\nu}(\mathbf{m}):= & (p-1)(\nu+1)+1 \\
& \quad-\#\left\{(j, i) \in \mathbb{Z}^{2} ; i \geq 0,0 \leq j \leq p, \widetilde{m}_{j, i} \geq n-\nu\right\}, \\
& \widetilde{m}_{j, i}:=\sum_{\nu=1}^{n_{j}} \max \left\{m_{j, \nu}-i, 0\right\} .
\end{aligned}
$$

See the Young diagram in 8.32 ) and its explanation for an interpretation of the number $\widetilde{m}_{j, i}$. 
Lemma 8.2. We assume that $\mathbf{m}=\left(m_{j, \nu}\right)_{\substack{j=0, \ldots, p \\ 1 \leq \nu \leq n_{j}}} \in \mathcal{P}_{p+1}^{(n)}$ satisfies

$$
m_{j, 1} \geq m_{j, 2} \geq \cdots \geq m_{j, n_{j}}>0 \text { and } n>m_{0,1} \geq m_{1,1} \geq \cdots \geq m_{p, 1}
$$

and

$$
m_{0,1}+\cdots+m_{p, 1} \leq(p-1) n .
$$

Then

$$
N_{\nu}(\mathbf{m}) \geq 0 \quad(\nu=2,3, \ldots, n-1)
$$

if and only if $\mathbf{m}$ is not any one of

$$
(k, k ; k, k ; k, k ; k, k), \quad(k, k, k ; k, k, k ; k, k, k),
$$

$(2 k, 2 k ; k, k, k, k ; k, k, k, k)$

and $(3 k, 3 k ; 2 k, 2 k, 2 k ; k, k, k, k, k, k)$ with $k \geq 2$.

Proof. Put

$$
\phi_{j}(t):=\sum_{\nu=1}^{n_{j}} \max \left\{m_{j, \nu}-t, 0\right\} \text { and } \bar{\phi}_{j}(t):=n\left(1-\frac{t}{m_{j, 1}}\right) \text { for } j=0, \ldots, p .
$$

Then $\phi_{j}(t)$ and $\bar{\phi}_{j}(t)$ are strictly decreasing continuous functions of $t \in\left[0, m_{j, 1}\right]$ and

$$
\begin{aligned}
\phi_{j}(0) & =\bar{\phi}_{j}(0)=n, & \\
\phi_{j}\left(m_{j, 1}\right) & =\bar{\phi}_{j}\left(m_{j, 1}\right)=0, & \\
2 \phi_{j}\left(\frac{t_{1}+t_{2}}{2}\right) & \leq \phi_{j}\left(t_{1}\right)+\phi_{j}\left(t_{2}\right) & \left(0 \leq t_{1} \leq t_{2} \leq m_{j, 1}\right), \\
\phi_{j}^{\prime}(t) & =-n_{j} \leq-\frac{n}{m_{j, 1}}=\bar{\phi}_{j}^{\prime}(t) & (0<t<1) .
\end{aligned}
$$

Hence we have

$$
\begin{array}{ll}
\phi_{j}(t)=\bar{\phi}_{j}(t) & \left(0<t<m_{j, 1}, n=m_{j, 1} n_{j}\right), \\
\phi_{j}(t)<\bar{\phi}_{j}(t) & \left(0<t<m_{j, 1}, n<m_{j, 1} n_{j}\right)
\end{array}
$$

and for $\nu=2, \ldots, n-1$

$$
\begin{aligned}
\sum_{j=0}^{p} \#\left\{i \in \mathbb{Z}_{\geq 0} ; \phi_{j}(i) \geq n-\nu\right\} & =\sum_{j=0}^{p}\left[\phi_{j}^{-1}(n-\nu)+1\right] \\
& \leq \sum_{j=0}^{p}\left(\phi_{j}^{-1}(n-\nu)+1\right) \\
& \leq \sum_{j=0}^{p}\left(\bar{\phi}_{j}^{-1}(n-\nu)+1\right)=\sum_{j=0}^{p}\left(\frac{\nu m_{j, 1}}{n}+1\right) \\
& \leq(p-1) \nu+(p+1)=(p-1)(\nu+1)+2 .
\end{aligned}
$$

Here $[r]$ means the largest integer which is not larger than a real number $r$.

Suppose there exists $\nu$ with $2 \leq \nu \leq n-1$ such that (8.5) doesn't hold. Then the equality holds in the above each line, which means

$$
\begin{aligned}
\phi_{j}^{-1}(n-\nu) & \in \mathbb{Z} & & (j=0, \ldots, p), \\
n & =m_{j, 1} n_{j} & & (j=0, \ldots, p), \\
(p-1) n & =m_{0,1}+\cdots+m_{p, 1} . & &
\end{aligned}
$$

Note that $n=m_{j, 1} n_{j}$ implies $m_{j, 1}=\cdots=m_{j, n_{j}}=\frac{n}{n_{j}}$ and $p-1=\frac{1}{n_{0}}+\cdots+\frac{1}{n_{p}} \leq$ $\frac{p+1}{2}$. Hence $p=3$ with $n_{0}=n_{1}=n_{2}=n_{3}=2$ or $p=2$ with $1=\frac{1}{n_{0}}+\frac{1}{n_{1}}+\frac{1}{n_{2}}$. If 
$p=2,\left\{n_{0}, n_{1}, n_{2}\right\}$ equals $\{3,3,3\}$ or $\{2,4,4\}$ or $\{2,3,6\}$. Thus we have (8.6) with $k=1,2, \ldots$. Moreover since

$$
\phi_{j}^{-1}(n-\nu)=\bar{\phi}_{j}^{-1}(n-\nu)=\frac{\nu m_{j, 1}}{n}=\frac{\nu}{n_{j}} \in \mathbb{Z} \quad(j=0, \ldots, p),
$$

$\nu$ is a common multiple of $n_{0}, \ldots, n_{p}$ and thus $k \geq 2$. If $\nu$ is the least common multiple of $n_{0}, \ldots, n_{p}$ and $k \geq 2$, then (8.7) is valid and the equality holds in the above each line and hence (8.5) is not valid.

Corollary 8.3 (Kostov Ko3]). Let $\mathbf{m} \in \mathcal{P}$ satisfying idx $\mathbf{m}=0$ and $d_{\max }(\mathbf{m}) \leq 0$. Then $\mathbf{m}$ is isomorphic to one of the tuples in (8.6) with $k=1,2,3, \ldots$..

Proof. Remark 7.8 assures that $d_{\max }(\mathbf{m})=0$ and $n=m_{j, 1} n_{j}$. Then the proof of the final part of Lemma 8.2 shows the corollary.

Lemma 8.4. Let $c_{0}, \ldots, c_{p}$ be $p+1$ distinct points in $\mathbb{C} \cup\{\infty\}$. Let $n_{0}, n_{1}, \ldots, n_{p}$ be non-negative integers and let $a_{j, \nu}$ be complex numbers for $j=0, \ldots, p$ and $\nu=$ $1, \ldots, n_{j}$. Put $\tilde{n}:=n_{0}+\cdots+n_{p}$. Then there exists a unique polynomial $f(x)$ of degree $\tilde{n}-1$ such that

$$
\begin{gathered}
f(x)=a_{j, 1}+a_{j, 2}\left(x-c_{j}\right)+\cdots+a_{j, n_{j}}\left(x-c_{j}\right)^{n_{j}-1} \\
+o\left(\left|x-c_{j}\right|^{n_{j}-1}\right) \quad\left(x \rightarrow c_{j}, c_{j} \neq \infty\right), \\
x^{1-\tilde{n}} f(x)=a_{j, 1}+a_{j, 2} x^{-1}+a_{j, n_{j}} x^{1-n_{j}}+o\left(|x|^{1-n_{j}}\right) \\
\quad\left(x \rightarrow \infty, c_{j}=\infty\right) .
\end{gathered}
$$

Moreover the coefficients of $f(x)$ are linear functions of the $\tilde{n}$ variables $a_{j, \nu}$.

Proof. We may assume $c_{p}=\infty$ with allowing $n_{p}=0$. Put $\tilde{n}_{i}=n_{0}+\cdots+n_{i-1}$ and $\tilde{n}_{0}=0$. For $k=0, \ldots, \tilde{n}-1$ we define

$$
f_{k}(x):= \begin{cases}\left(x-c_{i}\right)^{k-\tilde{n}_{i}} \prod_{\nu=0}^{i-1}\left(x-c_{\nu}\right)^{n_{\nu}} & \left(\tilde{n}_{i} \leq k<\tilde{n}_{i+1}, 0 \leq i<p\right), \\ x^{k-\tilde{n}_{p}} \prod_{\nu=0}^{n_{p-1}}\left(x-c_{\nu}\right)^{n_{\nu}} & \left(\tilde{n}_{p} \leq k<\tilde{n}\right) .\end{cases}
$$

Since $\operatorname{deg} f_{k}(x)=k$, the polynomials $f_{0}(x), f_{1}(x), \ldots, f_{\tilde{n}-1}(x)$ are linearly independent over $\mathbb{C}$. Put $f(x)=\sum_{k=0}^{\tilde{n}-1} u_{k} f_{k}(x)$ with $c_{k} \in \mathbb{C}$ and

$$
v_{k}= \begin{cases}a_{i, k-\tilde{n}_{i}+1} & \left(\tilde{n}_{i} \leq k<\tilde{n}_{i+1}, 0 \leq i<p\right), \\ a_{p, \tilde{n}-k} & \left(\tilde{n}_{p} \leq k<\tilde{n}\right)\end{cases}
$$

by 8.8 . The correspondence which maps the column vectors $u:=\left(u_{k}\right)_{k=0, \ldots, \tilde{n}-1} \in$ $\mathbb{C}^{\tilde{n}}$ to the column vectors $v:=\left(v_{k}\right)_{k=0, \ldots, \tilde{n}-1} \in \mathbb{C}^{\tilde{n}}$ is given by $v=A u$ with a square matrix $A$ of size $\tilde{n}$. Then $A$ is an upper triangular matrix of size $\tilde{n}$ with non-zero diagonal entries and therefore the lemma is clear.

\subsection{Existence theorem.}

Definition 8.5 (top term). Let

$$
P=a_{n}(x) \frac{d^{n}}{d x^{n}}+a_{n-1}(x) \frac{d^{n-1}}{d x^{n-1}}+\cdots+a_{1}(x) \frac{d}{d x}+a_{0}(x)
$$

be a differential operator with polynomial coefficients. Suppose $a_{n} \neq 0$. If $a_{n}(x)$ is a polynomial of degree $k$ with respect to $x$, we define Top $P:=a_{n, k} x^{k} \partial^{n}$ with the coefficient $a_{n, k}$ of the term $x^{k}$ of $a_{n}(x)$. We put Top $P=0$ when $P=0$.

Theorem 8.6. Suppose $\mathbf{m} \in \mathcal{P}_{p+1}^{(n)}$ satisfies (8.3). Retain the notation in Definition 8.1. 
i) We have $N_{1}(\mathbf{m})=p-2$ and

$$
\sum_{\nu=1}^{n-1} N_{\nu}(\mathbf{m})=\operatorname{Pidx} \mathbf{m}
$$

ii) Suppose $p \geq 2$ and $N_{\nu}(\mathbf{m}) \geq 0$ for $\nu=2, \ldots, n-1$. Put

$$
\begin{aligned}
q_{\nu}^{0} & :=\#\left\{i ; \widetilde{m}_{0, i} \geq n-\nu, i \geq 0\right\} \\
I_{\mathbf{m}} & :=\left\{(j, \nu) \in \mathbb{Z}^{2} ; q_{\nu}^{0} \leq j<q_{\nu}^{0}+N_{\nu}(\mathbf{m}) \text { and } 1 \leq \nu \leq n-1\right\} .
\end{aligned}
$$

Then there uniquely exists a Fuchsian differential operator $P$ of the normal form (6.43) which has the Riemann scheme 6.15) with $c_{0}=\infty$ under the Fuchs relation (6.16) and satisfies

$$
\frac{1}{(\operatorname{deg} P-j-\nu) !} \frac{d^{\operatorname{deg} P-j-\nu} a_{n-\nu-1}}{d x^{\operatorname{deg} P-j-\nu}}(0)=g_{j, \nu} \quad\left(\forall(j, \nu) \in I_{\mathbf{m}}\right)
$$

Here $\left(g_{j, \nu}\right)_{(j, \nu) \in I_{\mathbf{m}}} \in \mathbb{C}^{\mathrm{Pidx} \mathbf{m}}$ is arbitrarily given. Moreover the coefficients of $P$ are polynomials of $x, \lambda_{j, \nu}$ and $g_{j, \nu}$ and satisfy

$$
x^{j+\nu} \operatorname{Top}\left(\frac{\partial P}{\partial g_{j, \nu}}\right) \partial^{\nu+1}=\operatorname{Top} P \quad \text { and } \quad \frac{\partial^{2} P}{\partial g_{j, \nu}^{2}}=0 .
$$

Fix the characteristic exponents $\lambda_{j, \nu} \in \mathbb{C}$ satisfying the Fuchs relation. Then all the Fuchsian differential operators of the normal form with the Riemann scheme 6.15) are parametrized by $\left(g_{j, \nu}\right) \in \mathbb{C}^{\mathrm{Pidx} \mathbf{m}}$. Hence the operators are unique if and only if $\operatorname{Pidx} \mathbf{m}=0$.

Proof. i) Since $\widetilde{m}_{j, 1}=n-n_{j} \leq n-2, N_{1}(\mathbf{m})=2(p-1)+1-(p+1)=p-2$ and

$$
\begin{aligned}
\sum_{\nu=1}^{n-1} \#\left\{(j, i) \in \mathbb{Z}^{2} ; i \geq 0,0 \leq j \leq p, \tilde{m}_{j, i} \geq n-\nu\right\} \\
=\sum_{j=0}^{p}\left(\sum_{\nu=0}^{n-1} \#\left\{i \in \mathbb{Z}_{\geq 0} ; \tilde{m}_{j, i} \geq n-\nu\right\}-1\right) \\
=\sum_{j=0}^{p}\left(\sum_{i=0}^{m_{j, 1}} \tilde{m}_{j, i}-1\right)=\sum_{j=0}^{p}\left(\sum_{i=0}^{m_{j, 1}} \sum_{\nu=1}^{n_{j}} \max \left\{m_{j, \nu}-i, 0\right\}-1\right) \\
=\sum_{j=0}^{p}\left(\sum_{\nu=1}^{n_{j}} \frac{m_{j, \nu}\left(m_{j, \nu}+1\right)}{2}-1\right) \\
=\frac{1}{2}\left(\sum_{j=0}^{p} \sum_{\nu=1}^{n_{j}} m_{j, \nu}^{2}+(p+1)(n-2)\right), \\
\sum_{\nu=1}^{n-1} N_{\nu}(\mathbf{m})= \\
=\frac{1}{2}\left((p-1)\left(\frac{n(n+1)}{2}-1\right)+(n-1)-\frac{1}{2}\left(\sum_{j=0}^{p} \sum_{\nu=1}^{n_{j}} m_{j, \nu}^{2}+(p+1)(n-2)\right)\right. \\
\left.n^{2}+2-\sum_{j=0}^{p} \sum_{\nu=1}^{n_{j}} m_{j, \nu}^{2}\right)=\operatorname{Pidx} \mathbf{m} .
\end{aligned}
$$


ii) Put

$$
\begin{aligned}
& P=\sum_{\ell=0}^{p n} x^{p n-\ell} p_{0, \ell}^{P}(\vartheta) \\
& =\sum_{\ell=0}^{p n}\left(x-c_{j}\right)^{\ell} p_{j, \ell}^{P}\left(\left(x-c_{j}\right) \partial\right) \quad(1 \leq j \leq n), \\
& h_{j, \ell}(t):= \begin{cases}\prod_{\nu=1}^{n_{0}} \prod_{0 \leq i<m_{0, \nu}-\ell}\left(t+\lambda_{0, \nu}+i\right) & (j=0), \\
\prod_{\nu=1}^{n_{j}} \prod_{0 \leq i<m_{j, \nu}-\ell}\left(t-\lambda_{j, \nu}-i\right) & (1 \leq j \leq p),\end{cases} \\
& p_{j, \ell}^{P}(t)=q_{j, \ell}^{P}(t) h_{j, \ell}(t)+r_{j, \ell}^{P}(t) \quad\left(\operatorname{deg} r_{j, \ell}^{P}(t)<\operatorname{deg} h_{j, \ell}(t)\right) .
\end{aligned}
$$

Here $p_{j, \ell}^{P}(t), q_{j, \ell}^{P}(t), r_{j, \ell}^{P}(t)$ and $h_{j, \ell}(t)$ are polynomials of $t$ and

$$
\operatorname{deg} h_{j, \ell}=\sum_{\nu=1}^{n_{j}} \max \left\{m_{j, \nu}-\ell, 0\right\} .
$$

The condition that $P$ of the form (6.43) have the Riemann scheme (6.15) if and only if $r_{j, \ell}^{P}=0$ for any $j$ and $\ell$. Note that $a_{n-k}(x) \in \mathbb{C}[x]$ should satisfy

(8.15) $\operatorname{deg} a_{n-k}(x) \leq p n-k$ and $a_{n-k}^{(\nu)}\left(c_{j}\right)=0 \quad(0 \leq \nu \leq n-k-1,1 \leq k \leq n)$, which is equivalent to the condition that $P$ is of the Fuchsian type.

Put $P(k):=\left(\prod_{j=1}^{p}\left(x-c_{j}\right)^{n}\right) \frac{d^{n}}{d x^{n}}+a_{n-1}(x) \frac{d^{n-1}}{d x^{n-1}}+\cdots+a_{n-k}(x) \frac{d^{n-k}}{d x^{n-k}}$.

Assume that $a_{n-1}(x), \ldots, a_{n-k+1}(x)$ have already defined so that $\operatorname{deg} r_{j, \ell}^{P(k-1)}<$ $n-k+1$ and we will define $a_{n-k}(x)$ so that $\operatorname{deg} r_{j, \ell}^{P(k)}<n-k$.

When $k=1$, we put

$$
a_{n-1}(x)=-a_{n}(x) \sum_{j=1}^{p}\left(x-c_{j}\right)^{-1} \sum_{\nu=1}^{n_{j}} \sum_{i=0}^{m_{j, \nu}-1}\left(\lambda_{j, \nu}+i\right)
$$

and then we have $\operatorname{deg} r_{j, \ell}^{P(1)}<n-1$ for $j=1, \ldots, p$. Moreover we have $\operatorname{deg} r_{0, \ell}^{P(1)}<$ $n-1$ because of the Fuchs relation.

Suppose $k \geq 2$ and put

$$
a_{n-k}(x)=\left\{\begin{array}{l}
\sum_{\ell \geq 0} c_{0, k, \ell} x^{p n-k-\ell}, \\
\sum_{\ell \geq 0} c_{j, k, \ell}\left(x-c_{j}\right)^{n-k+\ell} \quad(j=1, \ldots, p)
\end{array}\right.
$$

with $c_{i, j, \ell} \in \mathbb{C}$. Note that

$$
\begin{aligned}
a_{n-k}(x) \partial^{n-k} & =\sum_{\ell \geq 0} c_{0, k, \ell} x^{(p-1) n-\ell} \prod_{i=0}^{n-k-1}(\vartheta-i) \\
& =\sum_{\ell \geq 0} c_{j, k, \ell}\left(x-c_{j}\right)^{\ell} \prod_{i=0}^{n-k-1}\left(\left(x-c_{j}\right) \partial-i\right) .
\end{aligned}
$$

Then $\operatorname{deg} r_{j, \ell}^{P(k)}<n-k$ if and only if $\operatorname{deg} h_{j, \ell} \leq n-k$ or

$$
c_{j, k, \ell}=-\left.\frac{1}{(n-k) !}\left(\frac{d^{n-k}}{d t^{n-k}} r_{j, \ell}^{P(k-1)}(t)\right)\right|_{t=0} .
$$

Namely, we impose the condition (8.16) for all $(j, \ell)$ satisfying

$$
\widetilde{m}_{j, \ell}=\sum_{\nu=1}^{n_{j}} \max \left\{m_{j, \nu}-\ell, 0\right\}>n-k .
$$


The number of the pairs $(j, \ell)$ satisfying this condition equals $(p-1) k+1-N_{k-1}(\mathbf{m})$. Together with the conditions $a_{n-k}^{(\nu)}\left(c_{j}\right)=0$ for $j=1, \ldots, p$ and $\nu=0, \ldots, n-k-1$, the total number of conditions imposing to the polynomial $a_{n-k}(x)$ of degree $p n-k$ equals

$$
p(n-k)+(p-1) k+1-N_{k-1}(\mathbf{m})=(p n-k+1)-N_{k-1}(\mathbf{m}) .
$$

Hence Lemma 8.4 shows that $a_{n-k}(x)$ is uniquely defined by giving $c_{0, k, \ell}$ arbitrarily for $q_{k-1}^{0} \leq \ell<q_{k-1}^{0}+N_{k-1}(\mathbf{m})$ because $q_{k-1}^{0}=\#\left\{\ell \geq 0 ; \widetilde{m}_{0, \ell}>n-k\right\}$. Thus we have the theorem.

Remark 8.7. The numbers $N_{\nu}(\mathbf{m})$ don't change if we replace a $(p+1)$-tuple $\mathbf{m}$ of partitions of $n$ by the $(p+2)$-tuple of partitions of $n$ defined by adding a trivial partition $n=n$ of $n$ to $\mathbf{m}$.

Example 8.8. We will examine the number $N_{\nu}(\mathbf{m})$ in Theorem 8.6. In the case of the Simpson's list (cf. $\$ 15.2$ ) we have the following.

$\left(H_{n}\right.$ : hypergeometric family)

$$
\begin{array}{ll} 
& \mathbf{m}=n-11,1^{n}, 1^{n} \\
& \widetilde{\mathbf{m}}=n, n-2, n-3, \ldots 1 ; n ; n \\
\left(E O_{2 m}:\right. \text { even family) } & \mathbf{m}=m m, m m-11,1^{2 m} \\
& \widetilde{\mathbf{m}}=2 m, 2 m-2, \ldots, 2 ; 2 m, 2 m-3, \ldots, 1 ; 2 m \\
\left(E O_{2 m+1} \text { : odd family }\right) & \mathbf{m}=m+1 m, m m 1,1^{2 m+1} \\
& \widetilde{\mathbf{m}}=2 m+1,2 m-1, \ldots, 1 ; 2 m+1,2 m-2, \ldots, 2 ; 2 m+1 \\
\left(X_{6}: \text { extra case }\right) & \mathbf{m}=42,222,1^{6} \\
& \widetilde{\mathbf{m}}=6,4,2,1 ; 6,3 ; 6
\end{array}
$$

In these cases $p=2$ and we have $N_{\nu}(\mathbf{m})=0$ for $\nu=1,2, \ldots, n-1$ because

$$
\begin{aligned}
\widetilde{\mathbf{m}}: & =\left\{\widetilde{m}_{j, \nu} ; \nu=0, \ldots, m_{j, 1}-1, j=0, \ldots, p\right\} \\
& =\{n, n, n, n-2, n-3, n-4, \ldots, 2,1\} .
\end{aligned}
$$

See Proposition 8.16 ii) for the condition that $N_{\nu}(\mathbf{m}) \geq 0$ for $\nu=1, \ldots$, ord $\mathbf{m}-1$.

We give other examples:

\begin{tabular}{|c|c|l|l|}
\hline $\mathbf{m}$ & Pidx & \multicolumn{1}{|c|}{$\widetilde{\mathbf{m}}$} & $N_{1}, N_{2}, \ldots, N_{\text {ord } \mathbf{m}-1}$ \\
\hline \hline $221,221,221$ & 0 & $52,52,52$ & $0,1,-1,0$ \\
\hline $21,21,21,21\left(P_{3}\right)$ & 0 & $31,31,31,31$ & $1,-1$ \\
\hline $22,22,22$ & -3 & $42,42,42$ & $0,-2,-1$ \\
\hline $11,11,11,11\left(\tilde{D}_{4}\right)$ & 1 & $2,2,2,2$ & 1 \\
\hline $111,111,111\left(\tilde{E}_{6}\right)$ & 1 & $3,3,3$ & 0,1 \\
\hline $22,1111,1111\left(E_{7}\right)$ & 1 & $42,4,4$ & $0,0,1$ \\
\hline $33,222,111111\left(\tilde{E}_{8}\right)$ & 1 & $642,63,6$ & $0,0,0,0,1$ \\
\hline $21,21,21,111$ & 1 & $31,31,31,3$ & 1,0 \\
\hline $222,222,222$ & 1 & $63,63,63$ & $0,1,-1,0,1$ \\
\hline $11,11,11,11,11$ & 2 & $2,2,2,2,2$ & 2 \\
\hline $55,3331,22222$ & 2 & $10,8,6,4,2 ; 10,6,3 ; 10,5$ & $0,0,1,0,0,0,0,0,1$ \\
\hline $22,22,22,211$ & 2 & $42,42,42,41$ & $1,0,1$ \\
\hline $22,22,22,22,22$ & 5 & $42,42,42,42,42$ & $2,0,3$ \\
\hline $32111,3221,2222$ & 8 & $831,841,84$ & $0,1,2,1,1,2,1$ \\
\hline
\end{tabular}

Note that if Pidx $\mathbf{m}=0$, in particular, if $\mathbf{m}$ is rigid, then $\mathbf{m}$ doesn't satisfy (8.4). The tuple 222, 222, 222 of partitions is the second case in (8.6) with $k=2$. 
Remark 8.9. Note that [06, Proposition 8.1] proves that there exit only finite basic tuples of partitions with a fixed index of rigidity.

Those with index of rigidity 0 are of only 4 types, which are $\tilde{D}_{4}, \tilde{E}_{6}, \tilde{E}_{7}$ and $\tilde{E}_{8}$ given in the above (cf. Corollary 8.3, Kostov Ko3]). Namely, those are in the $S_{\infty}$-orbit of

$$
\{11,11,11,11 \quad 111,111,111 \quad 22,1111,1111 \quad 33,222,111111\}
$$

and the operator $P$ in Theorem 8.6 with any one of this spectral type has one accessory parameter in its 0 -th order term.

The equation corresponding to $11,11,11,11$ is called Heun's equation (cf. SW, WW], which is given by the operator

$$
\begin{aligned}
P_{\alpha, \beta, \gamma, \delta, \lambda}= & x(x-1)(x-c) \partial^{2}+(\gamma(x-1)(x-c)+\delta x(x-c) \\
& +(\alpha+\beta+1-\gamma-\delta) x(x-1)) \partial+\alpha \beta x-\lambda
\end{aligned}
$$

with the Riemann scheme

$$
\left\{\begin{array}{ccccc}
x=0 & 1 & c & \infty & \\
0 & 0 & 0 & \alpha & ; x \\
1-\gamma & 1-\delta & \gamma+\delta-\alpha-\beta & \beta & ; \lambda
\end{array}\right\} .
$$

Here $\lambda$ is an accessory parameter. Our operation cannot decrease the order of $P_{\alpha, \beta, \gamma, \delta, \lambda}$ but gives the following transformation.

$$
\begin{aligned}
& \operatorname{Ad}\left(\partial^{1-\alpha}\right) P_{\alpha, \beta, \gamma, \delta, \lambda}=P_{\alpha^{\prime}, \beta^{\prime}, \gamma^{\prime}, \delta^{\prime}, \lambda^{\prime}} \\
& \left\{\begin{array}{l}
\alpha^{\prime}=2-\alpha, \beta^{\prime}=\beta-\alpha+1, \gamma^{\prime}=\gamma-\alpha+1, \delta^{\prime}=\delta-\alpha+1 \\
\lambda^{\prime}=\lambda+(1-\alpha)(\beta-\delta+1+(\gamma+\delta-\alpha) c)
\end{array}\right.
\end{aligned}
$$

Proposition 8.10. ([06, Proposition 8.4]). The basic tuples of partitions with index of rigidity -2 are in the $S_{\infty}$-orbit of the set of the 13 tuples

$$
\begin{aligned}
& \{11,11,11,11,1121,21,111,111 \quad 31,22,22,1111 \quad 22,22,22,211 \\
& 211,1111,1111 \quad 221,221,11111 \quad 32,11111,11111 \quad 222,222,2211 \\
& \text { 33,2211, } 11111144,2222,22211 \text { 44,332, } 11111111 \quad 55,3331,22222 \\
& 66,444,2222211\} \text {. }
\end{aligned}
$$

Proof. Here we give the proof in $\mathrm{O} 6$.

Assume that $\mathbf{m} \in \mathcal{P}_{p+1}$ is basic and monotone and idx $\mathbf{m}=-2$. Note that (7.41) shows

$$
0 \leq \sum_{j=0}^{p} \sum_{\nu=2}^{n_{j}}\left(m_{j, 1}-m_{j, \nu}\right) \cdot m_{j, \nu} \leq-\mathrm{idx} \mathbf{m}=2 .
$$

Hence (7.41) implies $\sum_{j=0}^{p} \sum_{\nu=2}^{n_{j}}\left(m_{j, 1}-m_{j, \nu}\right) m_{j, \nu}=0$ or 2 and we have only to examine the following 5 possibilities.

(A) $m_{0,1} \cdots m_{0, n_{0}}=2 \cdots 211$ and $m_{j, 1}=m_{j, n_{j}}$ for $1 \leq j \leq p$.

(B) $m_{0,1} \cdots m_{0, n_{0}}=3 \cdots 31$ and $m_{j, 1}=m_{j, n_{j}}$ for $1 \leq j \leq p$.

(C) $m_{0,1} \cdots m_{0, n_{0}}=3 \cdots 32$ and $m_{j, 1}=m_{j, n_{j}}$ for $1 \leq j \leq p$.

(D) $m_{i, 1} \cdots m_{i, n_{0}}=2 \cdots 21$ and $m_{j, 1}=m_{j, n_{j}}$ for $0 \leq i \leq 1<j \leq p$.

(E) $m_{j, 1}=m_{j, n_{j}}$ for $0 \leq j \leq p$ and ord $\mathbf{m}=2$.

Case (A). If $2 \cdots 211$ is replaced by $2 \cdots 22, \mathbf{m}$ is transformed into $\mathbf{m}^{\prime}$ with $\mathrm{idx} \mathbf{m}^{\prime}=0$. If $\mathbf{m}^{\prime}$ is indivisible, $\mathbf{m}^{\prime}$ is basic and $\mathrm{idx} \mathbf{m}^{\prime}=0$ and therefore $\mathbf{m}$ is $211,1^{4}, 1^{4}$ or $33,2211,1^{6}$. If $\mathbf{m}^{\prime}$ is not indivisible, $\frac{1}{2} \mathbf{m}^{\prime}$ is basic and $\mathrm{idx} \frac{1}{2} \mathbf{m}^{\prime}=0$ and hence $\mathbf{m}$ is one of the tuples in

$\{211,22,22,22 \quad 2211,222,222 \quad 22211,2222,442222211,444,66\}$. 
Put $m=n_{0}-1$ and examine the identity

$$
\sum_{j=0}^{p} \frac{m_{j, 1}}{\operatorname{ord} \mathbf{m}}=p-1+(\operatorname{ord} \mathbf{m})^{-2}\left(\operatorname{idx} \mathbf{m}+\sum_{j=0}^{p} \sum_{\nu=1}^{n_{j}}\left(m_{j, 1}-m_{j, \nu}\right) m_{j, \nu}\right)
$$

Case (B). Note that ord $\mathbf{m}=3 m+1$ and therefore $\frac{3}{3 m+1}+\frac{1}{n_{1}}+\cdots+\frac{1}{n_{p}}=p-1$. Since $n_{j} \geq 2$, we have $\frac{1}{2} p-1 \leq \frac{3}{3 m+1}<1$ and $p \leq 3$.

If $p=3$, we have $m=1$, ord $\mathbf{m}=4, \frac{1}{n_{1}}+\frac{1}{n_{2}}+\frac{1}{n_{3}}=\frac{5}{4},\left\{n_{1}, n_{2}, n_{3}\right\}=\{2,2,4\}$ and $\mathbf{m}=31,22,22,1111$.

Assume $p=2$. Then $\frac{1}{n_{1}}+\frac{1}{n_{2}}=1-\frac{3}{3 m+1}$. If $\min \left\{n_{1}, n_{2}\right\} \geq 3, \frac{1}{n_{1}}+\frac{1}{n_{2}} \leq \frac{2}{3}$ and $m \leq 2$. If $\min \left\{n_{1}, n_{2}\right\}=2, \max \left\{n_{1}, n_{2}\right\} \geq 3$ and $\frac{3}{3 m+1} \geq \frac{1}{6}$ and $m \leq 5$. Note that $\frac{1}{n_{1}}+\frac{1}{n_{2}}=\frac{13}{16}, \frac{10}{13}, \frac{7}{10}, \frac{4}{7}$ and $\frac{1}{4}$ according to $m=5,4,3,2$ and 1 , respectively. Hence we have $m=3,\left\{n_{1}, n_{2}\right\}=\{2,5\}$ and $\mathbf{m}=3331,55,22222$.

Case (C). We have $\frac{3}{3 m+2}+\frac{1}{n_{1}}+\cdots+\frac{1}{n_{p}}=p-1$. Since $n_{j} \geq 2, \frac{1}{2} p-1 \leq \frac{3}{3 m+2}<1$ and $p \leq 3$. If $p=3$, then $m=1$, ord $\mathbf{m}=5$ and $\frac{1}{n_{1}}+\frac{1}{n_{2}}+\frac{1}{n_{3}}=\frac{7}{5}$, which never occurs.

Thus we have $p=2, \frac{1}{n_{1}}+\frac{1}{n_{2}}=1-\frac{3}{3 m+2}$ and hence $m \leq 5$ as in Case (B). Then $\frac{1}{n_{1}}+\frac{1}{n_{2}}=\frac{14}{17}, \frac{11}{14}, \frac{8}{11}, \frac{5}{8}$ and $\frac{2}{5}$ according to $m=5,4,3,2$ and 1 , respectively. Hence we have $m=1$ and $n_{1}=n_{2}=5$ and $\mathbf{m}=32,11111,11111$ or $m=2$ and $n_{1}=2$ and $n_{2}=8$ and $\mathbf{m}=332,44,11111111$.

Case (D). We have $\frac{2}{2 m+1}+\frac{2}{2 m+1}+\frac{1}{n_{2}}+\cdots+\frac{1}{n_{p}}=p-1$. Since $n_{j} \geq 3$ for $j \geq 2$, we have $p-1 \leq \frac{3}{2} \frac{4}{2 m+1}=\frac{6}{2 m+1}$ and $m \leq 2$. If $m=1$, then $p=3$ and $\frac{1}{n_{2}}+\frac{1}{n_{3}}=2-\frac{4}{3}=\frac{2}{3}$ and we have $\mathbf{m}=21,21,111,111$. If $m=2$, then $p=2$, $\frac{1}{n_{2}}=1-\frac{4}{5}$ and $\mathbf{m}=221,221,11111$.

Case $(\mathrm{E})$. Since $m_{j, 1}=1$ and (7.41) means $-2=\sum_{j=0}^{p} 2 m_{j, 1}-4(p-1)$, we have $p=4$ and $\mathbf{m}=11,11,11,11,11$.

\subsection{Divisible spectral types.}

Proposition 8.11. Let $\mathbf{m}$ be any one of the partition of type $\tilde{D}_{4}, \tilde{E}_{6}, \tilde{E}_{7}$ or $\tilde{E}_{8}$ in Example 8.8 and put $n=$ ord $\mathbf{m}$. Then $\mathrm{km}$ is realizable but it isn't irreducibly realizable for $k=2,3, \ldots$ Moreover we have the operator $P$ of order $k$ ord $\mathbf{m}$ satisfying the properties in Theorem 8.6 ii) for the tuple $\mathrm{km}$.

Proof. Let $P(k, c)$ be the operator of the normal form with the Riemann scheme

$$
\left\{\begin{array}{cc}
x=c_{0}=\infty & x=c_{j}(j=1, \ldots, p) \\
{\left[\lambda_{0,1}-k(p-1) n+k m_{0,1}\right]_{\left(m_{0,1}\right)}} & {\left[\lambda_{j, 1}+k m_{j, 1}\right]_{\left(m_{j, 1}\right)}} \\
\vdots & \vdots \\
{\left[\lambda_{0, n_{1}}-k(p-1) n+k m_{0,1}\right]_{\left(m_{0, n_{1}}\right)}} & {\left[\lambda_{j, n_{j}}+k m_{j, n_{j}}\right]_{\left(m_{j, n_{j}}\right)}}
\end{array}\right\}
$$

of type $\mathbf{m}$. Here $\mathbf{m}=\left(m_{j, \nu}\right)_{\substack{j=0, \ldots, p \\ \nu=1, \ldots, n_{j}}}, n=$ ord $\mathbf{m}$ and $c$ is the accessory parameter contained in the coefficient of the 0 -th order term of $P(k, c)$. Since Pidx $\mathbf{m}=0$ means

$$
\sum_{j=0}^{p} \sum_{\nu=1}^{n_{j}} m_{j, \nu}^{2}=(p-1) n^{2}=\sum_{\nu=0}^{n_{0}}(p-1) n m_{0, \nu}
$$

the Fuchs relation (6.16) is valid for any $k$. Then it follows from Lemma 6.1 that the Riemann scheme of the operator $P_{k}\left(c_{1}, \ldots, c_{k}\right)=P\left(k-1, c_{k}\right) P(k-$ 
$\left.2, c_{k-1}\right) \cdots P\left(0, c_{1}\right)$ equals

$$
\left\{\begin{array}{cc}
x=c_{0}=\infty & x=c_{j}(j=1, \ldots, p) \\
{\left[\lambda_{0,1}\right]_{\left(k m_{0,1}\right)}} & {\left[\lambda_{j, 1}\right]_{\left(k m_{j, 1}\right)}} \\
\vdots & \vdots \\
{\left[\lambda_{0, n_{1}}\right]_{\left(k m_{0, n_{1}}\right)}} & {\left[\lambda_{j, n_{j}}\right]_{\left(k m_{j, n_{j}}\right)}}
\end{array}\right\}
$$

and it contain an independent accessory parameters in the coefficient of $\nu n$-th order term of $P_{k}\left(c_{1}, \ldots, c_{k}\right)$ for $\nu=0, \ldots, k-1$ because for the proof of this statement we may assume $\lambda_{j, \nu}$ are generic under the Fuchs relation.

Note that

$$
N_{\nu}(k \mathbf{m})= \begin{cases}1 & (\nu \equiv n-1 \quad \bmod n) \\ -1 & (\nu \equiv 0 \quad \bmod n), \\ 0 & (\nu \neq \equiv 0, n-1 \bmod n)\end{cases}
$$

for $\nu=1, \ldots, k n-1$ because

$$
\widetilde{k \mathbf{m}}=\left\{\begin{aligned}
\{2 i, 2 i, 2 i, 2 i ; i=1,2, \ldots, k\} \quad & \text { if } \mathbf{m} \text { is of type } \tilde{D}_{4}, \\
\{n i, n i, n i, n i-2, n i-3, \ldots, n i-n+1 ; i=1,2, \ldots, k\} & \text { if } \mathbf{m} \text { is of type } \tilde{E}_{6}, \tilde{E}_{7} \text { or } \tilde{E}_{8}
\end{aligned}\right.
$$

under the notation (8.2) and 8.17). Then the operator $P_{k}\left(c_{1}, \ldots, c_{k}\right)$ shows that when we inductively determine the coefficients of the operator with the Riemann scheme 8.22 ) as in the proof of Theorem 8.6. we have a new accessory parameter in the coefficient of the $((k-j) n)$-th order term and then the conditions for the coefficients of the $((k-j) n-1)$-th order term are overdetermined but they are automatically compatible for $j=1, \ldots, k-1$.

Thus we can conclude that the operators of the normal form with the Riemann scheme 8.22$)$ are $P_{k}\left(c_{1}, \ldots, c_{k}\right)$, which are always reducible.

Proposition 8.12. Let $k$ be a positive integer and let $\mathbf{m}$ be an indivisible $(p+1)$ tuple of partitions of $n$. Suppose $k \mathbf{m}$ is realizable and $\mathrm{idx} \mathbf{m}<0$. Then any Fuchsian differential equation with the Riemann scheme (8.22) is always irreducible if $\lambda_{j, \nu}$ is generic under the Fuchs relation

$$
\sum_{j=0}^{p} \sum_{\nu=1}^{n_{j}} m_{j, \nu} \lambda_{j, \nu}=\operatorname{ord} \mathbf{m}-k \frac{\mathrm{idx} \mathbf{m}}{2} .
$$

Proof. The above Fuchs relation follows from (6.32) with the identities ord $k \mathbf{m}=$ $k$ ord $\mathbf{m}$ and idx $k \mathbf{m}=k^{2}$ idx $\mathbf{m}$.

Suppose $P u=0$ is reducible. Then Remark 6.17 ii) says that there exist $\mathbf{m}^{\prime}$, $\mathbf{m}^{\prime \prime} \in \mathcal{P}$ such that $k \mathbf{m}=\mathbf{m}^{\prime}+\mathbf{m}^{\prime \prime}$ and $0<\operatorname{ord} \mathbf{m}^{\prime}<k$ ord $\mathbf{m}$ and $\left|\left\{\lambda_{\mathbf{m}^{\prime}}\right\}\right| \in$ $\{0,-1,-2, \ldots\}$. Suppose $\lambda_{j, \nu}$ are generic under 8.23$)$. Then the condition $\left|\left\{\lambda_{\mathbf{m}^{\prime}}\right\}\right| \in$ $\mathbb{Z}$ implies $\mathbf{m}^{\prime}=\ell \mathbf{m}$ with a positive integer satisfying $\ell<k$ and

$$
\begin{aligned}
\left|\left\{\lambda_{\ell \mathbf{m}}\right\}\right| & =\sum_{j=0}^{p} \sum_{\nu=1}^{n_{j}} \ell m_{j, \nu} \lambda_{j, \nu}-\operatorname{ord} \ell \mathbf{m}+\ell^{2} \mathrm{idx} \mathbf{m} \\
& =\ell\left(\operatorname{ord} \mathbf{m}-k \frac{\mathrm{idx} \mathbf{m}}{2}\right)-\ell \operatorname{ord} \mathbf{m}+\ell^{2} \mathrm{idx} \mathbf{m} \\
& =\ell(\ell-k) \mathrm{idx} \mathbf{m}>0 .
\end{aligned}
$$

Hence $\left|\left\{\lambda_{\mathbf{m}^{\prime}}\right\}\right|>0$. 
8.4. Universal model. Now we have a main result in $\$$ \& which assures the existence of Fuchsian differential operators with given spectral types.

Theorem 8.13. Fix a tuple $\mathbf{m}=\left(m_{j, \nu}\right)_{0 \leq j \leq p} \in \mathcal{P}_{p+1}^{(n)}$.

i) Under the notation in Definitions 6.10, 6.16 and 7.6, the tuple $\mathbf{m}$ is realizable if and only if there exists a non-negative integer $K$ such that $\partial_{\max }^{i} \mathbf{m}$ are well-defined for $i=1, \ldots, K$ and

$$
\begin{aligned}
& \operatorname{ord} \mathbf{m}>\operatorname{ord} \partial_{\max } \mathbf{m}>\operatorname{ord} \partial_{\max }^{2} \mathbf{m}>\cdots>\operatorname{ord} \partial_{\max }^{K} \mathbf{m}, \\
& d_{\max }\left(\partial_{\max }^{K} \mathbf{m}\right)=2 \operatorname{ord} \partial_{\max }^{K} \mathbf{m} \quad \text { or } \quad d_{\max }\left(\partial_{\max }^{K} \mathbf{m}\right) \leq 0 .
\end{aligned}
$$

ii) Fix complex numbers $\lambda_{j, \nu}$. If there exists an irreducible Fuchsian operator with the Riemann scheme (6.15) such that it is locally non-degenerate (cf. Definition 11.8), then $\mathbf{m}$ is irreducibly realizable.

Here we note that if $P$ is irreducible and $\mathbf{m}$ is rigid, $P$ is locally non-degenerate (cf. Definition 11.8).

Hereafter in this theorem we assume $\mathbf{m}$ is realizable.

iii) $\mathbf{m}$ is irreducibly realizable if and only if $\mathbf{m}$ is indivisible or $\operatorname{idx} \mathbf{m}<0$.

iv) There exists a universal model $P_{\mathbf{m}} u=0$ associated with $\mathbf{m}$ which has the following property.

Namely, $P_{\mathbf{m}}$ is the Fuchsian differential operator of the form

$$
\begin{aligned}
& P_{\mathbf{m}}=\left(\prod_{j=1}^{p}\left(x-c_{j}\right)^{n}\right) \frac{d^{n}}{d x^{n}}+a_{n-1}(x) \frac{d^{n-1}}{d x^{n-1}}+\cdots+a_{1}(x) \frac{d}{d x}+a_{0}(x), \\
& a_{j}(x) \in \mathbb{C}\left[\lambda_{j, \nu}, g_{1}, \ldots, g_{N}\right]
\end{aligned}
$$

such that $P_{\mathbf{m}}$ has regular singularities at $p+1$ fixed points $x=c_{0}=\infty, c_{1}, \ldots, c_{p}$ and the Riemann scheme of $P_{\mathbf{m}}$ equals (6.15) for any $g_{i} \in \mathbb{C}$ and $\lambda_{j, \nu} \in \mathbb{C}$ under the Fuchs relation (6.16). Moreover the coefficients $a_{j}(x)$ are polynomials of $x, \lambda_{j, \nu}$ and $g_{i}$ with the degree at most $(p-1) n+j$ for $j=0, \ldots, n$, respectively. Here $g_{i}$ are called accessory parameters and we call $P_{\mathbf{m}}$ the universal operator of type $\mathbf{m}$.

The non-negative integer $N$ will be denoted by Ridx $\mathbf{m}$ and given by

$$
N=\operatorname{Ridx} \mathbf{m}:= \begin{cases}0 & (\operatorname{idx} \mathbf{m}>0) \\ \operatorname{gcd} \mathbf{m} & (\text { idx } \mathbf{m}=0), \\ \operatorname{Pidx} \mathbf{m} & (\operatorname{idx} \mathbf{m}<0)\end{cases}
$$

Put $\overline{\mathbf{m}}=\left(\bar{m}_{j, \nu}\right)_{\substack{0 \leq j \leq p \\ 1 \leq \nu \leq n_{j}}}:=\partial_{\max }^{K} \mathbf{m}$ with the non-negative integer $K$ given in $\left.\mathrm{i}\right)$.

When idx $\mathbf{m} \leq 0$, we define

$$
\begin{aligned}
q_{\ell}^{0} & :=\#\left\{i ; \sum_{\nu=1}^{\bar{n}_{0}} \max \left\{\bar{m}_{0, \nu}-i, 0\right\} \geq \operatorname{ord} \overline{\mathbf{m}}-\ell, i \geq 0\right\}, \\
I_{\mathbf{m}} & :=\left\{(j, \nu) \in \mathbb{Z}^{2} ; q_{\nu}^{0} \leq j \leq q_{\nu}^{0}+N_{\nu}-1,1 \leq \nu \leq \operatorname{ord} \overline{\mathbf{m}}-1\right\} .
\end{aligned}
$$

When idx $\mathbf{m}>0$, we put $I_{\mathbf{m}}=\emptyset$.

Then $\# I_{\mathbf{m}}=\operatorname{Ridx} \mathbf{m}$ and we can define $I_{i}$ such that $I_{\mathbf{m}}=\left\{I_{i} ; i=1, \ldots, N\right\}$ and $g_{i}$ satisfy (8.13) by putting $g_{I_{i}}=g_{i}$ for $i=1, \ldots, N$.

v) Retain the notation in Definition 7.11. If $\lambda_{j, \nu} \in \mathbb{C}$ satisfy

$$
\left\{\begin{array}{l}
\sum_{j=0}^{p} \lambda(k)_{j, \ell(k)_{j}+\delta_{j, j_{o}}\left(\nu_{o}-\ell(k)_{j}\right)} \\
\notin\left\{0,-1,-2,-3, \ldots, m(k)_{j_{o}, \ell(k)_{j_{o}}}-m(k)_{j_{o}, \nu_{o}}-d(k)+2\right\} \\
\quad \text { for any } k=0, \ldots, K-1 \text { and }\left(j_{0}, \nu_{o}\right) \text { satisfying } \\
m(k)_{j_{o}, \nu_{o}} \geq m(k)_{j_{o}, \ell(k)_{j_{o}}}-d(k)+2,
\end{array}\right.
$$


any Fuchsian differential operator $P$ of the normal form which has the Riemann scheme (6.15) belongs to $P_{\mathbf{m}}$ with a suitable $\left(g_{1}, \ldots, g_{N}\right) \in \mathbb{C}^{N}$.

$$
\left\{\begin{array}{l}
\text { If } \mathbf{m} \text { is a scalar multiple of a fundamental tuple or simply reducible, } \\
8.27 \text { is always valid for any } \lambda_{j, \nu} .
\end{array}\right.
$$

$$
\left\{\begin{array}{l}
\text { Fix } \lambda_{j, \nu} \in \mathbb{C} \text {. Suppose there is an irreducible Fuchsian differential } \\
\text { operator with the Riemann scheme (6.15) such that the operator is } \\
\text { locally non-degenerate or } K \leq 1 \text {, then (8.27) is valid. }
\end{array}\right.
$$

Suppose $\mathbf{m}$ is monotone. Under the notation in \$9.1, the condition (8.27) is equivalent to

$$
\begin{aligned}
& (\Lambda(\lambda) \mid \alpha)+1 \notin\left\{0,-1, \ldots, 2-\left(\alpha \mid \alpha_{\mathbf{m}}\right)\right\} \\
& \quad \text { for any } \alpha \in \Delta(\mathbf{m}) \text { satisfying }\left(\alpha \mid \alpha_{\mathbf{m}}\right)>1 .
\end{aligned}
$$

Example 7.5 gives a Fuchsian differential operator with the rigid spectral type $21,21,21,21$ which doesn't belong to the corresponding universal operator.

The fundamental tuple and the simply reducible tuple are defined as follows.

Definition 8.14. i) (fundamental tuple) An irreducibly realizable tuple $\mathbf{m} \in \mathcal{P}$ is called fundamental if ord $\mathbf{m}=1$ or $d_{\max }(\mathbf{m}) \leq 0$.

For an irreducibly realizable tuple $\mathbf{m} \in \mathcal{P}$, there exists a non-negative integer $K$ such that $\partial_{\max }^{K} \mathbf{m}$ is fundamental and satisfies $(8.24)$. Then we call $\partial_{\max }^{K} \mathbf{m}$ is a fundamental tuple corresponding to $\mathbf{m}$ and define $f \mathbf{m}:=\partial_{\max }^{K} \mathbf{m}$.

ii) (simply reducible tuple) A tuple $\mathbf{m}$ is simply reducible if there exists a positive integer $K$ satisfying (8.24) and ord $\partial_{\max }^{K} \mathbf{m}=\operatorname{ord} \mathbf{m}-K$.

Proof of Theorem 8.19. i) We have proved that $\mathbf{m}$ is realizable if $d_{\max }(\mathbf{m}) \leq 0$. Note that the condition $d_{\max }(\mathbf{m})=2$ ord $\mathbf{m}$ is equivalent to the fact that $s \mathbf{m}$ is trivial. Hence Theorem 7.9 proves the claim.

iv) Now we use the notation in Definition 7.11. The existence of the universal operator is clear if $s \mathbf{m}$ is trivial. If $d_{\max }(\mathbf{m}) \leq 0$, Theorem 8.6 and Proposition 8.11 with Corollary 8.3 assure the existence of the universal operator $P_{\mathbf{m}}$ claimed in iii). Hence iii) is valid for the tuple $\mathbf{m}(K)$ and we have a universal operator $P_{K}$ with the Riemann scheme $\left\{\lambda(K)_{\mathbf{m}(K)}\right\}$.

The universal operator $P_{k}$ with the Riemann scheme $\left\{\lambda(k)_{\mathbf{m}(k)}\right\}$ are inductively obtained by applying $\partial_{\ell(k)}$ to the universal operator $P_{k+1}$ with the Riemann scheme $\left\{\lambda(k+1)_{\mathbf{m}(k+1)}\right\}$ for $k=K-1, K-2, \ldots, 0$. Since the claims in iii) such as (8.13) are kept by the operation $\partial_{\ell(k)}$, we have iv).

iii) Note that $\mathbf{m}$ is irreducibly realizable if $\mathbf{m}$ is indivisible (cf. Remark 6.17 ii)). Hence suppose $\mathbf{m}$ is not indivisible. Put $k=\operatorname{gcd} \mathbf{m}$ and $\mathbf{m}=k \mathbf{m}^{\prime}$. Then idx $\mathbf{m}=k^{2}$ idx $\mathbf{m}^{\prime}$.

If idx $\mathbf{m}>0$, then idx $\mathbf{m}>2$ and the inequality (7.19) in Lemma 7.3 implies that $\mathbf{m}$ is not irreducibly realizable. If $\operatorname{idx} \mathbf{m}<0$, Proposition 8.12 assures that $\mathbf{m}$ is irreducibly realizable.

Suppose idx $\mathbf{m}=0$. Then the universal operator $P_{\mathbf{m}}$ has $k$ accessory parameters. Using the argument in the first part of the proof of Proposition 8.11, we can construct a Fuchsian differential operator $\tilde{P}_{\mathbf{m}}$ with the Riemann scheme $\left\{\lambda_{\mathbf{m}}\right\}$. Since $\tilde{P}_{\mathbf{m}}$ is a product of $k$ copies of the universal operator $P_{\overline{\mathbf{m}}}$ and it has $k$ accessory parameters, the operator $P_{\mathbf{m}}$ coincides with the reducible operator $\tilde{P}_{\mathbf{m}}$ and hence $\mathbf{m}$ is not irreducibly realizable.

v) Fix $\lambda_{j, \nu} \in \mathbb{C}$. Let $P$ be a Fuchsian differential operator with the Riemann scheme $\left\{\lambda_{\mathbf{m}}\right\}$. Suppose $P$ is of the normal form.

Theorem 8.6 and Proposition 8.11 assure that $P$ belongs to $P_{\mathbf{m}}$ if $K=0$. 
Theorem 7.2 proves that if $\partial_{\max }^{k} P$ has the Riemann scheme $\left\{\lambda(k)_{\mathbf{m}(k)}\right\}$ and 8.27) is valid, then $\partial_{\max }^{k+1} P=\partial_{\ell(k)} \partial_{\max }^{k} P$ is well-defined and has the Riemann scheme $\left\{\lambda(k+1)_{\mathbf{m}(k+1)}\right\}$ for $k=0, \ldots, K-1$ and hence it follows from (7.26) that $P$ belongs to the universal operator $P_{\mathbf{m}}$ because $\partial_{\max }^{K} P$ belongs to the universal operator $P_{\mathbf{m}(K)}$.

If $\mathbf{m}$ is simply reducible, $d(k)=1$ and therefore (8.27) is valid because $m(k)_{j, \nu} \leq$ $m(k)_{j, \ell(k)_{\nu}}<m(k)_{j, \ell(k)_{\nu}}-d(k)+2$ for $j=0, \ldots, p$ and $\nu=1, \ldots, n_{j}$ and $k=$ $0, \ldots, K-1$.

The equivalence of the conditions (8.27) and (8.30) follows from the argument in 89.1 , Proposition 9.9 and Theorem 12.13.

ii) Suppose there exists an irreducible operator $P$ with the Riemann scheme (6.15). Let $\mathbf{M}=\left(M_{0}, \ldots, M_{p}\right)$ be the tuple of monodromy generators of the equation $P u=0$ and put $\mathbf{M}(0)=\mathbf{M}$. Let $\mathbf{M}(k+1)$ be the tuple of matrices applying the operations in 11.1 to $\mathbf{M}(k)$ corresponding to the operations $\partial_{\ell(k)}$ for $k=0,1,2, \ldots$.

Comparing the operations on $\mathbf{M}(k)$ and $\partial_{\ell(k)}$, we can conclude that there exists a non-negative integer $K$ satisfying the claim in i). In fact Theorem 11.3 proves that $\mathbf{M}(k)$ are irreducible, which assures that the conditions (7.6) and (7.7) corresponding to the operations $\partial_{\ell(k)}$ are always valid (cf. Corollary 12.12). Therefore $\mathbf{m}$ is realizable and moreover we can conclude that (8.29) implies (8.27). If idx $\mathbf{m}$ is divisible and idx $\mathbf{m}=0$, then $P_{\mathbf{m}}$ is reducible for any fixed parameters $\lambda_{j, \nu}$ and $g_{i}$. Hence $\mathbf{m}$ is irreducibly realizable.

Remark 8.15. i) The uniqueness of the universal operator in Theorem 8.13 is obvious. But it is not valid in the case of systems of Schlesinger canonical form (cf. Example 11.2).

ii) The assumption that $P u=0$ is locally non-degenerate seems to be not necessary in Theorem 8.13 ii) and (8.29). When $K=1$, this is clear from the proof of the theorem. For example, the rigid irreducible operator with the spectral type $31,31,31,31,31$ belongs to the universal operator of type $211,31,31,31,31$.

8.5. Simply reducible spectral type. In this subsection we characterize the tuples of the simply reducible spectral type.

Proposition 8.16. i) A realizable tuple $\mathbf{m} \in \mathcal{P}^{(n)}$ satisfying $m_{0, \nu}=1$ for $\nu=$ $1, \ldots, n$ is simply reducible if $\mathbf{m}$ is not fundamental.

ii) The simply reducible rigid tuple corresponds to the tuple in Simpson's list (cf. s15.2) or it is isomorphic to 21111,222,33.

iii) Suppose $\mathbf{m} \in \mathcal{P}_{p+1}$ is not fundamental. Then $\mathbf{m}$ satisfies the condition $N_{\nu}(\mathbf{m}) \geq 0$ for $\nu=2, \ldots$, ord $\mathbf{m}-1$ in Definition 8.1 if and only if $\mathbf{m}$ is realizable and simply reducible.

iv) Let $\mathbf{m} \in \mathcal{P}_{p+1}$ be a realizable monotone tuple. Suppose $\mathbf{m}$ is not fundamental. Then under the notation in $\$ 9.1, \mathbf{m}$ is simply reducible if and only if

$$
\left(\alpha \mid \alpha_{\mathbf{m}}\right)=1 \quad(\forall \alpha \in \Delta(\mathbf{m})),
$$

namely $[\Delta(\mathbf{m})]=1^{\# \Delta(\mathbf{m})}($ cf. Remark $9.11 \mathrm{ii})$ ).

Proof. i) The claim is obvious from the definition.

ii) Let $\mathbf{m}^{\prime}$ be a simply reducible rigid tuple. We have only to prove that $\mathbf{m}=$ $\partial_{\max } \mathbf{m}^{\prime}$ is in the Simpson's list or $21111,222,33$ and $\operatorname{ord} \mathbf{m}^{\prime}=\operatorname{ord} \mathbf{m}+1$ and $d_{\max }(\mathbf{m})=1$, then $\mathbf{m}^{\prime}$ is in Simpson's list or 21111,222, 33. The condition ord $\mathbf{m}^{\prime}=$ ord $\mathbf{m}+1$ implies $\mathbf{m} \in \mathcal{P}_{3}$. We may assume $\mathbf{m}$ is monotone and $\mathbf{m}^{\prime}=\partial_{\ell_{0}, \ell_{1}, \ell_{2}} \mathbf{m}$. The condition ord $\mathbf{m}^{\prime}=$ ord $\mathbf{m}+1$ also implies

$$
\left(m_{0,1}-m_{0, \ell_{0}}\right)+\left(m_{1,1}-m_{1, \ell_{0}}\right)+\left(m_{2,1}-m_{2, \ell_{0}}\right)=2 .
$$


Since $\partial_{\max } \mathbf{m}^{\prime}=\mathbf{m}$, we have $m_{j, \ell_{j}} \geq m_{j, 1}-1$ for $j=0,1,2$. Hence there exists an integer $k$ with $0 \leq k \leq 2$ such that $m_{j, \ell_{j}}=m_{j, 1}-1+\delta_{j, k}$ for $j=0,1,2$. Then the following claims are easy, which assures the proposition.

If $\mathbf{m}=11,11,11, \mathbf{m}^{\prime}$ is isomorphic to $1^{3}, 1^{3}, 21$.

If $\mathbf{m}=1^{3}, 1^{3}, 21, \mathbf{m}^{\prime}$ is isomorphic to $1^{4}, 1^{4}, 31$ or $1^{4}, 211,22$.

If $\mathbf{m}=1^{n}, 1^{n}, n-11$ with $n \geq 4, \mathbf{m}^{\prime}=1^{n+1}, 1^{n+1}, n 1$.

If $\mathbf{m}=1^{2 n}, n n-11, n n$ with $n \geq 2, \mathbf{m}^{\prime}=1^{2 n+1}, n n 1, n+1 n$.

If $\mathbf{m}=1^{5}, 221,32$, then $\mathbf{m}^{\prime}=1^{6}, 33,321$ or $1^{6}, 222,42$ or $21111,222,33$.

If $\mathbf{m}=1^{2 n+1}, n+1 n, n n 1$ with $n \geq 3, \mathbf{m}^{\prime}=1^{2 n+2}, n+1 n+1, n+1 n 1$.

If $\mathbf{m}=1^{6}, 222,42$ or $\mathbf{m}=21111,222,33, \mathbf{m}^{\prime}$ doesn't exists.

iii) Note that Theorem 8.6 assures that the condition $N_{\nu}(\mathbf{m}) \geq 0$ for $\nu=$ $1, \ldots$, ord $\mathbf{m}-1$ implies that $\mathbf{m}$ is realizable.

We may assume $\mathbf{m} \in \mathcal{P}_{p+1}^{(n)}$ is standard. Put $d=m_{0,1}+\cdots+m_{p, 1}-(p-1) n>0$ and $\mathbf{m}^{\prime}=\partial_{\max } \mathbf{m}$. Then $m_{j, \nu}^{\prime}=m_{j, \nu}-\delta_{\nu, 1} d$ for $j=0, \ldots, p$ and $\nu \geq 1$. Under the notation in Definition 8.1 the operation $\partial_{\max }$ transforms the sets

$$
\mathfrak{m}_{j}:=\left\{\widetilde{m}_{j, k} ; k=0,1,2, \ldots \text { and } \tilde{m}_{j, k}>0\right\}
$$

into

$$
\mathfrak{m}_{j}^{\prime}=\left\{\widetilde{m}_{j, k}-\min \left\{d, m_{j, 1}-k\right\} ; k=0, \ldots, \max \left\{m_{j, 1}-d, m_{j, 2}-1\right\}\right\},
$$

respectively because $\widetilde{m}_{j, i}=\sum_{\nu} \max \left\{m_{j, \nu}-i, 0\right\}$. Therefore $N_{\nu}\left(\mathbf{m}^{\prime}\right) \leq N_{\nu}(\mathbf{m})$ for $\nu=1, \ldots, n-d-1=$ ord $\mathbf{m}^{\prime}-1$. Here we note that

$$
\sum_{\nu=1}^{n-1} N_{\nu}(\mathbf{m})=\sum_{\nu=1}^{n-d-1} N_{\nu}\left(\mathbf{m}^{\prime}\right)=\operatorname{Pidx} \mathbf{m} .
$$

Hence $N_{\nu}(\mathbf{m}) \geq 0$ for $\nu=1, \ldots, n-1$ if and only if $N_{\nu}\left(\mathbf{m}^{\prime}\right)=N_{\nu}(\mathbf{m})$ for $\nu=$ $1, \ldots,(n-d)-1$ and moreover $N_{\nu}(\mathbf{m})=0$ for $\nu=n-d, \ldots, n-1$. Note that the condition that $N_{\nu}\left(\mathbf{m}^{\prime}\right)=N_{\nu}(\mathbf{m})$ for $\nu=1, \ldots,(n-d)-1$ equals

$$
m_{j, 1}-d \geq m_{j, 2}-1 \text { for } j=0, \ldots, p
$$

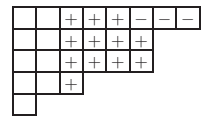

This is easy to see by using a Young diagram. For example, when $\{8,6,6,3,1\}=$ $\left\{m_{0,1}, m_{0,2}, m_{0,3}, m_{0,4}, m_{0,5}\right\}$ is a partition of $n=24$, the corresponding Young diagram is as above and then $\widetilde{m}_{0,2}$ equals 15 , namely, the number of boxes with the $\operatorname{sign}+$ or - . Moreover when $d=3$, the boxes with the sign - are deleted by $\partial_{\max }$ and the number $\widetilde{m}_{0,2}$ changes into 12 . In this case $m_{0}=\{24,19,15,11,8,5,2,1\}$ and $m_{0}^{\prime}=\{21,16,12,8,5,2\}$.

If $d \geq 2$, then $1 \in \mathfrak{m}_{j}$ for $j=0, \ldots, p$ and therefore $N_{n-2}(\mathbf{m})-N_{n-1}(\mathbf{m})=2$, which means $N_{n-1}(\mathbf{m}) \neq 0$ or $N_{n-2}(\mathbf{m}) \neq 0$. When $d=1$, we have $N_{\nu}(\mathbf{m})=$ $N_{\nu}\left(\mathbf{m}^{\prime}\right)$ for $\nu=1, \ldots, n-2$ and $N_{n-1}(\mathbf{m})=0$. Thus we have the claim.

iv) The claim follows from Proposition 9.9.

Example 8.17. We show the simply reducible tuples with index 0 whose fundamental tuple is of type $\tilde{D}_{4}, \tilde{E}_{6}, \tilde{E}_{7}$ or $\tilde{E}_{8}$ (cf. Example 8.8).

$\tilde{D}_{4}: 21,21,21,111 \quad 22,22,31,211 \quad 22,31,31,1111$

$\tilde{E}_{6}: 211,211,1111221,221,2111221,311,11111222,222,3111222,321,2211$

$222,411,111111322,331,2221 \quad 332,431,2222 \quad 333,441,3222$

$\tilde{E}_{7}: 11111,2111,32$ 111111,2211, 42 21111,2211,33 111111,3111,33

$22111,2221,43 \quad 1111111,2221,52 \quad 22211,2222,53 \quad 11111111,2222,62$

$32111,2222,4422211,3221,53$

$\tilde{E}_{8}: 1111111,322,43 \quad 11111111,332,53 \quad 2111111,332,44 \quad 11111111,422,44$

2211111,333,54 111111111,333, 63 2221111,433,55 2222111,443, 65

$3222111,444,662222211,444,75 \quad 2222211,543,66 \quad 2222221,553,76$ 
$222222,653,77$

In general, we have the following proposition.

Proposition 8.18. There exist only a finite number of standard and simply reducible tuples with any fixed non-positive index of rigidity.

Proof. First note that $\mathbf{m} \in \mathcal{P}_{p+1}$ if $d_{\max }(\mathbf{m})=1$ and ord $\mathbf{m}>3$ and $\partial_{\max } \mathbf{m} \in$ $\mathcal{P}_{p+1}$. Since there exist only finite basic tuples with any fixed index of rigidity (cf. Remark 9.15), we have only to prove the non-existence of the infinite sequence

$$
\mathbf{m}(0) \stackrel{\partial_{\max }}{\longleftarrow} \mathbf{m}(1) \stackrel{\partial_{\max }}{\longleftarrow} \cdots \cdots \stackrel{\partial_{\max }}{\longleftarrow} \mathbf{m}(k) \stackrel{\partial_{\max }}{\longleftarrow} \mathbf{m}(k+1) \stackrel{\partial_{\max }}{\longleftarrow} \cdots
$$

such that $d_{\max }(\mathbf{m}(k))=1$ for $k \geq 1$ and $\operatorname{idx} \mathbf{m}(0) \leq 0$.

Put

$$
\begin{aligned}
\bar{m}(k)_{j} & =\max _{\nu}\left\{m(k)_{j, \nu}\right\}, \\
a(k)_{j} & =\#\left\{\nu ; m(k)_{j, \nu}=\bar{m}(k)_{j}\right\}, \\
b(k)_{j} & = \begin{cases}\#\left\{\nu ; m(k)_{j, \nu}=\bar{m}(k)_{j}-1\right\} & \left(\bar{m}(k)_{j}>1\right), \\
\infty & \left(\bar{m}(k)_{j}=1\right) .\end{cases}
\end{aligned}
$$

The assumption $d_{\max }(\mathbf{m}(k))=d_{\max }(\mathbf{m}(k+1))=1$ implies that there exist indices $0 \leq j_{k}<j_{k}^{\prime}$ such that

$$
\left(a(k+1)_{j}, b(k+1)_{j}\right)= \begin{cases}\left(a(k)_{j}+1, b(k)_{j}-1\right) & \left(j=j_{k} \text { or } j_{k}^{\prime}\right), \\ \left(1, a(k)_{j}-1\right) & \left(j \neq j_{k} \text { and } j_{k}^{\prime}\right)\end{cases}
$$

and

$$
\bar{m}(k)_{0}+\cdots+\bar{m}(k)_{p}=(p-1) \operatorname{ord} \mathbf{m}(k)+1 \quad(p \gg 1)
$$

for $k=1,2, \ldots$ Since $a(k+1)_{j}+b(k+1)_{j} \leq a(k)_{j}+b(k)_{j}$, there exists a positive integer $N$ such that $a(k+1)_{j}+b(k+1)_{j}=a(k)_{j}+b(k)_{j}$ for $k \geq N$, which means

$$
b(k)_{j} \begin{cases}>0 & \left(j=j_{k} \text { or } j_{k}^{\prime}\right) \\ =0 & \left(j \neq j_{k} \text { and } j_{k}^{\prime}\right) .\end{cases}
$$

Putting $\left(a_{j}, b_{j}\right)=\left(a(N)_{j}, b(N)_{j}\right)$, we may assume $b_{0} \geq b_{1}>b_{2}=b_{3}=\cdots=0$ and $a_{2} \geq a_{3} \geq \cdots$. Moreover we may assume $j_{N+1}^{\prime} \leq 3$, which means $a_{j}=1$ for $j \geq 4$. Then the relations (8.33) and (8.35) for $k=N, N+1, N+2$ and $N+3$ prove that $\left(\left(a_{0}, b_{0}\right), \cdots,\left(a_{3}, b_{3}\right)\right)$ is one of the followings:

$$
\begin{aligned}
& \left(\left(a_{0}, \infty\right),\left(a_{1}, \infty\right),(1,0),(1,0)\right), \\
& \left(\left(a_{0}, \infty\right),(1,1),(2,0),(1,0)\right), \\
& ((2,2),(1,1),(4,0),(1,0)),((1,3),(3,1),(2,0),(1,0)), \\
& ((1,2),(2,1),(3,0),(1,0)), \\
& ((1,1),(1,1),(2,0),(2,0)) .
\end{aligned}
$$

In fact if $b_{1}>1, a_{2}=a_{3}=1$ and we have (8.36). Thus we may assume $b_{1}=1$. If $b_{0}=\infty, a_{3}=1$ and we have (8.37). If $b_{0}=b_{1}=1$, we have easily (8.40). Thus we may moreover assume $b_{1}=1<b_{0}<\infty$ and $a_{3}=1$. In this case the integers $j_{k}^{\prime \prime}$ satisfying $b(k)_{j_{k}^{\prime \prime}}=0$ and $0 \leq j_{k}^{\prime \prime} \leq 2$ for $k \geq N$ are uniquely determined and we have easily (8.38) or 8.39 .

Put $n=\operatorname{ord} \mathbf{m}(N)$. We may suppose $\mathbf{m}(N)$ is standard. Let $p$ be an integer such that $m_{j, 0}<n$ if and only if $j \leq p$. Note that $p \geq 2$. Then if $\mathbf{m}(N)$ satisfies 8.36) (resp. (8.37)), 8.34 implies $\mathbf{m}(N)=1^{n}, 1^{n}, n-11$ (resp. $1^{n}, m m-11, m m$ or $\left.1^{n}, m+1 m, m m 1\right)$ and $\mathbf{m}(N)$ is rigid. 
Suppose one of (8.38)- 8.40). Then it is easy to check that $\mathbf{m}(N)$ doesn't satisfy 8.34). For example, suppose 8.39). Then $3 m_{0,1}-2 \leq n, 3 m_{1,1}-1 \leq n$ and $3 m_{2,1} \leq n$ and we have $m_{0,1}+m_{1,1}+m_{2,1} \leq\left[\frac{n+2}{3}\right]+\left[\frac{n+1}{3}\right]+\left[\frac{n}{3}\right]=n$, which contradicts to 8.34). The relations $\left[\frac{n+2}{4}\right]+\left[\frac{n}{2}\right]+\left[\frac{n}{4}\right] \leq n$ and $2\left[\frac{n+1}{2}\right]+2\left[\frac{n}{2}\right]=2 n$ assure the same conclusion in the other cases.

\section{A KaC-Moody root System}

9.1. Correspondence with a Kac-Moody root system. We review a KacMoody root system to describe the combinatorial structure of middle convolutions on the spectral types. Its relation to Deligne-Simpson problem is first clarified by [CB].

Let

$$
I:=\{0,(j, \nu) ; j=0,1, \ldots, \nu=1,2, \ldots\} .
$$

be a set of indices and let $\mathfrak{h}$ be an infinite dimensional real vector space with the set of basis $\Pi$, where

$$
\Pi=\left\{\alpha_{i} ; i \in I\right\}=\left\{\alpha_{0}, \alpha_{j, \nu} ; j=0,1,2, \ldots, \nu=1,2, \ldots\right\} .
$$

Put

$$
\begin{aligned}
I^{\prime} & :=I \backslash\{0\}, \quad \Pi^{\prime}:=\Pi \backslash\left\{\alpha_{0}\right\}, \\
Q & :=\sum_{\alpha \in \Pi} \mathbb{Z} \alpha \supset Q_{+}:=\sum_{\alpha \in \Pi} \mathbb{Z}_{\geq 0} \alpha .
\end{aligned}
$$

We define an indefinite symmetric bilinear form on $\mathfrak{h}$ by

$$
\begin{aligned}
(\alpha \mid \alpha) & =2 \quad(\alpha \in \Pi), \\
\left(\alpha_{0} \mid \alpha_{j, \nu}\right) & =-\delta_{\nu, 1}, \\
\left(\alpha_{i, \mu} \mid \alpha_{j, \nu}\right) & = \begin{cases}0 & (i \neq j \text { or }|\mu-\nu|>1), \\
-1 & (i=j \text { and }|\mu-\nu|=1) .\end{cases}
\end{aligned}
$$

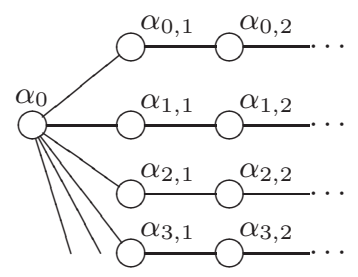

The element of $\Pi$ is called the simple root of a Kac-Moody root system and the Weyl group $W_{\infty}$ of this Kac-Moody root system is generated by the simple reflections $s_{i}$ with $i \in I$. Here the reflection with respect to an element $\alpha \in \mathfrak{h}$ satisfying $(\alpha \mid \alpha) \neq 0$ is the linear transformation

$$
s_{\alpha}: \mathfrak{h} \ni x \mapsto x-2 \frac{(x \mid \alpha)}{(\alpha \mid \alpha)} \alpha \in \mathfrak{h}
$$

and

$$
s_{i}=s_{\alpha_{i}} \text { for } i \in I \text {. }
$$

In particular $s_{i}(x)=x-\left(\alpha_{i} \mid x\right) \alpha_{i}$ for $i \in I$ and the subgroup of $W_{\infty}$ generated by $s_{i}$ for $i \in I \backslash\{0\}$ is denoted by $W_{\infty}^{\prime}$.

The Kac-Moody root system is determined by the set of simple roots $\Pi$ and its Weyl group $W_{\infty}$ and it is denoted by $\left(\Pi, W_{\infty}\right)$.

Denoting $\sigma\left(\alpha_{0}\right)=\alpha_{0}$ and $\sigma\left(\alpha_{j, \nu}\right)=\alpha_{\sigma(j), \nu}$ for $\sigma \in \mathfrak{S}_{\infty}$, we put

$$
\widetilde{W}_{\infty}:=\mathfrak{S}_{\infty} \ltimes W_{\infty},
$$

which is an automorphism group of the root system.

Remark $9.1([\mathrm{Kd}])$. The set $\Delta^{\text {re }}$ of real roots equals the $W_{\infty}$-orbit of $\Pi$, which also equals $W_{\infty} \alpha_{0}$. Denoting

$$
B:=\left\{\beta \in Q_{+} ; \operatorname{supp} \beta \text { is connected and }(\beta, \alpha) \leq 0 \quad(\forall \alpha \in \Pi)\right\},
$$


the set of positive imaginary roots $\Delta_{+}^{i m}$ equals $W_{\infty} B$. Here

$$
\operatorname{supp} \beta:=\left\{\alpha \in \Pi ; n_{\alpha} \neq 0\right\} \text { if } \beta=\sum_{\alpha \in \Pi} n_{\alpha} \alpha
$$

The set $\Delta$ of roots equals $\Delta^{r e} \cup \Delta^{i m}$ by denoting $\Delta_{-}^{i m}=-\Delta_{+}^{i m}$ and $\Delta^{i m}=$ $\Delta_{+}^{i m} \cup \Delta_{-}^{i m}$. Put $\Delta_{+}=\Delta \cap Q_{+}, \Delta_{-}=-\Delta_{+}, \Delta_{+}^{r e}=\Delta^{r e} \cap Q_{+}$and $\Delta_{-}^{r e}=-\Delta_{+}^{r e}$. Then $\Delta=\Delta_{+} \cup \Delta_{-}, \Delta_{+}^{i m} \subset \Delta_{+}$and $\Delta^{r e}=\Delta_{+}^{r e} \cup \Delta_{-}^{r e}$. The root in $\Delta$ is called positive if and only if $\alpha \in Q_{+}$.

A subset $L \subset \Pi$ is called connected if the decomposition $L_{1} \cup L_{2}=L$ with $L_{1} \neq \emptyset$ and $L_{2} \neq \emptyset$ always implies the existence of $v_{j} \in L_{j}$ satisfying $\left(v_{1} \mid v_{2}\right) \neq 0$. Note that $\operatorname{supp} \alpha \ni \alpha_{0}$ for $\alpha \in \Delta^{i m}$.

The subset $L$ is called classical if it corresponds to the classical Dynkin diagram, which is equivalent to the condition that the group generated by the reflections with respect to the elements in $L$ is a finite group.

The connected subset $L$ is called affine if it corresponds to affine Dynkin diagram and in our case it corresponds to $\tilde{D}_{4}$ or $\tilde{E}_{6}$ or $\tilde{E}_{7}$ or $\tilde{E}_{8}$ with the following Dynkin diagram, respectively.

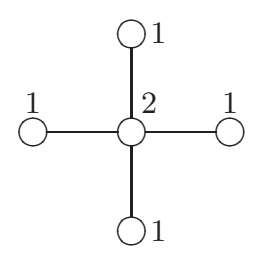

$11,11,11,11$

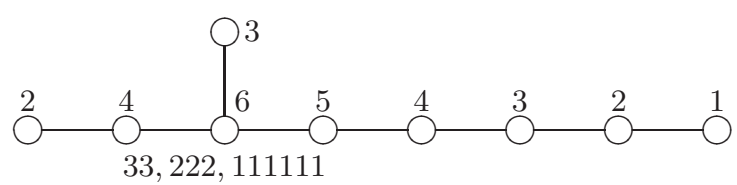

$33,222,111111$

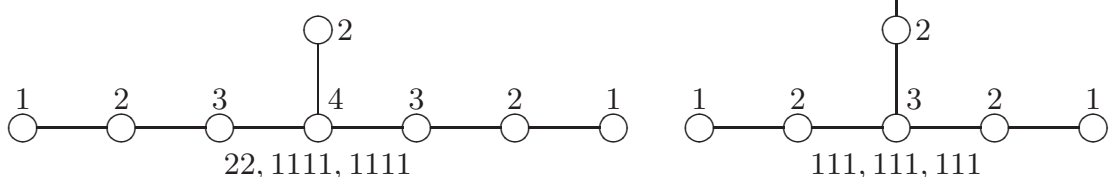

Here the circle correspond to simple roots and the numbers attached to simple roots are the coefficients $n$ and $n_{j, \nu}$ in the expression (9.15) of a root $\alpha$.

For a tuple of partitions $\mathbf{m}=\left(m_{j, \nu}\right)_{j \geq 0, \nu \geq 1} \in \mathcal{P}^{(n)}$, we define

$$
\begin{aligned}
n_{j, \nu} & :=m_{j, \nu+1}+m_{j, \nu+2}+\cdots, \\
\alpha_{\mathbf{m}} & :=n \alpha_{0}+\sum_{j=0}^{\infty} \sum_{\nu=1}^{\infty} n_{j, \nu} \alpha_{j, \nu} \in Q_{+}, \\
\kappa\left(\alpha_{\mathbf{m}}\right) & :=\mathbf{m} .
\end{aligned}
$$

As is given in $\mathrm{O} 6$, Proposition 2.22] we have

Proposition 9.2. i) $\quad \operatorname{idx}\left(\mathbf{m}, \mathbf{m}^{\prime}\right)=\left(\alpha_{\mathbf{m}} \mid \alpha_{\mathbf{m}^{\prime}}\right)$.

ii) Given $i \in I$, we have $\alpha_{\mathbf{m}^{\prime}}=s_{i}\left(\alpha_{\mathbf{m}}\right)$ with

$$
\mathbf{m}^{\prime}= \begin{cases}\partial \mathbf{m} & (i=0), \\ \left(m_{0,1} \ldots, m_{j, 1} \ldots m_{j, \nu+1}^{\nu} \underset{\nu+1}{\nu}\right. & (i=(j, \nu)) .\end{cases}
$$


Moreover for $\ell=\left(\ell_{0}, \ell_{1}, \ldots\right) \in \mathbb{Z}_{>0}^{\infty}$ satisfying $\ell_{\nu}=1$ for $\nu \gg 1$ we have

$$
\begin{gathered}
\alpha_{\ell}:=\alpha_{\mathbf{1}_{\ell}}=\alpha_{0}+\sum_{j=0}^{\infty} \sum_{\nu=1}^{\ell_{j}-1} \alpha_{j, \nu}=\left(\prod_{j \geq 0} s_{j, \ell_{j}-1} \cdots s_{j, 2} s_{j, 1}\right)\left(\alpha_{0}\right), \\
\alpha_{\partial_{\ell}(\mathbf{m})}=s_{\alpha_{\ell}}\left(\alpha_{\mathbf{m}}\right)=\alpha_{\mathbf{m}}-2 \frac{\left(\alpha_{\mathbf{m}} \mid \alpha_{\ell}\right)}{\left(\alpha_{\ell} \mid \alpha_{\ell}\right)} \alpha_{\ell}=\alpha_{\mathbf{m}}-\left(\alpha_{\mathbf{m}} \mid \alpha_{\ell}\right) \alpha_{\ell} .
\end{gathered}
$$

Note that

$$
\begin{gathered}
\alpha=n \alpha_{0}+\sum_{j \geq 0} \sum_{\nu \geq 1} n_{j, \nu} \alpha_{j, \nu} \in \Delta^{+} \text {with } n>0 \\
\Rightarrow n \geq n_{j, 1} \geq n_{j, 2} \geq \cdots \quad(j=0,1, \ldots) .
\end{gathered}
$$

In fact, for a sufficiently large $K \in \mathbb{Z}_{>0}$, we have $n_{j, \mu}=0$ for $\mu \geq K$ and

$$
s_{\alpha_{j, \nu}+\alpha_{j, \nu+1}+\cdots+\alpha_{j, K}} \alpha=\alpha+\left(n_{j, \nu-1}-n_{j, \nu}\right)\left(\alpha_{j, \nu}+\alpha_{j, \nu+1}+\cdots+\alpha_{j, K}\right) \in \Delta^{+}
$$

for $\alpha \in \Delta_{+}$in (9.15), which means $n_{j, \nu-1} \geq n_{j, \nu}$ for $\nu \geq 1$. Here we put $n_{j, 0}=n$ and $\alpha_{j, 0}=\alpha_{0}$. Hence for $\alpha \in \Delta_{+}$with $\operatorname{supp} \alpha \ni \alpha_{0}$, there uniquely exists $\mathbf{m} \in \mathcal{P}$ satisfying $\alpha=\alpha_{\mathbf{m}}$.

It follows from (9.14) that under the identification $\mathcal{P} \subset Q_{+}$with $(9.12)$, our operation $\partial_{\ell}$ corresponds to the reflection with respect to the root $\alpha_{\ell}$. Moreover the rigid (resp. indivisible realizable) tuple of partitions corresponds to the positive real root (resp. indivisible positive root) whose support contains $\alpha_{0}$, which were

\begin{tabular}{|c|c|}
\hline $\mathcal{P}$ & Kac-Moody root system \\
\hline $\mathbf{m}$ & $\alpha_{\mathbf{m}}(\mathrm{cf} .(9.12))$ \\
\hline m : monotone & $\alpha \in Q_{+}:(\alpha \mid \beta) \leq 0 \quad\left(\forall \beta \in \Pi^{\prime}\right)$ \\
\hline $\mathbf{m}$ : realizable & $\alpha \in \bar{\Delta}_{+}$ \\
\hline $\mathbf{m}$ : rigid & $\alpha \in \Delta_{+}^{r e}: \operatorname{supp} \alpha \ni \alpha_{0}$ \\
\hline m : monotone and fundamental & $\alpha \in Q_{+}: \alpha=\alpha_{0}$ or $(\alpha \mid \beta) \leq 0 \quad(\forall \beta \in \Pi)$ \\
\hline m : irreducibly realizable & $\begin{array}{c}\alpha \in \Delta_{+}, \quad \operatorname{supp} \alpha \ni \alpha_{0} \\
\text { indivisible or }(\alpha \mid \alpha)<0\end{array}$ \\
\hline $\mathbf{m}$ : basic and monotone & $\begin{aligned} \alpha \in Q_{+}: & (\alpha \mid \beta) \leq 0 \quad(\forall \beta \in \Pi) \\
& \text { indivisible }\end{aligned}$ \\
\hline $\mathbf{m}$ : simply reducible and monotone & $\begin{array}{c}\alpha \in \Delta_{+}:\left(\alpha \mid \alpha_{\mathbf{m}}\right)=1 \quad(\forall \alpha \in \Delta(\mathbf{m})) \\
\alpha_{0} \in \Delta(\mathbf{m}), \quad(\alpha \mid \beta) \leq 0 \quad\left(\forall \beta \in \Pi^{\prime}\right)\end{array}$ \\
\hline ord $\mathbf{m}$ & $n_{0}: \alpha=n_{0} \alpha_{0}+\sum_{i, \nu} n_{i, \nu} \alpha_{i, \nu}$ \\
\hline $\operatorname{idx}\left(\mathbf{m}, \mathbf{m}^{\prime}\right)$ & $\left(\alpha_{\mathbf{m}} \mid \alpha_{\mathbf{m}^{\prime}}\right)$ \\
\hline idx $\mathbf{m}$ & $\left(\alpha_{\mathbf{m}} \mid \alpha_{\mathbf{m}}\right)$ \\
\hline$d_{\ell}(\mathbf{m}) \quad(\mathrm{cf} .(7.24))$ & $\left(\alpha_{\ell} \mid \alpha_{\mathbf{m}}\right) \quad(\mathrm{cf.}(9.13))$ \\
\hline $\operatorname{Pidx} \mathbf{m}+\operatorname{Pidx} \mathbf{m}^{\prime}=\operatorname{Pidx}\left(\mathbf{m}+\mathbf{m}^{\prime}\right)$ & $\left(\alpha_{\mathbf{m}} \mid \alpha_{\mathbf{m}^{\prime}}\right)=-1$ \\
\hline$(\nu, \nu+1) \in G_{j} \subset S_{\infty}^{\prime} \quad($ cf. $(6.30))$ & $s_{j, \nu} \in W_{\infty}^{\prime}($ cf. 9.7$\left.)\right)$ \\
\hline$H \simeq \mathfrak{S}_{\infty}($ cf. 6.30$\left.)\right)$ & $\mathfrak{S}_{\infty}$ in 9.8 \\
\hline$\partial_{1}$ & $s_{0}$ \\
\hline
\end{tabular}
first established by $\mathrm{CB}$ in the case of Fuchsian systems of Schlesinger canonical form (cf. $\mathrm{O} 6$ ).

The corresponding objects with this identification are as follows, which will be clear in this subsection. Some of them are also explained in 06 . 


\begin{tabular}{|c|c|}
\hline$\partial_{\ell}$ & $s_{\alpha_{\ell}}$ (cf. (9.13)) \\
\hline$\left\langle\partial_{\mathbf{1}}, S_{\infty}\right\rangle$ & $\widetilde{W}_{\infty}($ cf. $(9.8))$ \\
\hline$\left\{\lambda_{\mathbf{m}}\right\}$ & $\left(\Lambda(\lambda), \alpha_{\mathbf{m}}\right)(\mathrm{cf.}$ (9.18) $)$ \\
\hline$\left|\left\{\lambda_{\mathbf{m}}\right\}\right|$ & $\left(\Lambda(\lambda)+\frac{1}{2} \alpha_{\mathbf{m}} \mid \alpha_{\mathbf{m}}\right)$ \\
\hline $\operatorname{Ad}\left(\left(x-c_{j}\right)^{\tau}\right)$ & $+\tau \Lambda_{0, j}^{0}(\mathrm{cf.}(9.18))$ \\
\hline
\end{tabular}

Here

$$
\bar{\Delta}_{+}:=\left\{k \alpha ; \alpha \in \Delta_{+}, k \in \mathbb{Z}_{>0}, \operatorname{supp} \alpha \ni \alpha_{0}\right\},
$$

$\Delta(\mathbf{m}) \subset \Delta_{+}^{r e}$ is given in 9.30 ) and $\Lambda(\lambda) \in \overline{\mathfrak{h}}_{p}$ is defined as follows.

Definition 9.3. Fix a positive integer $p$ which may be $\infty$. Put

$$
I_{p}:=\{0,(j, \nu) ; j=0,1, \ldots, p, \nu=1,2, \ldots\} \subset I
$$

for a positive integer $p$ and $I_{\infty}=I$.

Let $\mathfrak{h}_{p}$ be the $\mathbb{R}$-vector space of finite linear combinations the elements of $\Pi_{p}:=$ $\left\{\alpha_{i} ; i \in \Pi_{p}\right\}$ and let $\mathfrak{h}_{p}^{\vee}$ be the $\mathbb{C}$-vector space whose elements are linear combinations of infinite or finite elements of $\Pi_{p}$, which is identified with $\Pi_{i \in I_{p}} \mathbb{C} \alpha_{i}$ and contains $\mathfrak{h}_{p}$.

The element $\Lambda \in \mathfrak{h}_{p}^{\vee}$ naturally defines a linear form of $\mathfrak{h}_{p}$ by $(\Lambda \mid \cdot)$ and the group $\widetilde{W}_{\infty}$ acts on $\mathfrak{h}_{p}^{\vee}$. If $p=\infty$, we assume that the element $\Lambda=\xi_{0} \alpha_{0}+\sum \xi_{j, \nu} \alpha_{j, \nu} \in \mathfrak{h}_{\infty}^{\vee}$ always satisfies $\xi_{j, 1}=0$ for sufficiently large $j \in \mathbb{Z}_{\geq 0}$. Hence we have naturally $\mathfrak{h}_{p}^{\vee} \subset \mathfrak{h}_{p+1}^{\vee}$ and $\mathfrak{h}_{\infty}^{\vee}=\bigcup_{j \geq 0} \mathfrak{h}_{j}^{\vee}$.

Define the elements of $\mathfrak{h}_{p}^{\vee}$ :

$$
\begin{aligned}
\Lambda_{0} & :=\frac{1}{2} \alpha_{0}+\frac{1}{2} \sum_{j=0}^{p} \sum_{\nu=1}^{\infty}(1-\nu) \alpha_{j, \nu} \\
\Lambda_{j, \nu} & :=\sum_{i=\nu+1}^{\infty}(i-\nu) \alpha_{j, i} \quad(j=0, \ldots, p, \nu=0,1,2, \ldots) \\
\Lambda^{0} & :=2 \Lambda_{0}-2 \Lambda_{0,0}=\alpha_{0}+\sum_{\nu=1}^{\infty}(1+\nu) \alpha_{0, \nu}+\sum_{j=1}^{p} \sum_{\nu=1}^{\infty}(1-\nu) \alpha_{j, \nu}, \\
\Lambda_{j, k}^{0} & :=\Lambda_{j, 0}-\Lambda_{k, 0}=\sum_{\nu=1}^{\infty} \nu\left(\alpha_{k, \nu}-\alpha_{j, \nu}\right) \quad(0 \leq j<k \leq p), \\
\Lambda(\lambda) & :=-\Lambda_{0}-\sum_{j=0}^{p} \sum_{\nu=1}^{\infty}\left(\sum_{i=1}^{\nu} \lambda_{j, i}\right) \alpha_{j, \nu} \\
& =-\Lambda_{0}+\sum_{j=0}^{p} \sum_{\nu=1}^{\infty} \lambda_{j, \nu}\left(\Lambda_{j, \nu-1}-\Lambda_{j, \nu}\right) .
\end{aligned}
$$

Under the above definition we have

$$
\begin{aligned}
\left(\Lambda^{0} \mid \alpha\right) & =\left(\Lambda_{j, k}^{0} \mid \alpha\right)=0 \quad\left(\forall \alpha \in \Pi_{p}\right), \\
\left(\Lambda_{j, \nu} \mid \alpha_{j^{\prime}, \nu^{\prime}}\right) & =\delta_{j, j^{\prime}} \delta_{\nu, \nu^{\prime}} \quad\left(j, j^{\prime}=0,1, \ldots, \nu, \nu^{\prime}=1,2, \ldots\right) \\
\left(\Lambda_{0} \mid \alpha_{i}\right) & =\left(\Lambda_{j, 0} \mid \alpha_{i}\right)=\delta_{i, 0} \quad\left(\forall i \in \Pi_{p}\right), \\
\left|\left\{\lambda_{\mathbf{m}}\right\}\right| & =\left(\Lambda(\lambda)+\frac{1}{2} \alpha_{\mathbf{m}} \mid \alpha_{\mathbf{m}}\right),
\end{aligned}
$$




$$
\begin{aligned}
s_{0}(\Lambda(\lambda))= & -\left(\sum_{j=0}^{p} \lambda_{j, 1}-1\right) \alpha_{0}+\Lambda(\lambda) \\
= & -\mu \Lambda^{0}-\Lambda_{0}-\sum_{\nu=1}^{\infty}\left(\sum_{i=1}^{\nu}\left(\lambda_{0, i}-\left(1+\delta_{i, 0}\right) \mu\right) \alpha_{0, \nu}\right. \\
& -\sum_{j=1}^{p} \sum_{\nu=1}^{\infty}\left(\sum_{i=1}^{\nu}\left(\lambda_{j, i}+\left(1-\delta_{i, 0}\right) \mu\right)\right) \alpha_{j, \nu}
\end{aligned}
$$

with $\mu=\sum_{j=0}^{p} \lambda_{j, 1}-1$.

We identify the elements of $\mathfrak{h}_{p}^{\vee}$ if their difference are in $\mathbb{C} \Lambda^{0}$, namely, consider them in $\overline{\mathfrak{h}}_{p}:=\mathfrak{h}_{p}^{\vee} / \mathbb{C} \Lambda^{0}$. Then the elements have the unique representatives in $\mathfrak{h}_{p}^{\vee}$ whose coefficients of $\alpha_{0}$ equal $-\frac{1}{2}$.

Remark 9.4. i) If $p<\infty$, we have

$$
\left\{\Lambda \in \mathfrak{h}_{p}^{\vee} ;(\Lambda \mid \alpha)=0 \quad\left(\forall \alpha \in \Pi_{p}\right)\right\}=\mathbb{C} \Lambda^{0}+\sum_{j=1}^{p} \mathbb{C} \Lambda_{0, j}^{0} .
$$

ii) The invariance of the bilinear form ( $\mid$ ) under the Weyl group $W_{\infty}$ proves (7.15)

iii) The addition given in Theorem 7.2 i) corresponds to the map $\Lambda(\lambda) \mapsto \Lambda(\lambda)+$ $\tau \Lambda_{0, j}^{0}$ with $\tau \in \mathbb{C}$ and $1 \leq j \leq p$.

iii) Combining the action of $s_{j, \nu}$ on $\mathfrak{h}_{p}^{\vee}$ with that of $s_{0}$, we have

$$
\Lambda\left(\lambda^{\prime}\right)-s_{\alpha_{\ell}} \Lambda(\lambda) \in \mathbb{C} \Lambda^{0} \text { and } \alpha_{\mathbf{m}^{\prime}}=s_{\alpha_{\ell}} \alpha_{\mathbf{m}} \text { when }\left\{\lambda_{\mathbf{m}^{\prime}}^{\prime}\right\}=\partial_{\ell}\left\{\lambda_{\mathbf{m}}\right\}
$$

because of $(7.29)$ and $(9.23)$.

Thus we have the following theorem.

Theorem 9.5. Under the above notation we have the commutative diagram

$\left\{P_{\mathbf{m}}:\right.$ Fuchsian differential operators with $\left.\left\{\lambda_{\mathbf{m}}\right\}\right\} \rightarrow\left\{\left(\Lambda(\lambda), \alpha_{\mathbf{m}}\right) ; \alpha_{\mathbf{m}} \in \bar{\Delta}_{+}\right\}$

$$
\downarrow \text { fractional operations } \quad \circlearrowright \quad \downarrow W_{\infty} \text {-action, }+\tau \Lambda_{0, j}^{0}
$$

$\left\{P_{\mathbf{m}}:\right.$ Fuchsian differential operators with $\left.\left\{\lambda_{\mathbf{m}}\right\}\right\} \rightarrow\left\{\left(\Lambda(\lambda), \alpha_{\mathbf{m}}\right) ; \alpha_{\mathbf{m}} \in \bar{\Delta}_{+}\right\}$.

Here the defining domain of $w \in W_{\infty}$ is $\left\{\alpha \in \bar{\Delta}_{+} ; w \alpha \in \bar{\Delta}_{+}\right\}$.

Proof. Let $T_{i}$ denote the corresponding operation on $\left\{\left(P_{\mathbf{m}},\left\{\lambda_{\mathbf{m}}\right\}\right)\right\}$ for $s_{i} \in W_{\infty}$ with $i \in I$. Then $T_{0}$ corresponds to $\partial_{1}$ and when $i \in I^{\prime}, T_{i}$ is naturally defined and it doesn't change $P_{\mathbf{m}}$. The fractional transformation of the Fuchsian operators and their Riemann schemes corresponding to an element $w \in W_{\infty}$ is defined through the expression of $w$ by the product of simple reflections. It is clear that the transformation of their Riemann schemes do not depend on the expression.

Let $i \in I$ and $j \in I$. We want to prove that $\left(T_{i} T_{j}\right)^{k}=i d$ if $\left(s_{i} s_{j}\right)^{k}=i d$ for a non-negative integer $k$. Note that $T_{i}^{2}=i d$ and the addition commutes with $T_{i}$. Since $T_{i}=i d$ if $i \in I^{\prime}$, we have only to prove that $\left(T_{j, 1} T_{0}\right)^{3}=i d$. Moreover Proposition 7.7 assures that we may assume $j=0$.

Let $P$ be a Fuchsian differential operator with the Riemann scheme (6.15). Applying suitable additions to $P$, we may assume $\lambda_{j, 1}=0$ for $j \geq 1$ to prove $\left(T_{0,1} T_{0}\right)^{3} P=P$ and then this easily follows from the definition of $\partial_{\mathbf{1}}$ (cf. (7.25)) 
and the relation

$$
\begin{aligned}
& \left\{\begin{array}{cc}
\infty & c_{j}(1 \leq j \leq p) \\
{\left[\lambda_{0,1}\right]_{\left(m_{0,1}\right)}} & {[0]_{\left(m_{j, 1}\right)}} \\
{\left[\lambda_{0,2}\right]_{\left(m_{0,2}\right)}} & {\left[\lambda_{j, 2}\right]_{\left(m_{j, 2}\right)}} \\
{\left[\lambda_{0, \nu}\right]_{\left(m_{0, \nu}\right)}} & {\left[\lambda_{j, \nu}\right]_{\left(m_{j, \nu}\right)}}
\end{array}\right\} \quad\left(d=m_{0,1}+\cdots+m_{p, 1}-\text { ord } \mathbf{m}\right) \\
& \underset{\partial^{1-\lambda_{0,1}}}{\stackrel{T_{0,1} T_{0}}{\longrightarrow}}\left\{\begin{array}{cc}
\infty & c_{j}(1 \leq j \leq p) \\
{\left[\lambda_{0,2}-\lambda_{0,1}+1\right]_{\left(m_{0,1}\right)}} & {[0]_{\left(m_{j, 1}-d\right)}} \\
{\left[-\lambda_{0,1}+2\right]_{\left(m_{0,2}-d\right)}} & {\left[\lambda_{j, 2}+\lambda_{0,1}-1\right]_{\left(m_{j, 2}\right)}} \\
{\left[\lambda_{0, \nu}-\lambda_{0,1}+1\right]_{\left(m_{0, \nu}\right)}} & {\left[\lambda_{j, \nu}+\lambda_{0,1}-1\right]_{\left(m_{j, \nu}\right)}}
\end{array}\right\} \\
& \underset{\partial^{\lambda_{0,1}-\lambda_{0,2}}}{\stackrel{T_{0,1} T_{0}}{\longrightarrow}}\left\{\begin{array}{cc}
\infty & c_{j}(1 \leq j \leq p) \\
{\left[-\lambda_{0,2}+2\right]_{\left(m_{0,1}-d\right)}} & {[0]_{\left(m_{j, 1}+m_{0,1}-m_{0,2}-d\right)}} \\
{\left[\lambda_{0,1}-\lambda_{0,2}+1\right]_{\left(m_{0,1}\right)}} & {\left[\lambda_{j, 2}+\lambda_{0,2}-1\right]_{\left(m_{j, 2}\right)}} \\
{\left[\lambda_{0, \nu}-\lambda_{0,2}+1\right]_{\left(m_{0, \nu}\right)}} & {\left[\lambda_{j, \nu}+\lambda_{0,2}-1\right]_{\left(m_{j, \nu}\right)}}
\end{array}\right\} \\
& \underset{\partial^{\lambda_{0,2}-1}}{\stackrel{T_{0,1} T_{0}}{\longrightarrow}}\left\{\begin{array}{cc}
\infty & c_{j}(1 \leq j \leq p) \\
{\left[\lambda_{0,1}\right]_{\left(m_{0,1}\right)}} & {[0]_{\left(m_{j, 1}\right)}} \\
{\left[\lambda_{0,2}\right]_{\left(m_{0,2}\right)}} & {\left[\lambda_{j, 2}\right]_{\left(m_{j, 2}\right)}} \\
{\left[\lambda_{0, \nu}\right]_{\left(m_{0, \nu}\right)}} & {\left[\lambda_{j, \nu}\right]_{\left(m_{j, \nu}\right)}}
\end{array}\right\}
\end{aligned}
$$

and $\left(T_{0,1} T_{0}\right)^{3} P \in \mathbb{C}[x] \operatorname{Ad}\left(\partial^{\lambda_{0,2}-1}\right) \circ \operatorname{Ad}\left(\partial^{\lambda_{0,2}-\lambda_{0,1}}\right) \circ \operatorname{Ad}\left(\partial^{1-\lambda_{0,1}}\right) \mathrm{R} P=\mathbb{C}[x] \mathrm{R} P$.

Definition 9.6. For an element $w$ of the Weyl group $W_{\infty}$ we put

$$
\Delta(w):=\Delta_{+}^{r e} \cap w^{-1} \Delta_{-}^{r e} .
$$

If $w=s_{i_{1}} s_{i_{2}} \cdots s_{i_{k}}$ with $i_{\nu} \in I$ is the minimal expression of $w$ as the products of simple reflections which means $k$ is minimal by definition, we have

$$
\Delta(w)=\left\{\alpha_{i_{k}}, s_{i_{k}}\left(\alpha_{i_{k-1}}\right), s_{i_{k}} s_{i_{k-1}}\left(\alpha_{i_{k-2}}\right), \ldots, s_{i_{k}} \cdots s_{i_{2}}\left(\alpha_{i_{1}}\right)\right\} .
$$

The number of the elements of $\Delta(w)$ equals the number of the simple reflections in the minimal expression of $w$, which is called the length of $w$ and denoted by $L(w)$. The equality (9.27) follows from the following lemma.

Lemma 9.7. Fix $w \in W_{\infty}$ and $i \in I$. If $\alpha_{i} \in \Delta(w)$, there exists a minimal expression $w=s_{i_{1}^{\prime}} s_{i_{2}^{\prime}} \cdots s_{i_{k}^{\prime}}$ with $s_{i_{k}^{\prime}}=s_{i}$ and $L\left(w s_{i}\right)=L(w)-1$ and $\Delta\left(w s_{i}\right)=$ $s_{i}\left(\Delta(w) \backslash\left\{\alpha_{i}\right\}\right)$. If $\alpha_{i} \notin \Delta(w), L\left(w s_{i}\right)=L(w)+1$ and $\Delta\left(w s_{i}\right)=s_{i} \Delta(w) \cup\left\{\alpha_{i}\right\}$. Moreover if $v \in W_{\infty}$ satisfies $\Delta(v)=\Delta(w)$, then $v=w$.

Proof. The proof is standard as in the case of classical root system, which follows from the fact that the condition $\alpha_{i}=s_{i_{k}} \cdots s_{i_{\ell+1}}\left(\alpha_{i_{\ell}}\right)$ implies

$$
s_{i}=s_{i_{k}} \cdots s_{i_{\ell+1}} s_{i_{\ell}} s_{i_{\ell+1}} \cdots s_{i_{k}}
$$

and then $w=w s_{i} s_{i}=s_{i_{1}} \cdots s_{i_{\ell-1}} s_{i_{\ell+1}} \cdots s_{i_{k}} s_{i}$.

Definition 9.8. For $\alpha \in Q$, put

$$
h(\alpha):=n_{0}+\sum_{j \geq 0} \sum_{\nu \geq 1} n_{j, \nu} \text { if } \alpha=n_{0} \alpha_{0}+\sum_{j \geq 0} \sum_{\nu \geq 1} n_{j, \nu} \alpha_{j, \nu} \in Q .
$$

Suppose $\mathbf{m} \in \mathcal{P}_{p+1}$ is irreducibly realizable. Note that $s f \mathbf{m}$ is the monotone fundamental element determined by $\mathbf{m}$, namely, $\alpha_{s f \mathbf{m}}$ is the unique element of $W \alpha_{\mathbf{m}} \cap\left(B \cup\left\{\alpha_{0}\right\}\right)$. We inductively define $w_{\mathbf{m}} \in W_{\infty}$ satisfying $w_{\mathbf{m}} \alpha_{\mathbf{m}}=\alpha_{s f \mathbf{m}}$. We may assume $w_{\mathbf{m}^{\prime}}$ has already defined if $h\left(\alpha_{\mathbf{m}^{\prime}}\right)<h\left(\alpha_{\mathbf{m}}\right)$. If $\mathbf{m}$ is not monotone, there exists $i \in I \backslash\{0\}$ such that $\left(\alpha_{\mathbf{m}} \mid \alpha_{i}\right)>0$ and then $w_{\mathbf{m}}=w_{\mathbf{m}^{\prime}} s_{i}$ with $\alpha_{\mathbf{m}^{\prime}}=s_{i} \alpha_{\mathbf{m}}$. If $\mathbf{m}$ is monotone and $\mathbf{m} \neq f \mathbf{m}, w_{\mathbf{m}}=w_{\partial \mathbf{m}} s_{0}$. 
We moreover define

$$
\Delta(\mathbf{m}):=\Delta\left(w_{\mathbf{m}}\right) .
$$

Suppose $\mathbf{m}$ is monotone, irreducibly realizable and $\mathbf{m} \neq s f \mathbf{m}$. We define $w_{\mathbf{m}}$ so that there exists $K \in \mathbb{Z}_{>0}$ and $v_{1}, \ldots, v_{K} \in W_{\infty}^{\prime}$ satisfying

$$
\begin{aligned}
w_{\mathbf{m}} & =v_{K} s_{0} \cdots v_{2} s_{0} v_{1} s_{0}, \\
\left(v_{k} s_{0} \cdots v_{1} s_{0} \alpha_{\mathbf{m}} \mid \alpha\right) & \leq 0 \quad(\forall \alpha \in \Pi \backslash\{0\}, k=1, \ldots, K),
\end{aligned}
$$

which uniquely characterizes $w_{\mathbf{m}}$. Note that

$$
v_{k} s_{0} \cdots v_{1} s_{0} \alpha_{\mathbf{m}}=\alpha_{(s \partial)^{k} \mathbf{m}} \quad(k=1, \ldots, K) .
$$

The following proposition gives the correspondence between the reduction of realizable tuples of partitions and the minimal expressions of the elements of the Weyl group.

Proposition 9.9. Definition 9.8 naturally gives the product expression $w_{\mathbf{m}}=$ $s_{i_{1}} \cdots s_{i_{k}}$ with $i_{\nu} \in I \quad(1 \leq \nu \leq k)$.

i) We have

$$
\begin{aligned}
L\left(w_{\mathbf{m}}\right) & =k, \\
\left(\alpha \mid \alpha_{\mathbf{m}}\right) & >0 \quad(\forall \alpha \in \Delta(\mathbf{m})), \\
h\left(\alpha_{\mathbf{m}}\right) & =h\left(\alpha_{s f \mathbf{m}}\right)+\sum_{\alpha \in \Delta(\mathbf{m})}\left(\alpha \mid \alpha_{\mathbf{m}}\right) .
\end{aligned}
$$

Moreover $\alpha_{0} \in \operatorname{supp} \alpha$ for $\alpha \in \Delta(\mathbf{m})$ if $\mathbf{m}$ is monotone.

ii) Suppose $\mathbf{m}$ is monotone and $f \mathbf{m} \neq \mathbf{m}$. Fix maximal integers $\nu_{j}$ such that $m_{j, 1}-d_{\max }(\mathbf{m})<m_{j, \nu_{j}+1}$ for $j=0,1, \ldots$ Then

$$
\begin{aligned}
& \Delta(\mathbf{m})=s_{0}\left(\prod_{\substack{j \geq 0 \\
\nu_{j}>0}} s_{j, 1} \cdots s_{j, \nu_{j}}\right) \Delta(s \partial \mathbf{m}) \cup\left\{\alpha_{0}\right\} \\
& \cup\left\{\alpha_{0}+\alpha_{j, 1}+\cdots+\alpha_{j, \nu} ; 1 \leq \nu \leq \nu_{j} \text { and } j=0,1, \ldots\right\}, \\
& \left(\alpha_{0}+\alpha_{j, 1}+\cdots+\alpha_{j, \nu} \mid \alpha_{\mathbf{m}}\right)=d_{\max }(\mathbf{m})+m_{j, \nu+1}-m_{j, 1} \quad(\nu \geq 0) .
\end{aligned}
$$

iii) Suppose $\mathbf{m}$ is not rigid. Then $\Delta(\mathbf{m})=\left\{\alpha \in \Delta_{+}^{r e} ;\left(\alpha \mid \alpha_{\mathbf{m}}\right)>0\right\}$.

iv) Suppose $\mathbf{m}$ is rigid. Let $\alpha \in \Delta_{+}^{r e}$ satisfying $\left(\alpha \mid \alpha_{\mathbf{m}}\right)>0$ and $s_{\alpha}\left(\alpha_{\mathbf{m}}\right) \in \Delta_{+}$. Then

$$
\begin{cases}\alpha \in \Delta(\mathbf{m}) & \text { if }\left(\alpha \mid \alpha_{\mathbf{m}}\right)>1 \\ \#\left(\left\{\alpha, \alpha_{\mathbf{m}}-\alpha\right\} \cap \Delta(\mathbf{m})\right)=1 & \text { if }\left(\alpha \mid \alpha_{\mathbf{m}}\right)=1 .\end{cases}
$$

Moreover if a root $\gamma \in \Delta(\mathbf{m})$ satisfies $\left(\gamma \mid \alpha_{\mathbf{m}}\right)=1$, then $\alpha_{\mathbf{m}}-\gamma \in \Delta_{+}^{r e}$ and $\alpha_{0} \in$ $\operatorname{supp}\left(\alpha_{\mathbf{m}}-\gamma\right)$.

v) $w_{\mathbf{m}}$ is the unique element with the minimal length satisfying $w_{\mathbf{m}} \alpha_{\mathbf{m}}=\alpha_{s f \mathbf{m}}$.

Proof. Since $h\left(s_{i^{\prime}} \alpha\right)-h(\alpha)=-\left(\alpha_{i^{\prime}} \mid \alpha\right)=\left(s_{i^{\prime}} \alpha_{i^{\prime}} \mid \alpha\right)$, we have

$$
\begin{aligned}
h\left(s_{i_{\ell}^{\prime}} \cdots s_{i_{1}^{\prime}} \alpha\right)-h(\alpha) & =\sum_{\nu=1}^{\ell}\left(h\left(s_{i_{\nu}^{\prime}} \cdots s_{i_{1}^{\prime}} \alpha\right)-h\left(s_{i_{\nu-1}^{\prime}} \cdots s_{i_{1}^{\prime}} \alpha\right)\right) \\
& =\sum_{\nu=1}^{\ell}\left(\alpha_{i_{\nu}^{\prime}} \mid s_{i_{\nu}^{\prime}} \cdots s_{i_{1}^{\prime}} \alpha\right)=\sum_{\nu=1}^{\ell}\left(s_{i_{\ell}^{\prime}} \cdots s_{i_{\nu+1}^{\prime}} \alpha_{i_{\nu}^{\prime}} \mid s_{i_{\ell}^{\prime}} \cdots s_{i_{1}^{\prime}} \alpha\right)
\end{aligned}
$$

for $i^{\prime}, i_{\nu}^{\prime} \in I$ and $\alpha \in \Delta$.

i) We show by the induction on $k$. We may assume $k \geq 1$. Put $w^{\prime}=s_{i_{1}} \cdots s_{i_{k-1}}$ and $\alpha_{\mathbf{m}^{\prime}}=s_{i_{k}} \alpha_{\mathbf{m}}$ and $\alpha(\nu)=s_{i_{k-1}} \cdots s_{i_{\nu+1}} \alpha_{i_{\nu}}$ for $\nu=1, \ldots, k-1$. The hypothesis of the induction assures $L\left(w^{\prime}\right)=k-1, \Delta\left(\mathbf{m}^{\prime}\right)=\{\alpha(1), \ldots, \alpha(k-1)\}$ 
and $\left(\alpha(\nu) \mid \alpha_{\mathbf{m}^{\prime}}\right)>0$ for $\nu=1, \ldots, k-1$. If $L\left(w_{\mathbf{m}}\right) \neq k$, there exists $\ell$ such that $\alpha_{i_{k}}=\alpha(\ell)$ and $w_{\mathbf{m}}=s_{i_{1}} \cdots s_{i_{\ell-1}} s_{i_{\ell+1}} \cdots s_{i_{k-1}}$ is a minimal expression. Then $h\left(\alpha_{\mathbf{m}}\right)-h\left(\alpha_{\mathbf{m}^{\prime}}\right)=-\left(\alpha_{i_{k}} \mid \alpha_{\mathbf{m}^{\prime}}\right)=-\left(\alpha(\ell) \mid \alpha_{\mathbf{m}^{\prime}}\right)<0$, which contradicts to the definition of $w_{\mathbf{m}}$. Hence we have i). Note that (9.34) implies $\operatorname{supp} \alpha \ni \alpha_{0}$ if $\alpha \in \Delta(\mathbf{m})$ and $\mathbf{m}$ is monotone.

ii) The equality (9.36) follows from

$$
\Delta(\partial \mathbf{m}) \cap \sum_{\alpha \in \Pi \backslash\{0\}} \mathbb{Z} \alpha=\left\{\alpha_{j, 1}+\cdots+\alpha_{j, \nu_{j}} ; \nu=1, \ldots, \nu_{j}, \nu_{j}>0 \text { and } j=0,1, \ldots\right\}
$$

because $\Delta(\mathbf{m})=s_{0} \Delta(\partial \mathbf{m}) \cup\left\{\alpha_{0}\right\}$ and $\left(\prod_{\nu_{j}>0} s_{j, \nu_{j}} \cdots s_{j, 1}\right) \alpha_{\partial \mathbf{m}}=\alpha_{s \partial \mathbf{m}}$.

The equality 9.37$)$ is clear because $\left(\alpha_{0} \mid \alpha_{\mathbf{m}}\right)=d_{\mathbf{1}}(\mathbf{m})=d_{\max }(\mathbf{m})$ and $\left(\alpha_{j, \nu} \mid \alpha_{\mathbf{m}}\right)=$ $m_{j, \nu+1}-m_{j, \nu}$.

iii) Note that $\gamma \in \Delta(\mathbf{m})$ satisfies $\left(\gamma \mid \alpha_{\mathbf{m}}\right)>0$.

Put $w_{\nu}=s_{i_{\nu+1}} \cdots s_{i_{k-1}} s_{i_{k}}$ for $\nu=0, \ldots, k$. Then $w_{\mathbf{m}}=w_{0}$ and $\Delta(\mathbf{m})=$ $\left\{w_{\nu}^{-1} \alpha_{i_{\nu}} ; \nu=1, \ldots, k\right\}$. Moreover $w_{\nu^{\prime}} w_{\nu}^{-1} \alpha_{i_{\nu}} \in \Delta_{-}^{r e}$ if and only if $0 \leq \nu^{\prime}<\nu$.

Suppose $\mathbf{m}$ is not rigid. Let $\alpha \in \Delta_{+}^{r e}$ with $\left(\alpha \mid \alpha_{\mathbf{m}}\right)>0$. Since $\left(w_{\mathbf{m}} \alpha \mid \alpha_{\overline{\mathbf{m}}}\right)>0$, $w_{\mathbf{m}} \alpha \in \Delta_{-}^{r e}$. Hence there exists $\nu$ such that $w_{\nu} \alpha \in \Delta_{+}$and $w_{\nu-1} \alpha \in \Delta_{-}$, which implies $w_{\nu} \alpha=\alpha_{i_{\nu}}$ and the claim.

iv) Suppose $\mathbf{m}$ is rigid. Let $\alpha \in \Delta_{+}^{r e}$. Put $\ell=\left(\alpha \mid \alpha_{\mathbf{m}}\right)$. Suppose $\ell>0$ and $\beta:=s_{\alpha} \alpha_{\mathbf{m}} \in \Delta_{+}$. Then $\alpha_{\mathbf{m}}=\ell \alpha+\beta, \alpha_{0}=\ell w_{\mathbf{m}} \alpha+w_{\mathbf{m}} \beta$ and $\left(\beta \mid \alpha_{\mathbf{m}}\right)=$ $\left(\alpha_{\mathbf{m}}-\ell \alpha \mid \alpha_{\mathbf{m}}\right)=2-\ell^{2}$. Hence if $\ell \geq 2, \mathbb{R} \beta \cap \Delta(\mathbf{m})=\emptyset$ and the same argument as in the proof of iii) assures $\alpha \in \Delta(\mathbf{m})$.

Suppose $\ell=1$. There exists $\nu$ such that $w_{\nu} \alpha$ or $w_{\nu} \beta$ equals $\alpha_{i_{\nu}}$. We may assume $w_{\nu}^{-1} \alpha=\alpha_{i_{\nu}}$. Then $\alpha \in \Delta(\mathbf{m})$.

Suppose there exists $w_{\nu^{\prime}} \beta=\alpha_{i_{\nu^{\prime}}}$. We may assume $\nu^{\prime}<\nu$. Then $w_{\nu^{\prime}} \alpha_{\mathbf{m}}=$ $w_{\nu^{\prime}-1} \alpha+w_{\nu^{\prime}-1} \beta \in \Delta_{-}^{r e}$, which contradicts to the definition of $w_{\nu}$. Hence $w_{\nu^{\prime}} \beta=$ $\alpha_{i_{\nu^{\prime}}}$ for $\nu^{\prime}=1, \ldots, k$ and therefore $\beta \notin \Delta(\mathbf{m})$.

Let $\gamma=w_{\nu}^{-1} \alpha_{i_{\nu}} \in \Delta(\mathbf{m})$ and $\left(\gamma \mid \alpha_{\mathbf{m}}\right)=1$. Put $\beta=\alpha_{\mathbf{m}}-\alpha=s_{\alpha} \alpha_{\mathbf{m}}$. Then $w_{\nu-1} \alpha_{\mathbf{m}}=w_{\nu} \beta \in \Delta_{+}^{r e}$. Since $\beta \notin \Delta(\mathbf{m})$, we have $\beta \in \Delta_{+}^{r e}$.

Replacing $\mathbf{m}$ by $s \mathbf{m}$, we may assume $\mathbf{m}$ is monotone to prove $\alpha_{0} \in \operatorname{supp} \beta$. Since $\left(\beta \mid \alpha_{\mathbf{m}}\right)=1$ and $\left(\alpha_{i} \mid \alpha_{\mathbf{m}}\right) \leq 0$ for $i \in I \backslash\{0\}$, we have $\alpha_{0} \in \operatorname{supp} \beta$.

v) The uniqueness of $w_{\mathbf{m}}$ follows from iii) when $\mathbf{m}$ is not rigid. It follows from (9.34), Theorem 17.1 and Corollary 17.3 when $\mathbf{m}$ is rigid.

Corollary 9.10. Let $\mathbf{m}, \mathbf{m}^{\prime}, \mathbf{m}^{\prime \prime} \in \mathcal{P}$ and $k \in \mathbb{Z}_{>0}$ such that

$$
\mathbf{m}=k \mathbf{m}^{\prime}+\mathbf{m}^{\prime \prime} \text {, idx } \mathbf{m}=\mathrm{idx} \mathbf{m}^{\prime \prime} \text { and } \mathbf{m}^{\prime} \text { is rigid. }
$$

Then $\mathbf{m}$ is irreducibly realizable if and only if so is $\mathbf{m}^{\prime \prime}$.

Suppose $\mathbf{m}$ is irreducibly realizable. If idx $\mathbf{m} \leq 0$ or $k>1$, then $\mathbf{m}^{\prime} \in \Delta(\mathbf{m})$. If $\operatorname{idx} \mathbf{m}=2$, then $\left\{\alpha_{\mathbf{m}^{\prime}}, \alpha_{\mathbf{m}^{\prime \prime}}\right\} \cap \Delta(\mathbf{m})=\left\{\alpha_{\mathbf{m}^{\prime}}\right\}$ or $\left\{\alpha_{\mathbf{m}^{\prime \prime}}\right\}$.

Proof. The assumption implies $\left(\alpha_{\mathbf{m}} \mid \alpha_{\mathbf{m}}\right)=2 k^{2}+2 k\left(\alpha_{\mathbf{m}^{\prime}} \mid \alpha_{\mathbf{m}^{\prime \prime}}\right)+\left(\alpha_{\mathbf{m}^{\prime \prime}} \mid \alpha_{\mathbf{m}^{\prime \prime}}\right)$ and hence $\left(\alpha_{\mathbf{m}^{\prime}} \mid \alpha_{\mathbf{m}^{\prime \prime}}\right)=-k$ and $s_{\alpha_{\mathbf{m}^{\prime}}} \alpha_{\mathbf{m}^{\prime \prime}}=\alpha_{\mathbf{m}}$. Thus we have the first claim (cf. Theorem 9.5). The remaining claims follow from Proposition 9.9.

Remark 9.11. i) In general, $\gamma \in \Delta(\mathbf{m})$ does not always imply $s_{\gamma} \alpha_{\mathbf{m}} \in \Delta_{+}$.

Put $\mathbf{m}=32,32,32,32, \mathbf{m}^{\prime}=10,10,10,10$ and $\mathbf{m}^{\prime \prime}=01,01,01,01$. Putting $v=s_{0,1} s_{1,1} s_{2,1} s_{3,1}$, we have $\alpha_{\mathbf{m}^{\prime}}=\alpha_{0}, \alpha_{\mathbf{m}^{\prime \prime}}=v \alpha_{0},\left(\alpha_{\mathbf{m}^{\prime}} \mid \alpha_{\mathbf{m}^{\prime \prime}}\right)=-2, s_{0} \alpha_{\mathbf{m}^{\prime \prime}}=$ $2 \alpha_{\mathbf{m}^{\prime}}+\alpha_{\mathbf{m}^{\prime \prime}}, v s_{0} \alpha_{\mathbf{m}^{\prime \prime}}=\alpha_{0}+2 \alpha_{\mathbf{m}^{\prime \prime}}$ and $s_{0} v s_{0} v \alpha_{0}=s_{0} v s_{0} \alpha_{\mathbf{m}^{\prime \prime}}=3 \alpha_{\mathbf{m}^{\prime}}+2 \alpha_{\mathbf{m}^{\prime \prime}}=\alpha_{\mathbf{m}}$.

Then $\gamma:=s_{0} v \alpha_{0}=2 \alpha_{\mathbf{m}^{\prime}}+\alpha_{\mathbf{m}^{\prime \prime}} \in \Delta(\mathbf{m}),\left(\gamma \mid \alpha_{\mathbf{m}}\right)=\left(s_{0} v \alpha_{\mathbf{m}^{\prime}} \mid s_{0} v s_{0} v \alpha_{\mathbf{m}^{\prime}}\right)=$ $\left(\alpha_{\mathbf{m}^{\prime}} \mid s_{0} v \alpha_{\mathbf{m}^{\prime}}\right)=\left(\alpha_{\mathbf{m}^{\prime}} \mid 2 \alpha_{\mathbf{m}^{\prime}}+\alpha_{\mathbf{m}^{\prime \prime}}\right)=2$ and $s_{\gamma}\left(\alpha_{\mathbf{m}}\right)=\left(3 \alpha_{\mathbf{m}^{\prime}}+2 \alpha_{\mathbf{m}^{\prime \prime}}\right)-2\left(2 \alpha_{\mathbf{m}^{\prime}}+\right.$ $\left.\alpha_{\mathbf{m}^{\prime \prime}}\right)=-\alpha_{\mathbf{m}^{\prime}} \in \Delta_{-}$. 
ii) Define

$$
[\Delta(\mathbf{m})]:=\left\{\left(\alpha \mid \alpha_{\mathbf{m}}\right) ; \alpha \in \Delta(\mathbf{m})\right\} .
$$

Then $[\Delta(\mathbf{m})]$ gives a partition of the non-negative integer $h\left(\alpha_{\mathbf{m}}\right)-h(s f \mathbf{m})$, which we call the type of $\Delta(\mathbf{m})$. It follows from (9.35) that

$$
\# \Delta(\mathbf{m}) \leq h\left(\alpha_{\mathbf{m}}\right)-h(s f \mathbf{m})
$$

for a realizable tuple $\mathbf{m}$ and the equality holds in the above if $\mathbf{m}$ is monotone and simply reducible. Moreover we have

$$
\begin{aligned}
{[\Delta(\mathbf{m})] } & =[\Delta(s \partial \mathbf{m})] \cup\{d(\mathbf{m})\} \cup \bigcup_{j=0}^{p}\left\{m_{j, \nu}-m_{j, 1}-d(\mathbf{m}) \in \mathbb{Z}_{>0} ; \nu>1\right\}, \\
\# \Delta(\mathbf{m}) & =\# \Delta(s \partial \mathbf{m})+\sum_{j=0}^{p}\left(\min \left\{\nu ; m_{j, \nu}>m_{j, 1}-d(\mathbf{m})\right\}-1\right)+1, \\
h(\mathbf{m}) & =h(s f \mathbf{m})+\sum_{i \in[\Delta(\mathbf{m})]} i
\end{aligned}
$$

if $\mathbf{m} \in \mathcal{P}_{p+1}$ is monotone, irreducibly realizable and not fundamental. Here we use the notation in Definitions 6.11, 7.6 and 8.14. For example,

\begin{tabular}{|c|c|c|c|}
\hline type & $\mathbf{m}$ & $h\left(\alpha_{\mathbf{m}}\right)$ & $\# \Delta(\mathbf{m})$ \\
\hline \hline$H_{n}$ & $1^{n}, 1^{n}, n-11$ & $n^{2}+1$ & $n^{2}$ \\
\hline$E O_{2 m}$ & $1^{2 m}, m m, m m-11$ & $2 m^{2}+3 m+1$ & $\left(\begin{array}{c}2 m \\
2\end{array}\right)+4 m$ \\
\hline$E O_{2 m+1}$ & $1^{2 m+1}, m+1 m, m m 1$ & $2 m^{2}+5 m+3$ & $\left(\begin{array}{c}2 m+1 \\
2\end{array}\right)+4 m+2$ \\
\hline$X_{6}$ & $111111,222,42$ & 29 & 28 \\
\hline & $21111,222,33$ & 25 & 24 \\
\hline$P_{n}$ & $n-11, n-11, \ldots \in \mathcal{P}_{n+1}^{(n)}$ & $2 n+1$ & {$[\Delta(\mathbf{m})]: 1^{n+1} \cdot(n-1)$} \\
\hline$P_{4,2 m+1}$ & $m+1 m, m+1 m, m+1 m, m+1 m$ & $6 m+1$ & {$[\Delta(\mathbf{m})]: 1^{4 m} \cdot 2^{m}$} \\
\hline
\end{tabular}

Suppose $\mathbf{m} \in \mathcal{P}_{p+1}$ is basic. We may assume (3.3). Suppose $\left(\alpha_{\mathbf{m}} \mid \alpha_{0}\right)=0$, which is equivalent to $\sum_{j=0}^{p} m_{j, 1}=(p-1)$ ord $\mathbf{m}$. Let $k_{j}$ be positive integers such that

$$
\left(\alpha_{\mathbf{m}} \mid \alpha_{j, \nu}\right)=0 \text { for } 1 \leq \nu<k_{j} \text { and }\left(\alpha_{\mathbf{m}} \mid \alpha_{j, k_{j}}\right)<0,
$$

which is equivalent to $m_{j, 1}=m_{j, 2}=\cdots=m_{j, k_{j}}>m_{j, k_{j}+1}$ for $j=0, \ldots, p$. Then

$$
\sum_{j=0}^{p} \frac{1}{k_{j}} \geq \sum_{j=0}^{p} \frac{m_{j, 1}}{\operatorname{ord} \mathbf{m}}=p-1 \text {. }
$$

If the equality holds in the above, we have $k_{j} \geq 2$ and $m_{j, k_{j}+1}=0$ and therefore $\mathbf{m}$ is of one of the types $\tilde{D}_{4}$ or $\tilde{E}_{6}$ or $\tilde{E}_{7}$ or $\tilde{E}_{8}$. Hence if idx $\mathbf{m}<0$, the set $\left\{k_{j} ; 0 \leq j \leq p, k_{j}>1\right\}$ equals one of the set $\emptyset,\{2\},\{2, \nu\}$ with $2 \leq \nu \leq 5,\{3, \nu\}$ with $3 \leq \nu \leq 5,\{2,2, \nu\}$ with $2 \leq \nu \leq 5$ and $\{2,3, \nu\}$ with $3 \leq \nu \leq 5$. In this case the corresponding Dynkin diagram of $\left\{\alpha_{0}, \alpha_{j, \nu} ; 1 \leq \nu<k_{j}, j=0, \ldots, p\right\}$ is one of the types $A_{\nu}$ with $1 \leq \nu \leq 6, D_{\nu}$ with $4 \leq \nu \leq 7$ and $E_{\nu}$ with $6 \leq \nu \leq 8$. Thus we have the following remark.

Remark 9.12. Suppose a tuple $\mathbf{m} \in \mathcal{P}_{p+1}^{(n)}$ is basic and monotone. The subgroup of $W_{\infty}$ generated by reflections with respect to $\alpha_{\ell}(\mathrm{cf}$. $(9.13))$ which satisfy $\left(\alpha_{\mathbf{m}} \mid \alpha_{\ell}\right)=0$ is infinite if and only if idx $\mathbf{m}=0$.

For a realizable monotone tuple $\mathbf{m} \in \mathcal{P}$, we define

$$
\Pi(\mathbf{m}):=\left\{\alpha_{j, \nu} \in \operatorname{supp} \alpha_{\mathbf{m}} ; m_{j, \nu}=m_{j, \nu+1}\right\} \cup \begin{cases}\left\{\alpha_{0}\right\} & \left(d_{\mathbf{1}}(\mathbf{m})=0\right), \\ \emptyset & \left(d_{\mathbf{1}}(\mathbf{m}) \neq 0\right) .\end{cases}
$$


Note that the condition $\left(\alpha_{\mathbf{m}} \mid \alpha_{\ell}\right)=0$, which is equivalent to say that $\alpha_{\ell}$ is a root of the root space with the fundamental system $\Pi(\mathbf{m})$, means that the corresponding middle convolution $\partial_{\ell}$ keeps the spectral type invariant.

9.2. Fundamental tuples. We will prove some inequalities (9.48) and (9.49) for fundamental tuples which are announced in [O6].

Proposition 9.13. Let $\mathbf{m} \in \mathcal{P}_{p+1} \backslash \mathcal{P}_{p}$ be a fundamental tuple. Then

$$
\begin{aligned}
\operatorname{ord} \mathbf{m} & \leq 3|\operatorname{idx} \mathbf{m}|+6, \\
\operatorname{ord} \mathbf{m} & \leq|\operatorname{idx} \mathbf{m}|+2 \quad \text { if } p \geq 3, \\
p & \leq \frac{1}{2}|\operatorname{idx} \mathbf{m}|+3 .
\end{aligned}
$$

Example 9.14. For a positive integer $m$ we have special 4 elements

$$
\begin{array}{ll}
D_{4}^{(m)}: m^{2}, m^{2}, m^{2}, m(m-1) 1 & E_{6}^{(m)}: m^{3}, m^{3}, m^{2}(m-1) 1 \\
E_{7}^{(m)}:(2 m)^{2}, m^{4}, m^{3}(m-1) 1 & E_{8}^{(m)}:(3 m)^{2},(2 m)^{3}, m^{5}(m-1) 1
\end{array}
$$

with orders $2 m, 3 m, 4 m$ and $6 m$, respectively, and index of rigidity $2-2 m$.

Note that $E_{8}^{(m)}, D_{4}^{(m)}$ and $11,11,11, \cdots \in \mathcal{P}_{p+1}^{(2)}$ attain the equalities (9.48), (9.49) and (9.50), respectively.

Remark 9.15. It follows from the Proposition 9.13 that there exist only finite basic tuples $\mathbf{m} \in \mathcal{P}$ with a fixed index of rigidity under the normalization (3.3). This result is given in $\mathrm{O} 6$, Proposition 8.1].

Hence there exist only finite fundamental universal Fuchsian differential operators with a fixed number of accessory parameters. Here a fundamental universal Fuchsian differential operator means a universal operator given in Theorem 8.13 whose spectral type is fundamental (cf. Definition 8.14).

Now we prepare a lemma.

Lemma 9.16. Let $a \geq 0, b>0$ and $c>0$ be integers such that $a+c-b>0$. Then

$$
\frac{b+k c-6}{(a+c-b) b} \begin{cases}<k+1 & (0 \leq k \leq 5), \\ \leq 7 & (0 \leq k \leq 6) .\end{cases}
$$

Proof. Suppose $b \geq c$. Then

$$
\frac{b+k c-6}{(a+c-b) b} \leq \frac{b+k b-6}{b}<k+1 .
$$

Next suppose $b<c$. Then

$$
\begin{aligned}
(k+1)(a+c-b) b-(b+k c-6) & \geq(k+1)(c-b) b-b-k c+6 \\
& \geq(k+1) b-b-k(b+1)+6=6-k .
\end{aligned}
$$

Thus we have the lemma.

Proof of Proposition 9.13. Since idx $k \mathbf{m}=k^{2}$ idx $\mathbf{m}$ for a basic tuple $\mathbf{m}$ and $k \in$ $\mathbb{Z}_{>0}$, we may assume that $\mathbf{m}$ is basic and idx $\mathbf{m} \leq-2$ to prove the proposition.

Fix a basic monotone tuple $\mathbf{m}$. Put $\alpha=\alpha_{\mathbf{m}}$ under the notation (9.12) and $n=\operatorname{ord} \mathbf{m}$. Note that

$$
(\alpha \mid \alpha)=n\left(\alpha \mid \alpha_{0}\right)+\sum_{j=0}^{p} \sum_{\nu=1}^{n_{j}} n_{j, \nu}\left(\alpha \mid \alpha_{j, \nu}\right), \quad\left(\alpha \mid \alpha_{0}\right) \leq 0, \quad\left(\alpha \mid \alpha_{j, \nu}\right) \leq 0 .
$$

We first assume that (9.48) is not valid, namely,

$$
3|(\alpha \mid \alpha)|+6<n
$$


In view of (8.18), we have $(\alpha \mid \alpha)<0$ and the assumption implies $\left|\left(\alpha \mid \alpha_{0}\right)\right|=0$ because $|(\alpha \mid \alpha)| \geq n\left|\left(\alpha \mid \alpha_{0}\right)\right|$.

Let $\Pi_{0}$ be the connected component of $\left\{\alpha_{i} \in \Pi ;\left(\alpha \mid \alpha_{i}\right)=0\right.$ and $\left.\alpha_{i} \in \operatorname{supp} \alpha\right\}$ containing $\alpha_{0}$. Note that $\operatorname{supp} \alpha$ generates a root system which is neither classical nor affine but $\Pi_{0}$ generates a root system of finite type.

Put $J=\left\{j ; \exists \alpha_{j, \nu} \in \operatorname{supp} \alpha_{\mathbf{m}}\right.$ such that $\left.\left(\alpha \mid \alpha_{j, \nu}\right)<0\right\} \neq \emptyset$ and for each $j \in J$ define $k_{j}$ with the condition (9.45). Then we note that

$$
\left(\alpha \mid \alpha_{j, \nu}\right)= \begin{cases}0 & \left(1 \leq \nu<k_{j}\right) \\ 2 n_{j, k_{j}}-n_{j, k_{j-1}}-n_{j, k_{j+1}} \leq-1 & \left(\nu=k_{j}\right)\end{cases}
$$

Applying the above lemma to $\mathbf{m}$ by putting $n=b+k_{j} c$ and $n_{j, \nu}=b+\left(k_{j}-\nu\right) c$ $\left(1 \leq \nu \leq k_{j}\right)$ and $n_{j, k_{j}+1}=a$, we have

$$
\frac{n-6}{\left(n_{j, k_{j-1}}+n_{j, k_{j+1}}-2 n_{j, k_{j}}\right) n_{j, k_{j}}} \begin{cases}<k_{j}+1 & \left(1 \leq k_{j} \leq 5\right), \\ \leq 7 & \left(1 \leq k_{j} \leq 6\right) .\end{cases}
$$

Here $\left(\alpha \mid \alpha_{j, k_{j}}\right)=b-c-a \leq-1$ and we have $|(\alpha \mid \alpha)| \geq\left|\left(\alpha \mid \alpha_{j, \nu}\right)\right|>\frac{n-6}{k_{j}+1}$ if $k_{j}<6$ and therefore $k_{j} \geq 3$.

It follows from the condition $k_{j} \geq 3$ that $\mathbf{m} \in \mathcal{P}_{3}$ because $\Pi_{0}$ is of finite type and moreover that $\Pi_{0}$ is of exceptional type, namely, of type $E_{6}$ or $E_{7}$ or $E_{8}$ because $\operatorname{supp} \alpha$ is not of finite type.

Suppose $\# J \geq 2$. We may assume $\{0,1\} \subset J$ and $k_{0} \leq k_{1}$. Since $\Pi_{0}$ is of exceptional type and $\operatorname{supp} \alpha$ is not of finite type, we may assume $k_{0}=3$ and $k_{1} \leq 5$. Owing to (9.52) and (9.54), we have

$$
\begin{aligned}
|(\alpha \mid \alpha)| & \geq n_{0,3}\left(n_{0,2}+n_{0,4}-2 n_{0,3}\right)+n_{1, k_{1}}\left(n_{1, k_{1}-1}+n_{1, k_{1}+1}-2 n_{1, k_{1}}\right) \\
& >\frac{n-6}{3+1}+\frac{n-6}{5+1}>\frac{n-6}{3}
\end{aligned}
$$

which contradicts to the assumption.

Thus we may assume $J=\{0\}$. For $j=1$ and 2 let $n_{j}$ be the positive integer such that $\alpha_{j, n_{j}} \in \operatorname{supp} \alpha$ and $\alpha_{j, n_{j}+1} \notin \operatorname{supp} \alpha$. We may assume $n_{1} \geq n_{2}$.

Fist suppose $k_{0}=3$. Then $\left(n_{1}, n_{2}\right)=(2,1),(3,1)$ or $(4,1)$ and the Dynkin diagram of $\operatorname{supp} \alpha$ with the numbers $m_{j, \nu}$ is one of the diagrams:
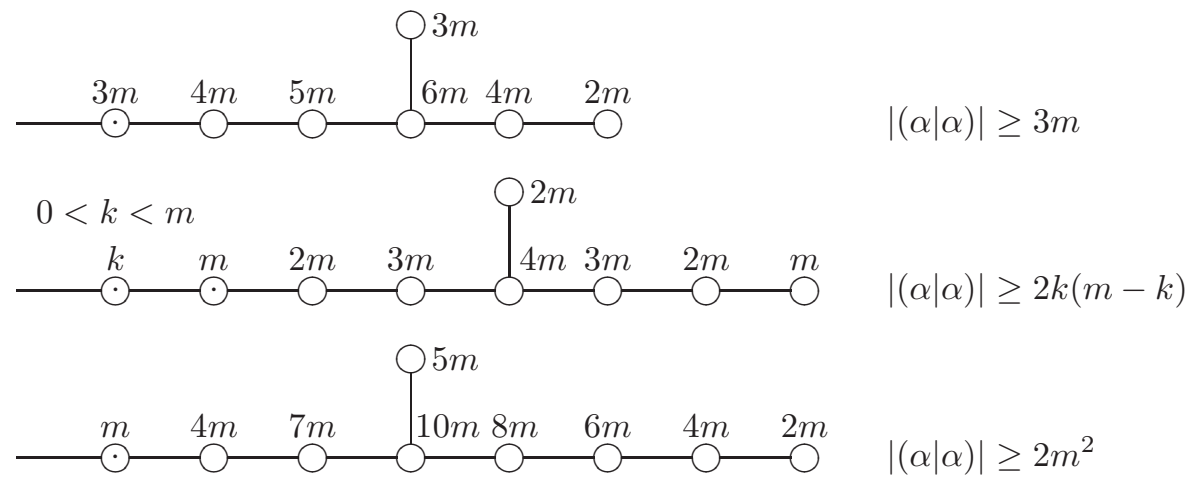

For example, when $\left(n_{1}, n_{2}\right)=(3,1)$, then $k:=m_{0,4} \geq 1$ because $\left(\alpha \mid \alpha_{0,3}\right) \neq 0$ and therefore $0<k<m$ and $|(\alpha \mid \alpha)| \geq k(m-2 k)+m(2 m+k-2 m)=2 k(m-k) \geq 2 m-2$ and $3|(\alpha \mid \alpha)|+6-4 m \geq 3(2 m-2)+6-4 m>0$. Hence (9.53) doesn't hold.

Other cases don't happen because of the inequalities $3 \cdot 3 m+6-6 m>0$ and $3 \cdot 2 m^{2}+6-10 m>0$. 
Lastly suppose $k_{0}>3$. Then $\left(k_{0}, n_{1}, n_{2}\right)=(4,2,1)$ or $(5,2,1)$.

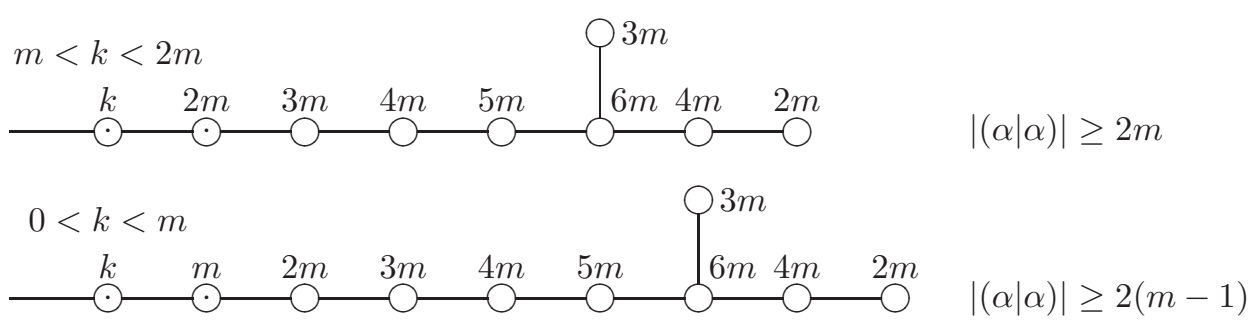

In the above first case we have $(\alpha \mid \alpha) \mid \geq 2 m$, which contradicts to (9.53). Note that $(|\alpha| \alpha) \mid \geq k \cdot(m-2 k)+m \cdot k=2 k(m-k) \geq 2(m-1)$ in the above last case, which also contradicts to $(9.53)$ because $3 \cdot 2(m-1)+6=6 m$.

Thus we have proved (9.48).

Assume $\mathbf{m} \notin \mathcal{P}_{3}$ to prove a different inequality (9.49). In this case, we may assume $\left(\alpha \mid \alpha_{0}\right)=0,|(\alpha \mid \alpha)| \geq 2$ and $n>4$. Note that

$$
2 n=n_{0,1}+n_{1,1}+\cdots+n_{p, 1} \text { with } p \geq 3 \text { and } n_{j, 1} \geq 1 \text { for } j=0, \ldots, p \text {. }
$$

If there exists $j$ with $1 \leq n_{j, 1} \leq \frac{n}{2}-1$, (9.49) follows from 9.52) and $\left|\left(\alpha \mid \alpha_{j, 1}\right)\right|=$ $n_{j, 1}\left(n+n_{j, 2}-2 n_{j, 1}\right) \geq 2 n_{j, 1}\left(\frac{n}{2}-n_{j, 1}\right) \geq n-2$.

Hence we may assume $n_{j, 1} \geq \frac{n-1}{2}$ for $j=0, \ldots, p$. Suppose there exists $j$ with $n_{j, 1}=\frac{n-1}{2}$. Then $n$ is odd and (9.55) means that there also exists $j^{\prime}$ with $j \neq j^{\prime}$ and $n_{j^{\prime}, 1}=\frac{n-1}{2}$. In this case we have (9.49) since

$\left|\left(\alpha \mid \alpha_{j, 1}\right)\right|+\left|\left(\alpha \mid \alpha_{j^{\prime}, 1}\right)\right|=n_{j, 1}\left(n+n_{j, 2}-2 n_{j, 1}\right)+n_{j^{\prime}, 1}\left(n+n_{j^{\prime}, 2}-2 n_{j, 1}\right) \geq \frac{n-1}{2}+\frac{n-1}{2}$.

Now we may assume $n_{j, 1} \geq \frac{n}{2}$ for $j=0, \ldots, p$. Then (9.55) implies that $p=3$ and $n_{j, 1}=\frac{n}{2}$ for $j=0, \ldots, 3$. Since $(\alpha \mid \alpha)<0$, there exists $j$ with $n_{j, 2} \geq 1$ and

$$
\begin{aligned}
\left|\left(\alpha \mid \alpha_{j, 1}\right)\right|+\left|\left(\alpha \mid \alpha_{j, 2}\right)\right| & =n_{j, 1}\left(n+n_{j, 2}-2 n_{j, 1}\right)+n_{j, 2}\left(n_{j, 1}+n_{j, 3}-2 n_{j, 2}\right) \\
& =\frac{n}{2} n_{j, 2}+n_{j, 2}\left(\frac{n}{2}+n_{j, 3}-2 n_{j, 2}\right) \\
& \begin{cases}\geq n & \left(n_{j, 2} \geq 1\right), \\
=n-2 & \left(n_{j, 2}=1 \text { and } n_{j, 3}=0\right) .\end{cases}
\end{aligned}
$$

Thus we have completed the proof of (9.49).

There are 4 basic tuples with the index of the rigidity 0 and 13 basic tuples with the index of the rigidity -2 , which are given in (8.18) and Proposition 8.10. They satisfy (9.50).

Suppose that (9.50) is not valid. We may assume that $p$ is minimal under this assumption. Then idx $\mathbf{m}<-2, p \geq 5$ and $n=\operatorname{ord} \mathbf{m}>2$. We may assume $n>n_{0,1} \geq n_{1,1} \geq \cdots \geq n_{p, 1}>0$. Since $\left(\alpha \mid \alpha_{0}\right) \leq 0$, we have

$$
n_{0,1}+n_{1,1}+\cdots+n_{p, 1} \geq 2 n>n_{0,1}+\cdots+n_{p-1,1} .
$$

In fact, if $n_{0,1}+\cdots+n_{p-1,1} \geq 2 n$, the tuple $\mathbf{m}^{\prime}=\left(\mathbf{m}_{0}, \ldots, \mathbf{m}_{p-1}\right)$ is also basic and $|(\alpha \mid \alpha)|-\left|\left(\alpha_{\mathbf{m}^{\prime}}, \alpha_{\mathbf{m}^{\prime}}\right)\right|=n^{2}-\sum_{\nu \geq 1} n_{p, \nu}^{2} \geq 2$, which contradicts to the minimality.

Thus we have $2 n_{j, 1}<n$ for $j=3, \ldots, p$. If $n$ is even, $|\operatorname{idx} \mathbf{m}| \geq \sum_{j=3}^{p}\left|\left(\alpha \mid \alpha_{j, 1}\right)\right|=$ $\sum_{i=3}^{p}\left(n+n_{j, 2}-2 n_{j, 1}\right) \geq 2(p-2)$, which contradicts to the assumption. If $n=3$, (9.56) assures $p=5$ and $n_{0,1}=\cdots=n_{5,0}=1$ and therefore idx $\mathbf{m}=-4$, which also contradicts to the assumption. Thus $n=2 m+1$ with $m \geq 2$. Choose $k$ so that $n_{k-1,1} \geq m>n_{k, 1}$. Then $|\operatorname{idx} \mathbf{m}| \geq \sum_{j=k}^{p}\left(\alpha \mid \alpha_{j, 1}\right) \mid=\sum_{j=k}^{p}\left(n+n_{j, 2}-\right.$ $\left.2 n_{j, 1}\right) \geq 3(p-k+1)$. Owing to (9.56), we have $2(2 m+1)>k m+(p-k)$ and $k<\frac{4 m+2-p}{m-1} \leq \frac{4 m-3}{m-1} \leq 5$, which means $k \leq 4,|\operatorname{idx} \mathbf{m}| \geq 3(p-3) \geq 2 p-4$ and a contradiction to the assumption. 


\section{EXPRESSION OF LOCAL SOLUTIONS}

Fix $\mathbf{m}=\left(m_{j, \nu}\right)_{\substack{j=0, \ldots, p \\ 1<\nu<n_{j}}} \in \mathcal{P}_{p+1}$. Suppose $\mathbf{m}$ is monotone and irreducibly realizable. Let $P_{\mathbf{m}}$ be the universal operator with the Riemann scheme (6.15), which is given in Theorem 8.13. Suppose $c_{1}=0$ and $m_{1, n_{1}}=1$. We give expressions of the local solution of $P_{\mathbf{m}} u=0$ at $x=0$ corresponding to the characteristic exponent $\lambda_{1, n_{1}}$.

Theorem 10.1. Retain the notation above and in Definition 7.11. Suppose $\lambda_{j, \nu}$ are generic. Let

$$
v(x)=\sum_{\nu=0}^{\infty} C_{\nu} x^{\lambda(K)_{1, n_{1}}+\nu}
$$

be the local solution of $\left(\partial_{\max }^{K} P_{\mathbf{m}}\right) v=0$ at $x=0$ with the condition $C_{0}=1$. Put

$$
\lambda(k)_{j, \max }=\lambda(k)_{j, \ell(k)_{j}} .
$$

Note that if $\mathbf{m}$ is rigid, then

$$
v(x)=x^{\lambda(K)_{1, n_{1}}} \prod_{j=2}^{p}\left(1-\frac{x}{c_{j}}\right)^{\lambda(K)_{j, \max }} .
$$

The function

$$
\begin{aligned}
u(x):= & \prod_{k=0}^{K-1} \frac{\Gamma\left(\lambda(k)_{1, n_{1}}-\lambda(k)_{1, \max }+1\right)}{\Gamma\left(\lambda(k)_{1, n_{1}}-\lambda(k)_{1, \max }+\mu(k)+1\right) \Gamma(-\mu(k))} \\
\int_{0}^{s_{0}} & \cdots \int_{0}^{s_{K-1}} \prod_{k=0}^{K-1}\left(s_{k}-s_{k+1}\right)^{-\mu(k)-1} \\
& \cdot \prod_{k=0}^{K-1}\left(\left(\frac{s_{k}}{s_{k+1}}\right)^{\lambda(k)_{1, \max }} \prod_{j=2}^{p}\left(\frac{1-c_{j}^{-1} s_{k}}{1-c_{j}^{-1} s_{k+1}}\right)^{\lambda(k)_{j, \max }}\right) \\
& \left.\cdot v\left(s_{K}\right) d s_{K} \cdots d s_{1}\right|_{s_{0}=x}
\end{aligned}
$$

is the solution of $P_{\mathbf{m}} u=0$ so normalized that $u(x) \equiv x^{\lambda_{1, n_{1}}} \bmod x^{\lambda_{1, n_{1}}+1} \mathcal{O}_{0}$.

Here we note that

$$
\begin{aligned}
\prod_{k=0}^{K-1} & \left(\left(\frac{s_{k}}{s_{k+1}}\right)^{\lambda(k)_{1, \max }} \prod_{j=2}^{p}\left(\frac{1-c_{j}^{-1} s_{k}}{1-c_{j}^{-1} s_{k+1}}\right)^{\lambda(k)_{j, \max }}\right) \\
= & \frac{s_{0}^{\lambda(0)_{1, \max }}}{s_{K}^{\lambda(K-1)_{1, \max }}} \prod_{j=1}^{p} \frac{\left(1-c_{j}^{-1} s_{0}\right)^{\lambda(0)_{j, \max }}}{\left(1-c_{j}^{-1} s_{K}\right)^{\lambda(K-1)_{j, \max }}} \\
& \cdot \prod_{k=1}^{K-1}\left(s_{k}^{\lambda(k)_{1, \max }-\lambda(k-1)_{1, \max }} \prod_{j=2}^{p}\left(1-c_{j}^{-1} s_{k}\right)^{\lambda(k)_{j, \max }-\lambda(k-1)_{j, \max }}\right) .
\end{aligned}
$$


When $\mathbf{m}$ is rigid,

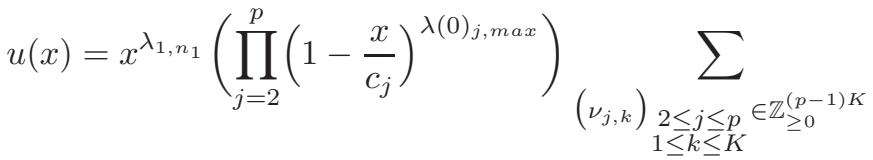

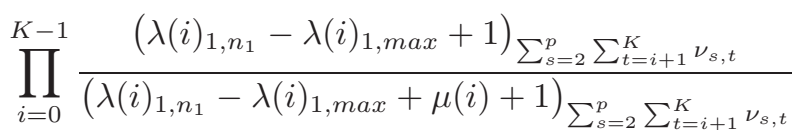

$$
\begin{aligned}
& \cdot \prod_{i=1}^{K} \prod_{s=2}^{p} \frac{\left(\lambda(i-1)_{s, \max }-\lambda(i)_{s, \max }\right)_{\nu_{s, i}}}{\nu_{s, i} !} \cdot \prod_{s=2}^{p}\left(\frac{x}{c_{s}}\right)^{\sum_{i=1}^{K} \nu_{s, i}} .
\end{aligned}
$$

When $\mathbf{m}$ is not rigid

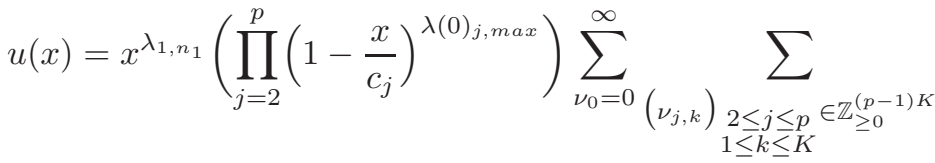

$$
\begin{aligned}
& \prod_{i=0}^{K-1} \frac{\left(\lambda(i)_{1, n_{1}}-\lambda(i)_{1, \max }+1\right)_{\nu_{0}+\sum_{s=2}^{p} \sum_{t=i+1}^{K} \nu_{s, t}}}{\left(\lambda(i)_{1, n_{1}}-\lambda(i)_{1, \max }+\mu(i)+1\right)_{\nu_{0}+\sum_{s=2}^{p} \sum_{t=i+1}^{K} \nu_{s, t}}} \\
& \cdot \prod_{s=2}^{p} \frac{\left(\lambda(K-1)_{s, \max }\right)_{\nu_{s, K}}}{\nu_{s, K} !} \cdot \prod_{i=1}^{K-1} \prod_{s=2}^{p} \frac{\left(\lambda(i-1)_{s, \max }-\lambda(i)_{s, \max }\right)_{\nu_{s, i}}}{\nu_{s, i} !} \\
& \text {. } C_{\nu_{0}} x^{\nu_{0}} \prod_{s=2}^{p}\left(\frac{x}{c_{s}}\right)^{\sum_{i=1}^{K} \nu_{s, i}} \text {. }
\end{aligned}
$$

Fix $j$ and $k$ and suppose

$$
\begin{cases}\ell(k-1)_{j}=\ell(k)_{\nu} & \text { when } \mathbf{m} \text { is rigid or } k<K, \\ \ell(k-1)_{j}=0 & \text { when } \mathbf{m} \text { is not rigid and } k=K .\end{cases}
$$

Then the terms satisfying $\nu_{j, k}>0$ vanish because $(0)_{\nu_{j, k}}=\delta_{0, \nu_{j, k}}$ for $\nu_{j, k}=$ $0,1,2, \ldots$.

Proof. The theorem follows from 77.25$),(7.26),(7.27),(4.2)$ and $(4.6)$ by the induction on $K$. Note that the integral representation of the normalized solution of $\left(\partial_{\max } P\right) v=0$ corresponding to the exponent $\lambda(1)_{n_{1}}$ equals

$$
\begin{aligned}
v(x):= & \prod_{k=1}^{K-1} \frac{\Gamma\left(\lambda(k)_{1, n_{1}}-\lambda(k)_{1, \max }+1\right)}{\Gamma\left(\lambda(k)_{1, n_{1}}-\lambda(k)_{1, \max }+\mu(k)+1\right) \Gamma(-\mu(k))} \\
& \cdot \int_{0}^{s_{1}} \cdots \int_{0}^{s_{K-1}} \prod_{k=0}^{K-1}\left(s_{k}-s_{k+1}\right)^{-\mu(k)-1} \\
& \cdot \prod_{k=0}^{K-1}\left(\left(\frac{s_{k}}{s_{k+1}}\right)^{\lambda(k)_{1, \max }} \prod_{j=2}^{p}\left(\frac{1-c_{j}^{-1} s_{k}}{1-c_{j}^{-1} s_{k+1}}\right)^{\lambda(k)_{j, \max }}\right) \\
& \left.\cdot v\left(s_{K}\right) d s_{K} \cdots d s_{1}\right|_{s_{1}=x} \\
\equiv & x^{\lambda(1)_{1, n_{1}}} \bmod x^{\lambda(1)_{1, n_{1}}+1} \mathcal{O}_{0}
\end{aligned}
$$


by the induction hypothesis and the normalized solution of $P u=0$ corresponding to the exponent $\lambda_{1, n_{1}}$ equals

$$
\begin{aligned}
& \frac{\Gamma\left(\lambda(0)_{1, n_{1}}-\lambda(0)_{1, \max }+1\right)}{\Gamma\left(\lambda(0)_{1, n_{1}}-\lambda(0)_{1, \max }+\mu(0)+1\right) \Gamma(-\mu(0))} \\
& \quad \cdot \int_{0}^{x}\left(x-s_{0}\right)^{-\mu(0)-1} \frac{x^{-\lambda(0)_{1, \max }}}{s_{0}^{-\lambda(0)_{1, \max }}} \prod_{j=2}^{p}\left(\frac{1-c_{j}^{-1} x}{1-c_{j}^{-1} s_{0}}\right)^{-\lambda(0)_{j, \max }} v\left(s_{0}\right) d s_{0}
\end{aligned}
$$

and hence we have (10.4). Then the integral expression (10.4) with (10.5), (4.2) and (4.6) inductively proves (10.6) and (10.7).

Example 10.2 (Gauss hypergeometric equation). The reduction (12.55) shows

$$
\begin{aligned}
\lambda(0)_{j, \nu} & =\lambda_{j, \nu}, m(0)_{j, \nu}=1 \quad(0 \leq j \leq 2,1 \leq \nu \leq 2), \mu(0)=-\lambda_{0,2}-\lambda_{1,2}-\lambda_{2,2}, \\
m(1)_{j, 1} & =0, m(1)_{j, 2}=1 \quad(j=0,1,2), \\
\lambda(1)_{0,1} & =\lambda_{0,1}+2 \lambda_{0,2}+2 \lambda_{1,2}+2 \lambda_{2,2}, \lambda(1)_{1,1}=\lambda_{1,1}, \lambda(1)_{2,1}=\lambda_{2,1}, \\
\lambda(1)_{0,2} & =2 \lambda_{0,2}+\lambda_{1,2}+\lambda_{2,2}, \lambda(1)_{1,2}=-\lambda_{0,2}-\lambda_{2,2}, \lambda(1)_{2,2}=-\lambda_{0,2}-\lambda_{1,2}
\end{aligned}
$$

and therefore

$$
\begin{aligned}
\lambda(0)_{1, n_{1}}-\lambda(0)_{1, \max }+\mu(0)+1 & =\lambda_{1,2}-\lambda_{1,1}-\left(\lambda_{0,2}+\lambda_{1,2}+\lambda_{2,2}\right)+1 \\
& =\lambda_{0,1}+\lambda_{1,2}+\lambda_{2,1}, \\
\lambda(0)_{2, \max }-\lambda(1)_{2, \max } & =\lambda(0)_{2,1}-\lambda(1)_{2,2}=\lambda_{2,1}+\lambda_{0,2}+\lambda_{1,2} .
\end{aligned}
$$

Hence (10.4) says that the normalized local solution corresponding to the characteristic exponent $\lambda_{1,2}$ with $c_{1}=0$ and $c_{2}=1$ equals

$$
\begin{aligned}
u(x)= & \frac{\Gamma\left(\lambda_{1,2}-\lambda_{1,1}+1\right) x^{\lambda_{1,1}}(1-x)^{\lambda_{2,1}}}{\Gamma\left(\lambda_{0,1}+\lambda_{1,2}+\lambda_{2,1}\right) \Gamma\left(\lambda_{0,2}+\lambda_{1,2}+\lambda_{2,2}\right)} \\
& \int_{0}^{x}(x-s)^{\lambda_{0,2}+\lambda_{1,2}+\lambda_{2,2}-1} s^{-\lambda_{0,2}-\lambda_{1,1}-\lambda_{2,2}}(1-s)^{-\lambda_{0,2}-\lambda_{1,2}-\lambda_{2,1}} d s
\end{aligned}
$$

and moreover (10.6) says

$$
u(x)=x^{\lambda_{1,2}}(1-x)^{\lambda_{2,1}} \sum_{\nu=0}^{\infty} \frac{\left(\lambda_{0,1}+\lambda_{1,2}+\lambda_{2,1}\right)_{\nu}\left(\lambda_{0,2}+\lambda_{1,2}+\lambda_{2,1}\right)_{\nu}}{\left(\lambda_{1,2}-\lambda_{1,1}+1\right)_{\nu} \nu !} x^{\nu} .
$$

Note that $u(x)=F(a, b, c ; x)$ when

$$
\left\{\begin{array}{ccc}
x=\infty & 0 & 1 \\
\lambda_{0,1} & \lambda_{1,1} & \lambda_{2,1} \\
\lambda_{0,2} & \lambda_{1,2} & \lambda_{2,2}
\end{array}\right\}=\left\{\begin{array}{ccc}
x=\infty & 0 & 1 \\
a & 1-c & 0 \\
b & 0 & c-a-b
\end{array}\right\} .
$$

The integral expression (10.9) is based on the minimal expression $w=s_{0,1} s_{1,1} s_{1,2} s_{0}$ satisfying $w \alpha_{\mathbf{m}}=\alpha_{0}$. Here $\alpha_{\mathbf{m}}=2 \alpha_{0}+\sum_{j=0}^{2} \alpha_{j, 1}$. When we replace $w$ and its minimal expression by $w^{\prime}=s_{0,1} s_{1,1} s_{1,2} s_{0} s_{0,1}$ or $w^{\prime \prime}=s_{0,1} s_{1,1} s_{1,2} s_{0} s_{2,1}$, we get the different integral expressions

$$
\begin{aligned}
u(x)= & \frac{\Gamma\left(\lambda_{1,2}-\lambda_{1,1}+1\right) x^{\lambda_{1,1}}(1-x)^{\lambda_{2,1}}}{\Gamma\left(\lambda_{0,2}+\lambda_{1,2}+\lambda_{2,1}\right) \Gamma\left(\lambda_{0,1}+\lambda_{1,2}+\lambda_{2,2}\right)} \\
& \int_{0}^{x}(x-s)^{\lambda_{0,1}+\lambda_{1,2}+\lambda_{2,2}-1} s^{-\lambda_{0,1}-\lambda_{1,1}-\lambda_{2,2}}(1-s)^{-\lambda_{0,1}-\lambda_{1,2}-\lambda_{2,1}} d s \\
= & \frac{\Gamma\left(\lambda_{1,2}-\lambda_{1,1}+1\right) x^{\lambda_{1,1}}(1-x)^{\lambda_{2,2}}}{\Gamma\left(\lambda_{0,1}+\lambda_{1,2}+\lambda_{2,2}\right) \Gamma\left(\lambda_{0,2}+\lambda_{1,2}+\lambda_{2,1}\right)} \\
& \int_{0}^{x}(x-s)^{\lambda_{0,2}+\lambda_{1,2}+\lambda_{2,1}-1} s^{-\lambda_{0,2}-\lambda_{1,1}-\lambda_{2,1}}(1-s)^{-\lambda_{0,2}-\lambda_{1,2}-\lambda_{2,2}} d s .
\end{aligned}
$$


These give different integral expressions of $F(a, b, c ; x)$ under (10.11).

Since $s_{\alpha_{0}+\alpha_{0,1}+\alpha_{0,2}} \alpha_{\mathbf{m}}=\alpha_{\mathbf{m}}$, we have

$$
\begin{aligned}
& \left\{\begin{array}{ccc}
x=\infty & 0 & 1 \\
a & 1-c & 0 \\
b & 0 & c-a-b
\end{array}\right\} \stackrel{x^{c-1}}{\longrightarrow}\left\{\begin{array}{ccc}
x=\infty & 0 & 1 \\
a-c+1 & 0 & 0 \\
b-c+1 & c-1 & c-a-b
\end{array}\right\} \\
& \stackrel{\partial^{c-d}}{\longrightarrow}\left\{\begin{array}{ccc}
x=\infty & 0 & 1 \\
a-d+1 & 0 & 0 \\
b-d+1 & d-1 & d-a-b
\end{array}\right\} \stackrel{x^{1-d}}{\longrightarrow}\left\{\begin{array}{ccc}
x=\infty & 0 & 1 \\
a & 1-d & 0 \\
b & 0 & d-a-b
\end{array}\right\}
\end{aligned}
$$

and hence (cf. (4.6))

$$
F(a, b, d ; x)=\frac{\Gamma(d) x^{1-d}}{\Gamma(c) \Gamma(d-c)} \int_{0}^{x}(x-s)^{d-c-1} s^{c-1} F(a, b, c ; s) d s .
$$

Remark 10.3. The integral expression of the local solution $u(x)$ as is given in Theorem 10.1 is obtained from the expression of the element $w$ of $W_{\infty}$ satisfying $w \alpha_{\mathbf{m}} \in B \cup\left\{\alpha_{0}\right\}$ as a product of simple reflections and therefore the integral expression depends on such element $w$ and the expression of $w$ as such product. The dependence on $w$ seems non-trivial as in the preceding example but the dependence on the expression of $w$ as a product of simple reflections is understood as follows.

First note that the integral expression doesn't depend on the coordinate transformations $x \mapsto a x$ and $x \mapsto x+b$ with $a \in \mathbb{C}^{\times}$and $b \in \mathbb{C}$. Since

$$
\begin{aligned}
\int_{c}^{x}(x-t)^{\mu-1} \phi(t) d t & =-\int_{\frac{1}{c}}^{\frac{1}{x}}\left(x-\frac{1}{s}\right)^{\mu-1} \phi\left(\frac{1}{s}\right) s^{-2} d s \\
& =-(-1)^{\mu-1} x^{\mu-1} \int_{\frac{1}{c}}^{\frac{1}{x}}\left(\frac{1}{x}-s\right)^{\mu-1}\left(\frac{1}{s}\right)^{\mu+1} \phi\left(\frac{1}{s}\right) d s,
\end{aligned}
$$

we have

$$
I_{c}^{\mu}(\phi)=-\left.(-1)^{\mu-1} x^{\mu-1}\left(\left.I_{\frac{1}{c}}^{x}\left(x^{\mu+1} \phi(x)\right)\right|_{x \mapsto \frac{1}{x}}\right)\right|_{x \rightarrow \frac{1}{x}},
$$

which corresponds to (7.11). Here the value $(-1)^{\mu-1}$ depends on the branch of the value of $\left(x-\frac{1}{s}\right)^{\mu-1}$ and that of $x^{\mu-1} x^{1-\mu}\left(\frac{1}{x}-s\right)^{\mu-1}$.

Hence the argument as in the proof of Theorem 9.5 shows that the dependence on the expression of $w$ by a product of simple reflections can be understood by the identities (10.14) and $I_{c}^{\mu_{1}} I_{c}^{\mu_{2}}=I_{c}^{\mu_{1}+\mu_{2}}$ (cf. (4.4)) etc.

\section{Monodromy}

The transformation of monodromy generators for irreducible Fuchsian systems of Schlesinger canonical form under the middle convolution or the addition is studied by [Kz] and [DR, DR2] etc. A non-zero homomorphism of an irreducible single Fuchsian differential equation to an irreducible system of Schlesinger canonical form induces the isomorphism of their monodromies of the solutions (cf. Remark 2.12). In particular since any rigid local system is realized by a single Fuchsian differential equation, their monodromies naturally coincide with each other through the correspondence of their monodromy generators. The correspondence between the local monodromies and the global monodromies is described by DR2, which we will review. 
11.1. Middle convolution of monodromies. For given matrices $A_{j} \in M(n, \mathbb{C})$ for $j=1, \ldots, p$ the Fuchsian system

$$
\frac{d v}{d x}=\sum_{j=1}^{p} \frac{A_{j}}{x-c_{j}} v
$$

of Schlesinger canonical form (SCF) is defined. Put $A_{0}=-A_{1}-\cdots-A_{p}$ and $\mathbf{A}=\left(A_{0}, A_{1}, \ldots, A_{p}\right)$ which is an element of

$$
M(n, \mathbb{C})_{0}^{p+1}:=\left\{\left(C_{0}, \ldots, C_{p}\right) \in M(n, \mathbb{C})^{p+1} ; C_{0}+\cdots+C_{p}=0\right\},
$$

The Riemann scheme of (11.1) is defined by

$$
\left\{\begin{array}{cccc}
x=c_{0}=\infty & c_{1} & \cdots & c_{p} \\
{\left[\lambda_{0,1}\right]_{m_{0,1}}} & {\left[\lambda_{1,1}\right]_{m_{1,1}}} & \cdots & {\left[\lambda_{p, 1}\right]_{m_{p, 1}}} \\
\vdots & \vdots & \vdots & \vdots \\
{\left[\lambda_{0, n_{0}}\right]_{m_{0, n_{0}}}} & {\left[\lambda_{1, n_{1}}\right]_{m_{1, n_{1}}}} & \cdots & {\left[\lambda_{p, n_{p}}\right]_{m_{p, 1}}}
\end{array}\right\}, \quad[\lambda]_{k}:=\left(\begin{array}{c}
\lambda \\
\vdots \\
\lambda
\end{array}\right) \in M(1, k, \mathbb{C})
$$

if

$$
A_{j} \sim L\left(m_{j, 1}, \ldots, m_{j, n_{j}} ; \lambda_{j, 1}, \ldots, \lambda_{j, n_{j}}\right) \quad(j=0, \ldots, p)
$$

under the notation (6.33). Here the Fuchs relation equals

$$
\sum_{j=0}^{p} \sum_{\nu=1}^{n_{j}} m_{j, \nu} \lambda_{j, \nu}=0 .
$$

We define that $\mathbf{A}$ is irreducible if a subspace $V$ of $\mathbb{C}^{n}$ satisfies $A_{j} V \subset A_{j}$ for $j=0, \ldots, p$, then $V=\{0\}$ or $V=\mathbb{C}^{n}$. In general, $\mathbf{A}=\left(A_{0}, \ldots, A_{p}\right), \mathbf{A}^{\prime}=$ $\left(A_{0}^{\prime}, \ldots, A_{p}^{\prime}\right) \in M(n, \mathbb{C})^{p+1}$, we denote by $\mathbf{A} \sim \mathbf{A}^{\prime}$ if there exists $U \in G L(n, \mathbb{C})$ such that $A_{j}^{\prime}=U A_{j} U^{-1}$ for $j=0, \ldots, p$.

For $\left(\mu_{0}, \ldots, \mu_{p}\right) \in \mathbb{C}^{p+1}$ with $\mu_{0}+\cdots+\mu_{p}=0$, the addition $\mathbf{A}^{\prime}=\left(A_{0}^{\prime}, \ldots, A_{p}^{\prime}\right) \in$ $M(n, \mathbb{C})_{0}^{p+1}$ of $\mathbf{A}$ with respect to $\left(\mu_{0}, \ldots, \mu_{p}\right)$ is defined by $A_{j}^{\prime}=A_{j}+\mu_{j}$ for $j=0, \ldots, p$.

For a complex number $\mu$ the middle convolution $\overline{\mathbf{A}}:=m c_{\mu}(\mathbf{A})$ of $\mathbf{A}$ is defined by $\bar{A}_{j}=\bar{A}_{j}(\mu)$ for $j=1, \ldots, p$ and $\bar{A}_{0}=-\bar{A}_{1}-\cdots-\bar{A}_{p}$ under the notation in $\S 2.5$. Then we have the following theorem.

Theorem 11.1 ([DR, DR2]). Suppose that A satisfies the conditions

$$
\begin{aligned}
& \bigcap_{\substack{1 \leq j \leq p \\
j \neq i}} \operatorname{ker} A_{j} \cap \operatorname{ker}\left(A_{0}-\tau\right)=\{0\} \quad(i=1, \ldots, p, \forall \tau \in \mathbb{C}), \\
& \bigcap_{\substack{1 \leq j \leq p \\
j \neq i}} \operatorname{ker}{ }^{t} A_{j} \cap \operatorname{ker}\left({ }^{t} A_{0}-\tau\right)=\{0\} \quad(i=1, \ldots, p, \forall \tau \in \mathbb{C}) .
\end{aligned}
$$

i) The tuple $m c_{\mu}(\mathbf{A})=\left(\bar{A}_{0}, \ldots, \bar{A}_{p}\right)$ also satisfies the same conditions as above with replacing $A_{\nu}$ by $\bar{A}_{\nu}$ for $\nu=0, \ldots, p$, respectively. Moreover we have

$$
\begin{aligned}
m c_{\mu}(\mathbf{A}) & \sim m c_{\mu}\left(\mathbf{A}^{\prime}\right) \text { if } \mathbf{A} \sim \mathbf{A}^{\prime}, \\
m c_{\mu^{\prime}} \circ m c_{\mu}(\mathbf{A}) & \sim m c_{\mu+\mu^{\prime}}(\mathbf{A}), \\
m c_{0}(\mathbf{A}) & \sim \mathbf{A}
\end{aligned}
$$

and $\mathbf{A}$ is irreducible if and only if $\mathbf{A}^{\prime}$ is irreducible.

ii) (cf. O6, Theorem 5.2]) Assume

$$
\mu=\lambda_{0,1} \neq 0 \text { and } \lambda_{j, 1}=0 \text { for } j=1, \ldots, p
$$


and

$$
\lambda_{j, \nu}=\lambda_{j, 1} \quad \text { implies } m_{j, \nu} \leq m_{j, 1}
$$

for $j=0, \ldots, p$ and $\nu=2, \ldots, n_{j}$. Then the Riemann scheme of $m c_{\mu}(\mathbf{A})$ equals

$$
\left\{\begin{array}{cccc}
x=\infty & c_{1} & \cdots & c_{p} \\
{[-\mu]_{m_{0,1}-d}} & {[0]_{m_{1,1}-d}} & \cdots & {[0]_{m_{p, 1}-d}} \\
{\left[\lambda_{0,2}-\mu\right]_{m_{0,2}}} & {\left[\lambda_{1,2}+\mu\right]_{m_{1,2}}} & \cdots & {\left[\lambda_{p, 2}+\mu\right]_{m_{p, 2}}} \\
\vdots & \vdots & \vdots & \vdots \\
{\left[\lambda_{0, n_{0}}-\mu\right]_{m_{0, n_{0}}}} & {\left[\lambda_{1, n_{1}}+\mu\right]_{m_{1, n_{1}}}} & \cdots & {\left[\lambda_{p, n_{p}}+\mu\right]_{m_{p, 1}}}
\end{array}\right\}
$$

with

$$
d:=m_{0,1}+\cdots+m_{p, 1}-(p-1) \text { ord } \mathbf{m} .
$$

Example 11.2. The addition of

$$
m c_{-\lambda_{0,1}-\lambda_{1,2}-\lambda_{2,2}}\left(\left\{\lambda_{0,2}-\lambda_{0,1}, \lambda_{0,1}+\lambda_{1,1}+\lambda_{2,2}, \lambda_{0,1}+\lambda_{1,2}+\lambda_{2,1}\right\}\right)
$$

with respect to $\left(-\lambda_{1,2}-\lambda_{2,2}, \lambda_{1,2}, \lambda_{2,2}\right)$ give the Fuchsian system of Schlesinger canonical form

$$
\begin{gathered}
\frac{d u}{d x}=\frac{A_{1}}{x} u+\frac{A_{2}}{x-1} u, \\
A_{1}=\left(\begin{array}{cc}
\lambda_{1,1} & \lambda_{0,1}+\lambda_{1,2}+\lambda_{2,1} \\
\lambda_{1,2}
\end{array}\right) \text { and } A_{2}=\left(\begin{array}{cc}
\lambda_{2,2} \\
\lambda_{0,1}+\lambda_{1,1}+\lambda_{2,2} & \lambda_{2,1}
\end{array}\right) .
\end{gathered}
$$

with the Riemann scheme

$$
\left\{\begin{array}{ccc}
x=\infty & 0 & 1 \\
\lambda_{0,1} & \lambda_{1,1} & \lambda_{2,1} \\
\lambda_{0,2} & \lambda_{1,2} & \lambda_{2,2}
\end{array}\right\} \quad\left(\lambda_{0,1}+\lambda_{0,2}+\lambda_{1,1}+\lambda_{1,2}+\lambda_{2,1}+\lambda_{2,2}=0\right) .
$$

The system is invariant as $W\left(x ; \lambda_{j, \nu}\right)$-modules under the transformation $\lambda_{j, \nu} \mapsto$ $\lambda_{j, 3-\nu}$ for $j=0,1,2$ and $\nu=1,2$.

Suppose $\lambda_{j, \nu}$ are generic complex numbers under the condition $\lambda_{0,1}+\lambda_{1,2}+\lambda_{2,1}=$ $\lambda_{0,2}+\lambda_{1,1}+\lambda_{2,2}=0$. Then $A_{1}$ and $A_{2}$ have a unique simultaneous eigenspace. In fact, $A_{1}\left(\begin{array}{l}0 \\ 1\end{array}\right)=\lambda_{1,2}\left(\begin{array}{l}0 \\ 1\end{array}\right)$ and $A_{2}\left(\begin{array}{l}0 \\ 1\end{array}\right)=\lambda_{2,1}\left(\begin{array}{l}0 \\ 1\end{array}\right)$. Hence the system is not invariant as $W(x)$-modules under the transformation above and $\mathbf{A}$ is not irreducible in this case.

To describe the monodromies, we review the multiplicative version of these operations.

Let $\mathbf{M}=\left(M_{0}, \ldots, M_{p}\right)$ be an element of

$$
G L(n, \mathbb{C})_{1}^{p+1}:=\left\{\left(G_{0}, \ldots, G_{p}\right) \in G L(n, \mathbb{C})^{p+1} ; G_{p} \cdots G_{0}=I_{n}\right\} .
$$

For $\left(\rho_{0}, \ldots, \rho_{p}\right) \in \mathbb{C}^{p+1}$ satisfying $\rho_{0} \cdots \rho_{p}=1$, the multiplication of $\mathbf{M}$ with respect to $\rho$ is defined by $\left(\rho_{0} M_{0}, \ldots, \rho_{p} M_{p}\right)$.

For a given $\rho \in \mathbb{C}^{\times}$, we define $\tilde{M}_{j}=\left(M_{j, \nu, \nu^{\prime}}\right)_{\substack{1 \leq \nu \leq n \\ 1 \leq \nu^{\prime} \leq p}} \in G L(p n, \mathbb{C})$ by

$$
\tilde{M}_{j, \nu, \nu^{\prime}}= \begin{cases}\delta_{\nu, \nu^{\prime}} I_{n} & (\nu \neq j) \\ M_{\nu^{\prime}}-1 & \left(\nu=j, 1 \leq \nu^{\prime} \leq j-1\right) \\ \rho M_{j} & \left(\nu=\nu^{\prime}=j\right) \\ \rho\left(M_{\nu^{\prime}}-1\right) & \left(\nu=j, j+1 \leq \nu^{\prime} \leq p\right)\end{cases}
$$


Let $\bar{M}_{j}$ denote the quotient $\left.\tilde{M}_{j}\right|_{\mathbb{C}^{p n} / V}$ of

$$
\tilde{M}_{j}=\left(\begin{array}{ccccc}
I_{n} & & & & \\
& \ddots & & & \\
M_{1}-1 & \cdots & \rho M_{j} & \cdots & \rho\left(M_{p}-1\right) \\
& & & \ddots & \\
& & & & I_{n}
\end{array}\right) \in G L(p n, \mathbb{C})
$$

for $j=1, \ldots, p$ and $M_{0}=\left(M_{p} \ldots M_{1}\right)^{-1}$. The tuple $\operatorname{MC}_{\rho}(\mathbf{M})=\left(\bar{M}_{0}, \ldots, \bar{M}_{p}\right)$ is called (the multiplicative version of) the middle convolution of $\mathbf{M}$ with respect to $\rho$. Here $V:=\operatorname{ker}(\tilde{M}-1)+\bigcap_{j=1}^{p} \operatorname{ker}\left(\tilde{M}_{j}-1\right)$ with

$$
\tilde{M}:=\left(\begin{array}{ccc}
M_{1} & & \\
& \ddots & \\
& & M_{p}
\end{array}\right) .
$$

Then we have the following theorem.

Theorem 11.3 (DR, DR2). Let $\mathbf{M}=\left(M_{0}, \ldots, M_{p}\right) \in G L(n, \mathbb{C})_{1}^{p+1}$. Suppose

$$
\begin{aligned}
& \bigcap_{\substack{1 \leq \nu \leq p \\
\nu \leq i}} \operatorname{ker}\left(M_{\nu}-1\right) \cap \operatorname{ker}\left(M_{i}-\tau\right)=\{0\} \quad\left(1 \leq i \leq p, \forall \tau \in \mathbb{C}^{\times}\right), \\
& \bigcap_{\substack{1 \leq \nu \leq p \\
\nu \leq i}} \operatorname{ker}\left({ }^{t} M_{\nu}-1\right) \cap \operatorname{ker}\left({ }^{t} M_{i}-\tau\right)=\{0\} \quad\left(1 \leq i \leq p, \forall \tau \in \mathbb{C}^{\times}\right) .
\end{aligned}
$$

i) The tuple $\mathrm{MC}_{\rho}(\mathbf{M})=\left(\bar{M}_{0}, \ldots, \bar{M}_{p}\right)$ also satisfies the same conditions as above with replacing $M_{\nu}$ by $\bar{M}_{\nu}$ for $\nu=0, \ldots, p$, respectively. Moreover we have

$$
\begin{aligned}
\operatorname{MC}_{\rho}(\mathbf{M}) & \sim \mathrm{MC}_{\rho}\left(\mathbf{M}^{\prime}\right) \text { if } \mathbf{M} \sim \mathbf{M}^{\prime}, \\
\operatorname{MC}_{\rho^{\prime}} \circ \mathrm{MC}_{\rho}(\mathbf{M}) & \sim \mathrm{MC}_{\rho \rho^{\prime}}(\mathbf{M}), \\
\mathrm{MC}_{1}(\mathbf{M}) & \sim \mathbf{M}
\end{aligned}
$$

and $\mathrm{MC}_{\rho}(\mathbf{M})$ is irreducible if and only if $\mathbf{M}$ is irreducible.

ii) Assume

$$
\begin{aligned}
M_{j} & \sim L\left(m_{j, 1}, \ldots, m_{j, n_{j}} ; \rho_{j, 1}, \ldots, \rho_{j, n_{j}}\right) \text { for } j=0, \ldots, p, \\
\rho & =\rho_{0,1} \neq 1 \text { and } \rho_{j, 1}=1 \text { for } j=1, \ldots, p
\end{aligned}
$$

and

$$
\rho_{j, \nu}=\rho_{j, 1} \quad \text { implies } m_{j, \nu} \leq m_{j, 1}
$$

for $j=0, \ldots, p$ and $\nu=2, \ldots, n_{j}$. In this case, we say that $\mathbf{M}$ has a spectral type $\mathbf{m}:=\left(\mathbf{m}_{0}, \ldots, \mathbf{m}_{p}\right)$ with $\mathbf{m}_{j}=\left(m_{j, 1}, \ldots, m_{j, n_{j}}\right)$.

Putting $\left(\bar{M}_{0}, \ldots, \bar{M}_{p}\right)=\mathrm{MC}_{\rho}\left(M_{0}, \ldots, M_{p}\right)$, we have

$$
\bar{M}_{j} \sim \begin{cases}L\left(m_{0,1}-d, m_{0,2}, \ldots, m_{0, n_{0}} ; \rho^{-1}, \rho^{-1} \rho_{0,2}, \ldots \rho^{-1} \rho_{0, n_{0}}\right) & (j=0), \\ L\left(m_{j, 1}-d, m_{j, 2}, \ldots, m_{j, n_{j}} ; 1, \rho \rho_{j, 2}, \ldots \rho \rho_{j, n_{j}}\right) & (j=1, \ldots, p) .\end{cases}
$$

Here $d$ is given by (11.13).

Remark 11.4. i) We note that some $m_{j, 1}$ may be zero in Theorem 11.1 and Theorem 11.3 .

ii) It follows from Theorem 11.1 (resp. Theorem 11.3) and Scott's lemma that any irreducible tuple $\mathbf{A} \in M(n, \mathbb{C})_{0}^{p+1}$ (resp. $\mathbf{M} \in G L(n, \mathbb{C})_{1}^{p+1}$ ) can be connected by successive applications of middle convolutions and additions (resp. multiplications) 
to an tuple whose spectral type is fundamental (cf. Definition 8.14). In particular, the spectral type of $\mathbf{M}$ is an irreducibly realizable tuple if $\mathbf{M}$ is irreducible.

Definition 11.5. Let $\mathbf{M}=\left(M_{0}, \ldots, M_{p}\right) \in G L(n, \mathbb{C})_{1}^{p+1}$. Suppose (11.21). Fix $\ell=\left(\ell_{0}, \ldots, \ell_{p}\right) \in \mathbb{Z}_{>1}^{p+1}$ and define $\partial_{\ell} \mathbf{M}$ as follows.

$$
\begin{aligned}
\rho_{j} & := \begin{cases}\rho_{j, \ell_{j}} & \left(0 \leq j \leq p, 1 \leq \ell_{j} \leq n_{j}\right), \\
\text { any complex number } & \left(0 \leq j \leq p, n_{j}<\ell_{j}\right),\end{cases} \\
\rho & :=\rho_{0} \rho_{1} \ldots \rho_{p}, \\
\left(M_{0}^{\prime}, \ldots, M_{p}^{\prime}\right) & :=\operatorname{MC}_{\rho}\left(\rho_{1} \cdots \rho_{p} M_{0}, \rho_{1}^{-1} M_{1}, \rho_{2}^{-1} M_{2}, \ldots, \rho_{p}^{-1} M_{p}\right), \\
\partial_{\ell} \mathbf{M} & :=\left(\rho_{1}^{-1} \cdots \rho_{p}^{-1} M_{0}^{\prime}, \rho_{1} M_{1}^{\prime}, \rho_{2} M_{2},{ }^{\prime} \ldots, \rho_{p} M_{p}^{\prime}\right) .
\end{aligned}
$$

Here we note that if $\ell=(1, \ldots, 1)$ and $\rho_{j, 1}=1$ for $j=2, \ldots, p, \partial_{\ell} \mathbf{M}=\mathrm{MC}_{\rho}(\mathbf{M})$.

Let $u(1), \ldots, u(n)$ be independent solutions of (11.1) at a generic point $q$. Let $\gamma_{j}$ be a closed path around $c_{j}$ as in the following figure. Denoting the result of the analytic continuation of $\tilde{u}:=(u(1), \ldots, u(n))$ along $\gamma_{j}$ by $\gamma_{j}(\tilde{u})$, we have a monodromy generator $M_{j} \in G L(n, \mathbb{C})$ such that $\gamma_{j}(\tilde{u})=\tilde{u} M_{j}$. We call the tuple $\mathbf{M}=\left(M_{0}, \ldots, M_{p}\right)$ the monodromy of (11.1) with respect to $\tilde{u}$ and $\gamma_{0}, \ldots, \gamma_{p}$. The connecting path first going along $\gamma_{i}$ and then going along $\gamma_{j}$ is denoted by $\gamma_{i} \circ \gamma_{j}$.

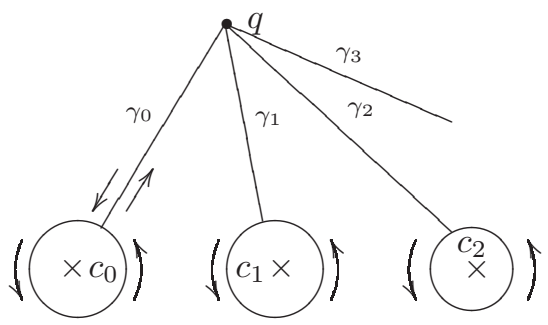

$$
\begin{aligned}
\gamma_{i} \circ \gamma_{j}(\tilde{u}) & =\gamma_{j}\left(\tilde{u} M_{i}\right) \\
& =\gamma_{j}(\tilde{u}) M_{i} \\
& =\tilde{u} M_{j} M_{i}, \\
M_{p} M_{p-1} \cdots & M_{1} M_{0}=I_{n} .
\end{aligned}
$$

The following theorem says that the monodromy of solutions of the system obtained by a middle convolution of the system (11.1) is a multiplicative middle convolution of that of the original system (11.1).

Theorem 11.6 (DR2]). Let $\mathrm{Mon}(\mathbf{A})$ denote the monodromy of the equation (11.1). Put $\mathbf{M}=\operatorname{Mon}(\mathbf{A})$. Suppose $\mathbf{M}$ satisfies (11.16) and (11.17) and

$$
\begin{aligned}
\operatorname{rank}\left(A_{0}-\mu\right) & =\operatorname{rank}\left(M_{0}-e^{2 \pi \sqrt{-1} \mu}\right), \\
\operatorname{rank}\left(A_{j}\right) & =\operatorname{rank}\left(M_{j}-1\right)
\end{aligned}
$$

for $j=1, \ldots, p$, then

$$
\operatorname{Mon}\left(m c_{\mu}(\mathbf{A})\right) \sim \operatorname{MC}_{e^{2 \pi \sqrt{-1} \mu}}(\operatorname{Mon}(\mathbf{A})) .
$$

Let $\mathcal{F}$ be a space of (multi-valued) holomorphic functions on $\mathbb{C} \backslash\left\{c_{1}, \ldots, c_{p}\right\}$ valued in $\mathbb{C}^{n}$ such that $\mathcal{F}$ satisfies (3.15), (3.16) and (3.17). For example the solutions of the equation (11.1) defines $\mathcal{F}$. Fixing a base $u=(u(1), \ldots, u(n))$ of $\mathcal{F}(U)$ with $U \ni q$, we can define monodromy generators $\left(M_{0}, \ldots, M_{p}\right)$. Fix $\mu \in \mathbb{C}$ and put $\rho=e^{2 \pi \sqrt{-1} \mu}$ and

$$
v_{j}(x)=\left(\begin{array}{cc}
\int^{\left(x+, c_{j}+, x-, c_{j}-\right)} & \frac{u(t)(x-t)^{\mu-1}}{t-c_{1}} d t \\
\vdots & \\
\int\left(x+, c_{j}+, x-, c_{j}-\right) & \frac{u(t)(x-t)^{\mu-1}}{t-c_{p}} d t
\end{array}\right) \text { and } v(x)=\left(v_{1}(x), \ldots, v_{p}(x)\right) .
$$

Then $v(x)$ is a holomorphic function valued in $M(p n, \mathbb{C})$ and the $p n$ column vectors of $v(x)$ define a convolution $\tilde{\mathcal{F}}$ of $\mathcal{F}$ and the following facts are shown by [DR2]. 
The monodromy generators of $\tilde{\mathcal{F}}$ with respect to the base $v(x)$ equals the convolution $\tilde{\mathbf{M}}=\left(\tilde{M}_{0}, \ldots, \tilde{M}_{1}\right)$ of $\mathbf{M}$ given by (11.15) and if $\mathcal{F}$ corresponds to the space of solutions of (2.74), $\tilde{\mathcal{F}}$ corresponds to that of the system of Schlesinger canonical form defined by $\left(\tilde{A}_{0}(\mu), \ldots, \tilde{A}_{p}(\mu)\right)$ in (2.77), which we denote by $\mathcal{M}_{\tilde{\mathbf{A}}}$.

The middle convolution $\mathrm{MC}_{\rho}(\mathbf{M})$ of $\mathbf{M}$ is the induced monodromy generators on the quotient space of $\mathbb{C}^{p n} / V$ where $V$ is the maximal invariant subspace such the restriction of $\tilde{\mathbf{M}}$ on $V$ is a direct sum of finite copies of 1-dimensional spaces with the actions $\left(\rho^{-1}, 1, \ldots, 1, \stackrel{j}{\rho}, 1, \ldots, 1\right) \in G L(1, \mathbb{C})_{1}^{p+1}(j=1, \ldots, p)$ and $(1,1, \ldots, 1)$. The system defined by the middle convolution $m c_{\mu}(\mathbf{A})$ is the quotient of the system $\mathcal{M}_{\tilde{\mathbf{A}}}$ by the maximal submodule such that the submodule is a direct sum of finite copies of the equations $\left(x-c_{j}\right) \frac{d w}{d x}=\mu w(j=1, \ldots, p)$ and $\frac{d w}{d x}=0$.

Suppose $\mathbf{M}$ and $\mathrm{MC}_{\rho}(\mathbf{M})$ are irreducible and $\rho \neq 1$. Assume $\phi(x)$ is a function belonging to $\mathcal{F}$ such that it is defined around $x=c_{j}$ and corresponds to the eigenvector of the monodromy matrix $M_{j}$ with the eigenvalue different from 1. Then the holomorphic continuation of $\Phi(x)=\int^{\left(x+, c_{j}+, x-, c_{j}-\right)} \frac{\phi(t)(t-x)^{\mu}}{t-c_{j}} d t$ defines the monodromy isomorphic to $\mathrm{MC}_{\rho}(\mathbf{M})$.

Remark 11.7. We can define the monodromy $\mathbf{M}=\left(M_{0}, \ldots, M_{p}\right)$ of the universal model $P_{\mathbf{m}} u=0$ (cf. Theorem 8.13) so that $\mathbf{M}$ is entire holomorphic with respect to the spectral parameters $\lambda_{j, \nu}$ and the accessory parameters $g_{i}$ under the normalization $u(j)^{(\nu-1)}(q)=\delta_{j, \nu}$ for $j, \nu=1, \ldots, n$ and $q \in \mathbb{C} \backslash\left\{c_{1}, \ldots, c_{p}\right\}$. Here $u(1), \ldots, u(n)$ are solutions of $P_{\mathbf{m}} u=0$.

Definition 11.8. Let $P$ be a Fuchsian differential operator with the Riemann scheme 6.15) and the spectral type $\mathbf{m}=\left(m_{j, \nu}\right)_{\substack{0 \leq j \leq p \\ 1 \leq \nu \leq n_{j}}}$. We define that $P$ is locally non-degenerate if the tuple of the monodromy generators $\mathbf{M}:=\left(M_{0}, \ldots, M_{p}\right)$ satisfies

$$
M_{j} \sim L\left(m_{j, 1}, \ldots, m_{j, n_{j}} ; e^{2 \pi \sqrt{-1} \lambda_{j, 1}}, \ldots, e^{2 \pi \sqrt{-1} \lambda_{j, n_{j}}}\right) \quad(j=0, \ldots, p),
$$

which is equivalent to the condition that

$$
\operatorname{dim} Z\left(M_{j}\right)=m_{j, 1}^{2}+\cdots+m_{j, n_{j}}^{2} \quad(j=0, \ldots, p) .
$$

Suppose $\mathbf{m}$ is irreducibly realizable. Let $P_{\mathbf{m}}$ be the universal operator with the Riemann scheme 6.15). We say that the parameters $\lambda_{j, \nu}$ and $g_{i}$ are locally nondegenerate if the corresponding operator is locally non-degenerate.

Note that the parameters are locally non-degenerate if

$$
\lambda_{j, \nu}-\lambda_{j, \nu^{\prime}} \notin \mathbb{Z} \quad\left(j=0, \ldots, p, \nu=1, \ldots, n_{j}, \nu^{\prime}=1, \ldots, n_{j}\right) .
$$

Define $P_{t}$ as in Remark 6.4 iv). Then we can define monodromy generator $M_{t}$ of $P_{t}$ at $x=c_{j}$ so that $M_{t}$ holomorphically depend on $t$ (cf. Remark 11.7). Then Remark 6.13 v) proves that (11.30) implies (11.29) for every $j$.

The following proposition gives a sufficient condition such that an operator is locally non-degenerate.

Proposition 11.9. Let $P$ be a Fuchsian differential operator with the Riemann scheme (6.15) and let $M_{j}$ be the monodromy generator at $x=c_{j}$. Fix an integer $j$ with $0 \leq j \leq p$. Then the condition

$$
\begin{gathered}
\lambda_{j, \nu}-\lambda_{j, \nu^{\prime}} \notin \mathbb{Z} \text { or }\left(\lambda_{j, \nu}-\lambda_{j, \nu^{\prime}}\right)\left(\lambda_{j, \nu}+m_{j, \nu}-\lambda_{j, \nu^{\prime}}-m_{j, \nu^{\prime}}\right) \leq 0 \\
\text { for } 1 \leq \nu \leq n_{j} \quad \text { and } 1 \leq \nu^{\prime} \leq n_{j}
\end{gathered}
$$

implies $\operatorname{dim} Z\left(M_{j}\right)=m_{j, 1}^{2}+\cdots+m_{j, n_{j}}^{2}$. In particular, $P$ is locally non-degenerate if (11.31) is valid for $j=0, \ldots, p$. 
Here we remark that the following condition implies (11.31).

$$
\lambda_{j, \nu}-\lambda_{j, \nu^{\prime}} \notin \mathbb{Z} \backslash\{0\} \quad \text { for } 1 \leq \nu \leq n_{j} \text { and } 1 \leq \nu^{\prime} \leq n_{j} .
$$

Proof. For $\mu \in \mathbb{C}$ we put

$$
N_{\mu}=\left\{\nu ; 1 \leq \nu \leq n_{j}, \mu \in\left\{\lambda_{j, \nu}, \lambda_{j, \nu}+1, \ldots, \lambda_{j, \nu}+m_{j, \nu}-1\right\}\right\} .
$$

If $N_{\mu}>0$, we have a local solution $u_{\mu, \nu}(x)$ of the equation $P u=0$ such that

$$
u_{\mu, \nu}(x)=\left(x-c_{j}\right)^{\mu} \log ^{\nu}\left(x-c_{j}\right)+\mathcal{O}_{c_{j}}\left(\mu+1, L_{\nu}\right) \text { for } \nu=0, \ldots, N_{\mu}-1
$$

Here $L_{\nu}$ are positive integers and if $j=0$, then $x$ and $x-c_{j}$ should be replaced by $y=\frac{1}{x}$ and $y$, respectively.

Suppose (11.31). Put $\rho=e^{2 \pi \mu i}, \mathbf{m}_{\rho}^{\prime}=\left\{m_{j, \nu} ; \lambda_{j, \nu}-\mu \in \mathbb{Z}\right\}$ and $\mathbf{m}_{\rho}^{\prime}=$ $\left\{m_{\rho, 1}^{\prime}, \ldots, m_{\rho, n_{\rho}}^{\prime}\right\}$ with $m_{\rho, 1}^{\prime} \geq m_{\rho, 2}^{\prime} \geq \cdots \geq m_{\rho, n_{\rho}}^{\prime} \geq 1$. Then (11.31 implies

$$
n-\operatorname{rank}\left(M_{j}-\rho\right)^{k} \leq \begin{cases}m_{\rho, 1}^{\prime}+\cdots+m_{\rho, k}^{\prime} & \left(1 \leq k \leq n_{\rho}\right), \\ m_{\rho, 1}^{\prime}+\cdots+m_{\rho, n_{\rho}}^{\prime} & \left(n_{\rho}<k\right) .\end{cases}
$$

The above argument proving (11.29) under the condition (11.30) shows that the left hand side of (11.34) is not smaller than the right hand side of (11.34). Hence we have the equality in (11.34). Thus we have (11.30) and we can assume that $L_{\nu}=\nu$ in $(11.33$.

Theorem 11.3, Theorem 11.6 and Proposition 4.1 show the following corollary. One can also prove it by the same way as in the proof of [DR2, Theorem 4.7].

Corollary 11.10. Let $P$ be a Fuchsian differential operator with the Riemann scheme (6.15). Let $\operatorname{Mon}(P)$ denote the monodromy of the equation $P u=0$. Put $\operatorname{Mon}(P)=\left(M_{0}, \ldots, M_{p}\right)$. Suppose

$$
M_{j} \sim L\left(m_{j, 1}, \ldots, m_{j, n_{j}} ; e^{2 \pi \sqrt{-1} \lambda_{j, 1}}, \ldots, e^{2 \pi \sqrt{-1} \lambda_{j, n_{j}}}\right) \quad \text { for } j=0, \ldots, p .
$$

In this case, $P$ is said to be locally non-degenerate. Under the notation in Definition 7.6, we fix $\ell \in \mathbb{Z}_{\geq 1}^{p+1}$ and suppose (7.23). Assume moreover

$$
\begin{aligned}
\mu_{\ell} & \notin \mathbb{Z} \\
m_{j, \nu} \leq m_{j, \ell_{j}} \quad \text { or } \quad \lambda_{j, \ell_{j}}-\lambda_{j, \nu} & \notin \mathbb{Z} \quad\left(j=0, \ldots, p, \nu=1, \ldots, n_{j}\right) .
\end{aligned}
$$

Then we have

$$
\operatorname{Mon}\left(\partial_{\ell} P\right) \sim \partial_{\ell} \operatorname{Mon}(P) .
$$

In particular, $\operatorname{Mon}(P)$ is irreducible if and only if $\operatorname{Mon}\left(\partial_{\ell} P\right)$ is irreducible.

11.2. Scott's lemma and Katz's rigidity. The results in this subsection are known but we will review them with their proof for the completeness of this paper.

Lemma 11.11 (Scott $\mathrm{Sd})$. Let $\mathbf{M} \in G L(n, \mathbb{C})_{1}^{p+1}$ and $\mathbf{A} \in M(n, \mathbb{C})_{0}^{p+1}$ under the notation (11.2) and (11.14). Then

$$
\begin{gathered}
\sum_{j=0}^{p} \operatorname{codim} \operatorname{ker}\left(M_{j}-1\right) \geq \operatorname{codim} \bigcap_{j=0}^{p} \operatorname{ker}\left(M_{j}-1\right)+\operatorname{codim} \bigcap_{j=0}^{p} \operatorname{ker}\left({ }^{t} M_{j}-1\right) \\
\quad \sum_{j=0}^{p} \operatorname{codim} \operatorname{ker} A_{j} \geq \operatorname{codim} \bigcap_{j=0}^{p} \operatorname{ker} A_{j}+\operatorname{codim} \bigcap_{j=0}^{p} \operatorname{ker}^{t} A_{j} .
\end{gathered}
$$


In particular, if $\mathbf{M}$ and $\mathbf{A}$ are irreducible, then

$$
\begin{aligned}
\sum_{j=0}^{p} \operatorname{dim} \operatorname{ker}\left(M_{j}-1\right) & \leq(p-1) n, \\
\sum_{j=0}^{p} \operatorname{dim} \operatorname{ker} A_{j} & \leq(p-1) n .
\end{aligned}
$$

Proof. Consider the following linear maps:

$$
\begin{aligned}
V & =\operatorname{Im}\left(M_{0}-1\right) \times \cdots \times \operatorname{Im}\left(M_{p}-1\right) \subset \mathbb{C}^{n(p+1)} \\
\beta & : \mathbb{C}^{n} \rightarrow V, \quad v \mapsto\left(\left(M_{0}-1\right) v, \ldots,\left(M_{p}-1\right) v\right), \\
\delta: & V \rightarrow \mathbb{C}^{n}, \quad\left(v_{0}, \ldots, v_{p}\right) \mapsto M_{p} \cdots M_{1} v_{0}+M_{p} \cdots M_{2} v_{1}+\cdots+M_{p} v_{p-1}+v_{p} .
\end{aligned}
$$

Since $M_{p} \cdots M_{1}\left(M_{0}-1\right)+\cdots+M_{p}\left(M_{p-1}-1\right)+\left(M_{p}-1\right)=M_{p} \cdots M_{1} M_{0}-1=0$, we have $\delta \circ \beta=0$. Moreover we have

$$
\begin{aligned}
& \sum_{j=0}^{p} M_{p} \cdots M_{j+1}\left(M_{j}-1\right) v_{j}=\sum_{j=0}^{p}\left(1+\sum_{\nu=j+1}^{p}\left(M_{\nu}-1\right) M_{\nu-1} \cdots M_{j+1}\right)\left(M_{j}-1\right) v_{j} \\
& \quad=\sum_{j=0}^{p}\left(M_{j}-1\right) v_{j}+\sum_{\nu=1}^{p} \sum_{i=0}^{\nu-1}\left(M_{\nu}-1\right) M_{\nu-1} \cdots M_{i+1}\left(M_{i}-1\right) v_{i} \\
& \quad=\sum_{j=0}^{p}\left(M_{j}-1\right)\left(v_{j}+\sum_{i=0}^{j-1} M_{j+1} \cdots M_{i-1}\left(M_{i}-1\right) v_{i}\right)
\end{aligned}
$$

and therefore $\operatorname{Im} \delta=\sum_{j=0}^{p} \operatorname{Im}\left(M_{j}-1\right)$. Hence

and

$$
\operatorname{dim} \operatorname{Im} \delta=\operatorname{rank}\left(M_{0}-1, \ldots, M_{p}-1\right)=\operatorname{rank}\left(\begin{array}{c}
{ }^{t} M_{0}-1 \\
\vdots \\
{ }^{t} M_{p}-1
\end{array}\right)
$$

$$
\begin{aligned}
\sum_{j=0}^{p} \operatorname{codim} \operatorname{ker}\left(M_{j}-1\right) & =\operatorname{dim} V=\operatorname{dim} \operatorname{ker} \delta+\operatorname{dim} \operatorname{Im} \delta \\
& \geq \operatorname{dim} \operatorname{Im} \beta+\operatorname{dim} \operatorname{Im} \delta \\
& =\operatorname{codim} \bigcap_{j=0}^{p} \operatorname{ker}\left(M_{j}-1\right)+\operatorname{codim} \bigcap_{j=0}^{p} \operatorname{ker}\left({ }^{t} M_{j}-1\right) .
\end{aligned}
$$

Putting

$$
\begin{aligned}
& V=\operatorname{Im} A_{0} \times \cdots \times \operatorname{Im} A_{p} \subset \mathbb{C}^{n(p+1)}, \\
& \beta: \mathbb{C}^{n} \rightarrow V, \quad v \mapsto\left(A_{0} v, \ldots, A_{p} v\right), \\
& \delta: V \rightarrow \mathbb{C}^{n}, \quad\left(v_{0}, \ldots, v_{p}\right) \mapsto v_{0}+v_{1}+\cdots+v_{p},
\end{aligned}
$$

we have the claims for $\mathbf{A} \in M(n, \mathbb{C})^{p+1}$ in the same way as in the proof for $\mathbf{M} \in$ $G L(n, \mathbb{C})_{1}^{p+1}$.

Corollary 11.12 (Katz [K] and [SV]). Let $\mathbf{M} \in G L(n, \mathbb{C})_{1}^{p+1}$. The dimensions of the manifolds

$$
V_{1}:=\left\{\mathbf{H} \in G L(n, \mathbb{C})_{1}^{p+1} ; \mathbf{H} \sim \mathbf{M}\right\}
$$

and

$$
V_{2}:=\left\{\mathbf{H} \in G L(n, \mathbb{C})_{1}^{p+1} ; H_{j} \sim M_{j} \quad(j=0, \ldots, p)\right\}
$$


are give by

$$
\begin{aligned}
& \operatorname{dim} V_{1}=\operatorname{codim} Z(\mathbf{M}) \\
& \operatorname{dim} V_{2}=\sum_{j=0}^{p} \operatorname{codim} Z\left(M_{j}\right)-\operatorname{codim} Z(\mathbf{M}) .
\end{aligned}
$$

Here $Z(\mathbf{M}):=\bigcap_{j=0}^{p} Z\left(M_{j}\right)$ and $Z\left(M_{i}\right)=\left\{X \in M(n, \mathbb{C}) ; X M_{j}=M_{j} X\right\}$.

Suppose $\mathbf{M}$ is irreducible. Then $\operatorname{codim} Z(\mathbf{M})=n^{2}-1$ and

$$
\sum_{j=0}^{p} \operatorname{codim} Z\left(M_{j}\right) \geq 2 n^{2}-2
$$

Moreover $\mathbf{M}$ is rigid, namely, $V_{1}=V_{2}$ if and only if $\sum_{j=0}^{p} \operatorname{codim} Z\left(M_{j}\right)=2 n^{2}-2$.

Proof. The group $G L(n, \mathbb{C})$ transitively acts on $V_{1}$ as simultaneous conjugations and the isotropy group with respect to $\mathbf{M}$ equals $Z(\mathbf{M})$ and hence $\operatorname{dim} V_{1}=$ $\operatorname{codim} Z(\mathbf{M})$.

The group $G L(n, \mathbb{C})^{p+1}$ naturally acts on $G L(n, \mathbb{C})^{p+1}$ by conjugations. Putting $L=\left\{\left(g_{j}\right) \in G L(n, \mathbb{C})^{p+1} ; g_{p} M_{p} g_{p}^{-1} \cdots g_{0} M_{0} g_{0}^{-1}=M_{p} \cdots M_{0}\right\}, V_{2}$ is identified with $L / Z\left(M_{0}\right) \times \cdots \times Z\left(M_{p}\right)$. Denoting $g_{j}=\exp \left(t X_{j}\right)$ with $X_{j} \in M(n, \mathbb{C})$ and $t \in \mathbb{R}$ with $|t| \ll 1$ and defining $A_{j} \in \operatorname{End}(M(n, \mathbb{C}))$ by $A_{j} X=M_{j} X M_{j}^{-1}$, we can prove that the dimension of $L$ equals the dimension of the kernel of the map

$$
\gamma: M(n, \mathbb{C})^{p+1} \ni\left(X_{0}, \ldots, X_{p}\right) \mapsto \sum_{j=0}^{p} A_{p} \cdots A_{j+1}\left(A_{j}-1\right) X_{j}
$$

by looking at the tangent space of $L$ at the identity element because

$$
\begin{aligned}
& \exp \left(t X_{p}\right) M_{p} \exp \left(-t X_{p}\right) \cdots \exp \left(t X_{0}\right) M_{0}\left(-t X_{0}\right)-M_{p} \cdots M_{0} \\
& \quad=t\left(\sum_{j=0}^{p} A_{p} \cdots A_{j+1}\left(A_{j}-1\right) X_{j}\right) M_{p} \cdots M_{0}+o(t) .
\end{aligned}
$$

We have obtained in the proof of Lemma 11.11 that codim $\operatorname{ker} \gamma=\operatorname{dim} \operatorname{Im} \gamma=$ $\operatorname{dim} \sum_{j=0}^{p} \operatorname{Im}\left(A_{j}-1\right)=\operatorname{codim} \bigcap_{j=0}^{p} \operatorname{ker}\left({ }^{t} A_{j}-1\right)$. We will see that $\bigcap_{j=0}^{p} \operatorname{ker}\left({ }^{t} A_{j}-1\right)$ is identified with $Z(\mathbf{M})$ and hence $\operatorname{codim} \operatorname{ker} \gamma=\operatorname{codim} Z(\mathbf{M})$ and

$$
\operatorname{dim} V_{2}=\operatorname{dim} \operatorname{ker} \gamma-\sum_{j=0}^{p} \operatorname{dim} Z\left(M_{j}\right)=\sum_{j=0}^{p} \operatorname{codim} Z\left(M_{j}\right)-\operatorname{codim} Z(\mathbf{M})
$$

In general, fix $\mathbf{H} \in V_{1}$ and define $A_{j} \in \operatorname{End}(M(n, \mathbb{C}))$ by $X \mapsto M_{j} X H_{j}^{-1}$ for $j=0, \ldots, p$. Note that $A_{p} A_{p-1} \cdots A_{0}$ is the identity map. If we identify $M(n, \mathbb{C})$ with its dual by the inner product trace $X Y$ for $X, Y \in M(n, \mathbb{C}),{ }^{t} A_{j}$ are identified with the map $Y \mapsto H_{j}^{-1} Y M_{j}$, respectively.

Fix $P_{j} \in G L(n, \mathbb{C})$ such that $H_{j}=P_{j} M_{j} P_{j}^{-1}$. Then

$$
\begin{aligned}
A_{j}(X) & =X \Leftrightarrow M_{j} X H_{j}^{-1}=X \Leftrightarrow M_{j} X=X P_{j} M_{j} P_{j}^{-1} \Leftrightarrow M_{j} X P_{j}=X P_{j} M_{j}, \\
{ }^{t} A_{j}(X) & =X \Leftrightarrow H_{j}^{-1} X M_{j}=X \Leftrightarrow X M_{j}=P_{j} M_{j} P_{j}^{-1} X \Leftrightarrow P_{j}^{-1} X M_{j}=M_{j} P_{j}^{-1} X
\end{aligned}
$$

and codim $\operatorname{ker}\left(A_{j}-1\right)=\operatorname{codim} Z\left(M_{j}\right)$ and $\bigcap_{j=0}^{p} \operatorname{ker}\left({ }^{t} A_{j}-1\right) \simeq Z(\mathbf{M})$.

Suppose $\mathbf{M}$ is irreducible. Then $\operatorname{codim} Z(\mathbf{M})=n^{2}-1$ and the inequality (11.47) follows from $V_{1} \subset V_{2}$. Moreover suppose $\sum_{j=0}^{p} \operatorname{codim} Z\left(M_{i}\right)=2 n^{2}-2$. Then 
Scott's lemma proves

$$
\begin{aligned}
2 n^{2}-2= & \sum_{j=0}^{p} \operatorname{codim} \operatorname{ker}\left(A_{j}-1\right) \\
\geq & n^{2}-\operatorname{dim} \bigcap_{j=0}^{p}\left\{X \in M(n, \mathbb{C}) ; M_{j} X=X H_{j}\right\} \\
& +n^{2}-\operatorname{dim} \bigcap_{j=0}^{p}\left\{X \in M(n, \mathbb{C}) ; H_{j} X=X M_{j}\right\} .
\end{aligned}
$$

Hence there exists a non-zero matrix $X$ such that $M_{j} X=X H_{j}(j=0, \ldots, p)$ or $H_{j} X=X M_{j}(j=0, \ldots, p)$. If $M_{j} X=X H_{j}$ (resp. $\left.H_{j} X=X M_{j}\right)$ for $j=0, \ldots, p$, $\operatorname{ker} X($ resp. $\operatorname{Im} X)$ is $M_{j}$-stable for $j=0, \ldots, p$ and hence $X \in G L(n, \mathbb{C})$ because $\mathbf{M}$ is irreducible, Thus we have $V_{1}=V_{2}$ and we get all the claims in the corollary.

\section{ReducibiLIty}

12.1. Direct decompositions. For a realizable $(p+1)$-tuple $\mathbf{m} \in \mathcal{P}_{p+1}^{(n)}$, Theorem 8.13 gives the universal Fuchsian differential operator $P_{\mathbf{m}}\left(\lambda_{j, \nu}, g_{i}\right)$ with the Riemann scheme (6.15). Here $g_{1}, \ldots, g_{N}$ are accessory parameters and $N=\operatorname{Ridx} \mathbf{m}$.

First suppose $\mathbf{m}$ is basic. Choose positive numbers $n^{\prime}, n^{\prime \prime}, m_{j, 1}^{\prime}$ and $m_{j, 1}^{\prime \prime}$ such that

$$
\begin{gathered}
n=n^{\prime}+n^{\prime \prime}, \quad 0<m_{j, 1}^{\prime} \leq n^{\prime}, \quad 0<m_{j, 1}^{\prime \prime} \leq n^{\prime \prime}, \\
m_{0,1}^{\prime}+\cdots+m_{p, 1}^{\prime} \leq(p-1) n^{\prime}, \quad m_{0,1}^{\prime \prime}+\cdots+m_{p, 1}^{\prime \prime} \leq(p-1) n^{\prime \prime} .
\end{gathered}
$$

We choose other positive integers $m_{j, \nu}^{\prime}$ and $m_{j, \nu}^{\prime \prime}$ so that $\mathbf{m}^{\prime}=\left(m_{j, \nu}^{\prime}\right)$ and $\mathbf{m}^{\prime \prime}=$ $\left(m_{j, \nu}^{\prime \prime}\right)$ are monotone tuples of partitions of $n^{\prime}$ and $n^{\prime \prime}$, respectively, and moreover

$$
\mathbf{m}=\mathbf{m}^{\prime}+\mathbf{m}^{\prime \prime} .
$$

Theorem 8.6 shows that $\mathbf{m}^{\prime}$ and $\mathbf{m}^{\prime \prime}$ are realizable. If $\left\{\lambda_{j, \nu}\right\}$ satisfies the Fuchs relation

$$
\sum_{j=0}^{p} \sum_{\nu=1}^{n_{j}} m_{j, \nu}^{\prime} \lambda_{j, \nu}=n^{\prime}-\frac{\mathrm{idx} \mathbf{m}^{\prime}}{2}
$$

for the Riemann scheme $\left\{\left[\lambda_{j, \nu}\right]_{\left(m_{j, \nu}^{\prime}\right)}\right\}$, Theorem 6.19 shows that the operators

$$
P_{\mathbf{m}^{\prime \prime}}\left(\lambda_{j, \nu}+m_{j, \nu}^{\prime}-\delta_{j, 0}(p-1) n^{\prime}, g_{i}^{\prime \prime}\right) \cdot P_{\mathbf{m}^{\prime}}\left(\lambda_{j, \nu}, g_{i}^{\prime}\right)
$$

has the Riemann scheme $\left\{\left[\lambda_{j, \nu}\right]_{\left(m_{j, \nu}\right)}\right\}$. This shows that the equation $P_{\mathbf{m}}\left(\lambda_{j, \nu}, g_{i}\right) u=$ 0 is not irreducible when the parameters take the values corresponding to (12.4).

In this subsection, we study the condition

$$
\operatorname{Ridx} \mathbf{m}=\operatorname{Ridx} \mathbf{m}^{\prime}+\operatorname{Ridx} \mathbf{m}^{\prime \prime}
$$

for realizable tuples $\mathbf{m}^{\prime}$ and $\mathbf{m}^{\prime \prime}$ with $\mathbf{m}=\mathbf{m}^{\prime}+\mathbf{m}^{\prime \prime}$. Under this condition the Fuchs relation (12.3) assures that the universal operator is reducible for any values of accessory parameters.

Definition 12.1 (direct decomposition). If realizable tuples $\mathbf{m}, \mathbf{m}^{\prime}$ and $\mathbf{m}^{\prime \prime}$ satisfy (12.2) and (12.5), we define that $\mathbf{m}$ is the direct sum of $\mathbf{m}^{\prime}$ and $\mathbf{m}^{\prime \prime}$ and call $\mathbf{m}=\mathbf{m}^{\prime}+\mathbf{m}^{\prime \prime}$ a direct decomposition of $\mathbf{m}$ and express it as follows.

$$
\mathbf{m}=\mathbf{m}^{\prime} \oplus \mathbf{m}^{\prime \prime} .
$$


Theorem 12.2. Let (12.6) be a direct decomposition of a realizable tuple $\mathbf{m}$.

i) Suppose $\mathbf{m}$ is irreducibly realizable and $\mathrm{idx} \mathbf{m}^{\prime \prime}>0$. Put $\overline{\mathbf{m}}^{\prime}=\operatorname{gcd}\left(\mathbf{m}^{\prime}\right)^{-1} \mathbf{m}^{\prime}$. If $\mathbf{m}^{\prime}$ is indivisible or $\mathrm{idx} \mathbf{m} \leq 0$, then

$$
\alpha_{\mathbf{m}}=\alpha_{\mathbf{m}^{\prime}}-2 \frac{\left(\alpha_{\overline{\mathbf{m}}^{\prime \prime}} \mid \alpha_{\mathbf{m}^{\prime}}\right)}{\left(\alpha_{\overline{\mathbf{m}}^{\prime \prime}} \mid \alpha_{\overline{\mathbf{m}}^{\prime \prime}}\right)} \alpha_{\overline{\mathbf{m}}^{\prime \prime}}
$$

or $\mathbf{m}=\mathbf{m}^{\prime} \oplus \mathbf{m}^{\prime \prime}$ is isomorphic to one of the decompositions

$$
\begin{aligned}
32,32,32,221 & =22,22,22,220 \oplus 10,10,10,10,001 \\
322,322,2221 & =222,222,2220 \oplus 100,100,0001 \\
54,3222,22221 & =44,2222,22220 \oplus 10,1000,00001 \\
76,544,2222221 & =66,444,2222220 \oplus 10,100,0000001
\end{aligned}
$$

under the action of $\widetilde{W}_{\infty}$.

ii) Suppose idx $\mathbf{m} \leq 0$ and $\operatorname{idx} \mathbf{m}^{\prime} \leq 0$ and $\operatorname{idx} \mathbf{m}^{\prime \prime} \leq 0$. Then $\mathbf{m}=\mathbf{m}^{\prime} \oplus \mathbf{m}^{\prime \prime}$ or $\mathbf{m}=\mathbf{m}^{\prime \prime} \oplus \mathbf{m}^{\prime}$ is transformed into one of the decompositions

$$
\begin{aligned}
& \Sigma=11,11,11,11 \quad 111,111,111 \quad 22,1^{4}, 1^{4} \quad 33,222,1^{6} \\
& m \Sigma=k \Sigma \oplus \ell \Sigma \\
& m m, m m, m m, m(m-1) 1=k k, k k, k k, k(k-1) 1 \oplus \ell \ell, \ell \ell, \ell \ell, \ell \ell 0 \\
& m m m, m m m, m m(m-1) 1=k k k, k k k, k k k, k k(k-1) 1 \oplus \ell \ell \ell, \ell \ell \ell, \ell \ell \ell 0 \\
&(2 m)^{2}, m^{4}, m m m(m-1) 1=(2 k)^{2}, k^{4}, k^{4}, k k k(k-1) 1 \oplus(2 \ell)^{2}, \ell^{4}, \ell^{4} 0 \\
&(3 m)^{2},(2 m)^{3}, m^{5}(m-1) 1=(3 k)^{2},(2 k)^{3}, k^{5}(k-1) 1 \oplus(3 \ell)^{2},(2 \ell)^{3}, \ell^{6} 0
\end{aligned}
$$

under the action of $\widetilde{W}_{\infty}$. Here $m, k$ and $\ell$ are positive integers satisfying $m=k+\ell$. These are expressed by

$$
\begin{array}{lll}
m \tilde{D}_{4}=k \tilde{D}_{4} \oplus \ell \tilde{D}_{4}, & m \tilde{E}_{j}=k \tilde{E}_{j} \oplus \ell \tilde{E}_{j} & (j=6,7,8), \\
D_{4}^{(m)}=D_{4}^{(k)} \oplus \ell \tilde{D}_{4}, & E_{j}^{(m)}=E_{j}^{(k)} \oplus \ell \tilde{E}_{j} & (j=6,7,8) .
\end{array}
$$

Proof. Put $\mathbf{m}^{\prime}=k \overline{\mathbf{m}}^{\prime}$ and $\mathbf{m}^{\prime \prime}=\ell \overline{\mathbf{m}}^{\prime \prime}$ with indivisible $\overline{\mathbf{m}}^{\prime}$ and $\overline{\mathbf{m}}^{\prime \prime}$. First note that

$$
\left(\alpha_{\mathbf{m}} \mid \alpha_{\mathbf{m}}\right)=\left(\alpha_{\mathbf{m}^{\prime}} \mid \alpha_{\mathbf{m}^{\prime}}\right)+2\left(\alpha_{\mathbf{m}^{\prime}} \mid \alpha_{\mathbf{m}^{\prime \prime}}\right)+\left(\alpha_{\mathbf{m}^{\prime \prime}} \mid \alpha_{\mathbf{m}^{\prime \prime}}\right) .
$$

ii) Using Lemma 12.3, we will prove the theorem. If idx $\mathbf{m}=0$, then (12.11) and (12.12) show $0=\left(\alpha_{\mathbf{m}^{\prime}} \mid \alpha_{\mathbf{m}^{\prime \prime}}\right)=k \ell\left(\alpha_{\overline{\mathbf{m}}^{\prime}} \mid \alpha_{\overline{\mathbf{m}}^{\prime \prime}}\right)$, Lemma 12.3 proves idx $\mathbf{m}^{\prime}=0$ and $\overline{\mathbf{m}}^{\prime}=\overline{\mathbf{m}}^{\prime \prime}$ and we have the theorem.

Suppose idx $\mathbf{m}<0$.

If idx $\mathbf{m}^{\prime}<0$ and idx $\mathbf{m}^{\prime \prime}<0$, we have Pidx $\mathbf{m}=\operatorname{Pidx} \mathbf{m}^{\prime}+\operatorname{Pidx} \mathbf{m}^{\prime \prime}$, which implies $\left(\alpha_{\mathbf{m}^{\prime}} \mid \alpha_{\mathbf{m}^{\prime \prime}}\right)=-1$ and contradicts to Lemma 12.3 .

Hence we may assume idx $\mathbf{m}^{\prime \prime}=0$.

Case: idx $\mathbf{m}^{\prime}<0$. It follows from (12.11) that $2-2 \operatorname{Ridx} \mathbf{m}=2-2 \operatorname{Ridx} \mathbf{m}^{\prime}+$ $2 \ell(\mathbf{m}, \overline{\mathbf{m}})$. Since $\operatorname{Ridx} \mathbf{m}=\operatorname{Ridx} \mathbf{m}^{\prime}+\ell$, we have $\left(\alpha_{\mathbf{m}} \mid \alpha_{\overline{\mathbf{m}}^{\prime}}\right)=-1$ and the theorem follows from Lemma 12.3 .

Case: $\mathrm{idx} \mathbf{m}^{\prime}=0$. It follows from (12.11) that $2-2 \operatorname{Ridx} \mathbf{m}=2 k \ell\left(\alpha_{\overline{\mathbf{m}}^{\prime}} \mid \alpha_{\overline{\mathbf{m}}^{\prime \prime}}\right)$. Since the condition Ridx $\mathbf{m}=k+\ell$ shows $\left(\alpha_{\overline{\mathbf{m}}^{\prime}} \mid \alpha_{\overline{\mathbf{m}}^{\prime \prime}}\right)=\frac{1}{k \ell}-\frac{1}{k}-\frac{1}{\ell}$ and we have $\left(\alpha_{\overline{\mathbf{m}}^{\prime}} \mid \alpha_{\overline{\mathbf{m}}^{\prime \prime}}\right)=-1$. Hence the theorem also follows from Lemma 12.3 .

i) First suppose idx $\mathbf{m}^{\prime} \neq 0$. Note that $\mathbf{m}$ and $\mathbf{m}^{\prime}$ are rigid if idx $\mathbf{m}^{\prime}>0$. We have $\operatorname{idx} \mathbf{m}=\operatorname{idx} \mathbf{m}^{\prime}$ and idx $\mathbf{m}=\left(\alpha_{\mathbf{m}^{\prime}}+\ell \alpha_{\overline{\mathbf{m}}^{\prime \prime}} \mid \alpha_{\mathbf{m}^{\prime}}+\ell \alpha_{\overline{\mathbf{m}}^{\prime \prime}}\right)=\operatorname{idx} \mathbf{m}^{\prime}+2 \ell\left(\alpha_{\mathbf{m}} \mid \alpha_{\overline{\mathbf{m}}^{\prime \prime}}\right)+2 \ell^{2}$, which implies (12.7).

Thus we may assume idx $\mathbf{m}<0$ and idx $\mathbf{m}^{\prime}=0$. If $k=1$, idx $\mathbf{m}=\operatorname{idx} \mathbf{m}^{\prime}=0$ and we have (12.7) as above. Hence we may moreover assume $k \geq 2$. Then (12.11) 
and the assumption imply $2-2 k=2 k \ell\left(\alpha_{\overline{\mathbf{m}}^{\prime}} \mid \alpha_{\overline{\mathbf{m}}^{\prime \prime}}\right)+2 \ell^{2}$, which means

$$
-\left(\alpha \overline{\mathbf{m}}^{\prime} \mid \alpha_{\overline{\mathbf{m}}^{\prime \prime}}\right)=\frac{k-1+\ell^{2}}{k \ell} .
$$

Here $k$ and $\ell$ are mutually prime and hence there exists a positive integer $m$ with $k=m \ell+1$ and

$$
-\left(\alpha_{\overline{\mathbf{m}}^{\prime}} \mid \alpha_{\overline{\mathbf{m}}^{\prime \prime}}\right)=\frac{m+\ell}{m \ell+1}=\frac{1}{\ell+\frac{1}{m}}+\frac{1}{m+\frac{1}{\ell}}<2 .
$$

Thus we have $m=\ell=1, k=2$ and $\left(\alpha_{\overline{\mathbf{m}}^{\prime}} \mid \alpha_{\overline{\mathbf{m}}^{\prime \prime}}\right)=-1$. By the transformation of an element of $\widetilde{W}_{\infty}$, we may assume $\overline{\mathbf{m}}^{\prime} \in \mathcal{P}_{p+1}$ is a tuple in $(12.16)$. Since $\left(\alpha_{\overline{\mathbf{m}}^{\prime}} \mid \alpha_{\overline{\mathbf{m}}^{\prime \prime}}\right)=-1$ and $\alpha_{\overline{\mathbf{m}}^{\prime \prime}}$ is a positive real root, we have the theorem by a similar argument as in the proof of Lemma 12.3. Namely, $m_{p, n_{p}^{\prime}}^{\prime}=2$ and $m_{p, n_{p}^{\prime}+1}^{\prime}=0$ and we may assume $m_{j, n_{j}^{\prime}+1}^{\prime \prime}=0$ for $j=0, \ldots, p-1$ and $m_{p, n_{p}^{\prime}+1}^{\prime \prime}+m_{p, n_{p}^{\prime}+2}^{\prime \prime}+\cdots=1$, which proves the theorem in view of $\alpha_{\mathbf{m}^{\prime \prime}} \in \Delta_{+}^{r e}$.

Lemma 12.3. Suppose $\mathbf{m}$ and $\mathbf{m}^{\prime}$ are realizable and $\mathrm{idx} \mathbf{m} \leq 0$ and $\mathrm{idx} \mathbf{m}^{\prime} \leq 0$. Then

$$
\left(\alpha_{\mathbf{m}} \mid \alpha_{\mathbf{m}^{\prime}}\right) \leq 0
$$

If $\mathbf{m}$ and $\mathbf{m}^{\prime}$ are basic and monotone,

$$
\left(\alpha_{\mathbf{m}} \mid w \alpha_{\mathbf{m}^{\prime}}\right) \leq\left(\alpha_{\mathbf{m}} \mid \alpha_{\mathbf{m}^{\prime}}\right) \quad\left(\forall w \in W_{\infty}\right) .
$$

If $\left(\alpha_{\mathbf{m}} \mid \alpha_{\mathbf{m}^{\prime}}\right)=0$ and $\mathbf{m}$ and $\mathbf{m}^{\prime}$ are indivisible, then $\mathrm{idx} \mathbf{m}=0$ and $\mathbf{m}=\mathbf{m}^{\prime}$. If $\left(\alpha_{\mathbf{m}} \mid \alpha_{\mathbf{m}^{\prime}}\right)=-1$, then the pair is isomorphic to one of the pairs

$$
\begin{array}{ll}
\left(D_{4}^{(k)}, \tilde{D}_{4}\right):((k k, k k, k k, k(k-1) 1), & (11,11,11,110)) \\
\left(E_{6}^{(k)}, \tilde{E}_{6}\right):((k k k, k k k, k(k-1) 1), & (111,111,1110)) \\
\left(E_{7}^{(k)}, \tilde{E}_{7}\right):\left(\left((2 k)^{2}, k k k k, k k k(k-1) 1\right),\right. & (22,1111,11110)) \\
\left(E_{8}^{(k)}, \tilde{E}_{8}\right):\left(\left((3 k)^{2},(2 k)^{3}, k k k k k(k-1) 1\right),\right. & (33,222,1111110))
\end{array}
$$

under the action of $\widetilde{W}_{\infty}$.

Proof. We may assume that $\mathbf{m}$ and $\mathbf{m}^{\prime}$ are indivisible. Under the transformation of the Weyl group, we may assume that $\mathbf{m}$ is a basic monotone tuple in $\mathcal{P}_{p+1}$, namely, $\left(\alpha_{\mathbf{m}} \mid \alpha_{0}\right) \leq 0$ and $\left(\alpha_{\mathbf{m}} \mid \alpha_{j, \nu}\right) \leq 0$.

If $\mathbf{m}^{\prime}$ is basic and monotone, $w \alpha_{\mathbf{m}^{\prime}}-\alpha_{\mathbf{m}^{\prime}}$ is a sum of positive real roots, which proves $(12.13)$.

Put $\alpha_{\mathbf{m}}=n \alpha_{0}+\sum n_{j, \nu} \alpha_{j, \nu}$ and $\mathbf{m}^{\prime}=n_{0}^{\prime} \alpha_{0}+\sum n_{j, \nu}^{\prime} \alpha_{j, \nu}$. Then

$$
\begin{aligned}
\left(\alpha_{\mathbf{m}} \mid \alpha_{\mathbf{m}^{\prime}}\right) & =n_{0}^{\prime}\left(\alpha_{\mathbf{m}} \mid \alpha_{0}\right)+\sum n_{j, \nu}^{\prime}\left(\alpha_{\mathbf{m}} \mid \alpha_{j, \nu}\right), \\
\left(\alpha_{\mathbf{m}} \mid \alpha\right) & \leq 0 \quad\left(\forall \alpha \in \operatorname{supp} \alpha_{\mathbf{m}}\right) .
\end{aligned}
$$

Let $k_{j}$ be the maximal positive integer satisfying $m_{j, k_{j}}=m_{j, 1}$ and put $\Pi_{0}=$ $\left\{\alpha_{0}, \alpha_{j, \nu} ; 1 \leq \nu<k_{j}, j=0, \ldots, p\right\}$. Note that $\Pi_{0}$ defines a classical root system if idx $\mathbf{m}<0$ (cf. Remark 9.12).

Suppose $\left(\alpha_{\mathbf{m}} \mid \alpha_{\mathbf{m}^{\prime}}\right)=0$ and $\mathbf{m} \in \mathcal{P}_{p+1}$. Then $m_{0,1}+\cdots+m_{p, 1}=(p-1)$ ord $\mathbf{m}$ and $\operatorname{supp} \alpha_{\mathbf{m}^{\prime}} \subset \Pi_{0}$ because $\left(\alpha_{\mathbf{m}} \mid \alpha\right)=0$ for $\alpha \in \operatorname{supp} \alpha_{\mathbf{m}^{\prime}}$. Hence it follows from $\operatorname{idx} \mathbf{m}^{\prime} \leq 0$ that $\operatorname{idx} \mathbf{m}=0$ and we may assume that $\mathbf{m}$ is one of the tuples (12.16). Since $\operatorname{supp} \alpha_{\mathbf{m}^{\prime}} \subset \operatorname{supp} \alpha_{\mathbf{m}}$ and idx $\mathbf{m}^{\prime} \leq 0$, we conclude that $\mathbf{m}^{\prime}=\mathbf{m}$.

Lastly suppose $\left(\alpha_{\mathbf{m}} \mid \alpha_{\mathbf{m}^{\prime}}\right)=-1$.

Case: $\operatorname{idx} \mathbf{m}=\operatorname{idx} \mathbf{m}^{\prime}=0$. If $\mathbf{m}^{\prime}$ is basic and monotone and $\mathbf{m}^{\prime} \neq \mathbf{m}$, then it is easy to see that $\left(\alpha_{\mathbf{m}} \mid \alpha_{\mathbf{m}^{\prime}}\right)<-1$ (cf. Remark 9.1). Hence $(12.13)$ assures $\mathbf{m}^{\prime}=w \mathbf{m}$ with a certain $w \in W_{\infty}$ and therefore $\operatorname{supp} \mathbf{m} \subsetneq \operatorname{supp} \mathbf{m}^{\prime}$. Moreover there exists 
$j_{0}$ and $L \geq k_{j_{0}}$ such that $\operatorname{supp} m^{\prime}=\operatorname{supp} m \cup\left\{\alpha_{j_{0}, k_{j_{0}}}, \alpha_{j_{0}, k_{j}+1}, \ldots, \alpha_{j_{0}, L}\right\}$ and $m_{j_{0}, k_{j_{0}}}=1$ and $m_{j_{0}, k_{j_{0}}+1}^{\prime}=1$. Then by a transformation of an element of the Weyl group, we may assume $L=k_{j_{0}}$ and $\mathbf{m}^{\prime}=r_{i_{N}} \cdots r_{i_{1}} r_{\left(j_{0}, k_{j_{0}}\right)} \mathbf{m}$ with suitable $i_{\nu}$ satisfying $\alpha_{i_{\nu}} \in \operatorname{supp} \mathbf{m}$ for $\nu=1, \ldots, N$. Applying $r_{i_{1}} \cdots r_{i_{N}}$ to the pair $\left(\mathbf{m}, \mathbf{m}^{\prime}\right)$, we may assume $\mathbf{m}^{\prime}=r_{\left(j_{0}, k_{j_{0}}\right)} \mathbf{m}$. Hence the pair $\left(\mathbf{m}, \mathbf{m}^{\prime}\right)$ is isomorphic to one of the pairs in the list (12.14) with $k=1$.

Case: $\operatorname{idx} \mathbf{m}<0$ and idx $\mathbf{m}^{\prime} \leq 0$. There exists $j_{0}$ such that $\operatorname{supp} \alpha_{\mathbf{m}^{\prime}} \ni \alpha_{j_{0}, k_{j}}$. Then the fact $\operatorname{idx}\left(\mathbf{m}, \mathbf{m}^{\prime}\right)=-1$ implies $n_{j_{0}, k_{0}}^{\prime}=1$ and $n_{j, k_{j}}^{\prime}=0$ for $j \neq j_{0}$. Let $L$ be the maximal positive integer with $n_{j_{0}, L}^{\prime} \neq 0$. Since $\left(\alpha_{\mathbf{m}} \mid \alpha_{j_{0}, \nu}\right)=0$ for $k_{0}+1 \leq \nu \leq L$, we may assume $L=k_{0}$ by the transformation $r_{\left(j_{0}, k_{0}+1\right)} \circ \cdots \circ r_{\left(j_{0}, L\right)}$ if $L>k_{0}$. Since the Dynkin diagram corresponding to $\Pi_{0} \cup\left\{\alpha_{j_{0}, k_{j_{0}}}\right\}$ is classical or affine and $\operatorname{supp} \mathbf{m}^{\prime}$ is contained in this set, idx $\mathbf{m}^{\prime}=0$ and $\mathbf{m}^{\prime}$ is basic and we may assume that $\mathbf{m}^{\prime}$ is one of the tuples

$$
11,11,11,11 \quad 111,111,111 \quad 22,1111,1111 \quad 33,222,111111
$$

and $j_{0}=p$. In particular $m_{p, 1}^{\prime}=\cdots=m_{p, k_{p}}^{\prime}=1$ and $m_{p, k_{p}+1}^{\prime}=0$. It follows from $\left(\alpha_{\mathbf{m}} \mid \alpha_{p, k_{p}}\right)=-1$ that there exists an integer $L^{\prime} \geq k_{p}+1$ satisfying supp $\mathbf{m}=$ $\operatorname{supp} \mathbf{m}^{\prime} \cup\left\{\alpha_{p, \nu} ; k_{p} \leq \nu<L^{\prime}\right\}$ and $m_{p, k_{p}}=m_{p, k_{p}-1}-1$. In particular, $m_{j, \nu}=m_{j, 1}$ for $\nu=1, \ldots, k_{j}-\delta_{j, p}$ and $j=0, \ldots, p$. Since $\sum_{j=0}^{p} m_{j, 1}=(p-1)$ ord $\mathbf{m}$, there exists a positive integer $k$ such that

$$
m_{j, \nu}= \begin{cases}k m_{j, 1}^{\prime} & \left(j=0, \ldots, p, \nu=1, \ldots, k_{j}-\delta_{j, p}\right), \\ k m_{p, 1}^{\prime}-1 & \left(j=p, \nu=k_{p}\right) .\end{cases}
$$

Hence $m_{p, k_{p}+1}=1$ and $L^{\prime}=k_{p}+1$ and the pair $\left(\mathbf{m}, \mathbf{m}^{\prime}\right)$ is one of the pairs in the list (12.14) with $k>1$.

Remark 12.4. Let $k$ be an integer with $k \geq 2$ and let $P$ be a differential operator with the spectral type $D_{4}^{(k)}, E_{6}^{(k)}, E_{7}^{(k)}$ or $E_{8}^{(k)}$. It follows from Theorem 6.19 and Theorem 8.13 that $P$ is reducible for any values of accessory parameters when the characteristic exponents satisfy Fuchs relation with respect to the subtuple given in (12.14). For example, the Fuchsian differential operator $P$ with the Riemann scheme

$$
\left\{\begin{array}{cccc}
{\left[\lambda_{0,1}\right]_{(k)}} & {\left[\lambda_{1,1}\right]_{(k)}} & {\left[\lambda_{2,1}\right]_{(k)}} & {\left[\lambda_{3,1}\right]_{(k)}} \\
{\left[\lambda_{0,2}\right]_{(k)}} & {\left[\lambda_{1,2}\right]_{(k)}} & {\left[\lambda_{2,2}\right]_{(k)}} & {\left[\lambda_{3,2}\right]_{(k-1)}} \\
& & & \lambda_{3,2}+2 k-2
\end{array}\right\}
$$

is reducible.

Example 12.5. i) (generalized Jordan-Pochhammer) If $\mathbf{m}=k \mathbf{m}^{\prime} \oplus \ell \mathbf{m}^{\prime \prime}$ with a rigid tuples $\mathbf{m}, \mathbf{m}^{\prime}$ and $\mathbf{m}^{\prime \prime}$ and positive integers $k$ and $\ell$ satisfying $1 \leq k \leq \ell$, we have

$$
\left(\alpha_{\mathbf{m}^{\prime}} \mid \alpha_{\mathbf{m}^{\prime \prime}}\right)=-\frac{k^{2}+\ell^{2}-1}{k \ell} \in \mathbb{Z} .
$$

For positive integers $k$ and $\ell$ satisfying $1 \leq k \leq \ell$ and

$$
p:=\frac{k^{2}+\ell^{2}-1}{k \ell}+1 \in \mathbb{Z}
$$

we have an example of direct decompositions

$$
\begin{aligned}
\overbrace{\ell k, \ell k, \ldots, \ell k}^{p+1 \text { partitions }}= & 0 k, 0 k, \ldots, 0 k \oplus \ell 0, \ell 0, \ldots, \ell 0 \\
= & ((p-1) k-\ell) k,((p-1) k-\ell) k, \ldots,((p-1) k-\ell) k \\
& \oplus(2 \ell-(p-1) k) 0,(2 \ell-(p-1) k) 0, \ldots,(2 \ell-(p-1) k) 0 .
\end{aligned}
$$


Here $p=3+\frac{(k-\ell)^{2}-1}{k \ell} \geq 2$ and the condition $p=2$ implies $k=\ell=1$ and the condition $p=3$ implies $\ell=k+1$. If $k=1$, then $\left(\alpha_{\mathbf{m}^{\prime}} \mid \alpha_{\mathbf{m}^{\prime \prime}}\right)=-\ell$ and we have an example corresponding to Jordan-Pochhammer equation:

$$
\overbrace{\ell 1, \cdots, \ell 1}^{\ell+2 \text { partitions }}=01, \cdots, 01 \oplus \ell 0, \cdots, \ell 0 .
$$

When $\ell=k+1$, we have $\left(\alpha_{\mathbf{m}^{\prime}} \mid \alpha_{\mathbf{m}^{\prime \prime}}\right)=-2 k$ and an example

$$
\begin{aligned}
(k & +1) k,(k+1) k,(k+1) k,(k+1) k \\
& =0 k, 0 k, 0 k, 0 k \oplus(k+1) 0,(k+1) 0,(k+1) 0,(k+1) 0 \\
& =(k-1) k,(k-1) k,(k-1) k,(k-1) k \oplus 20,20,20,20 .
\end{aligned}
$$

We have another example

$$
\begin{aligned}
83,83,83,83,83 & =03,03,03,03,03 \oplus 80,80,80,80,80 \\
& =13,13,13,13,13 \oplus 70,70,70,70,70
\end{aligned}
$$

in the case $(k, \ell)=(3,8)$, which is a special case where $\ell=k^{2}-1, p=k+1$ and $\left(\alpha_{\mathbf{m}^{\prime}} \mid \alpha_{\mathbf{m}^{\prime \prime}}\right)=-k$.

When $p$ is odd, the equation (12.18) is equal to the Pell equation

$$
y^{2}-\left(m^{2}-1\right) x^{2}=1
$$

by putting $p-1=2 m, x=\ell$ and $y=m \ell-k$ and hence the reduction of the tuple of partition (12.19) by $\partial_{\max }$ and its inverse give all the integer solutions of this Pell equation.

The tuple of partitions $\ell k, \ell k, \ldots, \ell k \in \mathcal{P}_{p+1}^{(\ell+k)}$ with $(12.18)$ is called a generalized Jordan-Pochhammer tuple and denoted by $P_{p+1, \ell+k}$. In particular, $P_{n+1, n}$ is simply denoted by $P_{n}$.

ii) We give an example of direct decompositions of a rigid tuple:

$$
\begin{aligned}
3322,532,532 & =0022,202,202 \oplus 3300,330,330: 1 \\
& =1122,312,312 \oplus 2200,220,220: 1 \\
& =0322,232,232 \oplus 3000,300,300: 2 \\
& =3302,332,332 \oplus 0020,200,200: 2 \\
& =1212,321,321 \oplus 2110,211,211: 4 \\
& =2211,321,312 \oplus 1111,211,220: 2 \\
& =2212,421,322 \oplus 1110,111,210: 4 \\
& =2222,431,422 \oplus 1100,101,110: 2 \\
& =2312,422,422 \oplus 1010,110,110: 4 \\
& =2322,522,432 \oplus 1000,010,100: 4 .
\end{aligned}
$$

They are all the direct decompositions of the tuple $3322,532,532$ modulo obvious symmetries. Here we indicate the number of the decompositions of the same type.

Corollary 12.6. Let $\mathbf{m} \in \mathcal{P}$ be realizable. Put $\mathbf{m}=\operatorname{gcd}(\mathbf{m}) \overline{\mathbf{m}}$. Then $\mathbf{m}$ has no direct decomposition (12.6) if and only if

(12.24) ord $\mathbf{m}=1$

or

(12.25) $\operatorname{idx} \mathbf{m}=0$ and basic 
or

$\mathrm{idx} \mathbf{m}<0$ and $\overline{\mathbf{m}}$ is basic and $\mathbf{m}$ is not isomorphic to any one of tuples in Example 9.14 with $m>1$.

Moreover we have the following result.

Proposition 12.7. The direct decomposition $\mathbf{m}=\mathbf{m}^{\prime} \oplus \mathbf{m}^{\prime \prime}$ is called rigid decomposition if $\mathbf{m}, \mathbf{m}^{\prime}$ and $\mathbf{m}^{\prime \prime}$ are rigid. If $\mathbf{m} \in \mathcal{P}$ is rigid and ord $\mathbf{m}>1$, there exists a rigid decomposition.

Proof. We may assume that $\mathbf{m}$ is monotone and there exist a non-negative integer $p$ such that $m_{j, 2} \neq 0$ if and only if $0 \leq j<p+1$. If ord $\partial \mathbf{m}=1$, then we may assume $\mathbf{m}=(p-1) 1,(p-1) 1, \ldots,(p-1) 1 \in \mathcal{P}_{p+1}^{(p)}$ and there exists a decomposition

$$
(p-1) 1,(p-1) 1, \ldots,(p-1) 1=01,10, \ldots, 10 \oplus(p-1) 0,(p-2) 1, \ldots,(p-2) 1 .
$$

Suppose ord $\partial \mathbf{m}>1$. Put $d=\operatorname{idx}(\mathbf{m}, \mathbf{1})=m_{0,1}+\cdots+m_{p, 1}-(p-1) \cdot \operatorname{ord} \mathbf{m}>0$.

The induction hypothesis assures the existence of a decomposition $\partial \mathbf{m}=\overline{\mathbf{m}}^{\prime} \oplus \overline{\mathbf{m}}^{\prime \prime}$ such that $\overline{\mathbf{m}}^{\prime}$ and $\overline{\mathbf{m}}^{\prime \prime}$ are rigid. If $\partial \overline{\mathbf{m}}^{\prime}$ and $\partial \overline{\mathbf{m}}^{\prime \prime}$ are well-defined, we have the decomposition $\mathbf{m}=\partial^{2} \mathbf{m}=\partial \overline{\mathbf{m}}^{\prime} \oplus \partial \overline{\mathbf{m}}^{\prime \prime}$ and the proposition.

If ord $\overline{\mathbf{m}}^{\prime}>1, \partial \overline{\mathbf{m}}^{\prime}$ is well-defined. Suppose $\overline{\mathbf{m}}^{\prime}=\left(\delta_{\nu, \ell_{j}}\right)_{\substack{j=0, \ldots, p \\ \nu=1,2, \ldots}}$. Then

$$
\begin{aligned}
\operatorname{idx}(\partial \mathbf{m}, \mathbf{1})-\operatorname{idx}\left(\partial \mathbf{m}, \overline{\mathbf{m}}^{\prime}\right) & =\sum_{j=0}^{p}\left(\left(m_{j, 1}-d-\left(m_{j, \ell_{j}}-d \delta_{\ell_{j}, 1}\right)\right)\right. \\
& \geq-d \#\left\{j ; \ell_{j}>1,0 \leq j \leq p\right\} .
\end{aligned}
$$

Since $\operatorname{idx}(\partial \mathbf{m}, \mathbf{1})=-d$ and $\operatorname{idx}\left(\partial \mathbf{m}, \overline{\mathbf{m}}^{\prime}\right)=1$, we have $d \#\left\{j ; \ell_{j}>1,0 \leq j \leq p\right\} \geq$ $d+1$ and therefore $\#\left\{j ; \ell_{j}>1,0 \leq j \leq p\right\} \geq 2$. Hence $\partial \overline{\mathbf{m}}^{\prime}$ is well-defined.

Remark 12.8. The author's original construction of a differential operator with a given rigid Riemann scheme doesn't use the middle convolutions and additions but uses Proposition 12.7.

Example 12.9. We give direct decompositions of a rigid tuple:

$$
\begin{aligned}
721,3331,22222 & =200,2000,20000 \oplus 521,1331,02222: 15 \\
& =210,1110,11100 \oplus 511,2221,11122: 10 \\
& =310,1111,11110 \oplus 411,2220,11112: 5
\end{aligned}
$$

The following irreducibly realizable tuple has only two direct decompositions:

$$
\begin{aligned}
44,311111,311111 & =20,200000,200000 \oplus 24,111111,111111 \\
& =02,200000,200000 \oplus 42,111111,111111
\end{aligned}
$$

But it cannot be a direct sum of two irreducibly realizable tuples.

12.2. Reduction of reducibility. We give a necessary and sufficient condition so that a Fuchsian differential equation is irreducible, which follows from [Kz and [DR, DR2]. Note that a Fuchsian differential equation is irreducible if and only if its monodromy is irreducible.

Theorem 12.10. Retain the notation in $\$ 12.1$. Suppose $\mathbf{m}$ is monotone, realizable and $\partial_{\max } \mathbf{m}$ is well-defined and

$$
d:=m_{0,1}+\cdots+m_{p, 1}-(p-1) \text { ord } \mathbf{m} \geq 0 .
$$


Put $P=P_{\mathbf{m}}($ cf. $(8.25))$ and

$$
\begin{aligned}
\mu & :=\lambda_{0,1}+\lambda_{1,1}+\cdots+\lambda_{p, 1}-1, \\
Q & :=\partial_{\max } P, \\
P^{o} & :=\left.P\right|_{\lambda_{j, \nu}=\lambda_{j, \nu}^{o}, g_{i}=g_{i}^{o}}, \quad Q^{o}:=\left.Q\right|_{\lambda_{j, \nu}=\lambda_{j, \nu}^{o}, g_{i}=g_{i}^{o}}
\end{aligned}
$$

with some complex numbers $\lambda_{j, \nu}^{o}$ and $g_{i}^{o}$ satisfying the Fuchs relation $\left|\left\{\lambda_{\mathbf{m}}^{o}\right\}\right|=0$.

i) The Riemann scheme $\left\{\tilde{\lambda}_{\tilde{\mathbf{m}}}\right\}$ of $Q$ is given by

$$
\left\{\begin{array}{l}
\tilde{m}_{j, \nu}=m_{j, \nu}-d \delta_{\nu, 1} \\
\tilde{\lambda}_{j, \nu}=\lambda_{j, \nu}+\left((-1)^{\delta_{j, 0}}-\delta_{\nu, 1}\right) \mu
\end{array}\right.
$$

ii) Assume that the equation $P^{o} u=0$ is irreducible. If $d>0$, then $\mu \notin \mathbb{Z}$. If the parameters given by $\lambda_{j, \nu}^{o}$ and $g_{i}^{o}$ are locally non-degenerate, the equation $Q^{o} v=0$ is irreducible and the parameters are locally non-degenerate.

iii) Assume that the equation $Q^{\circ} v=0$ is irreducible and the parameters given by $\lambda_{j, \nu}^{o}$ and $g_{i}^{o}$ are locally non-degenerate. Then the equation $P^{o} v=0$ is irreducible if and only if

$$
\sum_{j=0}^{p} \lambda_{j, 1+\delta_{j, j_{o}}\left(\nu_{o}-1\right)}^{o} \notin \mathbb{Z} \text { for any }\left(j_{o}, \nu_{o}\right) \text { satisfying } m_{j_{o}, \nu_{o}}>m_{j_{o}, 1}-d .
$$

If the equation $P^{o} v=0$ is irreducible, the parameters are locally non-degenerate.

iv) Put $\mathbf{m}(k):=\partial_{\max }^{k} \mathbf{m}$ and $P(k)=\partial_{\max }^{k} P$. Let $K$ be a non-negative integer such that ord $\mathbf{m}(0)>\operatorname{ord} \mathbf{m}(1)>\cdots>\operatorname{ord} \mathbf{m}(K)$ and $\mathbf{m}(K)$ is fundamental. The operator $P(k)$ is essentially the universal operator of type $\mathbf{m}(k)$ but parametrized by $\lambda_{j, \nu}$ and $g_{i}$. Put $P(k)^{o}=\left.P(k)\right|_{\lambda_{j, \nu}=\lambda_{j, \nu}^{o}}$.

If the equation $P^{o} u=0$ is irreducible and the parameters are locally nondegenerate, so are $P(k)^{o} u=0$ for $k=1, \ldots, K$.

If the equation $P^{\circ} u=0$ is irreducible and locally non-degenerate, so is the equation $P(K)^{o} u=0$.

Suppose the equation $P(K)^{o} u=0$ is irreducible and locally non-degenerate, which is always valid when $\mathbf{m}$ is rigid. Then the equation $P^{o} u=0$ is irreducible if and only if the equation $P(k)^{o} u=0$ satisfy the condition (12.34) for $k=0, \ldots, K-1$. If the equation $P^{o} u=0$ is irreducible, it is locally non-degenerate.

Proof. The claim i) follows from Theorem 7.2 and the claims ii) and iii) follow from Lemma 7.3 and Corollary 11.10, which implies the claim iv).

Remark 12.11. i) In the preceding theorem the equation $P^{o} u=0$ may not be locally non-degenerate even if it is irreducible. For example the equation satisfied by ${ }_{3} F_{2}$ is contained in the universal operator of type $111,111,111$.

ii) It is also proved as follows that the irreducible differential equation with a rigid spectral type is locally non-degenerate.

The monodromy generators $M_{j}$ of the equation with the Riemann scheme at $x=c_{j}$ satisfy

$\operatorname{rank}\left(M_{j}^{\prime}-e^{2 \pi \sqrt{-1} \lambda_{j, 1}}\right) \cdots\left(M_{j}^{\prime}-e^{2 \pi \sqrt{-1} \lambda_{j, k}}\right) \leq m_{j, k+1}+\cdots+m_{j, n_{j}} \quad\left(k=1, \ldots, n_{j}\right)$

for $j=0, \ldots, p$. The equality in the above is clear when $\lambda_{j, \nu}-\lambda_{j, \nu^{\prime}} \notin \mathbb{Z}$ for $1 \leq \nu<\nu^{\prime} \leq n_{j}$ and hence the above is proved by the continuity for general $\lambda_{j, \nu}$. The rigidity index of $\mathbf{M}$ is calculated by the dimension of the centralizer of $M_{j}$ and it should be 2 if $\mathbf{M}$ is irreducible and rigid, the equality in the above is valid (cf. [Kz], OG]), which means the equation is locally non-degenerate. 
iii) The same results as in Theorem 12.10 are also valid in the case of the Fuchsian system of Schlesinger canonical form (11.1) since the same proof works. A similar result is given by a different proof (cf. $\mathrm{CB}$ ).

iv) Let $\left(M_{0}, \ldots, M_{p}\right)$ be a tuple of matrices in $G L(n, \mathbb{C})$ with $M_{p} M_{p-1} \cdots M_{0}=$ $I_{n}$. Then $\left(M_{0}, \ldots, M_{p}\right)$ is called rigid if for any $g_{0}, \ldots, g_{p} \in G L(n, \mathbb{C})$ satisfying $g_{p} M_{p} g_{p}^{-1} \cdot g_{p-1} M_{p-1} g_{p-1}^{-1} \cdots g_{0} M_{0} g_{0}^{-1}=I_{n}$, there exists $g \in G L(n, \mathbb{C})$ such that $g_{i} M_{i} g_{i}^{-1}=g M_{i} g^{-1}$ for $i=0, \ldots, p$. The tuple $\left(M_{0}, \ldots, M_{p}\right)$ is called irreducible if no subspace $V$ of $\mathbb{C}^{n}$ satisfies $\{0\} \varsubsetneqq V \varsubsetneqq \mathbb{C}^{n}$ and $M_{i} V \subset V$ for $i=0, \ldots, p$. Choose $\mathbf{m} \in \mathcal{P}_{p+1}^{(n)}$ and $\left\{\mu_{j, \nu}\right\}$ such that $L\left(\mathbf{m} ; \mu_{j, 1}, \ldots, \mu_{j, n_{j}}\right)$ are in the conjugacy classes containing $M_{j}$, respectively. Suppose $\left(M_{0}, \ldots, M_{p}\right)$ is irreducible and rigid. Then Katz [K] shows that $\mathbf{m}$ is rigid and gives a construction of irreducible and rigid $\left(M_{0}, \ldots, M_{p}\right)$ for any rigid $\mathbf{m}$ (cf. Remark $\left.11.4 \mathrm{ii}\right)$ ). It is an open problem given by Katz $\mathrm{Kz}$ ] whether the monodromy generators $M_{j}$ are realized by solutions of a single Fuchsian differential equations without an apparent singularity, whose affirmative answer is given by the following corollary.

Corollary 12.12. Let $\mathbf{m}=\left(m_{j, \nu}\right)_{\substack{0 \leq j \leq p \\ 1 \leq \nu \leq n_{j}}}$ be a rigid monotone $(p+1)$-tuple of partitions with ord $\mathbf{m}>1$. Retain the notation in Definition \%.11.

i) Fix complex numbers $\lambda_{j, \nu}$ for $0 \leq j \leq p$ and $1 \leq \nu_{j}$ such that it satisfies the Fuchs relation

$$
\sum_{j=0}^{p} \sum_{\nu=1}^{n_{j}} m_{j, \nu} \lambda_{j, \nu}=\operatorname{ord} \mathbf{m}-1
$$

The universal operator $P_{\mathbf{m}}(\lambda) u=0$ with the Riemann scheme (1.10) is irreducible if and only if the condition

$$
\begin{aligned}
\sum_{j=0}^{p} \lambda(k)_{j, \ell}(k)_{j}+\delta_{j, j_{o}}\left(\nu_{o}-\ell(k)_{j}\right) & \notin \mathbb{Z} \\
\text { for any }\left(j_{o}, \nu_{o}\right) & \text { satisfying } m(k)_{j_{o}, \nu_{o}}>m(k)_{j_{o}, \ell(k)_{j_{o}}}-d(k)
\end{aligned}
$$

is satisfied for $k=0, \ldots, K-1$.

ii) Define $\tilde{\mu}(k)$ and $\mu(k)_{j, \nu}$ for $k=0, \ldots, K$ by

$$
\begin{aligned}
\mu(0)_{j, \nu} & =\mu_{j, \nu} \quad\left(j=0, \ldots, p, \nu=1, \ldots, n_{j}\right), \\
\tilde{\mu}(k) & =\prod_{j=0}^{p} \mu(k)_{j, \ell(k)_{j}}, \\
\mu(k+1)_{j, \nu} & =\mu(k)_{j, \nu} \cdot \tilde{\mu}(k)^{(-1)^{\delta_{j, 0}-\delta_{\nu, 1}} .}
\end{aligned}
$$

Then there exists an irreducible tuple $\left(M_{0}, \ldots, M_{p}\right)$ of matrices satisfying

$$
\begin{gathered}
M_{p} \cdots M_{0}=I_{n}, \\
M_{j} \sim L\left(m_{j, 1}, \ldots, m_{j, n_{j}} ; \mu_{j, 1}, \ldots, \mu_{j, n_{j}}\right) \quad(j=0, \ldots, p)
\end{gathered}
$$

under the notation (6.33) if and only if

$$
\prod_{j=0}^{p} \prod_{\nu=1}^{n_{j}} \mu_{j, \nu}^{m_{j, \nu}}=1
$$

and the condition

$$
\begin{aligned}
& \prod_{j=0}^{p} \mu(k)_{j, \ell(k)_{j}+\delta_{j, j_{o}}\left(\nu_{o}-\ell(k)_{j}\right)} \neq 1 \\
& \quad \text { for any }\left(j_{o}, \nu_{o}\right) \text { satisfying } m(k)_{j_{o}, \nu_{o}}>m(k)_{j_{o}, \ell(k)_{j_{o}}}-d(k)
\end{aligned}
$$


is satisfied for $k=0, \ldots, K-1$.

iii) Let $\left(M_{0}, \ldots, M_{p}\right)$ be an irreducible tuple of matrices satisfying (12.40). Then there uniquely exists a Fuchsian differential equation $P u=0$ with $p+1$ singular points $c_{0}, \ldots, c_{p}$ and its local independent solutions $u_{1}, \ldots, u_{\text {ord } \mathbf{m}}$ in a neighborhood of a non-singular point $q$ such that the monodromy generators around the points $c_{j}$ with respect to the solutions equal $M_{j}$, respectively, for $j=0, \ldots, p$ (cf. (11.25)).

Proof. The clam i) is a direct consequence of Theorem 12.10 and the claim ii) is proved by Theorem 11.3 and Lemma 11.11 as in the case of the proof of Theorem 12.10 (cf. Remark 11.4 ii)).

iii) Since $\operatorname{gcd} \mathbf{m}=1$, we can choose $\lambda_{j, \nu} \in \mathbb{C}$ such that $e^{2 \pi \sqrt{-1} \lambda_{j, \nu}}=\mu_{j, \nu}$ and $\sum_{j, \nu} m_{j, \nu} \lambda_{j, \nu}=\operatorname{ord} \mathbf{m}-1$. Then we have a universal operator $P_{\mathbf{m}}\left(\lambda_{j, \nu}\right) u=0$ with the Riemann scheme (1.10). The irreducibility of $\left(M_{p}, \ldots, M_{0}\right)$ and Theorem 11.6 assure the claim.

Now we state the condition (12.36) using the terminology of the Kac-Moody root system. Suppose $\mathbf{m} \in \mathcal{P}$ is monotone and irreducibly realizable. Let $\left\{\lambda_{\mathbf{m}}\right\}$ be the Riemann scheme of the universal operator $P_{\mathbf{m}}$. According to Remark 7.8 iii) we may relax the definition of $\ell_{\max }(\mathbf{m})$ as is given by $(7.42)$ and then we may assume

$$
v_{k} s_{0} \cdots v_{1} s_{0} \Lambda(\lambda) \in W_{\infty}^{\prime} \Lambda(\lambda(k)) \quad(k=1, \ldots, K)
$$

under the notation in Definition 7.11 and (9.31). Then we have the following theorem.

Theorem 12.13. Let $\mathbf{m}=\left(m_{j, \nu}\right)_{\substack{0 \leq j \leq p \\ 1 \leq \nu \leq n_{j}}}$ be an irreducibly realizable monotone tuple of partition in $\mathcal{P}$. Under the notation in Corollary 12.12 and $\$ 9.1$, there uniquely exists a bijection

$$
\begin{gathered}
\varpi: \Delta(\mathbf{m}) \stackrel{\sim}{\rightarrow}\left\{\left(k, j_{0}, \nu_{0}\right) ; 0 \leq k<K, 0 \leq j_{0} \leq p, 1 \leq \nu_{0} \leq n_{j_{0}},\right. \\
\left.\nu_{0} \neq \ell(k)_{j_{0}} \text { and } m(k)_{j_{0}, \nu_{0}}>m(k)_{j_{0}, \ell(k)_{j_{0}}}-d(k)\right\} \\
\cup\left\{\left(k, 0, \ell(k)_{0}\right) ; 0 \leq k<K\right\}
\end{gathered}
$$

such that

$$
(\Lambda(\lambda) \mid \alpha)=\sum_{j=0}^{p} \lambda(k)_{j, \ell(k)_{j}+\delta_{j, j_{o}}\left(\nu_{o}-\ell(k)_{j}\right)} \quad \text { when } \varpi(\alpha)=\left(k, j_{0}, \nu_{0}\right) .
$$

Moreover we have

$$
\begin{array}{r}
\left(\alpha \mid \alpha_{\mathbf{m}}\right)=m(k)_{j_{0}, \nu_{0}}-m(k)_{j_{0}, \ell(k)_{j_{0}}}+d(k) \\
\left(\alpha \in \Delta(\mathbf{m}),\left(k, j_{0}, \nu_{0}\right)=\varpi(\alpha)\right)
\end{array}
$$

and if the universal equation $P_{\mathbf{m}}(\lambda) u=0$ is irreducible, we have

$$
(\Lambda(\lambda) \mid \alpha) \notin \mathbb{Z} \quad \text { for any } \alpha \in \Delta(\mathbf{m}) .
$$

In particular, if $\mathbf{m}$ is rigid and (12.47) is valid, the universal equation is irreducible.

Proof. Assume ord $\mathbf{m}>1$ and use the notation in Theorem 12.10. Since $\tilde{\mathbf{m}}$ may not be monotone, we consider the monotone tuple $\mathbf{m}^{\prime}=s \tilde{\mathbf{m}}$ in $S_{\infty}^{\prime} \tilde{\mathbf{m}}$ (cf. Definition 6.11). First note that

$$
d-m_{j, 1}+m_{j, \nu}=\left(\alpha_{0}+\alpha_{j, 1}+\cdots+\alpha_{j, \nu-1} \mid \alpha_{\mathbf{m}}\right) .
$$

Let $\bar{\nu}_{j}$ be the positive integers defined by

$$
m_{j, \bar{\nu}_{j}+1} \leq m_{j, 1}-d<m_{j, \bar{\nu}_{j}}
$$


for $j=0, \ldots, p$. Then

$$
\alpha_{\mathbf{m}^{\prime}}=v^{-1} \alpha_{\tilde{\mathbf{m}}} \text { with } v:=\left(\prod_{j=0}^{p} s_{j, 1} \cdots s_{j, \bar{\nu}_{j}-1}\right)
$$

and $w(\mathbf{m})=s_{0} v s_{\alpha_{\tilde{\mathbf{m}}}}$ and

$$
\begin{aligned}
\Delta(\mathbf{m}) & =\Xi \cup s_{0} v \Delta\left(\mathbf{m}^{\prime}\right), \\
\Xi & :=\left\{\alpha_{0}\right\} \cup \underset{\substack{0 \leq j \leq p \\
\nu_{j} \neq 1}}{\bigcup}\left\{\alpha_{0}+\alpha_{j, 1}+\cdots+\alpha_{j, \nu} ; \nu=1, \ldots, \bar{\nu}_{j}-1\right\} .
\end{aligned}
$$

Note that $\ell(0)=(1, \ldots, 1)$ and the condition $m_{j_{0}, \nu_{0}}>m_{j_{0}, 1}-d(0)$ is valid if and only if $\nu_{0} \in\left\{1, \ldots, \bar{\nu}_{j_{0}}\right\}$. Since

$$
\sum_{j=0}^{p} \lambda(0)_{j, 1+\delta_{j, j_{0}}\left(\nu_{0}-1\right)}=\left(\Lambda(\lambda) \mid \alpha_{0}+\alpha_{j_{0}, 1}+\cdots+\alpha_{j_{0}, \nu_{0}-1}\right)+1,
$$

we have

$$
L(0)=\{(\Lambda(\lambda) \mid \alpha)+1 ; \alpha \in \Xi\}
$$

by denoting

$$
L(k):=\left\{\sum_{j=0}^{p} \lambda(k)_{j, \ell(k)_{j}+\delta_{j, j_{o}}\left(\nu_{o}-\ell(k)_{j}\right)} ; m(k)_{j_{o}, \nu_{o}}>m(k)_{j_{o}, \ell(k)_{j_{o}}}-d(k)\right\} .
$$

Applying $v^{-1} s_{0}$ to $\mathbf{m}$ and $\left\{\lambda_{\mathbf{m}}\right\}$, they changes into $\mathbf{m}^{\prime}$ and $\left\{\lambda_{\mathbf{m}^{\prime}}^{\prime}\right\}$, respectively, such that $\Lambda\left(\lambda^{\prime}\right)-v^{-1} s_{0} \Lambda(\lambda) \in \mathbb{C} \Lambda_{0}$. Hence we obtain the corollary by the induction as in the proof of Corollary 12.12 .

Remark 12.14. Let $\mathbf{m}$ be an irreducibly realizable monotone tuple in $\mathcal{P}$. Fix $\alpha \in$ $\Delta(\mathbf{m})$. We have $\alpha=\alpha_{\mathbf{m}^{\prime}}$ with a rigid tuple $\mathbf{m}^{\prime} \in \mathcal{P}$ and

$$
\left|\left\{\lambda_{\mathbf{m}^{\prime}}\right\}\right|=(\Lambda(\lambda) \mid \alpha) \text {. }
$$

Definition 12.15. Define an index $\operatorname{idx}_{\mathbf{m}}(\ell(\lambda))$ of the non-zero linear form $\ell(\lambda)=$ $\sum_{j=0}^{p} \sum_{\nu=1}^{n_{j}} k_{j, \nu} \lambda_{j, \nu}$ of with $k_{j, \nu} \in \mathbb{Z}_{\geq 0}$ as the positive integer $d_{i}$ such that

$$
\left\{\sum_{j=0}^{p} \sum_{\nu=1}^{n_{j}} k_{j, \nu} \epsilon_{j, \nu} ; \epsilon_{j, \nu} \in \mathbb{Z} \text { and } \sum_{j=0}^{p} \sum_{\nu=1}^{n_{j}} m_{j, \nu} \epsilon_{j, \nu}=0\right\}=\mathbb{Z} d_{i} .
$$

Proposition 12.16. For a rigid tuple $\mathbf{m}$ in Corollary 12.12, define rigid tuples $\mathbf{m}^{(1)}, \ldots, \mathbf{m}^{(N)}$ with a non-negative integer $N$ so that $\Delta(\mathbf{m})=\left\{\mathbf{m}^{(1)}, \ldots, \mathbf{m}^{(N)}\right\}$ and put

$$
\ell_{i}(\lambda):=\sum_{j=0}^{p} \sum_{\nu=1}^{n_{j}} m_{j, \nu}^{(i)} \lambda_{j, \nu} \quad(i=1, \ldots, N) .
$$

Here we note that Theorem 12.13 implies that $P_{\mathbf{m}}(\lambda)$ is irreducible if and only if $\ell_{i}(\lambda) \notin \mathbb{Z}$ for $i=1, \ldots, n$.

Fix a function $\ell(\lambda)$ of $\lambda_{j, \nu}$ such that $\ell(\lambda)=\ell_{i}(\lambda)-r$ with $i \in\{1, \ldots, N\}$ and $r \in \mathbb{Z}$. Moreover fix generic complex numbers $\lambda_{j, \nu} \in \mathbb{C}$ under the condition $\ell(\lambda)=$ $\left|\left\{\lambda_{\mathbf{m}}\right\}\right|=0$ and a decomposition $P_{\mathbf{m}}(\lambda)=P^{\prime \prime} P^{\prime}$ such that $P^{\prime}, P^{\prime \prime} \in W(x), 0<$ $n^{\prime}:=\operatorname{ord} P^{\prime}<n$ and the differential equation $P^{\prime} v=0$ is irreducible. Then there exists an irreducibly realizable subtuple $\mathbf{m}^{\prime}$ of $\mathbf{m}$ compatible to $\ell(\lambda)$ such that the monodromy generators $M_{j}^{\prime}$ of the equation $P^{\prime} u=0$ satisfies

$\operatorname{rank}\left(M_{j}-e^{2 \pi \sqrt{-1}} \lambda_{j, 1}\right) \cdots\left(M_{j}-e^{2 \pi \sqrt{-1}} \lambda_{j, k}\right) \leq m_{j, k+1}^{\prime}+\cdots+m_{j, n_{j}}^{\prime} \quad\left(k=1, \ldots, n_{j}\right)$ 
for $j=0, \ldots, p$. Here we define that the decomposition

$$
\mathbf{m}=\mathbf{m}^{\prime}+\mathbf{m}^{\prime \prime} \quad\left(\mathbf{m}^{\prime} \in \mathcal{P}_{p+1}^{\left(n^{\prime}\right)}, \mathbf{m}^{\prime \prime} \in \mathcal{P}_{p+1}^{\left(n^{\prime \prime}\right)}, 0<n^{\prime}<n\right)
$$

is compatible to $\ell(\lambda)$ and that $\mathbf{m}^{\prime}$ is a subtuple of $\mathbf{m}$ compatible to $\ell(\lambda)$ if the following conditions are valid

$$
\begin{aligned}
& \left|\left\{\lambda_{\mathbf{m}^{\prime}}\right\}\right| \in \mathbb{Z}_{\leq 0} \text { and }\left|\left\{\lambda_{\mathbf{m}^{\prime \prime}}\right\}\right| \in \mathbb{Z}, \\
& \mathbf{m}^{\prime} \text { is realizable if there exists }(j, \nu) \text { such that } m_{j, \nu}^{\prime \prime}=m_{j, \nu}>0, \\
& \mathbf{m}^{\prime \prime} \text { is realizable if there exists }(j, \nu) \text { such that } m_{j, \nu}^{\prime}=m_{j, \nu}>0 .
\end{aligned}
$$

Here we note $\left|\left\{\lambda_{\mathbf{m}^{\prime}}\right\}\right|+\left|\left\{\lambda_{\mathbf{m}^{\prime \prime}}\right\}\right|=1$ if $\mathbf{m}^{\prime}$ and $\mathbf{m}^{\prime \prime}$ are rigid.

Proof. The equation $P_{\mathbf{m}}(\lambda) u=0$ is reducible since $\ell(\lambda)=0$. We may assume $\lambda_{j, \nu}-\lambda_{j, \nu^{\prime}} \neq 0$ for $1 \leq \nu<\nu^{\prime} \leq n_{j}$ and $j=0, \ldots, p$. The solutions of the equation define the map $\mathcal{F}$ given by $(3.15)$ and the reducibility implies the existence of an irreducible submap $\mathcal{F}^{\prime}$ such that $\mathcal{F}^{\prime}(U) \subset \mathcal{F}(U)$ and $0<n^{\prime}:=\operatorname{dim} \mathcal{F}^{\prime}(U)<$ $n$. Then $\mathcal{F}^{\prime}$ defines a irreducible Fuchsian differential equation $P^{\prime} v=0$ which has regular singularities at $x=c_{0}=\infty, c_{1}, \ldots, c_{p}$ and may have other apparent singularities $c_{1}^{\prime}, \ldots, c_{q}^{\prime}$. Then the characteristic exponents of $P^{\prime}$ at the singular points are as follows.

There exists a decomposition $\mathbf{m}=\mathbf{m}^{\prime}+\mathbf{m}^{\prime \prime}$ such that $\mathbf{m}^{\prime} \in \mathcal{P}^{\left(n^{\prime}\right)}$ and $\mathbf{m}^{\prime \prime} \in \mathcal{P}^{\left(n^{\prime \prime}\right)}$ with $n^{\prime \prime}:=n-n^{\prime}$. The sets of characteristic exponents of $P^{\prime}$ at $x=c_{j}$ are $\left\{\lambda_{j, \nu, i}^{\prime} ; i=1, \ldots, m_{j, \nu}^{\prime}, \nu=1, \ldots, n\right\}$ which satisfy

$$
\lambda_{j, \nu, i}^{\prime}-\lambda_{j, \nu} \in\left\{0,1, \ldots, m_{j, \nu}-1\right\} \text { and } \lambda_{j, \nu, 1}^{\prime}<\lambda_{j, \nu, 2}^{\prime}<\cdots<\lambda_{j, \nu, m_{j, \nu}^{\prime}}^{\prime}
$$

for $j=0, \ldots, p$. The sets of characteristic exponents at $x=c_{j}^{\prime}$ are $\left\{\mu_{j, 1}, \ldots, \mu_{j, n^{\prime}}\right\}$, which satisfy $\mu_{j, i} \in \mathbb{Z}$ and $0 \leq \mu_{j, 1}<\cdots<\mu_{j, n^{\prime}}$ for $j=1, \ldots, q$. Then Remark 6.17 ii) says that the Fuchs relation of the equation $P^{\prime} v=0$ implies $\left|\left\{\lambda_{\mathbf{m}^{\prime}}\right\}\right| \in \mathbb{Z}_{\leq 0}$.

Note that there exists a Fuchsian differential operator $P^{\prime \prime} \in W(x)$ such that $P=$ $P^{\prime \prime} P^{\prime}$. If there exists $j_{o}$ and $\nu_{o}$ such that $m_{j_{o}, n_{o}}^{\prime}=0$, namely, $m_{j_{o}, \nu_{o}}^{\prime \prime}=m_{j_{o}, \nu_{o}}>0$, the exponents of the monodromy generators of the solution $P^{\prime} v=0$ are generic and hence $\mathbf{m}^{\prime}$ should be realizable. The same claim is also true for the tuple $\mathbf{m}^{\prime \prime}$. Hence we have the proposition.

Example 12.17. i) The reduction of the universal operator with the spectral type $11,11,11$ which is given by Theorem 12.10 is

$$
\begin{aligned}
& \left\{\begin{array}{ccc}
x=\infty & 0 & 1 \\
\lambda_{0,1} & \lambda_{1,1} & \lambda_{2,1} \\
\lambda_{0,2} & \lambda_{1,2} & \lambda_{2,2}
\end{array}\right\} \quad\left(\sum \lambda_{j, \nu}=1\right) \\
& \longrightarrow\left\{\begin{array}{ccc}
x=\infty & 0 & 1 \\
2 \lambda_{0,2}+\lambda_{1,1}+\lambda_{2,1} & -\lambda_{0,2}-\lambda_{2,2} & -\lambda_{0,2}-\lambda_{1,2}
\end{array}\right\}
\end{aligned}
$$

because $\mu=\lambda_{0,1}+\lambda_{1,1}+\lambda_{2,1}-1=-\lambda_{0,2}-\lambda_{1,2}-\lambda_{2,2}$. Hence the necessary and sufficient condition for the irreducibility of the universal operator given by (12.34) is

which is equivalent to

$$
\left\{\begin{array}{l}
\lambda_{0,1}+\lambda_{1,1}+\lambda_{2,1} \notin \mathbb{Z}, \\
\lambda_{0,2}+\lambda_{1,1}+\lambda_{2,1} \notin \mathbb{Z}, \\
\lambda_{0,1}+\lambda_{1,2}+\lambda_{2,1} \notin \mathbb{Z}, \\
\lambda_{0,1}+\lambda_{1,1}+\lambda_{2,2} \notin \mathbb{Z},
\end{array}\right.
$$

$$
\lambda_{0, i}+\lambda_{1,1}+\lambda_{2, j} \notin \mathbb{Z} \quad \text { for } i=1,2 \text { and } j=1,2 .
$$

The rigid tuple $\mathbf{m}=11,11,11$ corresponds to the real root $\alpha_{\mathbf{m}}=2 \alpha_{0}+\alpha_{0,1}+$ $\alpha_{1,1}+\alpha_{2,1}$ under the notation in 89.1 . Then $\Delta(\mathbf{m})=\left\{\alpha_{0}, \alpha_{0}+\alpha_{j, 1} ; j=0,1,2\right\}$ 
and $\left(\Lambda \mid \alpha_{0}\right)=\lambda_{0,1}+\lambda_{1,1}+\lambda_{2,1}$ and $\left(\Lambda \mid \alpha_{0}+\alpha_{0,1}\right)=\lambda_{0,2}+\lambda_{1,1}+\lambda_{2,1}$, etc. under the notation in Theorem 12.13 .

The Riemann scheme for the Gauss hypergeometric series ${ }_{2} F_{1}(a, b, c ; z)$ is given by $\left\{\begin{array}{ccc}x=\infty & 0 & 1 \\ a & 0 & 0 \\ b & 1-c & c-a-b\end{array}\right\}$ and therefore the condition for the irreducibility is

$$
a \notin \mathbb{Z}, b \notin \mathbb{Z}, c-b \notin \mathbb{Z} \text { and } c-a \notin \mathbb{Z} .
$$

ii) The reduction of the Riemann scheme for the equation corresponding to ${ }_{3} F_{2}\left(\alpha_{1}, \alpha_{2}, \alpha_{3}, \beta_{1}, \beta_{2} ; x\right)$ is

$$
\begin{aligned}
& \left\{\begin{array}{ccc}
x=\infty & 0 & 1 \\
\alpha_{1} & 0 & {[0]_{(2)}} \\
\alpha_{2} & 1-\beta_{1} & -\beta_{3} \\
\alpha_{3} & 1-\beta_{2} &
\end{array}\right\} \\
& \longrightarrow\left\{\begin{array}{ccc}
x=\infty & 0 & 1 \\
\alpha_{2}-\alpha_{1}+1 & \alpha_{1}-\beta_{1} & 0 \\
\alpha_{3}-\alpha_{1}+1 & \alpha_{1}-\beta_{2} & \alpha_{1}-\beta_{3}-1
\end{array}\right\}
\end{aligned}
$$

with $\mu=\alpha_{1}-1$. Hence Theorem 12.10 says that the condition for the irreducibility equals

$$
\begin{cases}\alpha_{i} \notin \mathbb{Z} & (i=1,2,3), \\ \alpha_{1}-\beta_{j} \notin \mathbb{Z} & (j=1,2)\end{cases}
$$

together with

$$
\alpha_{i}-\beta_{j} \notin \mathbb{Z} \quad(i=2,3, j=1,2) .
$$

Here the second condition follows from i). Hence the condition for the irreducibility is

$$
\alpha_{i} \notin \mathbb{Z} \text { and } \alpha_{i}-\beta_{j} \notin \mathbb{Z} \quad(i=1,2,3, j=1,2) .
$$

iii) The reduction of the even family is as follows:

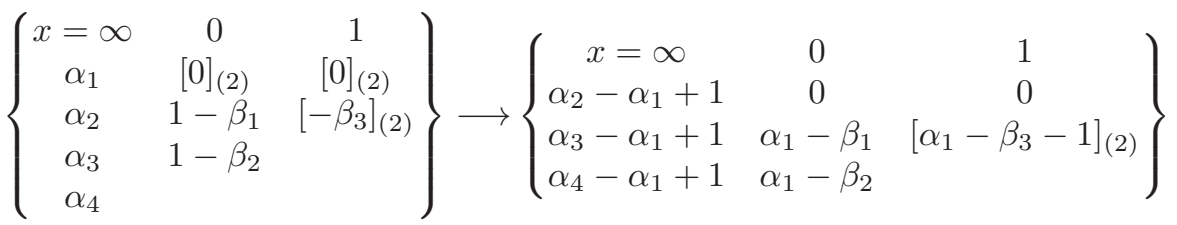

$$
\begin{aligned}
& \stackrel{(x-1)^{-\alpha_{1}+\beta_{3}+1}}{\longrightarrow}\left\{\begin{array}{ccc}
x=\infty & 0 & 1 \\
\alpha_{2}-\beta_{3} & 0 & -\alpha_{1}+\beta_{3}+1 \\
\alpha_{3}-\beta_{3} & \alpha_{1}-\beta_{1} & {[0]_{(2)}} \\
\alpha_{4}-\beta_{3} & \alpha_{1}-\beta_{2} &
\end{array}\right\} .
\end{aligned}
$$

Hence the condition for the irreducibility is

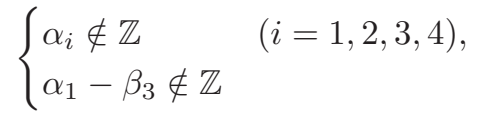

together with

$$
\begin{cases}\alpha_{i}-\beta_{3} \notin \mathbb{Z} & (i=2,3,4) . \\ \alpha_{1}+\alpha_{i}-\beta_{j}-\beta_{3} \notin \mathbb{Z} & (i=2,3,4, j=1,2)\end{cases}
$$


by the result in ii). Thus the condition is

$$
\begin{gathered}
\alpha_{i} \notin \mathbb{Z}, \alpha_{i}-\beta_{3} \notin \mathbb{Z} \text { and } \alpha_{1}+\alpha_{k}-\beta_{j}-\beta_{3} \notin \mathbb{Z} \\
(i=1,2,3,4, j=1,2, k=2,3,4) .
\end{gathered}
$$

Hence the condition for the irreducibility for the equation with the Riemann scheme

$$
\left\{\begin{array}{ccc}
\lambda_{0,1} & {\left[\lambda_{1,1}\right]_{(2)}} & {\left[\lambda_{2,1}\right]_{(2)}} \\
\lambda_{0,2} & \lambda_{1,2} & {\left[\lambda_{2,2}\right]_{(2)}} \\
\lambda_{0,3} & \lambda_{1,3} & \\
\lambda_{0,4} & &
\end{array}\right\}
$$

of type $1111,211,22$ is

$$
\begin{cases}\lambda_{0, \nu}+\lambda_{1,1}+\lambda_{2, k} \notin \mathbb{Z} & (\nu=1,2,3,4, k=1,2) \\ \lambda_{0, \nu}+\lambda_{0, \nu^{\prime}}+\lambda_{1,1}+\lambda_{1,2}+\lambda_{2,1}+\lambda_{2,2} \notin \mathbb{Z} & \left(1 \leq \nu<\nu^{\prime} \leq 4\right) .\end{cases}
$$

This condition corresponds to the rigid decompositions

$$
1^{4}, 21^{2}, 2^{2}=1,10,1 \oplus 1^{3}, 11^{2}, 21=1^{2}, 11,1^{2} \oplus 1^{2}, 11,1^{2},
$$

which are also important in the connection formula.

iv) (generalized Jordan-Pochhammer) The reduction of the universal operator of the rigid spectral type $32,32,32,32$ is as follows:

$$
\begin{aligned}
& \left\{\begin{array}{llll}
{\left[\lambda_{0,1}\right]_{(3)}} & {\left[\lambda_{1,1}\right]_{(3)}} & {\left[\lambda_{2,1}\right]_{(3)}} & {\left[\lambda_{3,1}\right]_{(3)}} \\
{\left[\lambda_{0,2}\right]_{(2)}} & {\left[\lambda_{1,2}\right]_{(2)}} & {\left[\lambda_{2,2}\right]_{(2)}} & {\left[\lambda_{3,2}\right]_{(2)}}
\end{array}\right\} \quad\left(3 \sum_{j=0}^{3} \lambda_{j, 1}+2 \sum_{j=0}^{3} \lambda_{j, 2}=4\right) \\
& \longrightarrow\left\{\begin{array}{cccc}
\lambda_{0,1}-2 \mu & \lambda_{1,1} & \lambda_{2,1} & \lambda_{3,1} \\
{\left[\lambda_{0,2}-\mu\right]_{(2)}} & {\left[\lambda_{1,2}+\mu\right]_{(2)}} & {\left[\lambda_{2,2}+\mu\right]_{(2)}} & {\left[\lambda_{3,2}+\mu\right]_{(2)}}
\end{array}\right\}
\end{aligned}
$$

with $\mu=\lambda_{0,1}+\lambda_{1,1}+\lambda_{2,1}+\lambda_{3,1}-1$. Hence the condition for the irreducibility is

$$
\begin{cases}\sum_{j=0}^{3} \lambda_{j, 1+\delta_{j, k}} \notin \mathbb{Z} & (k=0,1,2,3,4), \\ \sum_{j=0}^{3}\left(1+\delta_{j, k}\right) \lambda_{j, 1}+\sum_{j=0}^{3}\left(1-\delta_{j, k}\right) \lambda_{j, 2} \notin \mathbb{Z} & (k=0,1,2,3,4) .\end{cases}
$$

Note that under the notation defined by Definition 12.15 we have

$$
\operatorname{idx}_{\mathbf{m}}\left(\lambda_{0,1}+\lambda_{1,1}+\lambda_{2,1}+\lambda_{3,1}\right)=2
$$

and the index of any other linear form in (12.64) is 1.

In general, the universal operator with the Riemann scheme

$$
\begin{gathered}
\left\{\begin{array}{cccc}
{\left[\lambda_{0,1}\right]_{(k)}} & {\left[\lambda_{1,1}\right]_{(k)}} & {\left[\lambda_{2,1}\right]_{(k)}} & {\left[\lambda_{3,1}\right]_{(k)}} \\
{\left[\lambda_{0,2}\right]_{(k-1)}} & {\left[\lambda_{1,2}\right]_{(k-1)}} & {\left[\lambda_{2,2}\right]_{(k-1)}} & {\left[\lambda_{3,2}\right]_{(k-1)}}
\end{array}\right\} \\
\left(k \sum_{j=0}^{3} \lambda_{j, 1}+(k-1) \sum_{j=0}^{3} \lambda_{j, 2}=2 k\right)
\end{gathered}
$$

is irreducible if and only if

$$
\begin{cases}\sum_{j=0}^{3}\left(\nu-\delta_{j, k}\right) \lambda_{j, 1}+\sum_{j=0}^{3}\left(\nu-1+\delta_{j, k}\right) \lambda_{j, 1} \notin \mathbb{Z} & (k=0,1,2,3,4), \\ \sum_{j=0}^{3}\left(\nu^{\prime}+\delta_{j, k}\right) \lambda_{j, 1}+\sum_{j=0}^{3}\left(\nu^{\prime}-\delta_{j, k}\right) \lambda_{j, 2} \notin \mathbb{Z} & (k=0,1,2,3,4),\end{cases}
$$

for any integers $\nu$ and $\nu^{\prime}$ satisfying $1 \leq 2 \nu \leq k$ and $1 \leq 2 \nu^{\prime} \leq k-1$.

The rigid decomposition

$$
65,65,65,65=12,21,21,21 \oplus 53,44,44,44
$$

gives an example of the decomposition $\mathbf{m}=\mathbf{m}^{\prime} \oplus \mathbf{m}^{\prime \prime}$ with $\operatorname{supp} \alpha_{\mathbf{m}}=\operatorname{supp} \alpha_{\mathbf{m}^{\prime}}=$ $\operatorname{supp} \alpha_{\mathbf{m}^{\prime \prime}}$. 
v) The rigid Fuchsian differential equation with the Riemann scheme

$$
\left\{\begin{array}{ccccc}
x=0 & 1 & c_{3} & c_{4} & \infty \\
{[0]_{(9)}} & {[0]_{(9)}} & {[0]_{(9)}} & {[0]_{(9)}} & {\left[e_{0}\right]_{(8)}} \\
{[a]_{(3)}} & {[b]_{(3)}} & {[c]_{(3)}} & {[d]_{(3)}} & {\left[e_{1}\right]_{(3)}} \\
& & & & e_{2}
\end{array}\right\}
$$

is reducible when

$$
a+b+c+d+3 e_{0}+e_{1} \in \mathbb{Z}
$$

which is equivalent to $\frac{1}{3}\left(e_{0}-e_{2}-1\right) \in \mathbb{Z}$ under the Fuchs relation. At the generic point of this reducible condition, the spectral types of the decomposition in the Grothendieck group of the monodromy is

$$
93,93,93,93,831=31,31,31,31,211+31,31,31,31,310+31,31,31,31,310 .
$$

Note that the following reduction of the spectral types

$$
\begin{aligned}
& 93,93,93,93,831 \rightarrow 13,13,13,13,031 \rightarrow 10,10,10,10,001 \\
& 31,31,31,31,211 \rightarrow 11,11,11,11,011 \\
& 31,31,31,31,310 \rightarrow 01,01,01,01,010
\end{aligned}
$$

and $\operatorname{idx}(31,31,31,31,211)=-2$.

\section{SHIFT OPERATORS}

In this section we study an integer shift of spectral parameters $\lambda_{j, \nu}$ of the Fuchsian equation $P_{\mathbf{m}}(\lambda) u=0$. Here $P_{\mathbf{m}}(\lambda)$ is the universal operator (cf. Theorem 8.13) corresponding to the spectral type $\mathbf{m}=\left(m_{j, \nu}\right)_{\substack{j=0, \ldots, p \\ \nu=1, \ldots, n_{j}}}$. For simplicity, we assume that $\mathbf{m}$ is rigid in this section unless otherwise stated.

13.1. Construction of shift operators and recurrence relations. First we construct shift operators for general shifts.

Definition 13.1. For $\mathbf{m}=\left(m_{j, \nu}\right) \underset{\substack{j=0, \ldots, p \\ \nu=1, \ldots, n_{j}}}{\in} \in \mathcal{P}_{p+1}^{(n)}$, a set of integers $\left(\epsilon_{j, \nu}\right)_{\substack{j=0, \ldots, p \\ \nu=1, \ldots, n_{j}}}$ parametrized by $j$ and $\nu$ is called a shift compatible to $\mathbf{m}$ if

$$
\sum_{j=0}^{p} \sum_{\nu=1}^{n_{j}} \epsilon_{j, \nu} m_{j, \nu}=0
$$

Theorem 13.2 (shift operator). Fix a shift $\left(\epsilon_{j, \nu}\right)$ compatible to $\mathbf{m} \in \mathcal{P}_{p+1}^{(n)}$. Then there is a shift operator $R_{\mathbf{m}}(\epsilon, \lambda) \in W[x] \otimes \mathbb{C}\left[\lambda_{j, \nu}\right]$ which gives a homomorphism of the equation $P_{\mathbf{m}}\left(\lambda^{\prime}\right) v=0$ to $P_{\mathbf{m}}(\lambda) u=0$ defined by $v=R_{\mathbf{m}}(\epsilon, \lambda) u$. Here the Riemann scheme of $P_{\mathbf{m}}(\lambda)$ is $\left\{\lambda_{\mathbf{m}}\right\}=\left\{\left[\lambda_{j, \nu}\right]_{\left(m_{j, \nu}\right)}\right\}_{\substack{j=0, \ldots, p \\ \nu=1, \ldots, n_{j}}}$ and that of $P_{\mathbf{m}}\left(\lambda^{\prime}\right)$ is $\left\{\lambda_{\mathbf{m}}^{\prime}\right\}$ defined by $\lambda_{j, \nu}^{\prime}=\lambda_{j, \nu}+\epsilon_{j, \nu}$. Moreover we may assume ord $R_{\mathbf{m}}(\epsilon, \lambda)<$ ord $\mathbf{m}$ and $R_{\mathbf{m}}(\epsilon, \lambda)$ never vanishes as a function of $\lambda$ and then $R_{\mathbf{m}}(\epsilon, \lambda)$ is uniquely determined up to a constant multiple.

Putting

$$
\tau=\left(\tau_{j, \nu}\right) \underset{\substack{0 \leq j \leq p \\ 1 \leq \nu \leq n_{j}}}{\text { with }} \tau_{j, \nu}:=(2+(p-1) n) \delta_{j, 0}-m_{j, \nu}
$$

and $d=\operatorname{ord} R_{\mathbf{m}}(\epsilon, \lambda)$, we have

$$
P_{\mathbf{m}}(\lambda+\epsilon) R_{\mathbf{m}}(\epsilon, \lambda)=(-1)^{d} R_{\mathbf{m}}(\epsilon, \tau-\lambda-\epsilon)^{*} P_{\mathbf{m}}(\lambda)
$$

under the notation in Theorem 6.19 ii). 
Proof. We will prove the theorem by the induction on ord $\mathbf{m}$. The theorem is clear if ord $\mathbf{m}=1$.

We may assume that $\mathbf{m}$ is monotone. Then the reduction $\left\{\tilde{\lambda}_{\tilde{\mathbf{m}}}\right\}$ of the Riemann scheme is defined by (12.33). Hence putting

$$
\left\{\begin{array}{l}
\tilde{\epsilon}_{1}=\epsilon_{0,1}+\cdots+\epsilon_{p, 1}, \\
\tilde{\epsilon}_{j, \nu}=\epsilon_{j, \nu}+\left((-1)^{\delta_{j, 0}}-\delta_{\nu, 1}\right) \tilde{\epsilon}_{1} \quad\left(j=0, \ldots, p, \nu=1, \ldots, n_{j}\right),
\end{array}\right.
$$

there is a shift operator $R(\tilde{\epsilon}, \tilde{\lambda})$ of the equation $P_{\tilde{\mathbf{m}}}\left(\tilde{\lambda}^{\prime}\right) \tilde{v}=0$ to $P_{\tilde{\mathbf{m}}}(\tilde{\lambda}) \tilde{u}=0$ defined by $\tilde{v}=R(\tilde{\epsilon}, \tilde{\lambda}) \tilde{u}$. Note that

$$
\begin{aligned}
P_{\tilde{\mathbf{m}}}(\tilde{\lambda})= & \partial_{\max } P_{\mathbf{m}}(\lambda)=\operatorname{Ad}\left(\prod_{j=1}^{p}\left(x-c_{j}\right)^{\lambda_{j, 1}}\right) \prod_{j=1}^{p}\left(x-c_{j}\right)^{m_{j, 1}-d} \partial^{-d} \operatorname{Ad}\left(\partial^{-\mu}\right) \\
& \prod_{j=1}^{p}\left(x-c_{j}\right)^{-m_{j, 1}} \operatorname{Ad}\left(\prod_{j=1}^{p}\left(x-c_{j}\right)^{-\lambda_{j, 1}}\right) P_{\mathbf{m}}(\lambda), \\
P_{\tilde{\mathbf{m}}}\left(\tilde{\lambda}^{\prime}\right)= & \partial_{\max } P_{\mathbf{m}}\left(\lambda^{\prime}\right)=\operatorname{Ad}\left(\prod_{j=1}^{p}\left(x-c_{j}\right)^{\lambda_{j, 1}}\right) \prod_{j=1}^{p}\left(x-c_{j}\right)^{m_{j, 1}-d} \partial^{-d} \operatorname{Ad}\left(\partial^{-\mu^{\prime}}\right) \\
& \prod_{j=1}^{p}\left(x-c_{j}\right)^{-m_{j, 1}} \operatorname{Ad}\left(\prod_{j=1}^{p}\left(x-c_{j}\right)^{-\lambda_{j, 1}^{\prime}}\right) P_{\mathbf{m}}\left(\lambda^{\prime}\right) .
\end{aligned}
$$

Suppose $\lambda_{j, \nu}$ are generic. Let $u(x)$ be a local solution of $P_{\mathbf{m}}(\lambda) u=0$ at $x=c_{1}$ corresponding to a characteristic exponent different from $\lambda_{1,1}$. Then

$$
\tilde{u}(x):=\prod_{j=1}^{p}\left(x-c_{j}\right)^{\lambda_{j, 1}} \partial^{-\mu} \prod_{j=1}^{p}\left(x-c_{j}\right)^{-\lambda_{j, 1}} u(x)
$$

satisfies $P_{\tilde{\mathbf{m}}}(\tilde{\lambda}) \tilde{u}(x)=0$. Putting

$$
\begin{aligned}
\tilde{v}(x) & :=R(\tilde{\epsilon}, \tilde{\lambda}) \tilde{u}(x), \\
v(x) & :=\prod_{j=1}^{p}\left(x-c_{j}\right)^{\lambda_{j, 1}^{\prime}} \partial^{\mu^{\prime}} \prod_{j=1}^{p}\left(x-c_{j}\right)^{\lambda_{j, 1}^{\prime}} \tilde{v}(x), \\
\tilde{R}(\tilde{\epsilon}, \tilde{\lambda}) & :=\operatorname{Ad}\left(\prod_{j=1}^{p}\left(x-c_{j}\right)^{\lambda_{j, 1}}\right) R(\tilde{\epsilon}, \tilde{\lambda})
\end{aligned}
$$

we have $P_{\tilde{\mathbf{m}}}\left(\tilde{\lambda}^{\prime}\right) \tilde{u}(x)=0, P_{\mathbf{m}}\left(\lambda^{\prime}\right) v(x)=0$ and

$$
\prod_{j=1}^{p}\left(x-c_{j}\right)^{\epsilon_{j, 1}} \partial^{-\mu^{\prime}} \prod_{j=1}^{p}\left(x-c_{j}\right)^{-\lambda_{j, 1}^{\prime}} v(x)=\tilde{R}(\tilde{\epsilon}, \tilde{\lambda}) \partial^{-\mu} \prod_{j=1}^{p}\left(x-c_{j}\right)^{-\lambda_{j, 1}} u(x) .
$$

In general, if

$$
S_{2} \prod_{j=1}^{p}\left(x-c_{j}\right)^{\epsilon_{j, 1}} \partial^{-\mu^{\prime}} \prod_{j=1}^{p}\left(x-c_{j}\right)^{-\lambda_{j, 1}^{\prime}} v(x)=S_{1} \partial^{-\mu} \prod_{j=1}^{p}\left(x-c_{j}\right)^{-\lambda_{j, 1}} u(x)
$$

with $S_{1}, S_{2} \in W[x]$, we have

$$
R_{2} v(x)=R_{1} u(x)
$$


by putting

$$
\begin{aligned}
& R_{1}=\prod_{j=1}^{p}\left(x-c_{j}\right)^{\lambda_{j, \nu}+k_{1, j}} \partial^{\mu+\ell} \prod_{j=1}^{p}\left(x-c_{j}\right)^{k_{2, j}} S_{1} \prod_{j=1}^{\epsilon_{j, 1}} \partial^{-\mu} \prod_{j=1}^{p}\left(x-c_{j}\right)^{-\lambda_{j, \nu}}, \\
& R_{2}=\prod_{j=1}^{p}\left(x-c_{j}\right)^{\lambda_{j, \nu}+k_{1, j}} \partial^{\mu+\ell} \prod_{j=1}^{p}\left(x-c_{j}\right)^{k_{2, j}} S_{2} \prod_{j=1}^{\epsilon_{j, 1}} \partial^{-\mu^{\prime}} \prod_{j=1}^{p}\left(x-c_{j}\right)^{-\lambda_{j, \nu}^{\prime}}
\end{aligned}
$$

with suitable integers $k_{1, j}, k_{2, j}$ and $\ell$ so that $R_{1}, R_{2} \in W[x ; \lambda]$.

We choose a non-zero polynomial $S_{2} \in \mathbb{C}[x]$ so that $S_{1}=S_{2} \tilde{R}(\tilde{\epsilon}, \tilde{\lambda}) \in W[x]$. Since $P_{\mathbf{m}}\left(\lambda^{\prime}\right)$ is irreducible in $W(x ; \lambda)$ and $R_{2} v(x)$ is not zero, there exists $R_{3} \in W(x ; \xi)$ such that $R_{3} R_{2}-1 \in W(x ; \lambda) P_{\mathbf{m}}\left(\lambda^{\prime}\right)$. Then $v(x)=R u(x)$ with the operator $R=R_{3} R_{1} \in W(x ; \lambda)$.

Since the equations $P_{\mathbf{m}}(\lambda) u=0$ and $P_{\mathbf{m}}\left(\lambda^{\prime}\right) v=0$ are irreducible $W(x ; \lambda)$ modules, the correspondence $v=R u$ gives an isomorphism between these two modules. Since any solutions of these equations are holomorphically continued along the path contained in $\mathbb{C} \backslash\left\{c_{1}, \ldots, c_{p}\right\}$, the coefficients of the operator $R$ are holomorphic in $\mathbb{C} \backslash\left\{c_{1}, \ldots, c_{p}\right\}$. Multiplying $R$ by a suitable element of $\mathbb{C}(\lambda)$, we may assume $R \in W(x) \otimes \mathbb{C}[\lambda]$ and $R$ does not vanish at any $\lambda_{j, \nu} \in \mathbb{C}$.

Put $f(x)=\prod_{j=1}^{p}\left(x-c_{j}\right)^{n}$. Since $R_{\mathbf{m}}(\epsilon, \lambda)$ is a shift operator, there exists $S_{\mathbf{m}}(\epsilon, \lambda) \in W(x ; \lambda)$ such that

$$
f^{-1} P_{\mathbf{m}}(\lambda+\epsilon) R_{\mathbf{m}}(\epsilon, \lambda)=S_{\mathbf{m}}(\epsilon, \lambda) f^{-1} P_{\mathbf{m}}(\lambda) .
$$

Then Theorem 6.19 ii) shows

$$
\begin{aligned}
R_{\mathbf{m}}(\epsilon, \lambda)^{*}\left(f^{-1} P_{\mathbf{m}}(\lambda+\epsilon)\right)^{*} & =\left(f^{-1} P_{\mathbf{m}}(\lambda)\right)^{*} S_{\mathbf{m}}(\epsilon, \lambda)^{*}, \\
R_{\mathbf{m}}(\epsilon, \lambda)^{*} \cdot f^{-1} P_{\mathbf{m}}(\lambda+\epsilon)^{\vee} & =f^{-1} P_{\mathbf{m}}(\lambda)^{\vee} \cdot S_{\mathbf{m}}(\epsilon, \lambda)^{*}, \\
R_{\mathbf{m}}(\epsilon, \lambda)^{*} f^{-1} P_{\mathbf{m}}(\rho-\lambda-\epsilon) & =f^{-1} P_{\mathbf{m}}(\rho-\lambda) S_{\mathbf{m}}(\epsilon, \lambda)^{*}, \\
R_{\mathbf{m}}(\epsilon, \rho-\mu-\epsilon)^{*} f^{-1} P_{\mathbf{m}}(\mu) & =f^{-1} P_{\mathbf{m}}(\mu+\epsilon) S_{\mathbf{m}}(\epsilon, \rho-\mu-\epsilon)^{*} .
\end{aligned}
$$

Here we use the notation (6.52) and put $\rho_{j, \nu}=2(1-n) \delta_{j, 0}+n-m_{j, \nu}$ and $\mu=$ $\rho-\lambda-\epsilon$. Comparing (13.9) with (13.8), we see that $S_{\mathbf{m}}(\epsilon, \lambda)$ is a constant multiple of the operator $R_{\mathbf{m}}(\epsilon, \rho-\lambda-\epsilon)^{*}$ and $f R_{\mathbf{m}}(\epsilon, \rho-\lambda-\epsilon)^{*} f^{-1}=\left(f^{-1} R_{\mathbf{m}}(\epsilon, \rho-\lambda-\epsilon) f\right)^{*}=$ $R_{\mathbf{m}}(\epsilon, \tau-\lambda-\epsilon)^{*}$ and we have $(13.3)$.

Note that the operator $R_{\mathbf{m}}(\epsilon, \lambda)$ is uniquely defined up to a constant multiple.

The following theorem gives a recurrence relation among specific local solutions with a rigid spectral type and a relation between the shift operator $R_{\mathbf{m}}(\epsilon, \lambda)$ and the universal operator $P_{\mathbf{m}}(\lambda)$.

Theorem 13.3. Retain the notation in Corollary 12.12 and Theorem 13.8 with a rigid tuple $\mathbf{m}$. Assume $m_{j, n_{j}}=1$ for $j=0,1$ and 2 . Put $\epsilon=\left(\epsilon_{j, \nu}\right), \epsilon^{\prime}=\left(\epsilon_{j, \nu}^{\prime}\right)$,

$$
\epsilon_{j, \nu}=\delta_{j, 1} \delta_{\nu, n_{1}}-\delta_{j, 2} \delta_{\nu, n_{2}} \text { and } \epsilon_{j, \nu}^{\prime}=\delta_{j, 0} \delta_{\nu, n_{0}}-\delta_{j, 2} \delta_{\nu, n_{2}}
$$

for $j=0, \ldots, p$ and $\nu=1, \ldots, n_{j}$.

i) Define $Q_{\mathbf{m}}(\lambda) \in W(x ; \lambda)$ so that $Q_{\mathbf{m}}(\lambda) P_{\mathbf{m}}\left(\lambda+\epsilon^{\prime}\right)-1 \in W(x ; \lambda) P_{\mathbf{m}}(\lambda+\epsilon)$. Then

$$
R_{\mathbf{m}}(\epsilon, \lambda)-C(\lambda) Q_{\mathbf{m}}(\lambda) P_{\mathbf{m}}\left(\lambda+\epsilon^{\prime}\right) \in W(x ; \lambda) P_{\mathbf{m}}(\lambda)
$$

with a rational function $C(\lambda)$ of $\lambda_{j, \nu}$.

ii) Let $u_{\lambda}(x)$ be the local solution of $P_{\mathbf{m}}(\lambda) u=0$ such that $u_{\lambda}(x) \equiv\left(x-c_{1}\right)^{\lambda_{1, n_{1}}}$ $\bmod \left(x-c_{1}\right)^{\lambda_{1, n_{1}}+1} O_{c_{1}}$ for generic $\lambda_{j, \nu}$. Then we have the recurrence relation

$$
u_{\lambda}(x)=u_{\lambda+\epsilon^{\prime}}(x)+\left(c_{1}-c_{2}\right) \prod_{\nu=0}^{K-1} \frac{\lambda(\nu+1)_{1, n_{1}}-\lambda(\nu)_{1, \ell(\nu)_{1}}+1}{\lambda(\nu)_{1, n_{1}}-\lambda(\nu)_{1, \ell(\nu)_{1}}+1} \cdot u_{\lambda+\epsilon}(x) .
$$


Proof. Under the notation in Corollary 12.12, $\ell(k)_{j} \neq n_{j}$ for $j=0,1,2$ and $k=$ $0, \ldots, K-1$ and therefore the operation $\partial_{\max }^{K}$ on $P_{\mathbf{m}}(\lambda)$ is equals to $\partial_{m a x}^{K}$ on $P_{\mathbf{m}}(\lambda+\epsilon)$ if they are realized by the product of the operators of the form (7.25). Hence by the induction on $K$, the proof of Theorem 13.2 (cf. 13.5), (13.6) and (13.7)) shows

$$
P_{\mathbf{m}}\left(\lambda+\epsilon^{\prime}\right) u(x)=P_{\mathbf{m}}\left(\lambda+\epsilon^{\prime}\right) v(x)
$$

for suitable functions $u(x)$ and $v(x)$ satisfying $P_{\mathbf{m}}(\lambda) u(x)=P_{\mathbf{m}}(\lambda+\epsilon) v(x)=0$ and moreover (13.12) is calculated by (4.6). Note that the identities

$$
\begin{aligned}
\left(c_{1}-c_{2}\right) \prod_{j=1}^{p}\left(x-c_{j}\right)^{\lambda_{j}+\epsilon_{j}^{\prime}} & =\prod_{j=1}^{p}\left(x-c_{j}\right)^{\lambda_{j}}-\prod_{j=1}^{p}\left(x-c_{j}\right)^{\lambda_{j}+\epsilon_{j}}, \\
\left(\partial-\sum_{j=1}^{p} \frac{\lambda_{j}+\epsilon_{j}^{\prime}}{x-c_{j}}\right) \prod_{j=1}^{p}\left(x-c_{j}\right)^{\lambda_{j}} & =\left(\partial-\sum_{j=1}^{p} \frac{\lambda_{j}+\epsilon_{j}^{\prime}}{x-c_{j}}\right) \prod_{j=1}^{p}\left(x-c_{j}\right)^{\lambda_{j}+\epsilon_{j}}
\end{aligned}
$$

correspond to (13.12) and (13.13), respectively, when $K=0$.

Note that (13.13) may be proved by (13.12). The claim i) in this theorem follows from the fact $v(x)=Q_{\mathbf{m}}(\lambda) P_{\mathbf{m}}\left(\lambda+\epsilon^{\prime}\right) v(x)=Q_{\mathbf{m}}(\lambda) P_{\mathbf{m}}\left(\lambda+\epsilon^{\prime}\right) u(x)$.

In general, we have the following theorem for the recurrence relation.

Theorem 13.4 (recurrence relations). Let $\mathbf{m} \in \mathcal{P}^{(n)}$ be a rigid tuple with $m_{1, n_{1}}=$ 1 and let $u_{1}(\lambda, x)$ be the normalized solution of the equation $P_{\mathbf{m}}(\lambda) u=0$ with respect to the exponent $\lambda_{1, n_{1}}$ at $x=c_{1}$. Let $\epsilon^{(i)}$ be shifts compatible to $\mathbf{m}$ for $i=0, \ldots, n$. Then there exists polynomial functions $r_{i}(x, \lambda) \in \mathbb{C}[x, \lambda]$ such that $\left(r_{0}, \ldots, r_{n}\right) \neq 0$ and

$$
\sum_{i=0}^{n} r_{i}(x, \lambda) u_{1}\left(\lambda+\epsilon^{(i)}, x\right)=0 .
$$

Proof. There exist $R_{i} \in \mathbb{C}(\lambda) R_{\mathbf{m}}\left(\epsilon^{(i)}, \lambda\right)$ satisfying $u_{1}\left(\lambda+\epsilon^{(i)}, x\right)=R_{i} u_{1}(\lambda, x)$ and ord $R_{i}<n$. We have $r_{i}(x, \lambda)$ with $\sum_{i=0}^{n} r_{i}(x, \lambda) R_{i}=0$ and the claim.

Example 13.5 (Gauss hypergeometric equation). Let $P_{\lambda} u=0$ and $P_{\lambda^{\prime}} v=0$ be Fuchsian differential equations with the Riemann Scheme

$$
\left\{\begin{array}{ccc}
x=\infty & 0 & 1 \\
\lambda_{0,1} & \lambda_{1,1} & \lambda_{2,1} \\
\lambda_{0,2} & \lambda_{1,2} & \lambda_{2,2}
\end{array}\right\} \text { and }\left\{\begin{array}{ccc}
x=\infty & 0 & 1 \\
\lambda_{0,1}^{\prime}=\lambda_{0,1} & \lambda_{1,1}^{\prime}=\lambda_{1,1} & \lambda_{2,1}^{\prime}=\lambda_{2,1} \\
\lambda_{0,2}^{\prime}=\lambda_{0,2} & \lambda_{1,2}^{\prime}=\lambda_{1,2}+1 & \lambda_{2,2}=\lambda_{2,2}-1
\end{array}\right\},
$$

respectively. Here the operators $P_{\lambda}=P_{\lambda_{0,1}, \lambda_{0,2}, \lambda_{1,1}, \lambda_{1,2}, \lambda_{2,1}, \lambda_{2,1}}$ and $P_{\lambda^{\prime}}$ are given in (2.51). The normalized local solution $u_{\lambda}(x)$ of $P_{\lambda} u=0$ corresponding to the exponent $\lambda_{1,2}$ at $x=0$ is

By the reduction $\left\{\begin{array}{ccc}x=\infty & 0 & 1 \\ \lambda_{0,1} & \lambda_{1,1} & \lambda_{2,1} \\ \lambda_{0,2} & \lambda_{1,2} & \lambda_{2,2}\end{array}\right\} \rightarrow\left\{\begin{array}{ccc}x=\infty & 0 & 1 \\ \lambda_{0,2}-\mu & \lambda_{1,2}+\mu & \lambda_{2,2}+\mu\end{array}\right\}$ with $\mu=\lambda_{0,1}+\lambda_{1,1}+\lambda_{2,1}-1$, the recurrence relation (13.12) means

$$
\begin{aligned}
x^{\lambda_{1,2}}(1-x)^{\lambda_{2,1}} F\left(\lambda_{0,1}+\lambda_{1,2}+\lambda_{2,1}, \lambda_{0,2}+\lambda_{1,2}+\lambda_{2,1}, 1-\lambda_{1,1}+\lambda_{1,2} ; x\right) \\
=x^{\lambda_{1,2}}(1-x)^{\lambda_{2,1}} F\left(\lambda_{0,1}+\lambda_{1,2}+\lambda_{2,1}, \lambda_{0,2}+\lambda_{1,2}+\lambda_{2,1}+1,1-\lambda_{1,1}+\lambda_{1,2} ; x\right) \\
-\frac{\lambda_{0,1}+\lambda_{1,2}+\lambda_{2,1}}{1-\lambda_{1,1}+\lambda_{1,2}} x^{\lambda_{1,2}+1}(1-x)^{\lambda_{2,1}} \\
\quad \cdot F\left(\lambda_{0,1}+\lambda_{1,2}+\lambda_{2,1}+1, \lambda_{0,2}+\lambda_{1,2}+\lambda_{2,1}+1,2-\lambda_{1,1}+\lambda_{1,2} ; x\right),
\end{aligned}
$$


which is equivalent to the recurrence relation

$$
F(\alpha, \beta, \gamma, x)=F(\alpha, \beta+1, \gamma ; x)-\frac{\alpha}{\gamma} x F(\alpha+1, \beta+1, \gamma+1 ; x) .
$$

Using the expression 2.51), we have

$$
\begin{aligned}
P_{\lambda+\epsilon^{\prime}}-P_{\lambda}= & x^{2}(x-1) \partial+\lambda_{0,1} x^{2}-\left(\lambda_{0,1}+\lambda_{2,1}\right) x, \\
P_{\lambda+\epsilon^{\prime}}-P_{\lambda+\epsilon}= & x(x-1)^{2} \partial+\lambda_{0,1} x^{2}-\left(\lambda_{0,1}+\lambda_{1,1}\right) x-\lambda_{1,1}, \\
(x-1) P_{\lambda+\epsilon}= & \left(x(x-1) \partial+\left(\lambda_{0,2}-2\right) x+\lambda_{1,2}+1\right)\left(P_{\lambda+\epsilon^{\prime}}-P_{\lambda+\epsilon}\right) \\
& -\left(\lambda_{0,1}+\lambda_{1,1}+\lambda_{2,1}\right)\left(\lambda_{0,2}+\lambda_{1,2}+\lambda_{2,1}\right) x(x-1), \\
x^{-1}(x-1)^{-1}( & \left.x(x-1) \partial+\left(\lambda_{0,2}-2\right) x+\lambda_{1,2}+1\right)\left(P_{\lambda+\epsilon^{\prime}}-P_{\lambda}\right)-(x-1)^{-1} P_{\lambda} \\
= & -\left(\lambda_{0,1}+\lambda_{1,1}+\lambda_{2,1}\right)\left(x \partial-\lambda_{1,2}-\frac{\lambda_{2,1} x}{x-1}\right)
\end{aligned}
$$

and hence (13.11) says

$$
R_{\mathbf{m}}(\epsilon, \lambda)=x \partial-\lambda_{1,2}-\lambda_{2,1} \frac{x}{x-1} .
$$

In the same way we have

$$
R_{\mathbf{m}}(-\epsilon, \lambda+\epsilon)=(x-1) \partial-\lambda_{2,2}+1-\lambda_{1,1} \frac{x-1}{x} .
$$

Then

$$
\begin{aligned}
R_{\mathbf{m}}(-\epsilon, \lambda+\epsilon) R_{\mathbf{m}}(\epsilon, \lambda) & -x^{-1}(x-1)^{-1} P_{\lambda} \\
& =-\left(\lambda_{0,1}+\lambda_{1,2}+\lambda_{2,1}\right)\left(\lambda_{0,2}+\lambda_{1,2}+\lambda_{2,1}\right)
\end{aligned}
$$

and since $-R_{\mathbf{m}}(\epsilon, \tau-\lambda-\epsilon)^{*}=-\left(x \partial+\left(\lambda_{1,2}+2\right)+\left(\lambda_{2,1}+1\right) \frac{x}{x-1}\right)^{*}=x \partial-\lambda_{1,2}-$ $1-\left(\lambda_{2,1}+1\right) \frac{x}{x-1}$ with $\tau$ given by (13.2), the identity (13.3) means

$$
P_{\lambda} R_{\mathbf{m}}(\epsilon, \lambda)=\left(x \partial-\left(\lambda_{1,2}+1\right)-\left(\lambda_{2,1}+1\right) \frac{x}{x-1}\right) P_{\lambda+\epsilon} .
$$

Remark 13.6. Suppose $\mathbf{m}$ is irreducibly realizable but it is not rigid. If the reductions of $\left\{\lambda_{\mathbf{m}}\right\}$ and $\left\{\lambda_{\mathbf{m}}^{\prime}\right\}$ to Riemann schemes with a fundamental tuple of partitions are transformed into each other by suitable additions, we can construct a shift operator as in Theorem 13.2. If they are not so, we need a shift operator for equations whose spectral type are fundamental and such an operator is called a Schlesinger transformation.

Now we examine the condition that a universal operator defines a shift operator.

Theorem 13.7 (universal operator and shift operator). Let $\mathbf{m}=\left(m_{j, \nu}\right)_{\substack{0 \leq j \leq p \\ 1 \leq \nu \leq n_{j}}}$ and $\mathbf{m}^{\prime}=\left(m_{j, \nu}^{\prime}\right)_{\substack{0 \leq j \leq p \\ 1 \leq \nu \leq n_{j}}} \in \mathcal{P}_{p+1}$ be irreducibly realizable and monotone. They may not be rigid. Suppose ord $\mathbf{m}>\operatorname{ord} \mathbf{m}^{\prime}$. Fix $j_{0}$ with $0 \leq j_{0} \leq p$. Let $n_{j_{0}}^{\prime}$ be a positive integer such that $m_{j_{0}, n_{j_{0}}^{\prime}}^{\prime}>m_{j_{0}, n_{j_{0}+1}^{\prime}}^{\prime}=0$ and let $P_{\mathbf{m}}(\lambda)$ be the universal operator corresponding to $\left\{\lambda_{\mathbf{m}}\right\}$. Putting $\lambda_{j, \nu}^{\prime}=\lambda_{j, \nu}$ when $(j, \nu) \neq\left(j_{0}, n_{j_{0}}^{\prime}\right)$, we define the universal operator $P_{\mathbf{m}^{\prime}}^{j_{0}}(\lambda):=P_{\mathbf{m}^{\prime}}\left(\lambda^{\prime}\right)$ with the Riemann scheme $\left\{\lambda_{\mathbf{m}^{\prime}}^{\prime}\right\}$. Here $\lambda_{j_{0}, n_{j_{0}}^{\prime}}^{\prime}$ is determined by the Fuchs condition. Suppose

$$
\left(\alpha_{\mathbf{m}} \mid \alpha_{\mathbf{m}^{\prime}}\right)\left(=\sum_{j=0}^{p} \sum_{\nu=1}^{n_{j}} m_{j, \nu} m_{j, \nu}^{\prime}-(p-1) \operatorname{ord} \mathbf{m} \cdot \operatorname{ord} \mathbf{m}^{\prime}\right)=m_{j_{0}, n_{j_{0}}^{\prime}} m_{j_{0}, n_{j_{0}}^{\prime}}^{\prime}
$$


Then $\mathbf{m}^{\prime}$ is rigid and the universal operator $P_{\mathbf{m}^{\prime}}^{j_{0}}(\lambda)$ is the shift operator $R_{\mathbf{m}}(\epsilon, \lambda)$ :

$$
\begin{gathered}
\left\{\left[\lambda_{j, \nu}\right]_{\left(m_{j, \nu}\right)}\right\}_{\substack{0 \leq j \leq p \\
1 \leq \nu \leq n_{j}}} \stackrel{R_{\mathbf{m}}(\epsilon, \lambda)=P_{\mathbf{m}^{\prime}}^{j_{0}}(\lambda)}{\longrightarrow}\left\{\left[\lambda_{j, \nu}+\epsilon_{j, \nu}\right]_{\left(m_{j, \nu}\right)}\right\}_{\substack{0 \leq j \leq p \\
1 \leq \nu \leq n_{j}}} \\
\text { with } \epsilon_{j, \nu}=\left(1-\delta_{j, j_{0}} \delta_{\nu, n_{j_{0}}^{\prime}}\right) m_{j, \nu}^{\prime}-\delta_{j, 0} \cdot(p-1) \operatorname{ord} \mathbf{m}^{\prime} .
\end{gathered}
$$

Proof. We may assume $\lambda$ is generic. Let $u(x)$ be the solution of the irreducible differential equation $P_{\mathbf{m}}(\lambda) u=0$. Then

$$
\begin{aligned}
& P_{\mathbf{m}^{\prime}}\left(\lambda^{\prime}\right)\left(x-c_{j}\right)^{\lambda_{j, \nu}} \mathcal{O}_{c_{j}} \subset\left(x-c_{j}\right)^{\lambda_{j, \nu}+\left(1-\delta_{j, j_{0}} \delta_{\nu, n_{j_{0}}^{\prime}}\right) m_{j, \nu}^{\prime}} \mathcal{O}_{c_{j}}, \\
& P_{\mathbf{m}^{\prime}}\left(\lambda^{\prime}\right) x^{-\lambda_{0, \nu}} \mathcal{O}_{\infty} \subset x^{-\lambda_{0, \nu}-\left(1-\delta_{0, j_{0}} \delta_{\nu, n_{j_{0}}^{\prime}}\right) m_{0, \nu}^{\prime}+(p-1) \text { ord } \mathbf{m}^{\prime}} \mathcal{O}_{\infty}
\end{aligned}
$$

and $P_{\mathbf{m}^{\prime}}\left(\lambda^{\prime}\right) u(x)$ satisfies a Fuchsian differential equation. Hence the fact $R_{\mathbf{m}}(\epsilon, \lambda)=$ $P_{\mathbf{m}^{\prime}}\left(\lambda^{\prime}\right)$ is clear from the characteristic exponents of the equation at each singular points. Note that the left hand side of (13.21) is never larger than the right hand side and if they are not equal, $P_{\mathbf{m}^{\prime}}\left(\lambda^{\prime}\right) u(x)$ satisfies a Fuchsian differential equation with apparent singularities for the solutions $u(x)$ of $P_{\mathbf{m}}(\lambda) u=0$.

It follows from Lemma 12.3 that the condition (13.21) means that at least one of the irreducibly realizable tuples $\mathbf{m}$ and $\mathbf{m}^{\prime}$ is rigid and therefore if $\mathbf{m}$ is rigid, so is $\mathbf{m}^{\prime}$ because $R_{\mathbf{m}}(\epsilon, \lambda)$ is unique up to constant multiple.

If ord $\mathbf{m}^{\prime}=1$, the condition (13.21) means that $\mathbf{m}$ is of Okubo type, which will be examined in the next subsection. It will be interesting to examine other cases. When $\mathbf{m}=\mathbf{m}^{\prime} \oplus \mathbf{m}^{\prime \prime}$ is a rigid decomposition or $\alpha_{\mathbf{m}^{\prime}} \in \Delta(\mathbf{m})$, we easily have many examples satisfying (13.21).

Here we give examples of the pairs $\left(\mathbf{m} ; \mathbf{m}^{\prime}\right)$ with ord $\mathbf{m}^{\prime}>1$ :

$$
\begin{array}{ll}
\left(1^{n}, 1^{n}, n-11 ; 1^{n-1}, 1^{n-1}, n-21\right) & (221,32,32,41 ; 110,11,11,20) \\
\left(1^{2 m}, m m-11, m^{2} ; 1^{2}, 110,1^{2}\right) & \left(1^{2 m+1}, m^{2} 1, m+1 m ; 1^{2}, 1^{2} 0,11\right) \\
(221,221,221 ; 110,110,110) & (211,221,221 ; 110,110,110) .
\end{array}
$$

13.2. Relation to reducibility. In this subsection, we will examine whether the shift operator defines a $W(x)$-isomorphism or doesn't.

Theorem 13.8. Retain the notation in Theorem 13.2 and define a polynomial function $c_{\mathbf{m}}(\epsilon ; \lambda)$ of $\lambda_{j, \nu}$ by

$$
R_{\mathbf{m}}(-\epsilon, \lambda+\epsilon) R_{\mathbf{m}}(\epsilon, \lambda)-c_{\mathbf{m}}(\epsilon ; \lambda) \in(W[x] \otimes \mathbb{C}[\lambda]) P_{\mathbf{m}}(\lambda) .
$$

i) Fix $\lambda_{j, \nu}^{o} \in \mathbb{C}$. If $c_{\mathbf{m}}\left(\epsilon ; \lambda^{o}\right) \neq 0$, the equation $P_{\mathbf{m}}\left(\lambda^{o}\right) u=0$ is isomorphic to the equation $P_{\mathbf{m}}\left(\lambda^{o}+\epsilon\right) v=0$. If $c_{\mathbf{m}}\left(\epsilon ; \lambda^{o}\right)=0$, then the equations $P_{\mathbf{m}}\left(\lambda^{o}\right) u=0$ and $P_{\mathbf{m}}\left(\lambda^{o}+\epsilon\right) v=0$ are not irreducible.

ii) Under the notation in Proposition 12.16, there exists a set $\Lambda$ whose elements $(i, k)$ are in $\{1, \ldots, N\} \times \mathbb{Z}$ such that

$$
c_{\mathbf{m}}(\epsilon ; \lambda)=C \prod_{(i, k) \in \Lambda}\left(\ell_{i}(\lambda)-k\right)
$$

with a constant $C \in \mathbb{C}^{\times}$. Here $\Lambda$ may contain some elements $(i, k)$ with multiplicities.

Proof. Since $u \mapsto R_{\mathbf{m}}(-\epsilon, \lambda+\epsilon) R_{\mathbf{m}}(\epsilon, \lambda) u$ defined an endomorphism of the irreducible equation $P_{\mathbf{m}}(\lambda) u=0$, the existence of $c_{\mathbf{m}}(\epsilon ; \lambda)$ is clear.

If $c_{\mathbf{m}}\left(\epsilon ; \lambda^{o}\right)=0$, the non-zero homomorphism of $P_{\mathbf{m}}\left(\lambda^{o}\right) u=0$ to $P_{\mathbf{m}}\left(\lambda^{o}+\epsilon\right) v=0$ defined by $u=R_{\mathbf{m}}\left(\epsilon ; \lambda^{o}\right) v$ is not surjective nor injective. Hence the equations are not irreducible. If $c_{\mathbf{m}}\left(\epsilon ; \lambda^{o}\right) \neq 0$, then the homomorphism is an isomorphism and the equations are isomorphic to each other. 
The claim ii) follows from Proposition 12.16 .

Theorem 13.9. Retain the notation in Theorem 13.8 with a rigid tuple $\mathbf{m}$. Fix a linear function $\ell(\lambda)$ of $\lambda$ such that the condition $\ell(\lambda)=0$ implies the reducibility of the universal equation $P_{\mathbf{m}}(\lambda) u=0$.

i) If there is no irreducible realizable subtuple $\mathbf{m}^{\prime}$ of $\mathbf{m}$ which is compatible to $\ell(\lambda)$ and $\ell(\lambda+\epsilon), \ell(\lambda)$ is a factor of $c_{\mathbf{m}}(\epsilon ; \lambda)$.

If there is no dual decomposition of $\mathbf{m}$ with respect to the pair $\ell(\lambda)$ and $\ell(\lambda+\epsilon)$, $\ell(\lambda)$ is not a factor of $c_{\mathbf{m}}(\epsilon ; \lambda)$. Here we define that the decomposition (12.51) is dual with respect to the pair $\ell(\lambda)$ and $\ell(\lambda+\epsilon)$ if the following conditions are valid.

$$
\begin{aligned}
& \mathbf{m}^{\prime} \text { is an irreducibly realizable subtuple of } \mathbf{m} \text { compatible to } \ell(\lambda) \text {, } \\
& \mathbf{m}^{\prime \prime} \text { is a subtuple of } \mathbf{m} \text { compatible to } \ell(\lambda+\epsilon) .
\end{aligned}
$$

ii) Suppose there exists a decomposition $\mathbf{m}=\mathbf{m}^{\prime} \oplus \mathbf{m}^{\prime \prime}$ with rigid tuples $\mathbf{m}^{\prime}$ and $\mathbf{m}^{\prime \prime}$ such that $\ell(\lambda)=\left|\left\{\lambda_{\mathbf{m}}\right\}\right|+k$ with $k \in \mathbb{Z}$ and $\ell(\lambda+\epsilon)=\ell(\lambda)+1$. Then $\ell(\lambda)$ is a factor of $c_{\mathbf{m}}(\epsilon ; \lambda)$ if and only if $k=0$.

Proof. Fix generic complex numbers $\lambda_{j, \nu} \in \mathbb{C}$ satisfying $\ell(\lambda)=\left|\left\{\lambda_{\mathbf{m}}\right\}\right|=0$. Then we may assume $\lambda_{j, \nu}-\lambda_{j, \nu^{\prime}} \notin \mathbb{Z}$ for $1 \leq \nu<\nu^{\prime} \leq n_{j}$ and $j=0, \ldots, p$.

i) The shift operator $R:=R_{\mathbf{m}}(-\epsilon, \lambda+\epsilon)$ gives a non-zero $W(x)$-homomorphism of the equation $P_{\mathbf{m}}(\lambda+\epsilon) v=0$ to $P_{\mathbf{m}}(\lambda) u=0$ by the correspondence $v=R u$. Since the equation $P_{\mathbf{m}}(\lambda) u=0$ is reducible, we examine the decompositions of $\mathbf{m}$ described in Proposition 12.16. Note that the genericity of $\lambda_{j, \nu} \in \mathbb{C}$ assures that the subtuple $\mathbf{m}^{\prime}$ of $\mathbf{m}$ corresponding to a decomposition $P_{\mathbf{m}}(\lambda)=P^{\prime \prime} P^{\prime}$ is uniquely determined, namely, $\mathbf{m}^{\prime}$ corresponds to the spectral type of the monodromy of the equation $P^{\prime} u=0$.

If the shift operator $R$ is bijective, there exists a subtuple $\mathbf{m}^{\prime}$ of $\mathbf{m}$ compatible to $\ell(\lambda)$ and $\ell(\lambda+\epsilon)$ because $R$ indices an isomorphism of monodromy.

Suppose $\ell(\lambda)$ is a factor of $c_{\mathbf{m}}(\epsilon ; \lambda)$. Then $R$ is not bijective. We assume that the image of $R$ is the equation $P^{\prime \prime} \bar{u}=0$ and the kernel of $R$ is the equation $P_{\epsilon}^{\prime} \bar{v}=0$. Then $P_{\mathbf{m}}(\lambda)=P^{\prime \prime} P^{\prime}$ and $P_{\mathbf{m}}(\lambda+\epsilon)=P_{\epsilon}^{\prime} P_{\epsilon}^{\prime \prime}$ with suitable Fuchsian differential operators $P^{\prime}$ and $P_{\epsilon}^{\prime \prime}$. Note that the spectral type of the monodromy of $P^{\prime} u=0$ and $P_{\epsilon}^{\prime \prime} v=0$ corresponds to $\mathbf{m}^{\prime}$ and $\mathbf{m}^{\prime \prime}$ with $\mathbf{m}=\mathbf{m}^{\prime}+\mathbf{m}^{\prime \prime}$. Applying Proposition 12.16 to the decompositions $P_{\mathbf{m}}(\lambda)=P^{\prime \prime} P^{\prime}$ and $P_{\mathbf{m}}(\lambda+\epsilon)=P_{\epsilon}^{\prime} P_{\epsilon}^{\prime \prime}$, we have a dual decomposition (12.51) of $\mathbf{m}$ with respect to the pair $\ell(\lambda)$ and $\ell(\lambda+\epsilon)$.

ii) Since $P_{\mathbf{m}}(\lambda) u=0$ is reducible, we have a decomposition $P_{\mathbf{m}}(\lambda)=P^{\prime \prime} P^{\prime}$ with $0<\operatorname{ord} P^{\prime}<\operatorname{ord} P_{\mathbf{m}}(\lambda)$. We may assume $P^{\prime} u=0$ and let $\tilde{\mathbf{m}}^{\prime}$ be the spectral type of the monodromy of the equation $P^{\prime} u=0$. Then $\tilde{\mathbf{m}}^{\prime}=\ell_{1} \mathbf{m}^{\prime}+\ell_{2} \mathbf{m}^{\prime \prime}$ with integers $\ell_{1}$ and $\ell_{2}$ because $\left|\left\{\lambda_{\tilde{\mathbf{m}}^{\prime}}\right\}\right| \in \mathbb{Z}_{\leq 0}$. Since $P^{\prime} u=0$ is irreducible, $2 \geq \operatorname{idx} \tilde{\mathbf{m}}^{\prime}=$ $2\left(\ell_{1}^{2}-\ell_{1} \ell_{2}+\ell_{2}^{2}\right)$ and therefore $\left(\ell_{1}, \ell_{2}\right)=(1,0)$ or $(0,1)$. Hence the claim follows from i) and the identity $\left|\left\{\lambda_{\mathbf{m}^{\prime}}\right\}\right|+\left|\left\{\lambda_{\mathbf{m}^{\prime \prime}}\right\}\right|=1$

Remark 13.10. i) The reducibility of $P_{\mathbf{m}}(\lambda)$ implies that of the dual of $P_{\mathbf{m}}(\lambda)$.

ii) When $\mathbf{m}$ is simply reducible (cf. Definition 8.14), each linear form of $\lambda_{j, \nu}$ describing the reducibility uniquely corresponds to a rigid decomposition of $\mathbf{m}$ and therefore Theorem 13.9 gives the necessary and sufficient condition for the bijectivity of the shift operator $R_{\mathbf{m}}(\epsilon, \lambda)$.

Example 13.11 $\left(E O_{4}\right)$. Let $P(\lambda) u=0$ and $P\left(\lambda^{\prime}\right) v=0$ be the Fuchsian differential equation with the Riemann schemes

$$
\left\{\begin{array}{ccc}
\lambda_{0,1} & {\left[\lambda_{1,1}\right]_{(2)}} & {\left[\lambda_{2,1}\right]_{(2)}} \\
\lambda_{0,2} & \lambda_{1,2} & {\left[\lambda_{2,2}\right]_{(2)}} \\
\lambda_{0,3} & \lambda_{1,3} &
\end{array} \lambda_{0,4} \quad 3 \quad\left\{\begin{array}{ccc}
\lambda_{0,1} & {\left[\lambda_{1,1}\right]_{(2)}} & {\left[\lambda_{2,1}\right]_{(2)}} \\
\lambda_{0,2} & \lambda_{1,2} & {\left[\lambda_{2,2}\right]_{(2)}} \\
\lambda_{0,3} & \lambda_{1,3}+1 & \\
\lambda_{0,4}-1 &
\end{array}\right\}\right.
$$


respectively. Since the condition of the reducibility of the equation corresponds to rigid decompositions (12.63), it easily follows from Theorem 13.9 that the shift operator between $P(\lambda) u=0$ and $P\left(\lambda^{\prime}\right) v=0$ is bijective if and only if

$$
\begin{cases}\lambda_{0,4}+\lambda_{1,2}+\lambda_{2, \mu}-1 \neq 0 & (1 \leq \mu \leq 2), \\ \lambda_{0, \nu}+\lambda_{0, \nu^{\prime}}+\lambda_{1,1}+\lambda_{1,3}+\lambda_{2,1}+\lambda_{2,2}-1 \neq 0 & \left(1 \leq \nu<\nu^{\prime} \leq 3\right) .\end{cases}
$$

In general, for a shift $\epsilon=\left(\epsilon_{j, \nu}\right)$ compatible to the spectral type 1111,211,22, the shift operator between $P(\lambda) u=0$ and $P(\lambda+\epsilon) v=0$ is bijective if and only if the values of each function in the list

$$
\begin{array}{ll}
\lambda_{0, \nu}+\lambda_{1,1}+\lambda_{2, \mu} & (1 \leq \nu \leq 4,1 \leq \mu \leq 2), \\
\lambda_{0, \nu}+\lambda_{0, \nu^{\prime}}+\lambda_{1,1}+\lambda_{1,3}+\lambda_{2,1}+\lambda_{2,2}-1 & \left(1 \leq \nu<\nu^{\prime} \leq 4\right)
\end{array}
$$

are

$$
\left\{\begin{array}{l}
\text { not integers for } \lambda \text { and } \lambda+\epsilon \\
\text { or positive integers for } \lambda \text { and } \lambda+\epsilon \\
\text { or non-positive integers for } \lambda \text { and } \lambda+\epsilon .
\end{array}\right.
$$

Note that the shift operator gives a homomorphism between monodromies (cf. (3.23)).

The following conjecture gives $c_{\mathbf{m}}(\epsilon ; \lambda)$ under certain conditions.

Conjecture 13.12. Retain the assumption that $\mathbf{m}=\left(\lambda_{j, \nu}\right)_{\substack{0 \leq j \leq p \\ 1 \leq \nu \leq n_{j}}} \in \mathcal{P}_{p+1}^{(n)}$ is rigid.

i) If $\ell(\lambda)=\ell(\lambda+\epsilon)$ in Theorem 13.9, then $\ell(\lambda)$ is not a factor of $c_{\mathbf{m}}(\epsilon ; \lambda)$,

ii) Assume $m_{1, n_{1}}=m_{2, n_{2}}=1$ and

$$
\epsilon:=\left(\epsilon_{j, \nu}\right)_{\substack{0 \leq j \leq p \\ 1 \leq \nu \leq n_{j}}}, \quad \epsilon_{j, \nu}=\delta_{j, 1} \delta_{\nu, n_{1}}-\delta_{j, 2} \delta_{\nu, n_{2}},
$$

Then we have

$$
c_{\mathbf{m}}(\epsilon ; \lambda)=C \prod_{\substack{\mathbf{m}=\mathbf{m}^{\prime} \oplus \mathbf{m}^{\prime \prime} \\ m_{1, n_{1}}^{\prime}=m_{2, n_{2}}^{\prime \prime}=1}}\left|\left\{\lambda_{\mathbf{m}^{\prime}}\right\}\right|
$$

with $C \in \mathbb{C}^{\times}$.

Suppose the spectral type $\mathbf{m}$ is of Okubo type, namely,

$$
m_{1,1}+\cdots+m_{p, 1}=(p-1) \text { ord } \mathbf{m} .
$$

Then some shift operators are easily obtained as follows. By a suitable addition we may assume that the Riemann scheme is

$$
\left\{\begin{array}{cccc}
x=\infty & x=c_{1} & \cdots & x=c_{p} \\
{\left[\lambda_{0,1}\right]_{\left(m_{0}, 1\right)}} & {[0]_{\left(m_{1,1}\right)}} & \cdots & {[0]_{\left(m_{p, 1}\right)}} \\
{\left[\lambda_{0,2}\right]_{\left(m_{0,2}\right)}} & {\left[\lambda_{1,2}\right]_{\left(m_{1,2}\right)}} & \cdots & {\left[\lambda_{p, 2}\right]_{\left(m_{p, 2}\right)}} \\
\vdots & \vdots & \vdots & \vdots \\
{\left[\lambda_{0, n_{0}}\right]_{\left(m_{0, n_{0}}\right)}} & {\left[\lambda_{1, n_{1}}\right]_{\left(m_{1, n_{1}}\right)}} & \cdots & {\left[\lambda_{p, n_{p}}\right]_{\left(m_{p, n_{p}}\right)}}
\end{array}\right\}
$$

and the corresponding differential equation $P u=0$ is of the form

$$
P_{\mathbf{m}}(\lambda)=\prod_{j=1}^{p}\left(x-c_{j}\right)^{n-m_{j, 1}} \frac{d^{n}}{d x^{n}}+\sum_{k=0}^{n-1} \prod_{j=1}^{p}\left(x-c_{j}\right)^{\max \left\{k-m_{j, 1}, 0\right\}} a_{k}(x) \frac{d^{k}}{d x^{k}} .
$$

Here $a_{k}(x)$ is a polynomial of $x$ whose degree is not larger than $k-\sum_{j=1}^{n} \max \{k-$ $\left.m_{j, 1}, 0\right\}$. Moreover we have

$$
a_{0}(x)=\prod_{\nu=1}^{n_{0}} \prod_{i=0}^{m_{0, \nu}-1}\left(\lambda_{0, \nu}+i\right) .
$$


Define the differential operators $R_{1}$ and $R_{\mathbf{m}}(\lambda) \in W[x] \otimes \mathbb{C}[\lambda]$ by

$$
R_{1}=\frac{d}{d x} \text { and } P_{\mathbf{m}}(\lambda)=-R_{\mathbf{m}}(\lambda) R_{1}+a_{0}(x) .
$$

Let $P_{\mathbf{m}}\left(\lambda^{\prime}\right) v=0$ be the differential equation with the Riemann scheme

$$
\left\{\begin{array}{cccc}
x=\infty & x=c_{1} & \cdots & x=c_{p} \\
{\left[\lambda_{0,1}+1\right]_{\left(m_{0}, 1\right)}} & {[0]_{\left(m_{1,1}\right)}} & \cdots & {[0]_{\left(m_{p, 1}\right)}} \\
{\left[\lambda_{0,2}+1\right]_{\left(m_{0,2}\right)}} & {\left[\lambda_{1,2}-1\right]_{\left(m_{1,2}\right)}} & \cdots & {\left[\lambda_{p, 2}-1\right]_{\left(m_{p, 2}\right)}} \\
\vdots & \vdots & \vdots & \vdots \\
{\left[\lambda_{0, n_{0}}+1\right]_{\left(m_{0, n_{0}}\right)}} & {\left[\lambda_{1, n_{1}}-1\right]_{\left(m_{1, n_{1}}\right)}} & \cdots & {\left[\lambda_{p, n_{p}}-1\right]_{\left(m_{p, n_{p}}\right)}}
\end{array}\right\} .
$$

Then the correspondences $u=R_{\mathbf{m}}(\lambda) v$ and $v=R_{1} u$ give $W(x)$-homomorphisms between the differential equations.

Proposition 13.13. Let $\mathbf{m}=\left\{m_{j, \nu}\right\}_{\substack{0 \leq j \leq p \\ 1 \leq \nu \leq n_{j}}}$ be a rigid tuple of partitions satisfying (13.33). Putting

$$
\epsilon_{j, \nu}= \begin{cases}1 & \left(j=0,1 \leq \nu \leq n_{0}\right) \\ \delta_{\nu, 0}-1 & \left(1 \leq j \leq p, 1 \leq \nu \leq n_{j}\right)\end{cases}
$$

we have

$$
c_{\mathbf{m}}(\epsilon ; \lambda)=\prod_{\nu=1}^{n_{0}} \prod_{i=0}^{m_{0, \nu}-1}\left(\lambda_{0, \nu}+\lambda_{1,1}+\cdots+\lambda_{p, 1}+i\right) .
$$

Proof. By suitable additions the proposition follows from the result assuming $\lambda_{j, 1}=$ 0 for $j=1, \ldots, p$, which has been shown.

Example 13.14. The generalized hypergeometric equations with the Riemann schemes

$$
\left\{\begin{array}{ccc}
\lambda_{0,1} & \lambda_{1,1} & {\left[\lambda_{2,1}\right]_{(n-1)}} \\
\vdots & \vdots & \\
\lambda_{0, \nu} & \lambda_{1, \nu_{o}} & \\
\vdots & \vdots & \\
\lambda_{0, n} & \lambda_{1, n} & \lambda_{2,2}
\end{array}\right\} \text { and }\left\{\begin{array}{ccc}
\lambda_{0,1} & \lambda_{1,1} & {\left[\lambda_{2,1}\right]_{(n-1)}} \\
\vdots & \vdots & \\
\lambda_{0, \nu} & \lambda_{1, \nu_{o}}+1 & \\
\vdots & \vdots & \\
\lambda_{0, n} & \lambda_{1, n} & \lambda_{2,2}-1
\end{array}\right\}
$$

respectively, whose spectral type is $\mathbf{m}=1^{n}, 1^{n},(n-1) 1$ are isomorphic to each other by the shift operator if and only if

$$
\lambda_{0, \nu}+\lambda_{1, \nu_{o}}+\lambda_{2,1} \neq 0 \quad(\nu=1, \ldots, n) .
$$

This statement follows from Proposition 13.13 with suitable additions.

Theorem 13.9 shows that in general $P(\lambda) u=0$ with the Riemann scheme $\left\{\lambda_{\mathbf{m}}\right\}$ is $W(x)$-isomorphic to $P(\lambda+\epsilon) v=0$ by the shift operator if and only if the values of the function $\lambda_{0, \nu}+\lambda_{1, \mu}+\lambda_{2,1}$ satisfy (13.30) for $1 \leq \nu \leq n$ and $1 \leq \mu \leq n$. Here $\epsilon$ is any shift compatible to $\mathbf{m}$.

The shift operator between

$$
\left\{\begin{array}{ccc}
\lambda_{0,1} & \lambda_{1,1} & {\left[\lambda_{2,1}\right]_{(n-1)}} \\
\lambda_{0,2} & \lambda_{1,2} & \lambda_{2,2} \\
\vdots & \vdots & \\
\lambda_{0, n} & \lambda_{1, n} &
\end{array}\right\} \text { and }\left\{\begin{array}{ccc}
\lambda_{0,1} & \lambda_{1,1}+1 & {\left[\lambda_{2,1}\right]_{(n-1)}} \\
\lambda_{0,2} & \lambda_{1,2}-1 & \lambda_{2,2} \\
\vdots & \vdots & \\
\lambda_{0, n} & \lambda_{1, n} &
\end{array}\right\}
$$

is bijective if and only if

$$
\lambda_{0, \nu}+\lambda_{1,1}+\lambda_{2,1} \neq 0 \text { and } \lambda_{0, \nu}+\lambda_{1,2}+\lambda_{2,1} \neq 1 \text { for } \nu=1, \ldots, n .
$$


Hence if $\lambda_{1,1}=0$ and $\lambda_{1,2}=1$ and $\lambda_{0,1}+\lambda_{2,1}=0$, the shift operator defines a non-zero endomorphism which is not bijective and therefore the monodromy of the space of the solutions are decomposed into a direct sum of the spaces of solutions of two Fuchsian differential equations. The other parameters are generic in this case, the decomposition is unique and the dimension of the smaller space equals 1. When $n=2$ and $\left(c_{0}, c_{1}, c_{2}\right)=(\infty, 1,0)$ and $\lambda_{2,1}$ and $\lambda_{2,2}$ are generic, the space equals $\mathbb{C} x^{\lambda_{2,1}} \oplus \mathbb{C} x^{\lambda_{2,2}}$

13.3. Polynomial solutions. We characterize some polynomial solutions of a differential equation of Okubo type.

Proposition 13.15. Retain the notation in $\S 13.1$. Let $P_{\mathbf{m}}(\lambda) u=0$ be the differential equation with the Riemann scheme (13.34). Suppose that $\mathbf{m}$ is rigid and satisfies (13.33). Suppose moreover that there exists $j_{o}$ satisfying $m_{j_{o}, 1}=1$ and $0 \leq j_{0} \leq p$. Fix a complex number $C$. Suppose $\lambda_{0,1}=-C$ and $\lambda_{j, \nu} \notin \mathbb{Z}$ for $j=0, \ldots, p$ and $\nu=2, \ldots, n_{j}$. Then the equation has a polynomial solution of degree $k$ if and only if $C=k$.

We denote the polynomial solution by $p_{\lambda}$. Then $p_{\lambda}^{\prime}$ is a polynomial solution of $P_{\mathbf{m}}(\lambda+\epsilon) v=0$ under the notation (13.38). Moreover

$$
R_{\mathbf{m}}(\lambda) \circ R_{\mathbf{m}}(\lambda+\epsilon) \circ \cdots \circ R_{\mathbf{m}}(\lambda+(k-1) \epsilon) 1
$$

is a non-zero constant multiple of $p_{\lambda}$ under the notation (13.36).

Proof. Since $\mathbf{m}=\left(\delta_{1, \nu}\right)_{\substack{0 \leq j \leq p \\ 1 \leq \nu \leq n_{j}}} \oplus\left(m_{j, \nu}-\delta_{1, \nu}\right)_{\substack{0 \leq j \leq p \\ 1 \leq \nu \leq n_{j}}}$ is a rigid decomposition of $\mathbf{m}$, we have $P_{\mathbf{m}}(\lambda)=P_{1} \partial$ with suitable $P_{1} \in W(x)$ when $C=0$. Note that $R_{\mathbf{m}}(\lambda+\ell \epsilon)$ defines an isomorphism of the equation $P_{\mathbf{m}}(\lambda+(\ell+1) \epsilon) u_{k+1}=0$ to the equation $P_{\mathbf{m}}(\lambda+\ell \epsilon) u_{k}=0$ by $u_{k}=R_{\mathbf{m}}(\lambda+\ell \epsilon) u_{k+1}$ if $C \neq \ell$, the function (13.43) is a polynomial solution of $P_{\mathbf{m}}(\lambda) u=0$. The remaining part of the proposition is clear.

Remark 13.16. We have not used the assumption that $\mathbf{m}$ is rigid in Proposition 13.13 and Proposition 13.15 and hence the propositions are valid without this assumption.

\section{Connection problem}

14.1. Connection formula. For a realizable tuple $\mathbf{m} \in \mathcal{P}_{p+1}$, let $P_{\mathbf{m}} u=0$ be a universal Fuchsian differential equation with the Riemann scheme

$$
\left\{\begin{array}{cccccc}
x=0 & c_{1}=1 & \cdots & c_{j} & \cdots & c_{p}=\infty \\
{\left[\lambda_{0,1}\right]_{\left(m_{0,1}\right)}} & {\left[\lambda_{1,1}\right]_{\left(m_{1,1}\right)}} & \cdots & {\left[\lambda_{j, 1}\right]_{\left(m_{j, 1}\right)}} & \cdots & {\left[\lambda_{p, 1}\right]_{\left(m_{p, 1}\right)}} \\
\vdots & \vdots & \vdots & \vdots & \vdots & \vdots \\
{\left[\lambda_{0, n_{0}}\right]_{\left(m_{0, n_{0}}\right)}} & {\left[\lambda_{1, n_{1}}\right]_{\left(m_{1, n_{1}}\right)}} & \cdots & {\left[\lambda_{j, n_{j}}\right]_{\left(m_{j, n_{j}}\right)}} & \cdots & {\left[\lambda_{p, n_{p}}\right]_{\left(m_{p, n_{p}}\right)}}
\end{array}\right\}
$$

The singular points of the equation are $c_{j}$ for $j=0, \ldots, p$. In this subsection we always assume $c_{0}=0, c_{1}=1$ and $c_{p}=\infty$ and $c_{j} \notin[0,1]$ for $j=2, \ldots, p-1$. We also assume that $\lambda_{j, \nu}$ are generic.

Definition 14.1 (connection coefficients). Suppose $\lambda_{j, \nu}$ are generic under the Fuchs relation. Let $u_{0}^{\lambda_{0, \nu_{0}}}$ and $u_{1}^{\lambda_{1, \nu_{1}}}$ be normalized local solutions of $P_{\mathbf{m}}=0$ at $x=0$ and $x=1$ corresponding to the exponents $\lambda_{0, \nu_{0}}$ and $\lambda_{1, \nu_{1}}$, respectively, so that $u_{0}^{\lambda_{0, \nu_{0}}} \equiv x^{\lambda_{0, \nu_{0}}} \bmod x^{\lambda_{0, \nu_{0}}+1} \mathcal{O}_{0}$ and $u_{1}^{\lambda_{1, \nu_{1}}} \equiv(1-x)^{\lambda_{1, \nu_{1}}} \bmod (1-x)^{\lambda_{1, \nu_{1}}+1} \mathcal{O}_{1}$. Here $1 \leq \nu_{0} \leq n_{0}$ and $1 \leq \nu_{1} \leq n_{1}$. If $m_{0, \nu_{0}}=1, u_{0}^{\lambda_{0}, \nu_{0}}$ is uniquely determined and then the analytic continuation of $u_{0}^{\lambda_{0, \nu_{0}}}$ to $x=1$ along $(0,1) \subset \mathbb{R}$ defines a connection coefficient with respect to $u_{1}^{\lambda_{1, \nu_{1}}}$, which is denoted by $c\left(0: \lambda_{0, \nu_{0}} \rightsquigarrow 1: \lambda_{1, \nu_{1}}\right)$ 
or simply by $c\left(\lambda_{0, \nu_{0}} \rightsquigarrow \lambda_{1, \nu_{1}}\right)$. The connection coefficient $c\left(1: \lambda_{1, \nu_{1}} \rightsquigarrow 0: \lambda_{0 . \nu_{0}}\right)$ or $c\left(\lambda_{1, \nu_{1}} \rightsquigarrow \lambda_{0 . \nu_{0}}\right)$ of $u_{1}^{\lambda_{1, \nu_{1}}}$ with respect to $u_{0}^{\lambda_{0, \nu_{0}}}$ are similarly defined if $m_{1, \nu_{1}}=1$.

Moreover we define $c\left(c_{i}: \lambda_{i, \nu_{i}} \rightsquigarrow c_{j}: \lambda_{j, \nu_{j}}\right)$ by using a suitable linear fractional transformation $T$ of $\mathbb{C} \cup\{\infty\}$ which transforms $\left\{c_{i}, c_{j}\right\}$ to $\{0,1\}$ so that $T\left(c_{\nu}\right) \notin$ $(0,1)$ for $\nu=0, \ldots, p$. If $p=2$, we define the map $T$ so that $T\left(c_{k}\right)=\infty$ for the other singular point $c_{k}$. For example if $c_{j} \notin[0,1]$ for $j=2, \ldots, p-1$, we put $T(x)=\frac{x}{x-1}$ to define $c\left(0: \lambda_{0, \nu_{0}} \rightsquigarrow \infty: \lambda_{p, \nu_{p}}\right)$ or $c\left(\infty: \lambda_{p, \nu_{p}} \rightsquigarrow 0: \lambda_{0, \nu_{0}}\right)$.

In the definition $u_{0}^{\lambda_{0, \nu_{0}}}(x)=x^{\lambda_{0, \nu_{0}}} \phi(x)$ with analytic function $\phi(x)$ at 0 which satisfies $\phi(0)=1$ and if $\operatorname{Re} \lambda_{1, \nu_{1}}<\operatorname{Re} \lambda_{1, \nu}$ for $\nu \neq \nu_{1}$, we have

$$
c\left(\lambda_{0, \nu_{0}} \rightsquigarrow \lambda_{1, \nu_{1}}\right)=\lim _{x \rightarrow 1-0}(1-x)^{-\lambda_{1, \nu_{1}}} u_{0}^{\lambda_{0, \nu_{0}}}(x) \quad(x \in[0,1))
$$

by the analytic continuation. The connection coefficient $c\left(\lambda_{0 . \nu_{0}} \rightsquigarrow \lambda_{1, \nu_{1}}\right)$ meromorphically depends on spectral parameters $\lambda_{j, \nu}$. It also holomorphically depends on accessory parameters $g_{i}$ and singular points $\frac{1}{c_{j}}(j=2, \ldots, p-1)$ in a neighborhood of given values of parameters.

The main purpose in this subsection is to get the explicit expression of the connection coefficients in terms of gamma functions when $\mathbf{m}$ is rigid and $m_{0, \nu}=$ $m_{1, \nu^{\prime}}=1$.

Fist we prove the following key lemma which describes the effect of a middle convolution on connection coefficients.

Lemma 14.2. Using the integral transformation 2.37), we put

$$
\begin{aligned}
& \left(T_{a, b}^{\mu} u\right)(x):=x^{-a-\mu}(1-x)^{-b-\mu} I_{0}^{\mu} x^{a}(1-x)^{b} u(x), \\
& \left(S_{a, b}^{\mu} u\right)(x):=x^{-a-\mu} I_{0}^{\mu} x^{a}(1-x)^{b} u(x)
\end{aligned}
$$

for a continuous function $u(x)$ on $[0,1]$. Suppose $\operatorname{Re} a \geq 0$ and $\operatorname{Re} \mu>0$. Under the condition $\operatorname{Re} b+\operatorname{Re} \mu<0$ or $\operatorname{Re} b+\operatorname{Re} \mu>0,\left(T_{a, b}^{\mu} u\right)(x)$ or $S_{a, b}^{\mu}(u)(x)$ defines a continuous function on $[0,1]$, respectively, and we have

$$
\begin{aligned}
& T_{a, b}^{\mu}(u)(0)=S_{a, b}^{\mu}(u)(0)=\frac{\Gamma(a+1)}{\Gamma(a+\mu+1)} u(0), \\
& \frac{T_{a, b}^{\mu}(u)(1)}{T_{a, b}^{\mu}(u)(0)}=\frac{u(1)}{u(0)} C_{a, b}^{\mu}, \quad C_{a, b}^{\mu}:=\frac{\Gamma(a+\mu+1) \Gamma(-\mu-b)}{\Gamma(a+1) \Gamma(-b)}, \\
& \frac{S_{a, b}^{\mu}(u)(1)}{S_{a, b}^{\mu}(u)(0)}=\frac{1}{u(0)} \frac{\Gamma(a+\mu+1)}{\Gamma(\mu) \Gamma(a+1)} \int_{0}^{1} t^{a}(1-t)^{b+\mu-1} u(t) d t .
\end{aligned}
$$

Proof. Suppose $\operatorname{Re} a \geq 0$ and $0<\operatorname{Re} \mu<-\operatorname{Re} b$. Then

$$
\begin{aligned}
\Gamma(\mu) T_{a, b}^{\mu}(u)(x) \\
=x^{-a-\mu}(1-x)^{-b-\mu} \int_{0}^{x} t^{a}(1-t)^{b}(x-t)^{\mu-1} u(t) d t \quad\left(t=x s_{1}, 0 \leq x<1\right) \\
=(1-x)^{-b-\mu} \int_{0}^{1} s_{1}^{a}\left(1-s_{1}\right)^{\mu-1}\left(1-x s_{1}\right)^{b} u\left(x s_{1}\right) d s_{1} \\
\quad=\int_{0}^{1} s_{1}^{a}\left(\frac{1-s_{1}}{1-x}\right)^{\mu}\left(\frac{1-x s_{1}}{1-x}\right)^{b} u\left(x s_{1}\right) \frac{d s}{1-s_{1}} \\
=\int_{0}^{1}\left(1-s_{2}\right)^{a}\left(\frac{s_{2}}{1-x}\right)^{\mu}\left(1+\frac{x s_{2}}{1-x}\right)^{b} u\left(x-x s_{2}\right) \frac{d s_{2}}{s_{2}} \quad\left(s_{1}=1-s_{2}\right) \\
=\int_{0}^{\frac{1}{1-x}}(1-s(1-x))^{a} s^{\mu}(1+x s)^{b} u(x-x(1-x) s) \frac{d s}{s} \quad\left(s_{2}=(1-x) s\right) .
\end{aligned}
$$


Since

$$
\left|s_{1}^{a}\left(1-s_{1}\right)^{\mu-1}\left(1-x s_{1}\right)^{b} u\left(x s_{1}\right)\right| \leq \max \left\{\left(1-s_{1}\right)^{\operatorname{Re} \mu-1}, 1\right\} 3^{-\operatorname{Re} b} \max _{0 \leq t \leq 1}|u(t)|
$$

for $0 \leq s_{1}<1$ and $0 \leq x \leq \frac{2}{3}, T_{a, b}^{\mu}(u)(x)$ is continuous for $x \in\left[0, \frac{2}{3}\right)$. We have

$$
\left.\mid(1-s(1-x))^{a} s^{\mu-1}(1+x s)^{b} u(x-x(1-x) s)\right)\left|\leq s^{\operatorname{Re} \mu-1}\left(1+\frac{s}{2}\right)^{\operatorname{Re} b} \max _{0 \leq t \leq 1}\right| u(t) \mid
$$

for $\frac{1}{2} \leq x \leq 1$ and $0<s \leq \frac{1}{1-x}$ and therefore $T_{a, b}^{\mu}(u)(x)$ is continuous for $x \in\left(\frac{1}{2}, 1\right]$.

Hence $T_{a, b}^{\mu}(x)$ defines a continuous function on $[0,1]$ and

$$
\begin{aligned}
T_{a, b}^{\mu}(u)(0) & =\frac{1}{\Gamma(\mu)} \int_{0}^{1}\left(1-s_{2}\right)^{a} s_{2}^{\mu} u(0) \frac{d s_{2}}{s_{2}}=\frac{\Gamma(a+1)}{\Gamma(a+\mu+1)} u(0), \\
T_{a, b}^{\mu}(u)(1) & =\frac{1}{\Gamma(\mu)} \int_{0}^{\infty} s^{\mu}(1+s)^{b} u(1) \frac{d s}{s} \\
\left(t=\frac{s}{1+s}=1-\frac{1}{1+s},\right. & \left.\frac{1}{1+s}=1-t, 1+s=\frac{1}{1-t}, s=\frac{1}{1-t}-1=\frac{t}{1-t}, \frac{d s}{d t}=-\frac{1}{(1-t)^{2}}\right) \\
& =\frac{1}{\Gamma(\mu)} \int_{0}^{1}\left(\frac{t}{1-t}\right)^{\mu-1}(1-t)^{-b-2} u(1) d t=\frac{\Gamma(-\mu-b)}{\Gamma(-b)} u(1) .
\end{aligned}
$$

The claims for $S_{a, b}^{\mu}$ are clear from

$$
\Gamma(\mu) S_{a, b}^{\mu}(u)(x)=\int_{0}^{1} s_{1}^{a}\left(1-s_{1}\right)^{\mu-1}\left(1-x s_{1}\right)^{b} u\left(x s_{1}\right) d s_{1} .
$$

This lemma is useful for the middle convolution $m c_{\mu}$ not only when it gives a reduction but also when it doesn't change the spectral type.

Example 14.3. Applying Lemma 14.2 to the solution

$$
u_{0}^{\lambda_{0}+\mu}(x)=\int_{0}^{x} t^{\lambda_{0}}(1-t)^{\lambda_{1}}\left(\prod_{j=2}^{p-1}\left(1-\frac{t}{c_{j}}\right)^{\lambda_{j}}\right)(x-t)^{\mu-1} d t
$$

of the Jordan-Pochhammer equation (cf. Example 2.8 iii)) with the Riemann scheme

$$
\left\{\begin{array}{cccccc}
x=0 & c_{1}=1 & \cdots & c_{j} & \cdots & c_{p}=\infty \\
{[0]_{(p-1)}} & {[0]_{(p-1)}} & \cdots & {[0]_{(p-1)}} & \cdots & {[1-\mu]_{(p-1)}} \\
\lambda_{0}+\mu & \lambda_{1}+\mu & \cdots & \lambda_{j}+\mu & \cdots & -\sum_{\nu=0}^{p-1} \lambda_{\nu}-\mu
\end{array}\right\}
$$

we have

$$
\begin{aligned}
c\left(0: \lambda_{0}+\mu \rightsquigarrow 1: \lambda_{1}+\mu\right) & =\frac{\Gamma\left(\lambda_{0}+\mu+1\right) \Gamma\left(-\lambda_{1}-\mu\right)}{\Gamma\left(\lambda_{0}+1\right) \Gamma\left(-\lambda_{1}\right)} \prod_{j=2}^{p-1}\left(1-\frac{1}{c_{j}}\right)^{\lambda_{j}}, \\
c\left(0: \lambda_{0}+\mu \rightsquigarrow 1: 0\right) & =\frac{\Gamma\left(\lambda_{0}+\mu+1\right)}{\Gamma(\mu) \Gamma\left(\lambda_{0}+1\right)} \int_{0}^{1} t^{\lambda_{0}}(1-t)^{\lambda_{1}+\mu-1} \prod_{j=1}^{p-1}\left(1-\frac{t}{c_{j}}\right)^{\lambda_{j}} d t .
\end{aligned}
$$

Moreover the equation $P u=0$ with

$$
P:=\operatorname{RAd}\left(\partial^{-\mu^{\prime}}\right) \operatorname{RAd}\left(x^{\lambda^{\prime}}\right) \operatorname{RAd}\left(\partial^{-\mu}\right) \operatorname{RAd}\left(x^{\lambda_{0}}(1-x)^{\lambda_{1}}\right) \partial
$$

is satisfied by the generalized hypergeometric function ${ }_{3} F_{2}$ with the Riemann scheme

$$
\left\{\begin{array}{ccc}
x=0 & 1 & \infty \\
0 & {[0]_{(2)}} & 1-\mu^{\prime} \\
\lambda^{\prime}+\mu^{\prime} & & 1-\lambda^{\prime}-\mu-\mu^{\prime} \\
\lambda_{0}+\lambda^{\prime}+\mu+\mu^{\prime} & \lambda_{1}+\mu+\mu^{\prime} & -\lambda_{0}-\lambda_{1}-\lambda^{\prime}-\mu-\mu^{\prime}
\end{array}\right\}
$$


corresponding to $111,21,111$ and therefore

$$
\begin{aligned}
c\left(\lambda_{0}+\lambda^{\prime}+\mu+\mu^{\prime} \rightsquigarrow \lambda_{1}+\mu+\mu^{\prime}\right)=C_{\lambda_{0}, \lambda_{1}}^{\mu} \cdot C_{\lambda_{0}+\lambda^{\prime}+\mu, \lambda_{1}+\mu}^{\mu^{\prime}} & \\
= & \frac{\Gamma\left(\lambda_{0}+\mu+1\right) \Gamma\left(-\lambda_{1}-\mu\right)}{\Gamma\left(\lambda_{0}+1\right) \Gamma\left(-\lambda_{1}\right)} \cdot \frac{\Gamma\left(\lambda_{0}+\lambda^{\prime}+\mu+\mu^{\prime}+1\right) \Gamma\left(-\lambda_{1}-\mu-\mu^{\prime}\right)}{\Gamma\left(\lambda_{0}+\lambda^{\prime}+\mu+1\right) \Gamma\left(-\lambda_{1}-\mu\right)} \\
& =\frac{\Gamma\left(\lambda_{0}+\mu+1\right) \Gamma\left(\lambda_{0}+\lambda^{\prime}+\mu+\mu^{\prime}+1\right) \Gamma\left(-\lambda_{1}-\mu-\mu^{\prime}\right)}{\Gamma\left(\lambda_{0}+1\right) \Gamma\left(-\lambda_{1}\right) \Gamma\left(\lambda_{0}+\lambda^{\prime}+\mu+1\right)} .
\end{aligned}
$$

We further examine the connection coefficient.

In general, putting $c_{0}=0$ and $c_{1}=1$ and $\lambda_{1}=\sum_{k=0}^{p} \lambda_{k, 1}-1$, we have

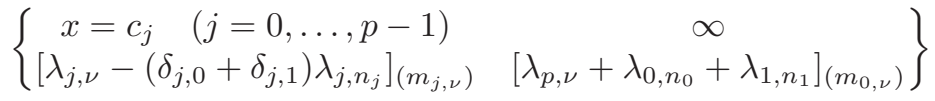

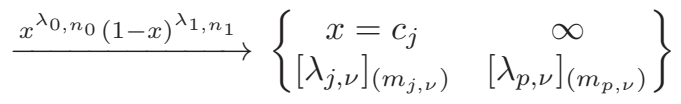

$$
\begin{aligned}
& \stackrel{x^{-\lambda_{0,1}} \prod_{j=1}^{p-1}\left(1-c_{j}^{-1} x\right)^{-\lambda_{j, 1}}}{\longrightarrow}\left\{\begin{array}{cl}
{[0]_{\left(m_{j, 1}\right)}} & {\left[\lambda_{p, 1}+\sum_{k=0}^{p-1} \lambda_{k, 1}\right]_{\left(m_{p, 1}\right)}} \\
{\left[\lambda_{j, \nu}-\lambda_{j, 1}\right]_{\left(m_{j, \nu}\right)}} & {\left[\lambda_{p, \nu}+\sum_{k=0}^{p-1} \lambda_{k, 1}\right]_{\left(m_{p, \nu}\right)}}
\end{array}\right\}
\end{aligned}
$$

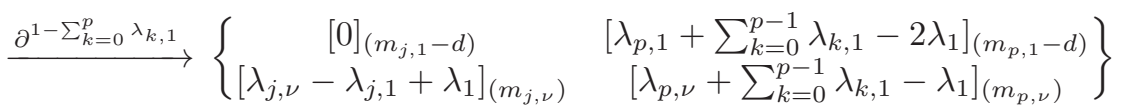

$$
\begin{aligned}
& \left(d=\sum_{k=0}^{p} m_{k, 1}-(p-1) n\right) \\
& \stackrel{x^{\lambda_{0,1}} \prod_{j=1}^{p-1}\left(1-c_{j}^{-1} x\right)^{\lambda_{j, 1}}}{\longrightarrow}\left\{\begin{array}{cc}
x=\frac{1}{c_{j}} & \infty \\
{\left[\lambda_{j, 1}\right]_{\left(m_{j, 1}-d\right)}} & {\left[\lambda_{p, 1}-2 \lambda_{1}\right]_{\left(m_{p, 1}-d\right)}} \\
{\left[\lambda_{j, \nu}+\lambda_{1}\right]_{\left(m_{j, \nu}\right)}} & {\left[\lambda_{p, \nu}-\lambda_{1}\right]_{\left(m_{p, \nu}\right)}}
\end{array}\right\}, \\
& C_{\lambda_{0, n_{1}}-\lambda_{0,1}, \lambda_{1, n_{1}}-\lambda_{1,1}}^{\lambda_{1}}=\frac{\Gamma\left(\lambda_{0, n_{0}}+\lambda_{1}-\lambda_{0,1}+1\right) \Gamma\left(\lambda_{1,1}-\lambda_{1, n_{1}}-\lambda_{1}\right)}{\Gamma\left(\lambda_{0, n_{0}}-\lambda_{0,1}+1\right) \Gamma\left(\lambda_{1,1}-\lambda_{1, n_{1}}\right)} .
\end{aligned}
$$

In general, the following theorem is a direct consequence of Definition 7.6 and Lemma 14.2 .

Theorem 14.4. Put $c_{0}=\infty, c_{1}=1$ and $c_{j} \in \mathbb{C} \backslash\{0\}$ for $j=3, \ldots, p-1$. By the transformation

$$
\operatorname{RAd}\left(x^{\lambda_{0,1}} \prod_{j=1}^{p-1}\left(1-\frac{x}{c_{j}}\right)^{\lambda_{j, 1}}\right) \circ \operatorname{RAd}\left(\partial^{1-\sum_{k=0}^{p} \lambda_{k, 1}}\right) \circ \operatorname{RAd}\left(x^{-\lambda_{0,1}} \prod_{j=1}^{p-1}\left(1-\frac{x}{c_{j}}\right)^{-\lambda_{j, 1}}\right)
$$

the Riemann scheme of a Fuchsian ordinary differential equation and its connection coefficient change as follows:

$$
\begin{aligned}
& \left\{\lambda_{\mathbf{m}}\right\}=\left\{\left[\lambda_{j, \nu}\right]_{\left(m_{j, \nu}\right)}\right\}_{\substack{0 \leq j \leq p \\
1 \leq \nu \leq n_{j}}}=\left\{\begin{array}{cc}
x=c_{j} & \infty \\
{\left[\lambda_{j, 1}\right]_{\left(m_{j, 1}\right)}} & {\left[\lambda_{p, 1}\right]_{\left(m_{p, 1}\right)}} \\
{\left[\lambda_{j, \nu}\right]_{\left(m_{j, \nu}\right)}} & {\left[\lambda_{p, \nu}\right]_{\left(m_{p, \nu}\right)}}
\end{array}\right\} \\
& \mapsto\left\{\lambda_{\mathbf{m}^{\prime}}^{\prime}\right\}=\left\{\left[\lambda_{j, \nu}^{\prime}\right]_{\left(m_{j, \nu}^{\prime}\right)}\right\}_{\substack{0 \leq j \leq p \\
1 \leq \nu \leq n_{j}}} \\
& =\left\{\begin{array}{cc}
x=c_{j} & \infty \\
{\left[\lambda_{j, 1}\right]_{\left(m_{j, 1}-d\right)}} & {\left[\lambda_{p, 1}-2 \sum_{k=0}^{p} \lambda_{k, 1}+2\right]_{\left(m_{p, 1}-d\right)}} \\
{\left[\lambda_{j, \nu}+\sum_{k=0}^{p} \lambda_{k, 1}-1\right]_{\left(m_{j, \nu}\right)}} & {\left[\lambda_{p, \nu}-\sum_{k=0}^{p} \lambda_{k, 1}+1\right]_{\left(m_{p, \nu}\right)}}
\end{array}\right\}
\end{aligned}
$$


with

$$
\begin{aligned}
& d=m_{0,1}+\cdots+m_{p, 1}-(p-1) \text { ord } \mathbf{m}, \\
& m_{j, \nu}^{\prime}=m_{j, \nu}-d \delta_{\nu, 1} \quad\left(j=0, \ldots, p, \nu=1, \ldots, n_{j}\right), \\
& \lambda_{j, 1}^{\prime}=\lambda_{j, 1} \quad(j=0, \ldots, p-1), \lambda_{p, 1}^{\prime}=-2 \lambda_{0,1}-\cdots-2 \lambda_{p-1,1}-\lambda_{p, 1}+2, \\
& \lambda_{j, \nu}^{\prime}=\lambda_{j, \nu}+\lambda_{0,1}+\lambda_{1,1}+\cdots+\lambda_{p, 1}-1 \quad\left(j=0, \ldots, p-1, \nu=2, \ldots, n_{j}\right), \\
& \lambda_{p, \nu}^{\prime}=\lambda_{p, \nu}-\lambda_{0,1}-\cdots-\lambda_{p, 1}+1
\end{aligned}
$$

and if $m_{0, n_{0}}=1$ and $n_{0}>1$ and $n_{1}>1$, then

$$
\frac{c^{\prime}\left(\lambda_{0, n_{0}}^{\prime} \rightsquigarrow \lambda_{1, n_{1}}^{\prime}\right)}{\Gamma\left(\lambda_{0, n_{0}}^{\prime}-\lambda_{0,1}^{\prime}+1\right) \Gamma\left(\lambda_{1,1}^{\prime}-\lambda_{1, n_{1}}^{\prime}\right)}=\frac{c\left(\lambda_{0, n_{0}} \rightsquigarrow \lambda_{1, n_{1}}\right)}{\Gamma\left(\lambda_{0, n_{0}}-\lambda_{0,1}+1\right) \Gamma\left(\lambda_{1,1}-\lambda_{1, n_{1}}\right)} .
$$

Applying the successive reduction by $\partial_{\max }$ to the above theorem, we obtain the following theorem.

Theorem 14.5. Suppose that a tuple $\mathbf{m} \in \mathcal{P}$ is irreducibly realizable and $m_{0, n_{0}}=$ $m_{1, n_{1}}=1$ in the Riemann scheme (14.1). Then the connection coefficient satisfies

$$
\begin{aligned}
& \frac{c\left(\lambda_{0, n_{0}} \rightsquigarrow \lambda_{1, n_{1}}\right)}{\bar{c}\left(\lambda(K)_{0, n_{0}} \rightsquigarrow \lambda(K)_{1, n_{1}}\right)} \\
& \quad=\prod_{k=0}^{K-1} \frac{\Gamma\left(\lambda(k)_{0, n_{0}}-\lambda(k)_{0, \ell(k)_{0}}+1\right) \cdot \Gamma\left(\lambda(k)_{1, \ell(k)_{1}}-\lambda(k)_{1, n_{1}}\right)}{\Gamma\left(\lambda(k+1)_{0, n_{0}}-\lambda(k+1)_{0, \ell(k)_{0}}+1\right) \cdot \Gamma\left(\lambda(k+1)_{1, \ell(k)_{1}}-\lambda(k+1)_{1, n_{1}}\right)}
\end{aligned}
$$

under the notation in Definitions 7.11. Here $\bar{c}\left(\lambda(K)_{0, n_{0}} \rightsquigarrow \lambda(K)_{1, n_{1}}\right)$ is a corresponding connection coefficient for the equation $\left(\partial_{\max }^{K} P_{\mathbf{m}}\right) v=0$ with the fundamental spectral type $f \mathbf{m}$. We note that

$$
\begin{aligned}
& \left(\lambda(k+1)_{0, n_{0}}-\lambda(k+1)_{0, \ell(k)_{0}}+1\right)+\left(\lambda(k+1)_{1, \ell(k)_{1}}-\lambda(k+1)_{1, n_{1}}\right) \\
& \quad=\left(\lambda(k)_{0, n_{0}}-\lambda(k)_{0, \ell(k)_{0}}+1\right)+\left(\lambda(k)_{1, \ell(k)_{1}}-\lambda(k)_{1, n_{1}}\right)
\end{aligned}
$$

for $k=0, \ldots, K-1$.

When $\mathbf{m}$ is rigid in the theorem above, we note that $\bar{c}\left(\lambda_{0, n_{0}}(K) \rightsquigarrow \lambda_{1, n_{1}}(K)\right)=1$ and we have the following more explicit result.

Theorem 14.6. Let $\mathbf{m} \in \mathcal{P}$ be a rigid tuple. Assume $m_{0, n_{0}}=m_{1, n_{1}}=1, n_{0}>1$ and $n_{1}>1$ in the Riemann scheme (14.1). Then

$$
\begin{gathered}
c\left(\lambda_{0, n_{0}} \rightsquigarrow \lambda_{1, n_{1}}\right)=\frac{\prod_{\nu=1}^{n_{0}-1} \Gamma\left(\lambda_{0, n_{0}}-\lambda_{0, \nu}+1\right) \cdot \prod_{\nu=1}^{n_{1}-1} \Gamma\left(\lambda_{1, \nu}-\lambda_{1, n_{1}}\right)}{\prod_{\substack{\mathbf{m}^{\prime} \oplus \mathbf{m}^{\prime \prime}=\mathbf{m} \\
m_{0, n_{0}}^{\prime}=m_{1, n_{1}}^{\prime \prime}=1}} \Gamma\left(\left|\left\{\lambda_{\mathbf{m}^{\prime}}\right\}\right|\right) \cdot \prod_{j=2}^{p-1}\left(1-\frac{1}{c_{j}}\right)^{-\lambda(K)_{j, \ell(K)_{j}}}}, \\
\sum_{\substack{\mathbf{m}^{\prime} \oplus \mathbf{m}^{\prime \prime}=\mathbf{m} \\
m_{0, n_{0}}^{\prime \prime}=m_{1, n_{1}=1}^{\prime \prime}=1}} m_{j, \nu}^{\prime}=\left(\begin{array}{c}
\left.n_{1}-1\right) m_{j, \nu}-\delta_{j, 0}\left(1-n_{0} \delta_{\nu, n_{0}}\right)+\delta_{j, 1}\left(1-n_{1} \delta_{\nu, n_{1}}\right) \\
\left(1 \leq \nu \leq n_{j}, 0 \leq j \leq p\right)
\end{array}\right.
\end{gathered}
$$

under the notation in Definitions 6.17 and 7.11 .

Proof. We may assume $\mathbf{m}$ is monotone and ord $\mathbf{m}>1$.

We will prove this theorem by the induction on ord $\mathbf{m}$. Suppose

$$
\mathbf{m}=\mathbf{m}^{\prime} \oplus \mathbf{m}^{\prime \prime} \quad \text { with } m_{0, n_{0}}^{\prime}=m_{1, n_{1}}^{\prime \prime}=1 .
$$

If $\partial_{\mathbf{1}} \mathbf{m}^{\prime}$ is not well-defined, then

$$
\operatorname{ord} \mathbf{m}^{\prime}=1 \text { and } m_{j, 1}^{\prime}=1 \text { for } j=1,2, \ldots, p
$$


and $1+m_{1,1}+\cdots+m_{p, 1}-(p-1)$ ord $\mathbf{m}=1$ because $\operatorname{idx}\left(\mathbf{m}, \mathbf{m}^{\prime}\right)=1$ and therefore

$$
d_{1}(\mathbf{m})=m_{0,1} .
$$

If $\partial_{1} \mathbf{m}^{\prime \prime}$ is not well-defined,

$$
\begin{aligned}
\operatorname{ord} \mathbf{m}^{\prime \prime} & =1 \text { and } m_{j, 1}^{\prime \prime}=1 \text { for } j=0,2, \ldots, p, \\
d_{\mathbf{1}}(\mathbf{m}) & =m_{1,1} .
\end{aligned}
$$

Hence if $d_{\mathbf{1}}(\mathbf{m})<m_{0,1}$ and $d_{\mathbf{1}}(\mathbf{m})<m_{1,1}, \partial_{\mathbf{1}} \mathbf{m}^{\prime}$ and $\partial_{\mathbf{1}} \mathbf{m}^{\prime \prime}$ are always welldefined and $\partial_{\mathbf{1}} \mathbf{m}=\partial_{\mathbf{1}} \mathbf{m}^{\prime} \oplus \partial_{\mathbf{1}} \mathbf{m}^{\prime \prime}$ and the direct decompositions (14.12) of $\mathbf{m}$ correspond to those of $\partial_{1} \mathbf{m}$ and therefore Theorem 14.4 shows $(14.10)$ by the induction because we may assume $d_{\mathbf{1}}(\mathbf{m})>0$. In fact, it follows from (7.15) that the gamma factors in the denominator of the fraction in the right hand side of (14.10) don't change by the reduction and the change of the numerator just corresponds to the formula in Theorem 14.4 .

If $d_{\mathbf{1}}(\mathbf{m})=m_{0,1}$, there exists the direct decomposition (14.12) with (14.13) which doesn't correspond to a direct decomposition of $\partial_{\mathbf{1}} \mathbf{m}$ but corresponds to the term $\Gamma\left(\left|\left\{\lambda_{\mathbf{m}^{\prime}}\right\}\right|\right)=\Gamma\left(\lambda_{0, n_{1}}+\lambda_{1,1}+\cdots+\lambda_{p, 1}\right)=\Gamma\left(\lambda_{0, n_{1}}^{\prime}-\lambda_{0,1}^{\prime}+1\right)$ in (14.8). Similarly if $d_{\mathbf{1}}(\mathbf{m})=m_{1,1}$, there exists the direct decomposition (14.12) with (14.15) and it corresponds to the term $\Gamma\left(\left|\left\{\lambda_{\mathbf{m}^{\prime}}\right\}\right|\right)=\Gamma\left(1-\left|\left\{\lambda_{\mathbf{m}^{\prime \prime}}\right\}\right|\right)=\Gamma\left(1-\lambda_{0,1}-\lambda_{1, n}-\lambda_{2,1}-\right.$ $\left.\cdots-\lambda_{p, 1}\right)=\Gamma\left(\lambda_{1,1}^{\prime}-\lambda_{1, n_{1}}^{\prime}\right)(\mathrm{cf}$. (14.21) $)$. Thus Theorem 14.4 assures (14.10) by the induction on ord $\mathbf{m}$.

Note that the above proof with (14.9) shows (14.18). Hence

$$
\begin{aligned}
\sum_{\substack{\mathbf{m}^{\prime} \oplus \mathbf{m}^{\prime \prime}=\mathbf{m} \\
m_{0, n_{0}}^{\prime}=m_{1, n_{1}}^{\prime \prime}=1}}\left|\left\{\lambda_{\mathbf{m}}\right\}\right|= & \sum_{\nu=1}^{n_{0}-1}\left(\lambda_{0, n_{0}}-\lambda_{0, \nu}+1\right)+\sum_{\nu=1}^{n_{1}-1}\left(\lambda_{1, \nu}-\lambda_{1, n_{1}}\right) \\
= & \left(n_{0}-1\right)+\left(n_{0}-1\right) \lambda_{0, n_{0}}-\sum_{\nu=1}^{n_{0}-1} \lambda_{0, \nu}+\sum_{\nu=1}^{n_{1}-1} \lambda_{1, \nu} \\
& +\left(n_{1}-1\right)\left(\sum_{j=0}^{p} \sum_{\nu=1}^{n_{j}-\delta_{j, 1}} m_{j, \nu} \lambda_{j, \nu}-n+1\right) \\
= & \left(n_{0}+n_{1}-2\right) \lambda_{0, n_{0}}+\sum_{\nu=1}^{n_{0}-1}\left(\left(n_{1}-1\right) m_{0, \nu}-1\right) \lambda_{0, \nu} \\
& +\sum_{\nu=1}^{n_{1}-1}\left(\left(n_{1}-1\right) m_{1, \nu}+1\right) \lambda_{1, \nu}+\sum_{j=2}^{p} \sum_{\nu=1}^{n_{2}}\left(n_{1}-1\right) m_{j, \nu} \lambda_{j, \nu} \\
& +\left(n_{0}+n_{1}-2\right)-\left(n_{1}-1\right) \operatorname{ord} \mathbf{m} .
\end{aligned}
$$

The left hand side of the above first equation and the right hand side of the above last equation don't contain the term $\lambda_{1, n_{1}}$ and therefore the coefficients of $\lambda_{j, \nu}$ in the both sides are equal, which implies (14.11).

Corollary 14.7. Retain the notation in Theorem 14.6. We have

$$
\begin{aligned}
& \#\left\{\mathbf{m}^{\prime} ; \mathbf{m}^{\prime} \oplus \mathbf{m}^{\prime \prime}=\mathbf{m} \text { with } m_{0, n_{0}}^{\prime}=m_{1, n_{1}}^{\prime \prime}=1\right\}=n_{0}+n_{1}-2, \\
& \sum_{\substack{\mathbf{m}^{\prime} \oplus \mathbf{m}^{\prime \prime}=\mathbf{m} \\
m_{0, n_{0}}^{\prime}=m_{1, n_{1}}^{\prime \prime}=1}} \operatorname{ord} \mathbf{m}^{\prime}=\left(n_{1}-1\right) \operatorname{ord} \mathbf{m} \\
& \sum_{\substack{\mathbf{m}^{\prime} \oplus \mathbf{m}^{\prime \prime}=\mathbf{m} \\
m_{0, n_{0}}^{\prime}=m_{1, n_{1}}^{\prime \prime}=1}}\left|\left\{\lambda_{m}^{\prime}\right\}\right|=\sum_{\nu=1}^{n_{0}-1}\left(\lambda_{0, n_{0}}-\lambda_{0, \nu}+1\right)+\sum_{\nu=1}^{n_{1}-1}\left(\lambda_{1, \nu}-\lambda_{1, n_{1}}\right) .
\end{aligned}
$$


Let $c\left(\lambda_{0, n_{0}}+t \rightsquigarrow \lambda_{1, n_{1}}-t\right)$ be the connection coefficient for the Riemann scheme $\left\{\left[\lambda_{j, \nu}+t\left(\delta_{j, 0} \delta_{\nu, n_{0}}-\delta_{j, 1} \delta_{\nu, n_{1}}\right)\right]_{\left(m_{j, \nu}\right)}\right\}$. Then

$$
\lim _{t \rightarrow+\infty} c\left(0: \lambda_{0, n_{0}}+t \rightsquigarrow 1: \lambda_{1, n_{1}}-t\right)=\prod_{j=2}^{p-1}\left(1-c_{j}\right)^{\lambda(K)_{j, \ell(K)_{j}}} .
$$

Under the notation in Theorem 12.13

$$
\begin{aligned}
& \left\{\mathbf{m}^{\prime} ; \mathbf{m}^{\prime} \oplus \mathbf{m}^{\prime \prime}=\mathbf{m} \text { with } m_{0, n_{0}}^{\prime}=m_{1, n_{1}}^{\prime \prime}=1\right\} \\
& =\left\{\mathbf{m}^{\prime} \in \mathcal{P} ; m_{0, n_{0}}^{\prime}=1, m_{1, n_{1}}^{\prime}=0, \alpha_{\mathbf{m}^{\prime}} \text { or } \alpha_{\mathbf{m}-\mathbf{m}^{\prime}} \in \Delta(\mathbf{m})\right\} .
\end{aligned}
$$

Proof. We have (14.18) in the proof of Theorem 14.4 and then Stirling's formula and (14.18) prove (14.19). Putting $(j, \nu)=\left(0, n_{0}\right)$ in (14.11) and considering the sum $\sum_{\nu}$ for (14.11) with $j=1$, we have (14.16) and (14.17), respectively.

Comparing the proof of Theorem 14.6 with that of Theorem 12.13, we have (14.20). Proposition 9.9 also proves (14.20).

Remark 14.8. i) When we calculate a connection coefficient for a given rigid partition $\mathbf{m}$ by (14.10), it is necessary to get all the direct decompositions $\mathbf{m}=\mathbf{m}^{\prime} \oplus \mathbf{m}^{\prime \prime}$ satisfying $m_{0, n_{0}}^{\prime}=m_{1, n_{1}}^{\prime \prime}=1$. In this case the equality (14.16) is useful because we know that the number of such decompositions equals $n_{0}+n_{1}-2$, namely, the number of gamma functions appearing in the numerator equals that appearing in the denominator in 14.10 ).

ii) A direct decomposition $\mathbf{m}=\mathbf{m}^{\prime} \oplus \mathbf{m}^{\prime \prime}$ for a rigid tuple $\mathbf{m}$ means that $\left\{\alpha_{\mathbf{m}^{\prime}}, \alpha_{\mathbf{m}^{\prime \prime}}\right\}$ is a fundamental system of a root system of type $A_{2}$ in $\mathbb{R} \alpha_{\mathbf{m}^{\prime}}+\mathbb{R} \alpha_{\mathbf{m}^{\prime \prime}}$ such that $\alpha_{\mathbf{m}}=\alpha_{\mathbf{m}^{\prime}}+\alpha_{\mathbf{m}^{\prime \prime}}$ and

$\left\{\begin{array}{l}\left(\alpha_{\mathbf{m}^{\prime}} \mid \alpha_{\mathbf{m}^{\prime}}\right)=\left(\alpha_{\mathbf{m}^{\prime \prime}} \mid \alpha_{\mathbf{m}^{\prime \prime}}\right)=2 \\ \left(\alpha_{\mathbf{m}^{\prime}} \mid \alpha_{\mathbf{m}^{\prime \prime}}\right)=-1\end{array}\right.$

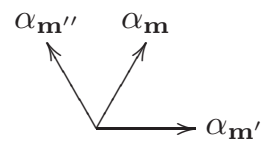

iii) In view of Definition 6.12, the condition $\mathbf{m}=\mathbf{m}^{\prime} \oplus \mathbf{m}^{\prime \prime}$ in (14.10) means

$$
\left|\left\{\lambda_{\mathbf{m}^{\prime}}\right\}\right|+\left|\left\{\lambda_{\mathbf{m}^{\prime \prime}}\right\}\right|=1 \text {. }
$$

Hence we have

$$
\begin{gathered}
c\left(\lambda_{0, n_{0}} \rightsquigarrow \lambda_{1, n_{1}}\right) \cdot c\left(\lambda_{1, n_{1}} \rightsquigarrow \lambda_{0, n_{0}}\right) \\
=\frac{\prod_{\substack{\mathbf{m}^{\prime} \oplus \mathbf{m}^{\prime \prime}=\mathbf{m} \\
m_{0, n_{0}}^{\prime}=m_{1, n_{1}}^{\prime \prime}=1}} \sin \left(\left|\left\{\lambda_{\mathbf{m}^{\prime}}\right\}\right| \pi\right)}{\prod_{\nu=1}^{n_{0}-1} \sin \left(\lambda_{0, \nu}-\lambda_{1, \nu}\right) \pi \cdot \prod_{\nu=1}^{n_{1}-1} \sin \left(\lambda_{1, \nu}-\lambda_{1, n_{1}}\right) \pi} .
\end{gathered}
$$

iv) By the aid of a computer, the author obtained the table of the concrete connection coefficients (14.10) for the rigid triplets $\mathbf{m}$ satisfying ord $\mathbf{m} \leq 40$ together with checking (14.11), which contains 4,111,704 independent cases (cf. \$15.11).

14.2. An estimate for large exponents. The Gauss hypergeometric series

$$
F(\alpha, \beta, \gamma ; x):=\sum_{k=0}^{\infty} \frac{\alpha(\alpha+1) \cdots(\alpha+k-1) \cdot \beta(\beta+1) \cdots(\beta+k-1)}{\gamma(\gamma+1) \cdots(\gamma+k-1) \cdot k !} x^{k}
$$

uniformly and absolutely converges for

$$
x \in \bar{D}:=\{x \in \mathbb{C} ;|x| \leq 1\}
$$

if $\operatorname{Re} \gamma>\operatorname{Re}(\alpha+\beta)$ and defines a continuous function on $\bar{D}$. The continuous function $F(\alpha, \beta, \gamma+n ; x)$ on $\bar{D}$ uniformly converges to the constant function 1 when $n \rightarrow+\infty$, which obviously implies

$$
\lim _{n \rightarrow \infty} F(\alpha, \beta, \gamma+n ; 1)=1
$$


and proves Gauss's summation formula $(1.3)$ by using the recurrence relation

$$
\frac{F(\alpha, \beta, \gamma ; 1)}{F(\alpha, \beta, \gamma+1 ; 1)}=\frac{(\gamma-\alpha)(\gamma-\beta)}{\gamma(\gamma-\alpha-\beta)}
$$

We will generalize such convergence in a general system of ordinary differential equations of Schlesinger canonical form.

Under the condition

$$
a>0, b>0 \text { and } c>a+b,
$$

the function $F(a, b, c ; x)=\sum_{k=0}^{\infty} \frac{(a)_{k}(b)_{k}}{(c)_{k} k !} x^{k}$ is strictly increasing continuous function of $x \in[0,1]$ satisfying

$$
1 \leq F(a, b, c ; x) \leq F(a, b, c ; 1)=\frac{\Gamma(c) \Gamma(c-a-b)}{\Gamma(c-a) \Gamma(c-b)}
$$

and it increases if $a$ or $b$ or $-c$ increases. In particular, if

$$
0 \leq a \leq N, 0 \leq b \leq N \text { and } c>2 N
$$

with a positive integer $N$, we have

$$
\begin{aligned}
0 & \leq F(a, b, c ; x)-1 \\
& \leq \frac{\Gamma(c) \Gamma(c-2 N)}{\Gamma(c-N) \Gamma(c-N)}-1=\frac{(c-N)_{N}}{(c-2 N)_{N}}-1=\prod_{\nu=1}^{N} \frac{c-\nu}{c-N-\nu}-1 \\
& \leq\left(\frac{c-N}{c-2 N}\right)^{N}-1=\left(1+\frac{N}{c-2 N}\right)^{N}-1 \\
& \leq N\left(1+\frac{N}{c-2 N}\right)^{N-1} \frac{N}{c-2 N} .
\end{aligned}
$$

Thus we have the following lemma.

Lemma 14.9. For a positive integer $N$ we have

$$
\begin{aligned}
& |F(\alpha, \beta, \gamma ; x)-1| \leq\left(1+\frac{N}{\operatorname{Re} \gamma-2 N}\right)^{N}-1 \\
& x \in \bar{D},|\alpha| \leq N,|\beta| \leq N \quad \text { and } \operatorname{Re} \gamma>2 N .
\end{aligned}
$$$$
\text { if }
$$

Proof. The lemma is clear because

$$
\left|\sum_{k=1}^{\infty} \frac{(\alpha)_{k}(\beta)_{k}}{(\gamma)_{k} k !} x^{k}\right| \leq \sum_{k=1}^{\infty} \frac{(|\alpha|)_{k}(|\beta|)_{k}}{(\operatorname{Re} \gamma)_{k} k !}|x|^{k}=F(|\alpha|,|\beta|, \operatorname{Re} \gamma-2 N ;|x|)-1
$$

For the Gauss hypergeometric equation

$$
x(1-x) u^{\prime \prime}+(\gamma-(\alpha+\beta+1) x) u^{\prime}-\alpha \beta u=0
$$

we have

$$
\begin{aligned}
\left(x u^{\prime}\right)^{\prime} & =u^{\prime}+x u^{\prime \prime}=\frac{x u^{\prime}}{x}+\frac{((\alpha+\beta+1) x-\gamma) u^{\prime}+\alpha \beta u}{1-x} \\
& =\frac{\alpha \beta}{1-x} u+\left(\frac{1}{x}-\frac{\gamma}{x(1-x)}+\frac{\alpha+\beta+1}{1-x}\right) x u^{\prime} \\
& =\frac{\alpha \beta}{1-x} u+\left(\frac{1-\gamma}{x}+\frac{\alpha+\beta-\gamma+1}{1-x}\right) x u^{\prime} .
\end{aligned}
$$

Putting

$$
\tilde{u}=\left(\begin{array}{l}
u_{0} \\
u_{1}
\end{array}\right):=\left(\begin{array}{c}
u \\
\frac{x u^{\prime}}{\alpha}
\end{array}\right)
$$


we have

$$
\tilde{u}^{\prime}=\frac{\left(\begin{array}{cc}
0 & \alpha \\
0 & 1-\gamma
\end{array}\right)}{x} \tilde{u}+\frac{\left(\begin{array}{cc}
0 & 0 \\
\beta & \alpha+\beta-\gamma+1
\end{array}\right)}{1-x} \tilde{u} .
$$

In general, for

$$
v^{\prime}=\frac{A}{x} v+\frac{B}{1-x} v
$$

we have

$$
\begin{aligned}
x v^{\prime} & =A v+\frac{x}{1-x} B v \\
& =A v+x\left(x v^{\prime}+(B-A) v\right) .
\end{aligned}
$$

Thus

$$
\left\{\begin{array}{l}
x u_{0}^{\prime}=\alpha u_{1} \\
x u_{1}^{\prime}=(1-\gamma) u_{1}+x\left(x u_{1}^{\prime}+\beta u_{0}+(\alpha+\beta) u_{1}\right)
\end{array}\right.
$$

and the functions

$$
\left\{\begin{array}{l}
u_{0}=F(\alpha, \beta, \gamma ; x) \\
u_{1}=\frac{\beta x}{\gamma} F(\alpha+1, \beta+1, \gamma+1 ; x)
\end{array}\right.
$$

satisfies (14.30).

Theorem 14.10. Let $n, n_{0}$ and $n_{1}$ be positive integers satisfying $n=n_{0}+n_{1}$ and let $A=\left(\begin{array}{ll}0 & A_{0} \\ 0 & A_{1}\end{array}\right), B=\left(\begin{array}{cc}0 & 0 \\ B_{0} & B_{1}\end{array}\right) \in M(n, \mathbb{C})$ such that $A_{1}, B_{1} \in M\left(n_{1}, \mathbb{C}\right)$, $A_{0} \in M\left(n_{0}, n_{1}, \mathbb{C}\right)$ and $B_{0} \in M\left(n_{1}, n_{0}, \mathbb{C}\right)$. Let $D(\mathbf{0}, \mathbf{m})=D\left(\mathbf{0}, m_{1}, \ldots, m_{n_{1}}\right)$ be the diagonal matrix of size $n$ whose $k$-th diagonal element is $m_{k-n_{0}}$ if $k>n_{0}$ and 0 otherwise. Let $u^{\mathrm{m}}$ be the local holomorphic solution of

$$
u=\frac{A-D(\mathbf{0}, \mathbf{m})}{x} u+\frac{B-D(\mathbf{0}, \mathbf{m})}{1-x} u
$$

at the origin. Then if $\operatorname{Re} m_{\nu}$ are sufficiently large for $\nu=1, \ldots, n_{1}$, the Taylor series of $u^{\mathbf{m}}$ at the origin uniformly converge on $\bar{D}=\{x \in \mathbb{C} ;|x| \leq 1\}$ and for a positive number $C$, the function $u^{\mathbf{m}}$ and their derivatives uniformly converge to constants on $\bar{D}$ when $\min \left\{\operatorname{Re} m_{1}, \ldots, \operatorname{Re} m_{n_{1}}\right\} \rightarrow+\infty$ with $\left|A_{i j}\right|+\left|B_{i j}\right| \leq C$. In particular, for $x \in \bar{D}$ and an integer $N$ satisfying

$$
\sum_{\nu=1}^{n_{1}}\left|\left(A_{0}\right)_{i \nu}\right| \leq N, \sum_{\nu=1}^{n_{1}}\left|\left(A_{1}\right)_{i \nu}\right| \leq N, \sum_{\nu=1}^{n_{0}}\left|\left(B_{0}\right)_{i \nu}\right| \leq N, \sum_{\nu=1}^{n_{1}}\left|\left(B_{1}\right)_{i \nu}\right| \leq N
$$

we have

$$
\max _{1 \leq \nu \leq n}\left|u_{\nu}^{\mathbf{m}}(x)-u_{\nu}^{\mathbf{m}}(0)\right| \leq \max _{1 \leq \nu \leq n_{0}}\left|u_{\nu}^{\mathbf{m}}(0)\right| \cdot \frac{2^{N}(N+1)^{2}}{\min _{1 \leq \nu \leq n_{1}} \operatorname{Re} m_{\nu}-4 N-1}
$$

if $\operatorname{Re} m_{\nu}>5 N+4$ for $\nu=1, \ldots, n_{1}$.

Proof. Use the method of majorant series and compare to the case of Gauss hypergeometric series (cf. (14.30) and (14.31)), namely, $\lim _{c \rightarrow+\infty} F(a, b, c ; x)=1$ on $\bar{D}$ with a solution of the Fuchsian system

$$
\begin{aligned}
u^{\prime} & =\frac{A}{x} u+\frac{B}{1-x} u, \\
A & =\left(\begin{array}{cc}
0 & A_{0} \\
0 & A_{1}
\end{array}\right), \quad B=\left(\begin{array}{cc}
0 & 0 \\
B_{0} & B_{1}
\end{array}\right), \quad u=\left(\begin{array}{l}
v_{0} \\
v_{1}
\end{array}\right),
\end{aligned}
$$




$$
\begin{aligned}
x v_{0}^{\prime} & =A_{0} v_{1}, \\
x v_{1}^{\prime} & =x^{2} v_{1}^{\prime}+(1-x) A_{1} v_{1}+x B_{0} v_{0}+x B_{1} v_{1} \\
& =A_{1} v_{1}+x\left(x v_{1}^{\prime}+B_{0} v_{0}+\left(B_{1}-A_{1}\right) v_{1}\right)
\end{aligned}
$$

or the system obtained by the substitution $A_{1} \mapsto A_{1}-D(\mathbf{m})$ and $B_{1} \mapsto B_{1}-D(\mathbf{m})$. Fix positive real numbers $\alpha, \beta$ and $\gamma$ satisfying

$$
\begin{aligned}
\alpha & \geq \sum_{\nu=1}^{n_{1}}\left|\left(A_{0}\right)_{i \nu}\right| \quad\left(1 \leq i \leq n_{0}\right), \quad \beta \geq \sum_{\nu=1}^{n_{0}}\left|\left(B_{0}\right)_{i \nu}\right| \quad\left(1 \leq i \leq n_{1}\right), \\
\alpha+\beta & \geq \sum_{\nu=1}^{n_{1}}\left|\left(B_{1}-A_{1}\right)_{i \nu}\right| \quad\left(1 \leq i \leq n_{0}\right), \\
\gamma & =\min \left\{\operatorname{Re} m_{1}, \ldots, \operatorname{Re} m_{n_{1}}\right\}-2 \max _{1 \leq i \leq n_{1}} \sum_{\nu=1}^{n_{1}}\left|\left(A_{1}\right)_{i \nu}\right|-1>\alpha+\beta .
\end{aligned}
$$

Then the method of majorant series with Lemma 14.11, 14.30) and (14.31) imply

$$
u_{i}^{\mathbf{m}} \ll \begin{cases}\max _{1 \leq \nu \leq n_{0}}\left|u_{\nu}^{\mathbf{m}}(0)\right| \cdot F(\alpha, \beta, \gamma ; x) & \left(1 \leq i \leq n_{0}\right), \\ \frac{\beta}{\gamma} \cdot \max _{1 \leq \nu \leq n_{0}}\left|u_{\nu}^{\mathbf{m}}(0)\right| \cdot F(\alpha+1, \beta+1, \gamma+1 ; x) & \left(n_{0}<i \leq n\right),\end{cases}
$$

which proves the theorem because of Lemma 14.9 with $\alpha=\beta=N$ as follows. Here $\sum_{\nu=0}^{\infty} a_{\nu} x^{\nu} \ll \sum_{\nu=0}^{\infty} b_{\nu} x^{\nu}$ for formal power series means $\left|a_{\nu}\right| \leq b_{\nu}$ for $\nu \in \mathbb{Z}_{\geq 0}$.

Put $\bar{m}=\min \left\{\operatorname{Re} m_{1}, \ldots, \operatorname{Re} m_{n_{1}}\right\}-2 N-1$ and $L=\max _{1 \leq \nu \leq n_{0}}\left|u_{\nu}^{\mathbf{m}}(0)\right|$. Then $\gamma \geq \bar{m}-2 N-1$ and if $0 \leq i \leq n_{0}$ and $x \leq \bar{D}$,

$$
\begin{aligned}
\left|u_{i}^{\mathbf{m}}(x)-u_{i}^{\mathbf{m}}(0)\right| & \leq L \cdot(F(\alpha, \beta, \gamma ;|x|)-1) \\
& \leq L\left(\left(1+\frac{N}{\bar{m}-4 N-1}\right)^{N}-1\right) \\
& \leq L\left(1+\frac{N}{\bar{m}-4 N-1}\right)^{N-1} \frac{N^{2}}{\bar{m}-4 N-1} \leq \frac{L 2^{N-1} N^{2}}{\bar{m}-4 N-1} .
\end{aligned}
$$

If $n_{0}<i \leq n$ and $x \in \bar{D}$,

$$
\begin{aligned}
\left|u_{i}^{\mathbf{m}}(x)\right| & \leq \frac{\beta}{\gamma} \cdot L F(\alpha+1, \beta+1, \gamma+1 ;|x|) \\
& \leq \frac{L N}{\bar{m}-2 N-1}\left(\left(1+\frac{N+1}{\bar{m}-4 N-3}\right)^{N+1}+1\right) \leq \frac{L N\left(2^{N+1}+1\right)}{\bar{m}-2 N-1} .
\end{aligned}
$$

Lemma 14.11. Let $A \in M(n, \mathbb{L})$ and put

$$
|A|:=\max _{1 \leq i \leq n} \sum_{\nu=1}^{n}\left|A_{i \nu}\right| .
$$

If positive real numbers $m_{1}, \ldots, m_{n}$ satisfy

$$
m_{\min }:=\min \left\{m_{1}, \ldots, m_{n}\right\}>2|A|,
$$

we have

$$
\left|\left(k I_{n}+D(\mathbf{m})-A\right)^{-1}\right| \leq\left(k+m_{\text {min }}-2|A|\right)^{-1} \quad(\forall k \geq 0) .
$$

Proof. Since

$$
\begin{aligned}
\left|(D(\mathbf{m})-A)^{-1}\right| & =\left|D(\mathbf{m})^{-1}\left(I_{n}-D(\mathbf{m})^{-1} A\right)^{-1}\right| \\
& =\left|D(\mathbf{m})^{-1} \sum_{k=0}^{\infty}\left(D(\mathbf{m})^{-1} A\right)^{k}\right| \\
& \leq m_{\min }^{-1} \cdot\left(1+\frac{2|A|}{m_{\min }}\right) \leq\left(m_{\min }-2|A|\right)^{-1},
\end{aligned}
$$


we have the lemma by replacing $m_{\nu}$ by $m_{\nu}+k$ for $\nu=1, \ldots, n$.

14.3. Zeros and poles of connection coefficients. In this subsection we examine the connection coefficients to calculate them in a different way from the one given in $\$ 14.1$.

First review the connection coefficient $c\left(0: \lambda_{0,2} \rightsquigarrow 1: \lambda_{1,2}\right)$ for the solution of Fuchsian differential equation with the Riemann scheme $\left\{\begin{array}{ccc}x=0 & 1 & \infty \\ \lambda_{0,1} & \lambda_{1,1} & \lambda_{2,1} \\ \lambda_{0,2} & \lambda_{1,2} & \lambda_{2,2}\end{array}\right\}$. Denoting the connection coefficient $c\left(0: \lambda_{0,2} \rightsquigarrow 1: \lambda_{1,2}\right)$ by $c\left(\left\{\begin{array}{lll}\lambda_{0,1} & \lambda_{1,1} & \lambda_{2,1} \\ \lambda_{0,2} \rightsquigarrow & \lambda_{1,2} & \lambda_{2,2}\end{array}\right\}\right)$, we have

$$
\begin{aligned}
& u_{0}^{\lambda_{0,2}}=c\left(\left\{\begin{array}{lll}
\lambda_{0,1} & \lambda_{1,1} & \lambda_{2,1} \\
\lambda_{0,2} \rightsquigarrow & \lambda_{1,2} & \lambda_{2,2}
\end{array}\right\}\right) u_{1}^{\lambda_{1,2}}+c\left(\left\{\begin{array}{ccc}
\lambda_{0,1} & \lambda_{1,2} & \lambda_{2,1} \\
\lambda_{0,2} \rightsquigarrow & \lambda_{1,1} & \lambda_{2,2}
\end{array}\right\}\right) u_{1}^{\lambda_{1,1}} .
\end{aligned}
$$

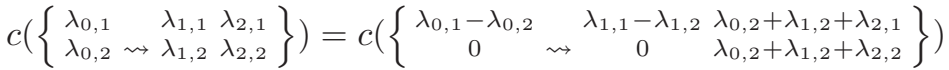

$$
\begin{aligned}
& =F\left(\lambda_{0,2}+\lambda_{1,2}+\lambda_{2,1}, \lambda_{0,2}+\lambda_{1,2}+\lambda_{2,2}, \lambda_{0,2}-\lambda_{0,1}+1 ; 1\right)
\end{aligned}
$$

under the notation in Definition 14.1. As was explained in the first part of $\$ 14.2$, the connection coefficient is calculated from

$$
\lim _{n \rightarrow \infty} c\left(\left\{\begin{array}{cccc}
\lambda_{0,1}-n & \lambda_{1,1}+n & \lambda_{2,1} \\
\lambda_{0,2} & \rightsquigarrow & \lambda_{1,2} & \lambda_{2,2}
\end{array}\right\}\right)=1
$$

and

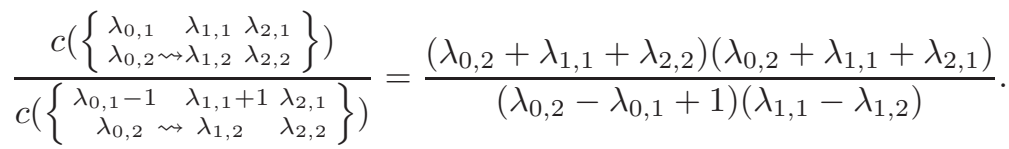

The relation $(14.40)$ is easily obtained from $(14.39)$ and $(14.24)$ or can be reduced to Theorem 14.10.

We will examine (14.41). For example, the relation 14.41 follows from the relation (14.25) which is obtained from

$$
\begin{aligned}
\gamma(\gamma-1-(2 \gamma-\alpha-\beta-1) x) F(\alpha, \beta, \gamma ; x)+ & (\gamma-\alpha)(\gamma-\beta) x F(\alpha, \beta, \gamma+1 ; x) \\
& =\gamma(\gamma-1)(1-x) F(\alpha, \beta, \gamma-1 ; x)
\end{aligned}
$$

by putting $x=1$ (cf. WW, $\S 14.1]$ ). We may use a shift operator as follows. Since

$$
\begin{aligned}
& \frac{d}{d x} F(\alpha, \beta, \gamma ; x)=\frac{\alpha \beta}{\gamma} F(\alpha+1, \beta+1, \gamma+1 ; x) \\
& =c\left(\left\{\begin{array}{ccc}
1-\gamma & \gamma-\alpha-\beta & \alpha \\
0 & 0 & \beta
\end{array}\right\}\right) \frac{d}{d x} u_{1}^{0}+c\left(\left\{\begin{array}{ccc}
1-\gamma & 0 & \alpha \\
0 & \rightsquigarrow \gamma-\alpha-\beta & \beta
\end{array}\right\}\right) \frac{d}{d x} u_{1}^{\gamma-\alpha-\beta}
\end{aligned}
$$

and

we have

$$
\frac{d}{d x} u_{1}^{\gamma-\alpha-\beta} \equiv(\alpha+\beta-\gamma)(1-x)^{\gamma-\alpha-\beta-1} \bmod (1-x)^{\gamma-\alpha-\beta} \mathcal{O}_{1},
$$

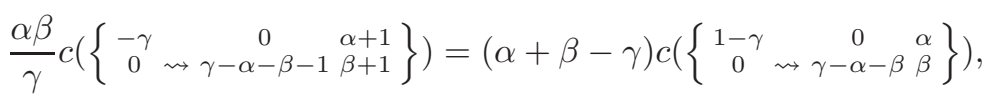

which also proves 14.41) because

$$
\frac{c\left(\left\{\begin{array}{lll}
\lambda_{0,1} & \lambda_{1,1} & \lambda_{2,1} \\
\lambda_{0,2} & \cdots \lambda_{1,2} & \lambda_{2,2}
\end{array}\right\}\right)}{c\left(\left\{\begin{array}{ccc}
\lambda_{0,1}-1 & \lambda_{1,1}+1 & \lambda_{2,1} \\
\lambda_{0,2} & \lambda_{1,2} & \lambda_{2,2}
\end{array}\right\}\right)}=\frac{c\left(\left\{\begin{array}{ccc}
\lambda_{0,1}-\lambda_{0,2} & 0 & \lambda_{0,2}+\lambda_{1,1}+\lambda_{2,1} \\
0 & \rightsquigarrow \lambda_{1,2}-\lambda_{1,1} & \lambda_{0,2}+\lambda_{1,1}+\lambda_{2,2}
\end{array}\right\}\right)}{c\left(\left\{\begin{array}{ccc}
\lambda_{0,1}-\lambda_{0,2}-1 & \begin{array}{c}
0 \\
0
\end{array} & \lambda_{0,2}+\lambda_{1,2}+\lambda_{2,1}+1 \\
0 & \lambda_{1,2}-\lambda_{1,1}-1 & \lambda_{0,2}+\lambda_{1,2}+\lambda_{2,2}+1
\end{array}\right\}\right)} .
$$

Furthermore each linear term appeared in the right hand side of (14.41) has own meaning, which is as follows.

Examine the zeros and poles of the connection coefficient $c\left(\left\{\begin{array}{lll}\lambda_{0,1} & \lambda_{1,1} & \lambda_{2,1} \\ \lambda_{0,2} \rightsquigarrow & \lambda_{1,2} & \lambda_{2,2}\end{array}\right\}\right)$. We may assume that the parameters $\lambda_{j, \nu}$ are generic in the zeros or the poles. 
Consider the linear form $\lambda_{0,2}+\lambda_{1,1}+\lambda_{2,2}$. The local solution $u_{0}^{\lambda_{0,2}}$ corresponding to the characteristic exponent $\lambda_{0,2}$ at 0 satisfies a Fuchsian differential equation of order 1 which has the characteristic exponents $\lambda_{2,2}$ and $\lambda_{1,1}$ at $\infty$ and 1 , respectively, if and only if the value of the linear form is 0 or a negative integer. In this case $c\left(\left\{\begin{array}{ccc}\lambda_{0,1} & \lambda_{1,1} & \lambda_{2,1} \\ \lambda_{0,2} & \lambda_{1,2} & \lambda_{2,2}\end{array}\right\}\right)$ vanishes. This explains the term $\lambda_{0,2}+\lambda_{1,1}+\lambda_{2,2}$ in the numerator of the right hand side of (14.41). The term $\lambda_{0,2}+\lambda_{1,2}+\lambda_{2,2}$ is similarly explained.

The normalized local solution $u_{0}^{\lambda_{0,2}}$ has poles where $\lambda_{0,1}-\lambda_{0,2}$ is a positive integer. The residue at the pole is a local solution corresponding to the exponent $\lambda_{0,2}$. This means that $c\left(\left\{\begin{array}{lll}\lambda_{0,1} & \lambda_{1,1} & \lambda_{2,1} \\ \lambda_{0,2} & \lambda_{1,2} & \lambda_{2,2}\end{array}\right\}\right)$ has poles where $\lambda_{0,1}-\lambda_{0,2}$ is a positive integer, which explains the term $\lambda_{0,2}-\lambda_{0,1}+1$ in the denominator of the right hand side of (14.41).

There exists a local solution $a(\lambda) u_{1}^{\lambda_{1,1}}+b(\lambda) u_{1}^{\lambda_{1,2}}$ such that it is holomorphic for $\lambda_{j, \nu}$ and $b(\lambda)$ has a pole if the value of $\lambda_{1,1}-\lambda_{1,2}$ is a non-negative integer, which means $c\left(\left\{\begin{array}{lll}\lambda_{0,1} & \lambda_{1,1} & \lambda_{2,1} \\ \lambda_{0,2} & \lambda_{1,2} & \lambda_{2,2}\end{array}\right\}\right)$ has poles where $\lambda_{1,2}-\lambda_{1,1}$ is non-negative integer. This explains the term $\lambda_{1,1}-\lambda_{1,2}$ in the denominator of the right hand side of (14.41). These arguments can be generalized, which will be explained in this subsection.

Fist we examine the possible poles of connection coefficients.

Proposition 14.12. Let $P u=0$ be a differential equation of order $n$ with a regular singularity at $x=0$ such that $P$ contains a holomorphic parameter $\lambda=\left(\lambda_{1}, \ldots, \lambda_{N}\right)$ defined in a neighborhood of $\lambda^{o}=\left(\lambda_{1}^{o}, \ldots, \lambda_{N}^{o}\right)$ in $\mathbb{C}^{N}$. Suppose that the set of characteristic exponents of $P$ at $x=0$ equals $\left\{\left[\lambda_{1}\right]_{\left(m_{1}\right)}, \ldots,\left[\lambda_{N}\right]_{\left(m_{N}\right)}\right\}$ with $n=$ $m_{1}+\cdots+m_{N}$ and

$$
\lambda_{2,1}^{o}:=\lambda_{2}^{o}-\lambda_{1}^{o} \in \mathbb{Z}_{\geq 0} \text { and } \lambda_{i}^{o}-\lambda_{j}^{o} \notin \mathbb{Z} \text { if } 1 \leq i<j \leq N \text { and } j \neq 2 \text {. }
$$

Let $u_{j, \nu}$ be local solutions of $P u=0$ uniquely defined by

$$
u_{j, \nu} \equiv x^{\lambda_{j}+\nu} \quad \bmod x^{\lambda_{j}+m_{j}} \mathcal{O}_{0} \quad\left(j=1, \ldots, m_{j} \text { and } \nu=0, \ldots, m_{j}-1\right)
$$

Note that $u_{j, \nu}=\sum_{k \geq 0} a_{k, j, \nu}(\lambda) x^{\lambda_{j}+\nu+k}$ with meromorphic functions $a_{k, j, \nu}(\lambda)$ of $\lambda$ which are holomorphic in a neighborhood of $\lambda^{o}$ if $\lambda_{2}-\lambda_{1} \neq \lambda_{2,1}^{o}$. Then there exist solutions $v_{j, \nu}$ with holomorphic parameter $\lambda$ in a neighborhood of $\lambda^{o}$ which satisfy the following relations. Namely

$$
v_{j, \nu}=u_{j, \nu} \quad\left(3 \leq j \leq N \text { and } \nu=0, \ldots, m_{j}-1\right)
$$

and when $\lambda_{1}^{o}+m_{1} \geq \lambda_{2}^{o}+m_{2}$,

$$
\begin{aligned}
& v_{1, \nu}=u_{1, \nu} \quad\left(0 \leq \nu<m_{1}\right), \\
& v_{2, \nu}=\frac{u_{2, \nu}-u_{1, \nu+\lambda_{2,1}^{o}}}{\lambda_{1}-\lambda_{2}+\lambda_{2,1}^{o}}-\sum_{m_{2}+\lambda_{2,1}^{o} \leq i<m_{1}} \frac{b_{\nu, i} u_{1, i}}{\lambda_{1}-\lambda_{2}+\lambda_{2,1}^{o}} \quad\left(0 \leq \nu<m_{2}\right)
\end{aligned}
$$

with the diagram $\begin{gathered}\lambda_{1}^{o} \lambda_{1}^{o}+1 \\ \circ-\ldots\end{gathered}$ 
which illustrates some exponents and when $\lambda_{1}^{o}+m_{1}<\lambda_{2}^{o}+m_{2}$,

$$
\begin{aligned}
& v_{2, \nu}=u_{2, \nu} \quad\left(0 \leq \nu<m_{2}\right), \\
& v_{1, \nu}=u_{1, \nu}-\sum_{\max \left\{0, m_{1}-\lambda_{2,1}^{o}\right\} \leq i<m_{2}} \frac{b_{\nu, i} u_{2, i}}{\lambda_{1}-\lambda_{2}+\lambda_{2,1}^{o}} \quad\left(0 \leq \nu<\min \left\{m_{1}, \lambda_{2,1}^{o}\right\}\right), \\
& v_{1, \nu}=\frac{u_{1, \nu}-u_{2, \nu-\lambda_{2,1}^{o}}}{\lambda_{1}-\lambda_{2}+\lambda_{2,1}^{o}}-\sum_{\max \left\{0, m_{1}-\lambda_{2,1}^{o}\right\} \leq i<m_{2}} \frac{b_{\nu, i} u_{2, i}}{\lambda_{1}-\lambda_{2}+\lambda_{2,1}^{o}} \quad\left(\lambda_{2,1}^{o} \leq \nu<m_{1}\right)
\end{aligned}
$$

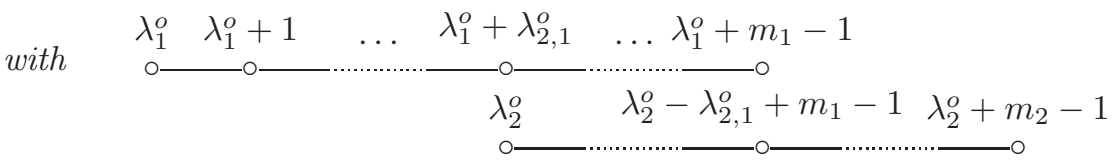

and here $b_{\nu, i} \in \mathbb{C}$. Note that $v_{j, \nu}\left(1 \leq j \leq N, 0 \leq \nu<m_{j}\right)$ are linearly independent for any fixed $\lambda$ in a neighborhood of $\lambda^{o}$.

Proof. See $\$ 3.1$ and the proof of Lemma 6.5 (and [03, Theorem 6.5] in a more general setting) for the construction of local solutions of $P u=0$.

Note that $u_{j, \nu}$ for $j \geq 3$ are holomorphic with respect to $\lambda$ in a neighborhood of $\lambda=\lambda^{\circ}$. Moreover note that the local monodromy generator $M_{0}$ of the solutions $P u=0$ at $x=0$ satisfies $\prod_{j=1}^{N}\left(M_{0}-e^{2 \pi \sqrt{-1}} \lambda_{j}\right)=0$ and therefore the functions $\left(\lambda_{1}-\lambda_{2}-\lambda_{2,1}^{o}\right) u_{j, \nu}$ of $\lambda$ are holomorphically extended to the point $\lambda=\lambda^{o}$ for $j=1$ and 2 , and the values of the functions at $\lambda=\lambda^{o}$ are solutions of the equation $P u=0$ with $\lambda=\lambda^{o}$.

Suppose $\lambda_{1}^{o}+m_{1} \geq \lambda_{2}^{o}+m_{2}$. Then $u_{j, \nu}(j=1,2)$ are holomorphic with respect to $\lambda$ at $\lambda=\lambda^{o}$ and there exist $b_{j, \nu} \in \mathbb{C}$ such that

$$
\left.u_{2, \nu}\right|_{\lambda=\lambda^{\circ}}=\left.u_{1, \nu+\lambda_{2,1}^{o}}\right|_{\lambda=\lambda^{\circ}}+\sum_{m_{2}+\lambda_{2,1}^{o} \leq \nu<m_{1}} b_{\nu, i}\left(\left.u_{1, i}\right|_{\lambda=\lambda^{\circ}}\right)
$$

and we have the proposition. Here

$$
\left.u_{2, \nu}\right|_{\lambda=\lambda^{o}} \equiv x^{\lambda_{2}^{o}}+\sum_{m_{2}+\lambda_{2,1}^{o} \leq \nu<m_{1}} b_{\nu, i} x^{\lambda_{1}^{o}+\nu} \bmod x^{\lambda_{1}^{o}+m_{1}} \mathcal{O}_{0}
$$

Next suppose $\lambda_{1}^{o}+m_{1}<\lambda_{2}^{o}+m_{2}$. Then there exist $b_{j, \nu} \in \mathbb{C}$ such that

$$
\begin{aligned}
\left.\left(\left(\lambda_{1}-\lambda_{2}+\lambda_{2,1}^{o}\right) u_{1, \nu}\right)\right|_{\lambda=\lambda^{o}} & =\sum_{\max \left\{0, m_{1}-\lambda_{2,1}^{o}\right\} \leq i<m_{2}} b_{\nu, i}\left(\left.u_{2, i}\right|_{\lambda=\lambda^{\circ}}\right) \\
\left.u_{1, \nu}\right|_{\lambda=\lambda^{o}} & =\sum_{\max \left\{0, m_{1}-\lambda_{2,1}^{o}\right\} \leq i<m_{2}} b_{\nu, i}\left(\left.u_{2, i}\right|_{\lambda=\lambda^{\circ}}\right) \quad\left(\lambda_{2,1}^{o} \leq \nu<m_{1}\right)
\end{aligned}
$$

and we have the proposition.

The proposition implies the following corollaries.

Corollary 14.13. Retain the notation and the assumption in Proposition 14.12.

i) Let $W_{j}(\lambda, x)$ be the Wronskian of $u_{j, 1}, \ldots, u_{j, m_{j}}$ for $j=1, \ldots, N$. Then $\left(\lambda_{1}-\lambda_{2}+\lambda_{2,1}^{o}\right)^{\ell_{1}} W_{1}(\lambda)$ and $W_{j}(\lambda)$ with $2 \leq j \leq N$ are holomorphic with respect to $\lambda$ in a neighborhood of $\lambda^{o}$ by putting

$$
\ell_{1}=\max \left\{0, \min \left\{m_{1}, m_{2}, \lambda_{2,1}^{o}, \lambda_{2,1}^{o}+m_{2}-m_{1}\right\}\right\} .
$$


ii) Let

$$
w_{k}=\sum_{j=1}^{N} \sum_{\nu=1}^{m_{j}} a_{j, \nu, k}(\lambda) u_{j, \nu, k}
$$

be a local solution defined in a neighborhood of 0 with a holomorphic $\lambda$ in a neighborhood of $\lambda^{o}$. Then

$$
\left(\lambda_{1}-\lambda_{2}+\lambda_{2,1}^{o}\right)^{\ell_{2, j}} \operatorname{det}\left(a_{j, \nu, k}(\lambda)\right)_{\substack{1 \leq \nu \leq m_{j} \\ 1 \leq k \leq m_{j}}}^{\substack{1 \\ j \leq \leq 1}}
$$

with

$$
\left\{\begin{array}{l}
\ell_{2,1}=\max \left\{0, \min \left\{m_{1}-\lambda_{2,1}^{o}, m_{2}\right\}\right\}, \\
\ell_{2,2}=\min \left\{m_{1}, m_{2}\right\}, \\
\ell_{2, j}=0 \quad(3 \leq j \leq N)
\end{array}\right.
$$

are holomorphic with respect to $\lambda$ in a neighborhood of $\lambda^{\circ}$.

Proof. i) Proposition 14.12 shows that $u_{j, \nu}\left(2 \leq j \leq N, 0 \leq \nu<m_{j}\right)$ are holomorphic with respect to $\lambda$ at $\lambda^{o}$. The functions $u_{1, \nu}$ for $\min \left\{m_{1}, \lambda_{2,1}^{o}\right\} \leq \nu \leq m_{1}$ are same. The functions $u_{1, \nu}$ for $0 \leq \nu<\min \left\{m_{1}, \lambda_{2,1}^{o}\right\}$ may have poles of order 1 along $\lambda_{2}-\lambda_{1}=\lambda_{2,1}^{o}$ and their residues are linear combinations of $\left.u_{2, i}\right|_{\lambda_{2}=\lambda_{1}+\lambda_{2,1}^{o}}$ with $\max \left\{0, m_{1}-\lambda_{2,1}^{o}\right\} \leq i<m_{2}$. Since

$$
\begin{aligned}
& \min \left\{\#\left\{\nu ; 0 \leq \nu<\min \left\{m_{1}, \lambda_{2,1}^{o}\right\}\right\}, \#\left\{i ; \max \left\{0, m_{1}-\lambda_{2,1}^{o}\right\} \leq i<m_{2}\right\}\right\} \\
& \quad=\max \left\{0, \min \left\{m_{1}, \lambda_{2,1}^{o}, m_{2}, m_{2}-m_{1}+\lambda_{2,1}^{o}\right\}\right\},
\end{aligned}
$$

we have the claim.

ii) A linear combination of $v_{j, \nu}\left(1 \leq j \leq N, 0 \leq \nu \leq m_{j}\right)$ may have a pole of order 1 along $\lambda_{1}-\lambda_{2}+\lambda_{2,1}^{o}$ and its residue is a linear combination of

$$
\begin{gathered}
\left.\left(u_{1, \nu}+\sum_{m_{2}+\lambda_{2,1}^{o} \leq i<m_{1}} b_{\nu+\lambda_{2,1}^{o}, i} u_{1, i}\right)\right|_{\lambda_{2}=\lambda_{1}+\lambda_{2,1}^{o}} \quad\left(\lambda_{2,1}^{o} \leq \nu<\min \left\{m_{1}, m_{2}+\lambda_{2,1}^{o}\right\}\right), \\
\left.\left(u_{2, \nu}+\sum_{\max \left\{0, m_{1}-\lambda_{2,1}^{o}\right\} \leq i<m_{2}} b_{\nu+\lambda_{2,1}^{o}, i} u_{2, i}\right)\right|_{\lambda_{2}=\lambda_{1}+\lambda_{2,1}^{o}} \quad\left(0 \leq \nu<m_{1}-\lambda_{2,1}^{o}\right), \\
\left.\sum_{\max \left\{0, m_{1}-\lambda_{2,1}^{o}\right\} \leq i<m_{2}} b_{\nu, i} u_{2, i}\right|_{\lambda_{2}=\lambda_{1}+\lambda_{2,1}^{o}} ^{o} \quad\left(0 \leq \nu<\min \left\{m_{1}, \lambda_{2,1}^{o}\right\}\right) .
\end{gathered}
$$

Since

$$
\begin{aligned}
& \#\left\{\nu ; \lambda_{2,1}^{o} \leq \nu<\min \left\{m_{1}, m_{2}+\lambda_{2,1}^{o}\right\}\right\}=\max \left\{0, \min \left\{m_{1}-\lambda_{2,1}^{o}, m_{2}\right\}\right\}, \\
& \#\left\{\nu ; 0 \leq \nu<m_{1}-\lambda_{2,1}^{o}\right\} \\
& \quad+\min \left\{\#\left\{i ; \max \left\{0, m_{1}-\lambda_{2,1}^{o}\right\} \leq i<m_{2}\right\}, \#\left\{\nu ; 0 \leq \nu<\min \left\{m_{1}, \lambda_{2,1}^{o}\right\}\right\}\right\} \\
& =\min \left\{m_{1}, m_{2}\right\},
\end{aligned}
$$

we have the claim.

Remark 14.14. If the local monodromy of the solutions of $P u=0$ at $x=0$ is locally non-degenerate, the value of $\left(\lambda_{1}-\lambda_{2}+\lambda_{2,1}^{o}\right)^{\ell_{1}} W_{1}(\lambda)$ at $\lambda=\lambda^{o}$ does not vanish.

Corollary 14.15. Let $P u=0$ be a differential equation of order $n$ with a regular singularity at $x=0$ such that $P$ contains a holomorphic parameter $\lambda=\left(\lambda_{1}, \ldots, \lambda_{N}\right)$ defined on $\mathbb{C}^{N}$. Suppose that the set of characteristic exponents of $P$ at $x=0$ equals $\left\{\left[\lambda_{1}\right]_{\left(m_{1}\right)}, \ldots,\left[\lambda_{N}\right]_{\left(m_{N}\right)}\right\}$ with $n=m_{1}+\cdots+m_{N}$. Let $u_{j, \nu}$ be the solutions of $P u=0$ defined by $(14.43)$. 
i) Let $W_{1}(x, \lambda)$ denote the Wronskian of $u_{1,1}, \ldots, u_{1, m_{1}}$. Then

$$
\frac{W_{1}(x, \lambda)}{\prod_{j=2}^{N} \prod_{0 \leq \nu<\min \left\{m_{1}, m_{j}\right\}} \Gamma\left(\lambda_{1}-\lambda_{j}+m_{1}-\nu\right)}
$$

is holomorphic for $\lambda \in \mathbb{C}^{N}$.

ii) Let

$$
v_{k}(\lambda)=\sum_{j=1}^{N} \sum_{\nu=1}^{m_{j}} a_{j, \nu, k}(\lambda) u_{j, \nu} \quad\left(1 \leq k \leq m_{1}\right)
$$

be local solutions of $P u=0$ defined in a neighborhood of 0 which have a holomorphic parameter $\lambda \in \mathbb{C}^{N}$. Then

$$
\begin{aligned}
& \operatorname{det}\left(a_{1, \nu, k}(\lambda)\right)_{\substack{1 \leq \nu \leq m_{1} \\
1 \leq k \leq m_{1}}} \\
& \frac{\prod_{j=2}^{N} \prod_{1 \leq \nu \leq \min \left\{m_{1}, m_{j}\right\}} \Gamma\left(\lambda_{j}-\lambda_{1}-m_{1}+\nu\right)}{1}
\end{aligned}
$$

is a holomorphic function of $\lambda \in \mathbb{C}^{N}$.

Proof. Let $\lambda_{j, 1}^{o} \in \mathbb{Z}$. The order of poles of (14.48) and that of (14.50) along $\lambda_{j}-\lambda_{1}=\lambda_{j, 1}^{o}$ are

$$
\begin{gathered}
\#\left\{\nu ; 0 \leq \nu<\min \left\{m_{1}, m_{j}\right\} \text { and } m_{1}-\lambda_{j, 1}^{o}-\nu \leq 0\right\} \\
=\#\left\{\nu ; \max \left\{0, m_{1}-\lambda_{j, 1}^{o}\right\} \leq \nu<\min \left\{m_{1}, m_{j}\right\}\right\} \\
=\max \left\{0, \min \left\{m_{1}, m_{j}, \lambda_{j, 1}^{o}, \lambda_{j, 1}^{o}+m_{j}-m_{1}\right\}\right\}
\end{gathered}
$$

and

$$
\begin{aligned}
& \#\left\{\nu ; 1 \leq \nu \leq \min \left\{m_{1}, m_{j}\right\} \text { and } \lambda_{j, 1}^{o}-m_{1}+\nu \leq 0\right\} \\
& \quad=\max \left\{0, \min \left\{m_{1}, m_{j}, m_{1}-\lambda_{j, 1}^{o}\right\}\right\},
\end{aligned}
$$

respectively. Hence Corollary 14.13 assures this corollary.

Remark 14.16. The product of denominator of (14.48) and that of (14.50) equals the periodic function

$$
\prod_{j=2}^{N}(-1)^{\left[\frac{\min \left\{m_{1}, m_{j}\right\}}{2}\right]+1}\left(\frac{\pi}{\sin \left(\lambda_{1}-\lambda_{j}\right) \pi}\right)^{\min \left\{m_{1}, m_{j}\right\}} .
$$

Definition 14.17 (generalized connection coefficient). Let $P_{\mathbf{m}} u=0$ be the Fuchsian differential equation with the Riemann scheme

$$
\left\{\begin{array}{ccccc}
x=c_{0}=0 & c_{1}=1 & c_{2} & \cdots & c_{p}=\infty \\
{\left[\lambda_{0,1}\right]_{\left(m_{0,1}\right)}} & {\left[\lambda_{1,1}\right]_{\left(m_{1,1}\right)}} & {\left[\lambda_{2,1}\right]_{\left(m_{2,1}\right)}} & \cdots & {\left[\lambda_{p, 1}\right]_{\left(m_{p, 1}\right)}} \\
\vdots & \vdots & \vdots & \vdots & \vdots \\
{\left[\lambda_{0, n_{0}}\right]_{\left(m_{0, n_{0}}\right)}} & {\left[\lambda_{1, n_{1}}\right]_{\left(m_{1, n_{1}}\right)}} & {\left[\lambda_{2, n_{2}}\right]_{\left(m_{2, n_{2}}\right)}} & \cdots & {\left[\lambda_{p, n_{p}}\right]_{\left(m_{p, n_{p}}\right)}}
\end{array}\right\} .
$$

We assume $c_{2}, \ldots, c_{p-1} \notin[0,1]$. Let $u_{0, \nu}^{\lambda_{0, \nu}+k}\left(1 \leq \nu \leq n_{0}, 0 \leq k<m_{0, \nu}\right)$ and $u_{1, \nu}^{\lambda_{1, \nu}+k}\left(1 \leq \nu \leq n_{1}, 0 \leq k<m_{1, \nu}\right)$ be local solutions of $P_{\mathbf{m}} u=0$ such that

$$
\left\{\begin{aligned}
u_{0, \nu}^{\lambda_{0, \nu}+k} & \equiv x^{\lambda_{0, \nu}+k} & & \bmod x^{\lambda_{0, \nu}+m_{0, \nu}} \mathcal{O}_{0}, \\
u_{1, \nu}^{\lambda_{1, \nu}+k} & \equiv(1-x)^{\lambda_{1, \nu}+k} & & \bmod (1-x)^{\lambda_{1, \nu}+m_{1, \nu}} \mathcal{O}_{1} .
\end{aligned}\right.
$$


They are uniquely defined on $(0,1) \subset \mathbb{R}$ when $\lambda_{j, \nu}-\lambda_{j, \nu^{\prime}} \notin \mathbb{Z}$ for $j=0,1$ and $1 \leq \nu<\nu^{\prime} \leq n_{j}$. Then the connection coefficients $c_{\nu, k}^{\nu^{\prime}, k^{\prime}}(\lambda)$ are defined by

$$
u_{0, \nu}^{\lambda_{0, \nu}+k}=\sum_{\nu^{\prime}, k^{\prime}} c_{\nu, k}^{\nu^{\prime}, k^{\prime}}(\lambda) u_{1, \nu^{\prime}}^{\lambda_{1, \nu^{\prime}}+k^{\prime}}
$$

Note that $c_{\nu, k}^{\nu^{\prime}, k^{\prime}}(\lambda)$ is a meromorphic function of $\lambda$ when $\mathbf{m}$ is rigid.

Fix a positive integer $n^{\prime}$ and the integer sequences $1 \leq \nu_{1}^{0}<\nu_{2}^{0}<\cdots<\nu_{L}^{0} \leq n_{0}$ and $1 \leq \nu_{1}^{1}<\nu_{2}^{1}<\cdots<\nu_{L^{\prime}}^{1} \leq n_{1}$ such that

$$
n^{\prime}=m_{0, \nu_{1}^{0}}+\cdots+m_{0, \nu_{L}^{0}}=m_{1, \nu_{1}^{1}}+\cdots+m_{1, \nu_{L^{\prime}}^{1}} .
$$

Then a generalized connection coefficient is defined by

$$
\begin{gathered}
c\left(0:\left[\lambda_{0, \nu_{1}^{0}}\right]_{\left(m_{0, \nu_{1}^{0}}\right)}, \ldots,\left[\lambda_{0, \nu_{L}^{0}}\right]_{\left(m_{0, \nu_{L}^{0}}\right)} \rightsquigarrow 1:\left[\lambda_{1, \nu_{1}^{1}}\right]_{\left(m_{1, \nu_{1}^{1}}\right)}, \ldots,\left[\lambda_{1, \nu_{L^{\prime}}^{1}}\right]_{\left(m_{1, \nu_{L}^{\prime}}\right)}\right) \\
:=\operatorname{det}\left(c_{\nu, k}^{\nu^{\prime}, k^{\prime}}(\lambda)\right)_{\substack{\nu \in\left\{\nu_{1}^{0}, \ldots, \nu_{L}^{0}\right\} \\
\nu^{\prime} \in\left\{\nu_{1}^{1}, \ldots, \nu_{L^{\prime}}^{\prime}\right\}}, 0 \leq k<m_{0, \nu}} . \\
0 \leq m_{1, \nu^{\prime}}
\end{gathered}
$$

The connection coefficient defined in $\$ 14.1$ corresponds to the case when $n^{\prime}=1$.

Remark 14.18. i) When $m_{0,1}=m_{1,1}$, Corollary 14.15 assures that

$$
\frac{c\left(0:\left[\lambda_{0,1}\right]_{\left(m_{0,1}\right)} \rightsquigarrow 1:\left[\lambda_{1,1}\right]_{\left(m_{1,1}\right)}\right)}{\prod_{\substack{2 \leq j \leq n_{0} \\ 0 \leq k<\min \left\{m_{0,1}, m_{0, j}\right\}}} \Gamma\left(\lambda_{0,1}-\lambda_{0, j}+m_{0,1}-k\right) \cdot \prod_{\substack{2 \leq j \leq n_{1} \\ 0<k \leq \min \left\{m_{1,1}, m_{1, j}\right\}}} \Gamma\left(\lambda_{1, j}-\lambda_{1,1}-m_{1,1}+k\right)}
$$

is holomorphic for $\lambda_{j, \nu} \in \mathbb{C}$.

ii) Let $v_{1}, \ldots, v_{n^{\prime}}$ be generic solutions of $P_{\mathbf{m}} u=0$. Then the generalized connection coefficient in Definition 14.17 corresponds to a usual connection coefficient of the Fuchsian differential equation satisfied by the Wronskian of the $n^{\prime}$ functions $v_{1}, \ldots, v_{n^{\prime}}$. The differential equation is of order $\left(\begin{array}{c}n \\ n^{\prime}\end{array}\right)$. In particular, when $n^{\prime}=n-1$, the differential equation is isomorphic to the dual of the equation $P_{\mathbf{m}}=0$ (cf. Theorem 6.19) and therefore the result in $\$ 14.1$ can be applied to the connection coefficient. The precise result will be explained in another paper.

Remark 14.19. The following procedure has not been completed in general. But we give a procedure to calculate the generalized connection coefficient (14.55), which we put $c(\lambda)$ here for simplicity when $\mathbf{m}$ is rigid.

(1) Let $\bar{\epsilon}=\left(\bar{\epsilon}_{j, \nu}\right)$ be the shift of the Riemann scheme $\left\{\lambda_{\mathbf{m}}\right\}$ such that

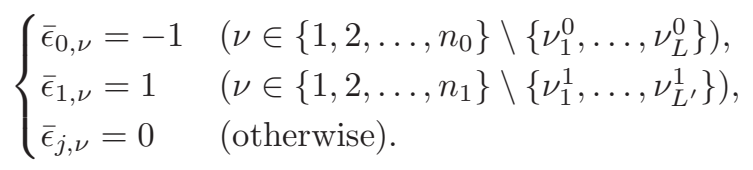

Then for generic $\lambda$ we show that the connection coefficient (14.55) converges to a non-zero meromorphic function $\bar{c}(\lambda)$ of $\lambda$ by the shift $\left\{\lambda_{\mathbf{m}}\right\} \mapsto\{(\lambda+$ $\left.k \bar{\epsilon})_{\mathbf{m}}\right\}$ when $\mathbb{Z}_{>0} \ni k \rightarrow \infty$.

(2) Choose suitable linear functions $b_{i}(\lambda)$ of $\lambda$ by applying Proposition 14.12 or Corollary 14.15 to $c(\lambda)$ so that $e(\lambda):=\prod_{i=1}^{N} \Gamma\left(b_{i}(\lambda)\right)^{-1} \cdot c(\lambda) \bar{c}(\lambda)^{-1}$ is holomorphic for any $\lambda$. 
In particular, when $L=L^{\prime}=1$ and $\nu_{1}^{0}=\nu_{1}^{1}=1$, we may put

$$
\begin{aligned}
\left\{b_{i}\right\}= & \bigcup_{j=2}^{n_{0}}\left\{\lambda_{0,1}-\lambda_{0, j}+m_{0,1}-\nu ; 0 \leq \nu<\min \left\{m_{0,1}, m_{0, j}\right\}\right\} \\
& \cup \bigcup_{j=2}^{n_{1}}\left\{\lambda_{1, j}-\lambda_{1,1}-m_{1,1}+\nu ; 1 \leq \nu \leq \min \left\{m_{1,1}, m_{1, j}\right\}\right\} .
\end{aligned}
$$

(3) Find the zeros of $e(\lambda)$ some of which are explained by the reducibility or the shift operator of the equation $P_{\mathbf{m}} u=0$ and choose linear functions $c_{i}(\lambda)$ of $\lambda$ so that $f(\lambda):=\prod_{i=1}^{N^{\prime}} \Gamma\left(c_{i}(\lambda)\right) \cdot e(\lambda)$ is still holomorphic for any $\lambda$.

(4) If $N=N^{\prime}$ and $\sum_{i} d_{i}(\lambda)=\sum_{i} c_{i}(\lambda)$, Lemma 14.20 assures $f(\lambda)=\bar{c}(\lambda)$ and

$$
c(\lambda)=\frac{\prod_{i=1}^{N} \Gamma\left(b_{i}(\lambda)\right)}{\prod_{i=1}^{N} \Gamma\left(c_{i}(\lambda)\right)} \cdot \bar{c}(\lambda)
$$

because $\frac{f(\lambda)}{f(\lambda+\epsilon)}$ is a rational function of $\lambda$, which follows from the existence of a shift operator assured by Theorem 13.2 .

Lemma 14.20. Let $f(t)$ be a meromorphic function of $t \in \mathbb{C}$ such that $r(t)=$ $\frac{f(t)}{f(t+1)}$ is a rational function and

$$
\lim _{\mathbb{Z}_{>0} \ni k \rightarrow \infty} f(t+k)=1 .
$$

Then there exists $N \in \mathbb{Z}_{\geq 0}$ and $b_{i}, c_{i} \in \mathbb{C}$ for $i=1, \ldots, n$ such that

$$
\begin{aligned}
b_{1}+\cdots+b_{N} & =c_{1}+\cdots+c_{N}, \\
f(t) & =\frac{\prod_{i=1}^{N} \Gamma\left(t+b_{i}\right)}{\prod_{i=1}^{N} \Gamma\left(t+c_{i}\right)} .
\end{aligned}
$$

Moreover, if $f(t)$ is an entire function, then $f(t)$ is the constant function 1.

Proof. Since $\lim _{k \rightarrow \infty} r(t+k)=1$, we may assume

$$
r(t)=\frac{\prod_{i=1}^{N}\left(t+c_{i}\right)}{\prod_{i=1}^{N}\left(t+b_{i}\right)}
$$

and then

$$
f(t)=\frac{\prod_{i=1}^{N} \prod_{\nu=0}^{n-1}\left(t+c_{i}+\nu\right)}{\prod_{i=1}^{N} \prod_{\nu=0}^{n-1}\left(t+b_{i}+\nu\right)} f(t+n) .
$$

Since

$$
\lim _{n \rightarrow \infty} \frac{n ! n^{x-1}}{\prod_{\nu=0}^{n-1}(x+\nu)}=\Gamma(x),
$$

the assumption implies (14.59) and (14.60).

We may assume $b_{i} \neq c_{j}$ for $1 \leq i \leq N$ and $1 \leq j \leq N$. Then the function (14.60) with (14.59) has a pole if $N>0$.

We have the following proposition for zeros of $c(\lambda)$.

Proposition 14.21. Retain the notation in Remark 14.19 and fix $\lambda$ so that

$$
\lambda_{j, \nu}-\lambda_{j, \nu^{\prime}} \notin \mathbb{Z} \quad\left(j=0,1 \text { and } 0 \leq \nu<\nu^{\prime} \leq n_{j}\right) .
$$

i) The relation $c(\lambda)=0$ is valid if and only if there exists a non-zero function

$$
v=\sum_{\substack{\nu \in\left\{\nu_{1}^{0}, \ldots, \nu_{L}^{0}\right\} \\ 0 \leq k<m_{0}, \nu}} C_{\nu, k} u_{0}^{\lambda_{0, \nu}+k}=\sum_{\substack{\nu \in\left\{1, \ldots, n_{1}\right\} \backslash\left\{\nu_{1}^{1}, \ldots, \nu_{L^{\prime}}^{1}\right\} \\ 0 \leq k<m_{1, \nu}}} C_{\nu, k}^{\prime} u_{1}^{\lambda_{1, \nu}+k}
$$

on $(0,1)$ with $C_{\nu, k}, C_{\nu, k}^{\prime} \in \mathbb{C}$. 
ii) Fix a shift $\epsilon=\left(\epsilon_{j, \nu}\right)$ compatible to $\mathbf{m}$ and let $R_{\mathbf{m}}(\epsilon, \lambda)$ be the shift operator in Theorem 13.2. Suppose $R_{\mathbf{m}}(\epsilon, \lambda)$ is bijective, namely, $c_{\mathbf{m}}(\epsilon ; \lambda) \neq 0$ (cf. Theorem 13.8). Then $c(\lambda+\epsilon)=0$ if and only if $c(\lambda)=0$

Proof. Assumption (14.61) implies that $\left\{u_{0}^{\lambda_{0, \nu}+k}\right\}$ and $\left\{u_{1}^{\lambda_{1, \nu}+k}\right\}$ define sets of basis of local solutions of the equation $P_{\mathbf{m}} u=0$. Hence the claim i) is clear from the definition of $c(\lambda)$.

Suppose $c(\lambda)=0$ and $R_{\mathbf{m}}(\epsilon, \lambda)$ is bijective. Then applying the claim i) to $R_{\mathbf{m}}(\epsilon, \lambda) v$, we have $c(\lambda+\epsilon)=0$. If $R_{\mathbf{m}}(\epsilon, \lambda)$ is bijective, so is $R_{\mathbf{m}}(-\epsilon, \lambda+\epsilon)$ and $c(\lambda+\epsilon)=0$ implies $c(\lambda)=0$.

Corollary 14.22. Let $\mathbf{m}=\mathbf{m}^{\prime} \oplus \mathbf{m}^{\prime \prime}$ be a rigid decomposition of $\mathbf{m}$ such that

$$
\sum_{\nu \in\left\{\nu_{1}^{0}, \ldots, \nu_{L}^{0}\right\}} m_{0, \nu}^{\prime}>\sum_{\nu \in\left\{\nu_{1}^{1}, \ldots, \nu_{L^{\prime}}^{1}\right\}} m_{1, \nu}^{\prime}
$$

Then $\Gamma\left(\left|\left\{\lambda_{\mathbf{m}^{\prime}}\right\}\right|\right) \cdot c(\lambda)$ is holomorphic under the condition (14.61).

Proof. When $\left|\left\{\lambda_{\mathbf{m}^{\prime}}\right\}\right|=0$, we have the decomposition $P_{\mathbf{m}}=P_{\mathbf{m}^{\prime \prime}} P_{\mathbf{m}^{\prime}}$ and hence $c(\lambda)=0$. There exists a shift $\epsilon$ compatible to $\mathbf{m}$ such that $\sum_{j=0}^{p} \sum_{\nu=1}^{n_{j}} m_{j, \nu}^{\prime} \epsilon_{j, \nu}=1$. Let $\lambda$ be generic under $\left|\left\{\lambda_{\mathbf{m}}\right\}\right|=0$ and $\left|\left\{\lambda_{\mathbf{m}^{\prime}}\right\}\right| \in \mathbb{Z} \backslash\{0\}$. Then Theorem 13.9) ii) assures $c_{\mathbf{m}}(\epsilon ; \lambda) \neq 0$ and Proposition 14.21 proves the corollary.

Remark 14.23. Suppose that Remark 14.19 (1) is established. Then Proposition 14.12 and Proposition 14.21 with Theorem 13.8 assure that the denominator and the numerator of the rational function which equals $\frac{c(\lambda)}{c(\lambda+\bar{\epsilon})}$ are products of certain linear functions of $\lambda$ and therefore (14.57) is valid with suitable linear functions $b_{i}(\lambda)$ and $c_{i}(\lambda)$ of $\lambda$ satisfying $\sum_{i=1}^{N} b_{i}(\lambda)=\sum_{i=1}^{N} c_{i}(\lambda)$.

Example 14.24 (generalized hypergeometric function). The generalized hypergeometric series (1.7) satisfies the equation $P_{n}(\alpha ; \beta) u=0$ given by (15.21) and $K h$, §4.1.2 Example 9] shows that the equation is isomorphic to the Okubo system

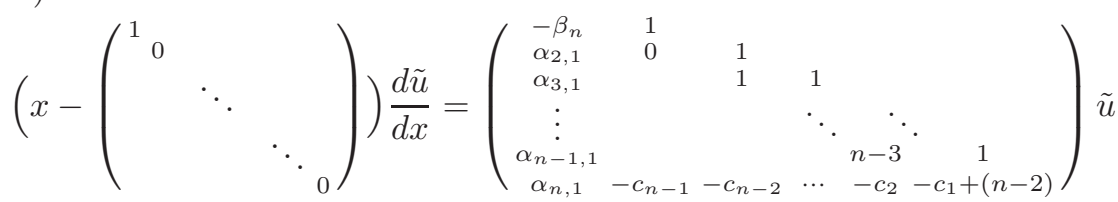

with

$$
u=\left(\begin{array}{c}
u_{1} \\
\vdots \\
u_{n}
\end{array}\right), u=u_{1} \text { and } \sum_{\nu=1}^{n} \alpha_{\nu}=\sum_{\nu=1}^{n} \beta_{\nu} .
$$

Let us calculate the connection coefficient

$c\left(0: 0 \rightsquigarrow 1:-\beta_{n}\right)=\lim _{x \rightarrow 1-0}(1-x)^{\beta_{n}} F_{n-1}\left(\alpha_{1}, \ldots, \alpha_{n} ; \beta_{1}, \ldots, \beta_{n-1} ; x\right) \quad\left(\operatorname{Re} \beta_{n}>0\right)$.

Applying Theorem 14.10 to the system of Schlesinger canonical form satisfied by $\operatorname{Ad}\left((1-x)^{\beta_{n}}\right)$, the connection coefficient satisfies Remark 14.19 i) with $\bar{c}(\lambda)=1$, namely,

$$
\left.\lim _{k \rightarrow+\infty} c\left(0: 0 \rightsquigarrow 1:-\beta_{n}\right)\right|_{\alpha_{j} \mapsto \alpha_{j}+k, \beta_{j} \mapsto \beta_{j}+k} \quad(1 \leq j \leq n)=1 .
$$

Then Remark 14.19 ii) shows that $\prod_{j=1}^{n} \Gamma\left(\beta_{j}\right)^{-1} \cdot c\left(0: 0 \rightsquigarrow 1:-\beta_{n}\right)$ is a holomorphic function of $(\alpha, \beta) \in \mathbb{C}^{n+(n-1)}$. 
Corresponding to the Riemann scheme (1.8), the existence of rigid decompositions

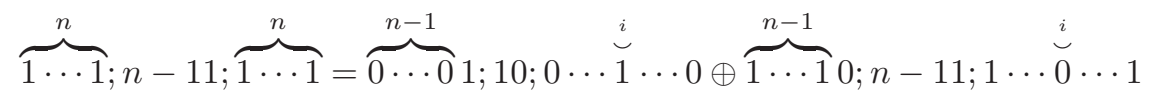

for $i=1, \ldots, n$ proves that $\prod_{i=1}^{n} \Gamma\left(\alpha_{i}\right) \cdot \prod_{j=1}^{n} \Gamma\left(\beta_{j}\right)^{-1} \cdot c\left(0: 0 \rightsquigarrow 1:-\beta_{n}\right)$ is also entire holomorphic. Then the procedure given in Remark 14.19 assures

$$
c\left(0: 0 \rightsquigarrow 1:-\beta_{n}\right)=\frac{\prod_{i=1}^{n} \Gamma\left(\beta_{i}\right)}{\prod_{i=1}^{n} \Gamma\left(\alpha_{i}\right)} .
$$

We can also prove (14.65) as in the following way. Since

$$
\frac{d}{d x} F(\alpha ; \beta ; x)=\frac{\alpha_{1} \cdots \alpha_{n}}{\beta_{1} \cdots \beta_{n-1}} F\left(\alpha_{1}+1, \ldots, \alpha_{n}+1 ; \beta_{1}+1, \ldots, \beta_{n-1}+1 ; x\right)
$$

and

$$
\frac{d}{d x}(1-x)^{-\beta_{n}}\left(1+(1-x) \mathcal{O}_{1}\right)=\beta_{n}(1-x)^{-\beta_{n}-1}\left(1+(1-x) \mathcal{O}_{1}\right),
$$

we have

$$
\frac{c\left(0: 0 \rightsquigarrow 1:-\beta_{n}\right)}{\left.c\left(0: 0 \rightsquigarrow 1:-\beta_{n}\right)\right|_{\alpha_{j} \mapsto \alpha_{j}+1, \beta_{j} \mapsto \beta_{j}+1}}=\frac{\alpha_{1} \ldots \alpha_{n}}{\beta_{1} \ldots \beta_{n}},
$$

which proves (14.65) because of (14.64).

A further study of generalized connection coefficients will be developed in another paper. In this paper we will only give some examples in $\$ 15.5$ and $\$ 15.7 .5$.

\section{EXAMPLES}

When we classify tuples of partitions in this section, we identify the tuples which are isomorphic to each other. For example, 21,111,111 is isomorphic to any one of $12,111,111$ and $111,21,111$ and 21,3,111,111.

Most of our results in this note are constructible and can be implemented in computer programs. Several reductions and constructions and decompositions of tuples of partitions and connections coefficients associated with Riemann schemes etc. can be computed by a program okubo written by the author (cf. \$15.11).

In $\$ 15.1$ and $\$ 15.2$ we list fundamental and rigid tuples respectively, most of which are obtained by the program okubo.

In $\S 15.3$ and $\S 15.4$ we apply our fractional calculus to Jordan-Pochhammer equations and a hypergeometric family (generalized hypergeometric equations), respectively. Most of the results in these sections are known but it will be useful to understand our unifying interpretation and apply it to general Fuchsian equations.

In $\$ 15.5$ we study an even family and an odd family corresponding to Simpson's list [Si]. The differential equations of an even family appear in suitable restrictions of Heckman-Opdam hypergeometric systems and in particular the explicit calculation of a connection coefficient for an even family was the original motivation for the study of Fuchsian differential equations developed in this note (cf. [OS]). We also calculate a generalized connection coefficient for an even family of order 4 .

In $\$ 15.7$, $\$ 15.8$ and $\$ 15.9$ we study the rigid Fuchsian differential equations of order not larger than 4 and those of order 5 or 6 and the equations belonging to 12 maximal series and some minimal series classified by [R0] which include the equations in Yokoyama's list [Y] $]$. We list sufficient data from which we get some connection coefficients and the necessary and sufficient conditions for the irreducibility of the equations as is explained in $\$ 15.9 .2$.

In $\$ 15.6$ we give some interesting identities of trigonometric functions as a consequence of the explicit value of connection coefficients. 
We examine Appell hypergeometric equations in $\$ 15.10$, which will be further discussed in another paper.

In $\$ 15.11$ we explain computer programs okubo and a library of risa/asir which calculate the results described in this paper.

15.1. Basic tuples. The number of basic tuples and fundamental tuples (cf. Definition 8.14 with a given Pidx are as follows.

\begin{tabular}{|c|r|r|r|r|r|r|r|r|r|r|r|r|}
\hline Pidx & 0 & 1 & 2 & 3 & 4 & 5 & 6 & 7 & 8 & 9 & 10 & 11 \\
\hline \hline \# fund. tuples & 1 & 4 & 13 & 36 & 67 & 103 & 162 & 243 & 305 & 456 & 578 & 720 \\
\hline \# basic tuples & 0 & 4 & 13 & 36 & 67 & 90 & 162 & 243 & 305 & 420 & 565 & 720 \\
\hline \# basic triplets & 0 & 3 & 9 & 24 & 44 & 56 & 97 & 144 & 163 & 223 & 291 & 342 \\
\hline \# basic 4-tuples & 0 & 1 & 3 & 9 & 17 & 24 & 45 & 68 & 95 & 128 & 169 & 239 \\
\hline maximal order & 6 & 12 & 18 & 24 & 30 & 36 & 42 & 48 & 54 & 60 & 66 & 72 \\
\hline
\end{tabular}

Note that if $\mathbf{m}$ is a basic tuple with $\operatorname{idx} \mathbf{m}<0$, then

$$
\operatorname{Pidx} k \mathbf{m}=1+k^{2}(\operatorname{Pidx} \mathbf{m}-1) \quad(k=1,2, \ldots) .
$$

Hence the non-trivial fundamental tuple $\mathbf{m}$ with $\operatorname{Pidx} \mathbf{m} \leq 4$ or equivalently idx $\mathbf{m} \geq$ -6 is always basic.

The tuple $2 \mathbf{m}$ with a basic tuple $\mathbf{m}$ satisfying Pidx $\mathbf{m}=2$ is a fundamental tuple and Pidx $2 \mathbf{m}=5$. The tuple 422, 44, 44, 44 is this example.

15.1.1. Pidx $\mathbf{m}=1$. There exist 4 basic tuples: (cf. Ko3], Corollary 8.3)

$$
\tilde{D}_{4}: 11,11,11,11 \quad \tilde{E}_{6}: 111,111,111 \quad \tilde{E}_{7}: 22,1111,1111 \quad \tilde{E}_{8}: 33,222,111111
$$

They are not of Okubo type. The tuples of partitions of Okubo type with minimal order which are reduced to the above basic tuples are as follows.

$$
\tilde{D}_{4}: 21,21,21,111 \quad \tilde{E}_{6}: 211,211,1111 \quad \tilde{E}_{7}: 32,2111,11111 \quad \tilde{E}_{8}: 43,322,1111111
$$

The list of simply reducible tuples of partitions whose indices of rigidity equal 0 is given in Example 8.17 .

We list the number of realizable tuples of partitions whose indices of rigidity equal 0 according to their orders and the corresponding fundamental tuple.

\begin{tabular}{|r|r|r|r|r|r|}
\hline ord & $11,11,11,11$ & $111,111,111$ & $22,1111,1111$ & $33,222,111111$ & total \\
\hline \hline 2 & 1 & & & & 1 \\
\hline 3 & 1 & 1 & & & 2 \\
\hline 4 & 4 & 1 & 1 & & 6 \\
\hline 5 & 6 & 3 & 1 & & 10 \\
\hline 6 & 21 & 8 & 5 & 1 & 35 \\
\hline 7 & 28 & 15 & 6 & 1 & 50 \\
\hline 8 & 74 & 31 & 21 & 4 & 130 \\
\hline 9 & 107 & 65 & 26 & 5 & 203 \\
\hline 10 & 223 & 113 & 69 & 12 & 417 \\
\hline 11 & 315 & 204 & 90 & 14 & 623 \\
\hline 12 & 616 & 361 & 205 & 37 & 1219 \\
\hline 13 & 808 & 588 & 256 & 36 & 1688 \\
\hline 14 & 1432 & 948 & 517 & 80 & 2977 \\
\hline 15 & 1951 & 1508 & 659 & 100 & 4218 \\
\hline 16 & 3148 & 2324 & 1214 & 179 & 6865 \\
\hline 17 & 4064 & 3482 & 1531 & 194 & 9271 \\
\hline 18 & 6425 & 5205 & 2641 & 389 & 14660 \\
\hline 19 & 8067 & 7503 & 3246 & 395 & 19211 \\
\hline 20 & 12233 & 10794 & 5400 & 715 & 29142 \\
\hline
\end{tabular}


15.1.2. Pidx $\mathbf{m}=2$. There are 13 basic tuples (cf. Proposition 8.10, [06, Proposition 8.4]):

$\begin{array}{cll}+2: 11,11,11,11,11 & 3: 111,111,21,21 & * 4: 211,22,22,22 \\ 4: 1111,22,22,31 & 4: 1111,1111,211 & 5: 11111,11111,32 \\ 5: 11111,221,221 & 6: 111111,2211,33 & * 6: 2211,222,222 \\ * 8: 22211,2222,44 & 8: 11111111,332,44 & 10: 22222,3331,55 \\ * 12: 2222211,444,66 & & \end{array}$

Here the number preceding to a tuple is the order of the tuple and the sign $*$ means that the tuple is the one given in Example $9.51\left(D_{4}^{(m)}, E_{6}^{(m)}, E_{7}^{(m)}\right.$ and $\left.E_{8}^{(m)}\right)$ and the sign + means $d(\mathbf{m})<0$.

15.1.3. Pidx $\mathbf{m}=3$. There are 36 basic tuples

$\begin{array}{ccc}+2: 11,11,11,11,11,11 & 3: 111,21,21,21,21 & 4: 22,22,22,31,31 \\ +3: 111,111,111,21 & +4: 1111,22,22,22 & 4: 1111,1111,31,31 \\ 4: 211,211,22,22 & 4: 1111,211,22,31 & * 6: 321,33,33,33 \\ 6: 222,222,33,51 & +4: 1111,1111,1111 & 5: 11111,11111,311 \\ 5: 11111,2111,221 & 6: 111111,222,321 & 6: 111111,21111,33 \\ 6: 21111,222,222 & 6: 111111,111111,42 & 6: 222,33,33,42 \\ 6: 111111,33,33,51 & 6: 2211,2211,222 & 7: 1111111,2221,43 \\ 7: 1111111,331,331 & 7: 2221,2221,331 & 8: 11111111,3311,44 \\ 8: 221111,2222,44 & 8: 22211,22211,44 & * 9: 3321,333,333 \\ 9: 111111111,333,54 & 9: 22221,333,441 & 10: 1111111111,442,55 \\ 10: 22222,3322,55 & 10: 222211,3331,55 & 12: 22221111,444,66 \\ * 12: 33321,3333,66 & 14: 2222222,554,77 & * 18: 3333321,666,99\end{array}$

15.1.4. Pidx $\mathbf{m}=4$. There are 67 basic tuples

$\begin{array}{ccc}+2: 11,11,11,11,11,11,11 & 3: 21,21,21,21,21,21 & +3: 111,111,21,21,21 \\ +4: 22,22,22,22,31 & 4: 211,22,22,31,31 & 4: 1111,22,31,31,31 \\ +3: 111,111,111,111 & +4: 1111,1111,22,31 & 4: 1111,211,22,22 \\ 4: 211,211,211,22 & 4: 1111,211,211,31 & 5: 11111,11111,41,41 \\ 5: 11111,221,32,41 & 5: 221,221,221,41 & 5: 11111,32,32,32 \\ 5: 221,221,32,32 & 6: 3111,33,33,33 & 6: 2211,2211,2211 \\ +6: 222,33,33,33 & 6: 222,33,33,411 & 6: 2211,222,33,51 \\ * 8: 431,44,44,44 & 8: 11111111,44,44,71 & 5: 11111,11111,221 \\ 5: 11111,2111,2111 & +6: 111111,111111,33 & +6: 111111,222,222 \\ 6: 111111,111111,411 & 6: 111111,222,3111 & 6: 21111,2211,222 \\ 6: 111111,2211,321 & 6: 2211,33,33,42 & 7: 1111111,1111111,52 \\ 7: 1111111,322,331 & 7: 2221,2221,322 & 7: 1111111,22111,43 \\ 7: 22111,2221,331 & 8: 11111111,3221,44 & 8: 11111111,2222,53 \\ 8: 2222,2222,431 & 8: 2111111,2222,44 & 8: 221111,22211,44 \\ 9: 33111,333,333 & 9: 3222,333,333 & 9: 22221,22221,54 \\ 9: 222111,333,441 & 9: 111111111,441,441 & 10: 22222,33211,55 \\ 10: 1111111111,433,55 & 10: 1111111111,4411,55 & 10: 2221111,3331,55 \\ 10: 222211,3322,55 & 12: 222111111,444,66 & 12: 333111,3333,66 \\ 12: 33222,3333,66 & 12: 222222,4431,66 & * 12: 4431,444,444 \\ 12: 111111111111,552,66 & 12: 3333,444,552 & 14: 33332,4442,77 \\ 14: 22222211,554,77 & 15: 33333,555,771 & * 16: 44431,4444,88 \\ 16: 333331,5551,88 & 18: 33333111,666,99 & 18: 3333222,666,99 \\ * 24: 4444431,888, c c & & \end{array}$

$* 24: 4444431,888, \mathrm{cc}$

Here a, b, c, . . represent $10,11,12, \ldots$, respectively.

15.1.5. Dynkin diagrams of basic tuples whose indices of rigidity equals -2 . We express the basic root $\alpha_{\mathbf{m}}$ for Pidx $\mathbf{m}=2$ using the Dynkin diagram (See (9.11) for $\operatorname{Pidx} \mathbf{m}=1)$. The circles in the diagram represent the simple roots in $\operatorname{supp} \alpha_{\mathbf{m}}$ and 
two circles are connected by a line if the inner product of the corresponding simple roots is not zero. The number attached to a circle is the corresponding coefficient $n$ or $n_{j, \nu}$ in the expression (9.12).

For example, if $\mathbf{m}=22,22,22,211$, then $\alpha_{\mathbf{m}}=4 \alpha_{0}+2 \alpha_{0,1}+2 \alpha_{1,1}+2 \alpha_{2,1}+$ $2 \alpha_{3,1}+\alpha_{3,2}$, which corresponds to the second diagram in the following.

The circle with a dot at the center means a simple root whose inner product with $\alpha_{\mathbf{m}}$ does not vanish. Moreover the type of the root system $\Pi(\mathbf{m})$ (cf. (9.47)) corresponding to the simple roots without a dot is given.

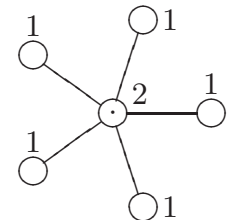

$11,11,11,11,11 \quad 5 A_{1}$

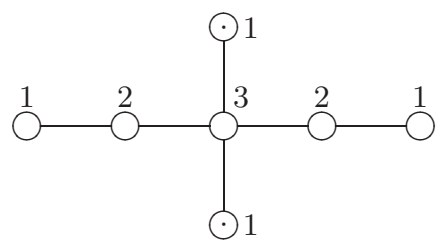

21, 21, 111, $111 \quad A_{5}$

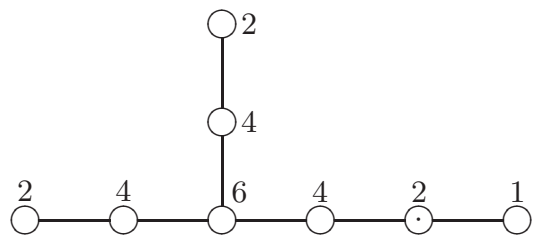

$222,222,2211 \quad E_{6}+A_{1}$
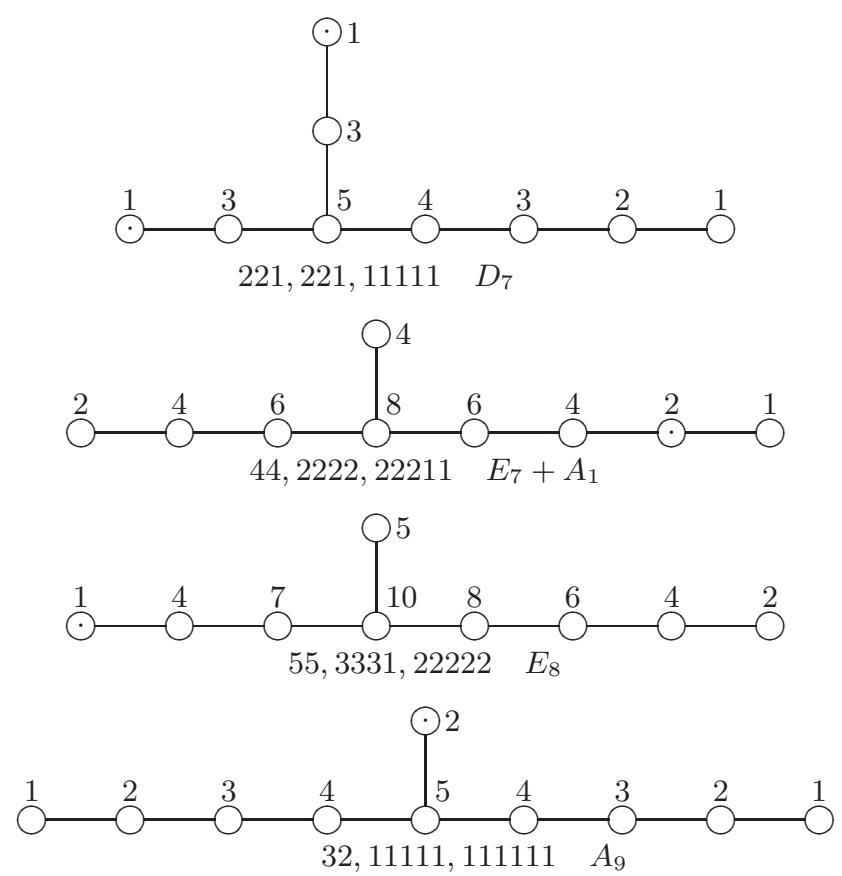

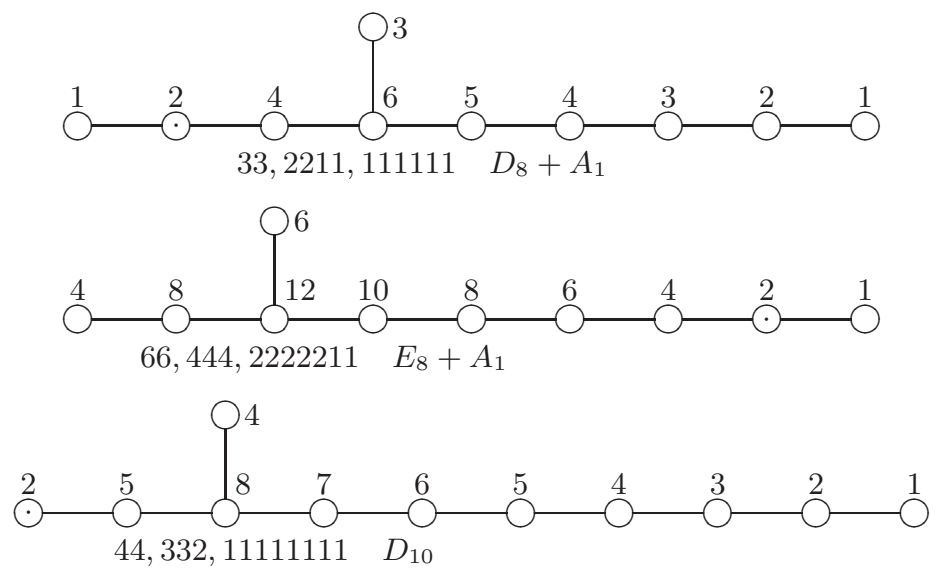

\subsection{Rigid tuples.}

15.2.1. Simpson's list. Simpson Si] classified the rigid tuples containing the partition $11 \cdots 1$ into 4 types (Simpson's list), which follows from Proposition 8.16 . They are $H_{n}, E O_{2 m}, E O_{2 m+1}$ and $X_{6}$ in the following table.

See Remark 9.11 ii) for $[\Delta(\mathbf{m})]$ with these rigid tuples $\mathbf{m}$.

The simply reducible rigid tuple (cf. 88.5 ) which is not in Simpson's list is isomorphic to $21111,222,33$.

\begin{tabular}{|c|c|c|c|}
\hline order & type & name & partitions \\
\hline \hline$n$ & $H_{n}$ & hypergeometric family & $1^{n}, 1^{n}, n-11$ \\
\hline $2 m$ & $E O_{2 m}$ & even family & $1^{2 m}, m m-11, m m$ \\
\hline $2 m+1$ & $E O_{2 m+1}$ & odd family & $1^{2 m+1}, m m 1, m+1 m$ \\
\hline 6 & $X_{6}=\gamma_{6,2}$ & extra case & $111111,222,42$ \\
\hline 6 & $\gamma_{6,6}$ & & $21111,222,33$ \\
\hline$n$ & $P_{n}$ & Jordan Pochhammer & $n-11, n-11, \ldots \in \mathcal{P}_{n+1}^{(n)}$ \\
\hline
\end{tabular}

15.2.2. Isomorphic classes of rigid tuples. Let $\mathcal{R}_{p+1}^{(n)}$ be the set of rigid tuples in $\mathcal{P}_{p+1}^{(n)}$. Put $\mathcal{R}_{p+1}=\bigcup_{n=1}^{\infty} \mathcal{R}_{p+1}^{(n)}, \mathcal{R}^{(n)}=\bigcup_{p=2}^{\infty} \mathcal{R}_{p+1}^{(n)}$ and $\mathcal{R}=\bigcup_{n=1}^{\infty} \mathcal{R}^{(n)}$. The sets of isomorphic classes of the elements of $\mathcal{R}_{p+1}^{(n)}$ (resp. $\mathcal{R}_{p+1}, \mathcal{R}^{(n)}$ and $\mathcal{R}$ ) are denoted $\overline{\mathcal{R}}_{p+1}^{(n)}$ (resp. $\overline{\mathcal{R}}_{p+1}, \overline{\mathcal{R}}^{(n)}$ and $\overline{\mathcal{R}}$ ). Then the number of the elements of $\overline{\mathcal{R}}^{(n)}$ are as follows.

\begin{tabular}{|r|r|r||r|r|r||r|r|r|}
\hline$n$ & $\# \overline{\mathcal{R}}_{3}^{(n)}$ & $\# \overline{\mathcal{R}}^{(n)}$ & $n$ & $\# \overline{\mathcal{R}}_{3}^{(n)}$ & $\# \overline{\mathcal{R}}^{(n)}$ & $n$ & $\# \overline{\mathcal{R}}_{3}^{(n)}$ & $\# \overline{\mathcal{R}}^{(n)}$ \\
\hline 2 & 1 & 1 & 15 & 1481 & 2841 & 28 & 114600 & 190465 \\
\hline 3 & 1 & 2 & 16 & 2388 & 4644 & 29 & 143075 & 230110 \\
\hline 4 & 3 & 6 & 17 & 3276 & 6128 & 30 & 190766 & 310804 \\
\hline 5 & 5 & 11 & 18 & 5186 & 9790 & 31 & 235543 & 371773 \\
\hline 6 & 13 & 28 & 19 & 6954 & 12595 & 32 & 309156 & 493620 \\
\hline 7 & 20 & 44 & 20 & 10517 & 19269 & 33 & 378063 & 588359 \\
\hline 8 & 45 & 96 & 21 & 14040 & 24748 & 34 & 487081 & 763126 \\
\hline 9 & 74 & 157 & 22 & 20210 & 36078 & 35 & 591733 & 903597 \\
\hline 10 & 142 & 306 & 23 & 26432 & 45391 & 36 & 756752 & 1170966 \\
\hline 11 & 212 & 441 & 24 & 37815 & 65814 & 37 & 907150 & 1365027 \\
\hline 12 & 421 & 857 & 25 & 48103 & 80690 & 38 & 1143180 & 1734857 \\
\hline 13 & 588 & 1177 & 26 & 66409 & 112636 & 39 & 1365511 & 2031018 \\
\hline 14 & 1004 & 2032 & 27 & 84644 & 139350 & 40 & 1704287 & 2554015 \\
\hline
\end{tabular}


15.2.3. Rigid tuples of order at most 8. We show all the rigid tuples whose orders are not larger than 8 .

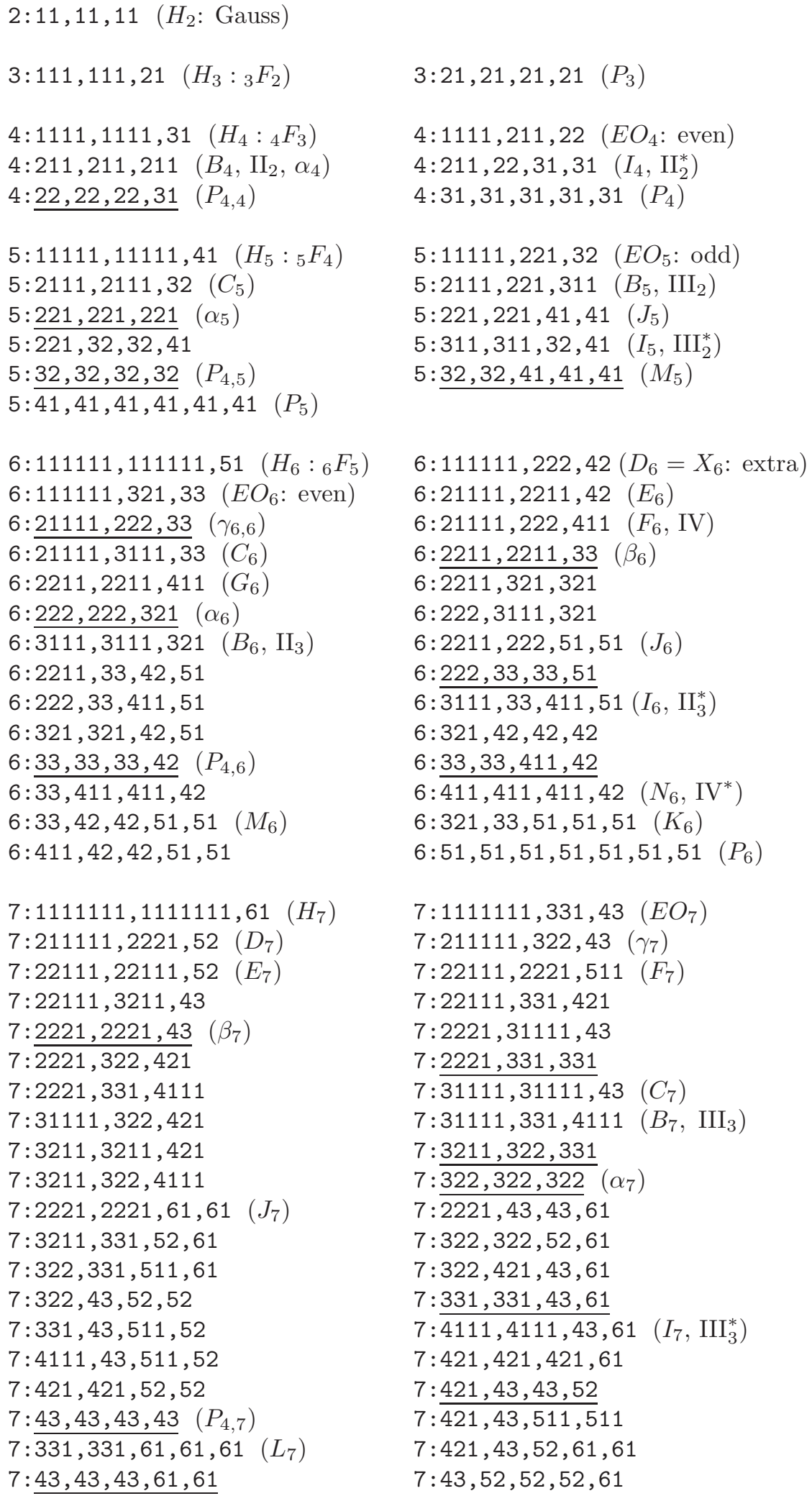


TOSHIO OSHIMA

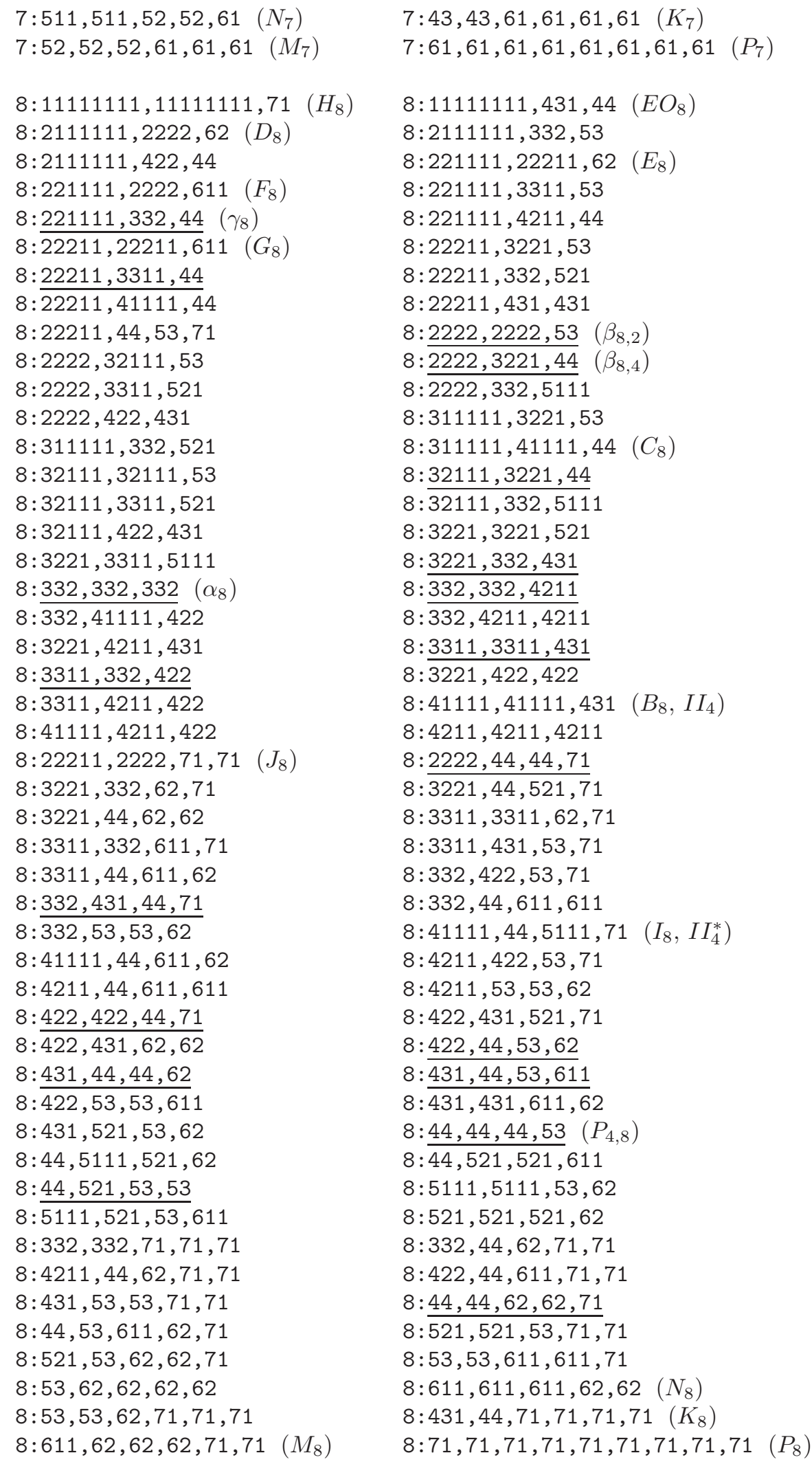

Here the underlined tuples are not of Okubo type (cf. (13.33)). 
The tuples $H_{n}, E O_{n}$ and $X_{6}$ are tuples in Simpson's list. The series $A_{n}=E O_{n}$, $B_{n}, C_{n}, D_{n}, E_{n}, F_{n}, G_{2 m}, I_{n}, J_{n}, K_{n}, L_{2 m+1}, M_{n}$ and $N_{n}$ are given in Ro and called submaximal series. The Jordan-Pochhammer tuples are denoted by $P_{n}$ and the series $H_{n}$ and $P_{n}$ are called maximal series by Ro. The series $\alpha_{n}, \beta_{n}, \gamma_{n}$ and $\delta_{n}$ are given in $[\mathrm{Ro}$ and called minimal series. See $\$ 15.9$ for these series introduced by Ro. Then $\delta_{n}=P_{4, n}$ and they are generalized Jordan-Pochhammer tuples (cf. Example 12.5 and $\$ 15.9 .13)$. Moreover $\mathrm{II}_{n}, \mathrm{II}_{n}^{*}, \mathrm{III}_{n}, \mathrm{III}_{n}^{*}, \mathrm{IV}$ and $\mathrm{IV}^{*}$ are in Yokoyama's list in $\mid \mathrm{YO}$ (cf. \$15.9.15).

Hierarchy of rigid triplets

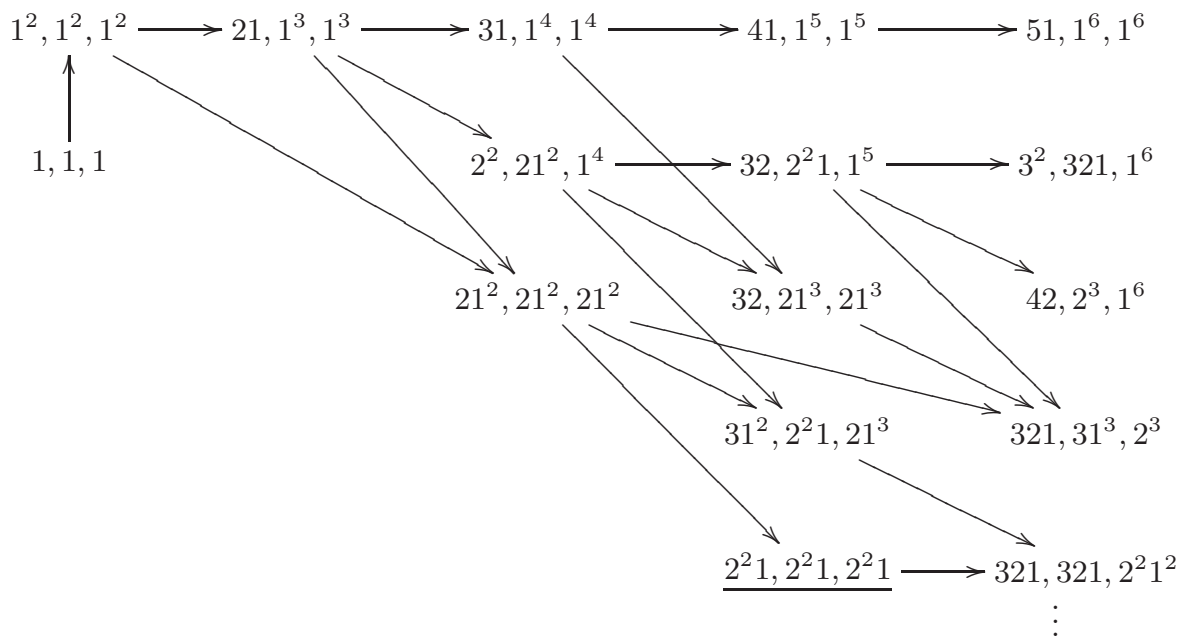

Here the arrows represent certain operations $\partial_{\ell}$ of tuples given by Definition 7.6.

15.3. Jordan-Pochhammer family. $P_{n}$

We have studied the the Riemann scheme of this family in Example 2.8 iii).

$\mathbf{m}=(p-11, p-11, \ldots, p-11) \in \mathcal{P}_{p+1}^{(p)}$

$$
\begin{aligned}
& \left\{\begin{array}{ccccc}
x=0 & 1=\frac{1}{c_{1}} & \cdots & \frac{1}{c_{p-1}} & \infty \\
{[0]_{(p-1)}} & {[0]_{(p-1)}} & \cdots & {[0]_{(p-1)}} & {[1-\mu]_{(p-1)}} \\
\lambda_{0}+\mu & \lambda_{1}+\mu & \cdots & \lambda_{p-1}+\mu & -\lambda_{0}-\cdots-\lambda_{p-1}-\mu
\end{array}\right\} \\
\Delta(\mathbf{m})= & \left\{\alpha_{0}, \alpha_{0}+\alpha_{j, 1} ; j=0, \ldots, p\right\} \\
{[\Delta(\mathbf{m})]=} & 1^{p+1} \cdot(p-1) \\
P_{p} & =H_{1} \oplus P_{p-1}: p+1=(p-1) H_{1} \oplus H_{1}: 1
\end{aligned}
$$

Here the number of the decompositions of a given type is shown after the decompositions. For example, $P_{p}=H_{1} \oplus P_{p-1}: p+1=(p-1) H_{1} \oplus H_{1}: 1$ represents the decompositions

$$
\begin{aligned}
\stackrel{\nu}{\nu} & \stackrel{\nu}{\mathrm{m}} \\
& =10, \ldots, 01, \ldots, 10 \oplus p-21, \ldots, p-10, \ldots, p-21 \quad(\nu=0, \ldots, p) \\
& =(p-1)(10, \ldots, 10) \oplus 01, \ldots, 01 .
\end{aligned}
$$

The differential equation $P_{P_{p}}(\lambda, \mu) u=0$ with this Riemann scheme is given by

$$
P_{P_{p}}(\lambda, \mu):=\operatorname{RAd}\left(\partial^{-\mu}\right) \circ \operatorname{RAd}\left(x^{\lambda_{0}} \prod_{j=1}^{p-1}\left(1-c_{j} x\right)^{\lambda_{j}}\right) \partial
$$


and then

$$
\begin{aligned}
P_{P_{p}}(\lambda, \mu) & =\sum_{k=0}^{p} p_{k}(x) \partial^{p-k} \\
p_{k}(x) & :=\left(\begin{array}{c}
-\mu+p-1 \\
k
\end{array}\right) p_{0}^{(k)}(x)+\left(\begin{array}{c}
-\mu+p-1 \\
k-1
\end{array}\right) q^{(k-1)}(x)
\end{aligned}
$$

with

$$
p_{0}(x)=x \prod_{j=1}^{p-1}\left(1-c_{j} x\right), \quad q(x)=p_{0}(x)\left(-\frac{\lambda_{0}}{x}+\sum_{j=1}^{p-1} \frac{c_{j} \lambda_{j}}{1-c_{j} x}\right) .
$$

It follows from Theorem 12.10 that the equation is irreducible if and only if

$$
\lambda_{j} \notin \mathbb{Z}(j=0, \ldots, p-1), \mu \notin \mathbb{Z} \text { and } \lambda_{0}+\cdots+\lambda_{p-1}+\mu \notin \mathbb{Z} .
$$

It follows from Proposition 13.13 that the shift operator defined by the map $u \mapsto \partial u$ is bijective if and only if

$$
\mu \notin\{1,2, \ldots, p-1\} \text { and } \lambda_{0}+\cdots+\lambda_{p-1}+\mu \neq 0 .
$$

The normalized solution at 0 corresponding to the exponent $\lambda_{0}+\mu$ is

$$
\begin{aligned}
& u_{0}^{\lambda_{0}+\mu}(x)=\frac{\Gamma\left(\lambda_{0}+\mu+1\right)}{\Gamma\left(\lambda_{0}+1\right) \Gamma(\mu)} \int_{0}^{x}\left(t^{\lambda_{0}} \prod_{j=1}^{p-1}\left(1-c_{j} t\right)^{\lambda_{j}}\right)(x-t)^{\mu-1} d t \\
& =\frac{\Gamma\left(\lambda_{0}+\mu+1\right)}{\Gamma\left(\lambda_{0}+1\right) \Gamma(\mu)} \int_{0}^{x} \sum_{m_{1}=0}^{\infty} \cdots \sum_{m_{p-1}=0}^{\infty} \frac{\left(-\lambda_{1}\right)_{m_{1}} \cdots\left(-\lambda_{p-1}\right)_{m_{p-1}}}{m_{1} ! \cdots m_{p-1} !} \\
& c_{2}^{m_{2}} \cdots c_{p-1}^{m_{p-1}} t^{\lambda_{0}+m_{1}+\cdots+m_{p-1}}(x-t)^{\mu-1} d t \\
& =\sum_{m_{1}=0}^{\infty} \cdots \sum_{m_{p-1}=0}^{\infty} \frac{\left(\lambda_{0}+1\right)_{m_{1}+\cdots+m_{p-1}}\left(-\lambda_{1}\right)_{m_{1}} \cdots\left(-\lambda_{p-1}\right)_{m_{p-1}}}{\left(\lambda_{0}+\mu+1\right)_{m_{1}+\cdots+m_{p-1}} m_{1} ! \cdots m_{p-1} !} \\
& c_{2}^{m_{2}} \cdots c_{p-1}^{m_{p-1}} x^{\lambda_{0}+\mu+m_{1}+\cdots+m_{p-1}} \\
& =x^{\lambda_{0}+\mu}\left(1-\frac{\left(\lambda_{0}+1\right)\left(\lambda_{1} c_{1}+\cdots+\lambda_{p-1} c_{p-1}\right)}{\lambda_{0}+\mu+1} x+\cdots\right) .
\end{aligned}
$$

This series expansion of the solution is easily obtained from the formula in 8 田 (cf. Theorem 10.1) and Theorem 13.3 gives the recurrence relation

$$
u_{0}^{\lambda_{0}+\mu}(x)=\left.u_{0}^{\lambda_{0}+\mu}(x)\right|_{\lambda_{1} \mapsto \lambda_{1}-1}-\left.\left(\frac{\lambda_{0}}{\lambda_{0}+\mu} u_{0}^{\lambda_{0}+\mu}(x)\right)\right|_{\substack{\lambda_{0} \mapsto \lambda_{0}+1 \\ \lambda_{1} \mapsto \lambda_{1}-1}} .
$$

Lemma 14.2 with $a=\lambda_{0}, b=\lambda_{1}$ and $u(x)=\prod_{j=2}^{p-1}\left(1-c_{j} x\right)^{\lambda_{j}}$ gives the following connection coefficients

$$
\begin{aligned}
c\left(0: \lambda_{0}+\mu \rightsquigarrow 1: \lambda_{1}+\mu\right)=\frac{\Gamma\left(\lambda_{0}+\mu+1\right) \Gamma\left(-\lambda_{1}-\mu\right)}{\Gamma\left(\lambda_{0}+1\right) \Gamma\left(-\lambda_{1}\right)} \prod_{j=2}^{p-1}\left(1-c_{j}\right)^{\lambda_{j}}, \\
c\left(0: \lambda_{0}+\mu \rightsquigarrow 1: 0\right)=\frac{\Gamma\left(\lambda_{0}+\mu+1\right)}{\Gamma(\mu) \Gamma\left(\lambda_{0}+1\right)} \int_{0}^{1} t^{\lambda_{0}}(1-t)^{\lambda_{1}+\mu-1} \prod_{j=2}^{p-1}\left(1-c_{j} t\right)^{\lambda_{j}} d t \\
=\frac{\Gamma\left(\lambda_{0}+\mu+1\right) \Gamma\left(\lambda_{1}+\mu\right)}{\Gamma(\mu) \Gamma\left(\lambda_{0}+\lambda_{1}+\mu+1\right)} F\left(\lambda_{0}+1,-\lambda_{2}, \lambda_{0}+\lambda_{1}+\mu+1 ; c_{2}\right) \quad(p=3) .
\end{aligned}
$$

Here we have

$$
u_{0}^{\lambda_{0}+\mu}(x)=\sum_{k=0}^{\infty} C_{k}(x-1)^{k}+\sum_{k=0}^{\infty} C_{k}^{\prime}(x-1)^{\lambda_{1}+\mu+k}
$$


for $0<x<1$ with $C_{0}=c\left(0: \lambda_{0}+\mu \rightsquigarrow 1: 0\right)$ and $C_{0}^{\prime}=c\left(0: \lambda_{0}+\mu \rightsquigarrow 1: \lambda_{1}+\mu\right)$. Since $\frac{d^{k} u_{0}^{\lambda_{0}+\mu}}{d x^{k}}$ is a solution of the equation $P_{P_{p}}(\lambda, \mu-k) u=0$, we have

$$
C_{k}=\frac{\Gamma\left(\lambda_{0}+\mu+1\right)}{\Gamma(\mu-k) \Gamma\left(\lambda_{0}+1\right) k !} \int_{0}^{1} t^{\lambda_{0}}(1-t)^{\lambda_{1}+\mu-k-1} \prod_{j=2}^{p-1}\left(1-c_{j} t\right)^{\lambda_{j}} d t .
$$

When $p=3$,

$$
C_{k}=\frac{\Gamma\left(\lambda_{0}+\mu+1\right) \Gamma\left(\lambda_{1}+\mu-k\right)}{\Gamma(\mu-k) \Gamma\left(\lambda_{0}+\lambda_{1}+\mu+1-k\right) k !} F\left(\lambda_{0}+1,-\lambda_{2}, \lambda_{0}+\lambda_{1}+\mu+1-k ; c_{2}\right) .
$$

Put

$$
\begin{aligned}
u_{\lambda, \mu}(x) & =\frac{1}{\Gamma(\mu)} \int_{0}^{x}\left(t^{\lambda_{0}} \prod_{j=1}^{p-1}\left(1-c_{j} t\right)^{\lambda_{j}}\right)(x-t)^{\mu-1} d t=\partial^{-\mu} v_{\lambda}, \\
v_{\lambda}(x): & =x^{\lambda_{0}} \prod_{j=1}^{p-1}\left(1-c_{j} x\right)^{\lambda_{j}} .
\end{aligned}
$$

We have

$$
\begin{aligned}
u_{\lambda, \mu+1} & =\partial^{-\mu-1} v_{\lambda}=\partial^{-1} \partial^{-\mu} v_{\lambda}=\partial^{-1} u_{\lambda, \mu}, \\
u_{\lambda_{0}+1, \lambda_{1}, \ldots, \mu} & =\partial^{-\mu} v_{\lambda_{0}+1, \lambda_{1}, \ldots}=\partial^{-\mu} x v_{\lambda}=-\mu \partial^{-\mu-1} v_{\lambda}+x \partial^{-\mu} v_{\lambda} \\
& =-\mu \partial^{-1} u_{\lambda, \mu}+x u_{\lambda, \mu}, \\
u_{\ldots, \lambda_{j}+1, \ldots} & =\partial^{-\mu}\left(1-c_{j} x\right) v_{\lambda}=\partial^{-\mu} v_{\lambda}+c_{j} \mu \partial^{-\mu-1} v_{\lambda}-c_{j} x \partial^{-\mu} v_{\lambda} \\
& =\left(1-c_{j} x\right) u_{\lambda, \mu}+c_{j} \mu \partial^{-1} u_{\lambda, \mu} .
\end{aligned}
$$

From these relations with $P_{P_{p}} u_{\lambda, \mu}=0$ we have all the contiguity relations. For example

$$
\begin{aligned}
\partial u_{\lambda_{0}, \ldots, \lambda_{p-1}, \mu+1} & =u_{\lambda, \mu}, \\
\partial u_{\lambda_{0}+1, \ldots, \lambda_{p-1}, \mu} & =(x \partial+1-\mu) u_{\lambda, \mu}, \\
\partial u_{\ldots, \lambda_{j}+1, \ldots, \mu} & =\left(\left(1-c_{j} x\right) \partial-c_{j}(1-\mu)\right) u_{\lambda, \mu}
\end{aligned}
$$

and

$$
\begin{aligned}
P_{P_{p}}(\lambda, \mu+1) & =\sum_{j=0}^{p-1} p_{j}(x) \partial^{p-j}+p_{n} \\
p_{n} & =(-1)^{p-1} c_{1} \ldots c_{p-1}\left((-\mu-1)_{p}+(-\mu)_{p-1} \sum_{j=0}^{p-1} \lambda_{j}\right) \\
& =c_{1} \cdots c_{p-1}(\mu+2-p)_{p-1}\left(\lambda_{0}+\cdots+\lambda_{p-1}-\mu-1\right)
\end{aligned}
$$

and hence

$$
\left(\sum_{j=0}^{p-1} p_{j}(x) \partial^{p-j-1}\right) u_{\lambda, \mu}=-p_{n} u_{\lambda, \mu+1}=-p_{n} \partial^{-1} u_{\lambda, \mu} .
$$

Substituting this equation to (15.9), we have $Q_{j} \in W(x ; \lambda, \mu)$ such that $Q_{j} u_{\lambda, \mu}$ equals $u_{\left(\lambda_{\nu}+\delta_{\nu, j}\right)_{\nu=0, \ldots, p-1, \mu}}$ for $j=0, \ldots, p-1$, respectively. The operators $R_{j} \in$ $W(x ; \lambda, \mu)$ satisfying $R_{j} Q_{j} u_{\lambda, \mu}=u_{\lambda, \mu}$ are calculated by the Euclid algorithm, namely, we find $S_{j} \in W(x ; \lambda, \mu)$ so that $R_{j} Q_{j}+S_{j} P_{P_{p}}=1$. Thus we also have $T_{j} \in W(x ; \lambda, \mu)$ such that $T_{j} u_{\lambda, \mu}$ equals $u_{\left(\lambda_{\nu}-\delta_{\nu, j}\right)_{\nu=0, \ldots, p-1}, \mu}$ for $j=0, \ldots, p-1$, respectively. 
As is shown in $\$ 3.4$ the Versal Jordan-Pochhammer operator $\tilde{P}_{P_{p}}$ is given by (15.2) with

$$
p_{0}(x)=\prod_{j=1}^{p}\left(1-c_{j} x\right), \quad q(x)=\sum_{k=1}^{p} \lambda_{k} x^{k-1} \prod_{j=k+1}^{p}\left(1-c_{j} x\right) .
$$

If $c_{1}, \ldots, c_{p}$ are different to each other, the Riemann scheme of $\tilde{P}_{P_{p}}$ is

$$
\left\{\begin{array}{cc}
x=\frac{1}{c_{j}}(j=1, \ldots, p) & \infty \\
\sum_{k=j}^{p} \frac{[0]_{(p-1)}}{\lambda_{k} \prod_{\substack{1 \leq \nu \leq k \\
\nu \neq j}}\left(c_{j}-c_{\nu}\right)}+\mu & \sum_{k=1}^{p} \frac{(-1)^{k} \lambda_{k}}{c_{1} \ldots c_{k}}-\mu
\end{array}\right\} .
$$

The solution of $\tilde{P}_{P_{p}} u=0$ is given by

$$
u_{C}(x)=\int_{C}\left(\exp \int_{0}^{t} \sum_{j=1}^{p} \frac{-\lambda_{j} s^{j-1}}{\prod_{1 \leq \nu \leq j}\left(1-c_{\nu} s\right)} d s\right)(x-t)^{\mu-1} d t .
$$

Here the path $C$ starting from a singular point and ending at a singular point is chosen so that the integration has a meaning. In particular when $c_{1}=\cdots=c_{p}=0$, we have

$$
u_{C}(x)=\int_{C} \exp \left(-\sum_{j=1}^{p} \frac{\lambda_{j} t^{j}}{j !}\right)(x-t)^{\mu-1} d t
$$

and if $\lambda_{p} \neq 0$, the path $C$ starts from $\infty$ to one of the $p$ independent directions $\lambda_{p}^{-1} e^{\frac{2 \pi \nu \sqrt{-1}}{p}+t}(t \gg 1, \nu=0,1, \ldots, p-1)$ and ends at $x$.

Suppose $n=2$. The corresponding Riemann scheme for the generic characteristic exponents and its construction from the Riemann scheme of the trivial equation $u^{\prime}=0$ is as follows:

$$
\begin{aligned}
& \left.\left\{\begin{array}{ccc}
x=0 & 1 & \infty \\
b_{0} & c_{0} & a_{0} \\
b_{1} & c_{1} & a_{1}
\end{array}\right\} \quad \text { (Fuchs relation: } a_{0}+a_{1}+b_{0}+b_{1}+c_{0}+c_{1}=1\right) \\
& \stackrel{x^{b_{0}}(1-x)^{c_{0}} \partial^{-a_{1}-b_{1}-c_{1}}}{\longleftarrow}\left\{\begin{array}{ccc}
x=0 & 1 & \infty \\
-a_{1}-b_{0}-c_{1} & -a_{1}-b_{1}-c_{0} & -a_{0}+a_{1}+1
\end{array}\right\} \\
& \stackrel{x^{-a_{1}-b_{0}-c_{1}}(1-x)^{-a_{1}-b_{1}-c_{0}}}{\longleftarrow}\left\{\begin{array}{ccc}
x=0 & 1 & \infty \\
0 & 0 & 0
\end{array}\right\} .
\end{aligned}
$$

Then our fractional calculus gives the corresponding equation

$$
\begin{aligned}
& x^{2}(1-x)^{2} u^{\prime \prime}-x(1-x)\left(\left(a_{0}+a_{1}+1\right) x+b_{0}+b_{1}-1\right) u^{\prime} \\
& \quad+\left(a_{0} a_{1} x^{2}-\left(a_{0} a_{1}+b_{0} b_{1}-c_{0} c_{1}\right) x+b_{0} b_{1}\right) u=0,
\end{aligned}
$$

the connection formula

$$
c\left(0: b_{1} \rightsquigarrow 1: c_{1}\right)=\frac{\Gamma\left(c_{0}-c_{1}\right) \Gamma\left(b_{1}-b_{0}+1\right)}{\Gamma\left(a_{0}+b_{1}+c_{0}\right) \Gamma\left(a_{1}+b_{1}+c_{0}\right)}
$$

and expressions of its solution by the integral representation

$$
\begin{gathered}
\int_{0}^{x} x^{b_{0}}(1-x)^{c_{0}}(x-s)^{a_{1}+b_{1}+c_{1}-1} s^{-a_{1}-c_{1}-b_{0}}(1-s)^{-a_{1}-b_{1}-c_{0}-} d s \\
\quad=\frac{\Gamma\left(a_{0}+b_{1}+c_{0}\right) \Gamma\left(a_{1}+b_{1}+c_{1}\right)}{\Gamma\left(b_{1}-b_{0}+1\right)} x^{b_{1}} \phi_{b_{1}}(x)
\end{gathered}
$$


and the series expansion

$$
\begin{aligned}
& \sum_{n \geq 0} \frac{\left(a_{0}+b_{1}+c_{0}\right)_{n}\left(a_{1}+b_{1}+c_{0}\right)_{n}}{\left(b_{1}-b_{0}+1\right)_{n} n !}(1-x)^{c_{0}} x^{b_{1}+n} \\
& \quad=(1-x)^{c_{0}} x^{b_{1}} F\left(a_{0}+b_{1}+c_{0}, a_{1}+b_{1}+c_{0}, b_{1}-b_{0}-1 ; x\right) .
\end{aligned}
$$

Here $\phi_{b_{1}}(x)$ is a holomorphic function in a neighborhood of 0 satisfying $\phi_{b_{1}}(0)=$ 1 for generic spectral parameters. We note that the transposition of $c_{0}$ and $c_{1}$ in (15.15) gives a nontrivial equality, which corresponds to Kummer's relation of Gauss hypergeometric function and the similar statement is true for (15.14). In general, different procedures of reduction of a equation give different expressions of its solution.

\subsection{Hypergeometric family. $H_{n}$}

We examine the hypergeometric family which corresponds to the equations satisfied by the generalized hypergeometric series (1.7). Its spectral type is in the Simpson's list (cf. \$15.2).

$$
\begin{aligned}
& \mathbf{m}=\left(1^{n}, n-11,1^{n}\right):{ }_{n} F_{n-1}(\alpha, \beta ; z) \\
& 1^{n}, n-11,1^{n}=1,10,1 \oplus 1^{n-1}, n-21,1^{n-1} \\
& \Delta(\mathbf{m})=\left\{\alpha_{0}+\alpha_{0,1}+\cdots+\alpha_{0, \nu}+\alpha_{2,1}+\cdots+\alpha_{2, \nu^{\prime}}\right. \\
&\left.0 \leq \nu<n, 0 \leq \nu^{\prime}<n\right\} \\
& {[\Delta(\mathbf{m})]=1^{n^{2}} } \\
& H_{n}=H_{1} \oplus H_{n-1}: n^{2} \\
& H_{n} \underset{R 2 E 0}{\stackrel{1}{\longrightarrow} H_{n-1}}
\end{aligned}
$$

Since $\mathbf{m}$ is of Okubo type, we have a system of Okubo normal form with the spectral type $\mathbf{m}$. Then the above $R 2 E 0$ represents the reduction of systems of equations of Okubo normal form due to Yokoyama Yo2. The number 1 on the arrow represents a reduction by a middle convolution and the number shows the difference of the orders.

$$
\begin{gathered}
\left\{\begin{array}{ccc}
x=0 & 1 & \infty \\
\lambda_{0,1} & {\left[\lambda_{1,1}\right]_{(n-1)}} & \lambda_{2,1} \\
\vdots & \vdots \\
\lambda_{0, n-1} & \lambda_{2, n-1} \\
\lambda_{0, n} & \lambda_{1,2} & \lambda_{2, n}
\end{array}\right\}, \quad\left\{\begin{array}{ccc}
x=0 & 1 & \infty \\
1-\beta_{1} & {[0]_{(n-1)}} & \alpha_{1} \\
\vdots & & \vdots \\
1-\beta_{n-1} & & \alpha_{n-1} \\
0 & -\beta_{n} & \alpha_{n}
\end{array}\right\} \\
\sum_{\nu=1}^{n}\left(\lambda_{0, \nu}+\lambda_{2, \nu}\right)+(n-1) \lambda_{1,1}+\lambda_{1,2}=n-1, \\
\alpha_{1}+\cdots+\alpha_{n}=\beta_{1}+\cdots+\beta_{n} .
\end{gathered}
$$

It follows from Theorem 13.7 that the universal operators

$$
P_{H_{1}}^{0}(\lambda), P_{H_{1}}^{2}(\lambda), P_{H_{n-1}}^{0}(\lambda), P_{H_{n-1}}^{1}(\lambda), P_{H_{n-1}}^{2}(\lambda) \text {. }
$$

are shift operators for the universal model $P_{H_{n}}(\lambda) u=0$.

The Riemann scheme of the operator

$$
P=\operatorname{RAd}\left(\partial^{-\mu_{n-1}}\right) \circ \operatorname{RAd}\left(x^{\gamma_{n-1}}\right) \circ \cdots \circ \operatorname{RAd}\left(\partial^{-\mu_{1}}\right) \circ \operatorname{RAd}\left(x^{\gamma_{1}}(1-x)^{\gamma^{\prime}}\right) \partial
$$


equals

$$
\left\{\begin{array}{ccc}
x=0 & 1 & \infty \\
0 & {[0]_{(n-1)}} & 1-\mu_{n-1} \\
\left(\gamma_{n-1}+\mu_{n-1}\right) & 1-\left(\gamma_{n-1}+\mu_{n-1}\right)-\mu_{n-2} \\
\sum_{j=n-2}^{n-1}\left(\gamma_{j}+\mu_{j}\right) & 1-\sum_{j=n-2}^{n-1}\left(\gamma_{j}+\mu_{j}\right)-\mu_{n-3} \\
\vdots & \vdots \\
\sum_{j=2}^{n-1}\left(\gamma_{j}+\mu_{j}\right) & 1-\sum_{j=2}^{n-1}\left(\gamma_{j}+\mu_{j}\right)-\mu_{1} \\
\sum_{j=1}^{n-1}\left(\gamma_{j}+\mu_{j}\right) & \gamma^{\prime}+\sum_{j=1}^{n-1} \mu_{j} & -\gamma^{\prime}-\sum_{j=1}^{n-1}\left(\gamma_{j}+\mu_{j}\right)
\end{array}\right\}
$$

which is obtained by the induction on $n$ with Theorem 7.2 and corresponds to the second Riemann scheme in (15.16) by putting

$$
\begin{aligned}
& \gamma_{j}=\alpha_{j+1}-\beta_{j} \quad(j=1, \ldots, n-2), \quad \gamma^{\prime}=-\alpha_{1}+\beta_{1}-1, \\
& \mu_{j}=-\alpha_{j+1}+\beta_{j+1} \quad(j=1, \ldots, n-1), \quad \mu_{n-1}=1-\alpha_{n} .
\end{aligned}
$$

The integral representation of the local solutions at $x=0$ (resp. 1 and $\infty$ ) corresponding to the exponents $\sum_{j=1}^{n-1}\left(\gamma_{j}+\mu_{j}\right)$ (resp. $\gamma^{\prime}+\sum_{j=1}^{n-1} \mu_{j}$ and $-\gamma^{\prime}-\sum_{j=1}^{n-1}\left(\gamma_{j}+\right.$ $\mu_{j}$ ) are given by

$$
I_{c}^{\mu_{n-1}} x^{\gamma_{n-1}} I_{c}^{\mu_{n-2}} \cdots I_{c}^{\mu_{1}} x^{\gamma_{1}}(1-x)^{\gamma^{\prime}}
$$

by putting $c=0$ (resp. 1 and $\infty$ ).

For simplicity we express this construction using additions and middle convolutions by

$$
u=\partial^{-\mu_{n-1}} x^{\gamma_{n-1}} \cdots \partial^{-\mu_{2}} x^{\gamma_{2}} \partial^{-\mu_{2}} x^{\gamma_{1}}(1-x)^{\gamma^{\prime}} .
$$

For example, when $n=3$, we have the solution

$$
\int_{c}^{x} t^{\alpha_{3}-\beta_{2}}(x-t)^{1-\alpha_{3}} d t \int_{c}^{t} s^{\alpha_{2}-\beta_{1}}(1-s)^{-\alpha_{1}+\beta_{1}-1}(t-s)^{-\alpha_{2}-\beta_{2}} d s .
$$

The operator corresponding to the second Riemann scheme is

$$
P_{n}(\alpha ; \beta):=\prod_{j=1}^{n-1}\left(\vartheta-\beta_{j}\right) \cdot \partial-\prod_{j=1}^{n}\left(\vartheta-\alpha_{j}\right) .
$$

This is clear when $n=1$. In general, we have

$$
\begin{aligned}
& \operatorname{RAd}\left(\partial^{-\mu}\right) \circ \operatorname{RAd}\left(x^{\gamma}\right) P_{n}(\alpha, \beta) \\
& =\operatorname{RAd}\left(\partial^{-\mu}\right) \circ \operatorname{Ad}\left(x^{\gamma}\right)\left(\prod_{j=1}^{n-1} x\left(\vartheta+\beta_{j}\right) \cdot \partial-\prod_{j=1}^{n} x\left(\vartheta+\alpha_{j}\right)\right) \\
& =\operatorname{RAd}\left(\partial^{-\mu}\right)\left(\prod_{j=1}^{n-1}\left(\vartheta+\beta_{j}-1-\gamma\right)(\vartheta-\gamma)-\prod_{j=1}^{n} x\left(\vartheta+\alpha_{j}-\gamma\right)\right) \\
& =\operatorname{Ad}\left(\partial^{-\mu}\right)\left(\prod_{j=1}^{n-1}\left(\vartheta+\beta_{j}-\gamma\right) \cdot(\vartheta-\gamma+1) \partial-\prod_{j=1}^{n}(\vartheta+1)\left(\vartheta+\alpha_{j}-\gamma\right)\right) \\
& =\prod_{j=1}^{n-1}\left(\vartheta+\beta_{j}-\gamma-\mu\right) \cdot(\vartheta-\gamma-\mu+1) \partial-\prod_{j=1}^{n}(\vartheta+1-\mu) \cdot\left(\vartheta+\alpha_{j}-\gamma-\mu\right)
\end{aligned}
$$


and therefore we have (15.21) by the correspondence of the Riemann schemes with $\gamma=\gamma_{n}$ and $\mu=\mu_{n}$.

Suppose $\lambda_{1,1}=0$. We will show that

$$
\begin{aligned}
& \sum_{k=0}^{\infty} \frac{\prod_{j=1}^{n}\left(\lambda_{2, j}-\lambda_{0, n}\right)_{k}}{\prod_{j=1}^{n-1}\left(\lambda_{0, n}-\lambda_{0, j}+1\right)_{k} k !} x^{\lambda_{0, n}+k} \\
& \quad=x^{\lambda_{0, n}}{ }_{n} F_{n-1}\left(\left(\lambda_{2, j}-\lambda_{0, n}\right)_{j=1, \ldots, n},\left(\lambda_{0, n}-\lambda_{0, j}+1\right)_{j=1, \ldots, n-1} ; x\right)
\end{aligned}
$$

is the local solution at the origin corresponding to the exponent $\lambda_{0, n}$. Here

$$
{ }_{n} F_{n-1}\left(\alpha_{1}, \ldots, \alpha_{n}, \beta_{1}, \ldots, \beta_{n-1} ; x\right)=\sum_{k=0}^{\infty} \frac{\left(\alpha_{1}\right)_{k} \cdots\left(\alpha_{n-1}\right)_{k}\left(\alpha_{n}\right)_{k}}{\left(\beta_{1}\right)_{k} \cdots\left(\beta_{n-1}\right)_{k} k !} x^{k} .
$$

We may assume $\lambda_{0,1}=0$ for the proof of (15.22). When $n=1$, the corresponding solution equals $(1-x)^{-\lambda_{2,1}}$ and we have (15.22). Note that

$$
\begin{aligned}
& I_{0}^{\mu} x^{\gamma} \sum_{k=0}^{\infty} \frac{\prod_{j=1}^{n}\left(\lambda_{2, j}-\lambda_{0, n}\right)_{k}}{\prod_{j=1}^{n-1}\left(\lambda_{0, n}-\lambda_{0, j}+1\right)_{k} k !} x^{\lambda_{0, n}+k} \\
& =\sum_{k=0}^{\infty} \frac{\prod_{j=1}^{n}\left(\lambda_{2, j}-\lambda_{0, n}\right)_{k}}{\prod_{j=1}^{n-1}\left(\lambda_{0, n}-\lambda_{0, j}+1\right)_{k} k !} \frac{\Gamma\left(\lambda_{0, n}+\gamma+k+1\right)}{\Gamma\left(\lambda_{0, n}+\gamma+\mu+k+1\right)} x^{\lambda_{0, n}+\gamma+\mu+k} \\
& =\frac{\Gamma\left(\lambda_{0, n}+\gamma+1\right)}{\Gamma\left(\lambda_{0, n}+\gamma+\mu+1\right)} \sum_{k=0}^{\infty} \frac{\prod_{j=1}^{n}\left(\lambda_{2, j}-\lambda_{0, n}\right)_{k} \cdot\left(\lambda_{0, n}+\gamma+1\right)_{k} \cdot x^{\lambda_{0, n}+\gamma+\mu+k}}{\prod_{j=1}^{n-1}\left(\lambda_{0, n}-\lambda_{0, j}+1\right)_{k} \cdot\left(\lambda_{0, n}+\gamma+\mu+1\right)_{k} k !} .
\end{aligned}
$$

Comparing (15.17) with the first Riemann scheme under $\lambda_{0,1}=\lambda_{1,1}=0$ and $\gamma=\gamma_{n}$ and $\mu=\mu_{n}$, we have the solution (15.22) by the induction on $n$. The recurrence relation in Theorem 13.3 corresponds to the identity

$$
\begin{aligned}
& { }_{n} F_{n-1}\left(\alpha_{1}, \ldots, \alpha_{n-1}, \alpha_{n}+1 ; \beta_{1}, \ldots, \beta_{n-1} ; x\right) \\
& \quad={ }_{n} F_{n-1}\left(\alpha_{1}, \ldots, \alpha_{n} ; \beta_{1}, \ldots, \beta_{n-1} ; x\right) \\
& \quad+\frac{\alpha_{1} \cdots \alpha_{n-1}}{\beta_{1} \cdots \beta_{n-1}} x \cdot{ }_{n} F_{n-1}\left(\alpha_{1}+1, \ldots, \alpha_{n}+1 ; \beta_{1}+1, \ldots, \beta_{n-1}+1 ; x\right) .
\end{aligned}
$$

The series expansion of the local solution at $x=1$ corresponding to the exponent $\gamma^{\prime}+\mu_{1}+\cdots+\mu_{n-1}$ is a little more complicated.

For the Riemann scheme

$$
\left\{\begin{array}{ccc}
x=\infty & 0 & 1 \\
-\mu_{2}+1 & {[0]_{(2)}} & 0 \\
1-\gamma_{2}-\mu_{1}-\mu_{2} & & \gamma_{2}+\mu_{2} \\
-\gamma^{\prime}-\gamma_{1}-\gamma_{2}-\mu_{1}-\mu_{2} & \underline{\gamma^{\prime}+\mu_{1}+\mu_{2}} & \gamma_{1}+\gamma_{2}+\mu_{1}+\mu_{2}
\end{array}\right\},
$$

we have the local solution at $x=0$

$$
\begin{aligned}
& I_{0}^{\mu_{2}}(1-x)^{\gamma_{2}} I_{0}^{\mu_{1}} x^{\gamma^{\prime}}(1-x)^{\gamma_{1}}=I_{0}^{\mu_{2}}(1-x)^{\gamma_{2}} \sum_{n=0}^{\infty} \frac{\left(-\gamma_{1}\right)_{n}}{n !} x^{n} \\
& =I_{0}^{\mu_{2}} \sum_{n=0}^{\infty} \frac{\Gamma\left(\gamma^{\prime}+1+n\right)\left(-\gamma_{1}\right)_{n}}{\Gamma\left(\gamma^{\prime}+\mu_{1}+1+n\right) n !} x^{\gamma^{\prime}+\mu_{1}+n}(1-x)^{\gamma_{2}} \\
& =I_{0}^{\mu_{2}} \sum_{m, n=0}^{\infty} \frac{\Gamma\left(\gamma^{\prime}+1+n\right)\left(-\gamma_{1}\right)_{n}\left(-\gamma_{2}\right)_{m}}{\Gamma\left(\gamma^{\prime}+\mu_{1}+1+n\right) m ! n !} x^{\gamma^{\prime}+\mu_{1}+m+n} \\
& =\sum_{m, n=0}^{\infty} \frac{\Gamma\left(\gamma^{\prime}+\mu_{1}+1+m+n\right) \Gamma\left(\gamma^{\prime}+1+n\right)\left(-\gamma_{1}\right)_{n}\left(-\gamma_{2}\right)_{m} x^{\gamma^{\prime}+\mu_{1}+\mu_{2}+m+n}}{\Gamma\left(\gamma^{\prime}+\mu_{1}+\mu_{2}+1+m+n\right) \Gamma\left(\gamma^{\prime}+\mu_{1}+1+n\right) m ! n !}
\end{aligned}
$$




$$
=\frac{\Gamma\left(\gamma^{\prime}+1\right) x^{\gamma^{\prime}+\mu_{1}+\mu_{2}}}{\Gamma\left(\gamma^{\prime}+\mu_{1}+\mu_{2}+1\right)} \sum_{m, n=0}^{\infty} \frac{\left(\gamma^{\prime}+\mu_{1}+1\right)_{m+n}\left(\gamma^{\prime}+1\right)_{n}\left(-\gamma_{1}\right)_{n}\left(-\gamma_{2}\right)_{m} x^{m+n}}{\left(\gamma^{\prime}+\mu_{1}+\mu_{2}+1\right)_{m+n}\left(\gamma^{\prime}+\mu_{1}+1\right)_{n} m ! n !} .
$$

Applying the last equality in (4.8) to the above second equality, we have

$$
\begin{aligned}
I_{0}^{\mu_{2}} & (1-x)^{\gamma_{2}} I_{0}^{\mu_{1}} x^{\gamma^{\prime}}(1-x)^{\gamma_{1}} \\
= & \sum_{n=0}^{\infty} \frac{\Gamma\left(\gamma^{\prime}+1+n\right)\left(-\gamma_{1}\right)_{n}}{\Gamma\left(\gamma^{\prime}+\mu_{1}+1+n\right) n !} x^{\gamma^{\prime}+\mu_{1}+\mu_{2}+n}(1-x)^{-\gamma_{2}} \\
& \cdot \sum_{m=0}^{\infty} \frac{\Gamma\left(\gamma^{\prime}+\mu_{1}+1+n\right)}{\Gamma\left(\gamma^{\prime}+\mu_{1}+\mu_{2}+1+n\right)} \frac{\left(\mu_{2}\right)_{m}\left(-\gamma_{2}\right)_{m}}{\left(\gamma^{\prime}+\mu_{1}+n+\mu_{2}+1\right)_{m} m !}\left(\frac{x}{x-1}\right)^{m} \\
= & \frac{\Gamma\left(\gamma^{\prime}+1\right) x^{\gamma^{\prime}+\mu_{1}+\mu_{2}}(1-x)^{-\gamma_{2}}}{\Gamma\left(\gamma^{\prime}+\mu_{1}+\mu_{2}+1\right)} \sum_{m, n=0}^{\infty} \frac{\left(\gamma^{\prime}+1\right)_{n}\left(-\gamma_{1}\right)_{n}\left(-\gamma_{2}\right)_{m}\left(\mu_{2}\right)_{m}}{\left(\gamma^{\prime}+\mu_{1}+\mu_{2}+1\right)_{m+n} m ! n !} x^{n}\left(\frac{x}{x-1}\right)^{m} \\
= & \frac{\Gamma\left(\gamma^{\prime}+1\right)}{\Gamma\left(\gamma^{\prime}+\mu_{1}+\mu_{2}+1\right)} \\
& \cdot x^{\gamma^{\prime}+\mu_{1}+\mu_{2}}(1-x)^{-\gamma_{2}} F_{3}\left(-\gamma_{2},-\gamma_{1}, \mu_{2}, \gamma^{\prime}+1 ; \gamma^{\prime}+\mu_{1}+\mu_{2}+1 ; x, \frac{x}{x-1}\right),
\end{aligned}
$$

where $F_{3}$ is Appell's hypergeometric function (15.52).

Let $u_{1}^{-\beta_{n}}\left(\alpha_{1}, \ldots, \alpha_{n} ; \beta_{1}, \ldots, \beta_{n-1} ; x\right)$ be the local solution of $P_{n}(\alpha, \beta) u=0$ at $x=1$ such that $u_{1}^{-\beta_{n}}(\alpha ; \beta ; x) \equiv(x-1)^{-\beta_{n}} \bmod (x-1)^{1-\beta_{n}} \mathcal{O}_{1}$ for generic $\alpha$ and $\beta$. Since the reduction

$$
\left\{\begin{array}{ccc}
\lambda_{0,1} & {[0]_{(n-1)}} & \lambda_{2,1} \\
\vdots & & \vdots \\
\lambda_{0, n} & \lambda_{1,2} & \lambda_{2, n}
\end{array}\right\} \stackrel{\partial_{\max }}{\longrightarrow}\left\{\begin{array}{ccc}
\lambda_{0,1}^{\prime} & {[0]_{(n-2)}} & \lambda_{2,1}^{\prime} \\
\vdots & & \vdots \\
\lambda_{0, n-1}^{\prime} & \lambda_{1,2}^{\prime} & \lambda_{2, n-1}^{\prime}
\end{array}\right\}
$$

satisfies $\lambda_{1,2}^{\prime}=\lambda_{1,2}+\lambda_{0,1}+\lambda_{0,2}-1$ and $\lambda_{0, j}^{\prime}+\lambda_{2, j}^{\prime}=\lambda_{0, j+1}+\lambda_{2, j+1}$ for $j=$ $1, \ldots, n-1$, Theorem 13.3 proves

$$
\begin{aligned}
u_{1}^{-\beta_{n}}(\alpha ; \beta ; x)= & u_{1}^{-\beta_{n}}\left(\alpha_{1}, \ldots, \alpha_{n}+1 ; \beta_{1}, \ldots, \beta_{n-1}+1 ; x\right) \\
& +\frac{\beta_{n-1}-\alpha_{n}}{1-\beta_{n}} u_{1}^{1-\beta_{n}}\left(\alpha ; \beta_{1}, \ldots, \beta_{n-1}+1 ; x\right) .
\end{aligned}
$$

The condition for the irreducibility of the equation equals

$$
\lambda_{0, \nu}+\lambda_{1,1}+\lambda_{2, \nu^{\prime}} \notin \mathbb{Z} \quad\left(1 \leq \nu \leq n, 1 \leq \nu^{\prime} \leq n\right),
$$

which is easily proved by the induction on $n$ (cf. Example 12.17 ii)). The shift operator under a compatible shift $\left(\epsilon_{j, \nu}\right)$ is bijective if and only if

$$
\lambda_{0, \nu}+\lambda_{1,1}+\lambda_{2, \nu^{\prime}} \text { and } \lambda_{0, \nu}+\epsilon_{0, \nu}+\lambda_{1,1}+\epsilon_{1,1}+\lambda_{2, \nu^{\prime}}+\epsilon_{2, \nu^{\prime}}
$$

are simultaneously not integers or positive integers or non-positive integers for each $\nu \in\{1, \ldots, n\}$ and $\nu^{\prime} \in\{1, \ldots, n\}$.

Connection coefficients in this example are calculated by [L] and [OTY] etc. In this paper we get them by Theorem 14.6 .

There are the following direct decompositions $(\nu=1, \ldots, n)$.

$$
\begin{aligned}
1 \ldots 1 \overline{1} ; n-1 \underline{1} ; 1 \ldots 1 & =0 \ldots 0 \overline{1} ; \quad 1 \quad \underline{0} ; 0 \ldots 010 \ldots 0 \\
& \oplus 1 \ldots 1 \overline{0} ; n-2 \underline{1} ; 1 \ldots 101 \ldots 1 .
\end{aligned}
$$

These $n$ decompositions $\mathbf{m}=\mathbf{m}^{\prime} \oplus \mathbf{m}^{\prime \prime}$ satisfy the condition $m_{0, n_{0}}^{\prime}=m_{1, n_{1}}^{\prime \prime}=1$ in (14.10), where $n_{0}=n$ and $n_{1}=2$. Since $n_{0}+n_{1}-2=n$, Remark 14.8 i) shows 
that these decompositions give all the decompositions appearing in (14.10). Thus we have

$$
\begin{aligned}
c\left(\lambda_{0, n} \rightsquigarrow \lambda_{1,2}\right) & =\frac{\prod_{\nu=1}^{n-1} \Gamma\left(\lambda_{0, n}-\lambda_{0, \nu}+1\right) \cdot \Gamma\left(\lambda_{1,1}-\lambda_{1,2}\right)}{\prod_{\nu=1}^{n} \Gamma\left(\lambda_{0, n}+\lambda_{1,1}+\lambda_{2, \nu}\right)}=\prod_{\nu=1}^{n} \frac{\Gamma\left(\beta_{\nu}\right)}{\Gamma\left(\alpha_{\nu}\right)} \\
& =\lim _{x \rightarrow 1-0}(1-x)^{\beta_{n}}{ }_{n} F_{n-1}(\alpha, \beta ; x) \quad\left(\operatorname{Re} \beta_{n}>0\right) .
\end{aligned}
$$

Other connection coefficients are obtained by the similar way.

$$
\begin{aligned}
c\left(\lambda_{0, n} \rightsquigarrow \lambda_{2, n}\right): \quad \text { When } n=3, \text { we have } \\
11 \overline{1}, 21,11 \underline{1}=001,10,100 \quad 001,10,010 \quad 101,11,110 \quad 011,11,110 \\
\oplus 110,11,011=110,11,101=010,10,001=100,10,001
\end{aligned}
$$

In general, by the rigid decompositions

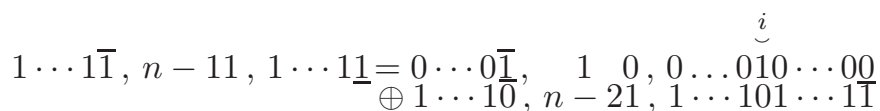

$$
\begin{aligned}
& \begin{array}{l}
\stackrel{i}{L} \\
=1 \cdots 101 \cdots 1 \overline{1}, n-21,1 \cdots 10 \\
\oplus 0 \ldots 010 \cdots 00, \quad 10,0 \cdots 0
\end{array}
\end{aligned}
$$

for $i=1, \ldots, n-1$ we have

$$
\begin{aligned}
& c\left(\lambda_{0, n} \rightsquigarrow \lambda_{2, n}\right)=\prod_{k=1}^{n-1} \frac{\Gamma\left(\lambda_{2, k}-\lambda_{2, n}\right)}{\Gamma\left(\left|\left\{\begin{array}{lll}
\lambda_{0, n} & \lambda_{1,1} & \lambda_{2, k}
\end{array}\right\}\right|\right)}
\end{aligned}
$$

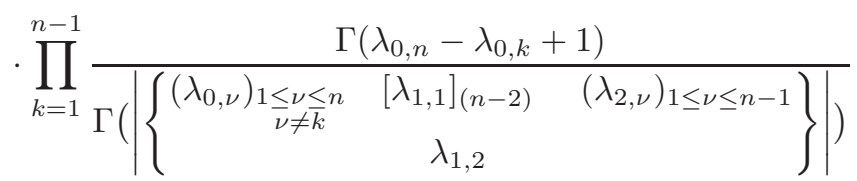

$$
\begin{aligned}
& =\prod_{k=1}^{n-1} \frac{\Gamma\left(\beta_{k}\right) \Gamma\left(\alpha_{k}-\alpha_{n}\right)}{\Gamma\left(\alpha_{k}\right) \Gamma\left(\beta_{k}-\alpha_{n}\right)} .
\end{aligned}
$$

Moreover we have

$$
\begin{aligned}
c\left(\lambda_{1,2} \rightsquigarrow \lambda_{0, n}\right) & =\frac{\Gamma\left(\lambda_{1,2}-\lambda_{1,1}+1\right) \cdot \prod_{\nu=1}^{n-1} \Gamma\left(\lambda_{0, \nu}-\lambda_{0, n}\right)}{\prod_{j=1}^{n} \Gamma\left(\mid\left\{\begin{array}{cc}
\left(\lambda_{0, \nu}\right)_{1 \leq \nu \leq n-1} & {\left[\lambda_{1,1}\right]_{(n-2)}} \\
\lambda_{1,2} & \left(\lambda_{2, \nu}\right)_{1 \leq \nu \leq n, \nu \neq j}
\end{array}\right\}\right)} \\
& =\prod_{\nu=1}^{n} \frac{\Gamma\left(1-\beta_{\nu}\right)}{\Gamma\left(1-\alpha_{\nu}\right)} .
\end{aligned}
$$

Here we denote

$$
\left(\mu_{\nu}\right)_{1 \leq \nu \leq n}=\left(\begin{array}{c}
\mu_{1} \\
\mu_{2} \\
\vdots \\
\mu_{n}
\end{array}\right) \in \mathbb{C}^{n} \text { and }\left(\mu_{\nu}\right)_{\substack{\leq \nu \leq n \\
\nu \neq i}}=\left(\begin{array}{c}
\mu_{1} \\
\vdots \\
\mu_{i-1} \\
\mu_{i+1} \\
\vdots \\
\mu_{n}
\end{array}\right) \in \mathbb{C}^{n-1}
$$

for complex numbers $\mu_{1}, \ldots, \mu_{n}$.

These connection coefficients were obtained by [Le] and [Yos] etc. 
We have

$$
\begin{aligned}
{ }_{n} F_{n-1}(\alpha, \beta ; x) & =\sum_{k=0}^{\infty} C_{k}(1-x)^{k}+\sum_{k=0}^{\infty} C_{k}^{\prime}(1-x)^{k-\beta_{n}}, \\
C_{0} & ={ }_{n} F_{n-1}(\alpha, \beta ; 1) \quad\left(\operatorname{Re} \beta_{n}<0\right), \\
C_{0}^{\prime} & =\prod_{\nu=1}^{n} \frac{\Gamma\left(\beta_{\nu}\right)}{\Gamma\left(\alpha_{\nu}\right)}
\end{aligned}
$$

for $0<x<1$ if $\alpha$ and $\beta$ are generic. Since

$$
\begin{aligned}
& \frac{d^{k}}{d x^{k}}{ }_{n} F_{n-1}(\alpha, \beta ; x) \\
& \quad=\frac{\left(\alpha_{1}\right)_{k} \cdots\left(\alpha_{n}\right)_{k}}{\left(\beta_{1}\right)_{k} \cdots\left(\beta_{n-1}\right)_{k}} F_{n-1}\left(\alpha_{1}+k, \ldots, \alpha_{n}+k, \beta_{1}+k, \ldots, \beta_{n-1}+k ; x\right),
\end{aligned}
$$

we have

$$
C_{k}=\frac{\left(\alpha_{1}\right)_{k} \cdots\left(\alpha_{n}\right)_{k}}{\left(\beta_{1}\right)_{k} \cdots\left(\beta_{n-1}\right)_{k} k !}{ }_{n} F_{n-1}\left(\alpha_{1}+k, \ldots, \alpha_{n}+k, \beta_{1}+k, \ldots, \beta_{n-1}+k ; 1\right) .
$$

We examine the monodromy generators for the solutions of the generalized hypergeometric equation. For simplicity we assume $\beta_{i} \notin \mathbb{Z}$ and $\beta_{i}-\beta_{j} \notin \mathbb{Z}$ for $i \neq j$. Then $u=\left(u_{0}^{\lambda_{0,1}}, \ldots, u_{0}^{\lambda_{0, n}}\right)$ is a base of local solution at 0 and the corresponding monodromy generator around 0 with respect to this base equals

$$
M_{0}=\left(\begin{array}{ccc}
e^{2 \pi \sqrt{-1} \lambda_{0,1}} & & \\
& \ddots & \\
& & e^{2 \pi \sqrt{-1} \lambda_{0, n}}
\end{array}\right)
$$

and that around $\infty$ equals

$$
\begin{aligned}
& M_{\infty}=\left(\sum_{k=1}^{n} e^{2 \pi \sqrt{-1} \lambda_{2, \nu}} c\left(\lambda_{0, i} \rightsquigarrow \lambda_{2, k}\right) c\left(\lambda_{2, k} \rightsquigarrow \lambda_{k, j}\right)\right)_{\substack{1 \leq i \leq n \\
1 \leq j \leq n}} \\
& =\left(\sum_{k=1}^{n} e^{2 \pi \sqrt{-1} \lambda_{2, \nu}} \prod_{\nu \in\{1, \ldots, n\} \backslash\{k\}} \frac{\sin 2 \pi\left(\lambda_{0, i}+\lambda_{1,1}+\lambda_{2, \nu}\right)}{\sin 2 \pi\left(\lambda_{0, k}-\lambda_{0, \nu}\right)}\right. \\
& \left.\cdot \prod_{\nu \in\{1, \ldots, n\} \backslash\{j\}} \frac{\sin 2 \pi\left(\lambda_{0, i}+\lambda_{1,1}+\lambda_{2, \nu}\right)}{\sin 2 \pi\left(\lambda_{2, j}-\lambda_{2, \nu}\right)}\right)_{\substack{1 \leq i \leq n \\
1 \leq j \leq n}} .
\end{aligned}
$$

Lastly we remark that the versal generalized hypergeometric operator is

$$
\begin{aligned}
& \tilde{P}=\operatorname{RAd}\left(\partial^{-\mu_{n-1}}\right) \circ \operatorname{RAd}\left(\left(1-c_{1} x\right)^{\frac{\gamma_{n-1}}{c_{1}}}\right) \circ \cdots \circ \operatorname{RAd}\left(\partial^{-\mu_{1}}\right) \\
& \circ \operatorname{RAd}\left(\left(1-c_{1} x\right)^{\frac{\gamma_{1}}{c_{1}}+\frac{\gamma^{\prime}}{c_{1}\left(c_{1}-c_{2}\right)}}\left(1-c_{2} x\right)^{\frac{\gamma^{\prime}}{c_{2}\left(c_{2}-c_{1}\right)}}\right) \partial \\
& =\operatorname{RAd}\left(\partial^{-\mu_{n-1}}\right) \circ \operatorname{RAdei}\left(\frac{\gamma_{n-1}}{1-c_{1} x}\right) \circ \cdots \circ \operatorname{RAd}\left(\partial^{-\mu_{1}}\right) \\
& \text { ○ RAdei }\left(\frac{\gamma_{1}}{1-c_{1} x}+\frac{\gamma^{\prime} x}{\left(1-c_{1} x\right)\left(1-c_{2} x\right)}\right) \partial
\end{aligned}
$$

and when $n=3$, we have the integral representation of the solutions

$$
\int_{c}^{x} \int_{c}^{t} \exp \left(-\int_{c}^{s} \frac{\gamma_{1}\left(1-c_{2} u\right)+\gamma^{\prime} u}{\left(1-c_{1} u\right)\left(1-c_{2} u\right)} d u\right)(t-s)^{\mu_{1}-1}\left(1-c_{1} t\right)^{\frac{\gamma_{2}}{c_{1}}}(x-t)^{\mu_{2}-1} d s d t .
$$

Here $c$ equals $\frac{1}{c_{1}}$ or $\frac{1}{c_{2}}$ or $\infty$. 
15.5. Even/Odd family. $E O_{n}$

The system of differential equations of Schlesinger canonical form belonging to an even or odd family is concretely given by [G]. We will examine concrete connection coefficients of solutions of the single differential equation belonging to an even or odd family. The corresponding tuples of partitions and their reductions and decompositions are as follows.

$$
\begin{aligned}
m+1 m, m^{2} 1,1^{2 m+1} & =10,10,1 \oplus m^{2}, m m-11,1^{2 m} \\
& =1^{2}, 1^{2} 0,1^{2} \oplus m m-1,(m-2)^{2} 1,1^{2 m-1} \\
m^{2}, m m-11,1^{2 m} & =1,100,1 \oplus m m-1,(m-1)^{2} 1,1^{2 m-1} \\
& =1^{2}, 110,1^{2} \oplus(m-1)^{2}, m-1 m-21,1^{2 m-2} \\
E O_{n} & =H_{1} \oplus E O_{n-1}: 2 n=H_{2} \oplus E O_{n-2}:\left(\begin{array}{c}
n \\
2
\end{array}\right) \\
{[\Delta(\mathbf{m})] } & =1^{\left(\begin{array}{c}
n \\
2
\end{array}\right)+2 n} \\
E O_{n} & \stackrel{R 1}{\stackrel{1}{E 0 R 0 E 0}} E O_{n-1} \\
E O_{2} & =H_{2}, \quad E O_{3}=H_{3}
\end{aligned}
$$

The following operators are shift operators of the universal model $P_{E O_{n}}(\lambda) u=0$ :

$$
P_{H_{1}}^{2}(\lambda), P_{E O_{n-1}}^{1}(\lambda), P_{E O_{n-1}}^{2}(\lambda), P_{H_{2}}^{2}(\lambda), P_{E O_{n-2}}^{1}(\lambda), P_{E O_{n-2}}^{2}(\lambda) .
$$

$E O_{2 m}\left(\mathbf{m}=\left(1^{2 m}, m m-11, m m\right):\right.$ even family $)$

$$
\begin{gathered}
\left\{\begin{array}{ccc}
x=\infty & 0 & 1 \\
\lambda_{0,1} & {\left[\lambda_{1,1}\right]_{(m)}} & {\left[\lambda_{2,1}\right]_{(m)}} \\
\vdots & {\left[\lambda_{1,2}\right]_{(m-1)}} & {\left[\lambda_{2,2}\right]_{(m)}} \\
\lambda_{0,2 m} & \lambda_{1,3} &
\end{array}\right\} \\
\sum_{\nu=1}^{2 m} \lambda_{0, \nu}+m\left(\lambda_{1,1}+\lambda_{2,1}+\lambda_{2,2}\right)+(m-1) \lambda_{1,2}+\lambda_{1,3}=2 m-1 .
\end{gathered}
$$

The rigid decompositions

$$
\begin{aligned}
& 1 \cdots 1 \overline{1}, m m-1 \underline{1}, m m \\
& =0 \cdots 0 \overline{1}, 10 \underline{0}, \stackrel{i}{1} 0 \oplus 1 \cdots 1 \overline{0}, m-1 m-1 \underline{1}, \stackrel{i}{0} 1 \\
& =0 \cdots \stackrel{j}{\stackrel{j}{1}} \overline{1}, 11 \underline{0}, 11 \oplus 1 \cdots \stackrel{j}{j} \overline{0}, m-1 m-2 \underline{1}, m-1 m-1 \text {, }
\end{aligned}
$$

which are expressed by $E O_{2 m}=H_{1} \oplus E O_{2 m-1}=H_{2} \oplus E O_{2 m-2}$, give

$$
\begin{aligned}
& c\left(\lambda_{0,2 m} \rightsquigarrow \lambda_{1,3}\right)=\prod_{i=1}^{2} \frac{\Gamma\left(\lambda_{1, i}-\lambda_{1,3}\right)}{\Gamma\left(\mid\left\{\begin{array}{lll}
\lambda_{0,2 m} & \lambda_{1,1} & \lambda_{2, i}
\end{array}\right\}\right)} \cdot \prod_{j=1}^{2 m-1} \frac{\Gamma\left(\lambda_{0,2 m}-\lambda_{0, j}+1\right)}{\Gamma\left(\left|\left\{\begin{array}{ccc}
\lambda_{0, j} & \lambda_{1,1} & \lambda_{2,1} \\
\lambda_{0,2 m} & \lambda_{1,2} & \lambda_{2,2}
\end{array}\right\}\right|\right)},
\end{aligned}
$$

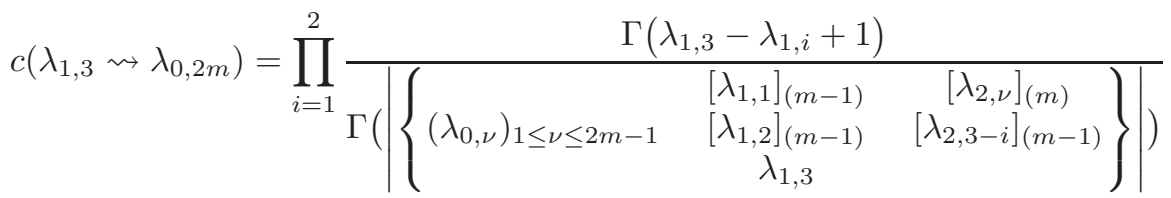

$$
\begin{aligned}
& \cdot \prod_{j=1}^{2 m-1} \frac{\Gamma\left(\lambda_{0, j}-\lambda_{0,2 m}\right)}{\Gamma\left(\left\{\begin{array}{ccc}
{\left[\lambda_{1,1}\right]_{(m-1)}} & {\left[\lambda_{2,1}\right]_{(m-1)}} \\
\left(\lambda_{0, \nu}\right)_{\substack{1 \leq \nu \leq 2 m-1 \\
\nu \neq j}} & {\left[\lambda_{1,2}\right]_{(m-2)}} & {\left[\lambda_{2,2}\right]_{(m-1)}} \\
\lambda_{1,3} &
\end{array}\right\}\right)}
\end{aligned}
$$


These formulas were obtained by the author in 2007 (cf. D6 ), which is a main motivation for the study in this paper. The condition for the irreducibility is

$$
\begin{cases}\lambda_{0, \nu}+\lambda_{1,1}+\lambda_{2, k} \notin \mathbb{Z} & (1 \leq \nu \leq 2 m, k=1,2), \\ \lambda_{0, \nu}+\lambda_{0, \nu^{\prime}}+\lambda_{1,1}+\lambda_{1,2}+\lambda_{2,1}+\lambda_{2,2}-1 \notin \mathbb{Z} & \left(1 \leq \nu<\nu^{\prime} \leq 2 m, k=1,2\right) .\end{cases}
$$

The shift operator for a compatible shift $\left(\epsilon_{j, \mu}\right)$ is bijective if and only if the values of each linear function in the above satisfy (13.30).

For the Fuchsian equation $\tilde{P} u=0$ of type $E O_{4}$ with the Riemann scheme

$$
\left\{\begin{array}{cccc}
x=\infty & 0 & 1 \\
{\left[a_{1}\right]_{(2)}} & b_{1} & {[0]_{(2)}} & ; x \\
{\left[a_{2}\right]_{(2)}} & b_{2} & c_{1} \\
& b_{3} & c_{2} & \\
& 0 &
\end{array}\right\}
$$

and the Fuchs relation

$$
2 a_{1}+2 a_{2}+b_{1}+b_{2}+b_{3}+c_{1}+c_{2}=3
$$

we have the connection formula

$$
c\left(0: 0 \rightsquigarrow 1: c_{2}\right)=\frac{\Gamma\left(c_{1}-c_{2}\right) \Gamma\left(-c_{2}\right) \prod_{\nu=1}^{3} \Gamma\left(1-b_{\nu}\right)}{\Gamma\left(a_{1}\right) \Gamma\left(a_{2}\right) \prod_{\nu=1}^{3} \Gamma\left(a_{1}+a_{2}+b_{\nu}+c_{1}-1\right)} .
$$

Let $\tilde{Q}$ be the Gauss hypergeometric operator with the Riemann scheme

$$
\left\{\begin{array}{ccc}
x=\infty & 0 & 1 \\
a_{1} & 1-a_{1}-a_{2}-c_{1} & 0 \\
a_{2} & 0 & c_{1}
\end{array}\right\} .
$$

We may normalize the operators by

$$
\tilde{P}=x^{3}(1-x) \partial^{4}+\cdots \text { and } \tilde{Q}=x(1-x) \partial^{2}+\cdots .
$$

Then

$$
\begin{aligned}
& \tilde{P}=\tilde{S} \tilde{Q}-\prod_{\nu=1}^{3}\left(a_{1}+a_{2}+b_{\nu}+c_{1}-1\right) \cdot \partial \\
& \tilde{Q}=\left(x(1-x) \partial+\left(a_{1}+a_{2}+c_{1}-\left(a_{1}+a_{2}+1\right) x\right)\right) \partial-a_{1} a_{2}
\end{aligned}
$$

with a suitable $\tilde{S}, \tilde{T} \in W[x]$ and $e \in \mathbb{C}$ and as is mentioned in Theorem 13.7, $\tilde{Q}$ is a shift operator satisfying

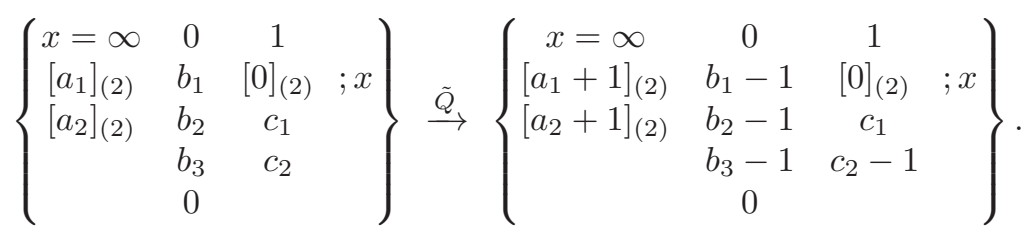

Let $u_{0}^{0}=1+\cdots$ and $u_{1}^{c_{2}}=(1-x)^{c_{2}}+\cdots$ be the normalized local solutions of $\tilde{P} u=0$ corresponding to the characteristic exponents 0 at 0 and $c_{2}$ at 1 , respectively. Then the direct calculation shows

$$
\begin{aligned}
\tilde{Q} u_{0}^{0} & =\frac{a_{1} a_{2} \prod_{\nu=1}^{3}\left(a_{1}+a_{2}+b_{\nu}+c_{1}-1\right)}{\prod_{\nu=1}^{3}\left(1-b_{\nu}\right)}+\cdots, \\
\tilde{Q} u_{1}^{c_{2}} & =c_{2}\left(c_{2}-c_{1}\right)(1-x)^{c_{2}-1}+\cdots .
\end{aligned}
$$


Denoting by $c\left(a_{1}, a_{2}, b_{1}, b_{2}, b_{3}, c_{1}, c_{2}\right)$ the connection coefficient $c\left(0: 0 \rightsquigarrow 1: c_{2}\right)$ for the equation with the Riemann scheme (15.30), we have

$$
\frac{c\left(a_{1}, a_{2}, b_{1}, b_{2}, b_{3}, c_{1}, c_{2}\right)}{c\left(a_{1}+1, a_{2}+1, b_{1}-1, b_{2}-1, b_{3}-1, c_{1}, c_{2}-1\right)}=\frac{a_{1} a_{2} \prod_{\nu=1}^{3}\left(a_{1}+a_{2}+b_{\nu}+c_{1}-1\right)}{\left(c_{1}-c_{2}\right)\left(-c_{2}\right) \prod_{\nu=1}^{3}\left(1-b_{\nu}\right)},
$$

which proves (15.32) since $\lim _{k \rightarrow \infty} c\left(a_{1}+k, a_{2}+k, b_{1}-k, b_{2}-k, b_{3}-k, c_{1}, c_{2}-k\right)=1$. Note that the shift operator $(15.33)$ is not bijective if and only if

$$
\tilde{Q} u=\prod_{\nu=1}^{3}\left(a_{1}+a_{2}+b_{\nu}+c_{1}-1\right) \cdot \partial u=0
$$

has a non-zero solution, which is equivalent to

$$
a_{1} a_{2} \prod_{\nu=1}^{3}\left(a_{1}+a_{2}+b_{\nu}+c_{1}-1\right)=0 .
$$

By the transformation $x \mapsto \frac{x}{x-1}$ we have

$$
\begin{aligned}
& \left\{\begin{array}{ccc}
x=\infty & 0 & 1 \\
{[0]_{(2)}} & 0 & {\left[a_{1}\right]_{(2)}} \\
c_{1} & b_{1} & {\left[a_{2}\right]_{(2)}} \\
c_{2} & b_{2} & \\
& b_{3} &
\end{array}\right\} \\
& \stackrel{(1-x)^{a_{1}} \partial^{1-a_{1}}(1-x)^{-a_{1}}}{\longrightarrow}\left\{\begin{array}{ccc}
x=\infty & 0 & 1 \\
2-2 a_{1} & & a_{1} \\
1+c_{1}-a_{1} & a_{1}+b_{1}-1 & {\left[a_{1}+a_{2}-1\right]_{(2)}} \\
1+c_{2}-a_{1} & a_{1}+b_{2}-1 & \\
& a_{1}+b_{3}-1 &
\end{array}\right\} \\
& \stackrel{x^{1-a_{1}-b_{1}}(1-x)^{1-a_{1}-a_{2}}}{\longrightarrow}\left\{\begin{array}{ccc}
x=\infty & 0 & 1 \\
a_{2}+b_{1} & & 1-a_{2} \\
a_{1}+a_{2}+b_{1}+c_{1}-1 & 0 & {[0]_{(2)}} \\
a_{1}+a_{2}+b_{1}+c_{2}-1 & b_{2}-b_{1} &
\end{array}\right\}
\end{aligned}
$$

and therefore Theorem 14.4 gives the following connection formula for (15.30):

$$
\begin{gathered}
c\left(0: b_{1} \rightsquigarrow \infty: a_{2}\right)=\frac{\Gamma\left(b_{1}+1\right) \Gamma\left(a_{1}-a_{2}\right)}{\Gamma\left(a_{1}+b_{1}\right) \Gamma\left(1-a_{2}\right)} \cdot{ }_{3} F_{2}\left(a_{2}+b_{1}, a_{1}+a_{2}+b_{1}+c_{1}-1,\right. \\
\left.a_{1}+a_{2}+b_{1}+c_{2}-1 ; b_{1}-b_{2}-1, b_{1}-b_{3}-1 ; 1\right) .
\end{gathered}
$$

In the same way, we have

$$
\begin{gathered}
c\left(1: c_{1} \rightsquigarrow \infty: a_{2}\right)=\frac{\Gamma\left(c_{1}+1\right) \Gamma\left(a_{1}-a_{2}\right)}{\Gamma\left(a_{1}+c_{1}\right) \Gamma\left(1-a_{2}\right)} \cdot{ }_{3} F_{2}\left(b_{1}-c_{1}, b_{2}-c_{1}, b_{3}-c_{1} ;\right. \\
\left.a_{1}+c_{1}, c_{1}-c_{2}+1 ; 1\right) .
\end{gathered}
$$

We will calculate generalized connection coefficients defined in Definition 14.17 . In fact, we get

$$
\begin{aligned}
& c\left(1:[0]_{(2)} \rightsquigarrow \infty:\left[a_{2}\right]_{(2)}\right)=\frac{\prod_{\nu=1}^{2} \Gamma\left(2-c_{\nu}\right) \cdot \prod_{i=1}^{2} \Gamma\left(a_{1}-a_{2}+i\right)}{\Gamma\left(a_{1}\right) \prod_{\nu=1}^{3} \Gamma\left(a_{1}+b_{\nu}\right)}, \\
& c\left(\infty:\left[a_{2}\right]_{(2)} \rightsquigarrow 1:[0]_{(2)}\right)=\frac{\prod_{\nu=1}^{2} \Gamma\left(c_{\nu}-1\right) \cdot \prod_{i=0}^{1} \Gamma\left(a_{2}-a_{1}-i\right)}{\Gamma\left(1-a_{1}\right) \prod_{\nu=1}^{3} \Gamma\left(1-a_{1}-b_{\nu}\right)}
\end{aligned}
$$


according to the procedure given in Remark 14.19, which we will explain.

The differential equation with the Riemann scheme $\left\{\begin{array}{ccc}x=\infty & 0 & 1 \\ \alpha_{1} & {[0]_{(2)}} & {[0]_{(2)}} \\ \alpha_{2} & {[\beta]_{(2)}} & \gamma_{1} \\ \alpha_{3} & & \gamma_{2} \\ \alpha_{4} & & \end{array}\right\}$ is

$P u=0$ with

$$
\begin{aligned}
P= & \prod_{j=1}^{4}\left(\vartheta+\alpha_{j}\right)+\partial(\vartheta-\beta)\left(\left(\partial-2 \vartheta+\gamma_{1}+\gamma_{2}-1\right)(\vartheta-\beta)\right. \\
& \left.+\sum_{1 \leq i<j \leq 3} \alpha_{i} \alpha_{j}-\left(\beta-2 \gamma_{1}-2 \gamma_{2}-4\right)(\beta-1)-\gamma_{1} \gamma_{2}+1\right) .
\end{aligned}
$$

The equation $P u=0$ is isomorphic to the system

$$
\begin{gathered}
\frac{d \tilde{u}}{d x}=\frac{A}{x} \tilde{u}+\frac{B}{x-1} \tilde{u} \\
A=\left(\begin{array}{llll}
0 & 0 & 1 & 0 \\
0 & 0 & 0 & 1 \\
0 & 0 & c & 0 \\
0 & 0 & 0 & c
\end{array}\right), B=\left(\begin{array}{llll}
0 & 0 & 0 & 0 \\
0 & 0 & 0 & 0 \\
s & 1 & a & 0 \\
r & t & 0 & b
\end{array}\right), \tilde{u}=\left(\begin{array}{l}
u_{1} \\
u_{2} \\
u_{3} \\
u_{4}
\end{array}\right)
\end{gathered}
$$

by the correspondence

$$
\left\{\begin{array}{l}
u_{1}=u \\
u_{2}=(x-1) x u^{\prime \prime}+((1-a-c) x+a-1) u^{\prime}-s u, \\
u_{3}=x u^{\prime}, \\
u_{4}=x^{2}(x-1) u^{\prime \prime \prime}+\left((3-a-c) x^{2}+(a-2) x\right) u^{\prime \prime}+(1-a-c-s) x u^{\prime},
\end{array}\right.
$$

where we may assume $\operatorname{Re} \gamma_{1} \geq \operatorname{Re} \gamma_{2}$ and

$$
\begin{gathered}
\beta=c, \gamma_{1}=a+1, \gamma_{2}=b+2, \\
\prod_{\nu=1}^{4}\left(\xi-\alpha_{\nu}\right)=\xi^{4}+(a+b+2 c) \xi^{3}+((a+c)(b+c)-s-t) \xi^{2} \\
-((b+c) s+(a+c) t) \xi+s t-r .
\end{gathered}
$$

Here $s, t$ and $r$ are uniquely determined from $\alpha_{1}, \alpha_{2}, \alpha_{3}, \alpha_{4}, \beta, \gamma_{1}, \gamma_{2}$ because $b+c \neq$ $a+c$. We remark that $\operatorname{Ad}\left(x^{-c}\right) \tilde{u}$ satisfies a system of Okubo normal form.

Note that the shift of parameters $\left(\alpha_{1}, \ldots, \alpha_{4}, \beta, \gamma_{1}, \gamma_{2}\right) \mapsto\left(\alpha_{1}, \ldots, \alpha_{4}, \beta-1, \gamma_{1}+\right.$ $\left.1, \gamma_{2}+1\right)$ corresponds to the shift $(a, b, c, s, t, r) \mapsto(a+1, b+1, c-1, s, t, r)$.

Let $u_{\alpha_{1}, \ldots, \alpha_{4}, \beta, \gamma_{1}, \gamma_{2}}^{j}(x)$ be local holomorphic solutions of $P u=0$ in a neighborhood of $x=0$ determined by

$$
\begin{aligned}
u_{\alpha_{1}, \ldots, \alpha_{4}, \beta, \gamma_{1}, \gamma_{2}}^{j}(0) & =\delta_{j, 0}, \\
\left(\frac{d}{d x} u_{\alpha_{1}, \ldots, \alpha_{4}, \beta, \gamma_{1}, \gamma_{2}}^{j}\right)(0) & =\delta_{j, 1}
\end{aligned}
$$

for $j=0$ and 1 . Then Theorem 14.10 proves

$$
\lim _{k \rightarrow \infty} \frac{d^{\nu}}{d x^{\nu}} u_{\alpha, \beta-k, \gamma_{1}+k, \gamma_{1}+k}^{0}(x)=\delta_{0, \nu} \quad(\nu=0,1,2, \ldots)
$$

uniformly on $\bar{D}=\{x \in \mathbb{C} ;|x| \leq 1\}$.

Put $u=v_{\alpha, \beta, \gamma_{1}, \gamma_{2}}=\left(\gamma_{1}-2\right)^{-1} u_{\alpha, \beta, \gamma}^{1}$. Then Theorem 14.10 proves

$$
\begin{aligned}
& \lim _{k \rightarrow \infty} \frac{d^{\nu}}{d x^{\nu}} v_{\alpha, \beta-k, \gamma_{1}+k, \gamma_{2}+k}(x)=0 \quad(\nu=0,1,2, \ldots), \\
& \lim _{k \rightarrow \infty}\left((x-1) x \frac{d^{2}}{d x^{2}}+\left(\left(2-\beta-\gamma_{1}\right) x+\gamma_{1}+k-2\right) \frac{d}{d x}-s\right) v_{\alpha, \beta-k, \gamma_{1}+k, \gamma_{2}+k}(x)=1
\end{aligned}
$$


uniformly on $\bar{D}$. Hence

$$
\lim _{k \rightarrow \infty}\left(\frac{d}{d x} u_{\alpha, \beta-k, \gamma+1, \gamma_{1}+k}^{1}\right)(x)=1
$$

uniformly on $\bar{D}$. Thus we obtain

$$
\left.\lim _{k \rightarrow \infty} c\left(\infty:\left[a_{2}\right]_{(2)} \rightsquigarrow 1:[0]_{(2)}\right)\right|_{a_{1} \mapsto a_{1}-k, c_{1} \mapsto c_{1}+k, c_{2} \mapsto c_{2}+k}=1
$$

for the connection coefficient in (15.35). Then the procedure given in Remark 14.19 and Corollary 14.22 with the rigid decompositions

$$
\begin{aligned}
2 \underline{2}, 1111, \overline{2} 11 & =1 \underline{2}, 0111, \overline{1} 11 \oplus \underline{10}, 1000,100=1 \underline{2}, 1011, \overline{1} 11 \oplus 1 \underline{0}, 0100, \overline{1} 00 \\
& =1 \underline{2}, 1101, \overline{1} 11 \oplus 1 \underline{0}, 0010, \overline{1} 00=1 \underline{2}, 1101, \overline{1} 11 \oplus 1 \underline{0}, 0010, \overline{1} 00
\end{aligned}
$$

prove (15.35). Corresponding to Remark 14.19 (4), we note

$$
\sum_{\nu=1}^{2}\left(c_{\nu}-1\right)+\sum_{i=0}^{1}\left(a_{2}-a_{1}-i\right)=\left(1-a_{1}\right)+\sum_{\nu=1}^{3}\left(1-a_{1}-b_{\nu}\right)
$$

because of the Fuchs relation (15.31). We can similarly obtain (15.34).

The holomorphic solution of $\tilde{P} u=0$ at the origin is given by

$$
\begin{aligned}
& u_{0}(x)=\sum_{m \geq 0, n \geq 0} \\
& \frac{\left(a_{1}+a_{2}+b_{3}+c_{2}-1\right)_{n} \prod_{\nu=1}^{2}\left(\left(a_{\nu}\right)_{m+n}\left(a_{1}+a_{2}+b_{\nu}+c_{1}-1\right)_{m}\right)}{\left(1-b_{1}\right)_{m+n}\left(1-b_{2}\right)_{m+n}\left(1-b_{3}\right)_{m} m ! n !} x^{m+n}
\end{aligned}
$$

and it has the integral representation

$$
\begin{aligned}
u_{0}(x)= & \frac{\prod_{\nu=1}^{3} \Gamma\left(1-b_{\nu}\right)}{\prod_{\nu=1}^{2}\left(\Gamma\left(a_{\nu}\right) \Gamma\left(1-a_{\nu}-b_{\nu}\right) \Gamma\left(b_{\nu}+c_{\nu}+a_{1}+a_{2}-1\right)\right)} \\
& \int_{0}^{x} \int_{0}^{s_{0}} \int_{0}^{s_{1}} x^{b_{1}}\left(x-s_{0}\right)^{-b_{1}-a_{1}} s_{0}^{b_{2}+a_{1}-1}\left(s_{0}-s_{1}\right)^{-b_{2}-a_{2}} \\
& \cdot s_{1}^{b_{3}+a_{2}-1}\left(1-s_{1}\right)^{-b_{3}-c_{1}-a_{2}-a_{1}+1}\left(s_{1}-s_{2}\right)^{c_{1}+b_{1}+a_{2}+a_{1}-2} \\
& \cdot s_{2}^{b_{2}+c_{2}+a_{2}+a_{1}-2}\left(1-s_{2}\right)^{-c_{2}-b_{1}-a_{2}-a_{1}+1} d s_{2} d s_{1} d s_{0} .
\end{aligned}
$$

The equation is irreducible if and only if any value of the following linear functions is not an integer.

$$
\begin{aligned}
& a_{1} a_{2} \\
& a_{1}+b_{1} \quad a_{1}+b_{2} \quad a_{1}+b_{3} \quad a_{2}+b_{1} \quad a_{2}+b_{2} \quad a_{2}+b_{3} \\
& a_{1}+a_{2}+b_{1}+c_{1}-1 \quad a_{1}+a_{2}+b_{1}+c_{2}-1 \quad a_{1}+a_{2}+b_{2}+c_{1}-1 \\
& a_{1}+a_{2}+b_{2}+c_{2}-1 \quad a_{1}+a_{2}+b_{3}+c_{1}-1 \quad a_{1}+a_{2}+b_{2}+c_{2}-1 \text {. }
\end{aligned}
$$

In the same way we have the connection coefficients for odd family. $E O_{2 m+1}\left(\mathbf{m}=\left(1^{2 m+1}, m m 1, m+1 m\right)\right.$ : odd family $)$

$$
\left\{\begin{array}{ccc}
x=\infty & 0 & 1 \\
\lambda_{0,1} & {\left[\lambda_{1,1}\right]_{(m)}} & {\left[\lambda_{2,1}\right]_{(m+1)}} \\
\vdots & {\left[\lambda_{1,2}\right]_{(m)}} & {\left[\lambda_{2,2}\right]_{(m)}} \\
\lambda_{0,2 m+1} & \lambda_{1,3} &
\end{array}\right\}
$$




$$
\begin{aligned}
& \sum_{\nu=1}^{2 m+1} \lambda_{0, \nu}+m\left(\lambda_{1,1}+\lambda_{1,2}+\lambda_{2,2}\right)+(m+1) \lambda_{2,1}+\lambda_{1,3}=2 m .
\end{aligned}
$$

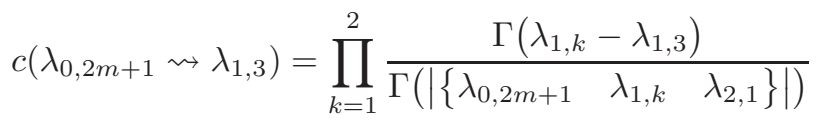

$$
\begin{aligned}
& \cdot \prod_{k=1}^{2 m} \frac{\Gamma\left(\lambda_{0,2 m+1}-\lambda_{0, k}+1\right)}{\Gamma\left(\left|\left\{\begin{array}{ccc}
\lambda_{0, k} & \lambda_{1,1} & \lambda_{2,1} \\
\lambda_{0,2 m+1} & \lambda_{1,2} & \lambda_{2,2}
\end{array}\right\}\right|\right)},
\end{aligned}
$$

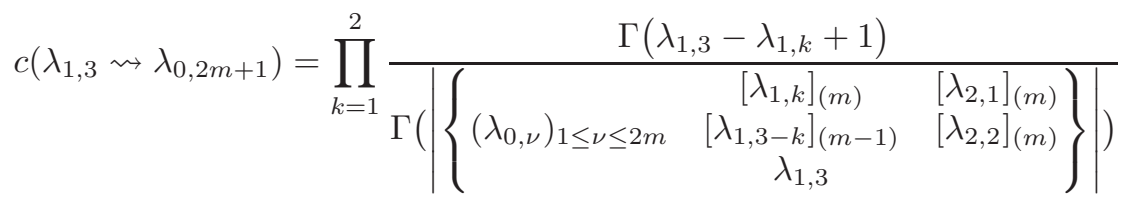

$$
\begin{aligned}
& \cdot \prod_{k=1}^{2 m} \frac{\Gamma\left(\lambda_{0, k}-\lambda_{0,2 m+1}\right)}{\Gamma\left(\left|\left\{\begin{array}{ccc}
\left(\lambda_{0, \nu}\right)_{\substack{1 \leq \nu \leq 2 m \\
\nu \neq k}} & \left.\left[\lambda_{1,1}\right]_{(m-1)}\right]_{(m-1)} & {\left[\lambda_{2,1}\right]_{(m)}} \\
\lambda_{1,3} & {\left[\lambda_{2,2}\right]_{(m-1)}}
\end{array}\right\}\right|\right)} \text {. }
\end{aligned}
$$

The condition for the irreducibility is

$$
\begin{cases}\lambda_{0, \nu}+\lambda_{1, k}+\lambda_{2,1} \notin \mathbb{Z} & (1 \leq \nu \leq 2 m+1, k=1,2), \\ \lambda_{0, \nu}+\lambda_{0, \nu^{\prime}}+\lambda_{1,1}+\lambda_{1,2}+\lambda_{2,1}+\lambda_{2,2}-1 \notin \mathbb{Z} & \left(1 \leq \nu<\nu^{\prime} \leq 2 m+1, k=1,2\right) .\end{cases}
$$

The same statement using the above linear functions as in the case of even family is valid for the bijectivity of the shift operator with respect to compatible shift $\left(\epsilon_{j, \nu}\right)$.

We note that the operation $\operatorname{RAd}\left(\partial^{-\mu}\right) \circ \operatorname{RAd}\left(x^{-\lambda_{1,2}}(1-x)^{-\lambda_{2,2}}\right)$ transforms the operator and solutions with the above Riemann scheme of type $E O_{n}$ into those of type $E O_{n+1}$ :

$$
\begin{aligned}
& \left\{\begin{array}{ccc}
\lambda_{0,1} & {\left[\lambda_{1,1}\right]_{\left(\left[\frac{n}{2}\right]\right)}} & {\left[\lambda_{2,1}\right]_{\left(\left[\frac{n+1}{2}\right]\right)}} \\
\vdots & {\left[\lambda_{1,2}\right]_{\left(\left[\frac{n-1}{2}\right]\right)}} & {\left[\lambda_{2,2}\right]_{\left(\left[\frac{n}{2}\right]\right)}} \\
\lambda_{0, n} & \lambda_{1,3} &
\end{array}\right\}
\end{aligned}
$$

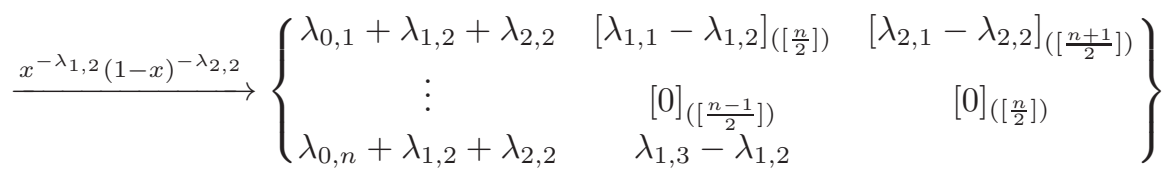

$$
\begin{aligned}
& \stackrel{\partial^{-\mu}}{\longrightarrow}\left\{\begin{array}{ccc}
\lambda_{0,1}+\lambda_{1,2}+\lambda_{2,2}-\mu & {\left[\lambda_{1,1}-\lambda_{1,2}+\mu\right]_{\left(\left[\frac{n}{2}\right]\right)}} & {\left[\lambda_{2,1}-\lambda_{2,2}+\mu\right]_{\left(\left[\frac{n+1}{2}\right]\right)}} \\
\vdots & {[\mu]_{\left(\left[\frac{n+1}{2}\right]\right)}} & {[\mu]_{\left(\left[\frac{n+2}{2}\right]\right)}} \\
\lambda_{0, n}+\lambda_{1,2}+\lambda_{2,2}-\mu & \lambda_{1,3}-\lambda_{1,2}+\mu & \\
1-\mu &
\end{array}\right\} \text {. }
\end{aligned}
$$

15.6. Trigonometric identities. The connection coefficients corresponding to the Riemann scheme of the hypergeometric family in $\$ 15.4$ satisfy

$$
\begin{gathered}
\sum_{\nu=1}^{n} c\left(1: \lambda_{1,2} \rightsquigarrow 0: \lambda_{0, \nu}\right) \cdot c\left(0: \lambda_{1, \nu} \rightsquigarrow 1: \lambda_{1,2}\right)=1, \\
\sum_{\nu=1}^{n} c\left(\infty: \lambda_{2, i} \rightsquigarrow 0: \lambda_{0, \nu}\right) \cdot c\left(0: \lambda_{0, \nu} \rightsquigarrow \infty: \lambda_{2, j}\right)=\delta_{i j} .
\end{gathered}
$$


These equations with Remark 14.8 iii) give the identities

$$
\begin{gathered}
\sum_{k=1}^{n} \frac{\prod_{\nu \in\{1, \ldots, n\}} \sin \left(x_{k}-y_{\nu}\right)}{\prod_{\nu \in\{1, \ldots, n\} \backslash\{k\}} \sin \left(x_{k}-x_{\nu}\right)}=\sin \left(\sum_{\nu=1}^{n} x_{\nu}-\sum_{\nu=1}^{n} y_{\nu}\right), \\
\sum_{k=1}^{n} \prod_{\nu \in\{1, \ldots, n\} \backslash\{k\}} \frac{\sin \left(y_{i}-x_{\nu}\right)}{\sin \left(x_{k}-x_{\nu}\right)} \prod_{\nu \in\{1, \ldots, n\} \backslash\{j\}} \frac{\sin \left(x_{k}-y_{\nu}\right)}{\sin \left(y_{j}-y_{\nu}\right)}=\delta_{i j} \quad(1 \leq i, j \leq n) .
\end{gathered}
$$

We have the following identity from the connection coefficients of even/odd families.

$$
\begin{aligned}
& \sum_{k=1}^{n} \sin \left(x_{k}+s\right) \cdot \sin \left(x_{k}+t\right) \cdot \prod_{\nu \in\{1, \ldots, n\} \backslash\{k\}} \frac{\sin \left(x_{k}+x_{\nu}+2 u\right)}{\sin \left(x_{k}-x_{\nu}\right)} \\
& = \begin{cases}\sin \left(n u+\sum_{\nu=1}^{n} x_{\nu}\right) \cdot \sin \left(s+t+(n-2) u+\sum_{\nu=1}^{n} x_{\nu}\right) & \text { if } n=2 m, \\
\sin \left(s+(n-1) u+\sum_{\nu=1}^{n} x_{\nu}\right) \cdot \sin \left(t+(n-1) u+\sum_{\nu=1}^{n} x_{\nu}\right) & \text { if } n=2 m+1 .\end{cases}
\end{aligned}
$$

The direct proof of these identities using residue calculus is given by $\mathrm{Od}$. It is interesting that similar identities of rational functions are given in [G] Appendix] which studies the systems of Schlesinger canonical form corresponding to Simpson's list (cf. $\$ 15.2$ ).

\subsection{Rigid examples of order at most 4 .}

15.7.1. order 1 . $1,1,1$

$$
u(x)=x^{\lambda_{1}}(1-x)^{\lambda_{2}} \quad\left\{\begin{array}{lll}
-\lambda_{1}-\lambda_{2} & \lambda_{1} & \lambda_{2}
\end{array}\right\}
$$

15.7.2. order 2. $11,11,11: H_{2}$ (Gauss) $\quad[\Delta(\mathbf{m})]=1^{4}$

$$
u_{H_{2}}=\partial^{-\mu_{1}} u(x) \quad\left\{\begin{array}{ccc}
-\mu_{1}+1 & 0 & 0 \\
-\lambda_{1}-\lambda_{2}-\mu_{1} & \lambda_{1}+\mu_{1} & \lambda_{2}+\mu_{1}
\end{array}\right\}
$$

15.7.3. order 3 . There are two types.

$$
\underline{111,21,111}: H_{3}\left({ }_{3} F_{2}\right) \quad[\Delta(\mathbf{m})]=1^{9}
$$

$$
\begin{aligned}
& u_{H_{3}}=\partial^{-\mu_{2}} x^{\lambda_{3}} u_{H_{2}} \\
& \left\{\begin{array}{ccc}
1-\mu_{2} & 0 & {[0]_{(2)}} \\
-\lambda_{3}-\mu_{1}-\mu_{2}+1 & \lambda_{3}+\mu_{2} & \\
-\lambda_{1}-\lambda_{2}-\lambda_{3}-\mu_{1}-\mu_{2} & \lambda_{1}+\lambda_{3}+\mu_{1}+\mu_{2} & \lambda_{2}+\mu_{1}+\mu_{2}
\end{array}\right\}
\end{aligned}
$$

$\underline{21,21,21,21}: P_{3}$ (Jordan-Pochhammer) $\quad[\Delta(\mathbf{m})]=1^{4} \cdot 2$

$$
\begin{aligned}
& u_{P_{3}}=\partial^{-\mu} x^{\lambda_{0}}(1-x)^{\lambda_{1}}\left(c_{2}-x\right)^{\lambda_{2}} \\
& \left\{\begin{array}{cccc}
{[1-\mu]_{(2)}} & {[0]_{(2)}} & {[0]_{(2)}} & {[0]_{(2)}} \\
-\lambda_{0}-\lambda_{1}-\lambda_{2}-\mu & \lambda_{0}+\mu & \lambda_{1}+\mu & \lambda_{2}+\mu
\end{array}\right\}
\end{aligned}
$$

15.7.4. order 4. There are 6 types.

$$
\begin{aligned}
& \frac{211,211,211: \alpha_{2}}{\partial^{-\mu_{2}} x^{\lambda_{3}}(1-x)^{\lambda_{4}} u_{H_{2}}}[\Delta(\mathbf{m})]=1^{10} \cdot 2 \\
& \left\{\begin{array}{ccc}
{\left[-\mu_{2}+1\right]_{(2)}} & {[0]_{(2)}} & {[0]_{(2)}} \\
-\mu_{1}-\lambda_{3}-\lambda_{4}-\mu_{2}+1 & \lambda_{3}+\mu_{2} & \lambda_{4}+\mu_{2} \\
-\lambda_{1}-\lambda_{2}-\lambda_{3}-\lambda_{4}-\mu_{1}-\mu_{2} & \lambda_{1}+\lambda_{3}+\mu_{1}+\mu_{2} & \lambda_{2}+\lambda_{4}+\mu_{1}+\mu_{2}
\end{array}\right\}
\end{aligned}
$$




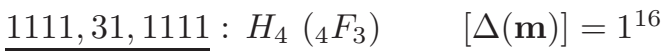

$$
\begin{aligned}
& \partial^{-\mu_{3}} x^{\lambda_{4}} u_{H_{3}} \\
& \left\{\begin{array}{ccc}
-\mu_{3}+1 & 0 & {[0]_{(3)}} \\
-\lambda_{4}-\mu_{2}-\mu_{3}+1 & \lambda_{4} & \\
-\lambda_{3}-\lambda_{4}-\mu_{1}-\mu_{2}-\mu_{3}+1 & \lambda_{3}+\lambda_{4}+\mu_{2}+\mu_{3} & \\
-\lambda_{1}-\cdots-\lambda_{4}-\mu_{1}-\mu_{2}-\mu_{3} & \lambda_{1}+\cdots+\lambda_{4}+\mu_{1}+\mu_{2}+\mu_{3} & \lambda_{2}+\mu_{1}+\mu_{2}+\mu_{3}
\end{array}\right\} \\
& \text { 211,22,1111: } E O_{4} \quad[\Delta(\mathbf{m})]=1^{14} \\
& \partial^{-\mu_{3}}(1-x)^{-\lambda^{\prime}} u_{H_{3}}, \quad \lambda^{\prime}=\lambda_{2}+\mu_{1}+\mu_{2} \\
& \left\{\begin{array}{ccc}
\lambda_{2}+\mu_{1}-\mu_{2}-\mu_{3}+1 & {[0]_{(2)}} & {\left[-\lambda_{2}-\mu_{1}-\mu_{2}+\mu_{3}\right]_{(2)}} \\
\lambda_{2}-\lambda_{3}-\mu_{3}+1 & \lambda_{3}+\mu_{2}+\mu_{3} & \\
-\lambda_{1}-\lambda_{3}-\mu_{3} & \lambda_{1}+\lambda_{3}+\mu_{1}+\mu_{2}+\mu_{3} & {[0]_{(2)}} \\
-\mu_{3}+1 &
\end{array}\right\}
\end{aligned}
$$

We have the integral representation of the local solution corresponding to the exponent at 0 :

$$
\begin{aligned}
& \int_{0}^{x} \int_{0}^{t} \int_{0}^{s}(1-t)^{-\lambda_{2}-\mu_{1}-\mu_{2}}(x-t)^{\mu_{3}-1} s^{\lambda_{3}}(t-s)^{\mu_{2}-1} u^{\lambda_{1}}(1-u)^{\lambda_{2}}(s-u)^{\mu-1} d u d s d t . \\
& \text { 211,22,31,31: } I_{4} \quad[\Delta(\mathbf{m})]=1^{6} \cdot 2^{2} \\
& \partial^{-\mu_{2}}\left(c_{2}-x\right)^{\lambda_{3}} u_{H_{2}} \\
& \left\{\begin{array}{cccc}
{\left[-\mu_{2}+1\right]_{(2)}} & {[0]_{(3)}} & {[0]_{(3)}} & {[0]_{(2)}} \\
-\lambda_{3}-\mu_{1}-\mu_{2}+1 & & & {\left[\lambda_{3}+\mu_{2}\right]_{(2)}} \\
-\lambda_{1}-\lambda_{2}-\lambda_{3}-\mu_{1}-\mu_{2} & \lambda_{1}+\mu_{1}+\mu_{2} & \lambda_{2}+\mu_{1}+\mu_{2} &
\end{array}\right\}
\end{aligned}
$$

31,31,31,31,31: $P_{4} \quad[\Delta(\mathbf{m})]=1^{5} \cdot 3$

$$
\begin{aligned}
& u_{P_{4}}=\partial^{-\mu} x^{\lambda_{0}}(1-x)^{\lambda_{1}}\left(c_{2}-x\right)^{\lambda_{2}}\left(c_{3}-x\right)^{\lambda_{3}} \\
& \left\{\begin{array}{ccccc}
{[-\mu+1]_{(3)}} & {[0]_{(3)}} & {[0]_{(3)}} & {[0]_{(3)}} & {[0]_{(3)}} \\
-\lambda_{0}-\lambda_{2}-\lambda_{3}-\mu & \lambda_{0}+\mu & \lambda_{1}+\mu & \lambda_{2}+\mu & \lambda_{3}+\mu
\end{array}\right\}
\end{aligned}
$$

22,22,22,31: $P_{4,4} \quad[\Delta(\mathbf{m})]=1^{8} \cdot 2$

$$
\begin{aligned}
& \partial^{-\mu^{\prime}} x^{-\lambda_{0}^{\prime}}(1-x)^{-\lambda_{1}^{\prime}}\left(c_{2}-x\right)^{-\lambda_{2}^{\prime}} u_{P_{3}}, \quad \lambda_{j}^{\prime}=\lambda_{j}+\mu, \quad \mu^{\prime}=\lambda_{0}+\lambda_{1}+\lambda_{2}+2 \mu \\
& \left\{\begin{array}{cccc}
{\left[1-\mu^{\prime}\right]_{(3)}} & {\left[\lambda_{1}+\lambda_{2}+\mu\right]_{(2)}} & {\left[\lambda_{0}+\lambda_{2}+\mu\right]_{(2)}} & {\left[\lambda_{0}+\lambda_{1}+\mu\right]_{(2)}} \\
-\lambda_{0}-\lambda_{1}-\lambda_{2} & {[0]_{(2)}} & {[0]_{(2)}} & {[0]_{(2)}}
\end{array}\right\}
\end{aligned}
$$

15.7.5. Tuple of partitions : 211,211,211. $\quad[\Delta(\mathbf{m})]=1^{10} \cdot 2$

$$
211,211,211=H_{1} \oplus H_{3}: 6=H_{2} \oplus H_{2}: 4=2 H_{1} \oplus H_{2}: 1
$$

From the operations

$$
\begin{aligned}
& \left\{\begin{array}{ccc}
x=\infty & 0 & 1 \\
1-\mu_{1} & 0 & 0 \\
-\alpha_{1}-\beta_{1}-\mu_{1} & \frac{\alpha_{1}+\mu_{1}}{x} & \beta_{1}+\mu_{1}
\end{array}\right\} \\
& \stackrel{x^{\alpha_{2}}(1-x)^{\beta_{2}}}{\longrightarrow}\left\{\begin{array}{ccc}
x=\infty \\
1-\alpha_{2}-\beta_{2}-\mu_{1}
\end{array}\right. \\
& \left.\begin{array}{ccc}
1-\alpha_{2}-\beta_{1}-\beta_{2}-\mu_{1} & \alpha_{1}+\alpha_{2}+\mu_{1} & \beta_{1}+\beta_{2}+\mu_{1}
\end{array}\right\} \\
& \stackrel{\partial^{-\mu_{2}} \longrightarrow}{x=\infty}\left\{\begin{array}{ccc}
0 & 1 \\
{\left[-\mu_{2}+1\right]_{(2)}} & {[0]_{(2)}} & {[0]_{(2)}} \\
1-\beta_{2}-\mu_{1}-\mu_{2} & \alpha_{2}+\mu_{2} & \beta_{2}+\mu_{2} \\
-\alpha_{1}-\beta_{1}-\beta_{2}-\mu_{1}-\mu_{2} & \frac{\alpha_{1}+\mu_{1}+\mu_{2}}{\longrightarrow} & \beta_{1}+\beta_{2}+\mu_{1}+\mu_{2}
\end{array}\right\}
\end{aligned}
$$




$$
\longrightarrow\left\{\begin{array}{ccc}
x=\infty & 0 & 1 \\
{\left[\lambda_{2,1}\right]_{(2)}} & {\left[\lambda_{0,1}\right]_{(2)}} & {\left[\lambda_{1,1}\right]_{(2)}} \\
\lambda_{2,2} & \lambda_{0,2} & \lambda_{1,2} \\
\lambda_{2,3} & \lambda_{0,3} & \lambda_{1,3}
\end{array}\right\} \quad \text { with } \sum_{j=0}^{2}\left(2 \lambda_{j, 1}+\lambda_{j, 2}+\lambda_{j, 3}\right)=3,
$$

we have the integral representation of the solutions as in the case of other examples we have explained and so here we will not discuss them. The universal operator of type $11,11,11$ is

$$
Q=x^{2}(1-x)^{2} \partial^{2}-(a x+b) x(1-x) \partial+\left(c x^{2}+d x+e\right) .
$$

Here we have

$$
\begin{array}{rlrl}
b & =\lambda_{0,1}^{\prime}+\lambda_{0,2}^{\prime}-1, & e & =\lambda_{0,1}^{\prime} \lambda_{0,2}^{\prime}, \\
-a-b & =\lambda_{1,1}^{\prime}+\lambda_{1,2}^{\prime}-1, & c+d+e & =\lambda_{1,1}^{\prime} \lambda_{1,2}^{\prime}, \\
c & =\lambda_{2,1}^{\prime} \lambda_{2,2}^{\prime}, \\
\lambda_{0,1}^{\prime} & =\alpha_{2}, & \lambda_{0,2}^{\prime} & =\alpha_{1}+\alpha_{2}+\mu_{1}, \\
\lambda_{1,1}^{\prime} & =\beta_{2}, & \lambda_{1,2}^{\prime} & =\beta_{1}+\beta_{2}+\mu_{2}, \\
\lambda_{2,1}^{\prime} & =1-\beta_{2}-\mu_{1}-\mu_{2}, & \lambda_{2,2}^{\prime} & =-\alpha_{1}-\beta_{1}-\beta_{2}-\mu_{1}-\mu_{2}
\end{array}
$$

corresponding to the above second Riemann scheme. The operator corresponding to the tuple $211,211,211$ is

$$
\begin{aligned}
P= & \operatorname{RAd}\left(\partial^{-\mu_{2}}\right) Q \\
= & \operatorname{RAd}\left(\partial^{-\mu_{2}}\right)\left(\left(\vartheta-\lambda_{0,1}^{\prime}\right)\left(\vartheta-\lambda_{0,2}^{\prime}\right)\right. \\
& +x\left(-2 \vartheta^{2}+\left(2 \lambda_{0,1}^{\prime}+2 \lambda_{0,2}^{\prime}+\lambda_{1,1}^{\prime}+\lambda_{1,2}^{\prime}-1\right) \vartheta+\lambda_{1,1}^{\prime} \lambda_{1,2}^{\prime}-\lambda_{0,1}^{\prime} \lambda_{0,2}^{\prime}-\lambda_{2,1}^{\prime} \lambda_{2,2}^{\prime}\right) \\
& \left.+x^{2}\left(\vartheta+\lambda_{2,1}^{\prime}\right)\left(\vartheta+\lambda_{2,2}^{\prime}\right)\right) \\
= & \partial^{2}\left(\vartheta-\lambda_{0,1}^{\prime}-\mu_{2}\right)\left(\vartheta-\lambda_{0,2}^{\prime}-\mu_{2}\right) \\
& +\partial\left(\vartheta-\mu_{2}+1\right)\left(-2\left(\vartheta-\mu_{2}\right)^{2}+\left(2 \lambda_{0,1}^{\prime}+2 \lambda_{0,2}^{\prime}+\lambda_{1,1}^{\prime}+\lambda_{1,2}^{\prime}-1\right)\left(\vartheta-\mu_{2}\right)\right. \\
& \left.+\lambda_{1,1}^{\prime} \lambda_{1,2}^{\prime}-\lambda_{0,1}^{\prime} \lambda_{0,2}^{\prime}-\lambda_{2,1}^{\prime} \lambda_{2,2}^{\prime}\right) \\
& +\left(\vartheta-\mu_{2}+1\right)\left(\vartheta-\mu_{2}+2\right)\left(\vartheta+\lambda_{2,1}^{\prime}-\mu_{2}\right)\left(\vartheta+\lambda_{2,2}^{\prime}-\mu_{2}\right) .
\end{aligned}
$$

The condition for the irreducibility:

$$
\left\{\begin{array}{l}
\lambda_{0,1}+\lambda_{1,1}+\lambda_{2,1} \notin \mathbb{Z}, \\
\lambda_{0, \nu}+\lambda_{1,1}+\lambda_{2,1} \notin \mathbb{Z}, \lambda_{0,1}+\lambda_{1, \nu}+\lambda_{2,1} \notin \mathbb{Z}, \lambda_{0,1}+\lambda_{1,1}+\lambda_{2, \nu} \notin \mathbb{Z} \quad(\nu=2,3), \\
\lambda_{0,1}+\lambda_{0,2}+\lambda_{1,1}+\lambda_{1, \nu}+\lambda_{2,1}+\lambda_{2, \nu^{\prime}} \notin \mathbb{Z} \quad\left(\nu, \nu^{\prime} \in\{2,3\}\right) .
\end{array}\right.
$$

There exist three types of direct decompositions of the tuple and there are 4 direct decompositions which give the connection coefficient $c\left(\lambda_{0,3} \rightsquigarrow \lambda_{1,3}\right)$ by the formula (14.10) in Theorem 14.6:

$$
\begin{aligned}
21 \overline{1}, 21 \underline{1}, 211 & =00 \overline{1}, 100,100 \oplus 210,11 \underline{1}, 111 \\
& =11 \overline{1}, 210,111 \oplus 100,00 \underline{1}, 100 \\
& =10 \overline{1}, 110,110 \oplus 110,10 \underline{1}, 101 \\
& =10 \overline{1}, 110,101 \oplus 110,10 \underline{1}, 110
\end{aligned}
$$


Thus we have

$$
\begin{aligned}
c\left(\lambda_{0,3} \rightsquigarrow \lambda_{1,3}\right)= & \frac{\prod_{\nu=1}^{2} \Gamma\left(\lambda_{0,3}-\lambda_{0, \nu}+1\right)}{\Gamma\left(\lambda_{0,3}+\lambda_{1,1}+\lambda_{2,1}\right) \cdot \Gamma\left(1-\lambda_{0,1}-\lambda_{1,3}-\lambda_{2,1}\right)} \\
& \cdot \frac{\prod_{\nu=1}^{2} \Gamma\left(\lambda_{1, \nu}-\Gamma_{1,3}\right)}{\prod_{\nu=2}^{3} \Gamma\left(\lambda_{0,1}+\lambda_{0,3}+\lambda_{1,1}+\lambda_{1,2}+\lambda_{2,1}+\lambda_{2, \nu}-1\right)} .
\end{aligned}
$$

We can also calculate generalized connection coefficient defined in Definition 14.17:

$$
c\left(\left[\lambda_{0,1}\right]_{(2)} \rightsquigarrow\left[\lambda_{1,1}\right]_{(2)}\right)=\frac{\prod_{\nu=2}^{3}\left(\Gamma\left(\lambda_{0,1}-\lambda_{0, \nu}+2\right) \cdot \Gamma\left(\lambda_{1, \nu}-\lambda_{1,1}-1\right)\right)}{\prod_{\nu=2}^{3}\left(\Gamma\left(\lambda_{0,1}+\lambda_{1, \nu}+\lambda_{2,1}\right) \cdot \Gamma\left(1-\lambda_{0, \nu}-\lambda_{1,1}-\lambda_{2,1}\right)\right)} .
$$

This can be proved by the procedure given in Remark 14.19 as in the case of the formula (15.35). Note that the gamma functions in the numerator of this formula correspond to Remark 14.19 (2) and those in the denominator correspond to the rigid decompositions

$$
\begin{aligned}
& \underline{2} 11, \overline{2} 11,211=\underline{100}, \overline{0} 10,100 \oplus \underline{1} 11, \overline{2} 01,111=\underline{100}, \overline{0} 01,100 \oplus \underline{111}, \overline{2} 10,111 \\
& =\underline{2} 10, \overline{1} 11,111 \oplus \underline{0} 01, \overline{1} 00,100=\underline{2} 01, \overline{1} 11,111 \oplus \underline{0} 10, \overline{1} 00,100 .
\end{aligned}
$$

The equation $P u=0$ with the Riemann scheme $\left\{\begin{array}{ccc}x=\infty & 0 & 1 \\ {\left[\lambda_{0,1}\right]_{(2)}} & {[0]_{(2)}} & {[0]_{(2)}} \\ \lambda_{0,2} & \lambda_{1,2} & \lambda_{2,2} \\ \lambda_{0,3} & \lambda_{1,3} & \lambda_{2,3}\end{array}\right\}$ is isomorphic to the system

$$
\begin{aligned}
\tilde{u}^{\prime} & =\frac{A}{x} \tilde{u}+\frac{B}{x-1} \tilde{u}, \quad \tilde{u}=\left(\begin{array}{l}
u_{1} \\
u_{2} \\
u_{3} \\
u_{4}
\end{array}\right), u_{1}=u, \\
A & =\left(\begin{array}{cccc}
0 & 0 & c_{1} & 0 \\
0 & 0 & 0 & c_{1} \\
0 & 0 & a_{1} & b_{1}-b_{2}-c_{2} \\
0 & 0 & 0 & a_{2}
\end{array}\right), \\
B & =\left(\begin{array}{llll}
0 & 0 & 0 & 0 \\
-a_{1}-b_{2}+c_{1} & -b_{1}+b_{2}+c_{2} & b_{2} & 0 \\
-a_{1}+a_{2}+c_{2} & -a_{2}-b_{1}+c_{1} & a_{1}-a_{2}-c_{2} & b_{1}
\end{array}\right), \\
& \left\{\begin{array}{l}
a_{1}=\lambda_{1,2}, \\
a_{2}=\lambda_{1,3}, \\
b_{1}=\lambda_{2,2}-2, \\
b_{2}=\lambda_{2,3}-1, \\
c_{1}=-\lambda_{0,1}, \\
c_{2}=\lambda_{0,1}+\lambda_{0,2}+\lambda_{1,2}+\lambda_{2,2}-1
\end{array}\right.
\end{aligned}
$$

when $\lambda_{0,1}\left(\lambda_{0,1}+\lambda_{2,2}\right)\left(\lambda_{0,1}+\lambda_{0,2}+\lambda_{1,2}+\lambda_{2,3}-2\right) \neq 0$. Let $u(x)$ be a holomorphic solution of $P u=0$ in a neighborhood of $x=0$. By a direct calculation we have

$$
\begin{aligned}
& u_{1}(0)=\frac{\left(a_{1}-1\right)\left(a_{2}-1\right)}{\left(b_{1}-c_{1}+1\right)\left(b_{1}-b_{2}-c_{2}\right) c_{1}} u^{\prime}(0)+ \\
& \frac{\left(a_{2}+b_{2}+c_{2}-1\right) a_{1}-\left(c_{1}+c_{2}\right) a_{2}+\left(a_{2}-a_{1}+c_{2}\right) b_{1}-\left(c_{2}+1\right) b_{2}-c_{2}^{2}+c_{1}}{\left(b_{1}-c_{1}+1\right)\left(b_{1}-b_{2}-c_{2}\right)} u(0) .
\end{aligned}
$$


Since the shift described in Remark 14.19 (1) corresponds to the shift

$$
\left(a_{1}, a_{2}, b_{1}, b_{2}, c_{1}, c_{2}\right) \mapsto\left(a_{1}-k, a_{2}-k, b_{1}+k, b_{2}+k, c_{1}, c_{2}\right),
$$

it follows from Theorem 14.10 that

$$
\left.\lim _{k \rightarrow \infty} c\left(\left[\lambda_{0,1}\right]_{(2)} \rightsquigarrow\left[\lambda_{1,1}\right]_{(2)}\right)\right|_{\substack{\lambda_{0,2} \mapsto \lambda_{0,2}-k, \lambda_{0,3} \mapsto \lambda_{0,3}-k \\ \lambda_{1,2} \mapsto \lambda_{1,2}+k, \lambda_{1,3} \mapsto \lambda_{1,3}+k}}=1
$$

as in the proof of $(15.35)$ because $u_{1}(0) \sim \frac{k}{\left(b_{1}-b_{2}-c_{2}\right) c_{1}} u^{\prime}(0)+C u(0)$ with $C \in \mathbb{C}$ when $k \rightarrow \infty$. Thus we can calculate this generalized connection coefficient by the procedure described in Remark 14.19.

Using (4.8), we have the series expansion of the local solution at $x=0$ corresponding to the exponent $\alpha_{1}+\mu_{1}+\mu_{2}$ for the Riemann scheme parametrized by $\alpha_{i}, \beta_{i}$ and $\mu_{i}$ with $i=1,2$.

$$
\begin{aligned}
I_{0}^{\mu_{2}} & x^{\alpha_{2}}(1-x)^{\beta_{2}} I_{0}^{\mu_{1}} x^{\alpha_{1}}(1-x)^{\beta_{1}} \\
= & I_{0}^{\mu_{2}} \frac{\Gamma\left(\alpha_{1}+1\right)}{\Gamma\left(\alpha_{1}+\mu+1\right)} \sum_{n=0}^{\infty} \frac{\left(\alpha_{1}+1\right)_{n}\left(-\beta_{1}\right)_{n}}{\left(\alpha_{1}+\mu+1\right)_{n} n !} x^{\alpha_{2}}(1-x)^{\beta_{2}} x^{\alpha_{1}+\mu+n} \\
= & \frac{\Gamma\left(\alpha_{1}+1\right) \Gamma\left(\alpha_{1}+\alpha_{2}+\mu_{1}+1\right) x^{\alpha_{1}+\alpha_{2}+\mu_{1}+\mu_{2}}}{\Gamma\left(\alpha_{1}+\mu_{1}+1\right) \Gamma\left(\alpha_{1}+\alpha_{2}+\mu_{1}+\mu_{2}+1\right)} \\
& \cdot \sum_{m, n=0}^{\infty} \frac{\left(\alpha_{1}+1\right)_{n}\left(\alpha_{1}+\alpha_{2}+\mu_{1}+1\right)_{m+n}\left(-\beta_{1}\right)_{n}\left(-\beta_{2}\right)_{m}}{\left(\alpha_{1}+\mu_{1}+1\right)_{n}\left(\alpha_{1}+\alpha_{2}+\mu_{1}+\mu_{2}+1\right)_{m+n} n ! m !} x^{m+n} \\
= & \frac{\Gamma\left(\alpha_{1}+1\right) \Gamma\left(\alpha_{1}+\alpha_{2}+\mu_{1}+1\right) x^{\alpha_{1}+\alpha_{2}+\mu_{1}+\mu_{2}}(1-x)^{-\beta_{2}}}{\Gamma\left(\alpha_{1}+\mu_{1}+1\right) \Gamma\left(\alpha_{1}+\alpha_{2}+\mu_{1}+\mu_{2}+1\right)} \\
& \cdot \sum_{m, n=0}^{\infty} \frac{\left(\alpha_{1}+1\right)_{n}\left(\alpha_{1}+\alpha_{2}+\mu_{1}+1\right)_{n}\left(\mu_{2}\right)_{m}\left(-\beta_{1}\right)_{n}\left(-\beta_{2}\right)_{m}}{\left(\alpha_{1}+\mu_{1}+1\right)_{n}\left(\alpha_{1}+\alpha_{2}+\mu_{1}+\mu_{2}+1\right)_{m+n} m ! n !} x^{n}\left(\frac{x}{x-1}\right)^{m} .
\end{aligned}
$$

Note that when $\beta_{2}=0$, the local solution is reduced to a local solution of the equation at $x=0$ satisfied by the hypergeometric series ${ }_{3} F_{2}\left(\alpha_{1}^{\prime}, \alpha_{2}^{\prime}, \alpha_{3}^{\prime} ; \beta_{1}^{\prime}, \beta_{2}^{\prime} ; x\right)$ and when $\alpha_{2}=0$, it is reduced to a local solution of the equation corresponding to the exponent at $x=1$ with free multiplicity.

Let $u_{0}\left(\alpha_{1}, \alpha_{2}, \beta_{1}, \beta_{2}, \mu_{1}, \mu_{2} ; x\right)$ be the local solution normalized by

$$
u_{0}(\alpha, \beta, \mu ; x)-x^{\alpha_{1}+\alpha_{2}+\mu_{1}+\mu_{2}} \in x^{\alpha_{1}+\alpha_{2}+\mu_{1}+\mu_{2}+1} \mathcal{O}_{0}
$$

for generic $\alpha, \beta, \mu$. Then we have the recurrence relation

$$
\begin{gathered}
u_{0}\left(\alpha, \beta_{1}-1, \beta_{2}, \mu ; x\right)=u_{0}(\alpha, \beta, \mu ; x)+\frac{\left(\alpha_{1}+1\right)\left(\alpha_{1}+\alpha_{2}+\mu_{1}+1\right)}{\left(\alpha_{1}+\mu_{1}+1\right)\left(\alpha_{1}+\alpha_{2}+\mu_{1}+\mu_{2}+1\right)} \\
\cdot u_{0}\left(\alpha_{1}+1, \alpha_{2}, \beta_{1}-1, \beta_{2}, \mu ; x\right) .
\end{gathered}
$$

15.7.6. Tuple of partitions : $211,22,31,31 . \quad[\Delta(\mathbf{m})]=1^{6} \cdot 2$

$$
\begin{aligned}
211,22,31,31 & =H_{1} \oplus P_{3}: 4=H_{2} \oplus H_{2}: 2=2 H_{1} \oplus H_{2}: 2 \\
& =010,10,10,10 \oplus 201,12,21,21=010,01,10,10 \oplus 201,21,21,21 \\
& =001,10,10,10 \oplus 210,12,21,21=001,01,10,10 \oplus 210,21,21,21 \\
& =110,11,11,20 \oplus 101,11,20,11=110,11,20,11 \oplus 101,11,11,20 \\
& =200,20,20,20 \oplus 011,02,11,11
\end{aligned}
$$$$
\stackrel{\partial_{\max }}{\longrightarrow} 011,02,11,11
$$ 


$$
\begin{aligned}
& \left\{\begin{array}{cccc}
x=0 & \frac{1}{c_{1}} & \frac{1}{c_{2}} & \infty \\
{\left[\lambda_{0,1}\right]_{(3)}} & {\left[\lambda_{1,1}\right]_{(3)}} & {\left[\lambda_{2,1}\right]_{(2)}} & {\left[\lambda_{3,1}\right]_{(2)}} \\
\lambda_{0,2} & \lambda_{1,2} & \lambda_{2,2} & {\left[\lambda_{3,2}\right]_{(2)}} \\
& & \lambda_{2,3} &
\end{array}\right\} \\
& \stackrel{x^{-\lambda_{0,1}}\left(1-c_{1} x\right)^{-\lambda_{1,1}}\left(1-c_{2} x\right)^{-\lambda_{2,1}}}{\longrightarrow} \\
& \left\{\begin{array}{cccc}
x=0 & \frac{1}{c_{1}} & \frac{1}{c_{2}} & \infty \\
{[0]_{(3)}} & {[0]_{(3)}} & {[0]_{(2)}} & {\left[\lambda_{3,1}+\lambda_{0,1}+\lambda_{1,1}+\lambda_{2,1}\right]_{(2)}} \\
\lambda_{0,2}-\lambda_{0,1} & \lambda_{1,2}-\lambda_{1,1} & \lambda_{2,2}-\lambda_{2,1} & {\left[\lambda_{3,2}+\lambda_{0,1}+\lambda_{1,1}+\lambda_{2,1}\right]_{(2)}}
\end{array}\right\} \\
& \stackrel{\partial^{-\lambda_{1}^{\prime}}}{\longrightarrow} \\
& \left\{\begin{array}{cccc}
x=0 & \frac{1}{c_{1}} & \frac{1}{c_{2}} & \infty \\
0 & 0 & & \\
\lambda_{0,2}+\lambda_{1}^{\prime}-\lambda_{0,1} & \lambda_{1,2}+\lambda_{1}^{\prime}-\lambda_{1,1} & \lambda_{2,2}+\lambda_{1}^{\prime}-\lambda_{2,1} & {\left[\lambda_{3,2}-\lambda_{3,1}+1\right]_{(2)}} \\
& & \lambda_{2,3}+\lambda_{1}^{\prime}-\lambda_{2,1} &
\end{array}\right\}
\end{aligned}
$$

The condition for the irreducibility:

$$
\begin{aligned}
& \left\{\begin{array}{l}
\lambda_{0,1}+\lambda_{1,1}+\lambda_{2, \nu}+\lambda_{3, \nu^{\prime}} \notin \mathbb{Z} \quad\left(\nu \in\{1,2,3\}, \nu^{\prime} \in\{1,2\}\right), \\
\lambda_{0,1}+\lambda_{0,2}+2 \lambda_{1,1}+\lambda_{2,1}+\lambda_{2, \nu}+\lambda_{3,1}+\lambda_{3,2} \notin \mathbb{Z} \quad(\nu \in\{2,3\}),
\end{array}\right. \\
& c\left(\lambda_{0,2} \rightsquigarrow \lambda_{1,2}\right)=\frac{\Gamma\left(\lambda_{0,2}-\lambda_{0,1}+1\right) \Gamma\left(\lambda_{1,2}-\lambda_{1,1}\right)\left(1-\frac{c_{2}}{c_{1}}\right)^{\lambda_{2,1}}}{\prod_{\nu=2}^{3} \Gamma\left(\lambda_{0,1}+\lambda_{0,2}+2 \lambda_{1,1}+\lambda_{2,1}+\lambda_{2, \nu}+\lambda_{3,1}+\lambda_{3,2}-1\right)}, \\
& c\left(\lambda_{0,2} \rightsquigarrow \lambda_{2,3}\right)=\prod_{\nu=1}^{2} \frac{\Gamma\left(\lambda_{2,3}-\lambda_{2, \nu}\right)}{\Gamma\left(1-\lambda_{0,1}-\lambda_{1,1}-\lambda_{2,3}-\lambda_{3, \nu}\right)} \\
& \cdot \frac{\Gamma\left(\lambda_{0,2}-\lambda_{0,1}+1\right)\left(1-\frac{c_{1}}{c_{2}}\right)^{\lambda_{1,1}}}{\Gamma\left(\lambda_{0,1}+\lambda_{0,2}+2 \lambda_{1,1}+\lambda_{2,1}+\lambda_{2,2}+\lambda_{3,1}+\lambda_{3,2}-1\right)} .
\end{aligned}
$$

15.7.7. Tuple of partitions : $22,22,22,31 . \quad[\Delta(\mathbf{m})]=1^{8} \cdot 2$

$$
\begin{aligned}
22,22,22,31 & =H_{1} \oplus P_{3}: 8=2(11,11,11,20) \oplus 00,00,00,(-1) 1 \\
& =10,10,10,10 \oplus 12,12,12,21=10,10,01,10 \oplus 12,12,21,21 \\
& =10,01,10,10 \oplus 12,21,12,21=10,01,01,10 \oplus 12,21,21,21 \\
& =01,10,10,10 \oplus 21,12,12,21=01,10,01,10 \oplus 21,12,21,21 \\
& =01,01,10,10 \oplus 21,21,12,21=01,01,01,10 \oplus 21,21,21,21 \\
& \stackrel{2}{\rightarrow} 12,12,12,21
\end{aligned}
$$

The condition for the irreducibility:

$$
\left\{\begin{array}{l}
\lambda_{0, i}+\lambda_{1, j}+\lambda_{2, k}+\lambda_{3,1} \notin \mathbb{Z} \quad(i, j, k \in\{1,2\}), \\
\lambda_{0,1}+\lambda_{0,2}+\lambda_{1,1}+\lambda_{1,2}+\lambda_{2,1}+\lambda_{2,2}+\lambda_{3,1}+\lambda_{3,2} \notin \mathbb{Z} .
\end{array}\right.
$$

15.8. Other rigid examples with a small order. First we give an example which is not of Okubo type.

15.8.1. 221,221,221. The Riemann Scheme and the direct decompositions are

$$
\left\{\begin{array}{ccc}
x=0 & 1 & \infty \\
{\left[\lambda_{0,1}\right]_{(2)}} & {\left[\lambda_{1,1}\right]_{(2)}} & {\left[\lambda_{2,1}\right]_{(2)}} \\
{\left[\lambda_{0,2}\right]_{(2)}} & {\left[\lambda_{1,2}\right]_{(2)}} & {\left[\lambda_{2,2}\right]_{(2)}} \\
\lambda_{0,3} & \lambda_{1,3} & \lambda_{2,3}
\end{array}\right\}, \quad \sum_{j=0}^{2}\left(2 \lambda_{j, 1}+2 \lambda_{j, 2}+\lambda_{j, 3}\right)=4
$$




$$
\begin{array}{rlrl}
{[\Delta(\mathbf{m})]} & =1^{14} \cdot 2 & & \\
22 \overline{1}, 22 \underline{1}, 221 & =H_{1} \oplus 211,211,211: 8 & 6 & =|2,2,2| \\
& =H_{2} \oplus H_{3}: 6 & 11 & =|21,22,22| \\
& =2 H_{2} \oplus H_{1}: 1 & & \\
& =10 \overline{1}, 110,110 \oplus 120,11 \underline{1}, 111=01 \overline{1}, 110,110 \oplus 210,11 \underline{1}, 111 \\
& =11 \overline{1}, 120,111 \oplus 110,10 \underline{1}, 110=11 \overline{1}, 210,111 \oplus 110,01 \underline{1}, 110 \\
& \rightarrow 121,121,121 &
\end{array}
$$

and a connection coefficient is give by

$$
\begin{aligned}
c\left(\lambda_{0,3} \rightsquigarrow \lambda_{1,3}\right)= & \prod_{\nu=1}^{2}\left(\frac{\Gamma\left(\lambda_{0,3}-\lambda_{0, \nu}+1\right)}{\Gamma\left(\lambda_{0, \nu}+\lambda_{0,3}+\lambda_{1,1}+\lambda_{1,2}+\lambda_{2,1}+\lambda_{2,2}-1\right)}\right. \\
& \left.\cdot \frac{\Gamma\left(\lambda_{1, \nu}-\lambda_{1,3}\right)}{\Gamma\left(2-\lambda_{0,1}-\lambda_{0,2}-\lambda_{1, \nu}-\lambda_{1,3}-\lambda_{2,1}-\lambda_{2,2}\right)}\right) .
\end{aligned}
$$

Using this example we explain an idea to get all the rigid decompositions $\mathbf{m}=$ $\mathbf{m}^{\prime} \oplus \mathbf{m}^{\prime \prime}$. Here we note that $\operatorname{idx}\left(\mathbf{m}, \mathbf{m}^{\prime}\right)=1$. Put $\mathbf{m}=221,221,221$. We may assume ord $\mathbf{m}^{\prime} \leq$ ord $\mathbf{m}^{\prime \prime}$.

Suppose ord $\mathbf{m}^{\prime}=1$. Then $\mathbf{m}^{\prime}$ is isomorphic to $1,1,1$ and there exists tuples of indices $\left(\ell_{0}, \ell_{1}, \ell_{2}\right)$ such that $m_{j, \nu}^{\prime}=\delta_{j, \ell_{j}}$. Then $\operatorname{idx}\left(\mathbf{m}, \mathbf{m}^{\prime}\right)=m_{0, \ell_{0}}+m_{1, \ell_{1}}+$ $m_{1, \ell_{2}}-(3-2)$ ord $\mathbf{m} \cdot \operatorname{ord} \mathbf{m}^{\prime}$ and we have $m_{0, \ell_{0}}+m_{1, \ell_{1}}+m_{1, \ell_{2}}=6$. Hence $\left(m_{0, \ell_{0}}, m_{1, \ell_{1}}, m_{1, \ell_{2}}\right)=(2,2,2)$, which is expressed by $6=|2,2,2|$ in the above. Since $\ell_{j}=1$ or 2 for $0 \leq j \leq 2$, it is clear that there exist 8 rigid decompositions with ord $\mathbf{m}^{\prime}=1$.

Suppose ord $\mathbf{m}^{\prime}=2$. Then $\mathbf{m}^{\prime}$ is isomorphic to $11,11,11$ and there exists tuples of indices $\left(\ell_{0,1}, \ell_{0,2}, \ell_{1,1}, \ell_{1,2}, \ell_{2,1}, \ell_{2,2}\right)$ which satisfies $\sum_{j=0}^{2} \sum_{\nu=1}^{2} m_{j, \ell_{\nu}}=$ $(3-2)$ ord $\mathbf{m} \cdot \operatorname{ord} \mathbf{m}^{\prime}+1=11$. Hence we may assume $\left(\ell_{0,1}, \ell_{0,2}, \ell_{1,1}, \ell_{1,2}, \ell_{2,1}, \ell_{2,2}\right)=$ $(2,1,2,2,2,2)$ modulo obvious symmetries, which is expressed by $11=|21,22,22|$. There exist 6 rigid decompositions with ord $\mathbf{m}^{\prime}=2$.

In general, this method to get all the rigid decompositions of $\mathbf{m}$ is useful when ord $\mathbf{m}$ is not big. For example if ord $\mathbf{m} \leq 7, \mathbf{m}^{\prime}$ is isomorphic to $1,1,1$ or $11,11,11$ or $21,111,111$.

The condition for the irreducibility is given by Theorem 12.10 and it is

$$
\begin{cases}\lambda_{0, i}+\lambda_{1, j}+\lambda_{2, k} \notin \mathbb{Z} & (i, j, k \in\{1,2\}), \\ \sum_{j=0}^{2} \sum_{\nu=1}^{2} \lambda_{j, \nu}+\left(\lambda_{i, 3}-\lambda_{i, k}\right) \notin \mathbb{Z} & (i \in\{0,1,2\}, k \in\{1,2\}) .\end{cases}
$$

15.8.2. Other examples. Theorem 14.6 shows that the connection coefficients between local solutions of rigid differential equations which correspond to the eigenvalues of local monodromies with free multiplicities are given by direct decompositions of the tuples of partitions $\mathbf{m}$ describing their spectral types.

We list the rigid decompositions $\mathbf{m}=\mathbf{m}^{\prime} \oplus \mathbf{m}^{\prime \prime}$ of rigid indivisible $\mathbf{m}$ in $\mathcal{P}^{(5)} \cup \mathcal{P}_{3}^{(6)}$ satisfying $m_{0, n_{0}}=m_{1, n_{1}}=m_{0, n_{0}}^{\prime}=m_{1, n_{1}}^{\prime \prime}=1$. The positions of $m_{0, n_{0}}$ and $m_{1, n_{1}}$ in $\mathbf{m}$ to which Theorem 14.6 applies are indicated by an overline and an underline, respectively. The number of decompositions in each case equals $n_{0}+n_{1}-2$ and therefore the validity of the following list is easily verified.

We show the tuple $\partial_{\max } \mathbf{m}$ after $\rightarrow$. The type $[\Delta(\mathbf{m})]$ of $\Delta(\mathbf{m})$ is calculated by (9.42), which is also indicated in the following with this calculation. For example, when $\mathbf{m}=311,221,2111$, we have $d(\mathbf{m})=2, \mathbf{m}^{\prime}=\partial \mathbf{m}=111,021,0111$, $[\Delta(s(111,021,0111))]=1^{9},\left\{m_{j, \nu}^{\prime}-m_{j, 1}^{\prime} \in \mathbb{Z}_{>0}\right\} \cup\{2\}=\{1,1,1,1,2,2\}$ and hence $[\Delta(\mathbf{m})]=1^{9} \times 1^{4} \cdot 2^{2}=1^{13} \cdot 2^{2}$, which is a partition of $h(\mathbf{m})-1=17$. 
Here we note that $h(\mathbf{m})$ is the sum of the numbers attached the Dynkin diagram 1_2__ $\int_{0}^{1} 3$

All the decompositions of the tuple $\mathbf{m}$ corresponding to the elements in $\Delta(\mathbf{m})$ are given, by which we easily get the necessary and sufficient condition for the irreducibility (cf. Theorem 12.13 and $\$ 15.9 .2$ ).

$$
\begin{aligned}
& \text { ord } \mathbf{m}=5 \\
& 311,221,2111=100,010,0001 \oplus 211,211,2110 \\
& =100,001,1000 \oplus 211,220,1111 \\
& =101,110,1001 \oplus 210,111,1110 \\
& 6=|3,2,1| \\
& 6=|3,1,2| \\
& =2(100,100,1000) \oplus 111,021,0111 \\
& 11=|31,22,21| \\
& \stackrel{2}{\rightarrow} 111,021,0111 \\
& {[\Delta(\mathbf{m})]=1^{9} \times 1^{4} \cdot 2^{2}=1^{13} \cdot 2^{2}} \\
& \mathbf{m}=H_{1} \oplus 211,211,211: 6=H_{1} \oplus E O_{4}: 1=H_{2} \oplus H_{3}: 6=2 H_{1} \oplus H_{3}: 2 \\
& 311,22 \overline{1}, 211 \underline{1}=211,211,2110 \oplus 100,010,0001=211,121,2110 \oplus 100,100,0001 \\
& =100,001,1000 \oplus 211,220,1111 \\
& =210,111,1110 \oplus 101,110,1001=201,111,1110 \oplus 110,110,1001 \\
& 31 \overline{1}, 221,211 \underline{1}=211,211,2110 \oplus 100,010,0001=211,121,2110 \oplus 100,100,0001 \\
& =201,111,1110 \oplus 110,110,1001 \\
& =101,110,1010 \oplus 210,111,1101=101,110,1100 \oplus 210,111,1011 \\
& 32,211 \overline{1}, 211 \underline{1}=22,1111,2110 \oplus 10,1000,0001=10,0001,1000 \oplus 22,2110,1111 \\
& =11,1001,1010 \oplus 21,1110,1101=11,1001,1100 \oplus 21,1110,1011 \\
& =21,1101,1110 \oplus 11,1010,1001=21,1011,1110 \oplus 11,1100,1001 \\
& \stackrel{2}{\rightarrow} 12,0111,0111 \\
& {[\Delta(\mathbf{m})]=1^{9} \times 1^{7} \cdot 2=1^{16} \cdot 2} \\
& \mathbf{m}=H_{1} \oplus H_{4}: 1=H_{1} \oplus E O_{4}: 6=H_{2} \oplus H_{3}: 9=2 H_{1} \oplus H_{3}: 1 \\
& 22 \overline{1}, 22 \underline{1}, 41,41=001,100,10,10 \oplus 220,121,31,31=001,010,10,10 \oplus 220,211,31,31 \\
& =211,220,31,31 \oplus 010,001,10,10=121,220,31,31 \oplus 100,001,10,10 \\
& \stackrel{2}{\rightarrow} 021,021,21,21 \\
& {[\Delta(\mathbf{m})]=1^{4} \cdot 2 \times 1^{4} \cdot 2^{3}=1^{6} \cdot 2^{4}} \\
& \mathbf{m}=H_{1} \oplus 22,211,31,31: 4=H_{2} \oplus H_{3}: 2=2 H_{1} \oplus P_{3}: 4 \\
& 22 \overline{1}, 221,4 \underline{1}, 41=001,100,10,10 \oplus 220,121,31,31=001,010,10,10 \oplus 220,211,31,31 \\
& =111,111,30,21 \oplus 110,110,11,20 \\
& 22 \overline{1}, 32,32,4 \underline{1}=101,11,11,20 \oplus 120,21,21,21=011,11,11,20 \oplus 210,21,21,21 \\
& =001,10,10,10 \oplus 220,22,22,31 \\
& \stackrel{2}{\rightarrow} 021,12,12,21 \\
& {[\Delta(\mathbf{m})]=1^{4} \cdot 2 \times 1^{3} \cdot 2^{2}=1^{7} \cdot 2^{3}}
\end{aligned}
$$




$$
\begin{aligned}
\mathbf{m} & =H_{1} \oplus 22,22,22,31: 1=H_{1} \oplus 211,22,31,31: 4=H_{2} \oplus P_{3}: 2 \\
& =2 H_{1} \oplus P_{3}: 2
\end{aligned}
$$

$31 \overline{1}, 31 \underline{1}, 32,41=001,100,10,10 \oplus 310,211,22,31=211,301,22,31 \oplus 100,001,10,10$ $=101,110,11,20 \oplus 210,201,21,21=201,210,21,21 \oplus 110,101,11,20$ $\stackrel{3}{\rightarrow} 011,011,02,11$

$$
[\Delta(\mathbf{m})]=1^{4} \times 1^{4} \cdot 2 \cdot 3=1^{8} \cdot 2 \cdot 3
$$$$
\mathbf{m}=H_{1} \oplus 211,31,22,31: 4=H_{2} \oplus P_{3}: 4
$$$$
=2 H_{1} \oplus H_{3}: 1=3 H_{1} \oplus H_{2}: 1
$$

$31 \overline{1}, 311,32,4 \underline{1}=001,100,10,10 \oplus 301,211,22,31$ $=101,110,11,20 \oplus 210,201,21,21=101,101,11,20 \oplus 210,210,21,21$

$32,32,4 \overline{1}, 4 \underline{1}, 41=11,11,11,20,20 \oplus 21,21,30,21,21$ $=21,21,21,30,21 \oplus 11,11,20,11,20$ $\stackrel{3}{\rightarrow} 02,02,11,11,11$

$$
[\Delta(\mathbf{m})]=1^{4} \times 2^{2} \cdot 3=1^{4} \cdot 2^{2} \cdot 3
$$$$
\mathbf{m}=H_{1} \oplus P_{4}: 1=H_{2} \oplus P_{3}: 3=2 H_{1} \oplus P_{3}: 2=3 H_{1} \oplus H_{2}: 1
$$

$$
\text { ord } \mathbf{m}=6 \text { and } \mathbf{m} \in \mathcal{P}_{3}
$$

$321,3111,222=311,2111,221 \oplus 010,1000,001$

$$
=211,2110,211 \oplus 110,1001,011 \quad 13=|32,31,22|
$$$$
7=|2,3,2|
$$$$
=210,1110,111 \oplus 111,2001,111
$$$$
\stackrel{2}{\rightarrow} 121,1111,022 \rightarrow 111,0111,012
$$

$$
[\Delta(\mathbf{m})]=1^{14} \times 1 \cdot 2^{3}=1^{15} \cdot 2^{3}
$$$$
\mathbf{m}=H_{1} \oplus 311,2111,221: 3=H_{2} \oplus 211,211,211: 6=H_{3} \oplus H_{3}: 6
$$$$
=2 \mathrm{H}_{1} \oplus E \mathrm{O}_{4}: 3
$$

$32 \overline{1}, 311 \underline{1}, 222=211,2110,211 \oplus 110,1001,011=211,2110,121 \oplus 110,1001,101$ $=211,2110,112 \oplus 110,1001,110$ $=111,2100,111 \oplus 210,1011,111=111,2010,111 \oplus 210,1101,111$

$321,311 \overline{1}, 311 \underline{1}=221,2111,3110 \oplus 100,1000,0001=100,0001,1000 \oplus 221,3110,2111$ $=211,2101,2110 \oplus 110,1010,1001=211,2011,2110 \oplus 110,1101,1001$ $=110,1001,1100 \oplus 211,2110,2011=110,1001,1010 \oplus 211,2110,2101$ $\stackrel{3}{\rightarrow} 021,0111,0111$

$[\Delta(\mathbf{m})]=1^{9} \times 1^{7} \cdot 2 \cdot 3=1^{16} \cdot 2 \cdot 3$

$$
\begin{aligned}
\mathbf{m} & =H_{1} \oplus 221,2111,311: 6=H_{1} \oplus 32,2111,2111: 1 \\
& =H_{2} \oplus 211,211,211: 9=2 H_{1} \oplus H_{4}: 1=3 H_{1} \oplus H_{3}: 1
\end{aligned}
$$

$32 \overline{1}, 311 \underline{1}, 3111=221,3110,2111 \oplus 100,0001,1000=001,1000,1000 \oplus 320,2111,2111$ $=211,2110,2110 \oplus 110,1001,1001=211,2110,2011 \oplus 110,1001,1100$ $=211,2110,2011 \oplus 110,1001,1100$ 


$$
\begin{aligned}
32 \overline{1}, 321,2211 & =211,220,1111 \oplus 110,101,1100=101,110,1100 \oplus 220,211,1111 \\
& =111,210,1110 \oplus 210,111,1101=111,210,1101 \oplus 210,111,1110 \\
& \stackrel{2}{\rightarrow} 121,121,0211 \rightarrow 101,101,0011 \\
{[\Delta(\mathbf{m})] } & =1^{10} \cdot 2 \times 1^{4} \cdot 2^{2}=1^{14} \cdot 2^{3} \\
\mathbf{m} & =H_{1} \oplus 311,221,2111: 4=H_{1} \oplus 221,221,221: 2 \\
& =H_{2} \oplus E O_{4}: 2=H_{2} \oplus 211,211,211: 4=H_{3} \oplus H_{3}: 2 \\
& =2 H_{1} \oplus 211,211,211: 2=2(110,110,1100) \oplus 101,101,0011: 1
\end{aligned}
$$

$321,32 \overline{1}, 221 \underline{1}=221,221,2210 \oplus 100,100,0001=110,101,1100 \oplus 211,220,1111$ $=211,211,2110 \oplus 110,110,0101=211,211,1210 \oplus 110,110,1001$ $=210,111,1110 \oplus 111,210,1101$

$41 \overline{1}, 221 \underline{1}, 2211=311,2210,2111 \oplus 100,0001,0100=311,2210,1211 \oplus 100,0001,1000$ $=101,1100,1100 \oplus 310,1111,1111=201,1110,1110 \oplus 210,1101,1101$ $=201,1110,1101 \oplus 210,1101,1110$ $\stackrel{2}{\rightarrow} 211,0211,0211 \rightarrow 011,001,0011$

$$
[\Delta(\mathbf{m})]=1^{10} \cdot 2 \times 1^{4} \cdot 2^{3}=1^{14} \cdot 2^{4}
$$$$
\mathbf{m}=H_{1} \oplus 311,221,2211: 8=H_{2} \oplus H_{4}: 2=H_{3} \oplus H_{3}: 4
$$$$
=2 H_{1} \oplus 211,211,211: 4
$$

$411,221 \overline{1}, 221 \underline{1}=311,2111,2210 \oplus 100,0100,0001=311,1211,2210 \oplus 100,1000,0001$ $=100,0001,0100 \oplus 311,2210,2111=100,0001,1000 \oplus 311,2210,1211$ $=201,1101,1110 \oplus 210,1110,1101=210,1101,1110 \oplus 201,1110,1101$

$41 \overline{1}, 222,2111 \underline{1}=311,221,21110 \oplus 100,001,00001=311,212,21110 \oplus 100,010,00001$ $=311,122,21110 \oplus 100,100,00001=201,111,11100 \oplus 210,111,10011$ $=201,111,11010 \oplus 210,111,10101=201,111,10110 \oplus 210,111,11001$ $\stackrel{2}{\rightarrow} 211,022,01111 \rightarrow 111,012,00111$

$$
[\Delta(\mathbf{m})]=1^{14} \times 1^{4} \cdot 2^{3}=1^{18} \cdot 2^{3}
$$$$
\mathbf{m}=H_{1} \oplus 311,221,2111: 12=H_{3} \oplus H_{3}: 6=2 H_{1} \oplus E O_{4}: 3
$$

$42,221 \overline{1}, 2111 \underline{1}=32,2111,21110 \oplus 10,0100,00001=32,1211,21110 \oplus 10,1000,00001$ $=10,0001,10000 \oplus 32,2210,11111=31,1111,11110 \oplus 11,1100,10001$ $=21,1101,11100 \oplus 21,1110,10011=21,1101,11010 \oplus 21,1110,10101$ $=21,1101,10110 \oplus 21,1110,11001$

$\stackrel{2}{\rightarrow} 22,0211,01111 \rightarrow 12,0111,00111$

$[\Delta(\mathbf{m})]=1^{14} \times 1^{6} \cdot 2^{2}=1^{20} \cdot 2^{2}$

$$
\begin{aligned}
\mathbf{m} & =H_{1} \oplus 32,2111,2111: 8=H_{1} \oplus E O_{4}: 2=H_{2} \oplus H_{4}: 4 \\
& =H_{3} \oplus H_{3}: 6=2 H_{1} \oplus E O_{4}: 2
\end{aligned}
$$

$33,311 \overline{1}, 2111 \underline{1}=32,2111,21110 \oplus 01,1000,00001=23,2111,21110 \oplus 10,1000,00001$ $=22,2101,11110 \oplus 11,1010,10001=22,2011,11110 \oplus 11,1100,10001$ $=11,1001,11000 \oplus 22,2110,10111=11,1001,10100 \oplus 22,2110,11011$ 


$$
\begin{aligned}
& =11,1001,10010 \oplus 22,2110,11101 \\
& \stackrel{2}{\rightarrow} 13,1111,01111 \\
{[\Delta(\mathbf{m})] } & =1^{16} \times 1^{4} \cdot 2^{2}=1^{20} \cdot 2^{2} \\
\mathbf{m} & =H_{1} \oplus 32,2111,2111: 8=H_{2} \oplus E O_{4}: 12=2 H_{1} \oplus H_{4}: 2 \\
32 \overline{1}, 3111,3111 & =221,3110,2111 \oplus 100,0001,1000=001,1000,1000 \oplus 320,2111,2111 \\
& =211,2110,2110 \oplus 110,1001,1001=211,2110,2101 \oplus 110,1001,1010 \\
& =211,2110,2011 \oplus 110,1001,1100 \\
& \stackrel{3}{\rightarrow} 021,0111,0111 \\
{[\Delta(\mathbf{m})] } & =1^{9} \times 1^{7} \cdot 2 \cdot 3=1^{16} \cdot 2 \cdot 3 \\
\mathbf{m} & =H_{1} \oplus 221,2111,311: 6=H_{1} \oplus 32,2111,2111: 1 \\
& =H_{2} \oplus 211,211,211: 9=2 H_{1} \oplus H_{4}: 1=3 H_{1} \oplus H_{3}: 1 \\
321,311 \overline{1}, 311 \underline{1} & =100,0001,1000 \oplus 221,3110,2111=221,2111,3110 \oplus 100,1000,0001 \\
& =211,2101,2110 \oplus 110,1010,1001=211,2011,2110 \oplus 110,1100,1001 \\
& =110,1001,1100 \oplus 211,2110,2011=110,1001,1010 \oplus 211,2110,2101 \\
33,221 \overline{1}, 221 \underline{1} & =22,1111,2110 \oplus 11,1100,1001=22,1111,1210 \oplus 11,1100,0101 \\
& =21,1101,1110 \oplus 12,1110,1011=12,1101,1110 \oplus 21,1110,1011 \\
& =11,1001,1100 \oplus 22,1210,1111=11,0101,1100 \oplus 22,2110,1111 \\
& \stackrel{1}{\rightarrow} 23,1211,1211 \rightarrow 21,1011,1011 \\
{[\Delta(\mathbf{m})] } & =1^{16} \cdot 2 \times 1^{4}=1^{20} \cdot 2 \\
\mathbf{m} & =H_{1} \oplus 32,2111,2111: 8=H_{2} \oplus E O_{4}: 8=H_{3} \oplus H_{3}: 4 \\
& =2(11,1100,1100) \oplus 11,0011,0011: 1
\end{aligned}
$$

We show all the rigid decompositions of the following simply reducible partitions of order 6 , which also correspond to the reducibility of the universal models.

$$
\begin{aligned}
42,222,111111 & =32,122,011111 \oplus 10,100,100000 \\
& =21,111,111000 \oplus 21,111,000111 \\
& \stackrel{1}{\rightarrow} 32,122,011111 \rightarrow 22,112,001111 \rightarrow 12,111,000111 \\
{[\Delta(\mathbf{m})] } & =1^{28} \\
\mathbf{m} & =H_{1} \oplus E O_{5}: 18=H_{3} \oplus H_{3}: 10 \\
33,222,21111 & =23,122,11111 \oplus 10,100,10000 \\
& =22,112,10111 \oplus 11,110,11000 \\
& =21,111,11100 \oplus 12,111,10011 \\
& \stackrel{1}{\rightarrow} 23,122,11111 \rightarrow 22,112,01111 \rightarrow 12,111,00111 \\
{[\Delta(\mathbf{m})] } & =1^{24} \\
\mathbf{m} & =H_{1} \oplus E O_{5}: 6=H_{2} \oplus E O_{4}: 12=H_{3} \oplus H_{3}: 6
\end{aligned}
$$

15.9. Submaximal series and minimal series. The rigid tuples $\mathbf{m}=\left\{m_{j, \nu}\right\}$ satisfying

$$
\#\left\{m_{j, \nu} ; 0<m_{j, \nu}<\text { ord } \mathbf{m}\right\} \geq \operatorname{ord} \mathbf{m}+5
$$


are classified by Roberts $\left[\mathrm{R} d\right.$. They are the tuples of type $H_{n}$ and $P_{n}$ which satisfy

$$
\#\left\{m_{j, \nu} ; 0<m_{j, \nu}<\text { ord } \mathbf{m}\right\}=2 \operatorname{ord} \mathbf{m}+2
$$

and those of 13 series $A_{n}=E O_{n}, B_{n}, C_{n}, D_{n}, E_{n}, F_{n}, G_{2 m}, I_{n}, J_{n}, K_{n}, L_{2 m+1}$, $M_{n}, N_{n}$ called submaximal series which satisfy

$$
\#\left\{m_{j, \nu} ; 0<m_{j, \nu}<\operatorname{ord} \mathbf{m}\right\}=\operatorname{ord} \mathbf{m}+5 .
$$

The series $H_{n}$ and $P_{n}$ are called maximal series.

We examine these rigid series and give enough information to analyze the series, which will be sufficient to construct differential equations including their confluences, integral representation and series expansion of solutions and get connection coefficients and the condition of their reducibility.

In fact from the following list we easily get all the direct decompositions and Katz's operations decreasing the order. The number over an arrow indicates the difference of the orders. We also indicate Yokoyama's reduction for systems of Okubo normal form using extension and restriction, which are denoted $E_{i}$ and $R_{i}$ $(i=0,1,2)$, respectively (cf. Yo2 ). Note that the inverse operations of $E_{i}$ are $R_{i}$, respectively. In the following we put

$$
\begin{aligned}
u_{P_{m}} & =\partial^{-\mu} x^{\lambda_{0}}(1-x)^{\lambda_{1}}\left(c_{2}-x\right)^{\lambda_{2}} \cdots\left(c_{m-1}-x\right)^{\lambda_{m-1}}, \\
u_{H_{2}} & =u_{P_{2}}, \\
u_{H_{m+1}} & =\partial^{-\mu^{(m)}} x^{\lambda_{0}^{(m)}} u_{H_{m}} .
\end{aligned}
$$

We give all the decompositions

$$
\mathbf{m}=\left(\operatorname{idx}\left(\mathbf{m}^{\prime}, \mathbf{m}\right) \cdot \mathbf{m}^{\prime}\right) \oplus \mathbf{m}^{\prime \prime}
$$

for $\alpha_{\mathbf{m}^{\prime}} \in \Delta(\mathbf{m})$. Here we will not distinguish between $\mathbf{m}^{\prime} \oplus \mathbf{m}^{\prime \prime}$ and $\mathbf{m}^{\prime \prime} \oplus \mathbf{m}^{\prime}$ when $\operatorname{idx}\left(\mathbf{m}^{\prime}, \mathbf{m}\right)=1$. Moreover note that the inequality assumed for the formula $[\Delta(\mathbf{m})]$ below assures that the given tuple of partition is monotone.

15.9.1. $B_{n} .\left(B_{2 m+1}=\mathrm{III}_{m}, B_{2 m}=\mathrm{II}_{m}, B_{3}=H_{3}, B_{2}=H_{2}\right)$

$$
\begin{aligned}
u_{B_{2 m+1}} & =\partial^{-\mu^{\prime}}(1-x)^{\lambda^{\prime}} u_{H_{m+1}} \\
m^{2} 1, m+11^{m}, m 1^{m+1} & =10,10,01 \oplus m m-11, m 1^{m}, m 1^{m} \\
& =01,10,10 \oplus m^{2}, m 1^{m}, m-11^{m+1} \\
& =1^{2} 0,11,11 \oplus(m-1)^{2} 1, m 1^{m-1}, m-11^{m} \\
{\left[\Delta\left(B_{2 m+1}\right)\right] } & =1^{(m+1)^{2}} \times 1^{m+2} \cdot m^{2}=1^{m^{2}+3 m+3} \cdot m^{2} \\
B_{2 m+1} & =H_{1} \oplus B_{2 m} \quad: 2(m+1) \\
& =H_{1} \oplus C_{2 m} \quad: 1 \\
& =H_{2} \oplus B_{2 m-1} \quad: m(m+1) \\
& =m H_{1} \oplus H_{m+1} \quad: 2 \\
u_{B_{2 m}} & =\partial^{-\mu^{\prime}} x^{\lambda^{\prime}}(1-x)^{\lambda^{\prime \prime}} u_{H_{m}} \\
m m-11, m 1^{m}, m 1^{m} & =100,01,10 \oplus(m-1)^{2} 1, m 1^{m-1}, m-11^{m} \\
& =001,10,10 \oplus m m-10, m-11^{m}, m-11^{m} \\
& =110,11,11 \oplus m-1 m-21, m-11^{m-1}, m-11^{m-1}
\end{aligned}
$$




$$
\begin{array}{rlrl}
{\left[\Delta\left(B_{2 m}\right)\right]} & =1^{m^{2}} \times 1^{2 m+1} \cdot(m-1)=1^{(m+1)^{2}} \cdot(m-1) \cdot m \\
B_{2 m} & =H_{1} \oplus B_{2 m-1} & & : 2 m \\
& =H_{1} \oplus C_{2 m-2} & & : 1 \\
& =H_{2} \oplus B_{2 m-2} & & : m^{2} \\
& =(m-1) H_{1} \oplus H_{m+1} & : 1 & \\
& =m H_{1} \oplus H_{m} & : 1 & \\
B_{2 m+1} & \stackrel{m}{R 2 E 0} H_{m+1}, \quad B_{n} \stackrel{1}{\longrightarrow} B_{n-1}, \quad B_{n} \stackrel{1}{\longrightarrow} C_{n-1} \\
B_{2 m} & \stackrel{m}{R 1 E 0} H_{m}, \quad B_{2 m} \stackrel{m-1}{\longrightarrow} H_{m+1} &
\end{array}
$$

15.9.2. An example. Using the example of type $B_{2 m+1}$, we explain how we get explicit results from the data written in $\$ 5.9 .1$.

The Riemann scheme of type $B_{2 m+1}$ is

$$
\begin{gathered}
\left\{\begin{array}{ccc}
\infty & 0 & 1 \\
{\left[\lambda_{0,1}\right]_{(m)}} & {\left[\lambda_{1,1}\right]_{(m+1)}} & {\left[\lambda_{2,1}\right]_{(m)}} \\
{\left[\lambda_{0,2}\right]_{(m)}} & \lambda_{1,2} & \lambda_{2,2} \\
\lambda_{0,3} & \vdots & \vdots \\
\sum_{j=0}^{p} \sum_{\nu=1}^{n_{j}} m_{j, \nu} \lambda_{j, \nu}=2 m & \lambda_{1, m+1} & \lambda_{2, m+2}
\end{array}\right\}, \\
\text { (Fuchs relation). }
\end{gathered}
$$

Theorem 12.13 says that the corresponding equation is irreducible if and only if any value of the following linear functions is not an integer.

$$
\begin{aligned}
L_{i, \nu}^{(1)} & :=\lambda_{0, i}+\lambda_{1,1}+\lambda_{2, \nu} \quad(i=1,2, \quad \nu=2, \ldots, m+2), \\
L^{(2)} & :=\lambda_{0,3}+\lambda_{1,1}+\lambda_{2,1}, \\
L_{\mu, \nu}^{(3)} & :=\lambda_{0,1}+\lambda_{0,2}+\lambda_{1,1}+\lambda_{1, \mu}+\lambda_{2,1}+\lambda_{2, \nu}-1 \\
\quad(\mu=2, \ldots, m+1, \quad \nu=2, \ldots, m+2), & \\
L_{i}^{(4)} & :=\lambda_{0, i}+\lambda_{1,1}+\lambda_{2,1} \quad(i=1,2) .
\end{aligned}
$$

Here $L_{i, \nu}^{(1)}$ (resp. $L^{(2)}$ etc.) correspond to the terms 10,01, 01 and $H_{1} \oplus B_{2 m}: 2(m+1)$ (resp. 01,10,10 and $H_{1} \oplus C_{2 m}: 1$ etc.) in $\$ 15.9 .1$.

It follows from Theorem 8.13 and Theorem 12.13 that the Fuchsian differential equation with the above Riemann scheme belongs to the universal equation $P_{B_{2 m+1}}(\lambda) u=0$ if

$$
L_{i}^{(4)} \notin\{-1,-2, \ldots, 1-m\} \quad(i=1,2) .
$$

Theorem 14.6 says that the connection coefficient $c\left(\lambda_{1, m+1} \rightsquigarrow \lambda_{2, m+2}\right)$ equals

$$
\frac{\prod_{\mu=1}^{m} \Gamma\left(\lambda_{1, m+1}-\lambda_{1, \mu}+1\right) \cdot \prod_{\mu=1}^{m+1} \Gamma\left(\lambda_{2, \nu}-\lambda_{1, m+2}\right)}{\prod_{i=1}^{2} \Gamma\left(1-L_{i, m+2}^{(1)}\right) \cdot \prod_{\nu=2}^{m+1} \Gamma\left(L_{m+1, \nu}^{(3)}\right) \cdot \prod_{\mu=2}^{m} \Gamma\left(1-L_{\mu, m+2}^{(3)}\right)}
$$

and

$$
\begin{aligned}
& c\left(\lambda_{1, m+1} \rightsquigarrow \lambda_{0,3}\right)=\frac{\prod_{\mu=1}^{m} \Gamma\left(\lambda_{1, m+1}-\lambda_{1, \mu}+1\right) \cdot \prod_{i=1}^{2} \Gamma\left(\lambda_{0, i}-\lambda_{0,3}\right)}{\Gamma\left(1-L^{(2)}\right) \cdot \prod_{\nu=2}^{m+1} \Gamma\left(L_{m+1, \nu}^{(3)}\right)}, \\
& c\left(\lambda_{2, m+2} \rightsquigarrow \lambda_{0,3}\right)=\frac{\prod_{\nu=1}^{m+1} \Gamma\left(\lambda_{2, m+2}-\lambda_{1, \nu}+1\right) \cdot \prod_{i=1}^{2} \Gamma\left(\lambda_{0, i}-\lambda_{0,3}\right)}{\prod_{i=1}^{2} \Gamma\left(L_{m+2}^{(1)}\right) \cdot \prod_{\nu=2}^{m+1} \Gamma\left(L_{m+1, \nu}^{(3)}\right)} .
\end{aligned}
$$


It follows from Theorem 13.7 that the universal operators

$$
\begin{array}{lllllll}
P_{H_{1}}^{0}(\lambda) & P_{H_{1}}^{2}(\lambda) & P_{B_{2 m}}^{0}(\lambda) & P_{B_{2 m}}^{1}(\lambda) & P_{B_{2 m}}^{2}(\lambda) & P_{C_{2 m}}^{1}(\lambda) & P_{C_{2 m}}^{2}(\lambda) \\
P_{H_{2}}^{1}(\lambda) & P_{H_{2}}^{2}(\lambda) & P_{B_{2 m-1}}^{0}(\lambda) & P_{B_{2 m-1}}^{1}(\lambda) & P_{B_{2 m-1}}^{2}(\lambda) & &
\end{array}
$$

define shift operators $R_{B_{2 m+1}}(\epsilon, \lambda)$ under the notation in the theorem.

We also explain how we get the data in $\$ 15.9 .1$. Since $\partial_{\max }: B_{2 m+1}=\mathbf{m}:=$ $m m 1, m+11^{m}, m 1^{m+1} \rightarrow H_{m+1}=\mathbf{m}^{\prime}:=0 m 1,11^{m}, 01^{m+1}$, the equality (9.42) shows

$$
\begin{aligned}
{\left[\Delta\left(B_{2 m+1}\right)\right] } & =\left[\Delta\left(H_{m+1}\right)\right] \cup\left\{d_{1,1,1}(\mathbf{m})\right\} \cup\left\{m_{j, \nu}^{\prime}-m_{j, 1}^{\prime}>0\right\} \\
& =1^{(m+1)^{2}} \times m^{1} \times 1^{m+2} \cdot m^{1}=1^{(m+1)^{2}} \times 1^{m+2} \cdot m^{2}=1^{m^{2}+3 m+3} \cdot m^{2} .
\end{aligned}
$$

Here we note that $\left\{m_{j, \nu}^{\prime}-m_{j, 1}^{\prime}>0\right\}=\left\{m, 1,1^{m+1}\right\}=1^{m+2} \cdot m^{1}$ and $\left[\Delta\left(H_{m+1}\right)\right]$ is given in $\$ 15.4$.

We check (9.44) for $\mathbf{m}$ as follows:

$$
\begin{aligned}
& h(\mathbf{m})=2(1+\cdots+m)+(2 m+1)+2(m+1)+1 \\
& =m^{2}+5 m+4, \quad \oiint_{0}^{1} m+1
\end{aligned}
$$

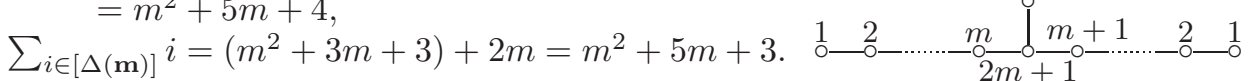

The decompositions $m H_{1} \oplus H_{m+1}$ and $H_{1} \oplus B_{2 m}$ etc. in $\$ 15.9 .1$ are easily obtained and we should show that they are all the decompositions (15.42), whose number is given by $\left[\Delta\left(B_{2 m+1}\right)\right]$. There are 2 decompositions of type $m H_{1} \oplus H_{m+1}$, namely, $B_{2 m+1}=m m 1, m+11^{m}, m 1^{m+1}=m(100,10,10) \oplus \cdots=m(010,10,10) \oplus \cdots$,

which correspond to $L_{i}^{(4)}$ for $i=1$ and 2 . Then the other decompositions are of type $\mathbf{m}^{\prime} \oplus \mathbf{m}^{\prime \prime}$ with rigid tuples $\mathbf{m}^{\prime}$ and $\mathbf{m}^{\prime \prime}$ whose number equals $m^{2}+3 m+3$. The numbers of decompositions $H_{1} \oplus B_{2 m}$ etc. given in $\$ 15.9 .1$ are easily calculated which correspond to $L_{i, \nu}^{(1)}$ etc. and we can check that they give the required number of the decompositions.

$$
\begin{aligned}
& \text { 15.9.3. } C_{n} .\left(C_{4}=E O_{4}, C_{3}=H_{3}, C_{2}=H_{2}\right) \\
& u_{C_{2 m+1}}=\partial^{-\mu^{\prime}} x^{\lambda^{\prime}} u_{H_{m+1}} \\
& m+1 m, m 1^{m+1}, m 1^{m+1}=10,01,10 \oplus m^{2}, m 1^{m}, m-11^{m+1} \\
& =11,11,11 \oplus m(m-1), m-11^{m} m-11^{m} \\
& {\left[\Delta\left(C_{2 m+1}\right)\right]=1^{(m+1)^{2}} \times 1^{2 m+2} \cdot m \cdot(m-1)} \\
& =1^{(m+1)(m+3)} \cdot m \cdot(m-1) \\
& C_{2 m+1}=H_{1} \oplus C_{2 m} \quad: 2 m+2 \\
& =H_{2} \oplus C_{2 m-2} \quad:(m+1)^{2} \\
& =m H_{1} \oplus H_{m+1} \quad: 1 \\
& =(m-1) H_{1} \oplus H_{m+2} \quad: 1 \\
& u_{C_{2 m}}=\partial^{-\mu^{\prime}} x^{\lambda^{\prime}}(1-x)^{-\lambda_{1}-\mu-\mu^{(2)}-\cdots-\mu^{(m)}} u_{H_{m+1}} \\
& m^{2}, m 1^{m}, m-11^{m+1}=1,10,01 \oplus m m-1, m-11^{m-1}, m-11^{m-1} \\
& =1^{2}, 11,11 \oplus(m-1)^{2}, m-11^{m-1}, m-21^{m} \\
& {\left[\Delta\left(C_{2 m}\right)\right]=1^{(m+1)^{2}} \times 1^{m+1} \cdot(m-1)^{2}=1^{m^{2}+3 m+2} \cdot(m-1)^{2}} \\
& C_{2 m}=H_{1} \oplus C_{2 m-1} \quad: 2 m+2 \\
& =H_{2} \oplus C_{2 m-2} \quad: m(m+1) \\
& =(m-1) H_{1} \oplus H_{m+1} \quad: 2
\end{aligned}
$$




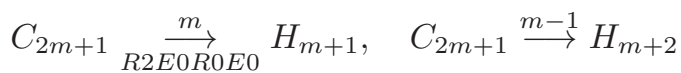

$$
\begin{aligned}
& C_{2 m} \underset{R 1 \stackrel{m-1}{\longrightarrow} H_{0 R E}}{\stackrel{m}{\longrightarrow}} H_{m+1}, \quad C_{n} \stackrel{1}{\longrightarrow} C_{n-1}
\end{aligned}
$$

15.9.4. $D_{n} \cdot\left(D_{6}=X_{6}\right.$ : Extra case, $\left.D_{5}=E O_{5}\right)$

$$
\begin{aligned}
u_{D_{5}} & =\partial^{-\mu_{5}}(1-x)^{-\lambda_{3}-\mu_{3}-\mu_{4}} u_{E_{4}} \\
u_{D_{6}} & =\partial^{-\mu_{6}}(1-x)^{-\lambda_{1}-\mu-\mu_{5}} u_{D_{5}} \\
u_{D_{n}} & =\partial^{-\mu_{n}}(1-x)^{-\lambda_{n}^{\prime}} u_{D_{n-2}} \quad(n \geq 7) \\
(2 m-1) 2,2^{m} 1,2^{m-2} 1^{5} & =10,01,10 \oplus(2 m-2) 2,2^{m}, 2^{m-3} 1^{6} \\
& =10,10,01 \oplus(2 m-2) 2,2^{m-1} 1^{2}, 2^{m-3} 1^{4} \\
& =(m-1) 1,1^{m} 0,1^{m-2} 1^{2} \oplus m 1,1^{m} 1,1^{m-2} 1^{3} \\
m \geq 2 \Rightarrow\left[\Delta\left(D_{2 m+1}\right)\right] & =1^{6 m+2} \cdot 2^{(m-1)(m-3)} \times 1^{6} \cdot 2^{2 m-3}=1^{6 m+8} \cdot 2^{m(m-2)} \\
D_{2 m+1} & =H_{1} \oplus D_{2 m} \quad: m-2 \\
& =H_{1} \oplus E_{2 m} \quad: 5 m \\
& =H_{m} \oplus H_{m+1} \quad: 10 \\
& =2 H_{1} \oplus D_{2 m-1} \quad: m(m-2) \\
(2 m-2) 2,2^{m}, 2^{m-3} 1^{6} & =10,1,01 \oplus(2 m-3) 2,2^{m-1} 1,2^{m-3} 1^{5} \\
& =(m-1) 1,1^{m}, 1^{m-3} 1^{3} \oplus(m-1) 1,1^{m}, 1^{m-3} 1^{3} \\
m \geq 3 \Rightarrow\left[\Delta\left(D_{2 m}\right)\right] & =1^{6 m+6} \cdot 2^{(m-1)(m-4)} \times 1^{6} \cdot 2^{2 m-4}=1^{6 m+10} \cdot 2^{m(m-3)} \\
D_{2 m} & =H_{1} \oplus D_{2 m-1} \quad: 6 m \\
& =H_{m} \oplus H_{m} \quad: 10 \\
& =2 H_{1} \oplus D_{2 m-2} \quad: m(m-3) \\
D_{n} & \stackrel{2}{R 2 E 0} D_{n-2}, \quad D_{n} \stackrel{1}{\longrightarrow} D_{n-1}, \quad D_{2 m+1} \stackrel{1}{\longrightarrow} E_{2 m}
\end{aligned}
$$

15.9.5. $E_{n} .\left(E_{5}=C_{5}, E_{4}=E O_{4}, E_{3}=H_{3}\right)$

$$
\begin{aligned}
u_{E_{3}} & =x^{-\lambda_{0}-\mu-\mu_{3}} \partial^{-\mu_{3}}(1-x)^{\lambda_{3}^{\prime}} u_{H_{2}} \\
u_{E_{4}} & =\partial^{-\mu_{4}} u_{E_{3}} \\
u_{E_{n}} & =\partial^{-\mu_{n}}(1-x)^{\lambda_{n}^{\prime}} u_{E_{n-2}} \quad(n \geq 5) \\
(2 m-1) 2,2^{m-1} 1^{3}, 2^{m-1} 1^{3} & =10,01,10 \oplus(2 m-2) 2,2^{m-1} 1^{2}, 2^{m-2} 1^{4} \\
& =(m-1) 1,1^{m-1} 1,1^{m-1} 1 \oplus m 1,1^{m-1} 1^{3}, 1^{m-1} 1^{2} \\
& =(m-2) 1,1^{m-1} 0,1^{m-1} 0 \oplus(m+1) 1,1^{m-1} 1^{2}, 1^{m-1} 1^{3} \\
m \geq 2 \Rightarrow\left[\Delta\left(E_{2 m+1}\right)\right] & =1^{6 m-2} \cdot 2^{(m-2)^{2}} \times 1^{6} \cdot 2^{2 m-3}=1^{6 m+4} \cdot 2^{(m-1)^{2}} \\
E_{2 m+1} & =H_{1} \oplus E_{2 m} \quad: 6(m-1) \\
& =H_{m-1} \oplus H_{m+2} \quad: 1 \\
& =H_{m} \oplus H_{m+1} \quad: 9 \\
& =2 H_{1} \oplus E_{2 m-1} \quad:(m-1)^{2} \\
(2 m-2) 2,2^{m-1} 1^{2}, 2^{m-2} 1^{4} & =10,10,01 \oplus(2 m-3) 2,2^{m-2} 1^{3}, 2^{m-2} 1^{3} \\
& =10,01,10 \oplus(2 m-3) 2,2^{m-1} 1,2^{m-3} 1^{5}
\end{aligned}
$$




$$
\begin{aligned}
& =(m-2) 1,1^{m-1} 0,1^{m-2} 1 \oplus m 1,1^{m-1} 1^{2}, 1^{m-2} 1^{3} \\
& =(m-1) 1,1^{m-1} 1,1^{m-2} 1^{2} \oplus(m-1) 1,1^{m-1} 1,1^{m-2} 1^{2} \\
& m \geq 2 \Rightarrow\left[\Delta\left(E_{2 m}\right)\right]=1^{6 m-4} \cdot 2^{(m-2)(m-3)} \times 1^{6} \cdot 2^{2 m-4}=1^{6 m+2} \cdot 2^{(m-1)(m-2)} \\
& E_{2 m}=H_{1} \oplus E_{2 m-1} \quad: 4(m-1) \\
& =H_{1} \oplus D_{2 m-1} \quad: 2(m-2) \\
& =H_{m-1} \oplus H_{m+1} \quad: 4 \\
& =H_{m} \oplus H_{m} \quad: 6 \\
& =2 H_{1} \oplus E_{2 m-2} \quad:(m-1)(m-2) \\
& E_{n} \underset{R 2 E 0}{\stackrel{2}{\longrightarrow}} E_{n-2}, \quad E_{n} \stackrel{1}{\longrightarrow} E_{n-1}, \quad E_{2 m} \stackrel{1}{\longrightarrow} D_{2 m-1}
\end{aligned}
$$

15.9.6. $F_{n} .\left(F_{5}=B_{5}, F_{4}=E O_{4}, F_{3}=H_{3}\right)$

$$
\begin{aligned}
u_{F_{3}} & =u_{H_{3}} \\
u_{F_{4}} & =\partial^{-\mu_{4}}(1-x)^{-\lambda_{1}-\lambda_{0}^{(3)}-\mu^{(3)}} u_{F_{3}} \\
u_{F_{n}} & =\partial^{-\mu_{n}}(1-x)^{\lambda_{n}^{\prime}} u_{F_{n-2}} \quad(n \geq 5) \\
(2 m-1) 1^{2}, 2^{m} 1,2^{m-1} 1^{3} & =10,10,01 \oplus(2 m-2) 1^{2}, 2^{m-1} 1^{2}, 2^{m-1} 1^{2} \\
& =10,01,10 \oplus(2 m-2) 1^{2}, 2^{m}, 2^{m-2} 1^{4} \\
& =(m-1) 1,1^{m} 0,1^{m-1} 1 \oplus m 1,1^{m} 1,1^{m-1} 1^{2} \\
m \geq 1 \Rightarrow\left[\Delta\left(F_{2 m+1}\right)\right] & \left.=1^{4 m+1} \cdot 2^{(m-1)(m-2)} \times 1^{4} \cdot 2^{2 m-2}=1^{4 m+5} \cdot 2^{m(m-1)}\right) \\
F_{2 m+1} & =H_{1} \oplus G_{2 m} \quad: 3 m \\
& =H_{1} \oplus F_{2 m} \quad: m-1 \\
& =H_{m} \oplus H_{m+1} \quad: 6 \\
& =2 H_{1} \oplus F_{2 m-1} \quad: m(m-1) \\
(2 m-2) 1^{2}, 2^{m}, 2^{m-2} 1^{4} & =10,1,01 \oplus(2 m-3) 1^{2}, 2^{m-1} 1,2^{m-2} 1^{3} \\
& =(m-1) 1,1^{m}, 1^{m-2} 1^{2} \oplus(m-1) 1,1^{m}, 1^{m-2} 1^{2} \\
m \geq 2 \Rightarrow\left[\Delta\left(F_{2 m}\right)\right] & =1^{4 m+2} \cdot 2^{(m-1)(m-3)} \times 1^{4} \cdot 2^{2 m-3}=1^{4 m+6} \cdot 2^{m(m-2)} \\
F_{2 m} & =H_{1} \oplus F_{2 m-1} \quad: 4 m \\
& =H_{m} \oplus H_{m} \quad: 6 \\
& =2 H_{1} \oplus F_{2 m-2} \quad: m(m-2) \\
F_{n} & \stackrel{2}{R 2 E 0} F_{n-2}, \quad F_{n} \stackrel{1}{\longrightarrow} F_{n-1}, \quad F_{2 m+1} \stackrel{1}{\longrightarrow} G_{2 m}
\end{aligned}
$$

15.9.7. $G_{2 m} \cdot\left(G_{4}=B_{4}\right)$

$$
\begin{aligned}
u_{G_{2}} & =u_{H_{2}} \\
u_{G_{2 m}} & =\partial^{-\mu_{2 m}}(1-x)^{\lambda_{2 m}^{\prime}} u_{G_{2 m-2}} \\
(2 m-2) 1^{2}, 2^{m-1} 1^{2}, 2^{m-1} 1^{2} & =10,01,01 \oplus(2 m-3) 1^{2}, 2^{m-1} 1,2^{m-2} 1^{3} \\
& =(m-2) 1,1^{m-1} 0,1^{m-1} 0 \oplus m 1,1^{m-1} 1^{2}, 1^{m-1} 1^{2} \\
m \geq 2 \Rightarrow\left[\Delta\left(G_{2 m}\right)\right] & =1^{4 m-2} \cdot 2^{(m-2)^{2}} \times 1^{4} \cdot 2^{2 m-3}=1^{4 m+2} \cdot 2^{(m-1)^{2}} \\
G_{2 m} & =H_{1} \oplus F_{2 m-1} \quad: 4 m \\
& =H_{m-1} \oplus H_{m+1} \quad: 2 \\
& =2 H_{1} \oplus G_{2 m-2} \quad:(m-1)^{2}
\end{aligned}
$$




$$
\begin{aligned}
& G_{2 m}=H_{1} \oplus F_{2 m-1}=H_{m-1} \oplus H_{m+1} \\
& G_{2 m} \underset{R 2 E 0}{\stackrel{2}{\longrightarrow}} G_{2(m-1)}, \quad G_{2 m} \stackrel{1}{\longrightarrow} F_{2 m-1}
\end{aligned}
$$

15.9.8. $I_{n} \cdot\left(I_{2 m+1}=\mathrm{III}_{m}^{*}, I_{2 m}=\mathrm{II}_{m}^{*}, I_{3}=P_{3}\right)$

$$
\begin{aligned}
u_{I_{2 m+1}} & =\partial^{-\mu^{\prime}} x^{\lambda^{\prime}}(c-x)^{\lambda^{\prime \prime}} u_{H_{m}} \\
(2 m) 1, & +1 m, m+11^{m}, m+11^{m} \\
& =10,10,10,01 \oplus(2 m-1) 1, m m, m 1^{m}, m+11^{m-1} \\
& =20,11,11,11 \oplus(2 m-2) 1, m m-1, m 1^{m-1}, m 1^{m-1} \\
{\left[\Delta\left(I_{2 m+1}\right)\right] } & =1^{m^{2}} \times 1^{2 m} \cdot m \cdot(m+1)=1^{m^{2}+2 m} \cdot m \cdot(m+1) \\
I_{2 m+1} & =H_{1} \oplus I_{2 m} \quad: 2 m \\
& =H_{2} \oplus I_{2 m-1} \quad: m^{2} \\
& =m H_{1} \oplus H_{m+1} \quad: 1 \\
& =(m+1) H_{1} \oplus H_{m} \quad: 1 \\
u_{I_{2 m}} & =\partial^{-\mu^{\prime}}(1-c x)^{\lambda^{\prime \prime}} u_{H_{m}} \\
(2 m-1) & 1, m m, m 1^{m}, m+11^{m-1} \\
& =10,01,01,10 \oplus(2 m-2) 1, m m-1, m 1^{m-1}, m 1^{m-1} \\
& =20,11,11,11 \oplus(2 m-3) 1, m-1 m-1, m-11^{m-1}, m 1^{m-2} \\
{\left[\Delta\left(I_{2 m}\right)\right] } & =1^{m^{2}} \times 1^{m} \cdot m^{2}=1^{m(m+1)} \cdot m^{2} \\
I_{2 m} & =H_{1} \oplus I_{2 m-1} \quad: 2 m \\
& =H_{2} \oplus I_{2 m-2} \quad: m(m-1) \\
& =m H_{1} \oplus H_{m} \quad: 2 \\
I_{2 m+1} & \stackrel{m+1}{\longrightarrow} H_{m}, \quad I_{2 m+1} \stackrel{m}{\longrightarrow} H_{m+1}, \quad I_{2 m} \stackrel{m}{\longrightarrow} H_{m}, \quad I_{n} \stackrel{1}{\longrightarrow} I_{n-1} \\
I_{2 m+1} & \stackrel{R 1 E 0}{\longrightarrow} I_{2 m} \underset{R 2 E 0}{\stackrel{I_{2 m-2}}{R}}
\end{aligned}
$$

15.9.9. $J_{n} .\left(J_{4}=I_{4}, J_{3}=P_{3}\right)$

$$
\begin{aligned}
u_{J_{2}} & =(c-x)^{\lambda^{\prime}} u_{H_{2}} \\
u_{J_{3}} & =u_{P_{3}} \\
u_{J_{n}} & =\partial^{-\mu_{n}^{\prime}} x^{\lambda_{n}^{\prime}} u_{J_{n-2}} \quad(n \geq 4) \\
(2 m) 1, & (2 m) 1,2^{m} 1,2^{m} 1 \\
& =10,10,01,10 \oplus(2 m-1) 1,(2 m-1) 1,2^{m}, 2^{m-1} 11 \\
& =(m-1) 1, m 0,1^{m} 0,1^{m} 0 \oplus(m+1), m 1,1^{m} 1,1^{m} 1 \\
{\left[\Delta\left(J_{2 m+1}\right)\right] } & =1^{2 m} \cdot 2^{(m-1)^{2}} \times 1^{2} \cdot 2^{2 m-1}=1^{2 m+2} \cdot 2^{m^{2}} \\
J_{2 m+1} & =H_{1} \oplus J_{2 m} \quad: 2 m \\
& =H_{m} \oplus H_{m+1} \quad: 2 \\
& =2 H_{1} \oplus J_{2 m-2} \quad: m^{2} \\
(2 m-1) & 1,(2 m-1) 1,2^{m}, 2^{m-1} 1^{2} \\
& =10,10,1,01 \oplus(2 m-2) 1,(2 m-2) 1,2^{m-1} 1,2^{m-1} 1 \\
& =(m-1) 1, m 0,1^{m}, 1^{m-1} 1 \oplus m 0,(m-1) 1,1^{m}, 1^{m-1} 1
\end{aligned}
$$




$$
\begin{aligned}
{\left[\Delta\left(J_{2 m}\right)\right] } & =1^{2 m} \cdot 2^{(m-1)(m-2)} \times 1^{2} \cdot 2^{2 m-2}=1^{2 m+2} \cdot 2^{m(m-1)} \\
J_{2 m} & =H_{1} \oplus J_{2 m-1} \quad: 2 m \\
& =H_{m} \oplus H_{m} \quad: 2 \\
& =2 H_{1} \oplus J_{2 m-2} \quad: m(m-1) \\
J_{n} & \underset{R 2 E 0}{\stackrel{2}{\longrightarrow}} J_{n-2}(n \geq 6), \quad J_{n} \stackrel{1}{\longrightarrow} J_{n-1}
\end{aligned}
$$

15.9.10. $K_{n} .\left(K_{5}=M_{5}, K_{4}=I_{4}, K_{3}=P_{3}\right)$

$$
\begin{aligned}
& u_{K_{2 m+1}}=\partial^{\mu+\lambda^{\prime}+\lambda^{\prime \prime}}\left(c^{\prime}-x\right)^{\lambda^{\prime}}\left(c^{\prime \prime}-x\right)^{\lambda^{\prime \prime}} u_{P_{m}} \\
& m+1 m, m+1 m,(2 m) 1,(2 m) 1,(2 m) 1, \ldots \in \mathcal{P}_{m+3}^{(2 m+1)} \\
& =11,11,11,20,20, \ldots \oplus m m-1, m m-1,(2 m-1) 0,(2 m-2) 1,(2 m-2) 1, \ldots \\
& {\left[\Delta\left(K_{2 m+1}\right)\right]=1^{m+1} \cdot(m-1) \times m^{2} \cdot(m+1)=1^{m+1} \cdot(m-1) \cdot m^{2} \cdot(m+1)} \\
& K_{2 m+1}=H_{2} \oplus K_{2 m-1} \quad: m+1 \\
& =(m-1) H_{1} \oplus P_{m+2} \quad: 1 \\
& =m H_{1} \oplus P_{m+1} \quad: 2 \\
& =(m+1) H_{1} \oplus P_{m} \quad: 1 \\
& u_{K_{2 m}}=\partial^{-\mu^{\prime}}\left(c^{\prime}-x\right)^{\lambda^{\prime}} u_{P_{m}} \\
& m m, m m-11,(2 m-1) 1,(2 m-1) 1, \ldots \in \mathcal{P}_{m+2}^{(2 m)} \\
& =01,001,10,10,10, \ldots \oplus m m-1, m m-10,(2 m-2) 1,(2 m-2) 1, \ldots \\
& =11,110,11,20,20, \ldots \oplus m-1 m-1, m-1 m-21,(2 m-2) 0,(2 m-3) 1, \ldots \\
& {\left[\Delta\left(K_{2 m}\right)\right]=1^{m+1} \cdot(m-1) \times 1 \cdot(m-1) \cdot m^{2}=1^{m+2} \cdot(m-1)^{2} \cdot m^{2}} \\
& K_{2 m}=H_{1} \oplus K_{2 m-1} \quad: 2 \\
& =H_{2} \oplus K_{2 m-2} \quad: m \\
& =(m-1) H_{1} \oplus P_{m+1} \quad: 2 \\
& =m H_{1} \oplus P_{m} \quad: 2 \\
& K_{2 m+1} \stackrel{m+1}{\longrightarrow} P_{m}, \quad K_{2 m+1} \stackrel{m}{R 1} P_{m+1}, \quad K_{2 m+1} \stackrel{m-1}{\longrightarrow} P_{m+2} \\
& K_{2 m} \underset{R 1}{\stackrel{m}{R}} P_{m}, \quad K_{2 m} \stackrel{m-1}{\longrightarrow} P_{m+1}, \quad K_{2 m} \stackrel{1}{\longrightarrow} K_{2 m-1} \\
& \text { 15.9.11. } L_{2 m+1} \cdot\left(L_{5}=J_{5}, L_{3}=H_{3}\right) \\
& u_{L_{2 m+1}}=\partial^{-\mu^{\prime}} x^{\lambda^{\prime}} u_{P_{m+1}} \\
& m m 1, m m 1,(2 m) 1,(2 m) 1, \ldots \in \mathcal{P}_{m+2}^{(2 m+1)} \\
& =001,010,10,10, \ldots \oplus m m 0, m m-11,(2 m-1) 1,(2 m-1) 1, \ldots \\
& =110,110,11,20, \ldots \oplus m-1 m-10, m-1 m-11,(2 m-1) 0,(2 m-2) 1, \ldots \\
& {\left[\Delta\left(L_{2 m+1}\right)\right]=1^{m+2} \cdot m \times 1^{2} \cdot m^{3}=1^{m+4} \cdot m^{4}} \\
& L_{2 m+1}=H_{1} \oplus K_{2 m} \quad: 4 \\
& =H_{2} \oplus L_{2 m-1} \quad: m \\
& =m H_{1} \oplus P_{m+1} \quad: 4 \\
& L_{2 m+1}=H_{1} \oplus K_{2 m}, \quad L_{2 m+1}=H_{2} \oplus L_{2 m-1} \\
& L_{2 m+1} \underset{R 2 E 0}{\stackrel{m}{\longrightarrow}} P_{m+1}, \quad L_{2 m+1} \stackrel{1}{\longrightarrow} K_{2 m}
\end{aligned}
$$


15.9.12. $M_{n} .\left(M_{5}=K_{5}, M_{4}=I_{4}, M_{3}=P_{3}\right)$

$$
\begin{aligned}
& u_{M_{2 m+1}}=\partial^{\mu+\lambda_{3}^{\prime}+\cdots+\lambda_{m+2}^{\prime}}\left(c_{3}-x\right)^{\lambda_{3}^{\prime}} \cdots\left(c_{m+2}-x\right)^{\lambda_{m+2}^{\prime}} u_{H_{2}} \\
& (2 m) 1,(2 m) 1,(2 m) 1,(2 m-1) 2,(2 m-1) 2, \ldots \in \mathcal{P}_{m+3}^{(2 m+1)} \\
& =m-11, m 0, m 0, m-11, m-11, \ldots \oplus m+10, m 1, m 1, m 1, m 1, \ldots \\
& =m-10, m-10, m-10, m-21, m-21, \ldots \\
& \oplus m+11, m+11, m+11, m+11, m+11, \ldots \\
& {\left[\Delta\left(M_{2 m+1}\right)\right]=1^{4} \times 2^{m} \cdot(2 m-1)=1^{4} \cdot 2^{m} \cdot(2 m-1)} \\
& M_{2 m+1}=P_{m-1} \oplus P_{m+2} \quad: 1 \\
& =P_{m} \oplus P_{m+1} \quad: 3 \\
& =2 H_{1} \oplus M_{2 m-1} \quad: m \\
& =(2 m-1) H_{1} \oplus H_{2} \quad: 1 \\
& u_{M_{2 m}}=\partial^{-\mu^{\prime}}\left(c_{3}-x\right)^{\lambda_{3}^{\prime}} \cdots\left(c_{m+1}-x\right)^{\lambda_{m+1}^{\prime}} u_{H_{2}} \\
& (2 m-2) 1^{2},(2 m-1) 1,(2 m-1) 1,(2 m-2) 2, \ldots \in \mathcal{P}_{m+2}^{(2 m)} \\
& =01,10,10,10, \ldots \oplus(2 m-2) 1,(2 m-2) 1,(2 m-2) 1,(2 m-3) 2, \ldots . \\
& =m-21, m-10, m-10, m-21, \ldots \oplus m 1, m 1, m 1, m 1, \ldots \\
& =m-11, m-11, m 0, m-11, \ldots \oplus m-11, m 0, m-11, m-11, \ldots \\
& {\left[\Delta\left(M_{2 m}\right)\right]=1^{4} \times 1^{2} \cdot 2^{m-1} \cdot(2 m-2)=1^{6} \cdot 2^{m-1} \cdot(2 m-2)} \\
& M_{2 m}=H_{1} \oplus M_{2 m-1} \quad: 2 \\
& =P_{m-1} \oplus P_{m+1} \quad: 2 \\
& =P_{m} \oplus P_{m} \quad: 2 \\
& =2 H_{1} \oplus M_{2 m-2} \quad: m-1 \\
& =(2 m-2) H_{1} \oplus H_{2} \quad: 1 \\
& M_{n} \stackrel{n-2}{\longrightarrow} H_{2}, \quad M_{n} \stackrel{2}{\longrightarrow} M_{n-2}, \quad M_{2 m} \underset{R 1 E 0}{\stackrel{1}{\longrightarrow}} M_{2 m-1} \underset{R 1}{\longrightarrow} M_{2 m-3}
\end{aligned}
$$

15.9.13. $N_{n} \cdot\left(N_{6}=\mathrm{IV}^{*}, N_{5}=I_{5}, N_{4}=G_{4}, N_{3}=H_{3}\right)$

$$
\begin{aligned}
& u_{N_{2 m+1}}=\partial^{-\mu^{\prime}} x^{\lambda^{\prime}}\left(c_{3}-x\right)^{\lambda_{3}^{\prime}} \cdots\left(c_{m+1}-x\right)^{\lambda_{m+1}^{\prime}} u_{H_{2}} \\
& (2 m-1) 1^{2},(2 m-1) 1^{2},(2 m) 1,(2 m-1) 2,(2 m-1) 2, \ldots \in \mathcal{P}_{m+2}^{(2 m+1)} \\
& =10,01,10,10,10 \ldots \\
& \oplus(2 m-2) 1^{2},(2 m-1) 1,(2 m-1) 1,(2 m-2) 2,(2 m-2) 2, \ldots \\
& =m-11, m-11, m 0, m-11, m-11, \ldots \oplus m 1, m 1, m 1, m 1, m 1, \ldots \\
& {\left[\Delta\left(N_{2 m+1}\right)\right]=1^{4} \times 1^{4} \cdot 2^{m-1} \cdot(2 m-1)=1^{8} \cdot 2^{m-1} \cdot(2 m-1)} \\
& N_{2 m+1}=H_{1} \oplus M_{2 m} \quad: 4 \\
& =P_{m} \oplus P_{m+1} \quad: 4 \\
& =2 H_{1} \oplus N_{2 m-1} \quad: m-1 \\
& =(2 m-1) H_{1} \oplus H_{2} \quad: 1 \\
& u_{N_{2 m}}=\partial^{-\mu^{\prime}} x^{\lambda_{0}^{\prime}}(1-x)^{\lambda_{1}^{\prime}}\left(c_{3}-x\right)^{\lambda_{3}^{\prime}} \cdots\left(c_{m}-x\right)^{\lambda_{m}^{\prime}} u_{H_{2}} \quad(m \geq 2) \\
& (2 m-2) 1^{2},(2 m-2) 1^{2},(2 m-2) 1^{2},(2 m-2) 2,(2 m-2) 2, \ldots \in \mathcal{P}_{m+1}^{(2 m)} \\
& =01,10,10,10,10 \ldots \\
& \oplus(2 m-2) 1,(2 m-3) 1^{2},(2 m-3) 1^{2},(2 m-3) 2,(2 m-3) 2, \ldots
\end{aligned}
$$




$$
\begin{aligned}
& \begin{aligned}
&=m-11, m-11, m-11, m-11, m-11, \ldots \\
& \oplus m-11, m-11, m-11, m-11, m-11, \ldots \\
& {\left[\begin{array}{rl}
\left.\Delta\left(N_{2 m}\right)\right] & =1^{4} \times 1^{6} \cdot 2^{m-2} \cdot(2 m-2)=1^{10} \cdot 2^{m-2} \cdot(2 m-2)
\end{array}\right.} \\
& N_{2 m}=H_{1} \oplus N_{2 m-1} \quad: 6 \\
&=P_{m} \oplus P_{m} \quad: 4 \\
&=2 H_{1} \oplus N_{2 m-2} \quad: m-2 \\
&=(2 m-2) H_{1} \oplus H_{2} \quad: 1
\end{aligned} \\
& N_{n} \stackrel{n-2}{\longrightarrow} H_{2}, \quad N_{n} \stackrel{2}{\longrightarrow} N_{n-2}, \quad N_{2 m+1} \underset{R 1 E 0}{\stackrel{1}{\longrightarrow}} M_{2 m}, \quad N_{2 m} \underset{R 1 E 0}{\stackrel{1}{\longrightarrow}} N_{2 m-1}
\end{aligned}
$$

15.9.14. minimal series. The tuple 11,11,11 corresponds to Gauss hypergeometric series, which has three parameters. Since the action of additions is easily analyzed, we consider the number of parameters of the equation corresponding to a rigid tuple $\mathbf{m}=\left(m_{j, \nu}\right)_{\substack{0 \leq j \leq p \\ 1 \leq \nu \leq n_{j}}} \in \mathcal{P}_{p+1}^{(n)}$ modulo additions and the Fuchs condition equals

$$
n_{0}+n_{1}+\cdots+n_{p}-(p+1) .
$$

Here we assume that $0<m_{j, \nu}<n$ for $1 \leq \nu \leq n_{j}$ and $j=0, \ldots, p$.

We call the number given by (15.43) the effective length of $\mathbf{m}$. The tuple 11,11,11 is the unique rigid tuple of partitions whose effective length equals 3 . Since the reduction $\partial_{\max }$ never increase the effective length and the tuple $\mathbf{m} \in \mathcal{P}_{3}$ satisfying $\partial_{\max }=11,11,11$ is $21,111,111$ or $211,211,211$, it is easy to see that the non-trivial rigid tuple $\mathbf{m} \in \mathcal{P}_{3}$ whose effective length is smaller than 6 is $H_{2}$ or $H_{3}$.

The rigid tuple of partitions with the effective length 4 is also uniquely determined by its order, which is

$$
\begin{aligned}
P_{4,2 m+1} & : m+1 m, m+1 m, m+1 m, m+1 m \\
P_{4,2 m} & : m+1 m-1, m m, m m, m m
\end{aligned}
$$

with $m \in \mathbb{Z}_{>0}$. Here $P_{4,2 m+1}$ is a generalized Jordan-Pochhammer tuple in Example 12.5 i).

In fact, if $\mathbf{m} \in \mathcal{P}$ is rigid with the effective length 4 , the argument above shows $\mathbf{m} \in \mathcal{P}_{4}$ and $n_{j}=2$ for $j=0, \ldots, 3$. Then $2=\sum_{j=0}^{3} m_{j, 1}^{2}+\sum_{j=0}^{3}\left(n-m_{j, 1}\right)^{2}-2 n^{2}$ and $\sum_{j=0}^{3}\left(n-2 m_{j, 1}\right)^{2}=4$ and therefore $\mathbf{m}=P_{4,2 m+1}$ or $P_{4,2 m}$.

We give decompositions of $P_{4, n}$ :

$$
\begin{aligned}
& m+1, m ; m+1, m ; m+1, m ; m+1, m \\
& =k, k+1 ; k+1, k ; k+1, k ; k+1, k \\
& \quad \oplus m-k+1, m-k-1 ; m-k, m-k ; m-k, m-k ; m-k, m-k \\
& =2(k+1, k ; k+1, k ; k+1, k ; \ldots) \\
& \quad \oplus m-2 k-1, m-2 k ; m-2 k-1, m-2 k ; m-2 k-1, m-2 k ; \ldots \\
& {\left[\begin{array}{rl}
\left.\Delta\left(P_{4,2 m+1}\right)\right] & =1^{4 m-4} \cdot 2^{m-1} \times 1^{4} \cdot 2=1^{4 m} \cdot 2^{m} \\
P_{4,2 m+1} & =P_{4,2 k+1} \oplus P_{4,2(m-k)} \quad: 4 \quad(k=0, \ldots, m-1) \\
& =2 P_{4,2 k+1} \oplus P_{4,2 m-4 k-1} \quad: 1 \quad(k=0, \ldots, m-1)
\end{array}\right.}
\end{aligned}
$$

Here $P_{k,-n}=-P_{k, n}$ and in the above decompositions there appear "tuples of partitions" with negative entries corresponding formally to elements in $\Delta^{r e}$ with (9.12) (cf. Remark 9.11 i)). 
It follows from the above decompositions that the Fuchsian equation with the Riemann scheme

$$
\begin{aligned}
& \left\{\begin{array}{cccc}
\infty & 0 & 1 & c_{3} \\
{\left[\lambda_{0,1}\right]_{(m+1)}} & {\left[\lambda_{1,1}\right]_{(m+1)}} & {\left[\lambda_{2,1}\right]_{(m+1)}} & {\left[\lambda_{3,1}\right]_{(m+1)}} \\
{\left[\lambda_{0,2}\right]_{(m)}} & {\left[\lambda_{1,2}\right]_{(m)}} & {\left[\lambda_{2,1}\right]_{(m)}} & {\left[\lambda_{3,2}\right]_{(m)}}
\end{array}\right\} \\
& \sum_{j=0}^{4}\left((m+1) \lambda_{j, 1}+m \lambda_{j, 2}\right)=2 m \quad \text { (Fuchs relation). }
\end{aligned}
$$

is irreducible if and only if

$$
\sum_{j=0}^{4} \sum_{\nu=1}^{2}\left(k+\delta_{\nu, 1}+\left(1-2 \delta_{\nu, 1}\right) \delta_{j, i}\right) \lambda_{j, \nu} \notin \mathbb{Z} \quad(i=0,1, \ldots, 5, k=0,1, \ldots, m) .
$$

When $\mathbf{m}=P_{4,2 m}$, we have the following.

$$
\begin{aligned}
& m+1, m-1 ; m, m ; m, m ; m, m \\
& =k+1, k ; k+1, k ; k+1, k ; k+1, k \\
& \oplus m-k, m-k-1 ; m-k-1, m-k ; m-k-1, m-k ; m-k-1, m-k \\
& =2(k+1, k-1 ; k, k ; k, k ; k, k) \\
& \quad \oplus m-2 k-1, m-2 k+1 ; m-2 k, m-2 k ; m-2 k, m-2 k ; m-2 k ; m-2 k \\
& {\left[\begin{array}{ll}
\left.\Delta\left(P_{4,2 m}\right)\right]=1^{4 m-4} \cdot 2^{m-1} \times 1^{4}=1^{4 m} \cdot 2^{m-1} & \\
P_{4,2 m}=P_{4,2 k+1}(=k+1, k ; k+1, k ; \ldots) \oplus P_{4,2 m-2 k+1} & : 4 \quad(k=0, \ldots, m-1) \\
=2 P_{4,2 k} \oplus P_{4,2 m-4 k} & : 1 \quad(k=1, \ldots, m-1) \\
P_{4, n} \stackrel{1}{\rightarrow} P_{4, n-1}, P_{4,2 m+1} \stackrel{2}{\rightarrow} P_{4,2 m-1}
\end{array}\right.}
\end{aligned}
$$

Roberts [R0] classifies the rigid tuples $\mathbf{m} \in \mathcal{P}_{p+1}$ so that

$$
\frac{1}{n_{0}}+\cdots+\frac{1}{n_{p}} \geq p-1
$$

They are tuples $\mathbf{m}$ in 4 series $\alpha, \beta, \gamma, \delta$, which are close to the tuples $r \tilde{E}_{6}, r \tilde{E}_{7}$, $r \tilde{E}_{8}$ and $r \tilde{D}_{4}$, namely, $\left(n_{0}, \ldots, n_{p}\right)=(3,3,3),(2,2,4),(2,3,6)$ and $(2,2,2,2)$, respectively (cf. (9.46)), and the series are called minimal series. Then $\delta_{n}=P_{4, n}$ and the tuples in the other three series belong to $\mathcal{P}_{3}$. For example, the tuples $\mathbf{m}$ of type $\alpha$ are

$$
\begin{aligned}
\alpha_{3 m} & =m+1 m m-1, m^{3}, m^{3}, & & \alpha_{3}=H_{3}, \\
\alpha_{3 m \pm 1} & =m^{2} m \pm 1, m^{2} m \pm 1, m^{2} m \pm 1, & \alpha_{4} & =B_{4},
\end{aligned}
$$

which are characterized by the fact that their effective lengths equal 6 when $n \geq 4$. As in other series, we have the following:

$$
\begin{aligned}
\alpha_{n} & \stackrel{1}{\rightarrow} \alpha_{n-1}, \quad \alpha_{3 m+1} \stackrel{2}{\rightarrow} \alpha_{3 m-1} \\
{\left[\Delta\left(\alpha_{3 m}\right)\right] } & =\left[\Delta\left(\alpha_{3 m-1}\right)\right] \times 1^{5},\left[\Delta\left(\alpha_{3 m-1}\right)\right]=\left[\Delta\left(\alpha_{3 m-2}\right)\right] \times 1^{4}, \\
{\left[\Delta\left(\alpha_{3 m-2}\right)\right] } & =\left[\Delta\left(\alpha_{3 m-4}\right)\right] \times 1^{6} \cdot 2 \\
{\left[\Delta\left(\alpha_{3 m-1}\right)\right] } & =\left[\Delta\left(\alpha_{2}\right)\right] \times 1^{10(m-1)} \cdot 2^{m-1}=1^{10 m-6} \cdot 2^{m-1} \\
{\left[\Delta\left(\alpha_{3 m}\right)\right] } & =1^{10 m-1} \cdot 2^{m-1} \\
{\left[\Delta\left(\alpha_{3 m-2}\right)\right] } & =1^{10 m-10} \cdot 2^{m-1}
\end{aligned}
$$




$$
\begin{aligned}
& \alpha_{3 m}=m+1 m m-1, m^{3}, m^{3} \\
& =k k k-1, k^{2} k-1, k^{2} k-1 \\
& \oplus(m-k+1)(m-k)(m-k),(m-k)^{2}(m-k+1),(m-k)^{2}(m-k+1) \\
& =k+1 k-1 k, k^{3}, k^{3} \\
& \oplus(m-k+1)(m-k)(m-k-1),(m-k)^{3},(m-k)^{3} \\
& =2\left(k+1 k k-1, k^{3}, k^{3}\right) \\
& \oplus(m-2 k-1)(m-2 k)(m-2 k+1),(m-2 k)^{3},(m-2 k)^{3} \\
& \alpha_{3 m}=\alpha_{3 k-1} \oplus \alpha_{3(m-k)+1} \quad: 9 \quad(k=1, \ldots, m) \\
& =\alpha_{3 k} \oplus \alpha_{3(m-k)} \quad: 1 \quad(k=1, \ldots, m-1) \\
& =2 \alpha_{3 k} \oplus \alpha_{3(m-2 k)} \quad: 1 \quad(k=1, \ldots, m-1) \\
& \alpha_{3 m-1}=m m m-1, m m m-1, m m m-1 \\
& =k k-1 k-1, k k-1 k-1, k k-1 k-1 \\
& \oplus(m-k)(m-k+1)(m-k),(m-k)(m-k+1)(m-k), \cdots \\
& =k+1 k k-1, k^{3}, k^{3} \\
& \oplus(m-k-1)(m-k)(m-k),(m-k)(m-k)(m-k-1), \cdots \\
& =2(k k k-1, k k k-1, k k k-1) \\
& \oplus(m-2 k)(m-2 k)(m-2 k+1),(m-2 k)(m-2 k)(m-2 k+1), \cdots \\
& \alpha_{3 m-1}=\alpha_{3 k-2}(=k, k-1, k-1 ; \cdots) \oplus \alpha_{3(m-k)+1} \quad: 4 \quad(k=1, \ldots, m) \\
& =\alpha_{3 k} \oplus \alpha_{3(m-k)-1} \quad: 6 \quad(k=1, \ldots, m-1) \\
& =2 \alpha_{3 k-1} \oplus \alpha_{3(m-2 k)+1} \quad: 1 \quad(k=1, \ldots, m-1) \\
& \alpha_{3 m-2}=m m-1 m-1, m m-1 m-1, m m-1 m-1 \\
& =k k k-1, k k k-1, k k k-1 \\
& \oplus(m-k)(m-k-1)(m-k),(m-k)(m-k-1)(m-k), \cdots \\
& =k+1 k k-1, k^{3}, k^{3} \\
& \oplus(m-k-1)(m-k-1)(m-k),(m-k)(m-k-1)(m-k-1), \cdots \\
& =2(k k-1 k-1, k k-1 k-1, k k-1 k-1) \\
& \oplus(m-2 k)(m-2 k+1)(m-2 k+1),(m-2 k)(m-2 k+1)(m-2 k+1), \cdots \\
& \alpha_{3 m-2}=\alpha_{3 k-1}(=k, k-1, k-1 ; \cdots) \oplus \alpha_{3(m-k)-1} \quad: 4 \quad(k=1, \ldots, m-1) \\
& =\alpha_{3 k} \oplus \alpha_{3(m-k)-2} \quad: 6 \quad(k=1, \ldots, m-1) \\
& =2 \alpha_{3 k-2} \oplus \alpha_{3(m-2 k)+2} \quad: 1 \quad(k=1, \ldots, m-1)
\end{aligned}
$$

The analysis of the other minimal series

$$
\begin{array}{rlrl}
\beta_{4 m, 2} & =(2 m+1)(2 m-1), m^{4}, m^{4} & & \beta_{4,2}=H_{4} \\
\beta_{4 m, 4} & =(2 m)^{2}, m^{4},(m+1) m^{2}(m-1) & \beta_{4,4}=E O_{4} \\
\beta_{4 m \pm 1} & =(2 m)(2 m \pm 1),(m \pm 1) m^{3},(m \pm 1) m^{3} & \beta_{5} & =C_{5}, \beta_{3}=H_{3} \\
\beta_{4 m+2} & =(2 m+1)^{2},(m+1)^{2} m^{2},(m+1)^{2} m^{2} & & \\
\gamma_{6 m, 2} & =(3 m+1)(3 m-1),(2 m)^{3}, m^{6} & \gamma_{6,2} & =D_{6}=X_{6} \\
\gamma_{6 m, 3} & =(3 m)^{2},(2 m+1)(2 m)(2 m-1), m^{6} & \gamma_{6,3} & =E O_{6} \\
\gamma_{6 m, 6} & =(3 m)^{2},(2 m)^{3},(m+1) m^{4}(m-1) & & \\
\gamma_{6 m \pm 1} & =(3 m)(3 m \pm 1),(2 m)^{2}(2 m \pm 1), m^{5}(m \pm 1) & \gamma_{5} & =E O_{5}
\end{array}
$$




$$
\begin{array}{ll}
\gamma_{6 m \pm 2}=(3 m \pm 1)(3 m \pm 1),(2 m)(2 m \pm 1)^{2}, m^{4}(m \pm 1)^{2} & \gamma_{4}=E O_{4} \\
\gamma_{6 m+3}=(3 m+2)(3 m+1),(2 m+1)^{3},(m+1)^{3} m^{3} & \gamma_{3}=H_{3}
\end{array}
$$

and general $P_{p+1, n}$ will be left to the reader as an exercise.

15.9.15. Relation between series. We have studied the following sets of families of spectral types of Fuchsian differential equations which are closed under the irreducible subquotients in the Grothendieck group.

$$
\begin{array}{ll}
\left\{H_{n}\right\} & \text { (hypergeometric family) } \\
\left\{P_{n}\right\} & \text { (Jordan-Pochhammer series) } \\
\left\{A_{n}=E O_{n}\right\} & \text { (even/odd family) } \\
\left\{B_{n}, C_{n}, H_{n}\right\} & (3 \text { singular points) } \\
\left\{C_{n}, H_{n}\right\} & (3 \text { singular points) } \\
\left\{D_{n}, E_{n}, H_{n}\right\} & (3 \text { singular points) } \\
\left\{F_{n}, G_{2 m}, H_{n}\right\} & (3 \text { singular points }) \\
\left\{I_{n}, H_{n}\right\} & (4 \text { singular points }) \\
\left\{J_{n}, H_{n}\right\} & (4 \text { singular points }) \\
\left\{K_{n}, P_{n}\right\} & \left(\left[\frac{n+5}{2}\right] \text { singular points }\right) \\
\left\{L_{2 m+1}, K_{n}, P_{n}\right\} & (m+2 \text { singular points }) \\
\left\{M_{n}, P_{n}\right\} & \left(\left[\frac{n+5}{2}\right] \text { singular points }\right) \quad \supset\left\{M_{2 m+1}, P_{n}\right\} \\
\left\{N_{n}, M_{n}, P_{n}\right\} & \left(\left[\frac{n+3}{2}\right] \text { singular points }\right) \quad \supset\left\{N_{2 m+1}, M_{n} . P_{n}\right\} \\
\left\{P_{4, n}=\delta_{n}\right\} & (4 \text { effective parameters }) \\
\left\{\alpha_{n}\right\} & (6 \text { effective parameters and } 3 \text { singular points })
\end{array}
$$

Yokoyama classified $\mathbf{m}=\left(m_{j, \nu}\right)_{\substack{0 \leq j \leq p \\ 1 \leq \nu \leq n_{j}}} \in \mathcal{P}_{p+1}$ such that

$$
\mathbf{m} \text { is irreducibly realizable, }
$$

$$
\begin{aligned}
& m_{0,1}+\cdots+m_{p-1,1}=(p-1) \text { ord } \mathbf{m} \quad(\mathbf{m} \text { is of Okubo type }) \\
& m_{j, \nu}=1 \quad\left(0 \leq j \leq p-1,2 \leq \nu \leq n_{j}\right)
\end{aligned}
$$

The tuple $\mathbf{m}$ satisfying the above conditions is in the following list given by $\mathrm{Y}$, Theorem 2] (cf. [Ro]).

\begin{tabular}{|c|c|c|c|c|}
\hline Yokoyama & type & order & $\mathrm{p}+1$ & tuple of partitions \\
\hline \hline $\mathrm{I}_{n}$ & $H_{n}$ & $n$ & 3 & $1^{n}, n-11,1^{n}$ \\
\hline $\mathrm{I}_{n}^{*}$ & $P_{n}$ & $n$ & $n+1$ & $n-11, n-11, \ldots, n-11$ \\
\hline $\mathrm{II}_{n}$ & $B_{2 n}$ & $2 n$ & 3 & $n 1^{n}, n 1^{n}, n n-11$ \\
\hline $\mathrm{II}_{n}^{*}$ & $I_{2 n}$ & $2 n$ & 4 & $n 1^{n}, n+11^{n-1}, 2 n-11, n n$ \\
\hline $\mathrm{III}_{n}$ & $B_{2 n+1}$ & $2 n+1$ & 3 & $n 1^{n+1}, n+11^{n}, n n 1$ \\
\hline $\mathrm{III}_{n}^{*}$ & $I_{2 n+1}$ & $2 n+1$ & 4 & $n+11^{n}, n+11^{n},(2 n) 1, n+1 n$ \\
\hline $\mathrm{IV}$ & $F_{6}$ & 6 & 3 & $21111,411,222$ \\
\hline $\mathrm{IV}^{*}$ & $N_{6}$ & 6 & 4 & $411,411,411,42$ \\
\hline
\end{tabular}

15.10. Appell's hypergeometric functions. First we recall the Appell hypergeometric functions.

$$
F_{1}\left(\alpha ; \beta, \beta^{\prime} ; \gamma ; x, y\right)=\sum_{m, n=0}^{\infty} \frac{(\alpha)_{m+n}(\beta)_{m}\left(\beta^{\prime}\right)_{n}}{(\gamma)_{m+n} m ! n !} x^{m} y^{n}
$$




$$
\begin{aligned}
F_{2}\left(\alpha ; \beta, \beta^{\prime} ; \gamma, \gamma^{\prime} ; x, y\right) & =\sum_{m, n=0}^{\infty} \frac{(\alpha)_{m+n}(\beta)_{m}\left(\beta^{\prime}\right)_{n}}{(\gamma)_{m}\left(\gamma^{\prime}\right)_{n} m ! n !} x^{m} y^{n}, \\
F_{3}\left(\alpha, \alpha^{\prime} ; \beta, \beta^{\prime} ; \gamma ; x, y\right) & =\sum_{m, n=0}^{\infty} \frac{(\alpha)_{m}\left(\alpha^{\prime}\right)_{n}(\beta)_{m}\left(\beta^{\prime}\right)_{n}}{(\gamma)_{m+n} m ! n !} x^{m} y^{n}, \\
F_{4}\left(\alpha ; \beta ; \gamma, \gamma^{\prime} ; x, y\right) & =\sum_{m, n=0}^{\infty} \frac{(\alpha)_{m+n}(\beta)_{m+n}}{(\gamma)_{m}\left(\gamma^{\prime}\right)_{n} m ! n !} x^{m} y^{n} .
\end{aligned}
$$

They satisfy the following equations

$$
\begin{array}{r}
\left(\left(\vartheta_{x}+\vartheta_{y}+\alpha\right)\left(\vartheta_{x}+\beta\right)-\partial_{x}\left(\vartheta_{x}+\vartheta_{y}+\gamma-1\right)\right) F_{1}=0, \\
\left(\left(\vartheta_{x}+\vartheta_{y}+\alpha\right)\left(\vartheta_{x}+\beta\right)-\partial_{x}\left(\vartheta_{x}+\gamma-1\right)\right) F_{2}=0, \\
\left(\left(\vartheta_{x}+\alpha\right)\left(\vartheta_{x}+\beta\right)-\partial_{x}\left(\vartheta_{x}+\vartheta_{y}+\gamma-1\right)\right) F_{3}=0, \\
\left(\left(\vartheta_{x}+\vartheta_{y}+\alpha\right)\left(\vartheta_{x}+\vartheta_{y}+\beta\right)-\partial_{x}\left(\vartheta_{x}+\gamma-1\right)\right) F_{4}=0 .
\end{array}
$$

Similar equations hold under the symmetry $x \leftrightarrow y$ with $(\alpha, \beta, \gamma) \leftrightarrow\left(\alpha^{\prime}, \beta^{\prime}, \gamma^{\prime}\right)$.

15.10.1. Appell's $F_{1}$. First we examine $F_{1}$. Put

$$
\begin{aligned}
u(x, y) & :=\int_{0}^{x} t^{\alpha}(1-t)^{\beta}(y-t)^{\gamma-1}(x-t)^{\lambda-1} d t \quad(t=x s) \\
& =\int_{0}^{1} x^{\alpha+\lambda+1} s^{\alpha}(1-x s)^{\beta}(y-x s)^{\gamma-1}(1-s)^{\lambda-1} d s \\
& =x^{\alpha+\lambda} y^{\gamma-1} \int_{0}^{1} s^{\alpha}(1-s)^{\lambda-1}(1-x s)^{\beta}\left(1-\frac{y}{x} s\right)^{\gamma-1} d s, \\
h_{x} & :=x^{\alpha}(x-1)^{\beta}(x-y)^{\gamma-1} .
\end{aligned}
$$

Since the left ideal of $\bar{W}[x, y]$ is not necessarily generated by a single element, we want to have good generators of $\operatorname{RAd}\left(\partial_{x}^{-\lambda}\right) \circ \operatorname{RAd}\left(h_{x}\right)\left(W[x, y] \partial_{x}+W[x, y] \partial_{y}\right)$ and we have

$$
\begin{aligned}
P & :=\operatorname{Ad}\left(h_{x}\right) \partial_{x}=\partial_{x}-\frac{\alpha}{x}-\frac{\beta}{x-1}-\frac{\gamma-1}{x-y}, \\
Q & :=\operatorname{Ad}\left(h_{x}\right) \partial_{y}=\partial_{y}+\frac{\gamma-1}{x-y}, \\
R & :=x P+y Q=x \partial_{x}+y \partial_{y}-(\alpha+\gamma-1)-\frac{\beta x}{x-1}, \\
S & :=\partial_{x}(x-1) R=\left(\vartheta_{x}+1\right)\left(\vartheta_{x}+\vartheta_{y}-\alpha-\beta-\gamma+1\right)-\partial_{x}\left(\vartheta_{x}+\vartheta_{y}-\alpha-\gamma+1\right) \\
T & :=\partial_{x}^{-\lambda} \circ S \circ \partial_{x}^{\lambda} \\
& =\left(\vartheta_{x}-\lambda+1\right)\left(\vartheta_{x}+\vartheta_{y}-\alpha-\beta-\gamma-\lambda+1\right)-\partial_{x}\left(\vartheta_{x}+\vartheta_{y}-\alpha-\gamma-\lambda+1\right)
\end{aligned}
$$

with

$$
a=-\alpha-\beta-\gamma-\lambda+1, b=1-\lambda, c=2-\alpha-\gamma-\lambda .
$$

This calculation shows the equation $T u(x, y)=0$ and we have a similar equation by changing $(x, y, \gamma, \lambda) \mapsto(y, x, \lambda, \gamma)$. Note that $T F_{1}\left(a ; b, b^{\prime} ; c ; x, y\right)=0$ with $b^{\prime}=1-\gamma$. 
Putting

$$
\begin{aligned}
v(x, z) & =I_{0, x}^{\mu}\left(x^{\alpha}(1-x)^{\beta}(1-z x)^{\gamma-1}\right) \\
& =\int_{0}^{x} t^{\alpha}(1-t)^{\beta}(1-z t)^{\gamma-1}(x-t)^{\mu-1} d t \\
& =x^{\alpha+\mu} \int_{0}^{1} s^{\alpha}(1-x s)^{\beta}(1-x z s)^{\gamma-1}(1-s)^{\mu-1} d s,
\end{aligned}
$$

we have

$$
\begin{gathered}
u(x, y)=y^{\gamma-1} v\left(x, \frac{1}{y}\right), \\
t^{\alpha}(1-t)^{\beta}(1-z t)^{\gamma-1}=\sum_{m, n=0}^{\infty} \frac{(-\beta)_{m}(1-\gamma)_{n}}{m ! n !} t^{\alpha+m+n} z^{n}, \\
v(x, z)=\sum_{m, n=0}^{\infty} \frac{\Gamma(\alpha+m+n+1)(-\beta)_{m}(1-\gamma)_{n}}{\Gamma(\alpha+\mu+m+n+1) m ! n !} x^{\alpha+\gamma+m+n} z^{n} \\
=x^{\alpha+\mu} \frac{\Gamma(\alpha+1)}{\Gamma(\alpha+\mu+1)} \sum_{m, n=0}^{\infty} \frac{(\alpha+1)_{m+n}(-\beta)_{m}(1-\gamma)_{n}}{(\alpha+\mu+1)_{m+n} m ! n !} x^{m+n} z^{n} \\
=x^{\alpha+\mu} \frac{\Gamma(\alpha+1)}{\Gamma(\alpha+\mu+1)} F_{1}(\alpha+1 ;-\beta, 1-\gamma ; \alpha+\mu+1 ; x, x z) .
\end{gathered}
$$

Using a versal addition to get the Kummer equation, we introduce the functions

$$
\begin{aligned}
v_{c}(x, y) & :=\int_{0}^{x} t^{\alpha}(1-c t)^{\frac{\beta}{c}}(y-t)^{\gamma-1}(x-t)^{\lambda-1}, \\
h_{c, x} & :=x^{\alpha}(1-c x)^{\frac{\beta}{c}}(x-y)^{\gamma-1} .
\end{aligned}
$$

Then we have

$$
\begin{aligned}
R & :=\operatorname{Ad}\left(h_{c, x}\right)\left(\vartheta_{x}+\vartheta_{y}\right)=\vartheta_{x}+\vartheta_{y}-(\alpha+\gamma-1)+\frac{\beta x}{1-c x}, \\
S & :=\partial_{x}(1-c x) R \\
& =\left(\vartheta_{x}+1\right)\left(\beta-c\left(\vartheta_{x}+\vartheta_{y}-\alpha-\gamma+1\right)\right)+\partial_{x}\left(\vartheta_{x}+\vartheta_{y}-\alpha-\gamma+1\right), \\
T & :=\operatorname{Ad}\left(\partial^{-\lambda}\right) R \\
& =\left(\vartheta_{x}-\lambda+1\right)\left(\beta-c\left(\vartheta_{x}+\vartheta_{y}-\lambda-\alpha-\gamma+1\right)\right)+\partial_{x}\left(\vartheta_{x}+\vartheta_{y}-\lambda-\alpha-\gamma+1\right)
\end{aligned}
$$

and hence $u_{c}(x, y)$ satisfies the differential equation

$$
\begin{aligned}
& \left(x(1-c x) \partial_{x}^{2}+y(1-c x) \partial_{x} \partial_{y}\right. \\
& \quad+(2-\alpha-\gamma-\lambda+(\beta+\lambda-2+c(\alpha+\gamma+\lambda-1)) x) \partial_{x}+(\lambda-1) \partial_{y} \\
& \quad-(\lambda-1)(\beta+c(\alpha+\gamma+\lambda-1))) u=0 .
\end{aligned}
$$

15.10.2. Appell's $F_{4}$. To examine $F_{4}$ we consider the function

$$
v(x, y):=\int_{\Delta} s^{\lambda_{1}} t^{\lambda_{2}}(s t-s-t)^{\lambda_{3}}(1-s x-t y)^{\mu} d s d t
$$

and the transformation

$$
J_{x}^{\mu}(u)(x):=\int_{\Delta} u\left(t_{1}, \ldots, t_{n}\right)\left(1-t_{1} x_{1}-\cdots-t_{n} x_{n}\right)^{\mu} d t_{1} \cdots d t_{n}
$$


for function $u\left(x_{1}, \ldots, u_{n}\right)$. For example the region $\Delta$ is given by

$$
v(x, y)=\int_{s \leq 0, t \leq 0} s^{\lambda_{1}} t^{\lambda_{2}}(s t-s-t)^{\lambda_{3}}(1-s x-t y)^{\mu} d s d t .
$$

Putting $s \mapsto s^{-1}, t \mapsto t^{-1}$ and $|x|+|y|<c<\frac{1}{2}$, Aomoto Ad shows

$$
\begin{aligned}
& \int_{c-\infty i}^{c+\infty i} \int_{c-\infty i}^{c+\infty i} s^{-\gamma} t^{-\gamma^{\prime}}(1-s-t)^{\gamma+\gamma^{\prime}-\alpha-2}\left(1-\frac{x}{s}-\frac{y}{t}\right)^{-\beta} d s d t \\
& =-\frac{4 \pi^{2} \Gamma(\alpha)}{\Gamma(\gamma) \Gamma\left(\gamma^{\prime}\right) \Gamma\left(\alpha-\gamma-\gamma^{\prime}+2\right)} F_{4}\left(\alpha ; \beta ; \gamma, \gamma^{\prime} ; x, y\right),
\end{aligned}
$$

which follows from the integral formula

$$
\begin{aligned}
& \frac{1}{(2 \pi i)^{n}} \int_{\frac{1}{n+1}-\infty i}^{\frac{1}{n+1}+\infty i} \cdots \int_{\frac{1}{n+1}-\infty i}^{\frac{1}{n+1}+\infty i} \prod_{j=1}^{n} t_{j}^{-\alpha_{j}}\left(1-\sum_{j=1}^{n} t_{j}\right)^{-\alpha_{n+1}} d t_{1} \cdots d t_{n} \\
& \quad=\frac{\Gamma\left(\sum_{j=1}^{n+1} \alpha_{j}-n\right)}{\prod_{j=1}^{n+1} \Gamma\left(\alpha_{j}\right)} .
\end{aligned}
$$

Since

$$
J_{x}^{\mu}(u)=J_{x}^{\mu-1}(u)-\sum x_{\nu} J_{x}^{\mu-1}\left(x_{\nu} u\right)
$$

and

$$
\begin{aligned}
\frac{d}{d t_{i}}(u(t)(1 & \left.\left.-\sum t_{\nu} x_{\nu}\right)^{\mu}\right) \\
& =\frac{d u}{d t_{i}}(t)\left(1-\sum t_{\nu} x_{\nu}\right)^{\mu}-\mu u(t) x_{i}\left(1-\sum t_{\nu} x_{\nu}\right)^{\mu-1},
\end{aligned}
$$

we have

$$
\begin{aligned}
J_{x}^{\mu}\left(\partial_{i} u\right)(x) & =\mu x_{i} J_{x}^{\mu-1}(u)(x) \\
& =-x_{i} \int t_{i}^{-1} u(t) \frac{d}{d x_{i}}\left(1-\sum x_{\nu} t_{\nu}\right)^{\mu} d t \\
& =-x_{i} \frac{d}{d x_{i}} J_{x}^{\mu}\left(\frac{u}{x_{i}}\right)(x), \\
J_{x}^{\mu}\left(\partial_{i}\left(x_{i} u\right)\right) & =-x_{i} \partial_{i} J_{x}^{\mu}(u), \\
J_{x}^{\mu}\left(\partial_{i} u\right) & =\mu x_{i} J_{x}^{\mu-1}(u) \\
& =\mu x_{i} J_{x}^{\mu}(u)+\mu x_{i} \sum x_{\nu} J_{x}^{\mu-1}\left(x_{\nu} u\right) \\
& =\mu x_{i} J_{x}^{\mu}(u)+x_{i} \sum J_{x}^{\mu}\left(\partial_{\nu}\left(x_{\nu} u\right)\right) \\
& =\mu x_{i} J_{x}^{\mu}(u)-x_{i} \sum x_{\nu} \partial_{\nu} J_{x}^{\mu}(u)
\end{aligned}
$$

and therefore

$$
\begin{aligned}
J_{x}^{\mu}\left(x_{i} \partial_{i} u\right) & =\left(-1-x_{i} \partial_{i}\right) J_{x}^{\mu}(u), \\
J_{x}^{\mu}\left(\partial_{i} u\right) & =x_{i}\left(\mu-\sum x_{\nu} \partial_{\nu}\right) J_{x}^{\mu}(u) .
\end{aligned}
$$

Thus we have

Proposition 15.1. For a differential operator

$$
P=\sum_{\substack{\alpha=\left(\alpha_{1}, \ldots, \alpha_{n}\right) \in \mathbb{Z}_{\geq 0}^{n} \\ \beta=\left(\beta_{1}, \ldots, \beta_{n}\right) \in \mathbb{Z}_{\geq 0}^{n}}} c_{\alpha, \beta} \partial_{1}^{\alpha_{1}} \cdots \partial_{n}^{\alpha_{n}} \vartheta_{1}^{\beta_{1}} \cdots \vartheta_{n}^{\beta_{n}},
$$


we have

$$
\begin{aligned}
J_{x}^{\mu}(P u(x)) & =J_{x}^{\mu}(P) J_{x}^{\mu}(u(x)), \\
J_{x}^{\mu}(P) & :=\sum_{\alpha, \beta} c_{\alpha, \beta} \prod_{k=1}^{n}\left(x_{k}\left(\mu-\sum_{\nu=1}^{n} \vartheta_{\nu}\right)\right)^{\alpha_{k}} \prod_{k=1}^{n}\left(-\vartheta_{k}-1\right)^{\beta_{k}} .
\end{aligned}
$$

Using this proposition, we obtain the system of differential equations satisfied by $J_{x}^{\mu}(u)$ from that satisfied by $u(x)$. Denoting the Laplace transform of the variable $x=\left(x_{1}, \ldots, x_{n}\right)$ by $\mathrm{L}_{x}$ (cf. Definition 2.1), we have

$$
J_{x}^{\mu} L_{x}^{-1}\left(\vartheta_{i}\right)=\vartheta_{i}, \quad J_{x}^{\mu} L_{x}^{-1}\left(x_{i}\right)=x_{i}\left(\mu-\sum_{\nu=1}^{n} \vartheta_{\nu}\right) .
$$

We have

$$
\begin{aligned}
& \operatorname{Ad}\left(x^{\lambda_{1}} y^{\lambda_{2}}(x y-x-y)^{\lambda_{3}}\right) \partial_{x}=\partial_{x}-\frac{\lambda_{1}}{x}-\frac{\lambda_{3}(y-1)}{x y-x-y}, \\
& \operatorname{Ad}\left(x^{\lambda_{1}} y^{\lambda_{2}}(x y-x-y)^{\lambda_{3}}\right) \partial_{y}=\partial_{y}-\frac{\lambda_{2}}{y}-\frac{\lambda_{3}(x-1)}{x y-x-y}, \\
& \operatorname{Ad}\left(x^{\lambda_{1}} y^{\lambda_{2}}(x y-x-y)^{\lambda_{3}}\right)\left(x(x-1) \partial_{x}\right) \\
& =x(x-1) \partial_{x}-\lambda_{1}(x-1)-\frac{\lambda_{3}(x-1)(x y-x)}{x y-x-y}, \\
& \operatorname{Ad}\left(x^{\lambda_{1}} y^{\lambda_{2}}(x y-x-y)^{\lambda_{3}}\right)\left(x(x-1) \partial_{x}-y \partial_{y}\right) \\
& =x(x-1) \partial_{x}-y \partial_{y}-\lambda_{1}(x-1)-\lambda_{2}-\lambda_{3}(x-1) \\
& =x \vartheta_{x}-\vartheta_{x}-\vartheta_{y}-\left(\lambda_{1}+\lambda_{3}\right) x+\lambda_{1}-\lambda_{2}+\lambda_{3}, \\
& \partial_{x} \operatorname{Ad}\left(x^{\lambda_{1}} y^{\lambda_{2}}(x y-x-y)^{\lambda_{3}}\right)\left(x(x-1) \partial_{x}-y \partial_{y}\right) \\
& =\partial_{x} x\left(\vartheta_{x}-\lambda_{1}-\lambda_{3}\right)-\partial_{x}\left(\vartheta_{x}+\vartheta_{y}-\lambda_{1}+\lambda_{2}-\lambda_{3}\right)
\end{aligned}
$$

and

$$
\begin{aligned}
& J_{x, y}^{\mu}\left(\partial_{x} x\left(\vartheta_{x}-\lambda_{1}-\lambda_{3}\right)-\partial_{x}\left(\vartheta_{x}+\vartheta_{y}-\lambda_{1}+\lambda_{2}-\lambda_{3}\right)\right) \\
& \quad=\vartheta_{x}\left(1+\vartheta_{x}+\lambda_{1}+\lambda_{3}\right)-x\left(-\mu+\vartheta_{x}+\vartheta_{y}\right)\left(2+\vartheta_{x}+\vartheta_{y}+\lambda_{1}-\lambda_{2}+\lambda_{3}\right) .
\end{aligned}
$$

Putting

$$
T:=\left(\vartheta_{x}+\vartheta_{y}-\mu\right)\left(\vartheta_{x}+\vartheta_{y}+\lambda_{1}-\lambda_{2}+\lambda_{3}+2\right)-\partial_{x}\left(\vartheta_{x}+\lambda_{1}+\lambda_{3}+1\right)
$$

with

$$
\alpha=-\mu, \quad \beta=\lambda_{1}-\lambda_{2}+\lambda_{3}+2, \quad \gamma=\lambda_{1}+\lambda_{3}+2,
$$

we have $T v(x, y)=0$ and moreover it satisfies a similar equation by replacing $\left(x, y, \lambda_{1}, \lambda_{3}, \gamma\right)$ by $\left(y, x, \lambda_{3}, \lambda_{1}, \gamma^{\prime}\right)$. Hence $v(x, y)$ is a solution of the system of differential equations satisfied by $F_{4}\left(\alpha ; \beta ; \gamma, \gamma^{\prime} ; x, y\right)$.

In the same way we have

$$
\begin{gathered}
\operatorname{Ad}\left(x^{\beta-1} y^{\beta^{\prime}-1}(1-x-y)^{\gamma-\beta-\beta^{\prime}-1}\right) \vartheta_{x}=\vartheta_{x}-\beta+1+\frac{\left(\gamma-\beta-\beta^{\prime}-1\right) x}{1-x-y}, \\
\operatorname{Ad}\left(x^{\beta-1} y^{\beta^{\prime}-1}(1-x-y)^{\gamma-\beta-\beta^{\prime}-1}\right)\left(\vartheta_{x}-x\left(\vartheta_{x}+\vartheta_{y}\right)\right) \\
=\vartheta_{x}-x\left(\vartheta_{x}+\vartheta_{y}\right)-\beta+1+(\gamma-3) x \\
=\left(\vartheta_{x}-\beta+1\right)-x\left(\vartheta_{x}+\vartheta_{y}-\gamma+3\right), \\
J_{x, y}^{\mu}\left(\partial_{x}\left(\vartheta_{x}-\beta+1\right)-\partial_{x} x\left(\vartheta_{x}+\vartheta_{y}-\gamma+3\right)\right) \\
=x\left(-\vartheta_{x}-\vartheta_{y}+\mu\right)\left(-\vartheta_{x}-\beta\right)+\vartheta_{x}\left(-2-\vartheta_{x}-\vartheta_{y}-\gamma+3\right) \\
=x\left(\left(\vartheta_{x}+\vartheta_{y}-\mu\right)\left(\vartheta_{x}+\beta\right)-\partial_{x}\left(\vartheta_{x}+\vartheta_{y}+\gamma-1\right)\right) .
\end{gathered}
$$


which is a differential operator killing $F_{1}\left(\alpha ; \beta, \beta^{\prime} ; \gamma ; x, y\right)$ by putting $\mu=-\alpha$ and in fact we have

$$
\begin{aligned}
& \iint_{\substack{s \geq 0, t \geq 0 \\
1-s-t \geq 0}} s^{\beta-1} t^{\beta^{\prime}-1}(1-s-t)^{\gamma-\beta-\beta^{\prime}-1}(1-s x-t y)^{-\alpha} d s d t \\
& \quad=\iint_{\substack{s \geq 0, t \geq 0 \\
1-s-t \geq 0}} \sum_{m, n=0}^{\infty} s^{\beta+m-1} t^{\beta^{\prime}+n-1}(1-s-t)^{\gamma-\beta-\beta^{\prime}-1} \frac{(\alpha)_{m+n} x^{m} y^{n}}{m ! n !} d s d t \\
& \quad=\sum_{m, n=0}^{\infty} \frac{\Gamma(\beta+m) \Gamma\left(\beta^{\prime}+n\right) \Gamma\left(\gamma-\beta-\beta^{\prime}\right)}{\Gamma(\gamma+m+n)} \cdot \frac{(\alpha)_{m+n}}{m ! n !} x^{m} y^{n} \\
& \quad=\frac{\Gamma(\beta) \Gamma\left(\beta^{\prime}\right) \Gamma\left(\gamma-\beta-\beta^{\prime}\right)}{\Gamma(\gamma)} F_{1}\left(\alpha ; \beta, \beta^{\prime} ; \gamma ; x, y\right) .
\end{aligned}
$$

Here we use the formula

$$
\iint_{\substack{s \geq 0, t \geq 0 \\ 1-s-t \geq 0}} s^{\lambda_{1}-1} t^{\lambda_{2}-1}(1-s-t)^{\lambda_{3}-1} d s d t=\frac{\Gamma\left(\lambda_{1}\right) \Gamma\left(\lambda_{2}\right) \Gamma\left(\lambda_{3}\right)}{\Gamma\left(\lambda_{1}+\lambda_{2}+\lambda_{3}\right)} .
$$

15.10.3. Appell's $F_{3}$. Since

$$
\begin{aligned}
T_{3}: & =J_{y}^{-\alpha^{\prime}} x^{-1} J_{x}^{-\alpha}\left(\partial_{x}\left(\vartheta_{x}-\beta+1\right)-\partial_{x} x\left(\vartheta_{x}+\vartheta_{y}-\gamma+3\right)\right) \\
& =J_{y}^{-\alpha^{\prime}}\left(\left(-\vartheta_{x}-\alpha\right)\left(-\vartheta_{x}-\beta\right)+\partial_{x}\left(-\vartheta_{x}+\vartheta_{y}-\gamma+2\right)\right) \\
& =\left(\vartheta_{x}+\alpha\right)\left(\vartheta_{x}+\beta\right)-\partial_{x}\left(\vartheta_{x}+\vartheta_{y}+\gamma-1\right)
\end{aligned}
$$

with (15.67), the operator $T_{3}$ kills the function

$$
\begin{aligned}
& \iint_{\substack{s \geq 0, t \geq 0 \\
1-s-t \geq 0}} s^{\beta-1} t^{\beta^{\prime}-1}(1-s-t)^{\gamma-\beta-\beta^{\prime}-1}(1-x s)^{-\alpha}(1-y t)^{-\alpha^{\prime}} d s d t \\
& \quad=\iint_{\substack{s \geq 0, t \geq 0 \\
1-s-t \geq 0}} \sum_{m, n=0}^{\infty} s^{\beta+m-1} t^{\beta^{\prime}+n-1}(1-s-t)^{\gamma-\beta-\beta^{\prime}-1} \frac{(\alpha)_{m}\left(\alpha^{\prime}\right)_{n} x^{m} y^{n}}{m ! n !} d s d t \\
& \quad=\sum_{m, n=0}^{\infty} \frac{\Gamma(\beta+m) \Gamma\left(\beta^{\prime}+n\right) \Gamma\left(\gamma-\beta-\beta^{\prime}\right)(\alpha)_{m}\left(\alpha^{\prime}\right)_{n}}{\Gamma(\gamma+m+n) m ! n !} x^{m} y^{n} \\
& \quad=\frac{\Gamma(\beta) \Gamma\left(\beta^{\prime}\right) \Gamma\left(\gamma-\beta-\beta^{\prime}\right)}{\Gamma(\gamma)} F_{3}\left(\alpha, \alpha^{\prime} ; \beta, \beta^{\prime} ; \gamma ; x, y\right) .
\end{aligned}
$$

Moreover since

$$
\begin{aligned}
T_{3}^{\prime} & :=\operatorname{Ad}\left(\partial_{x}^{-\mu}\right) \operatorname{Ad}\left(\partial_{y}^{-\mu^{\prime}}\right)\left(\left(\vartheta_{x}+1\right)\left(\vartheta_{x}-\lambda_{1}-\lambda_{3}\right)-\partial_{x}\left(\vartheta_{x}+\vartheta_{y}-\lambda_{1}+\lambda_{2}-\lambda_{3}\right)\right) \\
& =\left(\vartheta_{x}+1-\mu\right)\left(\vartheta_{x}-\lambda_{1}-\lambda_{3}-\mu\right)-\partial_{x}\left(\vartheta_{x}+\vartheta_{y}-\lambda_{1}+\lambda_{2}-\lambda_{3}-\mu-\mu^{\prime}\right)
\end{aligned}
$$

with $(15.66)$ and

$$
\alpha=-\lambda_{1}-\lambda_{3}-\mu, \quad \beta=1-\mu, \quad \gamma=-\lambda_{1}+\lambda_{2}-\lambda_{3}-\mu-\mu^{\prime}+1,
$$

the function

$$
u_{3}(x, y):=\int_{\infty}^{y} \int_{\infty}^{x} s^{\lambda_{1}} t^{\lambda_{2}}(s t-s-t)^{\lambda_{3}}(x-s)^{\mu-1}(y-t)^{\mu^{\prime}-1} d s d t
$$

satisfies $T_{3}^{\prime} u_{3}(x, y)=0$. Hence $u_{3}(x, y)$ is a solution of the system of the equations that $F_{3}\left(\alpha, \alpha^{\prime} ; \beta, \beta^{\prime} ; \gamma ; x, y\right)$ satisfies. 
15.10.4. Appell's $F_{2}$. Since

$$
\begin{aligned}
\partial_{x} & \operatorname{Ad}\left(x^{\lambda_{1}-1}\left(1-x^{\lambda_{2}-1}\right)\right) x(1-x) \partial_{x} \\
& =\partial_{x} x(1-x) \partial_{x}-\left(\lambda_{1}-1\right) \partial_{x}+\partial_{x}\left(\lambda_{1}+\lambda_{2}-2\right) x \\
& =\partial_{x} x\left(-\vartheta_{x}+\lambda_{1}+\lambda_{2}-2\right)+\partial_{x}\left(\vartheta-\lambda_{1}+1\right)
\end{aligned}
$$

and

$$
\begin{aligned}
T_{2}: & =J_{x, y}^{\mu}\left(\partial_{x} x\left(-\vartheta_{x}+\lambda_{1}+\lambda_{2}-2\right)+\partial_{x}\left(\vartheta_{x}-\lambda_{1}+1\right)\right) \\
& =-\vartheta_{x}\left(\vartheta_{x}+1+\lambda_{1}+\lambda_{2}-2\right)+x\left(\mu-\vartheta_{x}-\vartheta_{y}\right)\left(-1-\vartheta_{x}-\lambda_{1}+1\right) \\
& =x\left(\left(\vartheta_{x}+\lambda_{1}\right)\left(\vartheta_{x}+\vartheta_{y}-\mu\right)-\partial_{x}\left(\vartheta_{x}+\lambda_{1}+\lambda_{2}-1\right)\right)
\end{aligned}
$$

with

the function

$$
\begin{aligned}
u_{2}(x, y) & :=\int_{0}^{1} \int_{0}^{1} s^{\lambda_{1}-1}(1-s)^{\lambda_{2}-1} t^{\lambda_{1}^{\prime}-1}(1-t)^{\lambda_{2}^{\prime}-1}(1-x s-y t)^{\mu} d s d t \\
& =\int_{0}^{1} \int_{0}^{1} \sum_{m, n=0}^{\infty} s^{\lambda_{1}+m-1}(1-s)^{\lambda_{2}-1} t^{\lambda_{1}^{\prime}+n-1}(1-t)^{\lambda_{2}^{\prime}-1} \frac{(-\mu)_{m+n}}{m ! n !} x^{m} y^{n} d s d t \\
& =\sum_{m, n=0}^{\infty} \frac{\Gamma\left(\lambda_{1}+m\right) \Gamma\left(\lambda_{2}\right)}{\Gamma\left(\lambda_{1}+\lambda_{2}+m\right)} \frac{\Gamma\left(\lambda_{1}^{\prime}+n\right) \Gamma\left(\lambda_{2}^{\prime}\right)}{\Gamma(-\mu)_{m+n}} \frac{\left(\lambda_{1}^{\prime}+\lambda_{2}^{\prime}+m\right)}{m ! n !} x^{m} y^{n} \\
& =\frac{\Gamma\left(\lambda_{1}\right) \Gamma\left(\lambda_{2}\right) \Gamma\left(\lambda_{1}^{\prime}\right) \Gamma\left(\lambda_{2}^{\prime}\right)}{\Gamma\left(\lambda_{1}+\lambda_{2}\right) \Gamma\left(\lambda_{1}^{\prime}+\lambda_{2}^{\prime}\right)} \sum_{m, n=0}^{\infty} \frac{\left(\lambda_{1}\right)_{m}\left(\lambda_{1}^{\prime}\right)_{n}(-\mu)_{m+n}}{\left(\lambda_{1}+\lambda_{2}\right)_{m}\left(\lambda_{1}^{\prime}+\lambda_{2}^{\prime}\right)_{n} m ! n !} x^{m} y^{n}
\end{aligned}
$$

is a solution of the equation $T_{2} u=0$ that $F_{2}\left(\alpha ; \beta, \beta^{\prime} ; \gamma, \gamma^{\prime} ; x, y\right)$ satisfies.

Note that the operator $\tilde{T}_{3}$ transformed from $T_{3}^{\prime}$ by the coordinate transformation $(x, y) \mapsto\left(\frac{1}{x}, \frac{1}{y}\right)$ equals

$$
\begin{aligned}
\tilde{T}_{3} & =\left(-\vartheta_{x}+\alpha\right)\left(-\vartheta_{x}+\beta\right)-x\left(-\vartheta_{x}\right)\left(-\vartheta_{x}-\vartheta_{y}+\gamma-1\right) \\
& =\left(\vartheta_{x}-\alpha\right)\left(\vartheta_{x}-\beta\right)-x \vartheta_{x}\left(\vartheta_{x}+\vartheta_{y}-\gamma+1\right)
\end{aligned}
$$

and the operator

$$
\operatorname{Ad}\left(x^{-\alpha} y^{-\alpha^{\prime}}\right) \tilde{T}_{3}=\vartheta_{x}\left(\vartheta_{x}+\alpha-\beta\right)-x\left(\vartheta_{x}+\alpha\right)\left(\vartheta_{x}+\vartheta_{y}+\alpha+\alpha^{\prime}-\gamma+1\right)
$$

together with the operator obtained by the transpositions $x \leftrightarrow y, \alpha \leftrightarrow \alpha^{\prime}$ and $\beta \leftrightarrow \beta^{\prime}$ defines the system of the equations satisfied by the functions

$$
\left\{\begin{array}{l}
F_{2}\left(\alpha+\alpha^{\prime}-\gamma+1 ; \alpha, \alpha^{\prime} ; \alpha-\beta+1, \alpha^{\prime}-\beta^{\prime}+1 ; x, y\right), \\
x^{-\alpha^{\prime}} y^{-\alpha^{\prime}} F_{3}\left(\alpha, \alpha^{\prime} ; \beta, \beta^{\prime} ; \gamma ; \frac{1}{x}, \frac{1}{y}\right),
\end{array}\right.
$$

which also follows from the integral representation (15.69) with the transformation $(x, y, s, t) \mapsto\left(\frac{1}{x}, \frac{1}{y}, \frac{1}{s}, \frac{1}{t}\right)$.

15.11. Okubo and Risa/Asir. Most of our results in this paper are constructible and they can be explicitly calculated and implemented in computer programs.

The computer program okubo [O8] written by the author handles combinatorial calculations in this paper related to tuples of partitions. It generates basic tuples (cf. s15.1) and rigid tuples (cf. \$15.2), calculates the reductions originated by Katz and Yokoyama, the position of accessory parameters in the universal operator (cf. Theorem 8.13 iv)) and direct decompositions etc.

The author presented Theorem 14.6 in the case when $p=3$ as a conjecture in the fall of 2007, which was proved in May in 2008 by a completely different way from the proof given in $\$ 14.1$, which is a generalization of the original proof 
of Gauss's summation formula of the hypergeometric series explained in 14.3 . The original proof of Theorem 14.6 in the case when $p=3$ was reduced to the combinatorial equality (14.16). The author verified (14.16) by okubo and got the concrete connection coefficients for the rigid tuples $\mathbf{m}$ satisfying ord $\mathbf{m} \leq 40$. Under these conditions ( $\operatorname{ord} \mathbf{m} \leq 40, p=3, m_{0, n_{0}}=m_{1, n_{1}}=1$ ) there are 4,111,704 independent connection coefficients modulo obvious symmetries and it took about one day to got all of them by a personal computer with okubo.

Several operations on differential operators such as additions and middle convolutions defined in $\$ 2$ can be calculated by a computer algebra and the author wrote a program for their results under Risa/Asir, which gives a reduction procedure of the operators (cf. Definition 7.11), integral representations and series expansions of the solutions (cf. Theorem 10.1), connection formulas (cf. Theorem 14.5), differential operators (cf. Theorem 8.13 iv)), the condition of their reducibility (cf. Corollary $12.12 \mathrm{i})$ ), recurrence relations (cf. Theorem 13.3 ii)) etc. for any given spectral type or Riemann scheme (1.10) and displays the results using $\mathrm{T}_{\mathrm{E} X}$. This program for Risa/Asir written by the author contains many useful functions calculating rational functions, Weyl algebra and matrices. These programs can be obtained from

http://www.math.kobe-u.ac.jp/Asir/asir.html

ftp://akagi.ms.u-tokyo.ac.jp/pub/math/muldif

ftp://akagi.ms.u-tokyo.ac.jp/pub/math/okubo.

\section{FuRThER PROBLEMS}

16.1. Multiplicities of spectral parameters. Suppose a Fuchsian differential equation and its middle convolution are given. Then we can analyze the corresponding transformation of a global structure of its local solution associated with an eigenvalue of the monodromy generator at a singular point if the eigenvalue is free of multiplicity.

When the multiplicity of the eigenvalue is larger than one, we have not a satisfactory result for the transformation (cf. Theorem 14.5). The value of a generalized connection coefficient defined by Definition 14.17 may be interesting. Is the procedure in Remark 14.19 always valid? In particular, is there a general result assuring Remark 14.19 (1) (cf. Remark 14.23)? Are the multiplicities of zeros of the generalized connection coefficients of a rigid Fuchsian differential equation free?

16.2. Schlesinger canonical form. Can we define a natural universal Fuchsian system of Schlesinger canonical form (2.74) with a given realizable spectral type? Here we recall Example 11.2 .

Let $P_{\mathbf{m}}$ be the universal operator in Theorem 8.13 . Is there a natural system of Schlesinger canonical form which is isomorphic to the equation $P_{\mathbf{m}} u=0$ together with the explicit correspondence between them?

16.3. Apparent singularities. Katz [K] proved that any irreducible rigid local system is constructed from the trivial system by successive applications of middle convolutions and additions and it is proved in this paper that the system is realized by a single differential equation without an apparent singularity.

In general, an irreducible local system cannot be realized by a single differential equation without an apparent singularity but it is realized by that with apparent singularities. Hence it is expected that there exist some natural operations of single differential equations with apparent singularities which correspond to middle convolutions of local systems or systems of Schlesinger canonical form.

The Fuchsian ordinary differential equation satisfied by an important special function often hasn't an apparent singularity even if the spectral type of the equation is not rigid. Can we understand the condition that a $W(x)$-module has a 
generator so that it satisfies a differential equation without an apparent singularity? Moreover it may be interesting to study the existing of contiguous relations among differential equations with fundamental spectral types which have no apparent singularity.

16.4. Irregular singularities. Our fractional operations defined in 8 目 give transformations of ordinary differential operators with polynomial coefficients, which have irregular singularities in general. The reduction of ordinary differential equations under these operations is a problem to be studied. Note that versal additions and middle convolutions construct such differential operators from the trivial equation.

A similar result as in this paper is obtained for certain classes of ordinary differential equations with irregular singularities (cf. [Hi]).

A "versal" path of integral in an integral representation of the solution and a "versal" connection coefficient and Stokes multiplier should be studied. Here "versal" means a natural expression corresponding to the versal addition.

We define a complete model with a given spectral type as follows. For simplicity we consider differential operators without singularities at the origin. For a realizable irreducible tuple of partitions $\mathbf{m}=\left(m_{j, \nu}\right)_{\substack{0 \leq j \leq p \\ 1 \leq \nu \leq n_{j}}}$ of a positive integer $n$ Theorem 8.13 constructs the universal differential operator

$$
P_{\mathbf{m}}=\prod_{j=1}^{p}\left(1-c_{j} x\right)^{n} \cdot \frac{d^{n}}{d x^{n}}+\sum_{k=0}^{n-1} a_{k}(x, c, \lambda, g) \frac{d^{k}}{d x^{k}}
$$

with the Riemann scheme

$$
\left\{\begin{array}{cccc}
x=\infty & \frac{1}{c_{1}} & \cdots & \frac{1}{c_{p}} \\
{\left[\lambda_{0,1}\right]_{\left(m_{0,1}\right)}} & {\left[\lambda_{1,1}\right]_{\left(m_{1,1}\right)}} & \cdots & {\left[\lambda_{p, 1}\right]_{\left(m_{p, 1}\right)}} \\
\vdots & \vdots & \vdots & \vdots \\
{\left[\lambda_{0, n_{0}}\right]_{\left(m_{0, n_{0}}\right)}} & {\left[\lambda_{1, n_{1}}\right]_{\left(m_{1, n_{1}}\right)}} & \cdots & {\left[\lambda_{p, n_{p}}\right]_{\left(m_{p, n_{p}}\right)}}
\end{array}\right\}
$$

and the Fuchs relation

$$
\sum_{j=0}^{p} \sum_{\nu=1}^{n_{j}} m_{j, \nu} \lambda_{j, \nu}=n-\frac{\operatorname{idx} \mathbf{m}}{2}
$$

Here $c=\left(c_{0}, \ldots, c_{p}\right), \lambda=\left(\lambda_{j, \nu}\right)$ and $g=\left(g_{1}, \ldots, g_{N}\right)$ are parameters. We have $c_{i} c_{j}\left(c_{i}-c_{j}\right) \neq 0$ for $0 \leq i<j \leq p$. The parameters $g_{j}$ are called accessory parameters and we have idx $\mathbf{m}=2-2 N$. We call the Zariski closure $\bar{P}_{\mathbf{m}}$ of $P_{\mathbf{m}}$ in $W[x]$ the complete model of differential operators with the spectral type $\mathbf{m}$, whose dimension equals $p+\sum_{j=0}^{p} n_{j}+N-1$. It is an interesting problem to analyze the complete model $\bar{P}_{\mathbf{m}}$.

When $\mathbf{m}=11,11,11$, the complete model equals

$$
\left(1-c_{1} x\right)^{2}\left(1-c_{2} x\right)^{2} \frac{d^{2}}{d x^{2}}-\left(1-c_{1} x\right)\left(1-c_{2} x\right)\left(a_{1,1} x+a_{1,0}\right) \frac{d}{d x}+a_{0,2} x^{2}+a_{0,1} x+a_{0,0},
$$

whose dimension equals 7. Any differential equation defined by the operator belonging to this complete model is transformed into a Gauss hypergeometric equation, a Kummer equation, an Hermite equation or an airy equation by a suitable gauge transformation and a coordinate transformation. A good understanding together with a certain completion of our operators is required even in this fundamental example. It is needless to say that the good understanding is important in the case when $\mathbf{m}$ is fundamental. 
16.5. Special parameters. Let $P_{\mathbf{m}}$ be the universal operator of the form (16.1) for an irreducible tuple of partition $\mathbf{m}$. When a decomposition $\mathbf{m}=\mathbf{m}^{\prime}+\mathbf{m}^{\prime \prime}$ with realizable tuples of partitions $\mathbf{m}^{\prime}$ and $\mathbf{m}^{\prime \prime}$ is given, Theorem 6.19 gives the values of the parameters of $P_{\mathbf{m}}$ corresponding to the product $P_{\mathbf{m}^{\prime}} P_{\mathbf{m}^{\prime \prime}}$. A $W(x, \xi)$-automorphism of $P_{\mathbf{m}} u=0$ gives a transformation of the parameters $(\lambda, g)$, which is a contiguous relation and called Schlesinger transformation in the case of systems of Schlesinger canonical form. How can we describe the values of the parameters obtained in this way and characterize their position in all the values of the parameters when the universal operator is reducible? In general, they are not all even in a rigid differential equation. A direct decomposition $32,32,32,32=12,12,12,12 \oplus 2(10,10,10,10)$ of a rigid tuples $32,32,32,32$ gives this example (cf. (12.65)).

Analyse the reducible differential equation with an irreducibly realizable spectral type. This is interesting even when $\mathbf{m}$ is a rigid tuple. For example, describe the monodromy of its solutions.

Describe the characteristic exponents of the generalized Riemann scheme with an irreducibly realizable spectral type such that there exists a differential operator with the Riemann scheme which is outside the universal operator (cf. Example 7.5 and Remark 8.15). In particular, when the spectral type is not fundamental nor simply reducible, does there exist such a differential operator?

The classification of rigid and simply reducible spectral types coincides with that of indecomposable objects described in [MWZ, Theorem 2.4]. Is there some meaning in this coincidence?

Has the condition (8.28) a similar meaning in the case of Schlesinger canonical form? What is the condition on the local system or a (single) Fuchsian differential equation which has a realization of a system of Schlesinger canonical form?

Give the condition so that the monodromy group is finite. Give the condition so that the centralizer of the monodromy is the set of scalar multiplications.

Suppose $\mathbf{m}$ is fundamental. Study the condition so that the connection coefficients is a quotient of the products of gamma functions as in Theorem 14.6 or the solution has an integral representation only by using elementary functions.

16.6. Shift operators. Calculate the polynomial function $c_{\mathbf{m}}(\epsilon ; \lambda)$ of $\lambda$ defined in Theorem 13.8. Is it square free? See Conjecture 13.12.

Is the shift operator $R_{\mathbf{m}}(\epsilon, \lambda)$ Fuchsian?

Study the shift operators given in Theorem 13.7.

Study the condition on the characteristic exponents and accessory parameters assuring the existence of a shift operator for a Fuchsian differential operator with a fundamental spectral type.

Study the shift operator or Schlesinger transformation of a system of Schlesinger canonical form with a fundamental spectral type. When is it not defined or when is it not bijective?

16.7. Several variables. We have analyzed Appell hypergeometric equations in $\$ 15.10$. What should be the geometric structure of singularities of more general system of equations when it has a good theory?

Describe or define operations of differential operators that are fundamental to analyze good systems of differential equations.

A series expansion of a local solution of a rigid ordinal differential equation indicates that it may be natural to think that the solution is a restriction of a solution of a system of differential equations with several variables (cf. Theorem 10.1 and 15.3 15.4). Study the system.

\subsection{Other problems.}


- For a rigid decomposition $\mathbf{m}=\mathbf{m}^{\prime} \oplus \mathbf{m}^{\prime \prime}$, can we determine whether $\alpha_{\mathbf{m}^{\prime}} \in$ $\Delta(\mathbf{m})$ or $\alpha_{\mathbf{m}^{\prime \prime}} \in \Delta(\mathbf{m})$ (cf. Proposition 9.9 iv))?

- Are there analyzable series $\mathcal{L}$ of rigid tuples of partitions different from the series given in $\$ 15.9$ ? Namely, $\mathcal{L} \subset \mathcal{P}$, the elements of $\mathcal{L}$ are rigid, the number of isomorphic classes of $\mathcal{L} \cap \mathcal{P}^{(n)}$ are bounded for $n \in \mathbb{Z}_{>0}$ and the following condition is valid.

Let $\mathbf{m}=k \mathbf{m}^{\prime}+\mathbf{m}^{\prime \prime}$ with $k \in \mathbb{Z}_{>0}$ and rigid tuples of partitions $\mathbf{m}, \mathbf{m}^{\prime}$ and $\mathbf{m}^{\prime \prime}$. If $\mathbf{m} \in \mathcal{L}$, then $\mathbf{m}^{\prime} \in \mathcal{L}$ and $\mathbf{m}^{\prime \prime} \in \mathcal{L}$. Moreover for any $\mathbf{m}^{\prime \prime} \in \mathcal{L}$, this decomposition $\mathbf{m}=k \mathbf{m}^{\prime}+\mathbf{m}^{\prime \prime}$ exists with $\mathbf{m} \in \mathcal{L}, \mathbf{m}^{\prime} \in \mathcal{L}$ and $k \in \mathbb{Z}_{>0}$. Furthermore $\mathcal{L}$ is indecomposable. Namely, if $\mathcal{L}=\mathcal{L}^{\prime} \cup \mathcal{L}^{\prime \prime}$ so that $\mathcal{L}^{\prime}$ and $\mathcal{L}^{\prime \prime}$ satisfy these conditions, then $\mathcal{L}^{\prime}=\mathcal{L}$ or $\mathcal{L}^{\prime \prime}=\mathcal{L}$.

- Characterize the ring of automorphisms and that of endomorphisms of the localized Weyl algebra $W(x)$.

- In general, different procedures of the reduction of the universal operator $P_{\mathbf{m}} u=0$ give different integral representations and series expansions of its solution (cf. Example 10.2, Remark 10.3 and the last part of $\$ 15.3$ ). Analyze the difference.

\section{ApPEndiX}

In this section we give a theorem which is proved by K. Nuida. The author greatly thanks to K. Nuida for allowing the author to put the theorem with its proof in this section.

Let $(W, S)$ be a Coxeter system. Namely, $W$ is a group with the set $S$ of generators and under the notation $S=\left\{s_{i} ; i \in I\right\}$, the fundamental relations among the generators are

(17.1) $s_{k}^{2}=\left(s_{i} s_{j}\right)^{m_{i, j}}=e$ and $m_{i, j}=m_{j, i}$ for $\forall i, j, k \in I$ satisfying $i \neq j$.

Here $m_{i, j} \in\{2,3,4, \ldots\} \cup\{\infty\}$ and the condition $m_{i, j}=\infty$ means $\left(s_{i} s_{j}\right)^{m} \neq e$ for any $m \in \mathbb{Z}_{>0}$. Let $E$ be a real vector space with the basis set $\Pi=\left\{\alpha_{i} ; i \in I\right\}$ and define a symmetric bilinear form ( $\mid$ ) on $E$ by

$$
\left(\alpha_{i} \mid \alpha_{i}\right)=2 \text { and }\left(\alpha_{i} \mid \alpha_{j}\right)=-2 \cos \frac{\pi}{m_{i, j}} .
$$

Then the Coxeter group $W$ is naturally identified with the reflection group generated by the reflections $s_{\alpha_{i}}$ with respect to $\alpha_{i}(i \in I)$. The set $\Delta_{\Pi}$ of the roots of $(W, S)$ equals $W \Pi$, which is a disjoint union of the set of positive roots $\Delta_{\Pi}^{+}:=\Delta_{\Pi} \cap \sum_{\alpha \in \Pi} \mathbb{Z}_{\geq 0} \alpha$ and the set of negative roots $\Delta_{\Pi}^{-}:=-\Delta_{\Pi}^{+}$. For $w \in W$ the length $L(w)$ is the minimal number $k$ with the expression $w=s_{i_{1}} s_{i_{2}} \cdots s_{i_{k}}$ $\left(i_{1}, \ldots, i_{k} \in I\right)$. Defining $\Delta_{\Pi}(w):=\Delta_{\Pi}^{+} \cap w^{-1} \Delta_{\Pi}^{-}$, we have $L(w)=\# \Delta_{\Pi}(w)$.

Fix $\beta$ and $\beta^{\prime} \in \Delta_{\Pi}$ and put

$$
W_{\beta^{\prime}}^{\beta}:=\left\{w \in W ; \beta^{\prime}=w \beta\right\} \text { and } W^{\beta}:=W_{\beta}^{\beta} .
$$

Theorem 17.1 (K. Nuida). Retain the notation above. Suppose $W_{\beta^{\prime}}^{\beta} \neq \emptyset$ and there exist no sequence $s_{i_{1}}, s_{i_{2}}, \ldots s_{i_{k}}$ of elements of $S$ such that

$$
\left\{\begin{array}{l}
k \geq 3, \\
s_{i_{\nu}} \neq s_{i_{\nu}^{\prime}} \quad\left(1 \leq \nu<\nu^{\prime} \leq k\right), \\
m_{i_{\nu}, i_{\nu+1}} \text { and } m_{i_{1}, i_{k}} \text { are odd integers } \quad(1 \leq \nu<k) .
\end{array}\right.
$$

Then an element $w \in W_{\beta^{\prime}}^{\beta}$ is uniquely determined by the condition

$$
L(w) \leq L(v) \quad\left(\forall v \in W_{\beta^{\prime}}^{\beta}\right) .
$$

Proof. Put $\Delta_{\Pi}^{\beta}:=\left\{\gamma \in \Delta_{\Pi}^{+} ;(\beta \mid \gamma)=0\right\}$. First note that the following lemma. 
Lemma 17.2. If $w \in W_{\beta^{\prime}}^{\beta}$ satisfies 17.5), then $w \Delta_{\Pi}^{\beta} \subset \Delta_{\Pi}^{+}$.

In fact, if $w \in W_{\beta^{\prime}}^{\beta}$ satisfies (17.5) and there exists $\gamma \in \Delta_{\Pi}^{\beta}$ satisfying $w \gamma \in$ $\Delta_{\Pi}^{-}$, then there exists $j$ for a minimal expression $w=s_{i_{1}} \cdots s_{i_{L_{\Pi}(w)}}$ such that $s_{i_{j+1}} \cdots s_{j_{L_{\Pi}(w)}} \gamma=\alpha_{i_{j}}$, which implies $W_{\beta^{\prime}}^{\beta} \ni v:=w s_{\gamma}=s_{i_{1}} \cdots s_{i_{j-1}} s_{i_{j+1}} \cdots s_{i_{L_{\Pi}(w)}}$ and contradicts to (17.5).

It follows from $[\mathrm{Br}]$ that the assumption (17.4) implies that $W^{\beta}$ is generated by $\left\{s_{\gamma} ; \gamma \in \Delta_{\Pi}^{\beta}\right\}$. Putting $\Pi^{\beta}=\Delta_{\Pi}^{\beta} \backslash\left\{r_{1} \gamma_{1}+r_{2} \gamma_{2} \in \Delta_{\Pi}^{\beta} ; \gamma_{2} \notin \mathbb{R} \gamma_{1}, \gamma_{j} \in \Delta_{\Pi}^{\beta}\right.$ and $r_{j}>$ 0 for $j=1,2\}$ and $S^{\beta}=\left\{s_{\gamma} ; \gamma \in \Pi^{\beta}\right\}$, the pair $\left(W^{\beta}, S^{\beta}\right)$ is a Coxeter system and moreover the minimal length of the expression of $w \in W^{\beta}$ by the product of the elements of $S^{\beta}$ equals $\#\left(\Delta_{\Pi}^{\beta} \cap w^{-1} \Delta_{\Pi}^{-}\right)$(cf. [Nu, Theorem 2.3]).

Suppose there exist two elements $w_{1}$ and $w_{2} \in W_{\beta^{\prime}}^{\beta}$ satisfying $L\left(w_{j}\right) \leq L(v)$ for any $v \in W_{\beta^{\prime}}^{\beta}$ and $j=1$, 2. Since $e \neq w_{1}^{-1} w_{2} \in W^{\beta}$, there exists $\gamma \in \Delta_{\Pi}^{\beta}$ such that $w_{1}^{-1} w_{2} \gamma \in \Delta_{\Pi}^{-}$. Since $-w_{1}^{-1} w_{2} \gamma \in \Delta_{\Pi}^{\beta}$, Lemma 17.2 assures $-w_{2} \gamma=$ $w_{1}\left(-w_{1}^{-1} w_{2} \gamma\right) \in \Delta_{\Pi}^{+}$, which contradicts to Lemma 17.2 .

The above proof shows the following corollary.

Corollary 17.3. Retain the assumption in Theorem 17.1. For an element $w \in$ $W_{\beta^{\prime}}^{\beta}$, the condition 17.5$)$ is equivalent to $w \Delta_{\Pi}^{\beta} \subset \Delta_{\Pi}^{+}$.

Let $w \in W_{\beta^{\prime}}^{\beta}$ satisfying (17.5). Then

$$
W_{\beta^{\prime}}^{\beta}=w\left\langle s_{\gamma} ;(\gamma \mid \beta)=0, \gamma \in \Delta_{\Pi}^{+}\right\rangle .
$$

\section{REFERENCES}

[Ao] Aomoto K., A personal communication, 2011.

[AK] Appell K. and J. Kampé de Fériet, Fonctions hypergéométriques et hypersphériques polynomes d'Hermite, Gauthier-Villars, 1926.

[BH] Beukers F. and G. Heckman, Monodromy for the hypergeometric function ${ }_{n} F_{n-1}$, Invent. Math. 95 (1989), 325-354.

[Br] Brink B,, On centralizers of reflections in Coxeter groups, Bull. London Math. Soc. 28 (1996) 465-470

[CB] Crawley-Boevey, W., On matrices in prescribed conjugacy classes with no common invariant subspaces and sum zero, Duke Math. J. 118 (2003), 339-352.

[DR] Dettweiler, M. and S. Reiter, An algorithm of Katz and its applications to the inverse Galois problems, J. Symbolic Comput. 30 (2000), 761-798.

[DR2] Middle convolution of Fuchsian systems and the construction of rigid differential systems, J. Algebra 318 (2007), 1-24.

[Dix] Dixmier, J., Sur les algèbres de Weyl, Bull. Soc. Math. France 96 (1968), 209-242.

[EMO] Erdelyi, A., W. Magnus, F. Oberhettinger and F. G. Tricomi, Higher Transcendental Functions, 3 volumes, McGraw-Hill Book Co., New York, 1953.

[Ge] Gelfand, I. M., General theory of hypergeometric functions, Soviet Math. Dokl. 33 (1986), $573-577$.

[Gl] Gleizer, O., Some explicit solutions of additive Deligne-Simpson problem and their applications, Adv. Math. 178 (2003), 311-374.

[Ha] Haraoka, Y., Integral representations of solutions of differential equations free from accessory parameters, Adv. Math. 169 (2002), 187-240.

[HF] Haraoka, Y. and G. M. Filipuk, Middle convolution and deformation for Fuchsian systems, J. Lond. Math. Soc. 76 (2007), 438-450.

[HY] Haraoka, Y. and T. Yokoyama, Construction of rigid local systems and integral representations of their sections, Math. Nachr. 279 (2006), 255-271.

[HO] Heckman, G. J. and E. M. Opdam, Root systems and hypergeometric functions I, Comp. Math. 64 (1987), 329-352.

[Hi] Hiroe, K., Twisted Euler transform of differential equations with an irregular singular point, preprint.

[Kc] Kac, V. C., Infinite dimensional Lie algebras, Third Edition, Cambridge Univ. Press 1990.

[Kz] Katz, N. M., Rigid Local Systems, Annals of Mathematics Studies 139, Princeton University Press 1995. 
[Kh] Kohno, M., Global analysis in linear differential equations, Kluwer Academic Publishers, 1999.

[Ko] Kostov, V. P., On the Deligne-Simpson problem, Proc. Steklov Inst. Math. 238 (2002), $148-185$.

[Ko2] - On some aspects of the Deligne-Simpson problem, J. Dynam. Control Systems 9 (2003), 303-436.

[Ko3] _ The Deligne-Simpson problem for zero index of rigidity, Perspective in Complex Analysis, Differential Geometry and Mathematical Physics, World Scientific 2001, 1-35.

[Ko4] - The Deligne-Simpson problem — a survey, J. Algebra 281 (2004), 83-108.

[Le] Levelt, A. H. M., Hypergeometric functions III, Indag. Math. 23 (1961), 386-403.

[MWZ] Magyar, P., J. Weyman and A. Zelevinski, Multiple flag variety of finite type, Adv. in Math. 141 (1999), 97-118.

$[\mathrm{Nu}]$ Nuida, K., On centralizers of parabolic subgroups in Coxeter groups, to appear in Journal of Group Theory.

[Oc] Ochiai, H., A personal communication, 2008.

[OTY] Okubo, K., K. Takano and S. Yoshida, A connection problem for the generalized hypergeometric equations, Funkcial. Ekvac. 31 (1988), 483-495.

[O1] Oshima, T., A definition of boundary values of solutions of partial differential equations with regular singularities, Publ. RIMS Kyoto Univ. 19 (1983), 1203-1230.

[O2] A quantization of conjugacy classes of matrices, Advances in Math. 196 (2005), 124-146.

[O3] Annihilators of generalized Verma modules of the scalar type for classical Lie algebras, 'Harmonic Analysis, Group Representations, Automorphic forms and Invariant Theory", in honor of Roger Howe, Vol. 12, Lecture Notes Series, National University of Singapore, 2007, 277-319.

[O4] Commuting differential operators with regular singularities, Algebraic Analysis of Differential Equations, Springer-Verlag, Tokyo, 2007, 195-224.

[O5] - Heckman-Opdam hypergeometric functions and their specializations, Harmonische Analysis und Darstellungstheorie Topologischer Gruppen, Mathematisches Forschungsinstitut Oberwolfach, Report 49 (2007), 38-40.

[O6] Classification of Fuchsian systems and their connection problem, preprint, arXiv:0811.2916, 2008, 29pp, to appear in RIMS Kôkyûroku Bessatsu.

[O7] Katz's middle convolution and Yokoyama's extending operation, preprint, arXiv:0812.1135, 2008, 18pp.

[O8] , Okubo, a computer program for Katz/Yokoyama/Oshima algorithms, ftp://akagi.ms.u-tokyo.ac.jp/pub/math/okubo/okubo.zip, 2007-8.

[OS] Oshima T. and N. Shimeno, Heckman-Opdam hypergeometric functions and their specializations, RIMS Kôkyûroku Bessatsu B20 (2010), 129-162.

[Ro] Roberts, D. P., Rigid Jordan Tuples, preprint, http://cda.morris.umn.edu/ roberts /research/rjt.ps.

[Sc] Scott, L. L., Matrices and cohomology, Ann. Math. 105(1977), 473-492.

[Si] Simpson, C. T., Products of Matrices, Canadian Math. Soc. Conference Proceedings 12, AMS, Providence RI (1991), 157-185.

[SV] Strambach, K. and Völkein H., On linearly rigid tuples, J. reine angrew. Math. 510 (1999), $57-62$.

[Sz] Szaboó S., Deformations of Fuchsian equations and logarithmic connections, arXiv:math/0703230v3, 2008, preprint.

[SW] Slavyanov S. Yu. and Lay W., Special Functions, A Unified Theory Based on Singularities, Oxford Univ. Press, Oxford New York, 2000.

[Wa] Watson, G. N., A Treatise on Bessel Functions, 2nd edition, Cambridge Univ. Press,. London, 1948.

[WW] Whittaker, E. T. and G. N. Watson, A Course of Modern Analysis, 4th edition, Cambridge University Press, London, 1955.

[Yo] Yokoyama, T., On an irreducibility condition for hypergeometric systems, Funkcialaj Ekvacioj 38 (1995), 11-19.

[Yo2] Construction of systems of differential equations of Okubo normal form with rigid monodromy, Math, Nachr. 279 (2006), 327-348.

[Yo3] , Recursive calculation of connection formulas for systems of differential equations of Okubo normal form, preprint, 2009

[Yos] Yoshida M., Construction of a Moduli Space of Gauss Hypergeometric Differential Equations, Funkcial. Ekvac. 24 (1981), 1-10. 